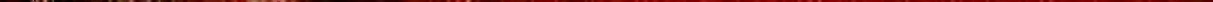




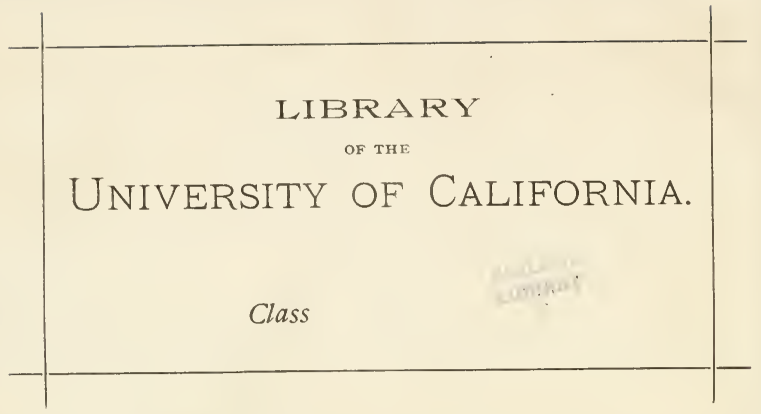





Digitized by the Internet Archive in 2008 with funding from Microsoft Corporation 


\title{
TEXT BOOK
}

OF

\section{COMPARATIVE}

\section{GENERAL PATHOLOGY}

FOR

Practitioners and Students of Veterinary Medicine

\author{
BY \\ PROFESSOR DR. TH. KITT \\ OF MUNICH \\ Authorized Translation \\ BY \\ DR. WILLIAM W. CADBURY \\ Asslstant Demonstrator of Pathology in the University of Pennsylvanla
}

Edited with Notes and Additional Illustrations

BY

DR. ALLEN J. SMITH

Professor of Pathology in the University of Pennsylvania

Illustrated with Four Colored Plates and One Hundred and Thirty-one Text lllustrations

CHICAGO

W. T. KEENER \& CO.

I 906 


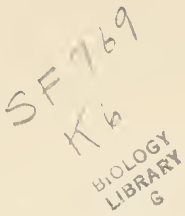

\section{CTMERAL'}

Copyrighted, I906

By W. T. Keener 


\section{PREFACE TO GERMAN EDITION}

The introduction of students of veterinary medicine to the study of pathology, because of the lack up to the present time of a German text book of general pathology adapted to diseases of animals, has depended chiefly upon works written for the practitioner of human medicine; the lectures of instructors in veterinary medicine and students' notes, made with more or less accuracy, supplying the necessary additions and explanations.

Only one work, the Text Book of General Pathological Anatomy by Birch-Hirschfeld, has been amended from the standpoint of our special branch of medicine, by the addition of veterinarymedical paragraphs from the facile pen of Johne, so as to seem adapted for students in veterinary schools; but it is confined to pathological anatomy, and the physiological and rtiological features, the manifestation of functional impairments, pathogenesis, etc., require further reference to special works.

As an introduction aud foundation for appreciation of the practice of veterinary medicine, to be studied, and put into application in the latter portions of the college curriculum, after completion of the courses in anatomy, physiology and the natural sciences, the student should be given a general idea of the meaning of disease, the rtiology of diseases, of the make-up of our medical knowledge and of the principles of classification, as well as a general familiarity with the alterations in structure and function met in disease. It is for this reason that lectures on general pathology are provided, introductory to the special applied branches of study. The need of a work concisely comprehending such features has determined me to undertake the task of preparing a condensed outline of the fundamental facts of pathology with special adlaptations to the requirements of veterinarians. Of course, it has been necessary to make use of much material from works intended for the student of human medicine, and I am vividly reminded of the old sentence in the Latin grammar-"Plinits 
nullum librum legit, ex quo non excerpserit." I therefore acknowledge at the ontset and at various places in the text, that I have freely employed in the preparation of the following textbook the works of Ribbert, Perls, Krehl, Durk, Samuel, Thoma, Birsch-Hirschfeld, Johne, Uhle and Wagner, as it is in fact practically impossible to write an authoritative work on general pathology without dependence upon other authors.

The scientific development of veterinary medicine depends primarily upon the same principles which obtain in human medicine. The propositions and methods of research which were originally employed in the study of human pathology, are for us, too, fundamentals for the appreciation and investigation of the nature of the animal diseases. Every new advance in human medicine in the fields of anatomy, physiology, the use of the microscope, technique of clinical study, surgical and therapeutic methods, bacteriological and practical hygiene, has been of advantage to comparative medicine, and, mutatis mutandis, has found an application in veterinary practice, and has pointed out the way for advanced work in our own branch. There has been much assistance afforded, too, in the fact that many of the discoveries of value to human medicine have been made from experimental studies upon animals, and the principles of general pathology particularly have been fundamentally proven by comparative study of the anatomy and physiology of animals; both branches of medicine, therefore, drawing from the same sources and having a common field of work.

Apart from these considerations, however, the representatives of veterinary medicine have, by their independent achievements, built up the pillars and walls of their own scientific temple, with such multiplicity of specialized purposes and requirements that the method and practice of veterinary medical instruction have come to have a peculiar character of their own. Consideration of these requirements is attempted in this volume.

It is well known to every teacher who is required to act as an examiner, how difficult it often is for the candidates in an examination to express what they well know and how, no matter how clearly the questions may be presented, or what statements are made suggesting a proper answer, this or that really capable student finds himself forced to struggle with words and is handicapped in expressing his ideas. In order to lessen these difficulties for students, I have endeavored to frame my definitions 
concisely, to present the positive facts in as clear and as brief a manner as possible, and to limit to what is absolutely essential the incomplete, uncertain and controversial points of study, the elaborate discussion of which is usually confusing and tends to weary the student. Of course, important objections and questioned points in theories have been noticed, lest the student be led to mere memorization and superficial study, and in order that he may be stimulated to think. I believe that the work will be found useful to the practising veterinarian as well, as a general presentation of the most recent position of the science.

I have omitted description of the individual types of animal parasites and bacteria and taken up only in this connection their bearings upon general pathology. The scope of the work would otherwise have been too large; and full details upon these subjects may be found in the part devoted to special pathology (Lehrbuch der pathol. Anatomie der Haustiere. II. Aufl. I90I. F. Enke, Stuttgart) and in my Lehrbuch der Baktericnkunde und pathol. Mikroskopic (IV. Aufl. M. Perles, Wien, 1903). The subject of malformations is also treated of at the beginning of the special section just mentioned, and a repetition is therefore superfluous.

The publisher has presented the volume in an excellence of style which places me under special obligation to him.

Numerous new illustrations are included, prepared by the artistic hand of K. Dirr; some of the cuts I have borrowed from the works of authors cited in the text. For a number of photographs I must thank for their kindness the veterinary physicians Dr. Jakob and Dr. W. Ernst, the latter of whom, a skillful microscopist and bacteriologist, has been engaged for a number of years in my institution and has aided me in a most valuable manner in carrying on investigations bearing upon my studies.

Munich.

TH. KITT. 



\section{PREFACE TO AMERICAN EDITION}

The work of translating and preparing for publication the following edition of Kitt's General Pathology has been a pleasure, because of the real value of the work and because both editor and translator have believed the labor a service to the profession. As Professor Kitt states in the preface to the German edition, it is practically the only work devoted to a discussion of general pathology from the standpoint of the veterinarian; and the need of such a work has long been manifest to every teacher of pathology in charge of students of veterinary medicine. The impressions given by any book upon pathology, even the best, which has been framed primarily for use in connection with human medicine, are often unfortunate from the inaptness of the descriptions of lesions for the needs of men studying comparative medicine. It is undoubtedly true that the general processes of disease are fundamentally the same in whatever subject they occur; but the varieties of appearances of one and the same type of lesion in different species may well be sufficiently marked to make the descriptions based upon the changes met in any given animal confusing and perhaps inadequate for students, whose experience in the earlier years is anything but extensive. The differences, for example, in the appearances of a tuberculous caseated area in man, in the cow, hog, horse, or in carnivora, well illustrate the point in mind; or the differences in bulk and in other characteristics of various tumors as met in man and in the large domestic animals serve as an example. The adaptation of the present volume to the needs of students who, as Professor Kitt points out, have had to accept, as a rule, the descriptions of lesions as seen in man and then amend them by notes from lectures, has strongly appealed to us as instructors of veterinary students, and will find, we believe, an equal appreciation from others whose work has fallen in similar lines.

The book reflects well the tendency of modern pathological 
teaching to devote considerable effort to direct the thought of the reader along lines of pathological physiology, to make the student reason for himself from given anatomical data and appearances as to the necessary functional faults and failures which would follow. Pathological anatomy is, of course, fundamental and finds its place on every page; but the author has had in mind the application of pathology to the living diseased animal, and the anatomical descriptions and discussions serve as a basis for explanation of the development of the processes and the functional faults the diseased animal must necessarily manifest. The chapters on disturbances of the circulatory, respiratory and other functions are, of course, brief, but they are by no means superficial and indicate well the lines of necessary study for the practicing physician and are stimulative to thought on the part of the student.

The value of such a work is not confined to the veterinarian; the intimate relations comparative pathology bears to the study of human medicine and the need that experimentalists should be in the best possible position to appreciate the reactions peculiar to various species of experiment animals require of pathologists broader and broader powers of recognition. Nuch of the criticism against animal experimentation has its only force in that in the hands of men unfamiliar with the animals utilized, both in their normal life and in their pathological reactions, the best results are not obtained, at least not appreciated, by such workers in return for the sacrifice entailed. The best is accomplished, other things being equal, by men whose training comprehends a broad biological and physiological experience and who are familiar with the peculiarities of physiological reaction and anatomical changes to be met in the animals employed under the operation of given pathogenic influences. Such works as the present volume, introductory to the fuller Special Pathology in the companion volumes of the German edition, have therefore a proper place on the shelves of every pathologist, whether his work deals with human or with comparative problems as its major field.

In presenting the book to our English-speaking colleagues and students, the editor and translator have endeavored to follow closely Professor Kitt's language, although no attempt to be absolutely literal in the translation has been made. Doubtless the effort to translate the German idiom to its English equivalent has often failed; and we both appreciate the fact that unwittingly the 
verbiage of the translation has sometimes, in the endeavor to kecp close to the original, come to be somewhat complicated for English text. The editorial notes, which are always enclosed in special brackets ([ ]), have purposely been kept within brief limits, because of our realization of the sufficiency of the work for the introductory purposes for which it is intended by the author, and because of a desire to keep the size of the book near the limits of the original edition. We feel that for use at the hands of graduate physicians the book as it stands meets the purpose for which it was written; for student purposes, as a handbook as well as a general text, it would perhaps have been regarded as an advantage to have added descriptive matter to the tables of the vegetable and animal parasitic organisms and to have expanded the text somewhat in connection with minute anatomy, thus giving it a fuller adaptation to laboratory studies. A number of illustrations have been generously added by the publishers with this latter view in mind. These illustrations, made by the well-known artist, Louis Schmidt, include figures 63, 82, 86, 89, 9I, 97, IOI, IO2, IO3, IO4, III and I25; and it is thought that the book, with stuch additions, will find a fuller place as an aid to the student in the laboratory of pathological histology.

The publishers have not spared expense to make this work possible in the translated form, and by their interest have, we feel, placed the profession under obligations. For ourselves the venture is in no sense a financial one, and if others will find, as we hope, that our efforts have aided in pressing forward the general good for both veterinary and human medicine, our compensation will be ample.

Allen J. Smith.

William W. Cadbury.

September, 1906. 



\section{TABLE OF CONTENTS}

PAGE

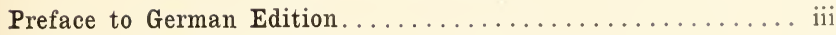

Preface to American Edition.................. vii

Introduction $\ldots \ldots \ldots \ldots \ldots \ldots \ldots \ldots \ldots \ldots \ldots \ldots \ldots \ldots \ldots \ldots$

Definition of Pathology; Conceptions of Disease; Processes of Disease.

History of Pathology ...................... Io

Disposition Toward Disease.................. I6

Predisposition and Immunity; Defensive Mechanisms of the Body.

Congenital and Inherited Disease.

Placental, Intrauterine Origin of Disease; Germinal

Tariation; Germinative Predisposition; Hereditary

Conditions.

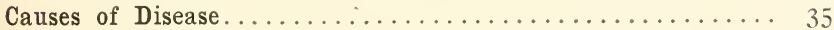

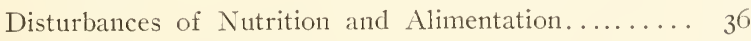

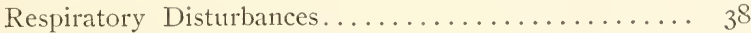

Excessive Functional Activity.............. 40

Thermic Influences.................. 4I

Chilling. ........................ 45

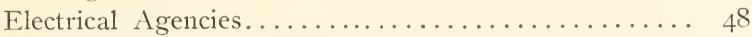

Mechanical and Traumatic Agencies........... 49

Chemical or Toxic Agents............... 50

Microbic or Infectious Agents............. 54

Animal Parasites....................... 76

Course and Termination of Disease............... 9I

Symptoms, Diagnosis, Natural and Artificial Means of Recovery, Death.

Circulatory Disturbances..................... I06

Disturbances of Cardiac Efficiency............ Io6

Local Variations in Amount of Blood in a part ( $\mathrm{Hy}$ peræmia, Anæmia).................... Iog 


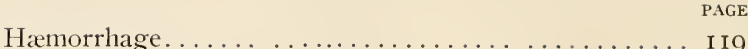

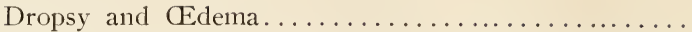

Occlusion of Blood Vessels (Thrombosis and Embolism) .

Alterations of the Blood (Plethora, the Anremias, Leukæmia) . . . . . . . . . . . . . . . . I 56

Disturbances of Metabolism, Retrograde Changes and Necrotic Processes......................... I65

Disturbances of Heat Regulation, Overheating, Fever. I65 Necrosis........................ I76

Atrophy........................... I 85

Cloudy Swelling..................... I90

The Fatty Changes..................... I92

Hyaline Degeneration. . . . . . . . . . . . . . . . I99

Mucoid Degeneration.................... 202

Colloid Degeneration.................... 204

Glycogenic Infiltration. . . . . . . . . . . . . . . . . . . 205

Amyloid Degeneration.................... 205

Pigmentation........................ 209

Calcification and Concrement Formation......... 2I6

Processes of Repair and New Formation.............. 232

Regeneration............... . . . . . . . . 232

Transplantation...................... 25 I

Hypertrophy........................... 254

Inflammation. ........................ 258

Tuberculosis ........................ 29I

Glanders........................ 3I I

Actinomycosis......................... 3 I8

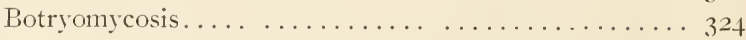

Tumors (Autoblastomata)................ 325

Fibroma (p. 3+1)-Lipoma (p. 346)-Myroma (p. 349)-Chondroma (p. 350)-Ostcoma (p. 353)Myoma (p. 356)-Nemroma, Glioma (p. 358)-Hamangioma, Lymphangioma (p. 362)-Sarcoma (p. 365) -Lymphoma (p. 372)-Mclanoma (1. 377)-Endo-. Peri-thelioma, Cholesteoma (p. 38o)-Papilloma (p. $3^{8} 3$ )-Adenoma (p. 388)-Cancers (p. 394)-Epithelial Cy'sts, Dermoid Cy'sts (p. 4 $\mathrm{I} 5)$-Adenocystoma (p. 4I8)-Odontoma (p. fIg)-Teratoma, Embryoma (p. $421)$. 
Functional Disturbances.................... 423

Nervous Disturbances; Disturbances of Motion...... 423

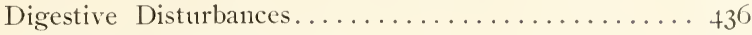

Respiratory Disturbances................... $4+8$

Disturbances of Urinary Excretion............. $45+$

Disturbances of the Thyroid Function........... 459

Disturbances of the Sexual Function............. 4 I

Disturbances of the Cutaneous Function.......... 462

Index.................................. 465 
4. 


\section{thent \\ .}

\section{INTRODUCTION}

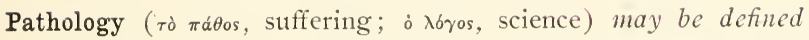
as the natural history of diseases, or as the scientific study of diseases. The terms "disease," "disordered" and "morbid" are employed to indicate the existence of disturbances of the physiological activity of the organs; that is, the presence of some deviation from the anatomical and chemical relations of the bodyconstituents which exceeds the limits of physiological variation and which calls forth such disturbances.

In ordinary life we designate as "health" a state of the body in which the vital manifestations (nutrition, metabolism, motility, sensation, psychical activity) are carried on in harmony; and it is assumed in addition that the various organs are of normal structure, and that the individual experiences a feeling of well-being. On the other hand the term "disease" carries with it a conception opposed to this so-called health, that of an irregularity of function and of impaiment of efficiency of the organs and systems of organs, accompanied as a rule by a feeling of discomfort.

The distinctions between these two conceptions merge, however, and are lost when we consider the infinite gradations between health and disease, and as we are required to employ the terms, either empirically or scientifically, in a narrower or broader sense. There might exist, for example, an organ of distinctly faulty structure in an individual without catsing any appreciable disturbance of the general health, as a malformed kidney, or a curvature of the spine. One of two paired organs, as a lobe of the thyroid or one of the kidneys, may be anatomically so altered and incapable of function as to merit being considered diseased; nevertheless the individual may feel well and live many years. Noreover in healthy bodies there may now and again be evinced deviations from the normal vital manifestations which may simulate morbid disturbances, but which are not felt or regarded as such because of their brief duration, because they are soon corrected by the protective and regulating mechanism of the body, or are at most expressions of such 
adjustments; for example, after marching there may be a brief elevation of body temperature even to fever heat. In considering a body corpulent from fat deposit, one may be at a loss to indicate whether the corpulence is but a normal condition, or whether a really morbid state of obesity has commenced; and, at least in the popular mind, intoxication from alcohol is certainly not to be considered as a disease although in the stricter interpretation we are forced to look upon it as a departure from the state of health.

Besides the term Disease, therefore, general convenience of speech requires the use of such terms as Sickness, Indisposition, Feeble Health, Defect, Fault or Damage, and limits the employment of "disease" to such conditions in which the structural and functional disturbances go so far as to hinder the more important vital processes, to cause pain and to impair in an essential manner the vital phenomena of the organism as a whole. By the expressions "feeble health," "debility" (imbecillitas) is to be understood a diminished power of bodily resistance to pathogenic influences; "sickness" or "indisposition" is used when there is a subjective feeling of impaired health and where but minor grades of disease actually exist. "Defects," "damages" and "faults" are conditions in which certain parts of the body appear structurally abnormal and do not properly functionate; conditions in which the derangements have come to a definite standstill, and only under special conditions are likely to give rise to further morbid manifestations. Should these be congenital, arising in the period of fotal life, they are spoken of as Malformations, Congenital Defects, Developmental Faults, Vitia Congenita; if acquired after birth as sequels of disease (wholly or partly removed), or if the results of mechanical influences, as Acquired Deformities, Mutilations, or Vitia Acquisita.

In its proper meaning the term Disease has no reference to any entity or thing, but to more or less complicated processes and conditions which are evidences of disturbances of the physiological constitution and activity of the body; this applying to the individual cells as well as to the whole group of cells making up the organism. Diseases are therefore to be distinguished as diseases of the cells or structural elements of the organs, as discases of the tissucs, of organs, of systems, and of the individual or organism as a whole. [Inasmuch as life is but an expression of a harmony of structural and functional relation between the cellular constituents of the body among themselves and of the maintenance of efficient adaptability to the bodily environment, disease should be thought of as being made 
up of any change of structure or modification of function which may impair this harmony and adaptability to cellular or bodily surroundings. And individual diseases may then be understood as including special groups of such modifications of structure and function, these groups varying in the isolated affections in type, distribution and number.] The processes which go to make up a disease are not fundamentally different from the physiological processes, are not features foreign to the operation and structure of the healthy body, but are to be directly referred to these; the only difference being that in disease they manifest themselves in a site, at a time or in a degree other than in the normal state of the body. Pathological processes may therefore, as by Virchow, be considered as hetcrotopic, hetcrochronic and hetcrometric physiologi-

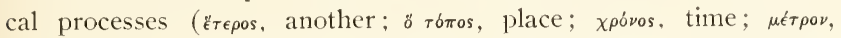
amount). As an illustration: a hæmorrhage into the ovary, caused by the bursting of a follicle and ovulation, is normal, but a hæmorrhage into the brain is abnormal; unconsciousness in sleep is normal, but at an unusual time and in pronounced degree, as in swooning, is pathological. Almost invariably physiological analogies are to be found, in comparison with which the alterations of disease are manifestly but quantitative variations or variations of place and time. The gastric mucous membrane, when the stomach is full and engorged during the process of digestion, is very full of blood and red; in another part of the body, as the conjunctival mucous membrane, this same redness and congestion would be pathological. The endometrium normally sheds epithelial cells and leucocytes in the lochial secretion in considerable numbers after the removal of the placenta; were this not merely temporary but of permanent duration the condition would constitute a pathological one, a catarrh. In the tumors or pathological new growths, which are apparently so foreign to the general organism, there are to be found only the same tissue elements as belong to the normal body, which however possess a power of growth which is abnormal. Even the phenomena of death have their physiological counterpart, as in the mummification of the umbilical cord.

The various pathological processes may be classed as follows: (I) Anatomical-pathological processes (gross pathological-anatomical or pathological-histological): Here are included all changes of structure or lesions whether of the gross organs or of the tissues and cells, as lesions of continuity of the skin and musculature (wounds), changes in consistence (as cerebral soften- 
ing or induration of the liver), occupation of spaces and formation of false membranes by fibrinous material (pleurisy, croup), microscopic changes in the cells (karyolysis, fatty infiltration). (2) Chemico-pathological processes: Quantitative and qualitative changes of the chemical constituents of the animal organism and its parts (metabolic faults), as the presence of bile in the blood, albumen in the urine, uric acid in the joints or abnormal proportion of water in the blood. [Here might well be included, too, the excessive formation or uric acid or its salts, of the faulty carbohydrate changes of diabetes, the development of faulty types of albumen in the blood and within the cells, the toxic changes of uremia and other intoxications, those essentially chemical processes which underlie in an important degree the changes produced by infections, as well as a wide group of more or less indefinite metabolic diseases.]

(3) Functional-pathological or symptomatic disturbances (sometimes spoken of as dynamic disturbances): These are faults in the nicéty of balance of the various physiological activities of the organism, as the occurrence of convulsions, pain, unconsciousness, labored breathing, diarrhœa or dribbling urine. Functional disturbances are usually caused by structural and metabolic faults. Commonly the latter are primary, as in physiological life the efficiency, growth and development of the organs are dependent upon the chemical processes of nutrition and metabolism. Our knowledge of the morphological and chemical basis of functional derangements is, however, incomplete.

Consideration, description and investigation of pathological variations may therefore be divided into (a) Pathological Morphology or Anatomy, (b) Pathological Chemistry and (c) Symptomatology, together with (d) considerations of the influences under which diseases develop, and the actual causes of disease (Aetiology: airia, cause). In the origin of disease some cause is apt to act so as to bring about material changes, chemical and physical, pathological lesions (ladere, to injure) of the component elements and organs, as well as the counteraction or pathological reaction on the part of these same elements and organs, both of which manifest themselves by functional disturbances (symptoms or signs of disease).

That phase of pathology which seeks to explain the development

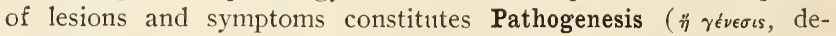
velopment, beginning); that which concerns the observation of the chronological succession of disease-events, the onset, course 
and termination of disease, is known as Nosology ( $\dot{\eta} \nu \delta \sigma o s$, sickness).

The object of pathology is primarily the acquisition of knowledge of the laws which govern pathological changes (Pathognomy, from $\gamma(\gamma \nu \omega \sigma \sigma \omega \omega$, to become acquainted with) ; in addition, to point how and why certain lesions and reactions must follow certain causes. Dealing in its broadest sense with life under abnormal conditions, pathology presents itself as Pathological Physiology; and moreover provides a scientific basis for the prevention and cure of disease, and thus the foundation of rational Prophylaxis ( $\pi \rho_{0-}$ $\phi v \lambda a ́ \sigma \sigma \omega$, to prevent or guard against something) and Therapy $\dot{\eta} \theta \epsilon \rho a \pi \epsilon i a$, therapeutics, from $\theta \epsilon \rho a \pi \epsilon \dot{\omega} \omega$, to attend, to heal).

For convenience in instruction pathology is separated into two divisions: General Pathology and Special Pathology. This is done with the purpose of facilitating a systenatic presentation of the wide scope of the study; general pathology dealing with the elementary pathological processes and their causes without reference to their site in the body, and being thus introductory to special pathology, in which the various individual phenomena of morbid change in the different organs and systems find expression.

With the healthy living individual as its starting point, and basing its comparisons upon the average normal structure and ordinary plysiological functions of the organism, pathology is dependent upon anatomy, physiology, chemistry and physics as its guides. A clear understanding or comprehension of its processes is impossible without a thorough knowledge of the organization of the animal body; a knowledge which includes the external configuration, the internal structure, the vital phenomena of health and the functions of each of the different organs. Chemistry and physics are essential for such a comprehension, both in their own immediate relations and, too, in connection with zoology and botany bearing upon the interpretation of disease causes. It is possible that one without this knowledge, a layman, may recognize various common morbid conditions (as wounds, fractures of bones, catarrhs, blisters, etc.), may name them correctly, and even treat them correctly on the basis of traditional information empirically acquired; and by frequent observance of such conditions may accumulate a fair amount of skill in their diagnosis and treatment. This is known as empirical knowledge. But to be able to scientifically interpret the complex picture presented by disease, to understand the whole trend of the process, its signs, its inception, and its recovery, and moreover to practice a 
rational method of treatment, is possible only to those who have become versed in these basic branches of learning. Only unprejudiced observation of nature and careful scientific study based upon anatomy and physiology, can give such insight. "To attempt to guess by speculation what is hard to learn even by thorough investigation has failed in this as in every other field of natural philosophy." (Samuel.) "The enigmas of disease are far too complex, the intermingling of forces in the higher forms of life are far too involved, that thought alone should successfully recognize such threads, not even to mention their absolute demonstration. The pages of general pathology are filled with the vanity of such attempts." (Samuel.)

Our knowledge of disease is primarily obtained from observation of the external appearances of diseased human beings and animals, from accumulation of experience with the external manifestations of disease (clinical obscriation). As long as autopsies were not permitted upon the bodies of the dead, diseases received consideration only from such external features; and there existed only a symptomatic classification of disease. It was customary to speak of jaundice, dropsy, ardent fevers, nervous fever, etc., as diseases, and to endeavor to explain by clever theories and all sorts of baseless ideas the origin of internal affections, whose cause and location were for the most part nnknown. They were regarded as mysterious occurrences, for which evil spirits or the influence of the stars on the lower world should be held responsible; and knowledge of pathology and the art of healing as well had to be groped for in darkness. Nothing beyond those morbid conditions caused by wounds, by gross external violence, or such affections of the skin or mucous surfaces which were patent to the eye, was dealt with in a less confused manner.

In many lines the physicians of antiquity, who sought to determine the nature of disease by dissection of human and animal cadavers, gained astonishing experience, practical skill and an acquaintance with the subjects, as is manifest from the clever experiments and methodical investigations of these early thinkers. But in the centuries of the Middle Ages, so barren of medical advancement, even long after the founding of the universities (which occasionally did succeed in making isolated discoveries of value in connection with physiology) medical science became stationary and fixed, hemmed in between philosophical systems on the one hand and all manner of outgrowths from a purely speculative and 
hypothesis-building science on the other. The learned doctors, with their schools of practically nothing more than mere dogma, who had no acquaintance with the structure and vital functions of the body save what conld be gained from its exterior, knew little more than the charlatans of their times. Veterinary medicine was practiced by butchers, farriers and grooms, whose information and practical knowledge were based upon the doctrines, conceptions and recipes handed down from former generations.

At the present time clinical observation is no longer limited to the mere detection of the external signs of disease, but seeks an explanation for them, searches for evidence of the internal morbid processes and for the precise location and for the causes of disease, calling to its aid every means of anatomical and physiological investigation and contributing materially to the complete developmental history of disease.

In point of time the real development of pathological science corresponds with the period when first the study of the exact location of disease was begun with the aid of the dissecting scalpel and the microscope. Morbid anatomy came to be recognized as the foundation stone of scientific medicine, and remains the most important landmark in experimental studies. It was recognized that definite symptoms are related with certain structural alterations, that it is possible to make inferences as to the altered state and structure of the organs from given disturbances of function, and that the symptomatology of a case may thus furnish a basis for the anatomical diagnosis and the anatomical conception of the disease in hand. Many of the alterations of disease are of such a character that the position, shape, consistence, color, weight and contents of the parts affected are strikingly different from the characteristics in health, and even the maided eye sees at once why symptoms must have been induced; and from the study of such evidence afforded by the dead bodies of diseased human beings and animals there has been accumulated a large amount of lata and statistics toward the establishment of a clear insight into the development, cause and termination of disease. Visceral anatomy along with visceral physiology has taught us what we know of the principles governing the action of the heart, the circulation of the blood, atmospheric interchange in the lungs, the functions of the digestive and urogenital tracts, and no little part of the pathology of the sensory organs: and it is quite possible for one to draw conclusions from given anatomical changes how the mechanism of the grosser 
parts of the body is interfered with, fails or becomes irregular, as in case of cardiac obstruction, obstruction of the blood vessels or intestinal canal by clots of blood or foreign bodies, or how intestinal displacement or unusual taxing of the organs must or may lead to disturbances. The entire group of physiological methods of investigation are of value in the solution of pathological problems; physics and chemistry aid in explanation and find the most varied application in their study. There has been developed in pathology along the lines of its special studies a series of practical methods of animal experimentation, known as Experimental Pathology, affording accurate information in many of the problems of pathogenesis and supplying valuable demonstrations of the correctness of our best established observations and conclusions. One may at will, by operative procedure upon living animals, by ligation or occlusion of blood vessels or ducts of glands, produce and imitate mechanical changes of various types; and it is quite possible by killing the experiment animal at any time to note precisely the anatomical changes and progress of the disease thus induced, from which the course of actual diseases of a corresponding type have come to be well appreciated. By operative removal of some organ or part of an organ, it is possible to obtain an idea as to the results which are likely to follow analogous disease processes in the same part, as in case of the thyroid gland, liver, kidney or adrenal. All our knowledge of regenerative growth of tissues, the healing of wounds, the union of fractured bones and of the general process of inflammation we owe directly to experimental studies on animals. By administering to animals various types of material of apparently poisonous character, it has been possible to study the action of a wide range of substances and to acquire the essential basis for diagnosis of the intoxications. The complete life history of the animal parasites, their development and multiplication in the human and animal body, and the diseases caused by them, have been worked out by means of animal experimentation in a really classical manner; and the practical application of this knowledge in food examination, in meat inspection and in other lines of prophylaxis against disease, are exceedingly numerous and have led to marked diminution of such diseases as hydatids, trichinosis and mange. But above all else animal experimentation has lifted the dark cloud which formerly obscured the nature of infectious diseases; and only from successful attempts in transmission by inoculation has it been possible to explain and obtain precise knowledge as to the real nature 
of plagues and their development. As long as these methods were not employed superstition and fear dominated mankind whenever a pestilence appeared. To-day, with realization of the conditions essential for infection and the modes of transmission, medical acumen has determined the surest preventive measures for controlling and combatting these diseases; and even the worst epidemics, as of plague and cholera, have largely lost their terrors. IVith confidence the work of extirpation is being carried forward, and medical science, crowned with success, has completely stamped out a number of these infections and is daily solving the difficulties in the prevention and cure of such evils.

To sympathetic persons it may indeed seem a serious thing that we be forced for our own advantage to make use of the sufferings of lower animals in order to avert such dangers from ourselves and to purchase by animal sacrifice the means of combatting contagion. But the instinct of self-preservation impels man, just as the necessity for food with any animal demands the death of other creatures. The slaughter of animals for sport is far worse, and productive of more pain to them; and many of the methods of killing in the kitchen of the epicure are much less excusable than any of the practices in the whole range of deplored animal inoculation, so unavoidable for the establishment of medical science. When it is realized that without the results obtainable by such work-Experimental Pathology-millions of people must forever be threatened by early death from pestilence, as of old, when countless numbers were sufferers in these epidemics and were hurried off before their time and when destructive cattle plagues forced heavy burdens on the land, whole hecatombs of animals for which the experimentalist must account must appear but a trifling matter. Prohibition of animal experimentation, as is sought by unrestrained zoomania, would be equivalent to prohibiting the cure of the sick; since nature affords for many affections no means for restoration other than the blood of inoculated animals. Human education and the high ethical tone of medical science will certainly be sufficient security that experimental pathology in pursuit of its purposes will not lend itself to useless animal torment. 


\section{HISTORY OF PATHOLOGY}

Much of our experience and knowledge of the diseases of man and of the lower animals has come down to us from antiquity. The gift of observation and the faculty of reasoning, as well as his desire to restore impaired health, impelled primitive man to formulate some kind of ideas regarding the origin and nature of disease. And as the grade of individual and popular intelligence advanced and as experience grew in value with its transmission from generation to generation by traditions and records, so our conceptions of disease expanded and our knowledge advanced, or on the other hand mistaken ideas took deeper hold or false notions once discarded again came into prominence. History informs us that for some thousands of years there had been attained considerable skill in the art of healing among the Babylonians, Persians, Egyptians, Hindoos, Israelites, Greeks and Romans both anong the priests and in the hands of a special class of physicians; and although religion and superstition, mysticism and philosophical speculation had much influence over it, medicine really was possessed of a very notable fund of information.

In the fourth and fifth centuries before Christ considerable work was done by physicians and naturalists like Alkmæon (B. C. 540). Hippocrates II, the son of Heraclides (B. C. $f(n-375)$ and Aristotle (B. C. 384-323) in the line of dissection of animals for inference to the supposedly similar structure of the human body; human cadavers being but rarely obtained for purposes of dissection. Dissemination of the knowledge of medicine which existed was advanced by the great schools of learning, like the Musem and Serapetum in Alexandria with their magnificent libraries (700,000 rolls of papyrus in the Museum and 300.000 in the Serapeum), and by the profuse literature of the Greeks and Romans. 'To some extent, doubtless, veterinary medicine shared in this; being practised both by the general physicians and by a special class of veterinarians,

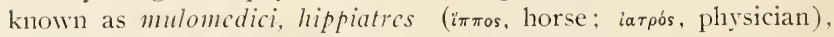

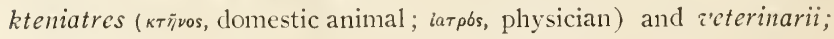


yet in a large measure it remained in its primitive condition in the hands of shepherds and farmers.

At the beginning of our present era medicine was chiefly dominated by the teachings formulated by Hippocrates and Aristotle in their general writings. The study of comparative natural science, instituted by Aristotle, laid the foundation of our knowledge of animal biology, of comparative anatomy and physiology; while Hippocrates and after him the physicians of his school established pathology. It that time it was held that there were in the animal and human body four cardinal humors, "blood, mucus, and yellow and black bile." It was tanglit that a proper relationship between these (crasis) insured health: and that disease depended upon the nccurrence of lack or excess of one or other, that is upon some modification of their relationship (dyscrasis). Next to the blood, the principal vital humor. Hippocrates placed mucus in order of importance, because it is often discharged in large quantities from the nose and was thought to come from the brain and to escape through the ethmoidal openings. Yellow bile was often seen in romit: but black bile was entirely a product of the imagination and was supposed to arise in the spleen. The basis of vital phenomena was supposed to be the inspired air (pncuma, the breath of life), which was thought to contribute warmth to the body. In conformity with the accepted theory of corruption of the lumors, therapentic measures were directed to a riddance from the system of the material which had caused the "dyscrasia;" and for this reason evacuants, diaphoretics, diuretics and renesection played an important part in those times. The importance which Hippocrates ascribed to these fluids or humors led to the application by later generations of the name Humoral Pathology to this system. The solid structures of the body were not entirely disregarded, but only vague ideas prevailed in relation to them. There was a theory (Democrites) that the solid parts were nade up of particles known as c.toms, between which there were pores [for the passage of air and hmmors], that the width of these pores varied with the varying density of deposition of the atoms, and that by some such method the state of the body was regulated. To this extent, therefore, there was a Solid Pathology, which, however, found but few adherents. Efforts toward such theoretical explanations found support particularly in the schools of philosophy: and tenacity of such views and the rigid 
adherence to authority, together with constant seeking after the keenest dialectic in argument, gave to one school of physicians of the day the character of "dogmatists ;" while others discarded entirely speculative reasoning and called themselves "empiricists," boasting that their methods of practice were based entirely on experience. They rejected the study of anatomy as superfluous; in which, however, the dogmatists, although essentially bookmen and theorists, made some progress.

In the middle of the second century Claudius Galenus (born in Pergamon, Asia Minor, A. D. I3 I ; educated in Smyrna, Corinth and Alexandria, and afterwards practicing his profession in Rome; died A. D. 206), noted both for his discoveries and as a practitioner, revolutionized medical science by his efforts to harmonize the prevailing theories with practical experience and thus give them a real value, and by his work in the establishment of rational scientific methods through comparative anatomy and experiments upon living animals. Throughout the middle ages, even into the fifteenth century, the theories of Galen, whose discoveries were numerous and whose extensive writings contain much that is of descriptive value, were held in esteem and respect, particularly his opinions upon anatomy and physiology.

Through studies of this character at the hands of physicians a close relationship developed between veterinary medicine and human medicine; but there were other" influences which aided in the development of comparative pathology. Agriculturists, like Xenophon, Cato, Columella, and Virgil, and veterinary specialists who in Roman times attained an independent standing, and among whom should he mentioned Apsyrtos (circa 290-350, A. D.) and Publius Vegetius Renatus (circa 540, A. D.) as especially distinguished, there were collected and recorded in literature their experiences with various diseases, especially epidemic affections among animals. Although there is much useless empiricism included in the dissertations of these writers, there are also no little excellence of observation and soundness of thought; and the works of the last-named author, like those of Galen, served for centuries as valuable spurces of information.

The general collapse of the sciences after the fall of the Roman Empire, during the time of migration of the European. peoples and the period of the Middle Ages, resulted in a long stagnation in medicine. Practically all that is worth considering was the preservation of the writings of the ancients, for which we 
are chiefly indebted to the monks, working as copyists in the monasteries. Technical medical skill made scarcely any noteworthy progress. With the founding of the universities in the thirteenth to fifteenth centuries, when a resumption of anatomical investigations gradually became possible, and with the discovery of the printing art, which stimulated the translation and wide -dissemination of the works of Grecian and Roman authors, there began a new epoch. The luminous works of the human anatomists like Vesalius, Failopius and Eustachius, the experimental researches of Servetus and Columbus Cesalpinus, and particularly William Harvey's ( $1578-1658$ ) discovery of the true conception of the circulation of the blood, led directly to the recognition of the errors in Galen's system and to a reorganization of medical science.* As usual pathology again fell into errow, ascribing, under the influence of prevailing views in natural science, the various processes of disease and their causation now to this, now to that physical or chemical factor. Speculative hypotheses took precedence of actual experiment, and attempts at proper explanation were quite lost in the fancies of the individuals. Some were followers of the "chemical school" founded by Sylvius, and endeavored to explain every fault by chemical changes in the composition of the body, as the introduction of "sharps" (Boerhaave); others, the "neuropathologists." when the importance of the nervous system became recognized, lair stress upon the influence of the nerves (William Cullen), or upon the effect of "stimuli" and the irritability of the tissues (the theory of excitability of Haller and Prown). Others, basing their views upon mechanics, believed the vital phenomena of morbid disturbances depended upon mechanical faults of relationship ("mechanical school," founded by Santoro, I561-1635, Borelli, I608-1677). Still others spoke of "vital spirits" circulating in the body, of "forces," of the power of the "entities" (Paracelsust), or made some injury to the immortal soul the real principle of disease (G. E. Stah1, I660-1734). These doctrines were denominated vitalism and animism. Such ideas have found atherents even as late as within the nineteenth century, clothed usually in high-sounding foreign verbiage to make the greater impression.

Along with these speculative theories, however, exact clinical

*Compare Eichbaum, Geschichte der Heilkunde: Berlin, Pareys' Verl., $18 \$ 5$. †Theophrastus Bombastus, whose proper name was Paracelsus, distinguished different forces, an ens astrale (power of the stars), an ens naturale, 8pirituale, veneni, etc., as factors in life and disease. 
observation, physiological experimentation and anatomical dissection grew more and more toward a plane of real excellence, affording an increasing clearness of insight into the processes which obtain in both healthy and diseased borlies. The anatomical changes shown in necropsies directed attention to the topography of disease. Morgagni (I682-I77I), a teacher of anatomy in Padua, in his work, "De Sedibus et Causis Morbormu" (г76r), outlined the first comprehensive and systematic exposition in this direction and came to be regarded as the founder of pathological anatomy. From efforts to correlate manifestations of disease of the various organs with the presence of anatomical changes, arose the school of Pathological-anatomical Diagnosis, to which is due the discovery of a number of valuable methods of diagnosis (percussion, thermometry, the microscope, chenical analysis), and which had as its founders men like Bichat, Pinel, Corvisart, Dupuytren, Auenbrügger, Laennec and Rokitansky.

The advances in physiology inaugurated by Johannes Müller (I8OI-1858) and the cellular theory formulated by Th. Schwann, along with the development of microscopic anatomy, are responsible for an important change from the older conceptions of disease, giving us as a basis for our ideas of morbic processes a cellular pathology', first proposed by Rudolph Virchow (1858), who referred the real seat of disease to the individual cells and the tissues, and regarded disease as depending upon the reaction of these to harmful influences. Although it is but about fifty years ago that Schönlein's school looked on disease as some sort of living thing of extra-corporeal origin, entering the bodies of men and animals like a parasite and expelled by our therapeutic measures; yet in the interim the study of Etiology has developed the definite view that disease is but the manifestation of morphological, chemical and functional changes which are induced by the most varied harmful influences upon the cells and tissues, chemical or physical; and thus to-day, through uniform and exact methods of objective research, clear conceptions and positive knowledge are in hand relative to most diseases.

As far as comparatioe pathology is concerned, it too was completely dormant from the fourth until the eighteenth century, at first because of the general depression in science, later because medical practitioners had but little interest in the diseases of animals when human medicine was developing along these newer lines, but especially because of the aversion which men came to 
hold for even mere contact with the dead bodies of animals. Among the rare publications appearing in the middle ages there is only one large work on the anatomy of the horse. (issued in I598 by the Venetian senator, Carlo Ruini, but probably the product of some physician) which is at all worthy of note as showing any advance in knowledge. At the same time the natural history of the domestic animals was considerably advanced by C. Gessner ( $5_{5} \mathrm{I} 6-\mathrm{I}_{5} 65$ ) and Aldrovandi (I522-I605). Practice of veterinary medicine was relegated morc and more to farriers, executioners and butchers and naturally drifted into the crudest sort of empiricism. However, when skill in riding and horses became more prized in the courts of princes and in war, there was a change for the better; and Italian, French and German masters of the stable (Pignatelli, Marx Fugger, Böhme, Winter von Adlersflügel, Robertson, J. von Sind, Solleysel, Pluvinel. Lafosse) published a number of works upon the diseases of the horse. From the eighteenth century physicians once more began to frequently pay attention to animal pathology; the necessities arising from devastating epidemics among cattle, particularly cattle plague, stimulating the members of the medical faculties to endeavor to stamp out these diseases and to publish numerous articles upon investigations bearing in this direction (Ramazzini, Lancisi, Schroeck, Gölike, Kamper, Sauvages, von Haller, Paulet). In the years from $1762-1790$, in most of the [European] states, schools of veterinary medicine were established, the first being inaugurated by Bourgelat in Lyons and Alfort. With this step comparative medicine found a place in scientific institutions, later, after various changes in organization, assuming the rank of independent colleges or becoming incorporated with the universities.

The first teachers of veterinary medicine were for the most part physicians: and even to the present the progress of the science is in close sympathy and relation with human medicine. However, the men who in the nineteenth century have been educated to the dignity of independent investigators and to a new standard as veterinarians, have broadened comparative medicine to a many sided field; and the results of their discoveries and their practical achievements have become of importance not only to the farming and cattle-raising industries, but to the general welfare of mankind as well when one takes into consideration the consumption of meat and the dangers of animal epidemics. 


\section{PREDISPOSITION TOIVARD DISEASE}

Any living being becomes affected by disease when no longer able to adapt itself to its environment and to the demands to which the functional ability of its cells and organs is subjected. As soon as such external influences exceed the limits of endurance, as soon as they so affect the cells and organs as to induce alteration of function, they become causes of disease. There is really nothing upon which the animal body is dependent or with which it is related, but may on occasion cause the onset of disease-nutriment, air, light, temperature, the various animal and vegetable organisms about it, the varied accidents of nature, its own active and passive relations, as well as every physiological process going on in its economy; and the very same factors, which are generally essential for the maintenance of existence and the well-being of the individual, may become agencies of harm and noxious (nocere, to harm) influences productive of disease.

Whether the individtial should experience the harmful possibilities of such factors or not depends upon the inherited potentiality of the organism, the functional capabilities of its cells and tissues, and upon the efficiency of its protective and regulative mechanism. Sensitiveness to noxions influences, known as Predisposition, and insensitiveness or insusceptibility, commonly spoken of under the terms Resistance or Immunity, are by nature widely different among different species and individuals and even among the different tissues in the same animal; and are subject to considerable variations and abnormalities. The polar bear, the Esquimau dog and the reindeer are accustomed to the cold of the north, but sicken in the temperature of warmer climes; many animals thoroughly adapted to the tropics, perish when transferred to cooler regions even though furnished, with their usual food. Individual animals of the same species often manifest differences of susceptibility, as where in herds of cattle or even, as is often observed, in a number of cattle kept in the same stable, some path- 
ogenic influence equally operative upon the whole group fails to produce its effect upon all, certain individuals resisting it successfully; or it is often noticed that here and there an animal sickens under conditions quite favorable for most of its species. The difference between different tissues in predisposition and immunity may be seen in comparing the skin and mucous membranes. The surface of the skin, with its hard epithelial covering, is much less sensitive to irritative substances than are the delicate mucous membranes; the mucous membrane of the stomach in the living animal is resistant to the action of acids, but that of the lower end of the intestinal tract may be injured by the acid of the gastric juice.

A number of poisons (snake venom, tetanus toxine) are entirely harmless when taken into the alimentary canal; aithough, if introduced by way of iesions of the skin into the tissues, they are extremely pathogenic.

Especial interest attaches to the differences in predisposition and immunity toward the viruses of infections diseases. In all infectious diseases we have to deal with poisonous materials caused by micro-organisms. These micio-organisms (microphytes, vegetable microscopic organisms; microzod, animal microscopic organisms) gain entrance to the body by alimentation, or by the respiratory path, or through a'ounds, or may even actively penetrate the tissues should they be in close relation with the skin or mucous surfaces. Within the body structure they multiply for a time and work harm to the tissues chiefly by the specific poisons existing in their protoplasm (that is, in the bodies of the microbes) or by poisons elaborated by them, diffused in the tissues and taken up by the blood. In the contest waged with these microscopic foreign invaders a twofold task is set for the animal body; it must in the first place destroy the nicrobes and at the same time must render their toxines inert. One animal may accomplish this with ease; another with difficulty. Some species of animals are by nature uninfluenced by the toxine of an infection which is sure to produce disease in another species; thus cattle are immune to glanders, the horse to pulmonary tuberculosis, and chickens bear large doses of tetanus toxine without injury to health.

Such absence of reaction to infections and their toxines in an animal species is spoken of as natural immunity. It is to be explained from one standpoint by the idea that the colls and tis- 
sues of the immune animal have no affinity, or but little affinity (chemical affinity), toriard the torines of the infection. The poisonons elements simply do not enter into combination with them. For example, the nervous system of the turtle is absolutely immune to the toxines of diphtheria and tetanus, and these substances may be injected into the animal entirely without effect. Yet the toxines thus introduced may remain in the bodies of the experiment animals for months without being rendered inert by the juices: and should the blood of such a turtle be injected into some sunceptible animal it will act in the same manner upon the latter as would the toxine itself.

From a second point of view it is to be recognized that immunity may depend upon the fact that some of the colls of the animal in question are able to take up and digest micro-organisms, and thus render them harmless (Phagocytosis). This power of seizing and ingesting small particles, organic and inorganic, or dead and living cells, is peculiar especially to the motile trpes of leucocytes (wandering cells, white blood cells), but is also possessed by giant cells, splenic and merlullary cells, and even fixed connective tissue cells (as endothelium); and plays an important part in the economy of the body. Its significance has been pointer out especially by the ingenious investigations of Metschnikoff, Leber and Bordet. These phagocytes act as scavengers, taking up and making away sometimes with blood debris, nuclear fragments, pigment, fat globules and all sorts of minute foreign particles with which they cone in contact. The ingestion of such corpuscular elenients is with them a simple process of feeding. The movement and approach of the phagocytes may be induced by a number of stimuli, as warnth or an acid, acting upon their own tactile or chemical sensitiveness. In coming in contact with foreign particles they attempt to increase their surface of contact as much as possible [applying their protoplasm more and more about the surface of the particle, and thus eventually enveloping it in their own naterial]: and are attracted by various chemical substances (chemota.ris). [It is but fair to add here that while the theory of cliemotaxis, as a part of the general theory of "tropisms" or of blind automatonlike response of living things to external forces or attractions, finds wide adherence among medical men and biologists, there are nevertheless others who do not accept such a view, the latter finding reason to believe that the manifestations which the former 
school attribute solely to non-intelligent attraction from without are really due to an inherent power of a low intelligent character of the organisms showing them. These latter would attribute even to individual cells as the phagocytes, a low but actual volitional power to either originate or refrain from efforts to approach the foreign particles referred to.] The protoplasm of the phagocytes apparently can secrete digestive juices [there is reason to think that certain granules seen in leucocytes are of the nature of ferments], through the action of which they are able to assimilate nutritive matter and dissolve foreign bodies.

In this latter manner, in part at least, it is possible that the virus of infectious diseases may be removed from the tissues. Nuttall first pointed out that in the blood plasma of healthy animals there exist certain substances which are capable of dissolving the body matter of bacteria, and of thus destroying them. These substances, called alexins by Buchner, and complements by Ehrlich (by others addiments and cytoses), are products of the cells, which are either given off by the living cells (Buchner) or are set free by cellular destruction (Metschnikoff): and are found in varying amount in the circulating blood of the individual animals. An excellent example of these substances is seen in the effect, discovered by Behring, of the serum of white rats upon the anthrax bacillus. If hundreds of thousands of anthrax germs are placed in some serum from a white rat (at $37^{\circ} \mathrm{C}$.) it will be noticed after ten or fifteen minutes that the bacilli have become swollen and degenerated (granular), and after from four to seven hours that they have completely disappeared. Canine or ovine serum does not possess this power, but should a few drops of rats' serum be added to sheep. serum the bactericidal action will immediately appear. The serum of the horse also has a strong bactericidal power. It must be clear that the cause of natural immunity cannot be attributed to the bactericidal action of the serum alone, for both rats and horses are susceptible to anthrax; it must be thought of, as already mentioned, as dependent in part upon the lack of affinity of the living cells for the toxines of the infection, and in part upon their phagocytic power.

Just as among different animal species and individuals there may be some one kind which is more highly resistant to poisons than the rest, so there may be met others exhibiting an excessive susceptibility to such 
influences. For example, cats are extremely sensitive to carbolic acid. It is well known that some persons possess so marked a susceptibility tc a number of substances and foods (as strawberries, mushrooms, crabs, lobsters, cocoa or alcohol) that after partaking of them they experience severe pain, vomiting and cutaneous eruptions, and in the same way are apt to be severely affected by certain medicaments (chloroform or morphine); they are influenced by such substances, as it were by poisons. Such extreme susceptibility is known as idiosyncrasy "(tôsos, peculiar;

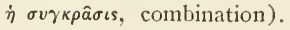

As a rule, the predisposition or immunity of individuals is only a relative one; that is, it is variable and of moderate degree, may be increased or decreased, and is often only temporary. Racial or specific immunity may also be but relative. Rabbits are ordinarily immune to symptomatic anthrax, but now and again a rabbit is found to be susceptible to this disease upon inoculation. Goats, horses and cattle are strongly resistant to swine-erysipelas, but if large amounts of the virus are injected intravenously they may show severe symptoms. Young dogs are somewhat susceptible to anthrax; older dogs less so. Young sucking calves are very rarely affected by "black leg" and usually resist inoculation tolerably well; but as soon as they begin to eat vegetable food they become very susceptible to the disease in question. Thus age and food are seen to have an influence upon predisposition and immunity. How intimately connected "the latter factor is may be inferred from the experiment, originally performed by Feser and afterward confirmed by others, in which rats, if fed upon meat alone, are found as a rule to be immune to anthrax, while if fed on bread they soon succumb to inoculation with the virus.

By experimentation it has been shown, moreover, that hunger is a predisposing cause, that bodily overexertion (overheated animals), mutrition of restricted quality and excessive amount ( as a diet too rich in fat) may have such depressing infuence that animals subjected to then readily succumb to injurious agencies which they would otherwise bear, and particularly become less resistant to infections. It is also true that certain altered conditions of the tissues afford especially vulnerable points for the attack of pathogenic influences; for example, gastric catarrhs, by diminishing the production of hydrochloric acid, favor the deposition and pathogenic action of bacteria which are otherwise destroyed by the gastric juice. As a rule, previous discase leaves as a sequel an increased disposition, the formerly affected tissues 
showing a diminished resistive power for a long time (local predisposition; locus minoris resistcntia), as, for example, mucous membranes after catarrhs.

Predisposition to disease and (a matter of extreme importance) immunity against diseasc may be acquired. It is well known that recovery from certain infectious diseases is accompanied by an insusceptibility to a repetition of the same affection. This immunity after provious attacks is at times only temporary, but a few weeks or months in duration, or it may extend over many years or for the entire lifetime. The alteration which has taken place in the condition of the body in such cases is chiefly a chemico-biological one, and although much remains enigmatical concerning it, some insight into the process has been obtained through experimental investigation. Besides the discoveries of Jenner and Pasteur, who gave to mankind facts and methods of the highest importance toward success in combating infections, by which it has become possible by artificial inoculation of an attenuated virus to produce a mild course of the infection and to obtain therefrom immunity from subsequent attacks of the same disease, there must also be recalled the important discovery that in the blood of man and animals, after attacks of infections, there appear certain substances which are of specific anti-toxic character and are destructive to the virus, and that upon the production of these substances depend protection and recovery from these diseases. The tissues of the animal body react to irritants which gain access to them. Should a particle of dust or a gnat happen to lodge upon the conjunctiva, such a foreign body acts as a stimulant to the nerves of the mucous membrane, this inducing a free secretion of tears, which usually wash away or dissolve the object. In an analogous manner there is a reaction on the part of the tissues should a toxine or some pathogenic germ (virus, bacterium) come into contact with them, not in this case with a simple secretion to wash away the poison, but nevertheless restraining the poison and giving origin to substarices which are apparently actual antitoxines, or which are able to destroy and thus render harmless the germs of disease. The conditions underlying the production of such antagonistic or protcctive substances, their mode of origin, manner of action and properties, are of extremely complex nature. The numerous experiments of such investigators as Behring, Ehrlich, Brieger, Kitasato, Wassermann, Buchner, Emmerich, Födor, Nuttall, Nirssen, Bordet, Morgenroth 
and Sachs, have, however, brought forward facts hitherto unsuspected and of the greatest interest in this connection, and have fointed out not merely theoretical hypotheses upon immunity, but also practical methods of extreme value in combating the infectious diseases.

As already pointed out, there already pre-exist in the normal blood of every animal certain substances capable of dissolving foreign material which has in some way gained entrance, and, by entering into combination with resultant toxic matter, of rendering it harmless. In some of the glands, as the thyroid and liver, neutralization of toxic matter and noxious metabolic products is being continually carried on; and when one considers the innumerable reactions and interactions which are taking place in metabolism, resulting in the most varied types of secretory material with destructive and digestive properties, it is easy to think of the body and each of its cells as a chemical laboratory of manifold productive ability.

In attempting an explanation of the mechanism involved in the production of these antibodies (antagonistic or protective substances), Ehrlich has proposed a brilliant, and, at first thought, a very convincing theory, the chief points of which are briefly included in the following. The hypothetical details of this theory have recently met such serious objection at the hands of Gruber that in a great measure it seems but doubtfully credible. The basic fact that antitoxines and protective substances are products of the cells and organs of the body is not endangered by this criticism, but the problem of how and where such substances are formed is renewed as a matter for further investigation.

According to this view the cells of the body may be thought of as organisms which consist of a basis of protoplasm, which has as its vital center a nucleus (vital nucleus of Ehrlich); this protoplasmic unit may be fancied as being possessed about its periphery of armlike processes, the receptors or side-chains of Ehrlich. [It is to be understood here that the author refers to the protoplasmic molecules as the basis of this theory, each molecule being composed of a more or less complex group of atoms or of combinations of atoms. The vital center does not refer to the nucleus seen as a structural part of the cell, but to the structural nucleus of such a complex molecule of its protoplasm. As is attempted in the graphic chemical formula of a complex organic molecule with the symbol $\mathrm{C}$ as its center, to indicate as 
"side-chains" the varions atoms or groups of atoms linked about this nuclens, so in Ehrlich's view the living protoplasmic molecule may be thought of as having as its elemental or basic compound a vital nucleus abont which are attached, and probably from which spring, a number of other atomic groups subsidiary to the center of the molecule and corresponding to the side-chains of the formula, but essential to the efficiency and integrity of the whole complex molecule, since they are its means of combination. These are the receptors or haptophores of the molecule. Every molecule possesses these haptophores in addition to its principal part, the centre or nucleus (in living protoplasmic molecules, the vital centre). Should these haptophores be lost in the living molecule, they are replaceable by others of like type because of the vital regenerative power of the molecule.]

The receptors are the principal instruments of [molecular, or in other words] intracellular metabolism and provide nutritive substance to the cells through their ability to nnite chemically with such material. However, just as proper food elements may thus combine, so other albuminous bodies or materials chemically allied to foods become linked to or combined with the receptors, as material extracted from bacteria, various poisons of animal, bacterial and vegetable origin (toxines, venom of bees, spiders or snakes, diphtheria toxine, ricin, abrin, the substance of blood cells, spermatozoa and nerrous tissue, and the milk albumen of different kinds of animals). Such union occurs because these substances contain in their molecular composition certain groups of atoms, which, like similar atomic groups in foods, have an affinity (chemical affinity) for the atomic groups of the cellular protoplasm; these Ehrlich speaks of as their haptophore groups. [Thus both the protoplasmic molecules of the body cells are provided with haptophores or combining chains, and, too, all nutritive or harmful molecules which come in contact with them. Should there be affinity between the haptophores of the cellular protoplasm and those of the introduced molecules a combination will result.] With such combination effected [the molecule and in proportionate degree the whole] cell comes under the influence of the substance chained to it. Should the latter possess properties actively harmful to the protoplasm, poisonous qualities (toxophore), the cellular protoplasm becomes more or less injured, and a pathological cellular lesion results. There may ensue complete death of the cell [protoplasm], 
or merely a condition of irritability or partial injury. If the injury be partial that part of the cell [protoplasmic molecule] which remains sound restores the defect. In this condition of irritability or stimulation there is a reaction on the part of the cellular protoplasm left with its vital centre intact to regenerate the receptors destroyed by the toxic influence. Following a law recognized by Weigert, regeneration in the cells of an organism is usually productive of more of the substance than originally existed at the site of loss; and so here the restoration is often so active that an excessive number of receptors are supplied to replace those which have been destroyed. "Such an excess of receptors constitutes a useless ballast for the cells and the extra ones are thrown out into the blood and circulate therein" (Ehrlich, Wechsberg). The presence of these free receptors in the blood, representing haptophores or chemically combining substances, affords opportunity for materials which have gotten into the blood and which have chemical affinity for them, having come in contact, to enter into" combination with the receptors. Thus held by these free receptors, such substances are prevented from involving the cellular protoplasm, and as a result the cells and the whole individual are protected from the disease.

This power of molecular combination differs in the different cells of the same individual and in the cells of different species of individuals. One species may possess no cells containing the elements essential for combining with a certain toxine; there would then be absolutely no chemical affinity shown between the cellular molecules and the toxic molecules, and a priori the latter must be without effect. In such a case the toxine, perhaps in large quantities, may remain for weeks in the blood and general circulation without any harm resulting to the individual. Such instances are examples of natural immunity. On the other hand, an individual may possess a vast number of receptors not merely in the general body, but each cell, each corpuscle, for example, may contain a mass of material capable of combining with substances of one or other kind. In classification of these receptors it is customary, following Ehrlich, to distinguish them as of I, II, III, etc., orders.

The first order (uniceptors) includes receptors having only one haptophore group for poisons (toxines) and utilizing this for combination with such substances; they are called antitoxines. The presence and formation of antitoxines is illustrated by the 
immunity to toxines acquired after infectious disease. The toxines are allied to the albumens* and enter the blood in a state of solution: thus dissolved, they here enter into combination and are therefore inert before they gain access to the tissues, as the nervous tissues-the cells (bacteria) which give origin to the toxines being remored from the economy by phagocytosis (as tetanus or diphtheria bacilli).

Another class of receptors [III order of Ehrlich] has the power of combining with foreign cellular elements and at the same time drawing into the combination the ferment-like alexines which naturally have pre-existed in the blood; these thus must possess two haptophore groups (a cytophile and a complementophile group), and for this reason are known as amboceptors. For this element which is the medinm (amboceptor) of the chemical union (anchoring together) the following terms are also employed: desmon ( $\delta \epsilon \omega=I$ bind), immune body, intermediary body, copula, immunisin, fixateur, sensitising body. The alexine is also known as the complement, addiment and cytose. When by the combined action of these two elements the destruction and solution of foreign cellular elements (the toxineproducing animal and vegetable microorganisms themselves) are accomplished, the compound antibody [amboceptor and complement] is spoken of as a cytolysin (E. S. London). The presence of the two elements allied to each other is shown by experiment. If one will heat a serum containing cytolysins to $5^{\circ} \mathrm{C}$. the complemental element will be destroyed, and the serum will be found to have lost its cytolytic power, is inert. If, however, to this serum there be added another, ordinary serum, containing only complement and inert by itself, the cytolytic power is restored to the first, and it is said to have been reactivated. So, too, the desmon or amboceptor in the cytolysin may be removed by adding to serum cells for which it has affinity [and by centrifugation these may be thrown down; while the serum continues to contain the complement]. There are a number of cytolysins of different kinds, each exerting its influence as a rule upon only one certain kind of cell [this depending upon the peculiar affinity of the amboceptor in the cytolysin combination]; thus we recognize among many those which act upon red blood cells (hamolysins), upon sperma-

* Poisons of other types, alkaloids, glucosides, saponines, which do not act by forming chemical combination do not cause the formation of antitoxines in the organlsm. (Ehrlich, H. Sachs.) 
tozoa (spermolysins), upon bacteria (bacteriolysins), upon white blood cells (leucolysins). Some dissolve several kinds of cells, as in the case of spermolysins, which destroy red blood cells as well as spermatozoa. From this it may be seen that in general cy'tolysins possess specificity of action.

The appearance of cytolysins in the body of any given animal is occasioned by the introduction of cells of a different species of animal into the first. If human blood be injected in increasing amounts under the skin of a rabbit or into its peritoneum, the serum of the rabbit will acquire the power of dissolving the human red blood cells, but not those of horses, cattle or guinea-pigs. If the spermatic fluid of guinea-pigs be introduced into rabbits, in the same way the serum of rabbits becomes solvent for the spermatozoa of guinea-pigs, but cannot influence the spermatozoa of another species of animal. If colon bacilli be injected into an animal a cytolysin will be developed destructive only to these microphytes, and in fact only to the particular strain of this bacterial group which was employed in the experiment.

From the foregoing it should be realized that the principal feature in the production of immunity against foreign cellular elements is the formation of the amboceptor or desmon, and that this alone is a new product of the cells of the invaded body; the other element, the alexine, naturally pre-exists in every body, its combination with the desmon forming the cytolysin. If, however, the necessary alexine be absent or present in insufficient amount, the cytolysin will not appear. In this latter way must be explained such occurrences as where, in spite of repeated introduction into an animal of some type of cells, the blood acquires no cytolytic power, but where on further addition of the serum (containing the required alexine) from another species of animal cytolytic activity is immediately produced.

The sources of the amboceptors are apparently the bonemarrow, spleen, lymph glands and perhaps the subcutaneous connective tissues.

In addition to the production of antitoxic and cytolytic substances the body may engage along the same line of reaction in the formation of substances [II order of Ehrlich] which cause foreign cells to aggregate in masses (clumping, agglutination), the so-called Agglutinins; and other substances which coagulate foreign types of albumens (Coagulins) and cause their precipitation (Precipitins). In illustration, if defibrinated human blood be injected into a rabbit there is developed in the blood of the latter a substance which will act on human blood to 
cause a flocculent precipitate, but which will not (as a rule, and never if certain modes of application are employed) exhibit such precipitating qualities with any other type of blood. It is interesting in this connection to add that the serum of a rabbit so treated will also precipitate the blood of anthropoid apes, gorillas, orang-outangs and chimpanzees, thus indicating a relationship of these animals to man. Cytolysins and hæmolysins also serve to show the relationships existing between animals in the zoological system of classification. Inasmuch as this reaction is applicable as well with a solution of old and dried blood, the discovery (which as an outcome of research in the field of immunity is due particularly to Bordet, Ehrlich and Morgenroth) has acquired especial importance as a means of information concerning the source of blood stains in forensic medicine.

Other albumens may also lead to the formation of specific antibodies in the animal organism, as milk albumen; thus by subcutaneous injection of goat's milk into rabbits, horses or cows a serum may be obtained which will immediately cause a precipitate if it be added to goat's milk. In the same way, by inoculation, coagulins may be obtained for cow's milk.

In the same way, too, in the course of an infections disease a series of substances are produced in the human or animal organism which render the infectious matter for the time harmless. Should the infectious products develop very rapidly, and by their. poisonous properties cause serious protoplasmic changes in the body cells, the disturbance is apt not to be limited or checked, and in spite of any reactive-products which may be formed the function of the cells becomes altered and the animal dies. Should the [protoplasmic molecules of these] cells be only partly injured the body prevails over the disease, the molecular defects of the cells becoming regenerated. The surplus of antibodies, the result of this regenerative action, are after recovery found circulating in the blood, and in some instances may even pass into the milk. The amount of protective bodies or antibodies in the body-fluids varies according to the intensity of the reaction and the time elapsed after recovery from the infection, the greater part being used up during the course of the disease and the remaining gradually disappearing in metabolism; while with the removal of the stimulus the supply from the cells tends to cease. If, however, infectious and toxic material be again introduced into the system, stimulating and injuring the cellular 
protoplasm, the production of antibodies will be again induced, receptors being again formed. This form of immunity, produced through cellular activity following the introduction of the infectious agents, is known as "active immunization." The discovery of the existence and production of specific antibodies in the serum, for which we are mainly indebted to the studies of Behring upon tetanus- and diphtheria-immunity, has led to extremely important methods of prophylactic and therapeutic inoculation against a number of the infectious diseases. This becomes possible from the fact that the blood serum of an animal which has survived a certain infection and is highly immunized against it can be employed by injection to produce in a second animal similar immunity against the same disease. The production of the protective material in the blood depends upon the introduction into the experiment animals, either intravenously or subcutaneously, of gradually increasing amounts of the infectious material against which immunization is sought. The animals in this way become more and more highly inmunized and their blood serum becomes correspondingly rich in antitoxine. By such a system of progressive inoculation it is possible to attain a degree of concentration in which a thimbleful of the serum contains more immunizing material than would exist in all the blood of the body after one single attack of the disease. The ability to produce such materials exists in the cells of all kinds of animals, both those susceptible to the disease in question and those naturally resistant to it. For example, by injecting the bacilli of swine-erysipelas, which in natural conditions are pathogenic only to the bristled kine, it is possible to inducc the reaction also in sheep, goats, cattle and horses and produce in their blood immunizing substances; and then, after withdrawal of the blood from animals thus prepared, to obtain from it a proplyylactic and curative serum in sufficient quantity for use in hogs. The production of active immunity in an individual requires some time, because the body cells must first form the protective substances; in other words, must first pass through the period of the disease, perhaps four to six weeks in duration. When, however, the prepared serum is introduced there is no such demand placed on the body cells: the protective substances, already formed, produced from the animal supplying the serum are introduced into the second animal. An immunity thus obtained is spoken of as "passive immunity." It comes on immediately or in a few hours after the inoculation. 
according to circumstances. As a definite cellular reaction in such a case does not occur at all, or at best only in a minor degree, and as the amount of protective antitoxic material introduced is gradually used up, destroyed or excreted (urine) and no new antitoxine is afforded because of lack of proper reaction, such passive immunity passes away after a short time (seven to fourteen days).

The recognition of this peculiarity has in practical application led to the coincident or successive employment of both methods of immunization in case of certain of the infectious diseases. Serum is first injected so as to induce a passive immunity in an animal, and living germs are next inoculated so as to transfer a passive into an active immunity; the previous introduction of the protective serum making the later inoculation with living microbes practically free from danger. 


\section{CONGENITAL AND INHERITED DISEASES}

If there exist at time of birth in an individual actual disease, or peculiarities of predisposition or immunity which also characterized the parents or ancestors, such conditions are said to be congenital (innate), and in the latter instance inherited (hereditary) as well. The genesis of such an occurrence is by no means always the same, although in a measure it is apparently dependent upon conditions of the parents.

During intrauterine life the embryo may experience injuries affecting its normal growth, causing deformity, interfering with the proper development of a limb or organ, or destroying some part already in stage of development. Such injuries are for the most part mechanical in type, as where anmiotic adhesions (or more rarely tumors of the uterus) by constriction or pressure of this or that part of the foctus (which in its movements might be entangled in the amnion) may compress, wound or otherwise injure it. Depending on the nature of such an injury, the embryo may in consequence present gross or trivial faults of one sort or another; these are spoken of as fatal or embryogenous anomalies, zitia congcnita, developmental defects or monstrosities. Except when a uterine lesion is the cause of the deformation of the fœutus, the mother has no influence upon the production of anomalies in the offspring.

There are a number of infectious diseases whose causative micro-organisms are capable of gaining access to the fotus indirectly through the placenta. In their multiplication in the placental tissue they may, by growth, penetrate it and obtain entrance to the blood of the fotal side. Under such circumstances the newly born animal carries into the world with it the same disease from which the naternal parent was suffering during the term of her pregnancy; the acquirement is here placontal. The most common example, of such a transmission is seen in congenital tuberculosis in cattle, occurring only in case the cow has uterine tuberculosis. The congenital pathological conditions of this type have 
therefore an intrauterine mode of origin, which, if strictly interpreted, is comparable to acquirement by an external influence, only that in this instance it operates within the womb.

It is reasonable to believe that injurions metabolic products which pass through the placenta of a mother animal suffering from some febrile condition (especially metabolic and nutritive disturbances which react from the mother upon the embryo, affecting primarily the ovum, or in the father's case affecting the spermatozoon in a similar manner) occasion morbid predispositions, as diminished developmental energy, or perhaps a tendency to excessive growth (dwarfism, foetal chondrodystrophy, gigantism), weak metabolic rower, tendency to fatty degeneration or fatty deposition (ozogenic or spermatogenic predisposition, congenital degeneratice inheritance).

According to Weissmann and Ziegler, it is quite probable that often predisposition to disease and congenital pathological characteristics are due to germinal variations; that is, that when two unadapted sexual cells unite there may be developed to some degree new and perhaps even abnormal peculiarities in the embryo from the union of the two elements (amplimixis of the ovum and spermatozoon). Thus healthy, strongly-constituted parents may often be seen who sometimes beget offspring of feeble constitution, weak-minded or of other morbid tendency. The offspring is never entirely like either parent, and the various members of the same generation are never alike in bodily structure and character. Only in case of twins, developing from one ovtum or from one act of coptulation, is there striking similarity of the bodily features. In the mingling of the maternal and paternal sexual elements two strains of hereditary tendency unite to produce new germinal variations. Should there be thus produced new peculiarities of value to the individual, or which we care to preserve, tỵpe-characteristics (precocity), these are not regarded as pathological; but it is readily conceivable that the variations might but poorly fit the offspring for life and render it but feebly resistant to given pathogenic influences or show from the first abnormal constitution of its tissues. In such cases is presented a germinatiz' $\mathrm{C}$ or constitutional predisposition.

A predisposition, no matter how dezeloped, as i'ell as acquired immunity, may be transmitted to the offspring. provided the individual is capable of begetting; yet not every tendency toward disease and not all the resistive powers of the parent are 
necessarily continued to succeeding generations. Only such conditions are transmitted as pre-existed in the germ, in the segmentation cells and the embryo, or those in which apparently the nature of the whole parental cellular structure is altered, as germinal variations and so-called constitutional tendencies and diseases.

Injuries and diseases which do not involve the sexual cells, as all simple traumatic lesions of the body, give rise to no transmission. For example, the common custom of cropping the ears and tails of dogs has never led to the birth of a short-eared or stub-tailed dog or of such a breed (just as the practice of circumcision, practiced for hundreds of years among various peoples, has never yet caused any congenital anomalies of the prepuce). If occasionally structural anomalies of this type are encountered careful investigation will show that some intrauterine mechanical fault is responsible for the defect in the fotus, as Bonnet has demonstrated in case of stump-tailed dogs that the caudal vertebræ have been bent or deformed simply by intrauterine pressure (amnion). This is also proved by the fact that the same anomaly occurs in cats (and in any other animals whose tails are unobjectionable to man and for the removal of which there is no occasion), showing that the habit of cropping can scarcely be held responsible.

Non-transmissible conditions and those which are truly inherited may very closely resemble each other in their anatomical features, and yet depend on entirely different causes; only the closest inquiry revealing the fundamental influences producing them. For example, supernumerary toes (polydactylism) may be an ataristic phenomenon, a, family trait, or may occur in a fœtus as a splitting of a digit because of amniotic adhesions; in the same way harelip (congenital fissure of the lip) may be caused by local amniotic trauma or may be an hereditary anomaly.

Heredity is best uncierstood when it incerns chemical infucnces. As indicated by Ribbert, it may be conceired that the whole body can suffer from the wide dissemination through it of some chemical substance and that, of course, under the circumstances the germ cell is also reached by the same noxious material. If the organism survive such disturbance and become immune through the changes called forth by the chemical poison, the germ cell may also survive with the same immunizing changes. Should such a process befall both parents, both spermatozoon 
and ovum are likely to be immune and the new cellular structure resulting from the copulation of these two (the embryo) will possess the same characteristic and transmit it again, because its own sexual cells are clearly the descendants of the rest of the group. In the same way a predisposition may be established and transmitted, because the chemical substance in some way weakens the germ cell and diminishes its resistive ability, and by the fusion of two similarly enfeebled sexual cells the progeny, as the product of both, partakes of their qualities. Similarly to such chemical influence as Ribbert stiggests, any exaggeration or diminution of the body temperature must affect the germ cells.

Inherited pathological conditions and properties of immunity need not necessarily manifest themselves in cach successiz' gencration of postcrity indefinitcly; for the most part they are linited to a few generations and then disappear. But they may recur in later gencrations. If but one of the progenitors possess a certain predisposition and come in sexual union with a nonpredisposed individual, the pathological condition will progressively diminish by half (Ribbert) because of its distribution in the two sexual cells; is proportionately corrected by the healthy cell of the copulation and by germinal variation; and grows weaker and weaker until it is no longer a factor in the posterity. Should the peculiarity reappear after several intervening generations, it may be assumed that this occurs as a result of the union of two germ cells, each possessed beforehand of a disposition which was hitherto latent, but which from the summation of the characteristics of both parent cells has again become sufficiently intensified to reappear as a pathological fault (Ribbert). Hence we distinguish: a direct heredity from father and mother to the offspring; a latent heredity if the offspring of affected parents are spared but in the next or later generations the disease should appear. arising from a transmitted tendency; a collateral heredity, should the disease manifest itself in the side lines of the family group; an atazistic horedity, when the origin of the disease suggests a reversion to the family ancestry.

In human medical practice the following are considered as diseases transmissible by heredity: Hæmophilia or hæmorrhagic diathesis, color blindness or Daltonism (so named after the Englishman, Dalton, who was himself color-blind), lenticular opacity or "gray cataract" (or cataract), near-sightedness or 
myopia, pigment-atrophy of the retina, polyuria (production of excessive amounts of urine), predisposition to mental diseases, to progressive muscular atrophy, to tumors, to obesity and to certain malformations (dwarfism, polydactylism, harelip, etc.). The anomalous conditions mentioned are due, perhaps, to germinal variations, possibly first brought into active manifestation by the introduction of some external disturbing factor. (Vide Ribbert, Lehrbuch der allgem. Pathologic. Ig0r.)

What parts respectively are taken by heiedity and by external influences in the development of congenital affections is in many instances difficult or even impossible to determine, as our knowledge of these matters is still deficient. This is particularly true regarding the so-called hereditary defects of the domestic animals. Among the diseases included in this category for the last century or more, some are surely not inherited and not transmissible but occur because of external influences; for example, intermittent ophthalmia, cataract, asthma, ringbone, frog-thrush. Others, as dumb-staggers ["blind-staggers"], hæmoglobinæmia, curb, spavin, deficient hoof-formation, may be caused directly by external influences, although it cannot be denied that inherited structural defects may indirectly favor their appearance.* As instances of direct inheritance and congenital origin may be mentioned many cases of goitre in animals.

*Cf. Dieckerhoff, Ueber d. Erbfehler bci Zuehtpferden: Zeitschr. $f$. Veterinärkunde, 1902, Feb., p. 53. 


\section{CAUSES OF DISEASE}

The influences which lead to bodily injury, to pathological lesions, and which are spoken of collectively as disease causes (causc morbi) may be arranged in a number of classes.

There are influences against which the strongest and most resistive constitution is powerless and which inevitably induce disease in the animal coming under their action; such absolute pathogenic influcnces include mechanical forces, intense thermic and electric agencies, mineral and vegetable poisons in their higher dosage and concentration, and, provided they can gain entrance to the intracorporeal structures, various animal parasites, bacteria and [animal] microorganisms. Other harmful agencies are pathogenic only under special circumstances and are therefore only relatice, depending. for example, on the quantity of the exciting agent, or upon special predisposition of the subject or upon the concurrence of several harmful influences (relatic'e causes of disease). The occasion which brings a harmful influence in relation with the body or subjects the latter to such influence, is spoken of as the predisposing cause (causa proxima). The principal factor in producing a lesion is known as the special, immediate or essential cause of disease (causa essentialis): among these it should be understood that either external harmful agencies which have gained entrance into the body or which operate upon it from without, or some already existing tendency toward disease may be included. For example, pasturing upon some upland infested with anthrax affords the opportunity by which the anthrax bacillus as the essential cause of anthrax gains entrance to the body: an injury, the favoring condition for wound infection by the essential cause of tetanus. Anything which promotes the action of an injurious agent may be regarded as a contributary cause (causa auxilians).

Pathological influences may be divided into the following groups : 
I. Disturbances of Nutrition and Alimentation: Abnormal states of nutrition depending on irregularity in the water or nutritive elements afforded.

2. Obstructions to Respiration: Interferences with oxygen convection.

3. Functional Disturbances: Depending upon fatigue, upon overexercise of the organs.

4. Thermic Influences: High or low temperatures.

5. Electric Agencies.

6. Mechanical or Traumatic Agencies.

7. Chemical or Toxic Agents: Poisons.

8. Microbic or Infectious Agents: Micro-organisms belonging to the protophytes and protozoa.

9. Animal Parasites: Of the class of worms and arthropoda.

\section{Disturbances of Alimentation and Nutrition.}

Total deprivation of food without water supply (complete inanition) leads, in case of the higher vertebrates, to rapid loss of body-weight, emaciation and death in the course of from one to four weeks. With absolute rest (as in case of imprisonment in a cared-in mine) an adult man may retain life without food and drink for about twenty days: exertion hastens the end. Strong dogs have lived under similar conditions for thirty-six days; horses and cats should live for about four weeks with absolute rest. Guinea-pigs and rats die within three to nine days. Liberge states that a well-conditioned cow, which had wandered into an out of the way place and had remained there forty days without food and without opportunity for much exercise, picked up quickly on a milk diet and was in tolerable condition eight days after being set free.

If water is obtainable abstinence from food can be endured without permanent harm by man and carniverous animals for from two to four weeks, and the fatal end may be postponed for a considerably longer period. A cat experimented on by Bidder, weighing two and one-half kilograms, died on the eighteenth day from starvation after haring lost I,I9J grams (water consumed, r 31.5 grams). Birds of prey (eagle) endure hunger and thirst for twenty to twenty-eight days; small birds only two to nine days. Mascagni has recorded a turkey's having fasted for tiventynine days. In case of cold-blooded animals the requirement for food is so small that water-salamanders and turtles may live for 
a year, snakes for six months and frogs for nine months, taking nothing but water.

When fasting an animal must furnish whatever energy is necessary for the maintenance of its proper temperature and for its organic functions (muscular activity and circulation) by the destruction of its body tissues, nourishment from external sources being impossible or insufficient. How the destruction of tissues, an actual self-combustion, takes place and how the vital organs live upon the less important structures, is well shown by the comprehensive studies of E. Voit. Fat and glycogen are first sacrificed, and as long as these substances are present the albuminous elements are not subject to the destructive process, the muscular structures afterwards bearing the greatest part of the loss. Loss of weight and atrophy are most marked in the omentum and the fleshy parts (also the fat and glycogen stored in the liver), and fat animals succumb to starvation later than lean ones According to Chossat, young, poorly nourished pigeons die after three days with a loss of one-third their body-weight; plump ones after thirteen days, with a loss of half their weight. The heart shows the least loss in weight (its constant activity and functional stimulation hindering its atrophy); the central nervous system similarly loses but little, and the diminution in the red corpuscles is comparatively unimportant. (Lipomata are unaffected in starvation; attempts to cause their removal by starvation have been unsuccessful.--Samuel.) Death from starvation takes place after the development of great muscular weakness, with complete loss of power and deep stupor (Samuel). In the bodies of animals dead from this cause are to be noted muscular and glandular atrophy, passive congestion and degenerative changes, together with scanty contents of the small intestine and diminished lumen of the latter.

Complete inhibition of water (as in feeding with material artificially deprived of its water) acts quite as effectively as hunger, death taking place in from eight to twelve days. Animals, unable to quench their thirst, refuse food, and the organism is unable to adequately supply the fluid secretions necessary for digestion. Death probably is due to the retention in the system of injurious metabolic products (with poisonous qualities), which cannot be flushed out. Actual drinking of water may very well be avoided by a number of animals (rabbits, guinea-pigs, cats or parrots) without injury to health, provided the food ingested contain water. 
Diminution in the amount of food ingested, undernutrition (relative or incomplete inanition), is often met with in connection with diseases of the alimentary tract; its consequences are precisely similar to those of total withdrawal of food, save that the progression of the case is slower. Usually the condition is accompanied by a diminution of erythrocytes in the blood (inanition ancmia), the alimentary disease producing wide disturbance through fluid-waste (diarrhœa) and other complications.

Faulty composition of food causes partial starvation, the body suffering loss in its fat, albumen or carbohydrate should its nourishment lack or contain but an insufficient amount of one of these substances, or should the animal by preference and exclusively feed upon only one of these types of nutritive material. Impoverishment of the diet in such manner brings about emaciation and physical weakness. If lime should be deficient in the food and water supply the skeleton will fail of its most essential constituent, that which gives it its rigid strength, and the bones become soft.

\section{Respiratory Faults.}

All animals die by asphyxia* if their supply of oxygen be prevented. A wide range of factors may bring about a diminution in the proportion of oxygen contained by the blood, associated as a rule with insufficient separation of the carbonic acid and its consequent high proportion in the blood. Primarily this may result from the insufficient access of atmospheric air to the respiratory organs, as by closure of the superior orifices [smothering] or by obstruction of the respiratory tube [choking] and constriction of the latter (strangulation, compression of the larynx or trachea) by fluids and foreign bodies (the latter also by lodging in the pharynx and occluding the trachea), pressure upon the larynx and trachea by tumors, obstruction by tumors or swelling of the mucous membrane in the folds of the glottis or in the bronchial tubes, collections of blood, fluid or coagulated exudates in the lungs and bronchi.

A second group of asphyxiating causes includes interference

*The editor is here taking the liberty of using the word asphyxia as the general term, including smothering as meaning respiratory obstruction, operative at the respiratory orifices, mouth and nose: choking. respiratory obstruction operative within the mouth, nasopharynx or cesophagus (pressing on the larynx or trachea) strangulation, respiratory obstruction by constriction or pressure from without upon the larynx or throat generally ; suffocation, respiratory obstruction by any type of cause operative below the level of the larynx, either within (as a suffocative gas) or without (as pressure upon the chest). In English this meaning is attached to asphyxia, although as Prof. Kitt indicates in the present section it really means pulselcss; his own general term is "Erstickung." 
with the respiratory movements, as in case of rupture of the diaphragm, the principal muscle of respiration, in case of its immobility or when it is forced forward by flatulence, in ease of marked pressure from without upon the chest walls (animals standing pressed together in railway cars), or in case of pressure upon the lungs by large fluid collections in the chest cavities. Failure of respiratory movement may also occur from nervous origin (pressure upon the brain, paralysis of the vagi, spasm of the bronchial museles). Premature separation of the piacenta or compression of the umbilical cord in the maternal canal prevents oxygen convection to the fœtus.

Furthermore, passive congestion of the lungs, interference with the emptying of the pulmonary veins and hæmic changes in which the blood corpuscles have lost their ability of taking up oxygen (carbon monoxide poisoning) render diffusion of the gases so difficult that the same result of lowering oxygenation arises, and the respiratory disturbance eventually advances to the stage of suffocation. The presence of irrespirable gas and the reduction of oxygen in the air of an inclosed space (instead of 20.8 per cent., perhaps only 2 to 3 per cent.) act in a similar manner.

In one or other of such a variety of ways asphytia may be the termination of a number of diseases and is the commonest cause of dcath. It is usually accompanied by the symptoms of dyspnca ( $\dot{\eta} \delta \dot{\sigma} \sigma \pi v o c a$, from $\pi \nu \epsilon \omega$, difficult breathing, shortness of breathing), labored respiratory movements (suffocative dyspnœa), marked increase in the cardiac movements, spasmodic twitching of the general musculature and loss of consciousness. Toward the close the respiratory movements become irregular, sometimes intermit and suddenly cease, the inspirations occasionally becoming very deep (terminal respiratory movement); and the spasms weaken the cardiac action (true asphyria, actual pulselessness, from $\dot{a}$ privitive and $\dot{\delta} \sigma \phi v \gamma \mu s_{s}$, the pulse). When the diminution in oxygen is gradual in its onset these symptoms appear less prominently; but as the tissues degenerate from the insufficient supply of oxygen (fatty degeneration of the heart, liver, kidneys) and as the respiratory nervous center loses its excitability, the patient dies from gradually increasing loss of conscionsness and cardiac failure. According to the rapidity of onset of the fatal end and the causes of the asphyxia, the post-mortem findings present different pictures. In addition to the local changes due to strangu- 
lation, obstruction to the respiratory passages or the rest of the causes mentioned, the most common features of this mode of death are found in the tar-like, uncoagulated, dark condition of the blood (excessive presence of carbonic acid, deficient decarbonization) and in the presence of hæmorrhages in the lung and pleura.

\section{Excessive Functional Stimulation.}

All organic activity is accompanied by consumption of the essentially functionating material of the organs and by the formation of metabolic products. Both of these factors, especially the accumulation of the latter substances in the tissues of the organ, lead to a gradual reduction in efficiency, that is, to fatigue. This is normally corrected (restoration or reconstitution of the part) during the intervals of rest, when the accession of arterial blood replaces what has been lost and the lymph current sweeps out the waste products (fatigue waste). Should the various organs be required to continue their activity without intervals of rest for a long time, or should they suddenly be overstrained, or called into functional effort beyond their physiological ability, their parenchymatous substance may be so affected by the heightened metabolism and so marked an accumulation take place of the waste products of fatigue (carbon dioxide, phosphoric acid, either free or combined as acid phosphates), that cxhaustion or wearing out results, with complete abolition of functional ability. This condition is clearly pathological if tissue changes can develop in the process and make the loss of function permanent (fatty degeneration or cellular atroplyy), or if the cessation of function, momentary though it be, can cause in the vital organs disturbances involving the general economy or the actual death of the individual.

Coincidence of other contributing causes (the weakened stage in fevers, traumatism or thrombosis) favors the development of such functional lesions from overexertion, as seen especially in the heart, the general muscles and in the nervous system.

General muscular fatigue and cardiac exhaustion may be the cause of death in overheated animals. Horses suffering from thrombosis of the abdominal aorta and its branches exhibit signs of functional disturbance of the muscles of the posterior limbs, even under moderate effort, because of their poor arterial supply and the accumulation of waste products from fatigue. The heart 
fatigued by prolonged effort may lose its power of contraction to such a degree that it becomes abnormally distended by the pressure of the contained blood (cardiac dilatation, insufficiency of the heart), with ensuing disturbance of the circulation. Overstimulation of the nervons system by sudden psychical shock is not only in man productive of serious results; fright palsy with cessation of cardiac action (paraplexis) has also been observed in the lower animals (fowls).

A gradually and only moderately increasing demand upon the muscles and glands acts as a stimulus to their functional activity, provided proper nutrition be afforded the tissue, and results in functional hypertrophy, that is, in an increased growth of the cellular elements corresponding to the demand for work. This may be noted especially in tubular structures provided with muscular walls (smooth muscle), where there is gradually and spontaneously developed an increasing competence for the work required of the muscle, as in the thickening of the muscular layers of the bladder in case of gradually narrowing stricture of the urethra (v. functional hypertrophy).

\section{Thermic Influences.}

High temperature of the surrounding atmosphere (above the body temperature peculiar to the animal) may lead to failure of heat loss (heat stasis) and hyperthermia of the entire body with fatal termination by so-called heat-stroke. This is frequently observed in logs when crowded in railway cars. The condition is most easily induced if the dissipation of the body heat by evaporation is diminished, and at the same time heat production increased in the animal by prolonged muscular effort or by rich feeding. (It is known from experiments that warm-blooded animals kept in well-ventilated warm clambers at 36 to $40^{\circ} \mathrm{C}$. die, some in from one to three days, others in from ten to thirty days. The body temperature of such animals-rabbits-rises to 39 to $42^{\circ} \mathrm{C}$; ; they become dyspnœic and there is increase in the frequency of the pulse; the hrmoglobin of the blood is reduced, and degenerative changes develop in the heart, the liver and kidneys.-Ziegler.) The cause of death is cardiac failure (heat rigidity) occasioned by the overheated blood, or may depend upon a thickening of the blood through excessive loss of fluid by sweating and respiration (fall of blood pressure, dimi- 
nution in vascular tone). As anatomical changes are found reddening of the skin (in hogs), failure of blood coagulation, dilatation of the right heart, venous hyperæmia of the lungs, liver, kidneys and brain. The names insolation and sun-stroke are applied to that form of heat-stroke which is caused by the influence of the direct heat-rays of the sun upon the body, particularly upon the head; it is thought to depend upon a paralytic dilatation of the ressels of the meninges and cortex of the brain, and the affection is accompanied by convulsions and marked symptoms of excitation (Birch-Hirschfeld).

The influence of heat locally applied occasions. lesions known as burns (combustio), of various grades according to the degree of temperature, the duration of application and the resistive powers of the tissues to heat. Burning may result from contact with solid, liquid or gaseous heated matter, or from direct action of a flame or radiant heat. Short exposure to a temperature of 40 to $50^{\circ}$, or longer exposure to 30 to $40^{\circ} \mathrm{C}$. is productive of an inflammatory reaction marked merely by dilatation of the capillaries (hyperæmia) and redness of the part (inflammatory burn, erythema, bum of the first degree or of mild degree). The process is only superficial, leading at most to desquamation of the epithelium. Exposure to heat of 60 to $80^{\circ}$ C. (burn of the second or intermediate degrec) is productive, in addition to the hyperæmia, of rapid exudation of serous fluid from the dilated vessels beneath the epithelial layer. The cells of the stratum Malpighii are pressed apart by this, become swollen, are loosened from the papillary bodies, and the firm, horny portion of the epidermis is raised up to form a blister. Through minute fissures, easily made in this bleb, pyogenic bacteria may enter, the fluid contents through further exudation [leucocytes] becoming turbid and purulent. Should the blister rupture the hyperæmic papillæ beneath are exposed, the repair of the epidermis starting from the epithelium at the margins of the lesions or from remnants of the Malpighian cells which were not destroyed. In case of mucous membranes, which are devoid of the horny epithelial layer, blister formation is not likely to take place, the epithelial layer desquamating in shreds; and the denuded surface is covered by a coagulating exudate, the so-called croupous or false membrane. The more severe infuences of heat, above $80^{\circ}$ C. (burns of the third degrec), produce searing and charring (eschar formation); the burned 
tissue coagulates; the blood within the capillarics is coagulated and stagnant, the tissue dying in consequence and being transformed into a brownish crust (or cschar). At the border of the actually involved and necrosed tissue inflammation ensues. Healing follows by the separation of the charred material and the formation of scars, which are apt to be of a radiating, reticular appearance, and which by their contraction and shrinkage may cause considerable disfiguration. All these degrees of burning may coexist as the heat has happened to influence in a greater or less measure one or more places.

In extensive burns of the skin (if as much as one-third of the body surface is involved) the subject is likely to die, even in case of burns of no more than the first or second degree. Death may take place within but a few hours after the occurrence of the accident with symptoms of impaired respiration, cardiac weakness and fall of the body temperature. In other cases the fatal end may take place after the course of a week, during which there may have been apparently favorable progress, pulmonary œdena and nephritis often developing in the meantime. Other cases pursue a course of sone weeks' duration before the lethal end. The explanation of the dangerous features and fatal termination of such burns is to be sought in part in functional disturbance of the skin in heat dissipation, fall of blood pressure, overheating and inspissation of the blood (cardiac paralysis) and in part by the changes which the blood corpuscles undergo. Different investigators (Salvioli, Maragliano, Castellino, Ponfick) have shown by experiment and observation upon human cases, that after burns of the skin the red blood corpuscles, partly degenerate (broken into small particles) and partly without any apparent structural changes, are incapable of convering oxygen, and give up their hæmoglobin (partly changed into methæmoglobin) into the serum, whence it is excreted by the liver and kidneys; that, further, the formation of hyaline thrombi is apt to take place and that these alterations of the blood may be regarded as the cause of deatl.

Low temperatures, which deprive the body of its proper warmth, may give rise to either local or general disturbances in warm-blooded animals. Sensitiveness to the power of withstanding cold varies very much in the different animal species. Fishes chilled to the freezing point may seem to be lifeless, their lymph frozen into solid ice; yet they may com- 
pletely recover from such a condition. Frogs are said to remain viable for hours subjected to a temperature of $-2.5^{\circ} \mathrm{C}$., with the heart frozen solid (anabiosis).

According to Koch, resuscitation is possible only by gradual thawing and providing only a part of the water present in the body has been actually frozen; in case of rapid thawing, violent diffusion currents appear between the water emerging from its crystalline form and the concentrated albuminous solutions of the blood and tissues, which may destroy the tissues (Koch, Ziegler).

Hibernating aninals sleep but lightly with a blood temperature of $6^{\circ} \mathrm{C}$., but soundly at $1.6^{\circ} \mathrm{C}$.; their cardiac beats sink to eight to ten each minute; breathing is almost suspended, the movement of the lungs caused by the heart action alone carrying on the feeble gas diffusion in the lungs (Samuel).

The hair and feathers (especially the winter pelt) of animals lessen the loss of heat by radiation and by convection of the [warm] air from the surface of the body; in consequence of which animals are seen to endure with ease the ordinary cold, providing they are well nourished, move abont freely and thus produce heat abundantly. [The loss of this protective element or the presence in the hair of much moisture favoring the ready convection of the heat from the body surface reduces tremendously the power of resistance to cold; and one finds the cattle in the prairies freezing to death in a rain at a temperature considerably above ice-forming temperature, although were the hair dry and capable of holding the layer of warmed air close to the skin the animals would have shown no signs of discomfort.] If there be diminution of heat-production because of insufficient nutrition, and extreme cold, even well-pelted animals (hares and deer) and birds may be frozen to death. Death by freezing takes place by loss of sensibility of the nervous system, with fall of body temperature, diminution in the frequence of the cardiac and respiratory movement, cerebral anæmia, loss of muscular power and blood coagulation.

The local action of cold, varying with duration of exposure and intensity, causes tissue changes of the same types as in burns (frosting, congelation). Primarily there results a constriction of the vessels of the part exposed (local anæmia), after which, if the cold continue, the nerves and muscle tissue of the vessel walls become paralyzed and dilatation of the vessels ensues with increased blood content, this condition usually returning to 
normal when the low temperature of the part is corrected. Temperatures below the freezing point are, however, likely to so disturb the vessel walls that inflammation of the tissue (swelling and redness of the skin, frostbite, chilblain) develops with or withont the formation of blisters; or after especially long and severe exposure the blood and lymph circulation cease and the tissue dies as a result. The necrotic area may be separated from the normal by inflammatory reaction, or if it remain moist may undergo putrefaction through the inftuence of invading bacteria. The extremities are the parts of the body most commonly subject to freezing, but it is secn rather seldom among animals, for example, the scrotum in bulls (Bang), the paws in dogs, in horses the thick skin of the hoof, particularly the crown and pastern. Jewsejenko and Cadiot have recorded instances of necrosis from freezing of the deeper part of the foot in horses (in the Russo-Turkish and Franco-German wars, as well as in Algeria).

By the term catching cold (chilling) is meant the pathogenic action of heat loss not sufficient to cause freezing, but productive of functional disturbances and inflammation of nerves, muscles, joints and internal organs. The laity commonly and primarily for almost all affections attributes chilling as the cause; and even the physician often evades the question as to the origin of some malady with the vague phrase of the possibility of catching cold, because of the obscurity of the retiology of the case. Many diseases formerly regarded as produced by exposure to cold are now recognized in the advanced state of atiological investigation as infectious, although undoubtedly there is a group of affections in which chilling of large areas of the skin and mucous membranes may with confidence be held responsible as the causal agency. Such a relation is evident in cases where, after unusual exposure to cold (thorough soaking, strong draughts, falling into icy water, heat loss by radiation to some neighboring cold object as a stone wall) there immediately develop in the chilled parts pains, functional disturbances and symptoms of inflammation, or where in a short time these phenonena without other demonstrable cause appear in the subjacent or more distant parts of the body. Cats almost invariably become sick if they become soaked by falling into the water, while flocks of sheep have been attacked by pleuro-pneumonia directly after wool washing if, while wet, they were left exposed to cold air. The 
occurrence of paroxysms of colic (dysperistalsis) in sweating horses after chilling of the surface cannot be denied. Rabbits and guinea pigs dipped into ice-cold water have been known to quickly sicken and die from pulmonary and renal inflammatory affections. As a further illustration it is well known that the peritoneum is very susceptible to the effects of lowered temperature, and that in case of extrusion of the viscera or in operations involving the exposure of the peritoneal cavity there often arise, even at a temperature of $2 \mathrm{I}^{\circ} \mathrm{C}$, , entirely from the chilling (under conditions of asepsis and in the absence of other causes), a general depression of temperature, attacks of colic, peritonitis and perhaps a fatal termination.

Hofer has observed that chilling may affect even fishes, provided they be suddenly changed from a warm water to a cold; and that such chilling causes changes in the skin (desquamation and necrosis of the epithelium).

Attempts to frame a theory explanatory of the real nature of chilling have as yet been unproductive of any definite information on the subject. If it be assumed that the blood in the cutaneous vessels is chilled, it remains unexplained why pract1cally only isolated parts of the body are affected, although the chilled blood flows on to other organs. Excretion of blood pigment in the urine (hæmoglobinuria) after exposure to cold might well be explained upon the idea of chill effects upon the blood causing destruction of the corpuscles, yet in such cases of hæmoglobinuria there is usually a previously developed myositis and the red color of the urine is looked upon as a result of the liberation of muscle pigment and not as depending solely on blood destruction. The chilling of the skin causes extensive vascular constriction and the blood is forced from the surface and accumulates in the internal or more deeply lying parts of the body. Why in these cases the blood is not evenly distributed in the body, but collects in special localities, is an open question. Sudden exposure of a cutaneous or mucous surface to cold undoubtedly causes an appreciable vascular tonic contraction, which may be ascribed to stimulation of the vaso-motor nerves. This vascular spasm is not limited to the area directly affected by the cold, but extends consensually or refle.rly to adjacent or symmetrical, or distant z'ascular areas (Samuel). If, for example, one dip a hand into very cold water the other hand also becomes paler (Samuel), and probably everybody has had the experience that oc- 
casionally a sudden chilling of the feet brings on directly a reflex sneezing and nasal catarrh. Rossbach has observed in experiments upon cats that by applying cold compresses over the abdomen vascular constriction passing over into vascular dilatation develops in the mucous membrane of the respiratory passages. There usually succeeds upon the vascular constriction a relaxation of the ressel walls with which is associated a marked congestion (vid. Hyperamia). Such disturbances are, of course, commonly corrected, the vascular constriction and internal congestion together with the vascular relaxation disappearing, and the chilling is realized but for a short time as a sense of cold or brief catarrhal affection by the subject. Why the same adjustment does not occur in all cases is not clear. We only know as a fact that the chilling leaves in the skin itself practically 110 anatomical alterations, that the sensitiveness of the nerves, contractility of the vessel walls, the circulation and perspiration are entirely restored, while in the deeper structures the vascular spasm and the succeeding vascular dilatation are apt at times to be prolonged. Sometimes, as further consequences, local engorgements, nutritive faults of the tissues, inflammatory exudates, excessive mucous glandular secretion are to be seen; sometimes catching cold may manifest itself only by nervous symptoms, functional disturbances and sensations of pain and may absolutely fail to give any idea concerning the anatomical changes of the tissues.

Affections which arise in consequence of catching cold are often but transient and are very apt to change their location in a system of tissues, appearing successively at different points along the larger nerves and in muscular regions and at different joints. For this reason they have been called rheumatic affections.

[While it is true, as the author indicates, that the varied effects of chilling of the cutaneous surface are not susceptible of a simple explanation, there are certain probable influences which can scarcely be overlooked. The development of congestive states in some mucous membrane in connection with the more or less widespread vascular changes beginning in the skin may be held as offering favorable conditions for the more active growth and penetration of some microorganisms, which perhaps in the normal condition, although present, were unable to advantageously invade the membrane, and many of the catarrhs which follow refrigeration undoubtedly show clear evidence of such in- 
fectious agencies. The old idea that by causing a more or less prolonged contraction of the cutaneous vessels the skin secretions are reduced or prevented and that in this way there tend to accumulate metabolic or other toxines in the tissues cannot be set aside. Such substances have been thought to perhaps possess irritant qualities which disturb the sensory nerves and muscle fibres and other structures, the rheumatic pains and stiffness supposedly arising in consequence. At least some weight is to be given to the readiness of disappearance of such symptoms when by warmth and exercise the general circulation is stimulated and skin secretion heightened, these toxines then perhaps finding more ready excretion from the body than could be afforded by the other excretory paths.]

\section{Electrical Influences.}

Powerful electrical discharges upon the animal body induce paralysis of the nerious apparatus (especially of the respiratory centre), electrolytic destruction of the red blood cells, local burns of the skin and laceration of the tissues. Death usually follows; but the paralysis and unconsciousness may, after shorter or longer duration, go on to recovery. The larger animals (catthe and horses in the stall or in the open) are especially liable to be struck by lightning: (Fröhner, Ziegenbein). Contact with live wires and completion of the circuit through the bodies of horses occasionally takes place when they step upon the contact points of an electric railway in the street pavements or on a broken overhead wire.* Horses have been killed by a current strength of 500 volts, I00 amperes (Puntigam, Mouquet, Blanchard); alternating currents of 160 volts are sufficient to kill dogs (Birch-Hirschfeld).

According to Leblanc, horses are exceptionally susceptible to electricity. A horse was killed, for example, by a relatively light current which the owner passed through the bit in order to divert the attention of the animal while being shod.

Anatomical changes may be entirely absent when death has been caused by electricity, or the hair may be found singed and the skin burned by the electric spark; and at the points of entrance and exit of the lightning or current, as well as in the internal organs, the tissues may be lacerated, with which lesions

* Birds sit on telegraph wires with impunity because they are not in contact with the earth. 
hæmorrhages of course occur. Along the entire course of the current tree-like, branching lines of singeing (hyperæmia and hæmorrhages), the so-called lightning pictures, may be seen in the skin and intestines. In addition the blood is dark and uncoagulated, the muscles dark brownish-red, the endocardium stained by the altered blood, and the heart muscle at times the seat of hæmorrhagic infarcts.

\section{Mechanical Influences.}

Pathological changes are very frequently produced by mechanical force. These are either lesions of tissue cohesion, ruptures (breaks in continuity, lasio continui), or of compression, as the constriction of a hollow organ, condensation of tissues or displacement of organs. If this be caused by external forces or foreign bodies the process is spoken of as traumatism (trauma,

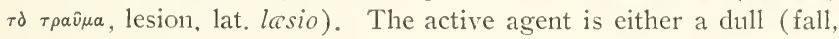
blow, jolt, pressure, pull, friction) or a pointed or sharp (stab, incising) solid body. In the same way internal mechanical disturbances may be caused by adhesions of the tissues, tumors and other pathological products producing constricting influences, or by excessive blood pressure, abnormal gas expansion or pressure by fluids; or may involve the functions of organs" (displacement of actively motile stomach, intestine, uterus or muscle). If the force gives rise to loss of continuity amounting to visible separation of the tissue the lesion is spoken of as a wound, hurt, tear (vulnus, trauma in restricted sense); if affecting dense structures (as bones, cartilage or teeth), as a break (fracture); if the tissue be destroyed by compression, as a crush, contusion or bruise. Displacements (dislocations) are met in joints, bones or muscles, as wrenching or luxations; in the intestines as ruptures (hernia), or twists (volvulus). The term stenosis is employed in connection with constriction and obliteration of hollow organs; where the closure is caused by some factor in the inside of the organ it is spoken of as an occlusion stenosis; where it is due to external pressure it is said to be a pressure stenosis.

The results of mechanical injury are extremely numerous and vary with the size and character of the producing agent. as well as of the local lesion, and with the relative importance of wounded tissue to the general organism. A wound is often 
complicated by other factors, as from the entrance of microbic or toxic agencies through the opening or breach made by the foreign body, with the result of further alteration of the tissues. The traumatic agent may be at the same time the conveyor of such substances, or may itself be a living parasite. Intense mechanical force may sometimes without any evident lesion, sometimes with manifest lesion, cause serious and perhaps fatal effects upon the nervous system. For example, this occurs in violent concussion of the body (commotio from com-moz'co), especially if the vertebral column be directly affected (concussion of brain and spinal cord-the former causing the loss of consciousness, the latter bilateral palsies of the extremities, the bladder and rectum). What the precise anatomical changes are which in such instances take place in the central nervous system has as yet not been determined, autopsy usually showing nothing that is characteristic. Possibly the alterations are molecular.

Death sometimes takes place suddenly, too, from blows upon the abdominal wall and viscera. Such instances are characterized by sudden loss of strength, fall in body temperature, cardiac failure and diminution of nervous excitability. This condition is known as shock, traumatic stupor, collapsc, traumatic reflex paralysis. It is thought that the paralysis and cardiac failure in such cases is due to vagus irritation.

\section{Chemical Agents; Poisons.}

Substances which harm the animal body by their chemical action are known as poisons; the actual process of introduction of the poison and its action, as poisoning or intorication. Probably nearly all chemical substances can, under proper conditions, act as poisons, or, in other words, have a deleterious infuence upon the tissues; the mode and power of combination of the chemical and the amount and degree of concentration in which it is present in the body being important items. For example, a substance as essential for the body as sodium chloride will in large doses cause vomiting and fatal poisoning in dogs. The most poisonous substances, as hydrocyanic acid or nicotine, have absolutely no effect when given in the minute doses of the homeopaths. Changes in the relation of the atoms and solubility may transform a non-poisonous substance into a poisonous one and vice versa; of the two forms of phosphorus, only the white is 
poisonous, the amorphous red form being harmless even in large quantities. A number of substances which are insoluble in water, and which have practically no effect when brought in contact with the skin or when introduced subcutaneously, are changed by the hydrochloric acid of the stomach so as to become serious poisons; for example, carbonate of barium is changed in the stomach into the soluble chloride of barimm. On the other hand, there are substances which are apparently energetic poisons when introduced into the blood, but which are inert in the stomach because they are completely neutralized by the albuminates of the gastric juice (Samuel).

Nany poisons belong to the mineral kingdom or are artificially made from minerals, as arsenic, mercury, iodine, bromine, chlorine, lead, copper and others, and the various compounds of these substances (oxides, sulphides, chlorates, chlorides, etc.). The organic compounds, as alcohol, chloroform, hydrocyanic acid, cyanide of potassium, carbolic acid and picric acid, especially [include many poisons].

The vegetable kingdom (Samtel) affords many examples of poisons. There are whole families of plants whose genera and species possess some poisonous principle common to them all. In many plants certain parts may contain a poisonous substance, while the rest may be quite free from it and edible (as is well known in case of potato plants, solanum tuberosum, whose leaves, blossoms, seed and immature fruit contain the poisonous solanin, while the tubers are quite free from it). A number of plants are innoctous, slightly or seriously poisonous, depending upon questions of climate, location, character of the soil and cultivation; the almond tree, for example, bears either sweet or bitter almonds, according to the location. The most powerful poisons contained in the higher plants are the vegetable alkaloids (morphine, atropine, colchicine, digitaline, etc.). In case of the lower plants also, especially the bacteria, there are products, some of which are marvelously toxic, fatal to large animals in the most minute and scarcely appreciable amounts; these substances, analogotts to the alkaloids and enzymes, or of albuninous nature. are embraced by the terms toxines, toxalbumens, toxcnaymes. (See also chapter on bacteria.)

Among animals there are a number known to be able to elaborate poisonous secretions within special glands, especially the venomous snakes (iipera berus, the common adder of Ger- 
many; vipera Redii and ammodytes in southern Europe, the different species of crotalus (or rattlesnake) in America, possessing poison glands in connection with the teeth or jaws. Scorpions, the females and neuters among honey bees, wasps and bumble bees possess poison glands and a sting at the posterior end of the abdomen; toads and salamanders, wart-like skin glands; hairy caterpillars, many biting flies, gnats and gadflies, salivary glands. There are certain species of fish, like the barbel, whose sexual glands contain a poisonous fluid and whose tins are provided with a poisonous substance derived from the skin glands. It is not certain whether the occasional poisonous qualities of edible mussels, oysters and star fish depend on transient gland secretions (sexual seasons), upon the food of these animals, or whether they arise from bacterial changes of the animal after death.

The changes caused by poisons are partly limited to certain localities, partly connected with general anatomical and physiological changes.*

The poisons may be arranged for classification in four groups, according to their modes of action: (I) Corrosize, locally irritative poisons; (2) Parenchymatous poisons; (3) Hamic poisons; (4) Nerve and Cardiac poisons. Many do not confine their influence to a simple type, but excite lesions and symptoms of multiple character simultaneously.

The corrosive and locally irritative poisons (caustica, irritantia) vary in their results with the dosage and concentration of application and with the character of the tissue with which they come in contact, ranging from simple hyperæmia and inflammation to coagulation, eschar-formation and solntion of the tissues. Such lesions depend upon special properties of the substances, as abstraction of water from the tissues, precipitation or solution of the albumens, formation of precipitates in mucus forming tissues, solution and decomposition of urea, conversion of fats and carbohydrates into acids, as well as production of a variety of chemical changes in the salines of the body or other destruction of the structure of the living protoplasm. Among these caustics and irritants (to the skin or mucous membranes by direct contact) are included the corrosive acids (sulphuric, nitric, hydrochloric, oxalic, osmic, acetic, carbolic, $\rightarrow$ etc.). the caustic compounds of the alkalies and alkaline earths (potassium and sodium hydrox-

\footnotetext{
-The following is taken from the works of Samuel, Zlegler and BirchHirschfeld.

†Carbolic acid or phenol is really an alcohol.
} 
ides, quicklime, barium chloride), the corrosive salts of the heavy metals (salts of antimony and mercury, zinc chloride, zinc sulphate, chromate of potassium, etc.), the poison of the beetle Lytta vesicatoria, cantharidin, snake venom, the poison of the sting glands of bees, wasps and hornets, the salivary secretion of gadflies, stinging flies and mosquitoes, and the so-called acria or acrid medicaments derived from a number of plants (croton oil, mezereum, etc.). A number of poisons, volatile or gaseous in form, may also cause dermal or mucous membrane irritation, especially to the lining of the respiratory tract during inhalation (irrespirable gases). Should these irritant and corrosive substances be absorbed and be conveyed by the lymph paths into the blood and internal organs, they may cause, in addition to their local effects, associated disturbances of the heart and nervous system, the liver and kidneys. Substances inducing degenerations of these parenchymatous organs may be spoken of as parenchymatous poisons, their effects depending chiefly upon alterations of tissue metabolism, regressive nutritive changes and formation of precipitates in the tissues. Among this group phosphorus belongs, capable of causing extensive fatty degeneration of tissues, especially the liver. Corrosive sublimate, chromic acid, cantharidin, which produce marked changes in the renal parenchyma, are also irritants. The production of argyria, the impregnation of the tissues with minute black particles of silver after long continued administration of nitrate of silver, may be thought of as a similar process; and lead is an excellent example of parenchymatous poisons, producing, as it does, a wide range of disturbances, palsies, degenerations and both local and general pathological results.

The so-called hamic poisons act principally by depriving the red blood corpuscles of their power to act as conveyors of oxygen, by inducing their disintegration, liberating and breaking up hæmoglobin or causing thrombosis. Some in addition may cause lesions at the point of introduction, and perhaps directly influence the nervous system. Some of them are gases entering the blood through inhalation, others are in solution and are derived perhaps from the intestine or from wounds. The best known blood poison is carbon monoxide, a constituent of illuminating gas, which enters into combination with hæmoglobin to form carbonoxyd-hæmoglobin, and thus prevents absorption of oxygen by the blood and induces a tissue asphyxia. A characteristic feature of $\mathrm{CO}$ poisoning is the bright, cherry-red color of the blood. Sul- 
phuretted hydrogen (in poisoning from the gas of manure pits) acts partly by paralyzing the nervous system, partly by forming sulphmethæmoglobin, giving a greenish (almost cadaveric) tint to the blood. Hydrocyanic acid and cyanide of potassium also cause a rapid paralysis of the central nervous system, in addition to interfering with oxygenation of the blood cells and tissues (cyanmethæmoglobin, bitter-almond odor in the organs). Potassium chlorate, nitrobenzole, potassium nitrate and amyl nitrite cause marked destruction of red corpuscles and transform hæmoglobin into methæmoglobin, in which the oxygen is more firmly fixed than in the oxyhrmoglobin. The blood, and of the organs in such instances the kidneys especially, take on a striking brown color. Extensive hæmocytolysis, with liberation and solution of the hæmoglobin in the blood plasma, giving a blood-red color to the urine (hrmoglobinuria), are caused by various toxines of fungous and bacterial origin (ptallin, helvellic acid), arsenuretted lydrogen, anilin, nitrous acid (fumes), carbolic acid and other poisons. Coagulation of the blood and its sequels are seen in poisoning with ricin (from the seeds of castor oil plant) and abrin (from the seeds of abrus precatorius).

\section{Infectious Agencies.}

$\mathrm{By}$ the term infection (inficerc, to put into, to inoculate, to taint) is meant the entrance into the system of a disease-producing microorganism capable of self-multiplication, a pathogenic microbe.

Nature is richly supplied with microorganisms; which on the borders between the animal and vegetable kingdoms represent primitive forms of living matter, in their minuteness are visible only with the aid of the microscope and are recognizable as consisting of but single cells. According to their classification as plants or animals, they are spoken of as protophytes or protozoa. There are forms which are only visible with a magnification of 2,000 diameters, and even then as barely perceptible points without definable cellular characters; and there are reasons for believing that still smaller organisms exist, invisible with our present optical instruments, but by no means unreal, being demonstrated as corpuscular entities by other methods, as by their detention in filtration or by inoculation (so-called invisible microbes). 
Even in ancient times the idea prevalled that devastating epidemic discases were caused by a living contagion (contagium cizum sive animatum). The nature of these contagions remained hidden, however, to the physicians of antiquity; and the impossibility of determination led, especially in the middle ages, to the wildest conjectures as to the nature and origin of epidemics. They were attributed to evil spirits, deemed punishments from on High, fancied the results of supermundane powers, of influences of the stars; their origin was sought in conditions of the weather, in magnetic and meteorological processes, in putrid gases and in peculiarities of the soil; and the hidden factor was characterized as a constitutio epidemica or pestilens. It is only about fifty years that our conceptions as to the real nature of epidemics began to becone clearer and an assured foundation became established by precise observation-when, with the aid of the microscope, it became possible to demonstrate the existence of low regetable organisms as foreign and invading elements in the diseased body and to determine the role which they play in the production of disease. In the last few decades this phase of science, stimulated and reorganized by the luminous work of Louis Pasteur and Robert Koch, improved by many technical aids and demonstrative methods, has fully disclosed the developmental history of many infectious diseases. Advances of tremendous significance for the whole of medical science, discoveries and experiences of the greatest consequence in the combating and cure of diseases, have been attained in this line of study.

The demonstration of the relation which a given microbe bears to a given disease has been especially facilitated by the success of artificial cultivation of microorganisms outside the body (in vitro, upon nutritive media) and of production at will at any time thereafter of the infectious disease by inoculation. Such experiments have been made not only in animals, but also in human beings many times: and anyone conversant with the strbject can convince himself by combined cultural and inoculation experiments that certain microbes cause certain diseases. The objection that the microorganisms are not the cause but the accompaniment or product of the disease can easily be proved worthless. Of course there are microbes in the body which have nothing to do with disease, merely surface inhabitants of the integument and mucous membranes, able to penetrate into the 
blood and internal organs only after death of the animal (mostly putrefactive organisms).

Those parts of the human or animal body which are freely accessible are exposed to the entrance of bacteria as of anything else from the exterior. Many of these microorganisms find favorable conditions for existence in the surface of the skin and mucous membranes, live and multiply there without doing harm, finding their nutrition in the secretions and refuse of such localities. They come from the air where they exist in the dust, from food and drink, and, in fact, from any objects in which they exist and with which contact is had. In our alimentary tracts there are always millions of bacteria* and other fungi, in the stomachs of ruminants countless infusoria, all of which partake of the nutrient fluid and aid by certain secretions of their own in the digestion of the food, but have no pathogenic action; they are our table guests (commensualists) and stand in a relation of symbiosis to us (living together for mutual profit). As long as these conditions are maintained and as long as they obtain their nutrition merely from the dead material they may

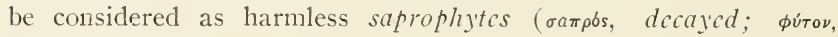
vegetable growth). Their harmlessness is explicable by the fact that these microorganisms are not in any way fitted for attacking the living substance of the animal body, and their metabolism gives rise to no products which might be toxic to the cells and tissues of the animal; or whatever toxines there may be are quickly neutralized by the body secretions. Besides, their increase is held in check by the various adjustment powers of the body. The protective epithelium of the skin and mucous membranes prevents their penetration into the tissues; they are expelled from the air passages by the activity of the cilia of the lining epithelium and from time to time by the expectoration of mucus; many are retarded in their growth by the acidity of the gastric juice and by the intestinal secretions, as well as by the bile; large numbers are expelled with the excrement from the intestines; many dry up upon the surface of the body. Moreover, the various organs and cells, as the liver and phagocytes and the blood particularly, contain substances of antitoxic and microbici-

- A stalned smear preparation should be made from the surface of the gums, tongue or throat of a convenient cadaver as proof of the interesting flora existing there and as evidence of the wandering corpuscles in our cellular constitution. Vid. for fuller consideration Kitt, Bakterienkande f. Tierärzte, IV. Aufl. Wien, 1903 
dal power; and even though they have gained access into the blood, bacteria may by these agencies be rendered harmless.

There must, therefore, be special conditions afforded before a microorganism can become the actual cause of infection. In the first place the microbe must possess peculiar toxic powers. All microbes carry on nietabolic processes and elaborate a variety of chemical substances in the medium in which they exist. If among these products there be any which are harmful to animal tissue, the possibility of toxic action exists. Since Brieger called attention to these substances and indicated their relation to disease occurrence, many of these microbic poisons have been proved to exist by various investigators and the process of infection is recognized as invariably connnected with intoxication.

In one class of microorganisms, the bacteria, poisons have been demonstrated which are apparently free, soluble secretory products of the bacterial cells, and which exhibit strong similarity to ferments. These poisons are as yet not well defined from a chemical standpoint; they are quickly rendered inert by being heated (to $50^{\circ}$ to $80^{\circ}$ C.) and are peculiar in that they do not manifest their activity at once, but require a certain period of incubation. They are, moreover, especially peculiar in their specificity and in the fact that when introduced into a susceptible animal there is invariably a spacific antitoxine gencratcd. They are, therefore, not a homogeneous product of all bacteria, but differ among themselves from their origin and the particular kind of microbe, each as a special product of a special process. These poisons are collectively called toxines.

According to Elrrlich's theory, a toxine is a poison which possesses two specific grotips of atoms, one of which, the haptophore, anchors the toxic molecule to the cellular protoplasm for which it has affinity; the second, or toxophore group, doing the harm, supplying the real poisonous agent. The toxines act then because they enter into chemical combination with the cells. (Vide Chapter on immunity, pp. I8 and 22.)

The proof that a microorganism secretes a toxine and through it produces disease of the animal body is shown by the following: Nany bacteria can be grown artificially upon nutritive culture media, as bouillon. If they secrete soluble toxines these will accumulate in the medium. If such a culture be passed through a filter impervious to bacteria, as a porcelain filter, the bacteria will be retained by the filter and the bonillon will pass through clear and quite free from bacteria. If there follow the injection 
of such a germ-free filtrate (into a suitable animal) evidence of toxic action, especially a specific one, then we must be dealing with a dissolved toxine yielded by the bacteria. The best and simplest example of this is afforded by cultures of tetanus bacilli, the filtrate from which produces typical and characteristic tetanus symptoms, the dry residue from the evaporation of the filtrate acting in the same manner.

A second proof that the immediate agent of bacteria is their soluble toxine may be had by artificially removing the latter from the germs. If water be allowed to run for several hours through the residue of bacteria upon the filter they will be washed free of the toxines and can be inoculated in enormous amounts, by the millions, without giving rise to apparent harm (the inert bacterial cells being quickly destroyed by phagocytosis in the animal body). Yet if these harmless bacteria be returned to a nutritive medium where they can again multiply, they produce poison anew, this collecting in the substance of the bacteria and in the fluid in such quantities that inoculation of even the smallest quantity, a very few of the bacteria, will produce fatal effects.

The virulence of toxines is remarkable; a hundred-thousandth of a cubic centimeter of the filtrate of a tetanus culture is sufficent to kill small animals, and a ten-thousandth of a milligram of the dried substance will do the same: less than one milligram would cause tetanus convulsions in a himman being. Such facts prove that some bacteria are provided with toxic agencies of frightfully dangerous power and explain why, when such causes of disease gain entrance into the human or animal body, they prove victorious in their conflict with the animal cells.

Toxins do not act uniformly upon all animals. Intoxication occurs only when they enter into chemical combination, only in bodies whose cells possess substances (receptors) capable of union and having chemical affinity for the toxine. In bodies in which such receptors do not exist the toxine behaves as an indifferent substance. This explains why certain animals show a natural inmunity against certain toxines and why the lacteria producing the latter are harmless to these animals, as chickens are insusceptible to tetanus toxine.

There are some germs which produce no toxine separable by filtration as a secretion, but whose toxicity is occasioned by substances enclosed in the body of the microbe and fixed there (endotoxines). Some of these substances are of albuminous na- 
ture (toxalbumen, mycoprotein, bacterial protein). They vary in their composition and modes of operation, some being peculiar to certain kinds of bacteria, others common to several forms. In general they act like albumens foreign to the animal, causing inflammatory changes, necrosis of cells and tissues and exciting febrile reactions. The dead bodies of the bacteria act in the same way to some degree, their toxic substances being freed only by the death and maceration of the microorganisms.

A number of bacteria produce pathological changes also by elaboration of acids and gas-forming substances, as sulphuretted hydrogen.

In addition to their chemical action it should be said that mechanical disturbances may be occasioned by bacteria which, in their multiplication, produce masses, perhaps for example obstructing the blood vessels.

The sum total of the pathogenic properties of a microbe is spoken of as its virulence. According to the quality of toxine produced and the energy of growth of the microbe in the animal body, there may be recognized gradations and differences of intensity of virulence of the various genera, species, strains and individual microbes; their power of disease production corressponding with the same factors. Just as in artificial culture in a number of nutrient media a bacterium will either elaborate much toxine and grow rapidly or will produce but little toxine and grow slowly; according to the composition, reaction and temperature of the medium; so in different kinds of animals there is a variation in capability of growth and toxine production of a given microbe. In adaptation to the conditions of nutrition afforded in a given body microorganisms may in greater or less measure lose the power of growth exhibited in some other body, may be altered in their pathogenic power; this is spoken of as change of virulcnce by transmission. Such change may manifest itself either as an attenuation or as an intensification of virulence. The oldest known example of attenuation by transmission is seen in the change of virulence of the germ of smallpox; variola, which in man is severe and marked by pock eruption all over the body, produces in the cow merely a local and mild eruption. In the cow, moreover, even in the first generation, it is permanently so attenuated that after reinoculation in man it gives rise to only a benign local eruption (not a general one). Wide differences of virulence exist between the different strains [growths of the 
same organism from different sources] of the tubercle bacillus; those obtained by culture from the human body have become almost non-virulent for cattle by their adaptation to man, although some strains are readily transmissible. Avian tubercle bacilli are innocuous for mammals (except rabbits) and conversely it is difficult to infect chickens with human tuberculosis. However, Nocard succeeded, by placing human tubercle bacilli, inclosed in collodion sacks containing bouillon, in the peritoneal cavity of chickens, in so adapting their growth to the avian body that thereafter (in later generations of the culture) they became pathogenic for birds as well as men.

In part, at least, the variability in the virulence of microbes of the same species must be recognized as a reason for the occurrence of either a comparatively mild or a severe course of an infectious disease (formerly called genius epizooticus) as well as for its self-limited termination. For example, mouth and foot disease in some of the epidemics which sweeps the country runs an unusually severe course with high mortality, although under ordinary circumstances it is not a fatal disease, recovery taking place in the course of two weeks. The investigations of Loeffler have shown that the virus of this plague, if inoculated from cow to cow, gradually loses its pathogenic powers, whereas if alternately transmitted from cow to swine in a long series the virulence is maintained or even increased. It is interesting, too, that pathogenic organisms may be influenced in artificial culture outside the body so that their virulence may be either increased or diminished (change of virulence by artificial culture methods). Toussaint and Pasteur, who were the first to establish this possibility, have demonstrated that anthrax bacilli, promptly fatal to cattle, sheep, rabbits and mice, may be so attenuated in virulence by artificial culture in bouillon at a temperature of $42^{\circ} \mathrm{C}$. that they produce fatal effects only in mice. Similar facts have since been established in connection with a number of microorganisms capable of artificial cultivation, culminating in efficient methods of prophylactic inoculation (Pasteur).

This is easily understood when we realize that the attenuated germs give rise to but a mild attack of the infection when inoculated, which, however, leaves behind an immunity to the disease. On the other hand, an intensification of the virulence, even of microorganisms ordinarily without pathogenic influence, has been obtained by other cultural methods, as in nutrient media rich in 
albumens and in the absence of oxygen (Wiener, Hueppe). From such considerations it may easily be appreciated that virulence is a very variable property.*

It is also essential for the development of an infectious disease that the infectious agent should be afforded a suitable path of entrance or atrium to the tissues. Mere contact with a microorganism does not necessarily result in disease; there are often pathogenic microbes upon the surface of the skin and mucous membranes, the host in no wise suffering in consequence. The virulent germs of tetanus and of spreading gangrene are very frequently present in the intestinal canal of herbivora and omnivora, but without inducing pathological results as long as the mucous membrane is intact, their toxines being neutralized and destroyed by the digestive juices. So, too, pyogenic and putrefactive microorganisms are found in large numbers in the intestinal contents and externally upon the skin in healthy human beings and animals, becoming pathogenic only in case of introduction into the lymph and blood through some tissue lesion.

The ordinary places through which microorganisms gain access to the tissues are the external skin with its gland pores, the digestive and respiratory tracts, the conjunctival mucous surfaces and the uro-genital passages. The protective epithelium of the skin and mucous membranes interferes with the penetration of most bacteria into the tissues and with their toxic action, partly because of the impenetrable barrier afforded (the horny epithelial layer), partly because the secretions of normal mucous membranes may wash off and destroy the microorganisms and dilute their toxines to such an extent that they are rendered inert or neutralized. This protective means is not an absolute one against some of the bacteria. Some may directly or by growth-extension penetrate the unaltered skin or mucous membranes; or, having gained entrance to sebaceous and sweat glands, especially the glandular ducts or lymph follicles (which are open upon the surface as in the pharynx and intestine), may be carried into the deeper structures by leucocytes (glandular or follicular infection). Many microbes are provided with means of motility (flagella) and are thus able to penetrate into canals, or from a surface may find their way into slight depressions or inappreciable defects in the epithelium, where, after local increase, their toxic metabolic

*See further Kitt, Bakterienkunde fur Tierärzte, M. Perle, publisher, Wien. 1903,4 th edition. 
products naturally come to reach such a degree of accumulation (concentration) that further tissue change is caused and opportunity thus afforded for further penetration by the germs. In this manner rats may be infected by plague by merely dropping upon the unaltered conjunctiva the plague bacteria; and the trypansome of dourine (horse) invades the body by its own motile power through the mucous membrane; various bacteria, if they can gain entrance into a duct in the nipple of the mammary glands, may multiply luxuriantly in the milk and give origin to intense suppurative inflammation.

Microbes gain access to the digestive tract along with food and drink or may be swallowed after being inhaled with dust. The special point of infection (so-called intestinal or food infection) in this case is usually the lymph glands of the mouth and pharynx, or of the intestine; by the same route it is possible that the toxic products of microorganisms, themselves confined to the interior of the intestinal canal, may be absorbed through the blood and lymph channels.

Microbes gain access to the respiratory tract by inhalation (inhalation infection) with dried dust particles or in the fine discharge coughed up by diseased animals and subsequently dried. They are in this case in part retained on the nasal mucous membrane, in part adhere to the pharyngeal surfaces and may thence pass into the lymph follicles and extend by route of the lymphatics or be swallowed (giving rise indirectly to an alimentary infection), or they may be carried by the currents of air into the lungs directly.

Infection of the urogenital mucous membrane usually takes place by transmission of the germs from one to another individual in the course of sexual congress (coital infection).

A most favorable opportunity for infection is afforded by wounds of the skin or mucous membrane (rvound infection), affording access to the microbes into the lymph spaces and channels of the connective tissue, to the subcutaneous and submucous structures, favoring penetration into the peritoneal cavity and eventually into the blood. The wounds may be so small as to be inappreciable to the unaided eye, as some tiny abrasion of the epithelium; or it may be that before the disease actually appears there may intervene a considerable period of time, the wound perhaps long healed, so that the point of entrance is en-

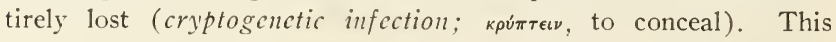


latter is often the case when we are dealing with a follicular infection. The various pathogenic microbes do not possess uniform powers of disease production, of multiplication and dissemination, granted that they gain entrance by one or other of the points suggested. Some give rise to infection, no matter from what point they are introduced; that is, they are capable of activity from various atria (tubercle bacilli). Others require introduction in certain situations in order that they may show pathogenic effects, a fixed point of access. For example, the tetanus bacillus, as already indicated, can only be actively pathogenic from wounds; the germs of vaginal catarrh and epidemic abortion naturally (partly effective also in case of intravascular inoculations) involve only the genital mucous membranes; the bacteria of mastitis invade the lactiferous ducts only; the bacteria of calf diarrhœe confine their activity to the stomach and intestines. These peculiarities probably depend upon the presence at the appropriate points of entrance of especially favorable conditions for multiplication of the germs, other parts presenting certain interfering conditions.

Many germs require some special underlying condition in order that they may live and multiply; For example, the piroplasmata must get into the blood, because they can only obtain their sustenance under the conditions afforded by the living blood and die out in the body fluids. Other organisms, as the colon bacillus and vibrio of Metschnikoff, thrive best in the chyme and intestinal mucous membrane.

Doubtless the condition of the cells and juices of the body play's some part in the question of decelopment of the infection. The biological and biochemical differences in cells and fluids, designated as tissue predisposition and tissue immunity, are indicated by the fact that a given species of animal is by nature completely resistant to a certain microbe and its toxine, surely lethal for some other species, and by the varying rates with which different tissues are involved, as where practically only one of the tissues offers resistance to the growth of the microbes (as the resistance of muscles to tuberculosis) or where but a single tissue is involved. Hence the question of development of an infectious disease depends not only upon the existence of a given essentially virulent microbe, but also upon the defensive powers of the body, upon the antitoxic and microbicidal properties of its organs and 
apon the mechanical obstruction to the penetration of the germ into the tissues (vide pp. I8, 2I).

The interval between the entrance of the microbes into the subject and the manifestation of appreciable symptoms is spoken of as the period of incubation. The length of this stage depends upon the vital peculiarities, the virulence and number of the microorganisms, and upon the site of the infection and the predisposing factors in the animal affected. If the microbes are capable of rapid multiplication and of generating large amounts of toxic material (as the bacteria of chicken-septicremia, which rapidly increase in the circulating blood, or the colon bacteria of mastitis which thrive luxuriantly in the milk of the udder) the period of incubation is usually of but a few hours' duration. Microorganisms of slow growth, as tubercle bacilli and actinomycetes, induce functional disturbances only after the structural changes which they bring about have attained a certain grade of development, and of necessity extend their period of incubation over weeks and months. Before the symptoms are appreciable clinically extensive anatomical changes may in many cases have developed, whence it follows that a discase may be latent or occult in its period of incubation, although if the animal be slaughtered it is clearly seen to have been present for some time. For example, in the case of pleuro-pneumonia in cattle there are often found characteristic appearances of the pulmonary inflammation in animals which have been slanghtered when apparently quite healthy; and in hogs affected with erysipelatous valvular endocarditis of intense grade, the disease may be discovered only on slaughtering, the animals having shown in life no symptoms of a character to have suggested the existence of their disease. In rabies and tetanus, in which the virus causes symptoms only after it has become fixed in the cerebral nervous system [the toxine in case of the latter rather than the germ itself], the disease manifests itself the more rapidly the closer the point of infection to the brain; if the virus be inoculated directly into the brain the incubation lasts but a few days, while in case of ordinary subcutaneous inoculation it may be prolonged to weeks, the virus being at first retained in the lymph glands. Cases of infection of human beings by the bites of rabid dogs manifest rabies in 8-I4 days where the wounds are in the face, but when the hands or feet have been bitten the period of incubation lasts usually one or two months, and sometimes the 
disease does not appear for a year. The influence exerted by the virulenec, the amount of toxine and the particular infectious germ upon the period of infection may be appreciated after introduction of tetanus toxine into mice: the simptoms of the disease, depending upon the dosage, appearing after varying intervals from 12 hours to $2-6$ days.

The dissemination and multiplication of pathogenic germs in the body exhibit a number of peculiarities. Siome micro-organisms remain closely confined to the immediate vicinity of the point of infection, giving rise from this localized situation to toxic results in proportion to their own disintegration and to the abstraction by the fluids of the body of their toxic substances. Thus they may cause lesions only in the neighboring tissues or induce intense general disturbance after absorption. The tetanus bacillus. for example, does not grow free in the body (except in the uterine carity, where, under anærobic conditions, the organism can accumulate in large quantities after having once been introduced) : in the course of a clay or two after inoculation it can no longer be found at the site of infection (wound). nor elsewhere in the body. It disappears because of its disintegration and through the agency of the phagocytes. Its toxine, however, is absorbed and causes the ganglion cells to undergo necrobiotic changes. Often bacteria, as the pyogenic organisms, increase by multiplication at the site of infection, but are prevented from further extension by the defensive properties of the body.

For the most part the germs are distributed from the point of infection along the lymph channels, in part at least because the newly developed germs are produced about the borders of the focus, are swept away in the lymph plasma or are taken up by the leucocytes and carried elsewhere. The local focus (local infection) enlarges directly into the surrounding regions (regional infection) ; and the microbes, conveyed by way of the lympli channels, are carried deep into the tissues to the lympl glands. from one site to another, from one lymph gland to another, until they finally reach the blood. They may also gain direct entrance into the capillaries. i'cins and arteries from the point of infection (as in wounds or ulcers, or by direct extension by growth through the vessel walls) and be carried along with the blood current (cmbolic or hamatoscnous infection). There thus are produced at a distance from the point of entrance or primary focus of infection new deposits of the infectious agents: and either new local areas of disease, 
secondary metastatic foci of infection, are caused, or the microorganisms are widely disseminated throughout the whole body (general infection, bacteriamia). As an illustration, streptococci of epidemic coryza in the horse gain access to the pharynx with the drinking water or with the inspired air; they here first give rise to a purulent catarrhal inflammation, penetrate by direct growth into the pharyngeal follicles, gradually make their way along the Eustachian tubes to the middle ear and here set np a similar purulent inflammation. Being carried away by the lymph current and by leucocytes, they give rise to abscess formation in the retropharyngeal lymph glands, and by going lower, in the cervical nodes; and, too, they may be swallowed and infect the mesenteric lymph glands as they pass along the chyliferous tract. Passing from the lymph glands to finally reach the blood, they are disseminated by the latter generally throughout the body, and cause a variable number of metastatic abscesses in such situations as the brain, lungs, liver and kidneys, eventually a septico-pyæmia. In similar way infection of the umbilical vein in the new-born by pyogenic bacteria causes primarily a local abscess-formation at the umbilicus, followed by multiple venous-embolic abscesses in the liver, later in the lungs, the joints and elsewhere.

It may happen that at the point of infection the local lesions are so slight as to be appreciable only by microscopic study, that the microbe passing from this point first lodges in the lymph glands or may perhaps even pass through several lymph-nodes without occasioning any disturbances, multiplying and manifesting its influences only after it has gained access to the blood and circulated in the body and become fixed in this or that position at a distance from the original site of entrance. The tubercle bacillus, for example, may be absorbed from the intestines without causing any alterations in them, but giving rise to caseation of the mesenteric lymph-nodes; or these may be traversed by it, the germ gaining access to the blood, and perhaps first deposited in the bone-marrow, where it gives origin to the primary tuberculous focus.

The physiological movements and changes of position of the intestines and muscles aid in great measure the spread and distribution of the invading and multiplying microorganisms: pyogenic microbes which have gained access to the peritoneal cavity are apt to be spread over the whole peritoneal surface by the peristaltic movement of the intestine, the resulting peri- 
tonitis as a consequence being generally a diffuse one. Tubercle bacilli having effected an entrance into the pleural or pericardial cavities multiply in the lymph and are actually rubber into the whole serous surface by the movements of the heart and lungs. For this reason there usually occurs in these regions a disseninated, dense eruption of tubercles involving the whole pleura, pericardium and epicardium. So, too, currents in the secretory fluids on mucous surfaces are favorable to the dissemination of microorganisms; and the covering of these surfaces permits migration and generalization, especially of the motile forms. Tubercle bacilli may thus reach the larynx in the bronchial secretions from eavities in the lungs, and if retained may oceasion laryngeal ulceration; pyogenic bacteria from the pharynx may pass to the Eustachian tubes and middle ear in horses; and in case of infection of the milk ducts of the nipple the different kinds of bacteria in the milk and milk passages advance into the parenchyma of the udder and give rise to mastitis.

The pathogenic action of bacteria is, as already indicated, for the most part a toric process, but also in some degree a mechanical one; it is governed both by the propertics and the quantity of the cxtremely zaried metabolic products elaborated by the individual microbes, but is also dependent upon factors determined by the place of infection and by the predisposition of the tissucs. Local and general effects are distinguishable; first by the fact that at the original point of infection and at metastatic localities anatomical lesions are originated (inflammations, degenerations, necroses, proliferations, depending upon the nature of the microbe, both in the sense of a mere foreign body and of the peculiarity of its constituent materials); and, second, by the development of general metabolic disturbances, especially fever, from the generalization of the infectious agents and their products. In each case the phenomena are expressions of tissue reaction to the noxious foreign microbe undergoing multiplication from time to time in the system (v. chapters on fever, limiting inflammation). Each kind of pathogenic microorganism manifests a fircd mode of action, determined by its manner of multiplication in the tissues and the particular nature of its toxic product (specific aciion), and therefore produces a spccific discasc. In case of other causes of disease there is no uniformity of action, but, on the contrary, a variability; or, better, it may be said that other or non-infectious diseases may occur in the same form from the 
effect of a variety of agencies. A nasal catarrh may be caused by the inhalation of dust, irritant gases, chemical fluids, the influence of cold upon the external surface of the body, or from infection: cold may produce in one subject painful peristalsis (colic), in another muscular rheumatism, in a third a coryza, a pneumonia, an intestinal catarrh. In infectious cliseases the body generally reacts with uniformity in a definite manner: the basic symptoms and lesions are invariably manifest, and it is possible to decide from the presence of certain signs of the disease that a definite type of infection exists. By specificity, therefore, we understand that each infectious disease is the result of fixed infectious agents and not of anything else. Anthrax is always caused by the anthrax bacillus, and never by any other microbe or noxious agency of any sort; glanders is caused by the glanders bacillus; smallpox by the smallpox contagium. There are, however, some micro-organisms which, because of similarity of toxic properties and analogous modes of multiplication, give rise to the same anatomical tissue changes, so that clinically the same anatomical types of disease are produced by several kinds of germs (the groups of the pyogenic bacteria, the micro-organisms of septicemia, of moist gangrene and of mastitis). Such infectious diseases are said to be polybactcrial.

I ariations in the duration of infections discases, modifications and degrees of anatomical changes, are proportioned in the seicrity of the infection to the grade of cirulence of the microbes, and are dependent as well upon the place of cutrance of the infection and the predisposition of the tissues. Just as a corrosive substance, an acid, depending upon whether it comes in contact with animal tissue in concentrated or dilute form, produces either an eschar, tissue necrosis, inflammation or mere lyperæmia, so a difference is appreciable in the action of microbes according as they are highly toxic or more or less attenuated. The importance of the factor exerted by the place of entrance of the infection is indicated by the studies of Arloing, Cornevin and Thomas upon the bacillus of symptonatic anthrax ("black leg"); this microorganism, if inoculated into the muscles and subcutaneously, kills animals after intense symptoms; but if the inoculation be made into the trachea or intravenously there follows merely an abortive course, which, however, is succeeded by an immunity. Variations of susceptibility and resistive powers in different animal species and individuals to precisely the same infectious agent may cause 
marked variations in the clinical and anatomical picture of the same infectious disease. Thus a certain strain of glanders bacilli has been known to cause in a horse a chronic glanders lasting over several years, but in guinea-pigs and field mice a very acute type of the disease; the bacillus of hog-erysipelas, producing in one hog an acute septicæmia, to cause in a second a slight urticaria, in a third a chronic cardiac valvular affection. The influence of tissue resistance upon the disease picture is especially apparent when inoculations of the organisms of chickencholera of high grade of virulence are made into rabbits previously treated by sertmin injection. Rabbits which have not been previonsly subjected to serum injection succumb to hypodermic inoculation of but a small number of bacteria as early as from 12 to 24 hours, dying of severe acute septicamia; thosc previously injected and then subjected to the same inoculation die only after 5 to $\mathrm{I} 4$ rays, and then show extensive purulent phleginons, purulent and fibrinous pleurisy and pericarditis, or merely an anæmia as a result of the prolonged infection.

Recovery from an infectious disease usually leaves thereafter a certain degree of immunity (v. active immunity, p. 27).

Sometimes several kinds of pathogenic organisms gain entrance at the same time through one point of infection, the resultant infection being thus a complicated one (mi.red infection); or after the individual is attacked by one infection other types may invade (secondary mired infection). The former condition is seen when the infective matter from the beginning containcd seieral zarieties of pathogenic germs, as where a wound is contaminated with earth containing both spores of tetanus bacilli and pus bacteria, or where a cow's nipple is soiled with filth and dung in which there are streptococci and colon bacilli. So, too, microorganisms which have existed indefinitely mpon the surface of some mucous membrane may, if occasion presents, penetrate into the tissues along with some other type of infection and combine with the latter in producing pathogenic effects; thus, should there be produced by some foreign body an injury to the tongue or pharynx of the cow, the various putrefactive organisms living as saprophytes in the mouth may occasion with pyogenic bacteria a mixed infection. The second form of mixed infection, the successive incasion of a second or third pathogenic germ, may be noted where local changes from a primary infection bring about conditions which facilitate the access and growth of other microbes: as 
when ulceration has occurred in the primary infection and the protective epithelium has been lost, or when in the course of a disease the bactericidal substances normally present in the fluids of the body have been exhausted and the resistance of the tissues reduced in consequence. Not infrequently such mixed infection is to be seen in hog-cholera, the micro-organisms of which cause necrotic and coagulative destructive changes in the intestinal mucous membrane and general wasting; there is thus prepared so suitable a culture medium for the sputum bacteria and necrosing bacilli present in the pharyngeal mucus and in the intestinal contents that these readily penetrate into the tissues and materially contribute to intensify the disease and hasten a fatal termination. Admixture with or contamination (association) of an infection by one or more other types of pathogenic microbes, each with its special properties, and the combined action of all, as a rule causes a severe course of the infectious disease and occasions unusual pathological processes (complications).

The mere presence and microscopic recognition of several types of microbes in any focus of disease or in the tissues of the cadaver by no means warrant, without other knowledge, the assumption that there has existed a mixed infection; after the death of the animal great numbers of saprophytic organisms living on the surface of the mucous membranes wander into the organs, and necrotic or ulcerating foci in the lungs, intestine or skin are apt to harbor the same sort of organisms from the air or food (so-called symbiotic bacteria). The association of such essentially nonpathogenic germs, or, too, of pathogenic microbes with another pathogenic variety, may (as especially pointed by Leclainche-Vallée in studies on symptomatic anthrax) determine actual infection; for example, a symbiotic bacterium may prevent phagocytosis by a negative chemotactic power and thus produce conditions favoring the increase of the other infecting agent. This should not be interpreted as meaning that a given microorganism cannot alone cause its special disease, and that only when in association with other microbes can produce its effect; it merely applies to special conditions in which the specific infectious agent is attenuated or is situated in an unsuitable point for infection. All known pathogenic microorganisms are individually capable of giving origin to their peculiar infections, and each is the specific bearer of such infection.

Those micro-organisms regarded as pathogenic may be classed in two groups: In the first group are microbes usually living in the external world, but inducing disease in the animal body if by accident they gain access to it (ectogenous infectious agents, facultative parasitic microbes). The diseases arising from this group affect individual animals here and there, or may attack a 
number, provided there be opportunity of convection from their habitat in the soil to the animal body with water, food, etc. They are essentially of tclluric origin, and it is customary to speak of

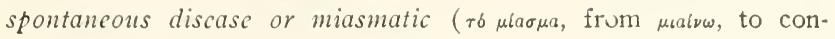
taminate) origin in this connection. In the second group are included microorganisms which, being exclusively adapted to the animal body from an indefinite period, live and thrive in it as their habitat (entogenous infcctious agents, obligate parasitic microbes). Diseases occasioned by these do not appear as spontaneous infections, but occur only when a human being or animal comes in contact with a previously infected individual or with desquamated or excretory material from such an individual in such manner that the infectious agent can pass to the former. Such instances are spoken of as contagious infectious diseascs. [Among many there is to-day a strong tendency to deny the existence of pure miasmatic infections, and thus to regard the terms contagions and infectious as synonymous, such persons holding that the so-called miasmatic diseases are caused by organisms which are essentially facultative parasites, and in more or less direct manner have come from previously diseased individuals. The difference of view is by no means a vital one, and the author's recognition of their immediate derivation from a source other than a previonsly diseased individual is correct. In the sense that these facultative parasitic microbes once affecting an individual may be further transmitted, his division, however, becomes unnecessary, since in this manner all infectious diseases are contagious.]

A certain number of infectious maladies may be acquired in both ways; originating primarily from the soil, the microorganisms multiply in the animal body and then are transmitted from the diseased to other individuals (contagio-miasmatic diseases).

The fact that some microorganisms thrive only in the animal body and die when in the external world is a phenomenon of adaptation. It may be assumed that all these obligate parasitic germs originally lived a free saprophytic life in nature, but having accidentally gained access to an animal body thrived therein and by rapid succession of generations usual to such low organisms acquired a special adaptation for the conditions thus afforded and lost their faculty of propagation outside.

Changes in nutritional conditions are likely to cause changes in their metabolism, and the influence which invading microbes have 
upun the tissues of the body depends in part upon their metabolic activity and metabolic products. From investigations of IViener it has been shown conclusively that a type of bacteria common to the human intestine and regarded as entirely harmless in this situation and in the excreta, can by special nutritive influence be so transformed as to acquire highly toxic properties (the so-called colon bacilli, living as saprophytes in the colon). If fed ordinarily to rats they give rise as a rule to no disturbances whatever, but if the colon bacilli have been cultivated for a few days under anærobic conditions on a medium specially rich in albumen (eggs) they acquire an intense virulence, and if fed to rats produce a serere and almost invariably fatal enteritis. Although sucin transformation of a non-toxic saprophyte into a toxic pathogen cannot off-hand be accomplished with other micro-organisms, this isolated fact proves that it is possible for infections of a claracter new and unknown to us to arise from time to time; and as a matter of fact spontaneous infectious diseases do now and again appear which lave not been previously observed and which are to be regarded as rarities. It is a pure accident when the special germs are brought into contact with the human and animal body : and it is further conditional upon special circumstances of nutrition whether the microbe possess virulent properties, as in case of the meat intoxications caused by various microorganisms, sausage poisoning, diarrhœea and vomiting and wound infections.

Some infectious diseases are of very frequent occurrence because the agent is widely disseminated in nature or because opportunities for transmission to the animal body are often afforded. For instance, the tetanus bacillus exists in the soil in many localities, and wounds are often contaminated by dirt, the opportunity for tetanus infection being correspondingly common. Such widespread microorganisms and their infections diseases are spoken of as ubiquitous. Is the alpine rose and reindeer moss, palms and cacti flourish only within certain geographical limits, and as poisonous snakes and the different kinds of insects have their habitats only in certain parts of the earth; so certain microbes occur only in certain lands, where alone, too, the corresponding infectious diseases arise as spontaneous affections. Such districts are foci in which anmually a certain number of cases are likely to occur, and it is said that the disease is established in such places (indigenous, endenic). Truly contagious diseases may also be endemic, provided in a certain district (irrespective of soil 
conditions) there are always a number of animals suffering from the special infections disease, so that others from these may in turn acquire the affection. When an infectious disease occurs in isolated cases here and there it is spoken of as a sporadic disease; if many or great numbers of individuals are affected, it is spoken of as a plague, an cpizootic (epidemic); if it extend over large areas, 'and if not merely one species, but many, are affected, it is called a panzootic (pandemic). Inimal diseases transmissible to man may be spoken of as androzoonoses.

The separation of spontaneous from contagious infections, the recognition whether a microbe is miasmatic or purely contagious, is of importance in dealing with epidemic diseases; the purely contagious diseases can be eradicated by measures protecting the well from the diseased animals and their excrementitious matters. This has been demonstrated in the extirpation of cattle plague, pleuro-pneumonia and hydrophobia from districts in which formerly they were rife.

SLMIMART OF THE MOST IMPORTANT INFECTIOLS DISEASES AND THEIR AGENTS.*

\section{Bacteriaemias, Septicaemias.}

Bacillus arisepticus (Bact. ariscpticum s. aricidum), septicrmia of birds and rabbits (chicken cholera).

Bac. pleuriscpticus, sporadic and epidemic septicremia of all domestic animals (bac. boriscpticus, suisepticus), deer and cattle plagues, pasteurcllosis boris.

Bac. anthracis, anthrax of domestic animals and man.

Bac. adematis maligri, spreading gangrene of domestic animals and man.

Bac. sarcophysematos bocis, symptomatic anthrax of cattle.

Bac. gastromycosis ci'is, bradsot of sheep. [Bradsot is a disease of sheep in northern Europe, by many regarded as a form of symptomatic anthrax.]

Bac. pestis tarandi, reindeer plagne.

Bac. rhusiopathice suis, swine erysipelas.

Bac. suipcstifcr, hog cholera.

Bac. pestis bubonica, human bubonic plague, transmissible to swine and rats.

*For detalled description and technique of investigations, v. Kitt, Bakterienkunde $f$. Tieräztc, IV. Aufl, Wien, 1903. 


\section{Toxic Infections.}

Bacillus tetam, tetanus in all animals and man.

Bac. botulinus, sausage poisoning in man.

Bac. enteritidis, meat poisoning in man.

Bac. dysenterice zitulormm, diarrhœa in calves.

Vibrio Metschnikovi, vibrio cholera of chickens [microspira Metschnikovi, irregular].

\section{Inflammatory and Pyogenic Organisms.}

Staphylococcus pyogcnes aurcus (albus, flazus, citreus), suppuration of wounds, furunculosis.

Streptococcus phlogogenes sive pyogencs (various forms), suppuration of wounds.

Micrococcus tetragenus, umbilical suppuration in calves.

Bacillus mallci, glanders in horses and man.

Bac. lymphangitidis ulcerose, lymphangitis in horses.

Saccharomyces farciminosus, turunculosis in horses.

Strcptothri.r farcini bor'is, furunculosis in cattle [Nocard's psendotuberculous nodules in the skin and viscera of cattle].

Streptococcus cqui, contagious coryza of the horse.

Bacillus acncos equi, contagious pustular dermatitis of horse.

Bacterium phlegmasice ubcris (a number of varieties), mastitis in the cow.

Streptococcus mastitidis (a number of varieties), mastitis in cow,

Micrococcus mastitidis gangrenosa or'is, mastitis of sheep.

Bacillus pycloncphritidis boris, nephritis of cow.

Bac. Pyocyancus, purulent inflammations.

Bac. coli communis (a number of varieties), various forms of cellulitis, mastitis, nephritis and enteritis.

Botryomyces ascoformans, suppuration and granuloma formation in horse.

Cladothrix canis, various suppurations in the dog.

\section{Diphtheritic Necroses.}

Bacillus necrophorus, traumatic necrosis, multiple coagulation necrosis of mucous membranes, liver and lungs in cow, horse and sheep.

Bac. diphtherice columbarum, pigeon diphtheria. 
Bac. diphtheria azimm, chicken diphtheria.

Bac. diphtheria hominis, diphtheria in man, exceptionally in cats and birds.

\section{Tuberculosis and Actinomycosis.}

Bacillus tuberculosis, tuberculosis of man and the lower animals (varieties in birds and cold-blooded animals).

Bac. bronchiolitidis vituli, cheesy psendotuberculosis of the lings in the cow.

Bac. pseudotuberculosis oris, in sheep.

Actinomyces bocis, actinomycosis of cow (with varieties).

Streptothrix capre, pseudotuberculosis of the goat.

\section{Specific Diseases of the Sexual Organs.}

Bacillus abortizus iaccarum, epidemic abortion of cows.

Micrococcus abortious equi, epidemic abortion of mares.

Streptocncous a asinalis accarmm, contagious vaginitis of cows.

(Trypanosoma equiperdum s. flagclose). dourine in horse.

[Spirochata pallida, syphilis in man?]

\section{Pleuropneumonia.}

The infectious agent of contagious pleuropneumonia of the cow.

\section{Epidemic Diseases Caused by Ultramicroscopic Agents.}

Contagium of foot and mouth disease, in cattle. swine, sheep, and transmissible to man.

Contagium of cattle plague.

Contagium of smallpox in man, transmissible to cow, horse, rabbits.

Contagium of sheep pox.

Contagium of rabies of $\log$. transmissible to man and all mammals.

Contagium of Lombardy chicken plague.

\section{Diseases Due to Flagellata, Piroplasmata and Sporozoa.}

Trypanosoma Eransi, surra in domestic animals in India.

" Brucei, nagana in African animals.

. cquiperdum, dourine in horse. 
Trypanosoma Theileri, African cattle plague.

[ . Gambiense, African sleeping sickness in man.]

Plasmodium malaric equorum, African equine malaria.

[Plasmodium of human malaria-tertian, quartan and estivoautumnal types.]

Piroplasma bigeminum, epidemic hæmoglobinuria of cattle, deer and sheep.

Piroplasma caninum, infectious jaundice of dog.

Sarcosporidia major and minor, parasites of muscle and cellular tissue in swine, cattle, sheep, goats and horses.

Coccidium oriforme, dysentery of cow, intestinal and biliary duct parasite of rabbit (also in man and $\operatorname{dog}$ ).

\section{Diseases Due to Mould Fungi.}

Monilia candida, thrush in man and birds.

Trichophyton tonsurans (numerous varieties), bald patches in cow, man and dog.

Achorion Schönlcinii (numerous varieties), favus in man and lower animals.

Dermatomyces gallinarum, scab of cock's comb.

Aspergillus fumigatus, pulmonary mycosis in birds (exceptionally in horse and cow).

\section{Animal Parasites.}

Food and conditions favorable to the existence of a variety of animals and plants are afforded by the human and animal bodies, both in the fluids and tissues, and, too, in the nutrient ingesta and in the excretory and waste matter. When some organism both acquires its nutrition and resides upon or in the body of another individual the condition is spoken of as parasitism (mapà along with; Gĩos, food). A parasite, therefore, is a form of life which either transiently or permanently lives upon or in the body of another organism for the purpose of obtaining its nutrition therefrom (Braun, Heller).

According to their origin from one or other of the two kingdoms in nature, they are separable into vegetable and animal parasites; those which live on the surface of the body are spoken of as ectoparasites or extcrnal parasites (epiphytes, epizoa), those whose habitats are within the body as internal parasites or entoparasites (entophytes, entozoa).

In the preceding chapters the discussion concerned infestment 
by microorganisms appreciable only with the aid of the microscope, the protoplyytes and protozora, a condition spoken of as infection when leading to disease. Some of these microbes well merit the name parasites. since the animal body in which they exist is the sonrce of their nutrition, but the toxic properties of the most of them are so conspicuons that we are likely to think of these not so much as parasites, but as comparable to poisonous plants.

A number of forms of worms and arthropoda (zooparasites) have gradually assumed the character of true parasites and actually live and subsist upon the body structures. Occupation of the body, either externally or internally, spoken of as inc'asion (incadere, to penetrate). is apt to give rise to a varied group of tissue alterations and disease processes (invasion diseases [infestment]). The individual in which such a zooparasite finds shelter is to be looked upon as the host of the parasite. Some infest the host only for a brief period as necessary for the acquirement of nutrition (transicnt or temporary parasites), or for the time essential for the completion of onc or more stages of their development, among which groups respectively fleas and the larve of gadflies are examples. Others are persistent and pass their entire existence as parasites (habitual or permanent parasites). The life of this latter type may be passed in some instances entirely in one host (mono.tenous parasites: $\mu$ bvos, one: $\xi \epsilon$ tvos, host), or may be distributed over several hosts (heteroxenous parasites).

Invasion of a host at times is merely the result of accident, in which case the parasite is nsually taken in with food: in other instances this or that parasite may by its own independent movements gain entrance into the body.

Parasitism may be referred to two underlying conditions, adaptability of the parasitic organism to the conditions of life afforded by the body of the host and the biological variability of the former. Worms and arthropods which have assumed parasitic characteristics undoubtedly at one time lived independently in nature. It may be imagined that one of these low types of animals by accident entered the body of some higher animal, and, finding conditions favorable for the acquirement of food, remained and multiplied; that the offspring, now native to the body of the host, did not again seek a free state, but continued as parasites, being from birth accustomed to the altered environments. Such supposition is justified by the fact that some parasites still show rudi- 
ments of organs worthless to them in their present form of life, but which were evidently inherited from their free-living ancestors. For example, in linguatula tcuioides, a permanent parasite of the nasal fossæ of the dog, there are rudimentary feet, probably better developed in the free-living predecessors, but stunted in these parasitic descendants from lack of 11 se. In the same way may be explained the absence of wings in parasitic insects; for example, sheep lice have probably developed through atrophy of their wings from disuse of these organs, for there are allied species of insects which are not parasitic and possess wings. On the other hand, gradual adaptation to life upon an animal host leads to the acquirement of new peculiarities. on the part of the parasite, as a transformation of masticating mouth parts into piercing and suctorial organs and the development of special types of organs for attachment and holding. Such adaptations and transformations of structure are of such common occurrence throughout nature, even in case of the human body (formation of a horny sole on the back of the foot, originally soft, in cases where from some disease the dorsum of the foot is turned down and walked on; transformation of the toes into prehensile organs by practice), that, as it is true in the higher animals, there is no wonder that it should occur in these lower invertebrates whose tissues are much more capable of such adaptive growth. The newly acquired characteristics become fixed in the succeeding generations because of heredity and because of uniformity in the vital conditions about them; some species which have gradually assumed parasitic nature are finally restricted entirely to a parasitic life and are unable to live in the external world; others pass only a part of their lives, a definite developmental stage, as parasites, and for the rest of their time live independently. There are certain filarial worms which ordinarily live in moist earth, but which now and again, as necessity arises, become parasitic. As the moisture disappears these worm retreat into depressions where the moisture is longest retained, but should a snail or earth-worm happen in their way they pick out the cavities of these animals and creep into them. In protracted drouths they are parasitic for a correspondingly long period, and it has been observed that in such parasitic existence they attain unusual size and produce a larger number of ova than their free-living fellows (Braun, Heller). It may be that the young brood continue in the snail and become completely transformed into parasites, but the original ones, when 
wet weather returns, abandon their parasitic hiding places. L. Oerley has succeeded in artificially causing a free species of worm (rhabditis pellio) to live temporarily as parasites by introducing them into the vagina of mice, where they continued to live and multiply, although when placed in the digestive canal they died or were expelled. Larvæ of flies often resort to parasitism as opportunity is afforded; meat flies and other insects which are ordinarily saprophagous often oviposit upon some animal in wounds or ulcers and the resultant maggots obtain their nutrition in the same location as parasites, although succeeding generations continue as usual under other conditions of existence. It may be supposed that frequently such assmmptions of a parasitic existence are brought about by the force of circumstances and end with the death of the organism if it be incapable of adaptation, cven if it should succeed in getting nourishment from the body of the animal host. Only in case the intruding worm (or other form of life) can successfully withstand the mechanical and chemical influences which oppose it, only in case it obtains a habitat where it is possible for it to live, is it likely to be changed into a parasite.

Some worms may be parasitic in different animal species; trichinella is parasitic in man, hog. rat, mouse, cat, fox, polecat and bear, and may be artificially induced to infest the dog, rabbit, guinea-pig, sheep, horse and other mammals. In the same way the liver fluke is found in a number of species. Other parasites, on the contrary, occur in only one fixed species. For example, tania solium and tania saginata are found only in the human intestine: crassicolis only in the cat: in the intestine of another species they die and it is impossible or only an exceptional case that they should persist. Such exceptions, however, do occur naturally as well as in artificial attempts to infest. For example, bothriocephalus latus, whose normal host is man, is occasionally found in the intestine of the dog; echynorhynchus gigas, a parasite of the intestine of the hog, is in rare instances met in man. Such cases are to be explained by exceptional opportunities for transmission.

The influence of parasites upon the host varies much according to their habits and situation. In many instances the presence of a parasite occasions no noticeable disturbances; dogs may have dozens of tapeworms in them without showing any evidence of sickness, although in other cases even a small number of the same tapeworms cause digestive disturbances. At one time it was supposed that some parasites were of use to the host, as the larvx 
of one of the gastric flies (gastrophilus salutaris-so named from this idea); it was thought that their presence in the stomach and small intestine of the horse promoted the secretion of gastric juice; or it was believed that some parasites consumed superfuons mucus. Althongh it is reasonable to suppose that some parasites obtain their nutriment from the waste matter (as is true of the infusoria and lacteria always present in the stomach in ruminants) and take part in the breaking up the intestinal contents. yet it is not to be concluded that the parasitic worms and arthropods have any value whatever to their host. On the contrary, the animal host, if rid of them. will thrive equally and better. All parasites exert from time to time some harmful influence, and some are death-bearing guests.

In the first place, many parasites are harmful to their host and productive of disease $\mathrm{ky}$ their wandering in the body and their movements. Undoubtedly the itching caused by the crawling parasites inhabiting the skin-surface, and often giving the host no rest day or night, has some influence upon the health; here may be mentioned the sheep-lice, itch-mites and the oxyurides inhabiting the rectum and causing continual tickling sensations, as perfect torments which often give rise to emaciation (due to the increase of metabolism following the incessant restlessness from the itching). Other parasites make their way through the tissues by boring, and thus where any considerable number penetrate into the organs cause tissue destruction and occasion hæmorrhages and inflammatory lesions (lesions produced by liver flukes, larval tapeworms and linguatulidx). They may give rise to a great variety of disturbances, just as any foreign bodies in a mechanical way, by compression of tissues, or by obstruction and narrowing of tubular passages, as sudden death by invading the brain, paralysis by pressure on the spinal cord, atrophy of the liver by growth in its parenchyma, blindness by penetrating into the eye, rupture or thrombosis of the larger blood vessels, respiratory interference and suffocation by lodgment in the air passages and lungs, and intestinal obstruction. The parasites adhering to the intestinal mucous membrane by hooklets or suctorial apparatus often produce reflexly marked irritative or perhaps nervous phenomena; and, too, a toxic influence is to be ascribed to the metabolic products of a number of worms which may be reasonably thought to explain the occurrence of nervous changes and general loss of flesh (as in 
case of echinococcus cysts, bothrioceplialus and truiæ**). Local inflammatory lesions of the skin and mucous membranes are the result of mechanical injuries caused by a variety of parasites.

The amount of nutritive substance abstracted by parasites from the host is to be regarded as ustually relatively insignificant. Where, however, the parasites are blood-suckers, the host is placed at special disadvantage; the smallest of the round worms, if present in large numbers, may cause serious loss of blood, and perhaps the death of the host. The roe, the common deer and the sheep, for instance, are miformly killed by an anæmia if bloodsucking strongylide infest the remnet-stomach. Whole flocks of ponltry may die out when the poultry mite, dermanyssus avium, gets into the hennery and infests the skin of the fowls in vast numbers, feeding daily upon the blood.

A century ago our appreciation of the life history of the parasites and of the diseases caused by them was but vague. It is true that most of the worms fotind in the human alimentary canal were known to the students of natural history in antiquity: and as early as the seventeenth century itchmites and their relation with the itch were fairly well known. Yet it was for the most part believerl that parasites had their origin in inspissated juices or pathologically altered parts of the human or animai body by a process of spontaneous generation (gencratio aquizoca). When the microscope canc into use and it became possible to closely observe their generative organs and their products (eggs), and particularly after attempts came to be made to artificially transmit the parasites by experimental feeding of the worms and their larve, the real history of their development was for the first time apprehended. Redi and Swammerdam, van Doeveren and Pallas, Pastor Goeze, C. Fr. Müller. Bojanus, Abildgard, toward the end of the I8th and the beginning of the Ioth century, Steenstrup, v. Baer, v. Siebold, van Beneden, Küchenmeister, in the middle of the latter century, corrected the mistakes and hiatuses of the earlier views and investigated the most interesting parasites; and in recent years the studies of a large number of zoologists, physicians and veterinarians (Leuckart. Zenker, M. Braun, Peroncito, Grassi, Railliet, Heller, Zürn, L. G. Neumann. v. Rátz and others) have contributed to extension of our knowledge in this field to such a degree that pathology and practical hygiene nave been decidedly benefited by their investigations.

* Persons handling ascaris megalocephala, the round worm of the horse, especially if engaged in mincing it, are apt to be directly affected by a conjunctivitis and urticarial eruption from a certain substance pxisting in the cutlcle of the worm (apparently a dermal secretion) whlch possesses irritatirn propertles. 
TABLE OF PARASITIC ARTHROPODA OF THE DOMESTIC ANIMALS.*

\section{Insects (Insecta).}

1-Blood-sucking Flies, attacking the Skin. (After Neumann.)

Culex pipicns (and other gnats) in man.

Simulia reptans, horse; mucous membranes of the head.

" maculatum, horse, ruminants, man.

Tabanus bovinus, morio, autumnalis, bromius, rusticus, fulvus, albipcs, horse, ruminants, man.

Hamatopota pluvialis, horse, ruminants, man.

Chrysops cacuticns, horse, ruminants, man.

Stomoxys calcitrans, horse.

Hamatobia stimulans, ferox, irritans, serrata, horse, ruminants.

Glossina morsitans, horse, ruminants.

Hippobosca equina, horse, ruminants, dog.

Mclophagus ovinus, sheep.

\section{2-Flies Parasitic Upon or Beneath the Skin in Larval Stage.}

Calliphora vomitoria, various domestic animals.

Sarcophaga camaria, magnifica, ITohlfarti.

Lucilia Casar, scricata, maccllaria.

Ochromyia anthropophaga, dog, cat, goat, man.

Hypodcrma lincata, sheep [cow].

" tarandi, reindeer.

" bovis, cow (even in the spinal canal).

" silcnus, horse, ass.

Dermatobia noxialis, cow, dog.

\section{3-Flies Whose Larvae are Parasitic in the Intestines and Body Cavities.}

Gastrophilus equi, stomach and pharynx of [horse].

" pecorum, stomach.

"duodenalis, duodenum of horse and exceptionally in dog.

- Details of the life-history and the pathological significance of the species enumerated may be found in Kitt's "Lehrbuch der Speziellen Pathol. Anatomie der Haustieve," [See also Friedberger and Fröhner, Fetcrinary Patholon!, Amer. Edition, W. T. Keener \& Co., Chicago, 1904.) 
Gastrophilus hamorrhoidalis, stomach, rectum of horse.

$$
\text { " flavipes, stomach of ass. }
$$

Oestrus oris (Cephalomyia oris), nasal and brain cavities of sheep.

\section{4 -Fleas.}

Pulex irritans, man, dog, cat.

" serraticeps, dog.

"goniocephalus, rabbit.

" avium, chickens, pigeons and other birds.

" penetrans, seu Sarcopsylla penetraits, man, domestic animals (tropical).

Sarcopsylla gallinacea, domestic fowl in tropics.

\section{5-Lice.}

Hamatopinus macroccphalus, horse.

$\begin{array}{ll}\text { " } & \text { eurysternus, cow. } \\ \text { " } & \text { stentirostris, cow. } \\ \text { " } & \text { cameli, camel. } \\ \text { " } & \text { piliferus, dog. } \\ \text { " } & \text { latus, dog. } \\ & \text { icutricosus, rabbit. }\end{array}$

\section{6-Parasites of Hair and Feathers.}

Trichodectes pilosus, horse.

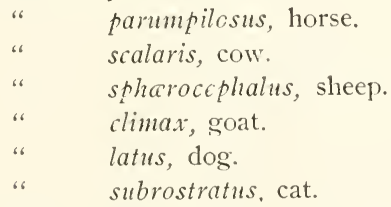

Melophagus orimus, vid. blood-sucking diptera.

Goniodes dissimilis, Burnetti, Goniocotes hologaster, gigas, Lipeurus variabilis, heterographus, Menopon pallidum, biseriatum, all in the hen.

Goniodes stylifer, polytrapeains, Menopon biseriatum, turkey.

Goniodes numidianus, rectangulatus, I,ip. numidia, Menop. numidia, guinea fowl.

Goniodes falcicornis, paricicps, rectangulatus, Menop. phaostommm. horse. 
Goniodes colchicus, truncatus, chrysocephalus, Lipeurus variabilis, Menop. productum, biscriatum, pheasant.

Goniodes minor, compar, Lip. barulus, Colpoceph. longicaudum, IIen. latum, biscriatum, pigeon.

Docophorus icterodes, adustus, Lipeurus jejunus, anscris, Trinoton conspurcatum, continuum, goose.

Docophorus ictcrodes, squalidus, Trinotum luridum, Mcnopon obscurum, duck.

Ornithobius buccphalus, swan.

\section{Spiders. (Arachnoida.)}

\section{1-Mites. Parasites Upon the Skin.}

Demodcr sive Acarns folliculorum canis, cati, suis, capre, ovis, etc. (in the sebaceous glands and hair follicles).

Sarcoptes major s. scabici communis, in and beneath the epithelium, in horse, dog, sheep, goat, camel, monkey, man.

Sarcoptes minor, cat, rabbit.

Dermatocoptes communis, horse, cow and sheep; also in goats. " cuniculi, in ear of rabbit.

Dermatophagus communis, horse, sheep.

" auricularum felis, cat.

" " canis, dog.

" " cuniculi, rabbit.

Dermatoryctes mutans, fowls, foot itch.

Sarcoptes lavis, pigeons and fowls.

Epidermoptes bilobatus and bifurcatus, fowls.

Harpirhynchus nidulans, pigeons, in the feather follicles.

Hypodcctes columbarum, pigeons, subcutaneous connective tissue and body cavities.

Laminosioptes cysticola, fowls, connective tissue.

Cystodites mudus, fowls, pheasants, air spaces, lungs, liver, kidneys.

Syringophilus bipectinatus, fowls, pigeons.

uncinatus, peacocks, quills of feathers.

Analges minor, fowls, quills of feathers.

Dimorphus columba, pigeon.

Leptus autumnalis, dog, man.

Dermanyssus avium, birds, man, dog, cat, horse, cow (ear).

Gamasus ptcropoides, rabbit, mole, field mouse, cow (ear).

Cheyletus parasitivorax, rabbit. 
2-Ticks. (Sucking blood from skin.)

Ixodes ricinus and hexagomus, man, dog, horse, cat, ruminants, birds.

Boophilus annulatus s. boris, cow (America).

Dermacentor reticulatus, sheep, cow, horse, man (America). " electus, dog (America).

Argas miniatus, cow.

Amblyomma americanum, cow, man.

Ornithodorus Megnim, man, cow, sheep, horse, ass, dog.

Argas reflerus s. marginatus, pigeons.

\section{3-Pentastomes of the Viscera.}

Linguatula tanioides, dog, wolf, horse, goat, man (nasal passages).

Larval form, Linguatula denticulata (mesenteric lymph nodes, lungs, liver), ruminants, horse, cat, rodents.

\section{LIST OF WORMS PARASITIC IN THE DOMESTIC ANIMALS.}

\section{Flatworms.}

\section{1-Tapeworms.}

Anoplocephala s. Tania plicata, horse. perfoliata, horse. mammillana, horse.

Moniesia expansa, ruminants.

. planissima, ruminants.

" Benedini, ruminants.

". Neumanni, ruminants.

. trigonophora, ruminants.

. denticulata, ruminants.

“ alba, ruminants.

Thy'sanosoma ortinoides, sheep.

" Giardi, cow and sheep (hog).

Stilesia centripunctata, sheep.

" globipunctata, sheep.

Tenia marginata, dog; bladder worm, cysticercus tenuicollis, ruminants and hog.

Tania serrata, dog; bladder worm, cy'sticercus pisiformis, hare, rabbit. 
Tania echinococcus, dog, jackal, wolf; bladder worm, echinococcus polymorphus, man, ruminants, hog.

Tania conurus, dog; bladder worm, canurus cerebralis, ruminants, horse.

Dipylidium caninum, (Tænia cucumerina), dog, cat, man; bladder worm, cryptocystis trichodectis et pulicis, larvæ of dog fleas and ticks.

Mesocestoides lineatus (Tænia litterata), dog, fox, cat.

Tania serialis, dog; bladder worm, cysticercus s. conurus serialis, rabbit.

Tania Krabbei, dog; bladder worm, cysticcrcus tarandi, reindeer.

Bothrioccphadus latus, man, dog. cat; larva, plerocercoid of fishes.

Bothriocephalus cordatus and fuscus, dog.

Ticilia crassicollis, cat; bladder worm, cysticercus fasciolaris, mouse.

Dipylidium Chyzeri, Pasquali, Trinchesii, cat.

Tania solium, man; bladder worm, cysticercus cellulosa, hog, dog, cat, sheep, roe, polar bear, rat, man.

Tania incrmis hom. s. mediocancllata s. saginata, man; bladder worm, cysticcrens incrmis, cow.

Drepanidiotania infundibuliformis, fowl.

Dicranotenia sphenoides, fowl.

Darainca proglottina.

. tetragona, cesticillus ectinobothrida, fowl; Friedbergeri, pheasant.

Bothriotenia longicollis, fowl.

Drcpanidiotenia lanccolata, sctigcra, fasciata, anatina, gracilis, Krabbi, tcnuirostris, goose and duck.

Dicranotania furcigera, megalops, coronula, duck.

Mesocestoides imbutiformis, duck.

Echinocotyle Rossctcri, duck.

Dazainea crassula, pigeon.

Bothrioccphalus columbarum, pigeons.

\section{2-Fluke-worms.}

Fasciola hepatica, distomum hepaticum, ruminants, hog, ass, horse, rodents, man (liver-errant examples in the lungs and musculature ). 
Fasciola lanceolata, distomum s. dicrocalium lanceolatum, ruminants, hog, rodents (liver).

Fasciola magna, cow, elk [deer], (liver), (America, Italy).

Distomum truncatum, albidum and felineum, cat (liver).

Agamodistomum suis, hog (muscle).

Distomum, echinostomum alatum, ducks and other water fowl, exceptionally dog (intestine).

Amplistoma conicum, ruminants (first stomach).

" Collinsi, horse (intestine), (Egypt).

Gastrodiscus Sonsinoi, horse (intestine), (Egypt).

Monostoma verrucosum, duck, fowl, goose.

" attemuatum, goose (intestine).

Distoma oxyccphalum, duck, fowl, goose (intestine).

“ dilatatum, armatum, lineare, ovatum, commutatum, fowl (intestine).

Holostoma erraticum, duck (intestine).

Distomum hamatobium s. crassum (Bilharsia crassa), cattle, sheep, monkey (urinary organs, intestine), (Mediterranean borders).

Distomum WVestermanni, cat [man] (lungs).

Distomum heterophycs, dog (intestine), (Japan).

Distomum pancreaticum, cow, sheep. (Japan).

\section{Round Worms.}

1-Acanthocephalid Worms (Thorn-headed worms).

Echinorrhynchus gigas, hog, man (intestine).

" polymorphus, goose, duck (intestine).

\section{2-Common Round Worms.}

Ascaris megaloccphala, horse, ass (intestine).

" mysta.t, dog, cat (stomach, intestine).

“ lumbricoides, man (intestine).

“. suilla, hog (intestine).

" vituli, cow (intestine).

" ovis, sheep (intestine).

Hetcrakis maculosa, pigeon (intestine).

" inflera, fowl, turkey (intestine).

turkey (intestine).

papillosa s. vesicularis, fowl, peacock, guinea fowl,

Hcterakis differens, fowl (intestine).

" dispar, duck, goose (intestine).

Gnathostoma hispidum, hog (stomach). 


\section{3-Pin Worms.}

O.ryturis aermiculuris, dog, man (rectum).

.. curaula, mastigodes, horse (colon and rectum).

.. ambigua, rabbit (сæcum).

\section{4-Strongylus Worms.}

Eustrongylus sigas, dog, horse, cow (kidneys, peritoneum, leart, liver).

Eustrongylus tubifert, duck (intestine).

Strongylus armatus s. sclcrostomum armatum, horse (intestine); peritonemu1 (larve and embryos wander into the arteries, brain, testes); str. armatus has recently bcen subdivided by Sticker into three species: Scler. edcntatum, bidentatum, quadridentatum.

Strongylus tetracanthus (sclerost. tetr.), horse (large intestine).

Strongylus contortus, ruminants (abomasum, small intestine).

.. Ostcrtugi, cow, sheep, goat (abomasum).

.. (urticëi, cow, sheep (abomasum, small intestine).

.. oncophorus, cow (abomasum, small intestine).

.. Harkeri, cow (abomasum).

.. retortcformis, ruminants, hare, rabbit (abomasum.

small intestine).

Strongylus filicollis, sheep (small intestine).

. radiatus, cow (small intestine).

.. inflatus, cow (colon).

. Arci, ass (stomach).

" lypostomus, sheep, goat (intestine).

.. filariu, sheep, goat, fallow deer, red deer (bronchi, lungs).

Strongylus paradorus, hog, sheep (bronchi, lungs).

“ commutatus, sheep, goat, hare (lungs, bronchi).

" micrurus, cow, horse, red deer, fallow deer (bron-

chi, lungs).

Strongylus Arnficldii, horse (lungs).

.. sagittatus, red deer (lungs).

" cupillaris, goat, sheep, chamois (bronchi, lungs).

. dentatus, hog (large intestine).

" rubidus, hog (America).

" annulatus (Strong. s. Filaria tracheo-bronchialis), dog (air passages). 
Strongylus iasorum canis, dog (blood vessels).

" pussilus, cat (lungs).

. tenuissimus, horse (stomach).

.. lcporum, hare, rabbit (stomach), (America).

" tonuis, goose (сæcum).

" nodularis, goose (pharynx).

.. $\quad$ s. Syngamus trachcalis, bronchialis, fowl, pheasant, horse, goose, luck, etc. (air passages).

Dochmius trigonoccphalus, dog, cat (intestine).

" stcnocephalus, dog (intestine).

" tubaformis, cat (intestine).

" bozis s. Esophagostoma z'estculosum, cow (intestine).

Esophagostoma columbianum, sheep, deer (intestine).

Uncinaria comua, sheep, goat (intestine).

Ollulanus tricuspis, cat (stomach, embryos in the lung).

Strongyloides intestinalis s. Anguillula stercoralis, man, fowl (intestine).

Anguillula vivipara, horse (colon).

Rhabdoncma longum, sheep, hog (intestine).

\section{5-Whip Worms.}

Tricoccphalus dispar, man, dog.

. affinis, ruminants (large intestine).

" cronatus, hog (large intestine).

“ depressiusculus, dog (large intestine).

Trichosoma crophilum, cat (trachea, bronchi).

" fclis cati, cat (urinary bladder).

. longicollc, fowls (cæcum and rectum).

" anmulatum, fowls (pharynx).

. contortum, duck (crop, pharynx).

.. collare, fockls (intestine).

. tonuissimum and brcicollc, pigeon (small intestine).

Trichina spiralis, hog, rat, man, all carnivora (intestine, embryos and undeveloped trichincllx in muscle).

\section{6-Filariae.}

Filaria papillosa s. cquina, horse (peritoneum, pleura).

" terebra, cow, deer (peritoneum, eye). 
Filaria lacrymalis, horse, cow (conjunctival sac).

" immitis, dog (heart) (Asia [America]).

" s. Spiroptera sanguinolenta, dog (stomach, aneurisms, pharynx).

Filaria s. Spiroptera strongylina, hog (stomach).

" s. Spiroptera megastoma and microstoma, horse (stomach); Gongylonema scutatum, sheep, cow, horse (pharynx); Gongylonema pulchrum, hog (pharynx, tongue).

Filaria s. Onchoccrca reticulata, horse (tendons, ligamentum nuchæ).

Filaria hamorrllagica, horse (skin).

Dermofiliaria irritans, horse (skin).

Filaria uncinata, goose (pharynx, crop, intestine).

" nasuta, fowl (crop).

“. $\quad$ s. Dispharagus spiralis, fowl (pharynx, crop).

Tropidocera inflata, duck (crop).

7-Annelides.

Hemopis sanguisuga, horse (pharynx). 


\section{COURSE AND TERMINATION OF DISEASE; SYMPTOMATOLOGY; DIAGNOSIS}

The disturbances of function which indicate the existence of disease, as well as anatomical or chemical organic changes, are spoken of as the signs or symptoms of clisease; the branch of study dealing with these as semeiology or symptomatology

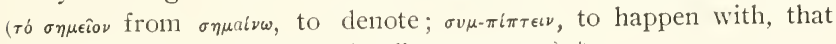
is, in connection with certain disease states).*

The art of concluding from symptoms the existence of definite morbid changes in the body, of determining the nature and location of disease, is known as Diagnosis or establishment of

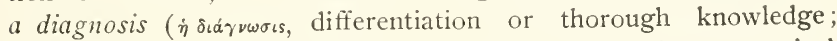
from $\gamma(\gamma \nu \omega, \sigma \kappa \iota \nu$, to recognize). Formerly when the anatomical and chemical faults which underlie disease were unknown it was deemed sufficient for the physician to merely appreciate the external manifestations of disease, and such terms as dropsy, jaundice, fever and marasmus were used without attempt at nearer approach to the causes of these symptoms. Even to-day there may now and again be times when it is necessary to rest satisfied with no more than such a symptomatic diagnosis. However, as far as it is possible to conclude from symptoms that definite anatomical changes exist, or as far as such are directly manifest, we are accustomed in these days to make anatomical diagnoses; and as far as it is possible to determine the development of any affection, to make also an atiological diagnosis. The aim of modern diagnosis is the study of the disease from all three of these points of view and the appreciation of the condition of all the organs in their relation to each other, so as to permit of the recognition of how a given local condition must influence the rest of the organs of the body generally.

- The principal features of this chapter have been in part adapted from Handbuch der allgem. Pathologie, ThI und Wagner (Lelpzlg, 1876). 
A purely symptomatic diagnosis is illustrated by such examples as the determination of varieties of convulsions, of palpitations, coughs; a symptomatic and anatomical diagnosis, by the recognition of a relation existing between jaundice and some hepatic affection; an ietiological diagnosis, by the conclusion that a jaundice depends upon the presence of some microorganisms in the liver and blood. [Very frequently diagnoses are classified as topographical, when indicating the location and extent of lesions, and as nosological, when indicating the nature of the process. No diagnosis can be regarded as complete unless embodying the recognition of both location and nature of an affection, as well as of its retiology, in so far as is possible. Incomplete, purely topographical diagnoses, as the declaration of "lung disease," "kidney disease," etc., are often made to serve the nonce, or by the careless as final; in the same way an incomplete, merely nosological diagnosis is often met with where it is said that an individual is the subject of "inflammation," "congestion," "dropsy," "fever," "tubcrculosis." The full diagnosis should declare that the subject has an inflammation of the pericardium, if possible indicating the cause of the pericarditis; "tuberculous caseation of the lungs" would embody all the desirable points of view.]

Symptoms immediately referring to changes in a given part, as abnormality of color, increased size, hardness, softness or unevenness of outline, are spoken of as direct symptoms; those which do not depend upon the diseased organ entirely, but which perhaps may be appreciable in the products of such an organ or become manifest in other organs in relation with the diseased part, are known as indirect symptoms. Thus a direct symptom of a lesion of one of the cardiac valves would be a cardiac murmur; an indirect symptom of pericarditis would be an cedema of the dewlap. There are certain symptoms called pathognomonic symptoms, which definitely indicate the existence of some particular disease, as the rusty nasal discharge in pneumonia, locking of the jaws in tetanus, crepitus at the site of a fracture of bone; however, as a rule a single symptom does not suffice for a diagnosis, but must be considered in its relations with a wider group of recognized features. Study of disease phenomena constitutes a large part of clinical pathology and pathological anatomy; the ability to recognize and to determine the importance of symptoms is one of the essentials of the medical art, and depends on the skill, experience, general knowledge and power of judgment of the individual conducting the examination of the subject. The majority of symptoms can be realized only by special methods of examination. He who has not acquired such methods cannot have a clear understanding about the morbid condition extant in the body of his patient, and is really groping about in the dark- 
ness. Eye, ear and sense of touch, partly unaided, partly aided by instruments of precision and special technical methods (employment of reflecting mirrors for illumination, of instruments used in percussion and auscultation, of methods of mensuration, weighing, thermometry, microscopy or chemical tests) contribute to our power of appreciation of the signs of disease. With experience it is possible that the examiner at a single glance (diagnosis at a distance) may recognize some types of disease, some forms which manifest themselves by sharply marked external features appreciable even at a hasty inspection. Other morbid conditions may be more or less correctly conjectured from the information given by some person from his observation

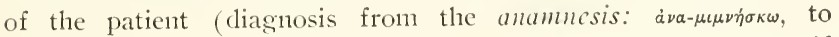
recollect). Both methods are open, however, to serious error if practiced alone, being based on incomplete data and imperfect investigation; and although practical experience with quick perception of frequently observed facts may often arrive at the truth by such methods, only careful objective study will guarantee an absolutely certain diagnosis. In order to gain a comprehensive idea of the pathological process presented, it is essential not only to consider the functional disturbances of one single part of the body, which is apparently the seat of the disease, but to systematically inquire into the condition of every part of the economy and every function.

(For fuller discussion of these points the reader is referred to Friedberger-Fröhner, Lehrbuch der klinischen Untersuchungsmethoden, or Möller, Klin. Diag. der äussern Krankheiten der Hausticre, F. Enke, publisher.)

The art of diagnosis concerns itself, after the proper recognition of the symptoms manifested, with the condition of the organs, finally reaching a definite conclusion through processes of comparison of the features appreciated with the commonly accepted pathology of known diseases. All possible affections are carefully considered, the symptoms presented by the subject contrasted with those of each different known disease, and determination of the affection attempted from the strongest features of correspondence.

[In systematic study of a given case it is well to follow some routine of investigation. Thus commonly one takes into consideration such general features as the age, sex, race or species of the subject, the general surroundings and habits of life and features 
bearing on questions of heredity; subsequently the general previous medical history of the subject, the anamnesis, is taken up, and a study of the known features of the present attack in its development. Thereafter is made a close and complete objective study of the subject, this including as thoroughly as possible the whole body, the condition of every organ by the methods of ordinary physical diagnosis and the more elaborate methods of clinical technology. Given such data one should be able by his pathological training to recognize the seat of the affection and the extent of its distribution in the body, the topographical diagnosis, and the nature of the affection, the nosological diagnosis.]

The establishment of a diagnosis leads directly to prognosis and therapentic application. By the term prognosis is meant the foretelling of the mode of development [the order of the events in the course, and the duration of the disease] and termination (whether favorable, prognosis fausta; unfavorable, prognosis infausta; or uncertain, prognosis anceps). The course and termination of many diseases may be predicted with more or less certainty, inasmuch as examples of the affections are constantly occurring and an experienced physician is quite familiar with their development and modes of progress. The prognosis must, however, in a measure depend upon each individual case, its special constitutional peculiarities, the degree of general involvement of the body and the distribution of the disease-changes, the vital importance of the affected organ, the variations of the disease from its usual trend, the complications and the possibility of employing proper remedial measures.

Therapeutics ( $\dot{\eta} \theta \epsilon \rho a \pi \epsilon l a$, from $\theta \epsilon \rho a \pi \epsilon \dot{\omega} \omega$, to serve or cure) or remedial treatment has to deal with efforts directed toward compensation for and removal of disease. Where it is possible by definitely conceived measures to remove the basic fault, the anatomical alterations and the causative influence underlying the morbid condition, therapy manifestly becomes rational or radical; where it is possible only to combat symptoms (pain, fever) it is said to be symptomatic treatment, which at best is but a temporary means or measure of relief (palliative, from pallium, a cloak or protection).

The duration and course of discases depend closely upon their causes and upon the extent and nature of their basic structural alterations. Some diseases are sudden in their onset, last but a few minutes or hours, and terminate in the death of the individual 
or in a rapid disappearance of the various lesions. The former type includes such conditions as wounds, lacerations of external or internal parts or the effects of poisons which rapidly and completely destroy the function of vital organs; the latter, characterized by rapid recovery, is met in disturbances which are not followed by structural changes, as convulsions, anæmic and hyperæmic states of the brain, of the skin and of mucous membranes. On the other hand, diseases may continue for weeks or months, or even years. They may begin suddenly or gradually and insidiously: may manifest alternately intensity and diminution in the severity of their symptoms, fluctuations in the morbid processes (remissions and cxacerbations); may invade suddenly (paroxysmal; paroxysm, invasion) and present interruptions (intcrmittent) in the course, sometimes ending with gradual improvement (lysis, resolution), sometimes in a rapid, abrupt change (crisis, decisize stage), leading to recovery or death. [By the course of the disease is meant the order of manifestation of the various stages or events of the disease. It may be definite, regular or acute on the one hand, when its events proceed in orderly manner and come to a definite termination, or it may be indefinite, irregular or chronic, when there is no fixed order of events and there is no set limitation. Among the different types of the regular course two major forms are recognized: (a) the continucd course, where there is but little variation in the intensity of the symptoms from time to time, and (b) periodic courses, in which at certain definite times special events manifest themselves. Among the periodic courses are met, intermittent forms, in which there alternate periods of absence of symptoms, known as intermissions, with periods of presence of the symptoms in their intensity or paroxysms; remittent forms, in which there alternate periods of intensity of symptoms, known as exacerbations, with periods of diminution in intensity, known as remissions; and recurrent forms in which there alternate comparatively long periods of absence of symptoms, the intermissions, with similarly prolonged periods of their presence, recurrences. The last form differs from the first in the length of the alternating periods.]

Diseases of brief duration are called acute diseases; those of prolonged course, chronic. The former may last for a variable period, up to fourteen days, while the latter extend over a course of more than forty days; any instances falling between these durations may be classed as sub-acute affections. In case of 
diseases which ordinarily continue for a year or more, a shortening of the course to a few months manifestly fixes such duration as an acute one, as in case of tuberculosis, glanders or rickets. [The terms acute and chronic have really a less. limited significance than the author here applies to them; they each have in one serise a reference to the length of the course, but in addition refer. perhaps without desirable clearness, to the possibility of recovery and the intensity of symptoms and the orler of events in the course. In the matter of time it is scarcely possible to give any fixed number of days, or even months, to the terms. Each disease is a law unto itself, and only in the broadest way may we say that an acute course is one of brief duration. But in addition, when we apply this term, we mean that whatever the actual duration, at any rate the disease will come to a definite limit; and, moreover, we expect the course to follow more or less closely a given order in the manifestation of its symptoms, and believe there is a chance of recovery. In case of chronic diseases again we cannot set a fixed limit of days, months, or even years, which shall declare the course to be a chronic one. (Thus, while alienists are inclined to set a limit of a year to cases of mania or melancholia, and to call all cases chronic if of longer duration, there really are no appreciable differences in many instances of even longer duration from their condition within the first few weeks of insanity.) Moreover, when a case is declared chronic, while there is no actual assertion to this effect, nevertheless there is a feeling of hopelessness as to the chance of recovery; the termination looked for is rather death, and that at an indefinite time. No exact order of symptom presentation is expected in such chronic cases. Finally, while there are often exceptions to this point, in general the severity of the symptoms of an acute case is apt to be greater than in a chronic form of the same disease.]

Diseases often show [especially those of an acute, regular or definite type of course] a succession of definite periods or stages in which certain phenomena appear, which are empiricaliy expected and whose development is awaited with the progression of the anatomical changes. These diseases are said to have a typical course. [A regular or definite course is well illustrated by the acute infectious fevers, in which the following periods may be recognized in the order named: (a) infection (time of entrance of the microbic cause), (b) incubation (a period without symptoms, but during which the germs are multiplying in the 
economy to sufficient number to excite their definite effects), (c) prodromes (a period of indefinite and usually slight symptoms, the first and as yet more or less obscure evidences of the presence of microbes in the systcm). (d) inzasion (the period of development of the specific syniptoms of the affection: this, it it occur rapidly, is said to be a frank incasion; if gradually, is called an insidious incasion), (e) acme or fastiginm (the period of fullest intensity of the disease), (f) occasionally an amplibolic stage (a period of uncertainty and marked variation, corresponding to the popular idea of crisis and used by the author above in this sense). (g) decline (period of disappearance of the disease; this, if it occur rapidly, is said to be "by crisis," if slowly, "by ly'sis"), (h) finally, not as a true stage of the disease, but a definite period before health is resumed, concalcscence (period of repair and rebuilding of structures destroyed or impaired in the course of the affection, and of resumption of efficient function by the various parts of the body). If the cause of an acute disease be not a vital one the course includes, of the above stages, only those of invasion, acme, decline and convalescence.]

However, in such well-known affections, as a result of special ætiological factors or the accidental interference of external influences, zariations (irrogularities) may occur, rendering the course an atypical one. In case the symptoms remain for a long time of miniform type and intensity, without appreciable amelioration or increase, the disease is said to be stationary.

Latcnt diseases are those which do not manifest themselves, either at all or in part, in certain phases of their development. This depends upon either the impossibility or difficulty of proper examination of the organs, which are the seat of the disease (as the pancreas or some parts of the lung), or upon the fact that the disease is so localized or has been so gradual in its development that the function of the organ has not been materially affected. Latency is often, therefore, only a temporary or transient feature, and it is often correct to speak of a latent stage and of a later manifested or open stage. Some diseases, cren though they be severe, remain latent because they are followed by adaptations (compensatory conditions) which entirely prevent their usual symptoms, as when a valvular lesion of the heart is followed by compensatory cardiac hypertrophy.

When a disease is characterized merely by local symptoms and alterations it is known as a local disease or discasc of This or 
that] organ; if, however, a number of organs are involved and their functions interfered with, and if the general economy is apparently affected, the condition is spoken of as a gencral discase or a disseminated (generalized) disease. (Examples of general disease are met with in such alterations as provoke general metabolic disturbances and abnormalities in the composition of the blood. Formerly the term constitutional discase or dyscrasia, decomposition of the blood, was used in this connection.) Fundamentally there is no distinction in these terms, but as a rule the term dissemination has special reference to a multiplication of local lesions, to an extension of the causes of the disease to a number of situations where new foci of the same type appear, or to a reaction of a functional disturbance of one organ upon the rest of the system. For example, a prolonged disturbance of the function of the kidneys will give rise to a disturbance in the cardiac action and retention of harmful products of metabolism; or, as in the case of tuberculosis, the infectious agencies pass from the original local fccus into the lympl and blood, extend by direct growth into the surrounding organs, and in various scattered foci in the body to which they have been conveyed; the disease thus becoming disseminated and generalized. Some affections, on the other hand, at first manifest general symptoms (fever), later, however, showing distinct evidence of their purely local type. Variations in the course of disease may also depend upon the predisposition characterizing the animal species in question, as, for example, is seen in case of glanders in field mice, an acute septicrmic affection, in contrast to the same disease in horses, where it is usually a chronic local affection, gradually extending through the system.

The local lesions which first arise from the operation of a pathogenic agency are known as primary lesions, those which follow as secondary. The action of such an agency may be confined to one locality, the lesions disappearing after its removal, as in case of corrosion, heat action or traumatisn. The generalization usually depends directly upon the spread of the pathogenic influences through the body, and may therefore take place (I) by continuity and contiguity of the tissues (per continuitatem, per contiguitatem), (2) by the blood or lymphatic tissues (hamatogenou's, lymphogenous), and (3) as already referred to, the functional interdependence of one organ upon the others may, in case of disease at one point, lead to further change in other parts of the 
system (sympathetic or consccutiz'c lesions). By the term cotension by continuity is understood the progress of the pathological process upon the surfaces in continuity and along uninterrupted tissues in the immediate vicinity of the original focus, as along the mucous membrane of the nose to the frontal and maxillary sinuses. from the pharynx to the Eustachian tubes and middle ears. Extension by contiguity occurs from one surface to that of an adjacent organ in contact with the first, as from the visceral pleura to the costal pleura, from the serous surface of the stomach to the liver. The explanation for such a mode of extension is mainly to be found in the fact that the pathogenic agent finds some particular opportunity for invading such adjacent tissues; thus bacteria may pass to an adjoining structure. be swept along by its fluids and spread widely. A suppurative or gangrenous focus in the lung may in some such manner gain access to the pleura; the latter may rupture and the microorganisms, spread over the entire pleural surface from the respiratory movements, may give rise to an extensive pleuritis. Hamatogenous cxtension of a disease occurs when the pathogenic agents gain access to the blood: lymphogenous e.rtcusion, when such influences, through the agency of the wandering cells, are mingled with the lymph (cf. embolism). Sympathetic disturbances in function arc apparently in part the result of sensory and motor reflex action, in part the result of primary changes, the efficiency of one organ depending upon the functional integrity of another, as a chronic hepatic cirrhosis induces cardiac hypertrophy and splenic cyanosis by the circulatory disturbances which it produces.

Coincidence of discascs may occur entirely independently of any relation between the processes, or may deperid upon some connection: where this occurs complications are said to exist. Tlus swine-erysipelas is often complicated by valvular endocarditis (the infectious germs invading the latter structures), or suppurative osteitis of the cranium may be complicated by a meningitis (through extension of the suppiration by contiguity to the meninges).

The termination of discasc may result in one of three ways:

I. Recoz'cry, cure or restoration, complete re-establishment of the disturbed function and condition of the organ;

2. Incomplete recorcry, with appearance of secondary affections, persistence of sequels or defects; or

3. Death of the individual. 
Tcrmination in recosery is accomplished through the reparative powers natural to the economy. The body is possessed of a variety of regulative mechanisms, whereby the influence of harmful agencies is neutralized, the loss of chemical substances and tissues is repaired, and noxious materials are elininated from the system. Such processes may in a compensatory fashion correct this or that fault, as by the discharge of toxic matter from the stomach or intestines, the removal of the products of fatigue from the muscles and nerves through the blood and lymph, the expulsion of exudations from the lungs through expectoration. Many corpuscular disturbing factors (as bacteria, dead cells, hæmorrhagic foci) are removed through the activity of the living cells (phagocytes) and digested, the body being thus freed of such substances. Other foreign bodies are encapsulated by a wall formed by the tissues of the organism for their own protection, and thus rendered harmless. Deficiency of nutritive material in the fluids and cells is corrected by the supply from the stomach and intestine; dead cells are replaced by new elements from existing formative areas (as new blood cells from the bone-marrow). Tissue losses are repaired by processes of regeneration. And, too, the production of antibodies of most varied type is possible for the protection of the animal body.

The therapeutic art is in no wise at variance with natural processes of recovery, but, on the contrary, employs the various regulating and compensatory mechanisms of the body in order to bring about an adjustment of the disturbances, and attempts to induce conditions more favorable to a rapid adjustment than is possible when no influence is brought to bear upon the pathogenic agent: at the same time it attempts to restrain further agencies which may retard the recovery. The practice of medicine is also directed to guard against the extension of contagious diseases and particularly against the inception of disease, so that in this phase, too, the lofty power of science holds nature in check, which, without the aid and skilled intervention of man. would threaten with annihilation, all too soon, the creatures of the earth. The significance and success of medical science is particularly impressed upon one when it is recalled how destructive epidemic diseases, which in times past carried off millions of arimals and men, have in some instances been wholly eradicated, in other instances greatly diminished. By appreciation of the nature of a varied group of affections, there has come the discovery of efficient methods for 
their cure and for prophylaxis against then. Of course, no remedy has been found for death, the inevitable end of development of all living beings, and the laws of nature can by no medicament, whatever its power, be broken. Yet the skillful use of the means of nature may hold off premature death and may assist the injured body to regain its health. Medical art can prevent fatal hæmorrhages, it loosens adhesions, cuts away what is unfit, dilates narrow canals, has antidotes at its disposal, can procure rapid evacuation of the bowel or emesis, remove foreign objects from the body, relieve promptly nervous strain, alleviate pain directly, often correct irregularity of cardiac action, reduce dangerous temperatures and accomplish a multitude of services without which a given discase might well end unfavorably or be protracted over a tedions course.

After recovery begins at the close of a general affection, there occurs a period, known as convalescence, the subject still evincing muscular weakness and marked sensitiveness to external influences. [During this period there are taking place various reparative and reconstructive processes in the economy, looking to the restoration of altered and destroyed elements, and, too, functional efficiency is being re-established, and all remaining factors of the previous disease are being finally eliminated from the system.]

Recovery is regarded as incomplete where, after a disease has run its course, there persist in the economy conditions preventing normal functional efficiency of the organs or where there is evident some permanent impairment of tissue [sequelc of disease]. As illustrative of this may be mentioned deformities of bones, cicatricial strictures of canals, kinks of the intestines, pericardial, pleural or other adhesions by bands of comnective tissue resulting from the previous disease, opacity of the normally transparent media of the eye, palsies of muscles and nerves, or the defects left after ulceration or burns.

Termination of disease in death (exilus lethalis or letalis, from letum, death; from $\Lambda \dot{\eta} \theta \eta$ forgetfulness, $\lambda a \nu \theta \dot{\alpha} \nu \omega$ to make forgetful) occurs when the organs which subserve the most important and necessary processes of vitality suspend their function. Inasmuch as continuance of life depends in an important measure upon the uninterrupted supply of blood containing oxygen to the medulla oblongata through the action of the heart, underlying which must be recognized the necessity for respiratory movements regulating the efficiency of the pulmonary surface in the intake of 
oxygen and output of carbon dioxide, it is essential to accept as causes of death of primary importance, various lesions and functional disturbances of the hind brain, the heart and the lungs. These parts are therefore spoken of as the atria mortis. Inhibition of their functions may be a direct result of chemical, mechanical or electrical influences (as from poisons which destroy or paralyze the nerve cells, concussion, the action of lightening), or may indirectly follow a wide variety of factors interfering with tissue nutrition, metabolism and gaseous interchange (anæmia, albuminous waste, closure of respiratory passages, elevation or depression of body-temperature, reflex palsies). The more important modes, therefore, by which this or that disease may induce death, are:

I. Cessation of cardiac action, caused by toxic or reflex nervous paralysis of the cardiac ganglia, fatigue and degeneration of the cardiac muscle, cardiac rupture, complete obstruction to the escape of blood from the cardiac chambers (formerly known as

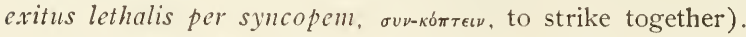

2. Asphyxiation, from interference with intrapulmonary respiratory interchange of gases, obstruction of the lungs or upper respiratory passages, compression of these tubes, spasm or palsy of the respiratory muscles, diaphragmatic rupture (exitus lethalis per suffocationem).

3. Medullary Paralysis, or paralysis of the respiratory centre in the medulla oblongata, from circulatory interference at the base of the brain, influence of toxic substances upon the nerve cells, concussion of the brain or reflex influences (exitus lethalis por apoplexiam, $\dot{\pi} \pi \circ \lambda \eta^{\prime} \sigma \sigma \epsilon \nu$, to strike down).

4. Hamorrhage, from rupture of important vessels, or extravasation of large amounts of blood into the somatic and visceral cavities.

5. Exhaustion, from inanition or consumption (diminished nutrition and exaggerated metabolism, accumulation of fatigue products and poisonous metabolites, auto-intoxication).

These modes of death may, of course, complicate each other and may be mutually causative of each other (as when hæmorrhage induces ischæmia of the heart and coronary vessels, with the result of cardiac cessation and at the same time the symptoms of suffocation, due to an anæmia of the medulla). Senile debility, more or less complicated by various diseases, is also to be regarded as a cause of death (senile marasmus, from $\mu a p a i v e \nu$, to 
weaken), a natural and necessary termination for each individual cell and for the entire cellular complex. In the individual cell, as well as in all the tissues of the organism of higher animals, changes are continually proceeding which lead to the destruction of the living substance and sooner or later result in the death of the individual. The living multinucleated organism is continually losing cells by their death, the substance of which is eliminated from the economy with the excretory material or is broken up and re-employed by the system. From causes inherent in the organism itself, but at present impossible of clear definition (vid. Verworn: Geschichte des Todes: Allgem. Physiologic) the power of multiplication ceases in the individual groups of cells and organs in varying rates in the different species of animals, and with varying swiftness the characteristics of old age and of death become apparent. Perpetual youth, immortality in the sense of reproduction, always renewing themselves, is possible only for the conjugated sexual cells (propagation cells), while in case of the somatic elements (and those sexual cells failing of copulation) the vegetal force sooner or later disappears. Whenever the decay of cells of vital importance reaches the grade in which it distinctly interferes with the rest of the economy, the mutual relations of the various parts is disturbed, and death ensues, just as a clock stops when its wheels wear out or are broken.

Transition from life to death may occur suddenly (mors subitanea); the animal falls from its standing position to the ground, becomes unconscious, and for a few moments at most is thrown into convulsions with groaning respirations. Such sudden termination may be noted in death from lightening stroke, sun stroke, rupture of the heart, cerebral concussion, massive internal hæmorrhages or cardiac and cerebral paralysis. In the majority of instances, however, death comes on gradually, with the manifestation of characteristic phenomena which predict the termination of life, and which together constitute what is known as the deathagony ( $\dot{\eta}$ arwía, the struggle, death struggle). They include the signs of a progressive paralysis of the nervous and muscular systems, together with those of the disease which causes death. Animals in the agonal state are unable to raise themselves from the ground, usually lie flat on one side, from time to time lifting the head and letting it fall heavily to the ground, with the feet rigidly extended and moved convulsively, at first violently, but gradually more and more weakly. Respiration is slowed and 
labored, deep groaning respirations following at irregular intervals the ordinary shallow breathing. With the appearance of pulmonary œdema, rales, heard even at a distance, are produced by the fluid exudate beaten into a foam in the air passages (death rattle). Involuntary discharges occur from the bowel and bladder, when paralysis of the sphincter muscles develops. The heart beats more rapidly, but the relaxed arteries are no longer able to propel the blood forward and the pulse in consequence, although quick, grows small and finally indistinguishable, and the skin is cool. The hair is bathed with a clammy sweat. The body temperature in the agonal period in diseases accompanied by loss of blood or inanition falls considerably below normal (hypothermia, to $3^{6-35^{\circ}}$ C.), while in other types there may be an antemortem rise in temperature. Extinction of life means complete cessation of metabolism, of cellular growth and the existence of the individual; and death therefore manifests itself by complete termination of the functions of all parts of the body. The last breath, naturally in the form of expiration, and the moment of complete cessation of the heart may ordinarily be looked upon as marking the actual end of life. However, the organs do not all perish at precisely the same moment; after the last breath the heart may flutter a few minutes; and after death from hæmorrhage, peristaltic movements of the intestines may sometimes be observed for perlaps fifteen minutes; and similarly after death from electricity muscular contractions may be elicited as long as rigor mortis does not set in (one to three hours after death).

The following are the characteristic signs of true somatic death :

I. Muscular rigidity (rigor mortis), sometimes setting in immediately after death, sometimes only after four to twenty hours later, and depending upon the coagulation of the muscle albumen. The muscles in this change become set, shortened and thickened, as in vital contractions; the extremities are rigid and either cannot be flexed or only with the application of considerable force, and the mouth cannot be opened by ordinary traction upon the lower jaw. After twenty-four hours, or perhaps later, the rigidity disappears [due to decomposition].

2. Cadazeric fall of temperature (algor mortis) develops in from half an hour to twenty-four hours after death, varying with the surrounding temperature and the degree of metabolic activity prevailing at the time of the death agony. In some affections, as in tetanus, because of the important heat production caused by the 
muscular contractions and because after death heat dissipation falls on account of the cessation of the circulation in the periphery of the body, a post-lethal internal temperature rise, to perhaps $42-44^{\circ}$ C., occurs, persisting a number of hours.

3. The eye in death: The eyelids of the cadaver are usually half open (rigidly); the ocular bulb is sunken and somewhat less tense than in life (evaporation of some of its fluids); the cornea becomes dull and opaque; the pupils are dilated. The ocular reflex movements upon touch are entirely absent.

4. Appearance of putrefaction: The bacteria in the digestive tract, producing all sorts of fermentative changes in the contents of the canal, shortly after the death of the tissues, penetrate the latter and cause their putrefaction. The gases, produced in large quantities from the fermentative processes, dilate the stomach and intestines, causing abdominal distension, sometimes to such a degree as to force the lower bowel out of the anus, rupture the diaphragm and allow the intruding intestines to distend the chest. The advance of putrefaction may be noted in the colorless parts of the skin by greenish discolorations (sulphur compounds of hæmoglobin), and also by the foul odors of cadaveric decomposition. These changes cause the disappearance of the rigidity of the body and progress the more rapidly the warmer the surrounding temperature. Measures which inhibit the growth of the putrefactive bacteria, preservation in alcohol or formaline (injection of the vessels with antiseptic or balsanic substances), refrigeration, drying, prevent putrefaction. With the advance of putrefaction all the organic matter of the body is broken up into ammoniacal compounds, carbonic acid and water, with the formation of a great variety of by-products (putrefactive alkaloids, acids, gas-forming matter, etc.) until finally only the solid calcified lones remain.

The name apparent dcath is applied to a condition in which all the vital functions are depressed to the 'owest possible degree, when only by great care in the examination of the seemingly dead body can there be detected feeble cardiac contractions and occasional faint respiratory movements, a condition accompanied by loss of consciousness and sensibility, and by reduction of the body temperature. In recently born animals this condition is seen comparatively frequently, lasting perhaps for hours (asphy.ria neonatorum); in this connection it is perhaps due to a premature separation of the placenta, aspiration of the amniotic fluid, compression of the umbilical cord, or to anæmia. 


\section{DISTURBANCES OF CIRCULATION}

Life and health are possible for the organs only if there be unimpaired circulation of a blood capable of supplying oxygen and nutrition to them. Disturbances of the circulation, as well as iaults of the blood and lymph, in other words, deficiency in the provision and passage of good blood through the organs, endanger both life and health.

The normal heart possesses a notable adaptabilty to the varyin's clemands upon its capacity for work; it accommodates itself immediately to the current of blood entering it by virtue of the elasticity of its walls; regulates the energy and rhythm of its contractions in conformity to the amount of blood in its chambers, the general circulatory resistance and the demand for blood in the active or resting organs. This power of accommodation enables it, to at certain extent, to overcome and compensate for pathological disturbances affecting the hæmic circulation; under such circumstances there is said to take place a compensation for these disturbances.

Where such conditions of resistance to the heart's action are of slow development and permanent, a thickening of the myocardium is assumed in connection with the increased requirement for work, consisting essentially in an increase in the number and size of the muscular elements (cardiac hypertrophy'). It may be said that even physiologically the size and muscular strength of the heart are adjustable to the demands made upon it in its function as a forcing-pump or as a suction pump. Certain animals, for example, which in the course of their lives perform especially intense muscular work, are likely to exhibit hearts larger in proportion to the body-weight than animals accustomed to but little muscular activity.* Pathological hypertrophy differs from this physiological form only in its cause, in the latter the excessive work being but a natural and customary condition in the life of the animal, in the former the result of abnormality of the vascular

* See also Kitt, Lehrbuch der pathol. Anatomie d. Haustiere. II. Aufl. 
system, of excessive blood pressure and of unusual quantities of blood within the organ. Such "work-hypertrophy" of the general organ is met in connection with epicardial adhesion to the parietal pericardium, compression of the base of the heart from pleuritic adhesion, tumors or similar conditions, because in such cases the propulsive effort of the entire organ has a greater opposition to overcome.

Hypertrophy of one side of the heart is seen especially in case of valvular lesions. These valvular lesions involve irregularities in the closure of the orifices of the heart and its great vessels; they are of two types, either narrowing of the orifices from proliferative changes, bloods clots, etc. (stenosis), or incompleteness of closure of the orifices by the valves (zaliular insufficioncy). Stenosis at the aortic opening increases the work of the left ventricle in order that the blood may be forced through the narrowed orifice, and in proportion there ensues a work-hypertrophy of the left ventricular wall. Following stenosis at the origin of the pulmonary artery there occurs a work-hypertrophy of the right heart (sometimes dilatation after special preceding strain). Valvular insufficiency also leads to hypertrophy, because where the valves are incompletely closed there first occurs a tendency to stasis because of the back pressure of the blood (regurgitation), which is likely to induce ventricular or auricular dilatation, and therefore the heart is required to work the harder in order to propel the larger quantity of blood. Although the so-called compensatory hypertrophy of the heart does in some measure contribute to the regulation of the blood distribution and is of some service, yet in reality it is only the expression of an increased blood-pressure in one of the ventricles or auricles or in the afferent or efferent vessels, and the compensation which it induces is only a relative one. As a matter of fact, the increase of pressure persists and the circulation does not become normal (Krehl). Even when the animal is at rest the continued heightened blood pressure causes the development of dilatation of the capillaries, pulmonary passive congestion and distension with consequent diminution in the pulmonary excursion, together with difficulty in breathing. The arteries show distinct changes of the pulse, the vessels being unusually tense and distended; they eventually lose their elasticity and may rupture. A very large heart may in addition mechanically interfere with the lungs. These consequences are still more evident if the individual exercises physically, the nuscular exertion raising the blood 
pressure still higher. (Powerful muscular contractions force larger amounts of blood into the right heart; the ventricles become engorged and the hæmic pressure rises.) Eventually the heart loses its force as a pathological hypertrophy is not, as might be supposed from the thickness of the walls, capable of indefinite response to increased functional demands, but on the contrary, is often unable to accomplish even the more moderate requirements made upon the strength of the heart. Perhaps the reason for this is that the influences which give rise to cardiac hypertrophy at the same time harm the myocardium in other respects. For example, valvular lesions, causing the heart to become hypertrophied in their train; are frequently caused by infectious substances. Such agencies may also cause a myocarditis; and if the inflammation be protracted and of low grade, it causes considerable reduction in the efficiency of the muscle. Moreover, the conditions which cause pathological cardiac hypertrophy are typically not stationary, but on the contrary the circulatory difficulties are apt to progress (the stenosed orifices are likely to become still more narrowed, thrombi which interfere with the vascular lumen become larger, capillary area; become contracted); and from such extra demands upon its ability the heart becomes fatigued. There must eventually, therefore, succeed upon compensatory hypertrophy a period of failure of compensation, a period of broken compensation. [There are numerous influences which combine to limit cardiac hypertrophy and to determine its eventual loss of compensatory power, and so certain and uniform are these results that it might well be declared a law that every pathological hypertrophy of the heart must necessarily reach a limit to its enlargement and must thereafter fail in its power of compensation and undergo degenerative changes. The limitations, aside from those set by the age of the muscle and its inherent power of increase, depend mainly upon the amount of proper blood which the coronary vessels are able to supply. In a great measure this is determined by the original size and construction of these vessels, although doubtless there is a possibility of true hypertrophy in these so as to accommodate the growing needs of the enlarging organ; yet if the original cause of the hypertrophy were a widespread arteriosclerosis the coronary arteries would be extremely likely to have been involved. Even were this not true, the mechanical infuence of a hypertrophied left ventricle upon the walls of these vessels in close functional and topographical relation with the pumping action, and the similar influences 
of the aortic recoil after closure of the aortic valve, must favor the development of a local coronary sclerosis on account of the possibility of fibrillary injuries to the walls. Given an arteriosclerosis there is every reason to expect an imperfection of the coronary circulation from the narrowing of the lumen and the increased rigidity of the tubes; and with the imperfection of circulation thus fixed upon the myocardium, its nutrition and ability to further enlarge are necessarily limited. The functional demands progressing. fatigue, degeneration an!l cardiac failure are the necessities of a not distant future.

Diminution of cardiac force is spoken of as cardiac failure. cardiac insufficicncy. It follows varions influences affecting the myocardium or its ganglia, among which as prominent examples may be mentioned excessive exertion or fatigue, numerous poisons and the analogous substances present in the system in the infections diseases, diminution in the blood supply to the myocardium, inflammations and degenerations of the muscle, excessive fatty deposits of the heart, and atrophy of the myocardium. All pathological changes which occasion cardiac insufficiency are followed by disturbances in the movement and distribution of the blood. A fatigued and weakened heart is incapable of normal contraction, expels from its chambers a smaller amount of blood than normally; in consequence the arteries are not properly filled, the blood pressure sinks in them and the movement of the current is slowed. The aspirating power of a weak heart is also low and the blood moves less freely from the venous side of the circulation, the veins becoming engorged and the pressure raised. The results of these irregularities differ somewhat as the left or right heart alone or the whole organ is especially involved; in one case the lesser circulation, in the other the greater being the more disturbed. Many variations, too, are occasioned by the grade of cardiac insufficiency and by the nature of the original causes (cf. ancmia and hypercmia). The myocardium being weak the cardiac cavities do not contract as in normal conditions, contain an excess of blood, and this engorgement gives occasion for dilatation of one or both sides of the organ.

Left ventricular insufficiency determines imperfect filling of the aorta and its branches, and in consequence deficiency in the amount of arterial blood going to the brain, skin, glands and elsewhere. The heart, however, remains full and the blood is dammed back into the pulmonary veins, and the right heart experiences marked difficulty in forcing the blood through the lungs. Insufficience of the right heart, on the contrary, causes incom: 
plete filling of the pulmonary artery, pulmonary anæmia, and, of course, the left heart in its turn receives unduly low amounts of blood. In the right auricle and larger veins engorgement and tension become marked, tending to cause a passive engorgement of the venous system throughout the body, a cyanotic liver, cyanotic spleen, etc.

A heart reduced in its propulsive power is itself but poorly supplied with blood because its own arteries are but incompletely supplied. Any condition of fatigue which prevails may therefore easily become crhaustion, complete functional inability, arrest of cardiac action (cardiac paralysis, cardiac sy'ncope, cardiac collapse). All these terminal changes interfering with circulatory integrity are occasioned by any influences which impair the contractile efficiency of the myocardium, any agencies which either directly or indirectly reduce its muscular power (rupture, fatty degeneration, inflammation, prevention of expansion of the heart from some external interference, anæmia); in the same way the action of some paralyzant toxine upon the cardiac ganglia, the analogous action of thermic and electric agencies, excessive reflex stimulation of the vagus or of the vagus nucleus in the medulla oblongata (the latter from deficiency of the blood oxygen, increased blood pressure in the brain, or toxines).

Just as in cases of diseases of the heart, affections of the arteries and veins are apt to occasion irregularity of distribution and movement of the blood in given organs and areas of the body. Narrowing of the arteries from vasoconstriction (arterial spasm), thrombus formation, inflammation and permanent shrinkage raise the intracardiac blood pressure and occasion dilatation and hypertrophy: the organ to which such a constricted artery is distributed is imperfectly supplied with blood and a variety of changes may in consequence occur in it (cf. ancmia and cmbolism). Decrease in the elasticity of the vascular walls or paralysis of the arterial musculature permits the vessels to dilate, the current to become slowed and the organ, congested. Reduction in the muscular tonus of the vessels of the splanchnic area from vasomotor paralysis, usually from toxic and infectious influences, causes a massive congestion of the abdominal vessels; in consequence of which the general blood pressure falls, cardiac labor from the small amount of blood coming to the organ ensues. and there is a fatal collapse (Krehl).

In the veins interference with the progression is occasioned not only by cardiac weakness, cardiac lesions and pulmonary 
affections which interfere with the aspiration factor of venous circulation and by thrombosis of the veins; but it should also be kept in mind that any abnormal compression of these thin-walled veins must interfere with the return flow of blood. The nearer the heart such compression occurs the greater the harm to the general body. Compression of the two venæ cave. which from the compressibility of these vessels is quite possible from pleural effusions, induce a reduction in the amount of blood entcring the cardiac chambers and venous congestion throughout the economy (cf. hypercmia).

The lymph supply and drainage are in close relation with the abnormalities of the blood vascular system and blood circulation; imperfections in the lymphatic circulation give rise to abnormal accumulations of fluid in the $1 \mathrm{ymph}$ vessels and serous cavities and occasion a wide group of organic lesions.

\section{Local Variations in Amount of Blood. Hyperaemia and Anaemia.}

The amount of blood in the organs of even healthy individuals is always subject to change and variation. The vessels are elastic tubes which are expanded by greater internal blood pressure and adjust themselves as their contents diminish. This is not merely a passive acconmodation, however; they are capable of active contraction and dilatation through the power of the smooth muscle in their structure, such alterations of lumen being primarily under control of nervous influences. The total quantity of blood in the body, similarly subject to continual variation, is never so great as to completely fill all the vessels were they fully dilated; there exists, however, a moderate fullness, varying here and there in the organs as the blood-current meets resistance to its progression or as the blood is drawn into special organs from dilatation of this or that vascular area. The quantity of blood in the organs, and the hæmic distribution in the body generally, physiologically accommodates itself to the existing demands. More blood enters an organ when functionating; the amount traversing it diminishes during periods of rest. The nervous stimulus which cause; an organ to actively functionate, at the same induces dilatation of its vessels, and in consequence a rich supply of blood is afforded. This is especially recognized in muscle. Ranke determined the blood content of the general musculature of rabbits at rest to be 36.6 per cent. of the total muscle bulk, and noted this proportion nearly doubled (66 per cent.) when the muscles 
were thrown into tetanic contraction. (Internal organs like the brain or intestine may thus be relieved of excess of blood by muscular movements.) When the stomach and intestine are filled with food during digestion they are much richer in blood than when empty. The influences which give rise to this stimulation are variable and multiple, acting sometimes directly upon the muscular coats of the blood vessel wall and the nervous apparatus inherent in the wall, sometimes upon the vascular centre in the medulla oblongata (mechanical, thermic, electric, chemical irritants).

It is but a step from the physiological to the pathological, and there is no sharp line of differentiation: change in the amount of blood in a part becomes a pathological condition when it occurs in an improper place, or at a wrong time, or if it exceeds the normal variations.

Increase in the quantity of blood in a part of the system, local engorgement, hypercmia, may be of one or other of two types, the result either of an excessive influx of blood to the part, or of a diminished escape of blood from the part.

The first form, the basic feature of which consists of excessive entrance of arterial blood into the tisstes, is spoken of as active hyperaemia, or, synonymously, as irritation-lypercmia, congestize hyperamia, flurion of blood or artcrial hypcramia. Increased supply of arterial blood may be caused by excessive blood pressure. This is met where an artery is occluded or some part of the body is for some reason prevented from receiving its proper supply: under which circumstances the blood propelled from the heart passes into the adjacent structures (collateral hypercmia), that is it rushes into the pervious branches of the artery near the obstruction, under the influence of the higher pressure. As a rule, however, instead of the blood being forced into the part in question, there is a lowering of the vascular tension, spoken of as a relaxation in the arterial distribution (relaxation-hypercmia), with a resultant widening of the channels. The blood freely pours into the dilated arterial branches and capillaries, and with increased force; in a word it is drawn into the part. The degree of relaxation depends largely upon the lack of tone of the smooth muscle of the vascular wall and the congestion is therefore in such instance a myoparalytic one. Every muscle being inseparable from its innervation, nervous influences must finally be regarded as responsible for changes in the vascular tension (tone). It is assumed that the vascular 
nervous apparatus comprises two types of nerve fibres, vasodilator and vasoconstrictor; theoretically, therefore, stimulation of the vasodilator nerves should occasion a widening of the blood channels (neurotonic congestion), and the same result should follow paralysis of the vasoconstrictors (ncuroparalytic congestion), both of these influences similarly reducing the tension of the muscular elements of the arterial wall. This relation with nervous influences is well seen in the fact that after section of the cervical sympathetic in rabbits the ear on the corresponding side becomes hyperæmic (Cl. Bernard), and after section of the splanchnics the vessels of all the abdominal viscera become distended and engorged with blood (paralysis of the vasoconstrictors). Stimulation of the nervi erigentes of the human penis causes a dilatation of the arteries of the organ; blushing in man is brought about by psychic influences upon the dilator centre through reflex action; certain poisons, as nicotine and alcohol, are apparently stimulating to the dilator centre. Generally the stimulation or paralysis of the vascular nervous apparatus indicated is the direct result of local influence by physical or chemical agents. Thus hyperemia may be induced mechanically by the removal of pressure acting upon the vessels; just as a sponge which has been squeezed and is then released takes up water, so the blood flows in large amount, immediately after removal of the pressure, into a tissue which has been deprived of its blood for a long time because of compression. The vessels here do not at once regain their contractility, but are relaxed.

Similarly friction and scratching can indnce hyperæmia mechanically, from the nervous stimulation thus originated. Thermic influences of both types give rise to hyperamia; heat by its direct relaxing influence upon the vessel walls, cold first causing a vasoconstriction which later gives place to a paralyysis of the vascular musculature. There is a special group of chemical irritants as oil of mustard, cantharides, ammonia, alcohol, chloroform, ether, saline solutions, which, either by stimulating or paralyzing the nerves of the vessel walls, cause a more or less intense arterial hyperæmia; these, because of the value of the hyperæmia in the treatment of various affections, are of therapentic importance (rubefacients, drugs which cause reddening of the skin). Probably in the erythemata which occur in various infectious diseases there also exists a similar toxic stimulation or paralysis of the vessel walls either from the bacteria or the microbic products. 
Active hyperæmia is essentially an arterial and capillary dilatation. The hyperæmic organ or tissue therefore manifests as one of its features a red color, which is the more striking the paler the tissue normally is, as in mucous and serous membranes, and may be so pronounced that the distended arteries appear as fine deepred lines (branched or injection erythema). The rapidity of the flow of the blood into and through the involved area, with but little output of its oxygen, causes it to remain bright red and pass thus into the veins. In peripheral parts of relatively lower temperature previous to the increased blood supply, there is a rise of temperature up to that of the blood. These peripheral parts, particularly the skin, because of their greater heat loss and their comparatively poor blood supply are lower in temperature than the internal organs; but with the access of more blood of the body temperature the skin with its rate of heat dissipation unchanged necessarily becomes warmer. The temperature attained does not, however, exceed that of the internal organs; and these, should they become hyperæmic, do not suffer any increase of temperature. Where there is marked capillary distension, provided the tissues are not rigid, there may also be induced some swelling.

The results of arterial hyperæmia vary with its duration and location. Hyperæmia of the brain is followed by unimportant or marked disturbances of consciousness, dizziness and general excitation (pressure of the engorged vessels on the nervous elements). Hyperæmia of the sympathetic nerve does not cause either lachrymation or salivation, and it is doubtful whether an uncomplicated hyperæmia causes any special lymph formation. In case of long continuance of the hyperæmia there becomes apparent an increased tissue proliferation: this is probably not due to the increased blood supply alone, the irritant which underlies the condition having also in all likelihood some stimulative influence in its causation.

As a rule uncomplicated hyperæmia is a transitory condition; with the disappearance of the cause for the vascular relaxation the contractility of the arteries returns. Otherwise the hyperæmia may be regarded as the precursor and concomitant of inflammation. Where large amounts of blood are drawn into extensive areas of hyperæmia, a deficiency of blood (collateral anæmia) may be occasioned in other regions.

Where the excess of blood exists in a part because of difficulty in its exit from the part it is known as passive hyperaemia or venous engorgement. This condition is encountered in connection 
with low arterial pressure, the blood not being properly propelled through the capillaries, in connection with deficiency in the propelling power of the heart and arteries or complete !oss of the arterial reactions (atonic hypercmia). When the blood is not kept moving onward, in compliance with the law of gravitation it settles into the dependent parts of the body. As the animal lies on one side the lowest parts become the seat of special engorgement (one half the lungs in the cadaver). This hypostatic or gravitation hyperamia (hypostasis) takes place becanse of the longer persistence of the blood in fluid condition in the capillaries as compared with the larger vessels. It may be noted in animals unable to raise themselves from the ground in long protracted diseases or in the agonal period, and may also develop after death. It may, however, develop in a nondependent organ because of reduction in its arterial supply, particularly if the blood pressure in the venous trunk be so great that the blood presses back from the veins into the capillaries no longer filled from the arterial side. Since the progression of the blood in the reins depends in part upon general muscular movements and the passage of blood from the venæ cavæ is particularly favored by the inspiratory expansion of the thorax, deficiency in body movements and in respiratory activity (pulmonary diseases), as may be expected, promote the development of hypostatic congestion. The most common obstacle to the venous circulation arises from compression of the delicate walls of the veins by external pressure, kinking as in intestinal volvulus, constriction or pressure from tumors; reduction of the venous lumen or actual obstruction from thrombosis should also be mentioned in the same connection.

Passive congestion of the portal vein and its branches, affecting the spleen, stomach and intestines [and pancreas] is occasioned by all diseases of the liver accompanied by shrinkage or reduction of the capillary network of the organ, the condition interfering with the progression of the portal blood through the liver.

Widespread passive hyperæmia is met in case of cardiac valvular lesions because of the interferences offered to the ready movement of the blood from the venæ cavæ and pulmonary veins.

A passive congestion caused by local interferences may have no further results provided the venous trunk in question or its tributary capillaries communicate by collateral circulation with other veins which are not affected, the blood then passing freely through such 
collateral anastomoses. This readily occurs if such paths are at all numerous and large; and where they are of narrow calibre they are gradually widened by the pressure of the obstructed blood, so that even very small branches and capillaries come to assume considerable dimensions permitting the blood to escape from the area of obstruction practically unhampered (anastomotic compensation). The possibility of such dilatation depends, however. upon the degree and rate of development of the venous obstruction and requires time. In the case of sudden venous obstruction and the existence of few anastomotic channels, before the collateral branches can adapt themselves to the congestion the disturbance of the circulation may well induce important functional and vital changes in the affected organs; and, of course, such occurrences are to be expected if the obstructed veins have no communication with other unobstructed channels (portal vein, the veins of the kidneys and lungs). Even compression under the finger (in phlebotomy or in a rabbit's ear) will show how the impeded blood causes the veins to dilate and swell up; all the rest of the phenomena of this form of hyperæmia may be followed up experimentally in the exposed tongue of a frog or under the microscope, after the ligation of the larger venous trunks so that the blood can pass out only through the venules. The web of the frog's foot may be employed for the same purpose after ligation of the femoral vein. At first the blood in the engorged and expanding veins and capillaries becomes slowed, then irregular, now flowing forward, now backward, and sometimes stagnates totally. It can be seen that in some places the current is reversed, that the blood is passing out of the engorged area through the collateral branches, these gradually dilating, and the stationary or slowly moving corpuscles gradually being drawn into the current and after a time the proper rate of circulation again assumed.

If a number of veins are occluded or cut off sufficient to entirely or largely prevent collateral compensation, stagnation or stasis of the blood takes place. In the affected parts of the vessel the red blood corpuscles are packed so closely together that their outlines can scarcely be distinguished and the blood completely fills the vessel as a uniform red mass. Stasis becomes especially well marked if, while the venous outlets are completely closed, the blood continues to be forced in from the arteries. The capillaries dilate to their fullest; their walls, permeable to the plasma even before their distention, can no longer hold the fluid blood, but allow 
it to escape more or less freely according to the pressure, and may in fact, rupture. However, even if the blood be not forced into the area from the arteries, exudation of the fluid part of the blood through the distended capillary walls may take place in ordinary atonic hypostatic congestion, and there may even occur a diapedesis of red blood cells. The endothelium and vessel walls remain intact only while they are being continually bathed with moving blood; and as soon as the circulation stops it may be accepted that a disturb. ance of the endothelial nutrition exists, these cells then shrinking and causing comparatively large open interstices, through which exudation takes place. For these reasons passive hyperæmia has frequently associated with it a congestion-transudation and hamorrhage. The latter may be sufficiently severe to make the whole tissue dark red in color, infiltrated and completely occupied by blood (hæmorrhagic stasis, hæmorrhagic infarction).

The pressure occasioned by the distended ressels and the fluid exudate, together with the impediment to circulation, may be followed by cessation of function of the affected tissues and finally by their death. The longer the blood remains in the capillaries, slowly giving off its oxygen and capable of but little or no renewal, the darker it becomes; the tissues in venous hyperemia are therefore of a dark bluish red (cyanotic) hue. Such a tint (diffuse cyanosis, black erythena) may be encountered (in hogs) extending over practically the whole surface of the body in case of general passive hyperæmia. The lack of oxygen and the excess of carbon dioxide in the venous blood are partly responsible for the functional disturbances arising from passive hyperæmia of the varions organs; these conditions act by stimulating the medulla, and occasion dyspnoea, dizziness, convulsions, disturbances of consciousness, muscular weakness and fatigue.

The superficial external parts of the body, when passively congested, become cool because of the impeded circulation of blood through them, because the blood is cooler than normal and the heat dissipation not diminished.

In contrast to what has been said above, a mild but persistent passive hyperamia is apt to cause not only a swelling of the organ affected (kidneys, lungs, liver, spleen) because of the permanent engorgement and distention of the capillaries, but in addition may induce an increase in size and induration from the production of an excess of connective tissue in the part (Cf. hypertrophy). 
Stasis and stagnation of the blood may at times be caused by substances which act by inducing a withdrawal of the fluid elements and thus a thickening of the blood (chemicals acting upon ressel walls). IThis can well be shown experimentally by bathing the mesentery of a frog, arranged for demonstrating the blood current, with a hypertonic saline solution, a rapid exosmosis of fluid taking place and the current being soon entirely checked, with the ressels choked with corpuscles.]

Deficiency of blood in an organ may be part of anæmia of the general body (general oligxemia or anæmia; $\dot{a}$ priv., $6 \lambda$ /ros, little, and ai $\mu a$, blood) or a consequence of local impediment to the

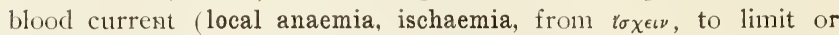
check). The blood supply to any part máy be impeded by:

I. Pressure upon the part (compression anamia) from increase in the bulk of adjacent organs, accumulations of fluid or any other compressing influence upon the part from without, or by narrowing of the capillaries by fat deposit, fluid or air in the parenchyma of the organ.

2. Narrowing, occlusion or fault of contractility of the arteries. Here may be mentioned external pressure upon the vessel, ligation, occlusion by solid bodies as by thrombi in the arterial lumen (cf. thrombosis and embolism), rigidity of the vascular walls from calcification or sclerosis and spasm of the arterial musculature with resultant contraction of the lumen (arterial anamia or ischamia, occlusion anamia, spastic anamia).

3. In addition, anæmia may involve a certain part of the body in case the general blood distribution is irregular because the general volume of blood passes to one large area to the deprivation of other parts (collateral ancmia); as when the posterior parts of the body are markedly infiltrated with blood (symptomatic anthrax) the fore parts are rendered anæmic, or as when internal hamorrhages occur the flesh is left anæmic, etc.

The organ or tissue deprived of its blood looks pale, the absence of the blood tint permitting the peculiar hue to become more apparent and more like the tissues of slaughtered animals or like a washed tissue deprived of its blood by the washing. It contains less fluid and but little blood appears on the sectioned surface, and its volume is diminished because of the emptiness and collapse of the ressels.

Anæmic parts at the exterior of the body, being exposed to the air and losing their heat, feel cool, because with the loss of blood their principal thermal substance is lost and they are capable of producing little or no heat in themselves under the circumstances; 
and with the existing diminution of nutritive and secretory substances disturbances of nutrition and diminution of functional ability must ensue. In addition the products of previous metabolic activity necessarily remain in the anæmic tissue and may act unfavorably upon it. According to the grade of oxygen deprivation, need for nutrition and rapidity of metabolism in the affected structure there must sooner or later result a gradual death of the anæmic part, because of the absence of blood. It should be kept in mind, too, that the integrity of the endothelial lining of the blood vessels depends upon the continuous flow of blood through the vessel and that these cells gradually degenerate and become permeable when the anæmia is complete. Should the blood again flow through such capillaries which have been empty for perhaps twenty-four hours it readily escapes through their walls, with consequent hæmorrhagic infiltration of the surrounding tissues. (After interruption for as much as two hours the renal epithelial cells become necrotic and consequently the renal function becomes checked or ceases entirely.) Suspension of activity in vital organs because of anæmia is a matter of extreme gravity. Cerebral anæmia is quickly followed by unconscionsness; and because of degeneration of the central nervous tissue, even after but brief periods of anæmia, this condition in the brain and spinal cord is likely to give rise to destruction of the ganglion cells and focal lesions with symptoms of paralysis. Complete anæmia of the myocardium necessarily causes cessation of the heart's action.

Other parts of the system, as the skin and muscles, are less sensitive to the effects of anæmia; their tissue may withstand the condition for some hours, as may readily be appreciated by the practice of artificially causing an anæmia in surgical operations by means of the Esmarch's elastic bandages. In case of such resistant parts, if the cause of the anæmia be eliminated the results may be but transient or only partial, as in case of the anæmia of cramps (spastic anæmia) or anæmia caused by external pressure. Obstruction of an artery, and more particularly the anæmia resulting therefrom, may be corrected if the blood supply can be quickly reestablished through the anastomotic vessels (so-called collateral paths).

\section{Loss of Blood; Haemorrhage.}

The escape of blood from the vessels into the tissue spaces, body cavities or to the free surfaces of the body is known as 
bleeding, haemorrhage (aĩa, blood; parás, rupture) or cxtravasation (extra, without: a'as, vessel).

The most common causes of hæmorrhage are wounds and lacerations of the vessel walls. Anything which severs the continuity of the vascular wall by puncture, incision, tearing, concussion or contusion affords opportunity for the effusion of blood (traumatic lamorrhage). In the same way all conditions which impair the resistive strength of the vessel walls to the pressure of the blood favor hæmorrhage, as the lesions cansed by the action of corrosive fluids, fatty degeneration of the walls of the vessels, vascular inflammations leading to brittleness and fragility of their tissues (corrosion hemorrhage, spontaneous hemorriage). Exaggeration of blood pressure (increase of pressure from sudden and powerful cardiac contraction and from hyperemia) permits the rupture of these weakened vessels, or perhaps even normal pressure may be sufficient. Rupture of the liver is not infrequently met with from such a cause after fatty degeneration of its vessels and tissues. ()r when the muscle of an artery (after chronic inflammation) is in a degenerate state its walls may be distended to form permanent dilatations [aneurisms]; here the wall comes to consist of but little more than connective tissuc, which tears when the distension becomes great.

Hæmorrhages of the types mentioned are spoken of as hæm-

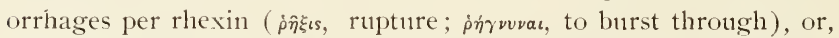
particularly where the wall has been weakened by pathological

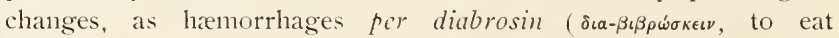
through).

The blood may, however, leak out of the smalier vessels, capillaries and veins, without the apparent existence of any lesion in the continuity of structure, because of some abnormal permeability of their walls. Such hrmorrhage is known under the

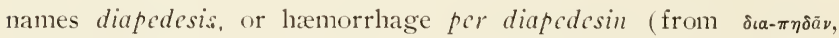
to burst through). The increased permeability of the vessel wall is explained by the fact that under the influence of toxic, infectious, thermic or other injurious agencies, as well as from marked engorgement and distension of the capillaries (passive hyperæmia, stasis), the cement substance between the endothelial cells becomes porous; for example, the endothelial cells may shrink into globular form and separate from each other, or openings may result from destruction of the cells. The red corpuscles then slip through between the cells or are forced out along with the plas- 
matic exudate; because of their elasticity these corpuscles are easily able to pass through the most minute clefts of the cement substance. There exist therefore in these cases minute microscopic tears. Hamorrhage by diapedesis, however, may assume important proportion and may be fatal because of its location (as in the brain or stomach) or of its duration.

Since it is the bathing of the endothelium with normal blood which is essential to its integrity, it may be easily apprehended that even a temporary interruption of the circulation to

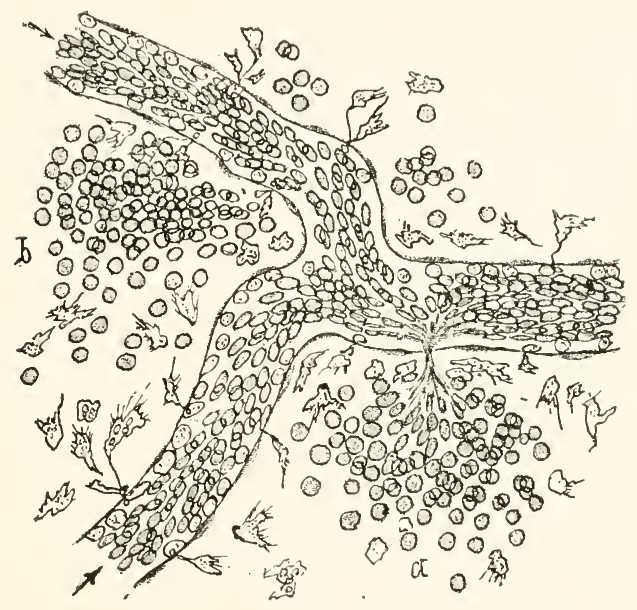

Fig. 1.

Mesentery of the dog. (a) Ilimorrhage by diapedesis, (b) Ecchrmosis occurring in a similar manner, the opening in the wall of the capillary having closed up agaill. The figure also shows an emigration of lencocytes. Ilagnified 2in times. (After Thoma.)

the capillaries may render them abnormally permeable and be the cause of diapedesis after restoration of the flow (cf. cmbolism).

Hæmorrhages are also classerl according to the type of ressel from which the blood escapes, as arterial, z'enous and capillary hamorrlages; where the blood oozes from a widely diffused fault in the softer tissues, as in the liver, and the type of the bleeding vessel cannot be made out, the term parenchymatous hamorrhage is appropriate.

The blood may escape freely to the exterior of the body or 
into one of the internal body cavities (cxtemal and internal hamorrhagc, surface hamorrhage and hamorrhage into a cavity). When the exuded blood fills the tissue spaces and completely saturates the tissues, the terms hamorrhagic infiltration or hamorrhagic infarction (infarcere, to stop up or fill up) are applied, the latter term being used particularly when the blood coagulates in the tissue and remains in it as a dense compact mass [usually in a definite circumscribed area of infiltration further described in connection with embolism]. If the bloody effusion causes an extensive, loose, gelatinous swelling of the tissue, diffusely filling the structure [applied particularly to infiltration beneath some surface], it is spoken of as a suffusion (suffundere, to pour) or suggillation. Small circumscribed foci of hæmorrhage, apparent as spots and points from which the blood cannot be discharged by pressure (especially in serous membranes), are called hamor-

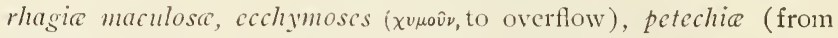
pidocchio, louse or its bite: or from pedicula flea-bites [pctigo, eruption]), or vibices when in streaks (cibcr, a streak). When the extravasated blood collects in a rounded mass, as when it dissects up the capsule of an organ or a connective tissue structure, or when it accumulates in a carity or becomes encapsulated by a surrounding inflammatory zone, the mass is spoken of as a hamatoma (blood boil) or hamorrhagic cyst. Special terms are also employed for these effusions of blood depending upon their location: an extravasation of blood into the pleural cavity is termed hamothorax; into the peritoneal sac, hamocolia ( $k$ oisia, the body cavity); into the pericardial cavity, hamopcricardium; into the uterus, hamatomctra ( $\mu \hat{\eta} \tau \rho a$, uterus); into the sac of the tunica vaginalis testis, hamatocclc ( $\kappa \dot{\eta} \lambda \eta$, rupture). In addition special names have always been made use of in connection with hæmorrhage from special organs: cpistaxis (i $\dot{\pi} \iota \cdot \sigma \tau \kappa \dot{\zeta} \epsilon \iota \nu$, to drip) for nasal hæmorrhage; lacmaturia (ovipov, urine) for the escape of blood with the urine; hamatcmesis (é $\mu \epsilon \hat{\nu}$, to vomit)

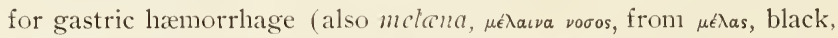
because of the dark brown color given the blood by the gastric juice); hamoptysis ( $\pi \tau \dot{v} \epsilon \omega$, to spit) for expectoration of blood from the lungs; metrorrhagia for hæmorrhage from the uterus;

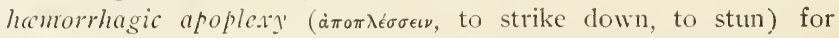
spontaneous hæmorrhage of the brain.

Symptoms and Results. From its characteristic blood tint an effusion of blood is easily recognizable anatomically as a dark red accumulation of blood, or spot which cannot be effaced and which 
is more or less sharply circumscribed. Upon surfaces or in the body cavities the extravasation presents a striking picture, in profuse, partly coagulated, partly fluid masses, or in a gelatinons, red, jelly-like tumor extending into the loose subcutaneous or sub-mucous tissue (hrenurrhage following contusions of the abdominal walls, hæmorrhagic stasis of the intestinal mucous membrane), or sharply outlined in numerous definite droplike flecks (not effaced on pressure) as seen in the sub-serous cellular tissue of the pleura, pericardium and epicardium. In the secretions it is easily appreciated by the more or less pronounced blood tint which it imparts to them: the intestinal contents may assume a slightly reddishgray, cafe-au-lait color up to a chocolate brown or pure blood-red from the admixture of blood; the gastric contents a sepiabrown, the urine a bloodred to black; the expectorate and nasal secretion a rusty, red-streaked or foamy red appearance (pulmonary œedema).

While it is usually easy to detect the ruptured ves-

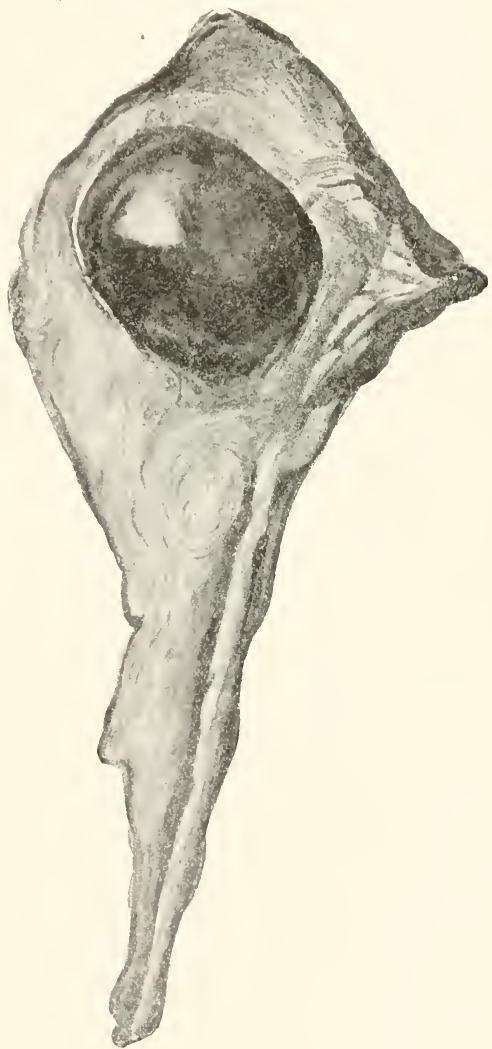

Fig. 2 .

Ilematoma of the spleen of horse. sel in case of massive hæmorrhages (as a ruptured aneurism, the eroded stump of a vessel in hæmorrhage from gastric ulcers), and the infiltration of blood surrounding the lesion in the organ points to the origin of the hæmorrhage and indicates its intravital occurrence, 
yet in capillary diapedesis from a mucous membrane, as the gastric or intestinal lining, the points of escape are invisible to the naked eye, and only the blood deposit on the surface and the pallor of the mucous membrane indicate the fact of extravasation. Arterial hæmorrhages are recognized in living animals by the bright red color of the arterial blood, by the spurting character of the escaping stream from the larger arteries, the spurts corresponding to the cardiac contractions; while the bleeding from the veins is continuous and without pulsation. However, an arterial spurting may be prevented by the interposed parenchyma (Samuel). If the larger vessels between the point of hæmorrhage and the heart be compressed the hæmorrhage will diminish if it be arterial; it will be increased if venous.

The results of hamorrhage depend upon the quantity and the duration of the bleeding, as well as the location of the lesion. In case of loss of over one-third of the total rolume of blood (about 3 to + per cent. of the body weight; the average total quantity being from one-tenth to one-twentieth of the weight of the body), the flow continting, the blood pressure falls. The ressel walls, although capable of wide adaptation to the volume of blood within, have become too wide for the diminished amount and cannot longer advantageously propel it. A general oligrmia has been established, indicated by the pallor of the mucous membranes, dizziness, fainting (anemia of the brain) and convulsions, and the animal may bleed to deatli. These results occur not only when the hæmorrhage is external, but also in copious hremorrhages into the intestinal lumen, peritoneal cavity, etc. (internal hæmorrhage).

Hæmorrhage gradually ceases by the closure of the opening in the vessel from the formation of a blood coagulum in the opening (adhesion thrombus), the closure being facilitated by constriction of the vascular lumen (contraction of the wall), external pressure (by the tissue engorged with blood), and by the fall of blood pressure. After such cessation the volume of the fluid portion of the blood is soon restored. The watery element first passes into the vessels from the tissues and for a time the blood is consequently more fluid (hydræmic) than before the hæmorrhage; but gradually the blood cells. regain their normal numbers as their regeneration (cf. chapter on regeneration) proceeds in the blood forming organs.

At the site of hemorrhagic infiltrations the tissues present more or less functional alteration. The presence of the extravasated 
mass of blood exerts a certain amount of pressure and in consequence the cells and tissues are forcel apart, displaced by the invading blood; and hollow viscera are occluded by the coagulated blood. Even small hæmorrhages in the brain, according to their precise location, occasion a loss of function in the area affected, perhaps even to the extent of complete obliteration of consciousness; in the case of spinal hemorthages palsies ensue; hemorrhage under the mucous lining of the air passages (fracture of the trachea and larynx) forces the same into the lumen to such a degree that the air passage is constricted and death from asphyxia may result.

The escaped blood usually coagulates, precisely as the blood in venesection ( $\mathrm{cf}$. chapter on thrombosis). Should this clot remain in the tissue or in one of the serous sacs it acts as an inflammatory excitant; it attracts the leucocytes by chemotaxis and causes a limiting zone of connective tissue proliferation about it. This gradually forms a capsule about the collection of blood, transforming it into a hæmorrhagic cyst, often called a hrematoma, in which the disintegrating blood exists, partly as soft, elastic, fibrinous masses, of a reddish or brownish color, partly as liquid serum expressed from the clot. Such hrematomata, sometimes approaching the size of a human head, are not infrequently seen in the spleen of domestic animals, around the kidneys in swine, and in the pelvic cavity of cattle as the results of contusion hrmorrhages. On the other hand the penetration of lencocytes to the mass and the proliferation of fibroplastic and angioplastic tissue leads to the resorption of the blood. Its disintegrating elements are taken up by the amoboid cells and carried away; the plasma is absorbed by the cells and may pass off through the lymph paths. The red corpuscles both in the tissue lymph and in the blood vessels begin to swell and lose their color or to become shriveled. The coloring matter, hæmoglobin, having been dissolved out may soak through the tissue diffusely or may become deposited in the form of flakes, granules or more rarely as crystals through the tissue. The crystalline form is especially apt to occur where the blood has been stagnant (hrematoma), appearing as ruby-red to brown, minute rhombic plates and needles (iron-free hæmatoidin); the granules are yellow and angular, sometimes give an iron reaction (hremosi(lerin), sometimes undergo a change like the crystals by which the iron is separated from the hamoglobin, iron-free hrenatoidin (hæmofuscin) remaining. 
This blood precipitate is found microscopically not only at the site of hremorrhage, especially in the amoboid cells which are loaded with it, but also in the nearest lymph glands which are often found tinted all through by a rusty, reddish-brown color from the quantity of blood pigment conveyed to them. In the dead body, on account of sulphur products (sulphuretted hydrogen) of the cadaveric bacteria, the site of a hemorrhagic infiltration may be found changed to a slate gray to black hue by the sulphur combinations; in life, too, in places where similar bacterial accumulations and fermentations occur (intestine, abscess cavities, fistulous paths, mycotic diseases of the bladder) the pigment may undergo the same changes in color and thereafter remain as evidence of previous hæmorrhage.

\section{Dropsy, Hydrops and 0edema.}

Excessive accumulation of the tissue fluid or lymph in the lymph spaces, lympli vessels and serous sacs (lymph sacs) is spoken of as dropsy, hy'drops (from $\ddot{w} \delta \omega \rho$, water) or hydropsia and, if the accumulation has occasioned swelling of the tissue, as oedema (from oĩoav, to swell). The process itself is described as a dropsical transulation (from sudare, to sweat); the accumulated fluid as a transudate. Neither the process nor the transuded fluid is essentially anything more than a quantitative disturbance of physiological lymph-production. Lymph is originally derived from the blood, and is regarded by many as a secretory product of the endothelimm of the capillaries, although the blood pressure is also maintained as a factor in its production and accumulation, affecting the filtration of the liquid part of the blood through the capillary walls. [Whether the physical processes of dialysis and osmosis should also be invoked here is debated. Lazarus-Barlow, in discussing the vital transudation of $1 \mathrm{ymph}$, urges the importance of tissue demands for lymph both for purposes of nutrition and for its influence in diluting and removing various products of their metabolic activities or of their degeneration, and points out that in this last case there exists a possible explanation for the persistence of an œdematous process in case the excretory organs as the kidneys fail of eliminating from the blood the various waste materials. Thus if, in a case of nephritis, the blood becomes surcharged with waste materials and if at the same time such waste in a local area of special activity or of degeneration is excessive, 
the tissue demands for increased transudation are increased in order to accomplish its removal by the lymph; yet if the blood already contain an excess of the very substance in question the increased transudation only serves to more nearly complete a vicious cycle, and adds to the existing accumulation.] Two reasons may be assumed for the pathological increase of this fluid in the tissues, either an obstruction to the lympl drainage, or an unusual, exaggerated transudation; both of which factors may be operative at the same time. In, view of the free anastomosis and the great abundance of lymph channels, together with the fact that lymph absorption is also accomplished by the capillaries and veins, obstruction of isolated lymph channels, it may be said, does not ordinarily occasion lymph stasis. Even in case of unusial formation of lympl there is 110 essential reason for determination of any abnormal condition, as within certain limits the paths of lympluatic drainage are capable of accommodating and carrying off large quantities of fluid, provided the tissues retain their proper elasticity (Landerer), and the propulsive forces (muscular movements, etc.) are of normal functional excellence. It is only when the increased transudation becomes extreme and the lymphatic flow fails to keep pace, that oedema develops.

The most common cause for such fault is passive hyperæmia. In case of passive congestion the blood pressure in the capillaries becomes raised and the current of blood slowed, as a result of which the endothelium of the walls of the capillaries becomes abnormally permeable and in consequence the fluid element of the blood exudes profusely. This exudate is usually not as rich in albumen as the normal lymph. Is examples of odema of this type may be suggested that caused by compression (as cedema of the lips by application of twitches to a horse's mouth), by venous thrombosis, by cardiac lesions which impair the current of blood in the venze cave, by pulmonary changes which prevent proper progression of blood from the right heart, by structural faults of the liver causing obstruction of the portal capillaries and producing portal congestion (dropsy or adcma from passiz'c congcstion). Coincident interference with lymphatic drainage may be determined perhaps by the pressure of fluid accumulations in the thoracic or peritoneal cavities upon the thoracic duct, perhaps by the loss of natural elasticity of the tissues, stretched and 
distended by the increased and persistent pressure of the lymphatic transudate.

Increased transudation is observed also in connection with inflammation and changes of the vascular walls caused by toxic chemical agents acting either locally or, when circulating in the blood, widely (irritatice or inflammatory dropsy). One should recall in this relation the influence of the slowing of the blood current and vascular dilatation, together with possible shrinkage and separation of the endothelial cells, combining to render the vessel walls more permeable; noreover, a number of poisons, as some of the products of metabolism, seem to directly stimulate the endothelium to increased secretory activity, to possess what might be called a "lymph-driving" (lymphogogue) action. In addition it may be pointed out that in inflamed tissues the lymph flow is impaired by the fact that the inflammation occasions coagulation of the lymph and impairs the elasticity of the tissues. Such features explain the occurrence of cedema around tissues which are the seat of purulent or hamorrhagic inflammation (collateral adema).

Dropsy is often concurrent with wasting diseases and abnormal states of the blood characterized by porerty of its cellular elements and increase in its proportion of water, the so-called cachectic or hydramic odcuia, as scen in cases of pulmonary verminosis, fluke disease of the liver and chronic parenchymatous nephritis. It is an open question in such cases whether the essential fault in determining the dropsy is the dilution of the blood which may perhaps make it filter more readily through the vessel walls, or whether we should ascribe it to the presence of metabolic products and other toxic matter in the circulating blood impairing the vascular endothelium and permitting the transudation because of an increased permeability. (Cohnheim and Lichtheim failed to obtain dropsy by introducing large quantities of sodium chloride solution into the blood of experiment animals, even when as much as half of the blood was replaced by the saline solution; Gärtner, however, succeeded in causing an œdema in dogs by long continued infusion of salt solution.)

No satisfactory explanation exists for the rather common congenital anasarca of aborted calves. According to the investigations of L. Franks the thoracic duct is sometimes missing in these "water calves" (or "Dunstkälbern") : while in other cases 
renal changes, perhaps the sequels of an intrauterine nephritis, are met which may occasion dropsies because of retention of water or on account of some infectious toxic intluence present.

The dropsical transudate is usually a colorless or wine-yellow water-like fluid, generally containing a smaller proportion of aibumen than the lymph and fluid exudates, as a rule showing only o. I to o.8 per cent. albumen, according to l'erls up to 5

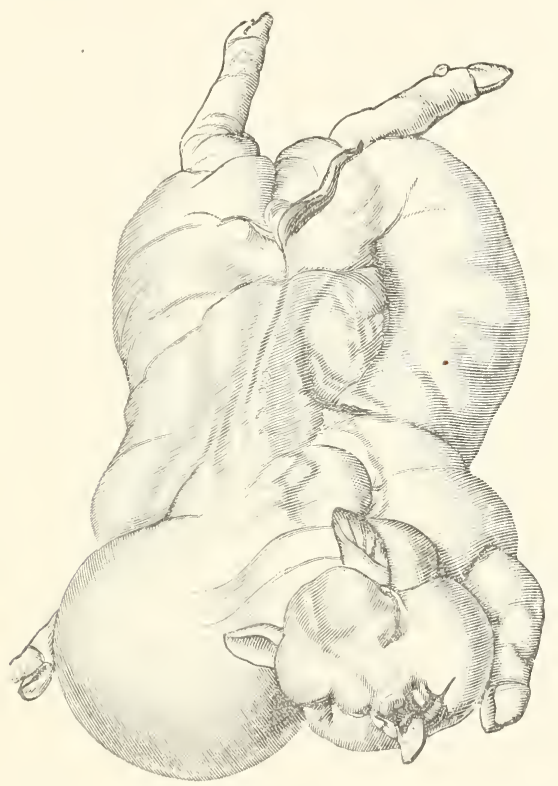

Fig 3 .

Anasarca universalis congen., so-called water calf.

per cent. (Lymph and blood plasma contain about 7 per cent.) The proportion of salines is the same as in the plasma (o.S per cent). [The precise composition, particularly as relates to proteid, varies in the different types of dropsical fluid; and eren in the same subject the amount of proteid is not identical in fluid from different parts of the body, that from the subcutaneous tissue being usually marked by the lowest proteid content whilc 
that from the serous cavities, especially the pleura, is apt to be richer in albumen.] In case of coincident escape of blood corpuscles (in passive hyperæmia) admixture of these elements with the transudate may occur to a sufficient extent to give it a blood-red color and occasion fibrin deposition (hydrops hamorrhagicus). In other instances admixture of fat from the blood or from fatty degeneration of the cellular elements macerated in the fluid may give a milky appearance to it (hydrops adiposus, chyliformis); and in abdominal dropsy a similar appearance may be occasioned by rupture of the chylous vessels and admixture of chyle with the fluid (hydrops chylosus).

The amount of fluid in the large body cavities may reach considerable volume, IO, 50 to Ioo liters in large domestic animals. Such quantities of fluid are naturally capable of exerting marked pressure upon the different adjacent organs, and distend the walls of these cavities. Consequently in case of ascites the peritoneal cavity is considerably enlarged and becomes a fluctuating sac; the pericardial sac attains a wide outline and fluctuates: and even the chest wall may be distended and barrel-shaped from the separation and outward displacement of the lower ribs. In the fœus, in which the fontanelles are still vielding, the corering of the brain becomes a huge sac, partly membranous, partly made up of the cranial bones forced apart and transformed into thin plates. When the transudate fills the spaces of a tissue the porous cellular structure becomes a gelatinous, amber-colored, swollen mass, from the cut-surface of which a serous fluid drips and whose doughy swelling retains for a long time the pressure marks of a finger. Sometimes bladder-like collections or cysts may form from the forcible distension of the connective tissue spaces.

Various terms have by custom been applied to these accumulations of transuded fluid. Edema of the skin is spoken of as

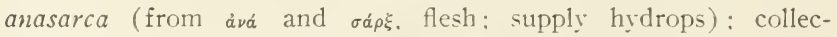
tions in the pleural cavities as hydrothorax; in the pericardial sac, $h y d r o p e r i c a r d i u m$; in the peritoneal cavity, ascites (from á $\sigma$ kós, belly; namely, hydrops ascites): in the tunica vaginalis testis, hydrocele (from $\tilde{\delta} \delta \omega-\kappa \eta \dot{\lambda} \eta$, water rupture): in the cranial cavity, hydrocephalus.

The result of the odema depends upon the location and cause of the condition. Local dropsies of the skin may last a long time, but eventually disappear after the removal of the cause and the resorption of the fluid. Edema of the tissues about the 
laryngeal entrance (cedema of the glottis) is dangerous, occasioning asphyxiation by narrowing the air passages; redema of the lungs and of the brain may be fatal by causing functional disturbances of these organs. The micchanical pressure effects of collections of fluid in the serous cavities upon the blood circulation, the compression and displacement of viscera (pressure on the diaphragmi), as secondary results of the dropsy, increase the severity of the primary disease which was the cause of the dropsy (affections of the heart, liver or kidneys).

\section{Obstruction of Blood Vessels. Thrombosis and Embolism.}

Obstruction of blood ressels may be due to the formation within them of relatively solid plugs made up of the blood constituents, this process being known as thrombosis ( $\dot{\theta} \theta \rho \dot{\rho} \mu \beta o s$, mass; from $\tau \rho \epsilon \phi \omega$, to make firm or compact), or to forcible lodgment in their lumen of materials conveyed by the blond current, this latter precess being known as embolism ( $\dot{\epsilon} \mu-\beta a ́ \lambda \lambda \epsilon \iota \nu$, to throw in).

It is well known that blood ontside the body clots in a few minutes after its escape from the ressels, as after venesection. There is formed from the blood a red jelly-like mass, the bloody cruor sanguinis; over the surface of which there collects a pale vellow fluid, the blood serum. The clot is primarily constituted of fibres of coagulated fibrin, which include the blood plaqnes. In case of slow coagulation the red corpuscles sink to the bottom of the containing ressel and thus the superior part of the coagulum is largely occupied by the lighter and therefore less readily sinking white blood corpuscles; for which reason the surface of the clot shows a vellowish-white layer composed of fibrin and lencocytes. the so-called buffy coat. The formation of fibrin is the main feature of extravascular coagulation, and can be directly followed in a drop of blood under the microscope. In a blood drop placed upon the cold slide the film can be observed to separate as fine threads, and these, with the platelets adhering, wnite with each other to form a network (Birch-Hirschfeld). Fibrin is an albuminous substance, existing in solution in the circulating blood in the plasma (the so-called fibrinogenous material or fibrinogen), which separates as a coagulating calcium compound with proteid when acted upon in the presence of calcium salts by a ferment known as the fibrin ferment.

Following the apt comparison of Birch-Hirschfeld, blood coagulation may be thought of as somewhat analogous to remnet coagulation of milk. 
In the presence of lime salts of the milk, casein (which is similarly a calcium proteid combination) is separated from the milk by the labferment, leaving a serum, the whey.

Coagulation of blood may be checked or inhibited by certain chemicals which destroy the ferment or which dissolve the fibrin immediately after it is formed (potassium citrate, or oxalate, extract of the cephalic glands of the leech). [Extract of the ceplalic end of the hookworms of the $\log$ possesses a similar property.] So, too, coagulation fails if the inner surfaces of the vessel in which the blood is caught be covered with vaseline, thus preventing adhesion (Freund).

After death the blood clots quickly and in a manner similar to extravascular coagulation, especially in the heart and larger vessels ; in the capillaries it remains fluid for a longer time. In the presence o: toxic substances having solvent action upon the blood and fibritn, too, coagulation is incomplete (hamolytic substances, as some of the bacterial products, presence of excessive amounts of carbon dioxide in the blood). Cada'cric clots are homogeneons, soft. elastic, gelatinous masses which look like casts of the ressels. are without lamination, and are either of a dark red color throughout or of a more or less yellowish gray to amber lute with jelly-like semi-transparent appearance. The latter form, comparable to the "buffy coat" are met in anæmic states and gradual cardiac failure, the blood cells in such instances settling and the fibrin containing a large amount of serum. [In these heart-clots the dark red homogeneous examples are commonly interpreted as indicating rapid death and quick coagulation before the corpuscles have had time to separate; they are thus referred to as post-mortem clots. On the other hand, the yellowish translucent coagula, occurring in slow circulatory failure, are often spoken of as ante-mortcm clots, from the idea that they form or start to form during the period of slowing of the current prior to death, the red cells at least sinking more or less from the general blood volume prior to the actual stoppage of the current and coagulation of the blood.]

Coagulation and thrombus formation within the vessels of the living takes place from the following causes or favoring conditions:

I. Entrance of chemicals into the circulation favoring coagulation of the blood (ferment thrombosis): By the injection of ether and other chemicals, the introduction of substances destructive to the leucocytes, injections of emulsions of various parenchymatous tissues (brain, adrenals) or of the expressed juices of the thymus 
body, testicles, etc., or of laked or altered bloorl, it is possible to bring about extensive coagulation with fatal effect. Such experimental findings warrant the assumption that the occurrence of tirrombosis is, at times, in infections and diseases accompanied by tissue destruction dependent upon the coagulative action of microbic foisons or tissue products permitting the formation of fibrin ferment by the disintegration of leucocytes.

2. Slowing and cessation of the blood current: It has been

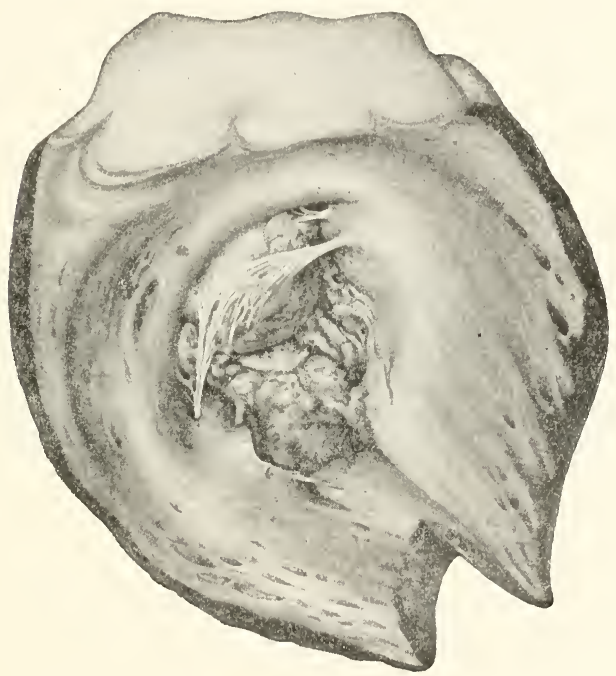

Fig. 4 .

Cardiac valvular thrombosis (right ventrical of heart of hog).

shown by experiment that after clouble ligation of a vein or artery, although the blood in the segment letween the ligatures is entirely stagnant, it may remain fluid for months (Baungarten. Senftleben and others). Nevertheless in situations where blood ressels are expanded, particularly when the seat of pathological dilatations, and when the flow of the blood is slackened, thrombi are particularly likely to form, because these ectases favor the agglutination of leucocytes and plaques from which arises the inception of the thrombotic process (stagnation thrombosis). 
3. Alterations of the lining of the vascular wall: Lesions of the vascular intima resulting in the destruction of the endothelium and thus causing a loss of the natural smoothness of its surface give opportunity for adhesion of platelets and white blood corpuscles to the roughened surfaces and give origin to thrombus formation (adhesion thrombosis). In lacerations, incised and puncture wounds as well as contusions of the vessels, thrombi invariably form, an important feature in wounds both as regards healing and preservation of life, the clot formation gradually closing the rent in continuity of the ressel and checking the loss of blood. Thrombosis also takes place in case of vascular changes caused bv bacterial invasion, parasites, perforating neoplasms, and in vessels in the neighborhood of active inflammatory lesions, as upon the valves in case of endocarditis, in the vessels of the lungs in pneu-

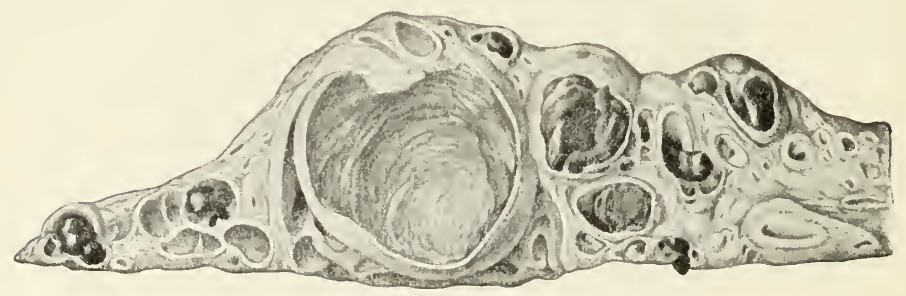

Fig. 5.

Thrombosis in a carernous angioma of the liver of a cow.

monia, in parasitism of the arteries by strongylus armatus, in case of invasion of pyogenic bacteria in the umbilical vein, etc.

As a rule two or three conditions favoring thrombus formation cooperate to cause it; thus when a thrombus is formed after bacterial invasion of a vessel, it is partly the result of special ferment production, partly due to injury of the intima.

The composition of thrombi varies with the rapidity of formation and the causes of production. Those rapidly formed and raused by special elaboration of ferment are often quite like the clots of external hremorrhage, homogeneous and of a dark red color therrighout, being made up mainly of fibrin and red blood corpuscles (homogcileous thrombi); microscopically the fibrin forms an intricate reticulum of delicate threads in a radiating or stellate form around the disintegrating leucocytes which furnish the fibrin ferment (coagulation centers of Al. Schmidt and Ribbert). Stratified 
or layered thrombi, usually forming upon a limited area of the vessel wall, legin by the adhesion of white blood corpuscles and plaques to the altered part of the vessel wall and their accumulation at such site; upon this basis fibrin is deposited in layers of varying extent and quantity, enclosing the corpuscular elements of the blood in its structure. The arrangement of this coagulum is often such that in microscopic sections the framework of the thrombus may be observed to be formed of way trabecula of granular substance made up of blood platelets (Ribbert): these bands always surrounded by a broad zone of letcocytes and between them the network of fibrin fibrils spread ont with the red corpuscles included in the reticulum (Ribbert). Corresponding with the greater or smaller amount of the constituent fibrin and cells and the types of formation described, the general appearance of thrombi and their color and consistence vary. There may be distinguished the following varieties from this viewpoint:

I. Red thrombi, showing a homogeneous, blood red, gelatinous mass, similar to the post-mortem clots ["currant-jelly thrombi"].

I. White and grayish-red thrombi, reminding one of bacon-fat or spinal-cord tissue or minced meat, not transparent, but opaque, dull, of the consistence of soft rubber or cooked minced meat.

3. Mi.ted and stratific thrombi, made up of alternating layers of red and white coagulum, well seen in cross-section, often arranged concentrically like the tunics of an onion and separable like the leaves of a book from each other. The reason for this lamination and alternating arrangement is to be found in the fact that new layers are not being continuously formed upon the primary clot but are deposited at intervals, the older layers thus attaining a certain degree of firmmess, and the new ones not adhering so closely to the previously formed strata and remaining easily separable from them. Where such mode of increment is frequently repeated the thrombus may exhibit quite a number of layers. The surface of these thrombi may be either smooth or uneven, often marked by ridges or ribs; the latter peculiarity is due to the trabecula of blood platelets and leucocytes extending to the surface and forming ledgelike projections with trough-like depressions between them (similar to the wavy deposit of river sand, and according to Zahn probably produced by the wave-like motion of the blood). [Such appearance may be produced, too, by the uneven contraction of the mass, the shrinkage being most marked in the fibrinous network lying between the coarser plaque-trabecula.] 
The clots appearing at the beginning of thrombosis are known as primary (autochthonous) thrombi; the coagula subsequently ceposited upon them as sccondary or consccutice thrombi. The former are more or less firmly attached to the vessel wall (parictul thrombi); the latter usually lie free in the lumen as cylindrical plugs with conical or rounded ends. The thrombus increases principally in the direction of the blood stream, in the arteries toward the periphery of the circulation, in the veins centripetally. The length may be quite considerable, as in those extending from the veins in the neck down into the heart, or from the aorta at the level of the renal artery down into the arteries of the popliteal space and leg with or without (discontinuous) continuity. The thickness depends upon the calibre of the blood vessel; parietal clots are at first flat, marked with wary ridges, villons, or in the pockets of the valves of the veins may form smoothly rounded plugs (zalitular thrombi); with growth the thrombus may canse complete occlusion of the vascular lumen (total occluding thrombi). In arteries thrombi are usually smaller than the calibre of the relaxed ressel, being compressed by the contraction of the pulsating ressel wall. In aneurisms and in the cardiac climbers there occur huge irregular nodulated thrombi, sometimes are large as the fist. Thrombi in the course of time undergo a number of changes (metamorphoses) as well might be supposed. They are but dead masses made up of coagulated and necrotic elements and may slirink, becoming firmer, drier and smaller; such changes depending upon changes in the structure with lomogenization of the fibrin and plaques and upon pressure exerted by the walls of the vessels. In clots which have undergone such changes there may occasionally take place a calcarecus deposit (vein stones, phleboliths). As a result of such shrinkage the vascular lumen may again become partially permeable to the blood. [The diminution in size of an old clot depends in an important degree upon the shrinkage of its own fibrinous network; this may be easily noted in the shrinkage which takes place in any clot ontside the body, with the separation of the serum originally present in the coagulum, the question of compression by the vessel walls being in such case entirely eliminated.] The most common change consists in a softening of the thrombus, the clot being transformed by fatty degeneration of its lencocytes [and liquefaction of any of its elements] into a gray or grayish-red mass of pultaceous consistence somewhat like minced meat. This change may be a favoring circumstance, but is open to serious consequences. In 
(ase the substance is broken up into a finely granular fatty material this may be taken up by the blood current and washed away and the lumen of the vessel may thus be again established, or through the softened clot the blood stream may sometimes force one or more passageways (canalization of the thrombus). Should, however, larger fragments, as a result of the degenerative changes, be loosened from the coagulum and swept onward by the current of blood they may find lodgment in some other part of the circulatory apparatus and give rise to embolism.

A thrombus acts as a foreign body upon the living vessel walls with which it is in contact; and its characteristic properties excite an invasion by lencocytes and phagocytic activity. The latter may, as is uniformly true in case of small tranmatic thrombi, bring about complete absorption of the clot Coincidently there is established an inflammation of the ressel wall with fibroplastic and angioplastic proliferation, result- $\omega$ ing in the formation of embryonic connective tissue and vascular buds from the endothelium and vasa vasorum and their penetration into the thrombus. These proliferating elements replace in time more or less completely the clot substance. In case the thrombus were a parietal one, occupying only one side of the vessel wall, as after venesection, after removal of the clot the connective tissue contracts and shrinks into a flat scat or mere thickening of the

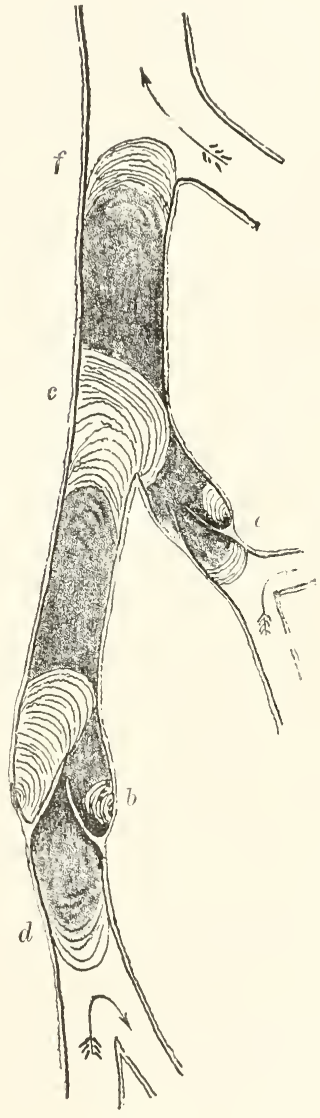

Fig. 6 .

Schematic section of a thrombosed rein; somewhat enlarged. (After Troma.) wall. Where the thrombus occupies the whole lumen the embryonic tissue develops about it, and in its growth pene- 
trates the mass; and in the site of the clot there is formed a mass of connective tissue. These changes are known under the term organization of the thrombus. The ressel in the situation in ciuestion is converted into a solid cord, its lumen completely obliterated; and as a result of shrinkage of the newly formed connective tissue the part eventually may be seen as a thin string-like obliterated section of the vascular tube. Now and again spaces

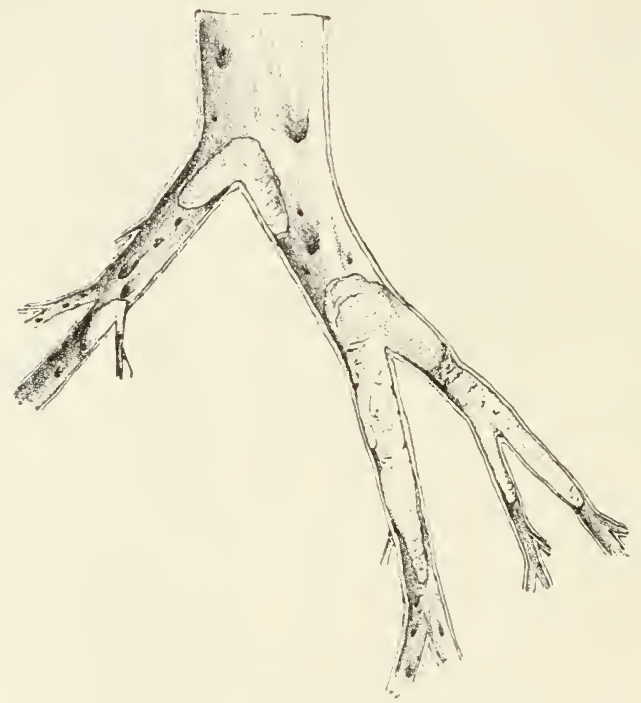

Fig. 7 .

Embolism of branches of the pulmonary artery; the superior embolus almost completely adhering to the ressel walls and shruken. Somewhat reduced. (After Thoma.)

are left in the connective tissue, however, through which blood in sone degree may pass.

If a thrombus or a portion of a thrombus should break away, it is carried forward with the blood current and must find lodgment at some point where the calibre of the artery is less than the diameter of the floating mass. The latter is then spoken of as an embolus, and the process as embolism. Detachment is especially likely to take place in connection with softening of a thrombus, in which process the clot may easily crumble; however, even entire 
thrombi, no matter whether loosely or firmly attached, may sometimes accidentally be torn loose by external force exerted upon the ressel through movements occasioning twisting of the vessels or increase of blood pressure. At the points of branching of the vascular tubes it may happen that a thrombus or embolus may strike upon the dividing angle and here again be broken into fragments or may remain fixed at the point of division. It is quite likely that an unusually long, flexible and tough thrombus may so iodge at these places of division as to extend into both branches of the vessel, riding as if in a saddle (riding cmbolus). A long cylindrical, flexible thrombus may, moreover, be thrown into folds. twisted in serpentine fashion and compressed into a mass by the force of the blood stream.

Although an embolus displaced from its original site is forced into a relatively narrow portion of the vessel, it does not necessarily at once completely occlude the lumen, since as a broken fragment of a thrombus it need not retain the cylindrical shape of the vascular tube, but may well be of irregular and angular outline. Such emboli, particularly when of the riding type, are apt to leave room free about them for the passage of blood. Cylindrical or round emboli are comparatively easily forced into the vascular lumen and give rise to complete obstruction. An embolus which has become lodged tends to further enlargement, either by the massive coagulation of the stagnant colmmn of blood adjacent or by the deposition of thrombotic layers. Sooner or later this leads to complete obstruction of the vessel. In these cases it is often difficult to determine whether there was primary thrombosis or whether an embolism formed the nucleus of the blood clot, which, from the formation of new layers, comes to completely fill out the lumen of the vessel, and the original embolus may, moreover, have been very small, and entirely hidden by the further depositions.

The course traversed by an embolus is determined by circulatory circumstances. An embolus carried from the veins must always pass into the right heart and thence into the pulmonary arteries; it may, however, adhere to the auriculo-ventricular valve leaflets in the heart. Its convection to the heart is easy, the veins becoming wider and wider from the periphery centrally to the venæ cavæ. If therefore a fragment become loosened from a thrombus in veins of the foot, thyroid, uterus or liver it would be swept by a continually widening current to the heart. Coming 
from the intestine, stomach or spleen in the portal vein, emboli must lodge in the liver; embolism of the latter organ arising also from the umbilical vein, inasmuch as there is no direct communica-

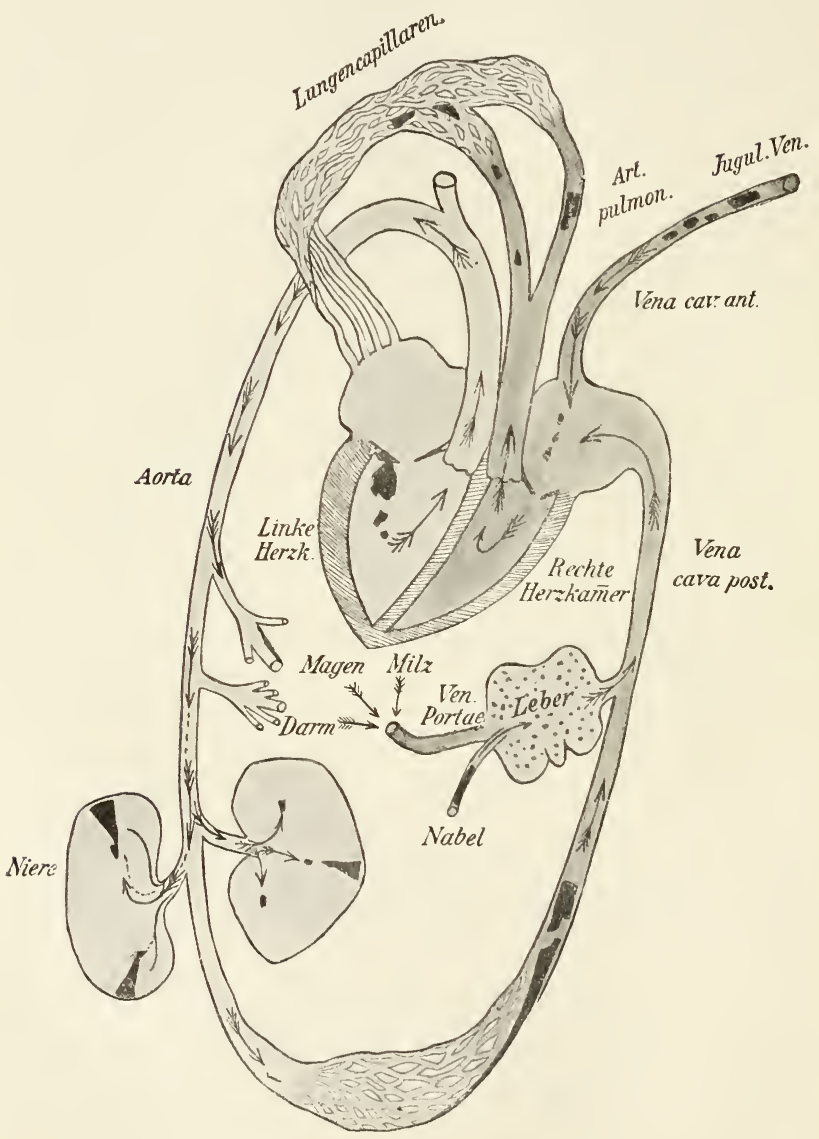

Fig. 8.

Schematic representation of the circulation with fragments of thrombi at different positions (cf. text). Lungencapillaren, pulmonary capillaries; Linke Herzk., left cardiac ventricle; Rechte Herzkammer, right cardiac ventricle; Magen, stomach; Milz, spleen ; Darm, intestine; Nabel, umbilicus ; Leber, liver ; Niere, kidney. 
tion of the portal and umbilical veins with the vena cava, their blood passing into the fine meshed capillary network of the portal vein within the liver substance. (In calves, however, where the ductus Arantii forms a direct passage between the umbilical vein and the vena cava the lung may stand equal chance of cmbolism

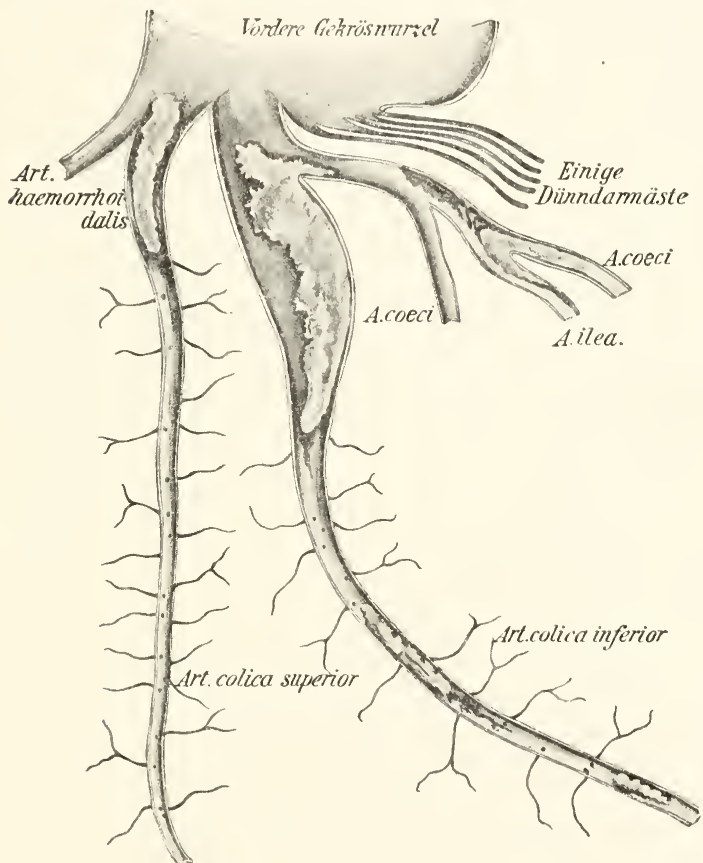

Fig. 9.

Thrombosis of the colic arteries of a horse; a riding embolus in arterit copi. Vordere Gekröswurzel, anterier mesenteric trunk; einige dïnndarmäste, branches to small intestine.

from the last named source.) Emboli from the left heart, from the lungs (pulmonary veins), entering the aorta and arterial tree always find their way into the peripheral arterial branches, as from the anterior portion of the aorta into the brain, from the postcrior aorta into the kidneys, pelvic arteries, etc., from the mesenteric trunk into the arteries of the intestines. As a rule blood clots are 
not so minute that they can be carried from the arteries through the capillaries into the veins, for which reason such emboli from the pulmonary arteries become fixed in their branches, that is, in the lungs; other types of emboli, however, as minute animal parasites or bacteria, may traverse a capillary area and may therefore pass from the greater into the lesser circulation and in turn arrive again in the greater.

In very rare instances in human beings, where there is a patulous foramen ovale, emboli have been encountered in the aorta and its branches which did not originate in the left heart or lungs, but which passed directly from the right into the left heart through the patulous opening. When first seen, before the condition of the foramen orale has been noticed, the existence of such embolism seems inexplicable and confusing. for which reason such an occurrence is spoken of as paradorical cmbolism. In addition, it should be mentioned that there is a possibility of retrograde cmbolism of embolic substances in the veins. This, according to Ribbert, may be explained on the supposition that clots or emboli of any type which happen to lie close to the inner surface of a comparatively large vein are always subject to slight backward repression by recurrent waves of the venous blood (venous pulsation in passive congestion), somewhat as at the shore the surging wave carries back with it a floating log into the sea. If the venous current to the heart be weak, as in congestive states, it may not carry the embolus forward with it, while the more powerful backward impulse forces it gradually back toward the periphery to the capillaries. [This is well seen sometimes in cises of valvular insufficiency and regurgitation.]

The results of thrombosis and embolism vary with the vascular conditions, the size and nature of the obstructive substance and the relative importance of the affected organ. In part they are purely mechanical, depending upon the degree of narrowing or obstruction of the lumen occasioned by the process and the consequent disturbance of circulation; in part the results may be spccific (peculiar), the nature of the thrombus involving some special properties (as pyogenic or putrefactive agencies, or tumor forming elements). Thrombosis of arteries occasions primarily an anæmia of the organ or area supplied by the artery affected. This anemia in vital organs like the brain or heart or lungs necessarily immediately induces disturbances of function dangerous to life itself (fainting, cessation of cardiac action, phenomena of asphyxia), if an important artery be involved and quick compensation by anastomotic blood supply is impossible. In case, however, branches of the occluded or obstructed artery are so connected with other adjacent arteries that a sufficient amount of blood is supplied to 
the organ by these anastomoses or collateral communications, the anæmia is likely to be only of short duration and without functional disturbance. In many organs such collateral paths are very abundant, and in some, as in the muscles and lungs, they are so numerous and of stuch calibre that sufficient blood is transmitted to the blocked area of the artery by the ordinary collateral circulation without any special effect. The blood which previously tra-

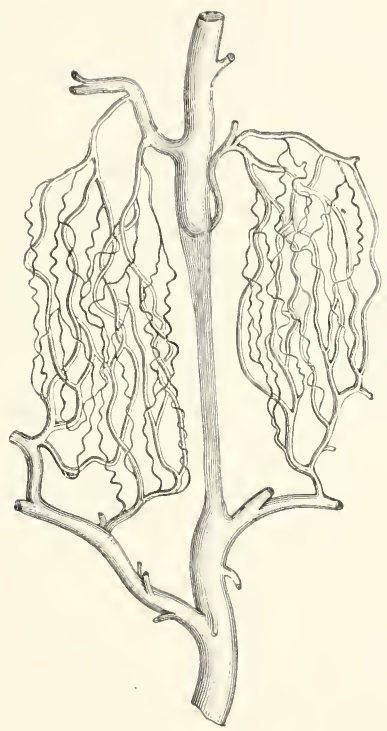

Fig. 10 .

Femoral artery of a large dog, injected three months atter ligation. iffer l'orta.)

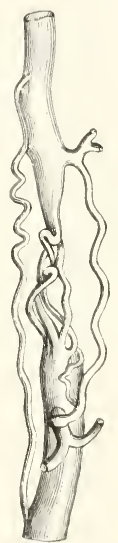

Fig. 11.

Carotid artery of a guat, injected thirty-five months a $i$ te $r$ ligation. (After Porta.)

versed the occluded vessel is of course distributed throughout the permeable branches arising proximal to the site of obstruction and driven into these by the arterial pressure. The blood pressure in these collateral paths must therefore be somewhat raised, for which reason, as well as from the probability that the anæmic area actually sucks in the blood from these paths, the blood in the collaterals flows with increased rapidity. This excess of pressure also determines an additional dilatation of the collateral vessels, 
corresponding at first merely to their distensibility; but after the dilatation has persisted for a time there also occurs a proliferation of the cells of the vessel walls in consequence of the altered conditions of tension, not only causing narrow arteries to assume wide calibre, but changing capillary vessels into arteries. It is in this manner that a tissue whose principal artery has been ligated or occluded by thrombosis has its blood supply restored. The development of collateral circulation, however, requires time before complete compensation is attained; and should the existing anasto-

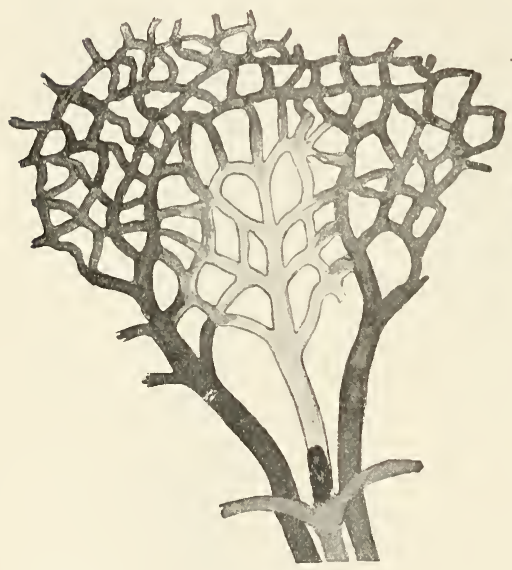

Fig. 12.

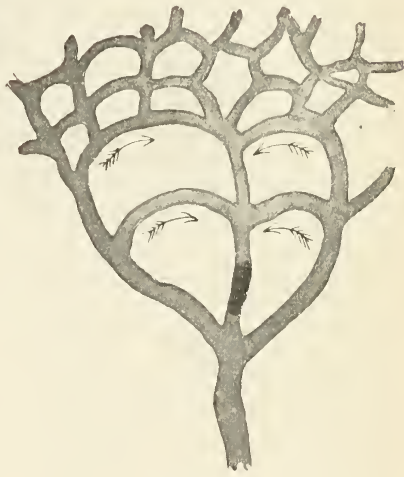

Fig. 13 .

Embolism of an artery with congestion of its two vense comites.

supply by anastomotic channels.

motic channels be very small, few or entirely absent, the tissue must in the interim suffer from the circulatory loss, be more or less completely deprived of its nutriment and perish. This is particularly true when there are absolutely no arterial anastomoses. Arteries which possess no communicating channels with neighboring arterial areas are commonly known as end-arteries, following Cohnheim.

Parts of organs supplied with no arterial anastomoses have of course capillary communications with the adjacent tissues, but the small amount of blood which passes through these minute channels is often insufficient to maintain the nutrition of the area deprived of its arterial supply; only in case of very small areas is the 
capillary supply sufficient for this purpose. Moreover it may happen that it is not one single artery that is blocked, but that at the same time its anastomosing vessels are also occluded, under which circumstances there necessarily result circulatory disturbances having serious consequences. The area which is deprived of its blood supply loses its blood color, and assumes a paler and drier appearance than the surrounding tissue; the blood remaining in it becoming decolorized because its hæmoglobin is dissolved, disintegrated or dispersed by diffusion, and the anæmic tissue, in which, too, the lymph is stagnant and coagulates, undergoing regressive metamorphoses. Such an anæmic and dead area is known as an ancmic infarct.

A tissue which is thus cut out of the circulation and deprived of its arterial blood supply is not necessarily anæmic but on the contrary may be the scat of a distinctly pathological engorgement as a result of regurgitation of blood from the veins. Particularly where the veins of the part whose arterial supply has been occluded anastomose with adjacent reins, the blood, which in the latter vessels is under a certain amount of pressure, flows over into the former and back into the anæmic network of capillaries: just as the water from a brook moves back into the side ditches of a stream if these have no fall. There is no force from the arteries in such a site capable of driving the blood through the affected area and the capillaries in which the renous blood collects become distended and their walls flaccid and permeable from the lack of normal bathing with flowing blood. The venous blood thus stagnating in the capillaries partly leaks through their walls, partly undergoes coagulation: and the whole area thus engorged with blood assumes a dark red hute. Such an area is spoken of as a hamorrhagic infarct (infarcirc, to stop up). [Others would refuse the importance attached by the author to the above idea of a venous regurgitation giving rise to the engorgement of the infarcted area with blood. Anastomotic communications are usually more numerous and free between veins than between arteries; and if this be true and the author's views correct, anrmic infarcts should be the exception rather than of as common occurrence as they actually appear. If venous reflux were as easy as supposed by the older adherents of this theory, anæmic infarcts should be practically unknown. From the editor's vicwpoint the process is more easily understood if we accept the belief, which seems reasonable, that there are no true end-arteries and that there are al- 
ways some small anastomotic channels between adjacent terminal arterial areas. Through such anastomoses, inappreciable under normal conditions but distended by the collateral rise of blood pressure when the embolism diverts the blood into the surrounding areas of distribution, the blood enters the infarct. The question whether in any given case an embolism should cause an anæmic or a hæmorrhagic type of infarct is largely determined by the texture of the tissue in which the lesion occurs, anæmic infarcts being met in relatively firm tissues as in the kidneys, and hæmorrhagic in looser structures as in the lungs. The explanation of such distribution is not entirely clear but probably rests in the facility afforded by the tissues at the border of the infarct for distension of the capillary anastomotic channels entering the infarcted area. Naturally the looser the texture, the greater the ease of such dilatation. It should however be realized that the collateral. congestion existing along the border of the infarct is productive of swelling and of pressure upon the periphery of the infarct. Granted a fairly firm structure, such pressure should compress the very channels of ingress into the interior of the infarct which are here supposed to give opportunity for the entrance of blood: and in such instances the part occluded should remain anæmic. If, however, the tissue be. loose in texture such compression cannot be of sufficient importance to occasion the interference with these anastomotic channels just suggested; and here the blood may by such paths enter the area from the zone of collateral hyperæmia and give rise to the appearance of a hæmorrhagic infarct. Infarcts of the lung are usually hremorrhagic in type in conformity with the natural looseness of this tissue; but the writer recalls a recent case of an anæmic infarct of a collapsed lung, bearing out the above theory. There is no objection to the author's views in so far as entrance of blood to the infarct through venous anastomoses may constitute a part of the process; but to the writer's mind it is excessive to suppose that this factor with reflux of blood into and through the venules and capillaries from the veins is the sole explanation of the phenomenon; and in his opinion both arterial and venous anastomoses may contribute, regulated however by the factors of border swelling, texture of tissue and pressure, to determine to what degree the entrance of blood may be possible].

Microscopically a hæmorrhagic infarct exhibits the capillaries of the part turgid with blood and the lympl spaces and all the tissues 
infiltrated with hemorrhagic effusion. In large infarcts the congestive reflux of venous blood is often restricted to the periphery of the area alone, the central portions remaining anæmic and having therefore on! y a red areola.

Infarcts as a rule are conical in shape, the apex of the cone at the position of arterial occlusion, the expansion from this point corresponding with the branching of the artery. From fusion of closely situated infarcts or in those of flat organs like the wall of the intestinal canal, the conical shape may be indefinite and the outlines suggestive of a nlap. Hæmorrhagic as well as anæmic infarcts occasion destruction of the tissue, the circulation being en-

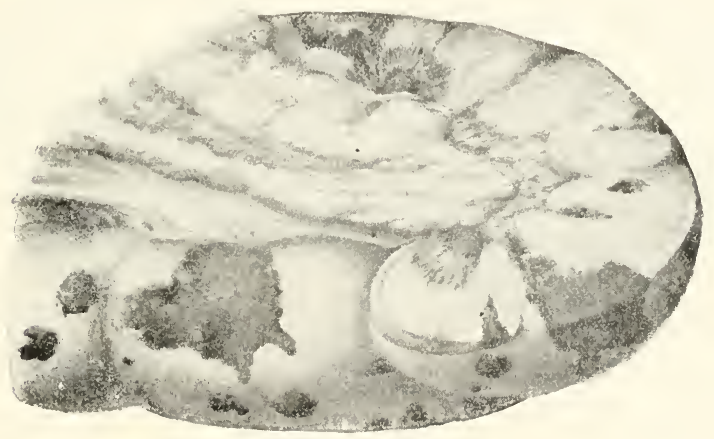

Fig. 14.

Conical hemorrhagic infarets in a portion of the kidney of a cow.

tirely ended in the area involved; the tissue with all the blood which has engorged it being reduced to a mass of coagulated necrotic material. Such an area excites an inflammation in the surrounding tissue, to the formation of an actual inflammatory zone of demarcation; a wall of lencocytes collecting around the area of coagulated material, fibroblastic and angioplastic cells from the surrounding healthy structure penetrating the infarct, whether it be anxmic or hæmorrhagic, the blood and disintegrating tissue of the infarct being removed by phagocytosis, and the site of the infarct being eventually occupied by a mass of contracting connective tissue (infarct scar). Such cicatrices are quite common in the kidneys (cicatricial kidneys). When either an anæmic or hæmorrhagic infarct is related to some surface infected with bacteria these in- 
variably invade every part of its tissue, causing its destruction by moist gangrene, as is regularly seen in such lesions of the intestine (septic destruction of the intestine).

- Thrombi, developing in the veins so as to cause their occlusion, of necessity interfere with the passage of the venous blood from the periphery, and of course give rise to passive congestion. The duration and termination of such cases also depend upon the presence or absence of proper collateral communications. In case of a partial or complete occlusion of unpaired venous trunks (vena

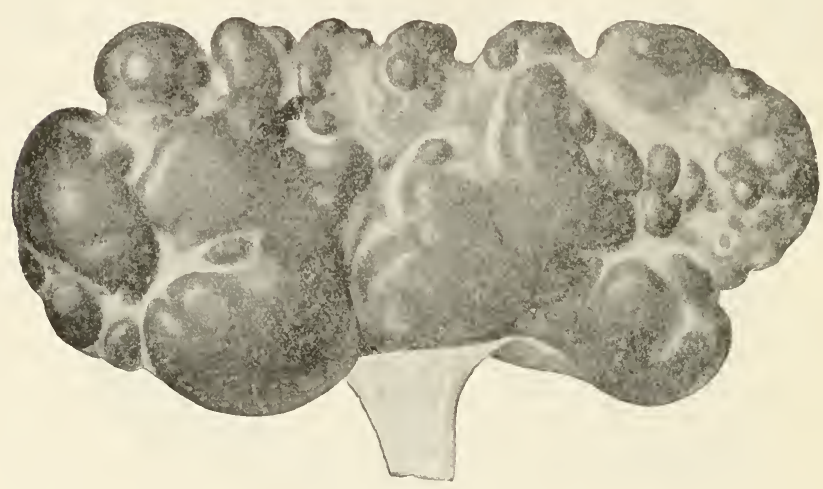

Fig. 15.

Cicatricial shrinkage after embolism (hamorrhagic infarcts) of the kidney of a hog.

cava, portal vein) or in case of synchronous thrombosis of a number of venous tributaries (veins of the lung) a serious passive congestion is occasioned, with stasis of the blood column, hæmorrhagic infiltrations or congestive transudations. Should there be a number of collateral branches of sufficient calibre the stagnation of blood will extend from the point of occlusion only to the first collateral branch, and the blood will pass without difficulty by the collateral route to the heart: should the anastomotic passageways be narrow they may become dilated by the pressure of the obstructed blood and the tissue be gradually relieved of its congestion, provided the degree of dilatation will accommodate the blood accumulated in the various venous channels of the structure. Very small veins and the capillaries everywhere in the part may be so distended as to serve the purposes of drainage, thus conducting 
the blood by a round-about way into one of the venæe cave. Clots and fragments of clots from the capillaries and veins pass as emboli to the right heart and thence to the lungs.

In the same way as clots, a variety of substances may, if once they have gained entrance to the blood, be carried along by the current and give rise to embolism. Virchow and Cohnheim, whose studies were fundamental to our knowledge of the embolic process, showed that small balls of wax introduced into the blood vessels are carried along by the current, lodge in some relatively narrower place in the vascular tube, and give rise to all the mechanical consequences of embolism. Sometimes parasitic worms which have succeeded in making their way from the intestine into the radicles of the portal vein are conveyed with the portal blood into the liver and may be carried thence onward as enboli to the heart and lungs; very minute animal parasites, as the rounded oncospheres, measuring only 20 to 40 micromillimeters in diameter, and larval trichince. may slip through the capillaries of the pulmonary circulation, often measuring O.I $\mathrm{mm}$. in diameter, and thus gaining access to the arterial stream be carried to any of the organs. It may be easily appreciated from this why the liver and lungs are the most common sites for echinococcus cysts; and the embolic mode of distribution of the parasites is recognized from thcir usual wide dissemination thronghont the whole organ. Of the larger parasites which the blood may aid in distributing, liver flukes deserve special mention; these sometimes in their migrations in the hepatic parenchyma penetrate into the branches of the hepatic vein and may pass with the blood through the right heart into the lungs. The mechanical consequences of embolism by these animal parasites are usually of little importance because of the minute size of the bodies and the relative certainty of sufficient anastomotic supply to the small areas affected: and disturbances are only oceasioned by pressure, etc., in ease the invading parasite increases in size. The larger parasites like strongy armatus are capable of directly cansing the same mechanical effects as described in connection with blood clots (as cerebral embolism with infarction).

The entrance of air and of droplets of fluid fat maty also occasion circulatory disturbances in a purely mechanical manner, in the same way as blood clots. Air cmbolism is of rare occurence and is met with in operations on the neck." The air which is drawn by the suction force of the heart through a valvular venesec-

*Traumatic anremia was first cbserved in the horse by the French veterinarian, Verrier $(1806)$. 
tion wound into the jugular vein, or which is accidentally introduced in intravenous injection, becomes mixed with the blood in the form of bubbles of various sizes. In case of healthy animals with normal blood pressure, moderately large quantities of air (5 to 10 cubic centimeters in dogs, sheep, horses) injected into the jugular vein are sometimes born without harmful results, the air disappearing quite rapidly by absorption in the circulating blood. In animals, with low pressure and a tendency to accumulation in the right heart (as after operations and after loss of blood in parturition) the air bubbles are apt to accumulate in the right heart and form a large elastic air cushion which is not forced onward by systole, being only compressed by cardiac contraction and expanding again in diastole. In consequence the blood is dammed back in the right heart, and there results a marked dilatation of the right ventricle, reaching two or three times its normal volume. (Death takes place from the cardiac dilatation and suffocation, the blood being prevented access to the lungs. According to François Franck it may also be possible that some of the air passes through the lungs, and, traversing the left heart into the coronary arteries, brings about embolic occlusion of the latter and anæmia of the myocardium.*)

Fat cmbolism is usually met in connection with severe concussive lesions of the bone marrow (Ribbert). The occurrence of fracture of a bone with such concussion allows lacerations of the delicate walls of the venous capillaries to take place, after which fat droplets from the fat cells abundantly present in the marrow may pass into the blood (Ribbert). Access of fat into the blood may also be thought possible from the liver cells in case of contusion of this organ, its cells also being usually rich in fat (Ribbert). The oil (lroplets are first carried with the venous blood to the lung, where they lodge in the small arteries and capillaries, the capillary network often appearing turgid as if filled with a fatty injection mass. From the occlusion thus brought about hæmorrhagic infarcts are formed in the lungs, nsually a transient condition (may be proved by injecting a fat emulsion into the jugular). The liquid fat may, however, pass through the pulmonary capillaries and be carried on into the left heart, whence it may be conveyed with the aortic blood into the cardiac musculature (coronary arteries), the renal glomeruli, or the brain and other organs. The fat lodged in the coronary arteries, just as any other

*Recueil vétér. 1903, p. 370. 
emboli, occasions anxmic degeneration of the myocardium; in the brain numerous small hemorrhages; and in such or other analogous manner fat embolism may prove fatal.

Sometimes after traumatic lesions of the tissues, cells are similarly swept into the blood, as giant cells from the marrow or liver cells, and lodge in the pulmonary capillaries because of their size. This type of cellular embolism occasions no special disturbance, the occlusive lesion being at the most a minute one and the cells soon broken up or removed by phagocytosis. However, the entrance of tumor cells or pathogenic bacteria into the blood, and the embolic transmission of such bodies, are of much more importance. Their mechanical effects in causing complete or partial occlusion of the

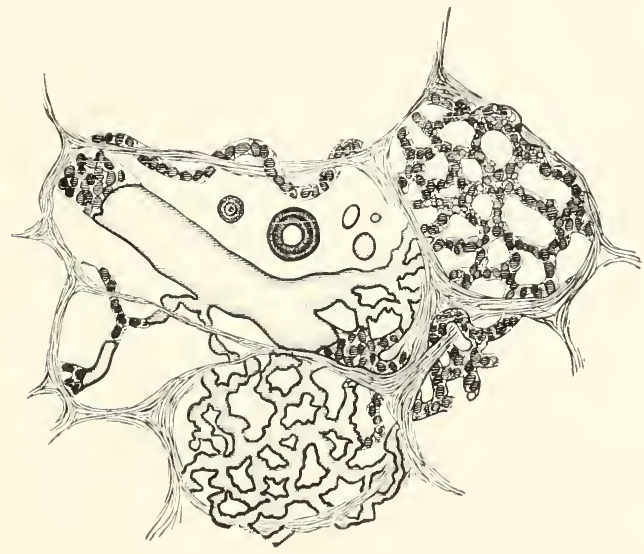

Fig. 16 .

Fat embolism of the lung, eight days after fracture of the leg. Drawn by Dr. Ziesing; $\times 250$.

The capillary network of the alveolus to the right is choked with blood. the lower on with fat and the artery hkewise Just above the latter are two air bubbles (showing as dark rings) and several droplets of fat which have escaped from it (with simple dark vutline). (After Perls.)

ressels are of minor significance because of their minute size; but because of their nature they induce specific changes, the process

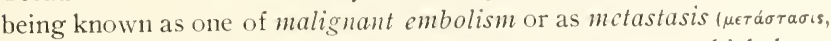
displacement). We have here to deal with substances which have already given rise to pathological processes in some part of the system, and which are now, by being transported by the blood into new situations in the body, inducing the same disturbances in the latter. Emboli composed of tumor cells originate from autoblas- 
tomata (neoplasms, tumors) whose cells in the growth of the mass are penetrating the walls of the blood vessels and, picked up by the blood flowing by, are carried along with the stream. Wherever they lodge a new autoblastic growth develops from the multiplication of the displaced cells, which retain their power of growtin. Since usually it is not one single cell which is thus swept from the primary site, but whole groups of cells being loosened and separated from each other in the circulating blood and scattered throughout the capillary network of the organ into which they are carried, the latter becomes riddled with tumor nodules (disseminated or multiple embolism). In dogs, for example, which are suffering from cancer of the thyroid gland the lungs are as a rule occupied by great numbers of nodules of the same carcinomatous character, the cells having penetrated from the cancerous alveoli of the gland into the thyroid veins and having of course been carried into the lings. In the same way from cancers of the manmary glands, the skin, the nose, etc., after invasion of the tributaries of the vena cava, metastatic nodules develop in the lungs. Intestinal, gastric and pancreatic cancers give rise to metastatic embolism in the liver, the tumor cells primarily gaining access to the portal vein; only in case they pass through this organ does the lung become involved by the tumor cells. [This is true in a general sense provided the hæmic route is followed in metastasis. It should be added however that these cells may find their way by the lymph stream through the thoracic duct to the lungs, the liver being entirely escaped. So, too, it is possible by the minor anastomotic communications between the portal system and the general venous system, as by way of the veins of the smaller gastric curvature, lower nesophageal and azygos veins, that the cells may be convered to the lungs directly, the liver being evaded.]

Malignant bactcrial cmbolism is determined by the entrance of pathogenic bacteria into the blood vessels, quite commonly the pyogenic organisus; more rarely the vegetable organisms like the spores of moulds enter into consideration. Such germs may gain entrance into the circulating blood through lesions of the skin or mucous membranes. Lesions which were practically imperceptible may serve as the point of entrance [it is also claimed by a number of persons that in a limited degree bacteria may pass, as it were be absorbed, through delicate mucous membranes without the actual existence of any structural disturbance of the membrane, as Ravenel has pointed out in case of tubercle bacilli passing through the normal intestinal wall to the mesenteric glands], 
the bacteria first multiplying at this point of invasion and then either by way of the lymph passages, conveyed to the blood, or directly growing through or actively penetrating the delicate walls of the capillaries. Some bacteria are motile and are able to actively progress because of this property, or because of their minute size may be passively conveyed through the lymph spaces. Generally at the point of entrance the local action of the bacteria causes a focus of destruction varying in type with the form of bacteria present, as a suppurative liquefaction or ichorous softening (furuncle, suppurative wounds of the skin): coincidently the destruction of the walls of the capillaries and veins permits the bacteria to gain access to the blood and circulate with it and to be carried to the heart and lung or from the intestine to the liver. Bacteria, too, because of their minute size may traverse capillary areas and therefore often pass from the lesser to the general circulation and become scattered widely throughout the system. In a strict sense they should be regarded as emboli, although primarily because of their minuteness they occasion no mechanical disturbances but remain suspended here and there in the vascular tubes, as at places where the blood current is temporarily. hindered (a joint) or where there are inequalities in the intima (valves, sharp bends in the capillaries, renal glomeruli). At such sites they induce the same type of changes as at the point of entrance, causing inflammatory reaction, suppuration, etc., and multiplying at first to the production of a definite metastatic focus and later making their mechanical obstructive effects appreciable when they have developed into masses and climps. In other cases the bacteria may from the first be disseminated in the blood in small clumps. or as is usually the case they may adhere to bits of thrombi which have been broken loose. Under such circumstances mechanical occlusion and specific action are to be expected coincidently or in close succession. Precisely as in case of simple embolism a smaller or larger infarction is produced and within the tissue of the infarct, filled up with coagulated blood and necrosed, the bacteria may multiply to great numbers, transforming the infarct, according to the specific properties of the bacteria of the embolus, into a suppurating focus or breaking it down into an ichorons mass.

The most common example of malignant embolism is met with in umbilical phlebitis, frequently seen in newly born animals. In case after section of the nmbilical cord, dirt infected with bacteria (from dung, pus, dust from the straw of the animal's bed, the 
coating of the tongue of the mother animal as she licks her young) comes in contact with the umbilical wound, germs capable of causing inflammation may penetrate into the umbilical vein (motile bacteria). These microorganisms rapidly multiply in the clotted blood which occupies the vein, penetrating it possibly up to the liver where the portal and umbilical veins unite. Thence by the portal current particles of the softened and infected blood clot

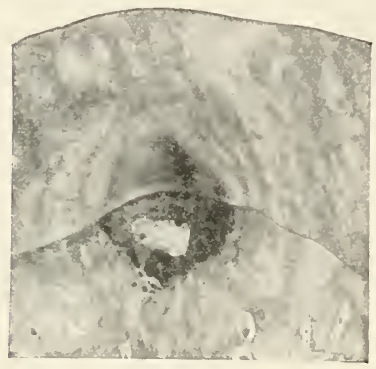

Fig. 17.

Malignant hæmorrhagic infarct of the lung of a horse, suppurating in the centre. are swept into the substance of the liver and give rise there to multiple abscess formation. Isolated bacteria or small clumps of the germs are carried onward from the liver through the hepatic ventules (in calves perhaps directly by way of the ductus Arantii) entering the heart and lungs with the blood of the rena cava, with production of further suppurative metastases in the latter. They mar moreover gain access to the general circulation (pulmonary reins, left heart, aorta), as the result of which extensive pyogenic foci of inflammation of hrenotogenous embolic origin are produced widely through the body, as in the kidners, joints, eves or brain. Another striking example of embolic metastasis is seen in case of tuberculosis, where the tubercle bacilli may be disseminated as above (cf. chapter on tuberculosis).

Lymphatic Thrombosis and Lymphogenous Embolism. The lymph flowing through the lymph vessels, the onward movement of which depends partly upon the pressure with which the fluid transudes through the capillaries, partly upon the compression upon the canals (just as a sponge is squeezed) by the movements and contractions of the organs, is also subject to coagulation. For the most part lymph coagulation is caused by the enzymes of infectious toxine-producing organisms and by lesions of the lymphressels with adhesion of necrosing lencocytes and separation of fibrin in the spaces. The lymph canals filled with lymph coagula are to be seen as broad strands occupied by the coagulated material, transparent and jelly-like, as in the interstices of the lung in croupous pnetmonia, and strikingly shown, too, in cutaneons 
lymphangitis. Because of the rich and intricate anastomosis of the lymphatics the escape of any excess of lymph is easy by the collateral paths, and usually the obstruction is in time removed by a fatty degeneration of the clot. Commonly the lymph stasis therefore, is but a transient feature. Extensive lymph thrombosis may however lead to a necrosis of the part involved, from failure of removal of its metabolic products and the stagnation of the lymph. Fragments of the thrombi loosened and carried away give rise to little of pathological importance, because of the rich collateral communication and the fact that the paths lead into the lymphatic glands, as well as because of the fine or molecular disintegration of the coagulated material. Only in case of the lymphatics of the mammary gland is there to be apprehended a direct passage of such material into the blood with consequent pulmonary embolism.

The lymph channels are important rontes of transportation of specific types of emboli, as tumor cells and bacteria as well as for the convection of all sorts of material arising from the disintegration of tissues, dust particles, pigment, and any other minute solid elements. Such substances are carried along, in part free in the lymphplasm with its slow current, partly in the motile wandering lymph corpuscles which load themselves with small particles: the pathological significance of their convection varying with the character of these bodies. Thus by the removal of disintegrated material, cellular fragments or dead bacteria, a tissue or organ may be restored to its proper functional capacity, a sort of street-cleaning being accomplished; but on the other hand the convection of toxic matter, of cells or microbes capable of multiplication, serves to establish more local points (or generalization) of the disease. A few examples may render this more clear. In case of himorrhagic extravasations, as after the fracture of a bone, the entire hæmorrhagic focus gradually disappears, the disintegrating blood corpuscles being taken up by the lymph corpuscles wandering about and carried to the lymph glands for further destruction (as a result of which the lymph glands in the neighborhood of such hremorrhagic areas become the seat of a rusty brown discoloration). Carbonaceous particles inhaled and lodged in the lung are carried along the !ymph passages to the bronchial lymph nodes, coloring them black. Virulent streptococci which have gained entrance to the mouth and pharynx are taken up by leucocytes wandering out and in the mucous lining, carried primarily to the pharyngeal 
lymphglands, where they may perhaps multiply and give rise to suppuration; thence they may travel from one lymph node to another (retropharyngeal, cervical, thoracic), in each setting up new foci of suppuration, until they finally reach the blood and produce pyæmia.

No matter how minute the lesion of the skin or mucous membrane, infectious bacteria may from some source gain access to the lymphatic radicles and occasion their specific processes in the lymph channels and at their points of deposit in the lymph glands (cf. tuberculosis and carcinoma). Lymphogenous extension or embolism is therefore to be regarded as an important factor in the generalization of local infectious processes.

\section{Pathological Changes of the Blood.}

The blood supplies the tissites with the oxygen essential to their life, besides the albuminates, carbohydrates and water; it removes their metabolic products and is the principal factor in the distribution of heat; it, therefore, maintains the functions of the nervous system, the heart and all of the organs as their liquid nutrient, their source of heat and their cleansing fluid. In its manifold relations to the tissues, in its function of giving and receiving on so many sides, there arises constant change in the quantity and composition of the blood, limited, of course, within certain bounds and relations set by the tissue requirements. When blood is lost, fluid is taken into the vessels from the tissues and corpuscles are supplied by the blood forming organs to the eventual restoration of the blood. When an excessive amount of water is absorbed there follows rapid excretion of the superfluous quantity through the kidneys; and in analogous fashion the variations in its chemical constitution are continually undergoing adjustment. We have ret to discover wherein lies the exact and delicate regulation of the quantity and quality of this complex liquid; at all events the changes in chemical composition involve continually repeated stimulation of the nerves of the blood vessel walls, of the cells of the hrmopoietic organs and all secretory cells upon which the hrmic composition depends. As soon as this regulation is disturbed the variations in the volume and proportionate composition of the blood deviate from the physiological limits, and hrmic anomalies* are said to exist.

*The word dyscrasia was formerly employed in this connection, but was without exact significance, being applied by some to any blood abnormality, by others to long persisting fauits in the proportionate presence of its constituents or simply to impurities of the blood from the presence of special substances. The word may be dispensed with. 
It can readily be appreciated why there are so many types and degrees of blood abnormalities when it is recalled that of necessity pathological changes in the blood must follow any alteration in the efficiency of the organs, the metabolic processes of which are constantly removing from or adding to the blood so many elements.

The amount of blood obtainable from the domestic animals at slaughter is subject to such wide variation, as Bollinger and Bergman have pointed out, that it is clear that we can have no safe basis for determining the existence of an excessive volume, of a condition which may be termed full bloodedness or plethora, in any subject, either from the quantity obtained at slaughter or deduced from symptoms. The healthy animal very quickly gets rid of excesses introduced experimentally into the body, whether it be blood of the identical species, blood serum or indifferent fluids; and the transient plethora caused by transfusion causes no disturbances worth mentioning. The blood vessels are in fact never filled to their full capacity collectively: the vast multitude of capillaries capalsle of further dilatation can accommodate a large volume of fluid in excess of their ordinary content. Experiment upon animals shows that in dogs the blood volume may be doubled by transfusion, a quantity representing eight to twelve per cent. of the body weight introduced, without injury to the animals; danger to life occurs only after the introduction of a still greater amount (Samuel). The excess of fluid transudes from the vessels and is eliminated from the body in the course of a few days with the urine; the superfluous blood cells are destroyed in the liver and other organs. Whether a plethora of actual pathological significance ever occurs in animals is quite unknown.

Diminution of the volume of the blood, known as blood impoverishment, oligaemia or general anaemia, has associated with it a decrease of the number of blood corpuscles, known technically as oligocythamia; usually, too, the converse is true. From a practical standpoint it is difficult to draw any fine distinctions as to whether the blood is normal as far as the volume of its fluid is concerned and only the cellular elements deficient, or whether the plasma, its salts and other constituents have undergone the greater reduction; for which we are accustomed to regard the loss and destruction of the erythrocytes, the most conspicuous of the blood constituents, as the principal pathological feature. Inasmuch, however, as there is a close interrelation of these alterntions, the one dependent upon the other, there are apt to exist many 
peculiar phenomena in these instances, which have thus far been imperfectly studied; for example, the red blood corpuscles may undergo reduction and disintegration because the plasma contains some unusual saline or an excess of water or because some specific hæmolytic substance has become mingled with it. Briefly speaking, it is customary to speak of general anæmia in a subject whose mucous membranes and skin persistently appear very pale, whose muscular tissue and viscera have at autopsy the pallor characteristic of a slaughtered animal and in which in life there existed a condition of general weakness. The contrast between the peculiar tint of the organs and the deep red color given by the full capillary blood serves better to indicate the reduction in the volume of the blood than the amount which can be obtained in the heart and large vessels and which can be estimated only with extreme difficulty. Oligocythæmia is determined for a cubic millimeter of blood by microscopic counts of the cells with special measuring apparatus; diminution of the hæmoglobin content can be determined only by chemical methods. [Clinically this latter is estimated by comparison of either the whole or the diluted blood with standard color scales, each graduation of tint representing a certain proportion of hæmoglobin. Natching of colors always involves a large personal equation and at best can only be approximate, even were it established that the supposedly standard reference scale were entirely correct-which is not the case. However, as an approximation only and as a fairly ready means of comparison, these color tests have at least a clinical value.]

The least complicated form of oligæmia is that occasioned by blood loss from injury to the vascular apparatus, hamorrhagic ancmia, following wounds and lacerations of the vessels, venesection or the removal of blood by blood sucking parasites. A blood loss exceeding three per cent. may be dangerous to life (external or internal injuries) and sudden hæmorrhages of still smaller volume from the large vessels may be fatal. [While exceptionally this statement may be true where there are other factors as nervous shock involved, the limits of non-fatal blood loss as here given are for ordinary cases quite too low. As much as one-third the volume of blood has often been withdrawn without fatal collapse.] The volume of fluid lost by non-fatal hæmorrhage is soon restored by absorption of water from the tissues and ingesta; while the corpuscles and albuminous material are reformed in the course of a few weeks or months. In fatal hæmorrhage there occur 
phenomena of suffocation, lowering of blood pressure and unconsciousness (anæmia of the brain). After hamorrhage proteid metabolism is increased; metabolism of fats is, however, decreased because of the diminished power of combustion. Hence repeated venesections are appropriate treatment where it is desired to increase the deposition of fat and they are employed as aids to fattening animals.

General anmenia may also be brought about by insufficient nutrition or improper assimilation of food, as well as by diseases and injuries of those tissues which supply definite blood constituents. Here are particularly included various affections of the stomach and intestine, hepatic diseases and alterations of the lymphatic tissues; but in addition to such disturbances there is a wide further possibility of the establishment of an ancmia from disease of the many other tissues which contribute to blood formation. A number of agencies are known to be capable of inducing the condition by causing the destruction of the blood corpuscles, perhaps to such a degree that regenerative effort can no longer efficiently restore the loss, as certain parasites of the blood (trypanosomes) and poisonous or toxic products of metabolism.

Among the most notable symptoms of many aniemias are changes in the form and structure of the erythrocytes. In place of the circular discs there may be met irregular pyriform, clubshaped or indented elements of various size, cells containing vacuoles (cavities, hollow spaces), and nucleated red corpuscles. A blood preparation showing such multiform cells of varying appearances is said to exhibit poikilocytosis ( $\pi$ ockinos, variable, confused, involved). Recently alterations in the staining reactions of the corpuscles have been made out. The normal cells invariably take up only one tint from certain staining combinations; the stroma of young forms, degenerating and dead cells, however, may assume several colors (polychromatophilia). The occurence of nucleated red corpuscles in the circulating blood is an evidence of active regeneration of these elements, and is often encountered in the anremias. Especially large cells of this type are sometimes met (megaloblasts, gigantoblasts), a peculiarity interpreted as evidence that regeneration is taking place with extreme laste, such cells, otherwise only seen in the bone marrow as early stages of the erythrocytes, entering the circulation in these cases as inmature elements (Krehl). The increased cellular multiplication in the bone marrow, the principal site of the formation of erythro- 
cytes, causes in anæmias large areas of this tissue to return to the condition of red marrow (normally only young growing animals have much red marrow; in adults medullary blood formation is limited to a few bones according to the amount of blood required to be formed; change of the marrow to the red state and production of this condition in many of the bones may be broinght about in animals by venesection-Naunyn, Litten and Orth).

In severe forms of anæmia not occasioncd by actual blood loss the proportion of iron in the liver, spleen and kidneys becomes abnormally high, this feature invariably indicating exaggerated destruction of the red cells.

From a variety of causes, particularly the influence of certain poisons (salts of the biliary acids, snake venom, bacterial toxines) and, too, the transfusion of heterogeneous blood (blood from a different species of animal), the hæmoglobin may undergo solution and separation from the erythrocytes into the plasma (hamoglobincmia). The coloring matter thus freed gives the plasma or serum a diffuse wine-red color. A portion of the hamoglobin is taken up by the liver and transformed into biliary pigment, and the remnants of the injured and therefore disintegrating blood corpuscles are to be found in the liver and in the spleen (also in the marrow), these organs sometimes becoming considerably swollen and excessive bile formation taking place. The bulk of the liberated hrmoglobin is removed by the kidneys, causing more or less harm to these organs and giving to the urine a blood red to black discoloration (hamoglobinamia). These changes are observed not only in association with severe infections and intoxications in cattle, but are also seen after muscular strain and chilling, especially in horses and, too, in human beings (infectious, toxic, paroxysmal, myogenous, rheumatic hremoglobinuria). Some of the hæmoglobin in hæmoglobinæmia which remains in the blood is converted into methæmoglobin; this change may also affect the coloring matter within the corpuscles, and is apt to result particularly from the action of certain poisons (antipyrin, potassium chlorate). It gives rise to an appreciable sepia-brown coloration of the blood (methamoglobincmia). There always coexist with this group of blood changes serious diseases of the subject; the hæmoglobin, freed from the cells and altered in its constitution, useless for respiratory purposes, is no longer capable of taking up and giving off oxygen (Krehl), and the disintegrating blood cells give origin to substances which may cause coagulation to take 
place here and there in the vessels, occlusion of the capillaries and serious lesions of the renal epithelial cells.

Oligæmia is occasionally associated with a truly watery condition of the blood, as where in case of hæmorrhage. blood impoverishment, inanition, or renal disease the blood becomes deficient in albumen, the ressels become filled with the fluid from the tissues in an effort to restore the deficiency; such is condition is termed hydramia (oligamia scrosa). So, too, the increase in the proportion of water with increase of the total volume of blood (plethora aquosa, scrosa) may be thought of as a pathological possibility; however, proper information is as yet lacking concerning these pathological conditions and the relation between quantitative clianges of the serum albumen to the proportion of water in the blood. Normally, large excesses of water known to be rapidly absorbed from the stomach and intestine into the blood are quickly passed off through the kidneys.

Of the pathological conditions of the lencocytes or white blood cells and their reduction or increase in numbers in the circulating blood our knowledge is only of the most superficial character. To what extent the morphological and tinctorial peculiarities of the individual forms and groups of leucocytes and lymphocytes normally found in the blood are characteristic of the origin and of the role of these cells is a matter requiring further investigation. These cells are of especial interest to the pathologist from the fact that they are either attracted or repelled by chemical materials in the tissues and by various particulate elements which may give origin to the former (positice and negatice chemotaxis). Because of the power possessed by these cells of approaching or receding from a given point like motile organisms, of taking into their substance small bodies which they encounter-an act which is to be regarded as an attempt to obtain nourishment (cellular devouring capacity, phagocytosis) - they may be thought of as playing the part of "scavengers of the organism" (products of disintegration, dust particles, dead cells and tissues, lead bacteria. etc., all being removed in this manner). They may however by identically the same mode of action come to serve as carriers of infection in case a living bacterium or animal microorganism happen to be taken up by them. A microorganism not necessarily destroyed in the substance of the leucocyte, but possibly actually multiplying there. may be carried away to a new situation and there renew its toxic influences, especially if the phagocytic cell be destroyed by its poison. [Metschnikoff urges strongly that after appropriation of a nicrobe by a phagocyte, the former mav be 
regarded as entirely destroyed or as seriously impaired in its vitality.] It is readily observed in suppurations undergoing metastasis (cf. mctastasis), in swine erysipelas and other bacteriæmias, that many of the lencocytes are loaded with the specific germs.

Negative chemotaxis, too, the movement of the lettcocytes away from certain poisons or more frequently the mere failure of attraction of the cell toward these substances, is capable of explaining a number of points connected with disease and immunity. For example, as shown by the extremely interesting studies of Pesson, Leclainche-Vallée, Vaillard and Vincent, non-virulent but living germs (spores) of tetanus, malignant nedema and blackleg are englobed by lencocytes and ingested and thus prevented from development and from becoming virulent; virulent germs of these same sorts, however, prevent the lencocytes from entering the tissues because of the toxines to which they give origin and are therefore not taken up by the phagocytes, but continue their harmful action upon the tissues in which they exist.

The general group of lencocytes in the blood is subject to considerable variation in number, even nuder normal conditions. After taking food or violent muscular exercise there may be uniformly observed an increase in their number (physiological leucocytosis or hypcrlcucocytosis), apparently occasioned by the attractive influence exerted by various proteid matters, fatigue stuffs, etc., which have entered the blood. In inflammations, suppurations, and infections fevers a similar increase in the number of leucocytes distributed throughout the blood occurs (pathological hyperleucocytosis), similarly ascribable, so far as indicated by experiment, to chemical attraction and the circulation in the blood of substances stimulating the leucocyte-forming tissues to greater production. (Injection of bacterial proteins and other proteids into the blood is particularly likely to be followed by hyperlencocytosis). [While there are a few exceptions to the usual occurrence of leucocytosis in acute infections, it is in a general way to be interpreted as an unfavorable feature when such increase fails to take place (leucopenia); either the subject being in such deteriorated state that the usual reaction is impossible and the resistance to the disease much below par, or the smallness of number of these protective cells itself determining the failure of sufficient antagonism. Cases marked by such unusual leucopenia, with the exception as above suggested of a few special forms of disease, are apt to terminate fatally. 
To a certain extent the increase of this or that particular form of letcocyte is of diagnostic importance. In brief we recognize as the most common form of white blood corpuscle the polymorphonuclear neutrophile cells (containing multiple nuclei or a single polymorphous nuclets, and having numerous fine granules in the cytoplasm which elect a combination of the acid and alkaline dyes in staining with such mixtures, becoming purplish with metlylene blue and eosin for example). An increase of these elements, spoken of simply as leucocytosis or hyperleucocytosis according to its grade, is commonly met in a number of infections, particularly those of the septic group. There are normally present in the blood a few similar letucocytes which have, however, in their cytoplasm rather coarse granules which, from an acid and basic combination of dyes, select the former, being therefore called oxyphile cells (or because eosin is the acid part of the common methylene blue and eosin solutions and their cell grantules appropriate the red of the eosin, these cells are often called eosinophile cells). These oxyphile cells may become increased in a number of conditions, but this increase, known as cosinophilia, is especially notable in the blood of animals or human beings subject to some of the animal parasites as the triclina or lookworm. In psendoleukæmia and lymplatic letkiemia the lymphocytes, cells with single large, deeply basic staining nuclei and with fine basophilic granules in the scanty cytoplasm, are especially increased. In the common lieno-medullary lenkemia there is special increase in a large type of lencocyte not normally present in the blood but natural to the bone-marrow, known as a myelocyte, with large nucleus taking the basic stain faintly and with fine nettrophilic granules in the cytoplasm.]

Where there is such an increase of lencocytes in the blood that they equal or exceed the number of erythrocytes in the drop from which the cell count is made, or if certain cells normally present in but meager numbers are unusually numerous the monontuclear cells above noted as myelocytes] the name lcukamia is employed to denote the condition. [The actual number has been known to equal that of the erythrocytes, but this is exceptional: and the present conception of leukæmia concerns rather the type of cell present in the blood, the myelocyte, than the actual numbers attained.] In this disease the blood is apt to be changed in its macroscopic appearance as well, beconing of a light red or raspberry color. There also occur hyperplastic changes in the various 
lymphoid structures of the body (lymph glands [in lymphatic leukæmia]: bone-marrow and spleen [in lieno-medullary leukæmia]); and the coincidence of a general debility, fever, and tendency to hæmorrhage mark the condition as a serious affection. Nothing definite is known of the rtiology of the affection either in man or the animals; it has been conjectured, however, that the cause may be of an infectious nature. 


\section{DISTURBANCES OF METABOLISM; RETRO- GRESSIVE AND NECROTIC PROCESSES}

\section{Faults of Heat Regulation; Hyperthermia; Fever.}

The temperature peculiar to the various warm-blooded animals is constantly maintained in lealth at the same level within narrow. limitation by the delicate and accurate regulatory mechanism of the body. With every change in the surrounding temperature and other external influences there occurs special adaptation of heat production and heat dissipation on the part of the animal borly. If an excess of heat be produced within the body from exaggeration of metabolism (nuscular activity, ingestion of large amounts of food), excessive heat dissipation follows; the cutaneous blood vessels dilate and the system loses in consequence a greater amount of heat from its surface by conduction, by radiation and if need be by perspiration, as well as by free evaporation of moisture in the lungs. In case the body suffers unusual heat loss (cold environment) heat dissipation is diminished by constriction of the cutaneous vessels, and the chemical activities in the tissues increase heat production by accelerating decomposition, that is, the combustion of carbohydrates and fats, particularly in the musculature. Rich food supply and bodily activity increase the production of heat; and by these means and by simple diminution of heat dissipation the warm blooded animal is enabled to maintain its proper temperature in spite of marked chilling.

The human being in state of health, possessing additional means of conserving his warmth by a number of layers of warmed air (clothes) about his body, maintains an average body temperature of $37.2^{\circ}-37.4^{\circ} \mathrm{C}$, the variation from morning to evening being about one or one and one-half degrees. The horse, according to Friedberger-Fröhner, has a normal temperature of $37.5^{\circ}-38.5^{\circ} \mathrm{C}$. ; the cow, $38^{\circ}-39^{\circ} \mathrm{C}$; the sheep and goat, $39^{\circ}-40.5^{\circ} \mathrm{C}$; hog, 
$38^{\circ}-40^{\circ}$ C.; $\operatorname{dog}, 37.5^{\circ}-39^{\circ}$ C.; cat, $38^{\circ}-39^{\circ}$ C.; birds, $41.5^{\circ}-42.5^{\circ} \mathrm{C}$.

The warm blooded animal maintains the constant physiological level of temperature by the continued operation of the heat dissipating powers; if there were no such loss, the amount of heat production would be sufficient to cause an increment of about one degree centigrade in the temperature of the blood every half hour.

Passive Hyperthermia. By limitation of heat dissipation the body may become overheated, particularly when the cause is some external physical influence which hinders heat loss by evaporation. Although in a dry atmosphere where there is no restriction to evaporation the body is capable of enduring even high temperatures of the surrounding air, the body heat increases rapidly in a hot, moist atmosphere, heat loss by evaporation being impossible, and all the more if in addition there be increased production from muscular exercise. In a warm bath of $40^{\circ}-41^{\circ} \mathrm{C}$. the human body temperature may even in half an hour reach $40^{\circ} \mathrm{C}$.; in water of $37^{\circ} \mathrm{C}$, it rises about $\mathrm{I}^{\circ} \mathrm{C}$. in an hour, and in an hour and a half about $2^{\circ} \mathrm{C}$.

Animals closely packed in hot railway cars experience considerable increase of temperature: in warm, dann air animals closely herded in long drives (droves of hogs, oxen yoked to wagons) may have their temperature reach more than $40^{\circ} \mathrm{C}$. Under such circumstances where only external physical conditions render the regulation of the body temperature impossible or where (perhaps coincidently) unusual accession of heat production occurs because of exceptional muscular exercise, there is really no true febrile temperature present, but rather only a passive heat accumulation, passive hyperthermia; the mechanism of heat dissipation is acting in its fullest capacity and is insufficient only because the denands upon it are excessive (Krehl). Thermolysis so far as the animal body is concerned is not at fault; but fails because of conditions existing in the external surroundings.

Whether pathological results follow such passive hyperthermia will depend upon the persistence of the condition and the subject's power of adaptation. Men and animals living in tropical countries give evidence upon this last point; and, too, it is commonly seen that when a number of individuals are subjected to excessive temperatures there are only certain ones who suffer pathologically, those probably whose thermo-regulatory mechanism is of inferior efficiency. Passive hyperthermia may reach $42^{\circ}-45^{\circ} \mathrm{C}$. and may 
terminate fatally (heat stroke). The occurrence of a heat stroke is marked by symptoms of general weakness, dizziness, fainting attacks, and marked acceleration of the pulse, these probably being mainly dependent upon disturbances of brain and myocardium, although upon this point there is need of further study.

Fever (fcbris, from fervere, to be hot) is a state of the body in which because of some disturbance of metabolism the regulation of the body temperature to a mean level has ceased. The chief and most constant evidence of fever is the abnormal increase of the temperature of the blood, or internal body temperature. This febrile hyperthermia or fever heat may exceed the normal temperature of the animal perhaps but half a degree or by from two to five degrees centigrade. It is characterized by marked resistance to influences causing heat dissipation and by considerable variation; and is accompanied by increased tissue waste, changes in the type of and increased frequency of the arterial pulse, increase in the rate of respiration, diminution and alterations in the various secretions, particularly in the urine, disturbances in the sensory apparatus, increased thirst and loss of appetite. This group of symptoms, which occur with the heightened temperature and with it go to make up the clinical picture of fever, are to a slight extent directly dependent upon the increased temperature of the blood (for example, the number of cardiac movements and respirations), but for the greater part are the results of the complex action of the causes of the fever. The main point in the conception of fever, with its febrile excess of temperature, is that the latter is not the mere result of external physical influences restricting thermolysis, or of muscular activity, but that there are certain metabolic processes in operation which are due to the presence of special temperature producing substances (pyrogenetic substances) which give origin to the hyperthermia. How the heat equilibrium is affected by such substances, whether they directly disengage the heat from the cells and celfular groups or whether they act indirectly through a heat centre, is not as yet certain and cannot even in a general way be explained. These pyrogenetic substances vary much among themselves and the reactions which they give rise to are by no means uniform. Fever may be thought of as an indicator (Wassermann) that some peculiar metabolic processes are in operation in the body accompanied by tissue destruction and the production of antitoxic and bactericidal substances and precipitins; and may be considered as an associated 
or partial manifestation of a complex reaction to which the organism is prompted by the invasion of substances foreign to the system. From this it may be appreciated that the idea of febrile temperature and of fever is in reality principally based on the retiology of the condition; the existence of proper causes of fever, the presence of pyrogenetic substances, is the factor determining whether we are or are not to regard and speak of a hyperthermia as febrile.

Microorganisms, both vegetable and animal, constitute a most important group of these pyrogenic substances, acting especially through their chemical toxic products. It is this fact which explains why the course of nearly all the acute infections is accompanied by fever. It can be promptly induced by infection with various specific germs; inoculation with anthrax bacilli or pyogenic cocci, with trypanosomes or piroplasmata, each occasions a febrile reaction. After Charrin and Ruffer pointed out that, by injecting into rabbits cultures of bacillus pyocyancus which had been sterilized and freed from living germs, febrile temperature accession may be induced, a number of observers have demonstrated that similar effects may be obtained by employing the metabolic products of a number of other bacteria. This means therefore that we are here dealing with a group of soluble substances passing from the substance of the microorganisms into the fluids and tissues of the affected body, either secreted by the living bacteria or representing some of the results of disintegration of the bacterial body (toxines, proteins, alkaloids and a variety of substances of different composition). According to E. Centanni, practically the entire group of bacteria produce a practically uniform fever toxine which he isolated from cultures and named pyrotorina bactcritica. and with which he was able to reproduce in rabbits all the main clinical features of the infectious fevers.

This thermotoxine is, however, produced in varying quantity by the individual types of bacteria; injection of definite doses of some forms of bacteria and culture filtrates occasioning no febrile reaction; some only after large doses; others causing high fever with the employment of extremely small amounts. Another feature to be considered is that various kinds of bacteria known to elaborate thermogenic substances do not act with uniformity in the production of fever in all kinds of animals, and different animals show even less uniformity in their susceptibility to the fever toxines (Krehl); from which it may be assumed that the 
pyrogenetic substances of the different bacteria also have a qualitative variation, are just as different and specific as for example diphtheria toxine and tetanus toxine (IVassermann). The pyrogenetic power of bacteria is not in any way related to their pathogenicity (Kraus); there are bacteria of high grade virulence which produce but little febrile disturbance (as tetanus bacillus), and on the contrary there are non-pathogenic microbes whose cultures nevertheless exhibit a (transitory) pyrotoxic activity, as those of bacillus prodigiosus and bacillus subtilis (Voges). A number of bacterial products when administered to the smaller experiment animals in but small doses give rise to increase of body temperature, while in large doses they cause it to fall below normal; and there is a notable peculiarity in the action of the substances obtained from cultures of the tubercle bacillus and the glanders bacillus, very small doses of extracts of these cultures causing fever to occur in tuberculous and glanders animals but not in healthy ones (in the latter, however, a rise in temperature is produced when the extracts are given in large doses, which would on the contrary produce depression of temperature in the diseased animals with symptoms of fatal intoxication).

According to the experiments of Matthes the sterile, salt-free hydration products of the albumen molecule, protalbumose, deuteroalbumose, peptone, act in the same manner as the last named agents, and it seems that in the protoplasm of a!l living cells there exist certain proteid substances capable of inducing temperature accession when injected or when set free in the bolly (Krats, Vassermann). By subcutaneous and intravenous injection of blood or blood sermu of a different species of animal, or by introduction of iodide of potash solution or pure distilled water it is possible to cause a rise of temperature, a phenomenon depending upon the hemolytic influence of these substances, either the haemoglobin thus freed or the fibrin ferment formed occasioning the increase of temperature. The injection of fibrin ferment promptly occasions marked increase of temperature, true also of other ferments (pepsin, rennet, pancreatin, invertin) as well as of the animal secretions (milk, urine). The purified enzymes are apparently inert: but the same ferments in impure state (but sterile), presumably containing some albuminous admixture, induce an elevation of temperature from which it may be inferred that the active principles are some type of albuminous body (Krehl). However, the influence is not the property of any one single albu- 
minous substance, various proteins, bacteria and other microorganisms, the products of disintegration of red and white blood corpuscles and other tissue elements all possessing the same influence.

Foreign albuminates always give rise to the development of protective processes, of phagocytosis and the production of specific antibodies. Certain organs like bone marrow, spleen and lymph glands are stimulated to special activity by the presence of these foreign substances manifesting cellular hyperplasia (mitotic nuclear figures) and probably producing special secretions. This increase of their physiological activity is necessarily associated with increased metabolism and the latter must occasion elevation of temperature. The relation between the production of antibodies and febrile attacks is very striking in many of the infectious diseases. For example in case of pneumonia in human beings it is known that in the course of the fever protective bodies are formed in the bone marrow and that the fever diminishes as these substances begin to manifest their bactericidal action. After the crisis these protective substances are found in considerable amounts in the blood (Klemperer, Wassermann). In recurrent fever of man the onset of the fever takes place as soon as the organisms appear in the blood; protective bodies are then formed by reaction of the body cells and with their accession to the blocd the microorganisms are destroyed and the fever falls; if these are removed the spirilla return from their foci of deposit to the blood and the fever returns. In other examples the beginning, rise and fall of the fever are apparently dependent upon definite stages of development of the infectious agents; in human malaria the fever begins as soon as the parasites reach the phase of sporulation and is checked if by the administration of quinine the maturation or ruptutre of the sportulating organism is prevented.

The connection of fever production with substances foreign to the body (infections and toxic) is so clear that if an animal manifest febrile symptoms it seems a permissible conclusion that there must be infectious or toxic substances circulating in its blood. Often the fever is the only manifestation of the disease appreciable during life to indicate the existence of an infection (essential infections fever) ; usually it appears as a precursor of other symptoms of the infections cause (prodromal fezer), or accompanies local or general disease processes (secondary fezer, traumatic ferer. pyomic, septicamic, hectic ferer, inflammatory ferer); and may also develop after the employment of therapeutic measures 
which introduce pyrogenetic substances into the body or occasion their production in the system (infusion fecer, defricalion fever).

The question has been asked whether fever is useful or liarmful to the body and whether it may not possibly have the definite purpose of rendering infections and toxic matters harmless. From the fact shown by various experiments that temperatures from 40 to $42^{\circ}$ C. may inhibit the growth of a number of bacteria, attenuate their virulence or actually destroy them it has been conjectured that the same effect is produced in the body by febrile hyperthermia, that the fever, "purifying by fire" as it were, is in reality a factor in causing recovery. There are a number of experiments which seem to uphold this iclea; small experiment animals have been shown to bear better (that is do not die as soon from) diphtheria, chicken cholera and other infections if kept in an incubator at $+\mathrm{I}$ to $42^{\circ} \mathrm{C}$. than when left in a lower atmosphere. The resistance. however, does not depend upon the direct action of the higher temperature on the microorganisms, as Wassermann has pointed out, but upon the fact that the body when kept warm is better able to permit the action of its natural protective mechanism (phagocytosis, antitoxine production). There is no evidence of any purpose for the increased temperature: this is only a symptom and a result of the exaggerated metabolism, and the favorable outcome is brought about by the reactive production of antibodies. In a similar manner the complications and sequels of a fever are not to be imputed to the elevated temperature. Of course, it does cause increased rapidity of cardiac action and of respiration, loss of appetite and perhaps parenchymatous changes; but the real point of the fever is in the severity of the infection, in the toxic action of the infectious microorganisms which contribute the real danger, and the elevation of temperature is only the index of the latter. The resinlt of measures having for their purpose the reduction of temperature (cold water treatment and antipyretics) is not in conflict with this conception of the relative inutility of the tenperature in fever; such measures manifest their beneficial influence not only by reducing the temperature but also by inhibiting the causes and by diminishing metabolism.

Stages of Fever. The febrile course is usually separable into a number of periods or stages. The inception of the fever, its initial period (pyrogenctic stage, stadium incrementi, chill stage) nanifests itself by general symptoms of fatigue, weakness, roughening of the hairy coat, loss of appetite and rise of the internal tempera- 
ture. In a number of affections it is marked by chills (trembling and shivering throughout the body): during the paroxysm, the internal temperature is elevated from the first and continues high, while the skin of the peripheral parts shows a lack of uniformity in temperature, the feet, for example, being cold, the muzzle in cattle and the nose in dogs, hot. The action of the heart is accelerated, the pulse correspondingly increased in frequency, small and tense; and the respirations increased in number. The cause of the chill is to be found in the spasmodic constriction of the cutaneous vessels occasioned by the poisonous products of the infectious agents: the loss of appetite and the occasional romiting (in dogs) may also be ascribed to a toxic influence acting upon the nervous centres (Blumenthal). The initial or chill stage lasts for from half an hour to two holirs, or, in cases where chills are absent, for several dars. The heated stage which follows, varying in duration with the particular disease from a few hours to a number of weeks, is that in which the temperature elevation reaches its maximum level; it is known as the fastiginm or acme (stadium acmeos). In the remittant type, characterized by alternations of depressions of the febrile temperature (remissions) and succeeding reaccessions (craccrbations), the elevation of temperature manifests a number of maximal points. The other symptoms keep pace with the hyperthermia (loss of appetite, increased thirst, general weakness. diminution of the secretions, acceleration of respiration and pulse rate, hot, dry skin). The intensity and duration of the fastigium are expressive of the severity of the febrile disease, and estimated by the height of the temperature and the strength of the heart's action. In a moderate feier the temperature should not rise beyond $4^{\circ} \mathrm{C}$. ; an intense feicr is characterized by an elevation exceeding $40^{\circ} \mathrm{C}$. A fever in which the cardiac action is strong and the pulse tension high is said to be sthenic; where the cardiac beat is weak and without force and the pulse soft the fever is spoken of as asthenic.

In the period of febrile decline (stadium decrementi or deferiesconce) the body temperature returns to normal and the associated symptoms diminish in their intensity. In case the fall of temperature takes place rapidly (in a few hours or at the most within three days), sometimes with marked perspiration and profuse excretion of urine, it is spoken of as crisis. Sometimes just before defervescence there are noted special exacerbations of the febrile state (perturbatio critica); in case there is a period of several days' 
duration in which there is alternation of notable depression and exacerbations of temperature it is spoken of as an amphibolic stage. The slow and gradual trpe of defervescence is called lysis or resolution. The decline of fever is believed to be due to the formation somewhere in the organism (bone marrow, spleen, liver, etc.) during the febrile process of a large quatitity of protective substances and to the destruction of the progenetic substances present in the srstem from the time when these protectives become predominant. Should the blood become rapidly filled with protective substances from the sites of antitoxine production or with phagocytic elements from hyperlencocytosis, the change in the case comes on suddenly (crisis) in the first, or gradually (lysis) in the second instance. The termination of the ferer, at which time the normal temperature again prevails or merely trifling variations continue, brings back the appetite: the pulse recovers its softness and evenness; the functions of all the organs proceed as before the attack: and there persist for a time only weakness and general debility. This is known as the period of contulescence. In case, however, the febrile disease go on to an unfavorable termination, and there appear irregular fluctuations in the temperature curve, a sudden rise far above normal or a subnormal depression, with signs of impending death, we are then dealing with what is known as the premortal [moribund] stage [cf. p. 95].

When the stages above mentioned succeed each other in the order indicated the course of the fever is said to be regular or typical; if there be variations, it is said to be irregular or atypical.

If in this regular conrse, a fever should continue for only a few hours or for a day, the whole process ending within this time, it is described as an chthmeral fecer. A ferer of one or more weeks' duration with a temperature curve showing daily an evening rise and a morning fall comparable to those of the normal body temperature, but at a level above that of the normal internal temperature, is said to be a continued fercr: where the depressions and exacerbations are more pronounced it is called discontinuous or remittent. When the variations in temperature are so great that between the times of high temperature accession, which take place periodically (fcbrile paro.rysms), there occur periods of absence of fever (apyreria), the fever is said to be of an intermittent or recurrent type.

Mctabolism in Ferer. The causes of fever are known to give rise as a rule to increased destruction of the albuminates, essen- 
tially an increase of oxidation (increased consumption of oxygen). As a result of this heat production is increased.

There is, however, a well defined type of fever in which there is no recognizable increase of heat production (Krell1), the febrile condition resulting solely from diminution of heat dissipation.

This increase exists at the time of the initial stage and is marked during the chill, the muscular contractions in this phenomenon adding to the heat production. Heat dissipation is also increased in fever; otherwise the temperature would continue to progressively rise throughout the attack (Krehl). Dissipation is, however, less than production. (It is only in the period of invasion that heat dissipation is with any uniformity diminished, in the chill, by the constriction of the cutaneous vessels.)

The increased albumen destruction by oxidation is shown by the increased nitrogenous output in the urine, the increased elimination of carbonic acid and the lessened oxygen elimination. Coincidently there is a retention of water; Senator has shown that of the ingested fluids, only one-third or less appears in the urine, a circumstance which cannot be explained by the amount lost by evaporation from the skin (v. Leyden). The water may be supposed to he used to keep in solution the large accumulation of products of the increased metabolism in the tissues: this idea receives corroboration in the fact that after the crisis when the retained substances are being eliminated there occurs a polyuria without increase of ingestion of water (Blumenthal). A large part of the nitrogen produced by the increased albumen destruction must come from the blood, the disintegration of the latter being the cause of the high proportion of potassium and urobilin met in the urine (Blumenthal). The uric acid excretion is also increased, from which may be inferred a marked destruction of the nucleo-albumens (Blumenthal).

Probably not all of the products of tissue disintegration are eliminated from the system or consumed; the urine does not contain everything which underwent disintegration in the organism. Probably during the fever some of its products are employed for tissue reconstruction or in the formation of antitoxines. The chlorides, phosphoric acid and sodium are found in the urine in smaller proportion than normally. With the critical decline of the fever, the tissue destruction ceases and the organism rapidly restores its nitrogen-containing structures, the nitrogenous excretion falling below its intake and a distinct increase in body weight ensuing (Blumenthal). Only in cases in which, because of re- 
sorption of exudates (pnemmonia), there is a large amout of nitrogen to be disposed of, is its elimination in the urine maintained above the usual proportion. As the result of the degenerative changes which have taken place, fatty acids appear for a time in the urine (fatty degeneration); and after a time solium chloride may be again found in large amounts.

In all febrile affections the kidneys are particularly likely to be affected. The poisonous bacterial products, and the substances of greater or less toxicity which come from tissue destruction are irritative to these organs. and render the glomeruli and renal epithelial cells permeable to albumen. As a rule an albuminuria of rariable importance is established, an evidence of the existence of a nephritis of an exudative type.

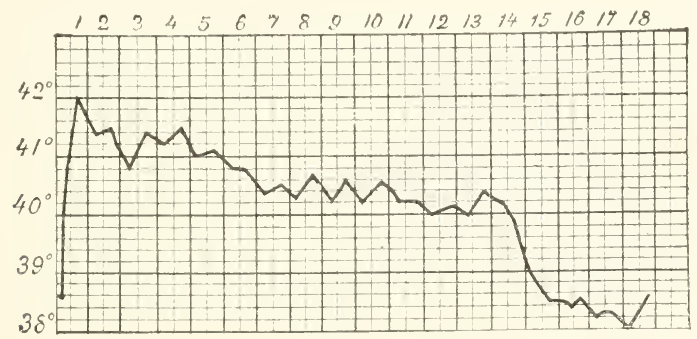

Fig. 18.

Continued fever; epidemic pleurisy in a five-year-old horse (after FriedbergerFröhner).

In some cases acetone is found in the urine, considered by some authorities as indicative of fat consumption, but regarded by Blumenthal as originating from proteid through bacterial influences and oxidation. [Acetone, diacetic acid and oxybutyric acid are all occasionally encountered in the urine in febrile states, just as in case of diabetes mellitus, and there is reason to suppose that in both conditions faults of carbohydrate metabolism underlie their occurrence, although it is possible that such substances may in less direct mamner be products of fat or proteid destruction, as above suggested.] The origin of diazo bodies (diazo reaction of urine) has been attributed (Ehrlich) likewise to bacterial influences (in man in tuberculosis, typhoid fever, measles).

The existence of fever is most efficiently detected by thermometric measurement of the body temperature. This is performed in animals by introducing a suitable thermometer into the rectum, or in females into the vagina, allowing it to remain for five minutes. after which the degree of temperature may be read off. A single 
observation may establish the presence of fever, but observations made at regular intervals afford more complete and certain information as to the existence and characteristic features of the fever and are necessary in following its course. The normal temperature characteristic of the animal must be known, together with the subnormal and hypernormal (non-febrile) ranges when in health; the differences peculiar to species and age, and the daily physiological fluctuations. These are dealt with in works on normal physiology, to which reference may be made (Cf. Friedberger and Fröhner, Lehrb. der klinischen Untersuchungsmethoden $f$. Tierär $\approx t c)$.

The course of the fever can be most satisfactorily followed by

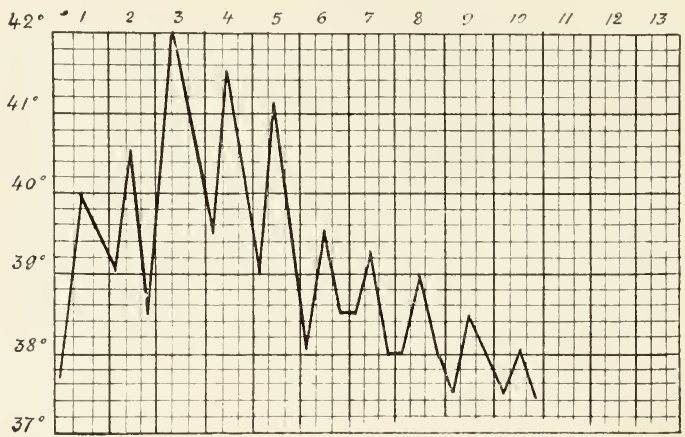

Fig. 19 .

liemittent fever: an infectious fever in an eight-year-old mare. (After Fried-

recording the degrees of temperature obtained from time to time by thermometric reading upon a chart marked out with abscissa lines, each as a point [with corresponding height from the normal line according to the number of degrees of temperature observed]. thereafter connecting these points so as to construct a scale or curve of the temperature range upon the chart, the temperatureor fever-curve.

\section{Necrosis.}

Complete death of organs or parts of organs within the living body, local death, is known as necrosis ( $\dot{\eta} v \epsilon \kappa \rho \omega \iota s$, death); when this process of disintegration has associated with it the features 
of degeneration it is also indicated by the term necrobiosis. Between the degenerations (the retrograde metamorphoses) and the necroses it is impossible to draw any sharp line of difierence. (There are in addition a number of other expressions in use for the special types of necrosis by which the death of a part in the living body is seen to occur, which are often employed indifferently as synonymsvide infra). Even in normal life in the interchange going on in the course of the physiological processes there is a certain amount of the cellular material which is being broken down in the tisstes, consumed and replaced by new cells. The epidermal cells of the skin are continually undergoing keratinization, later desquamating as dead structures, and newly formed cells from the stratum Malpighii take their place to undergo in their turn the same change. In the same way the epithelium of various glands die and contribute their own substance to their secretions, as the cells of the sebaceous and mammary glands. The blood cells are also perishable elements, some being all the time in course of disintegration and death, the formation of new cells continually proceeding in the hrmopoietic parts (bone marrow and lymph glands) to compensate for this loss. Other physiological instances of local death are also seen in the loss of the milk teeth and in the mummification of the umbilical cord.

Pathological conditions capable of causing local death are usually such as interfere with the mutrition of the cells and tissues and render the metabolic interchange between them and their surroundings impossible. The most common is some interruption of the blood supply; as thrombosis and embolism (both of which occlurle the vascular lumen), rupture of the vessels, separation of the cells or tissues from their sources of supply (trammatic, or the undermining of the skin in case of subcutaneous suppuration), or compression of the tissues which interferes with the entrance of blood to the part. If the blood is unable to gain access to an organ or some portion of an organ, no more than mere microscopic parts of it can be maintained by the lymph: with the excepion of the cornea and the cardiac valves, for which the lymphatic fluid is the principal source of nutrition, no organ can withstand a protracted interruption of its blood supply. This is especially true of gland cells; according to Litten two hours are sufficient after ligation of the renal arteries in rabbits to canse necrosis of the renal epithelium, even though after removal of the ligatures the circulation be re-established. Ehrlich and Brieger 1 cited from Pirch- 
Hirschfeld) observed a destruction of the gray matter as a result of withholding the blood supply for a single hour in case of the spinal cord. Interference with the venous outflow, if marked enough to cause stasis in the capillaries, is also followed by nutritive disturbances which terminate in gangrene.

The vitality of the tissues may also be overcome by chemical and thermic influences producing coagulation or liquefaction of the proteids and thus either directly or indirectly rendering the protoplasm incapable of appropriating nutrition and giving off its metabolic products, as well as interfering with the interchange of gases (burns, freezing, poisons like the acids and alkalies, the digestive juices, bacterial poisons). Evaporation of moisture, as in case of evisceration, causes necrosis of the exposed organs partly by drying the protoplasm of the cells, partly by desiccating and stagnating the blood and lymph. [An important adjuvant cause of necrosis is also seen in the destruction of innervation of a part. as may be observed in the massive and fulminating necrotic changes met with in cases of lesions of the spinal cord at points of pressure below the level of the spinal injury, the well known gangrenous decubitus of such individuals. In some measure here, disregarding the important pressure factor, the necrosis may depend upon vascular changes due to the nerrous lesion; but there are reasons for believing a more direct cellular failure results as well, from the loss of the nervous trophic control.]

As a general rule the occurrence of necrosis is to be attributed to a dual influence, the causative lesion having both a direct destructive power upon the tissue and at the same time interfering with the circulation of its fluids.

The characteristics of necrotic and dying parts vary with their structural peculiarities, the proportion of moisture in the organ. the relation with the uninvolved parts, and external influences.

Dense structures like bones and teeth are subject to a simple necrosis; these and the dense elastic tissues are extremely resistant to physical and chemical agencies, retaining long after complete death their form and general appearance as in life. Soft tissues also, like the epithelium of the stomach, if killed by poisons having disinfecting power (carbolic acid poisoning), may remain without material change, with but little alteration of form, just as anatomical specimens preserved in alcohol or formaldehyde solutions. In such instances the necrosis is primarily recognized by the reaction in the surrounding healthy structures in their effort to isolate the dead part (sequestration). 
Necrosis with desiccation and shrinkage (mortificatio sicca, mummification, dry gangrene) results from deprivation of moisture by evaporation, compression, chemical action or prevention of the entrance of fluids to the part. A physiological prototype is observed in the shriveling of the umbilical cord which is converted in the course of a few days after birth into a dry brown parchmentlike mass. On a small scale desiccation from loss of moisture may be seen in the formation of crusts (eschars) on the surface of wounds and ulcers, upon which usually the exuded fluid, pus, extravasated blood and a portion of the underlying tissue (cutis. mucous membrane) are dried up into brown or black crusts and scabs (eschars from caustics, scabs of wounds and tulcers). Mlummification of deeper and more extensive type, changing the tissues into a wrinkled, brown or black, tongh, leathery material, or into a completely dried hard mass, may affect the extremities, ears. feet, tail or quite frequently the skin, as the result of the action of poisons or complete exclusion of blood from the part involved. as in swine-erysipelas, ergotism or occlusion thrombosis. A common example of this variety of necrosis is the leather-like mummification which takes place in a dead fotus retained with its amnion in the uterus in case the amniotic fluid has all drained away or been absorbed, putrefaction being impossible because of the absence of the necessary bacteria, the fotus becoming a veritable mummy, enclosed and compressed in the uterine sac.

Necrosis with coagulation, coagulation necrosis. Where necrosed tissue assumes the character of an albuninous coagulate, being changed into an elastic, dense, dull white, grayish yellow or yellow, and more or less dry mass, the type of destruction is described, following Weigert, under the term coagulation necrosis or sometimes cascation. This metamorphosis is peculiar in that the dead area does not seem diminished or shrunken and has apparently not suffered loss of its fluids, but rather tends to be somewhat increased in volume, projecting slightly above the surrounding structures. This swelling of the dead tissues depends upon the diffusion of lymph from the adjacent parts through the necrotic substance, saturating it. After this has taken place the coagulation occurs in the same way as in the formation of a blood clot, by the action of an enzyme which is either derived from the necrotic cells or originates from the primary cause of the necrosis. This variety of necrosis is often induced by certain infectious germs (tubercle bacilli, necrosing bacilli, bacilli of swine plague) which seem to 
give off a ferment into the tissues; it is also frequently seen in infarcts caused by embolism and in new growths anæmic because of deprivation of their blood supply, in which necessarily it must be supposed that there is produced by the tissue disintegration some substance comparable to fibrin ferment.

The saturation of a necrosed tissue by lymph is quite possible if evaporation is prevented (for this reason coagulation-necrosis is most commonly seen in parts enclosed from the air, internal organs and mucous membranes); in moist, necrotic tissue the blood circulation is, it is true, completely in abeyance, but the diffusion of the lymph and the immigration of lymph corpuscles may be expected.

[Many, among whom the editor would include himself, differentiate between coagulation-necrosis and caseation, regarding the latter as essentially a further destructive change, an advanced fatty degeneration of the necrotic substance. Where an area of coagulation necrosis, or what is practically the same thing, hyaline degeneration (at least some forms of the latter), is retained in the tissue and is not further disintegrated by liquefying processes it becomes the seat of fatty degeneration and is broken down into a fine detritus, oil droplets, and often contains crystals of cholesterin and fatty acids. When this is of advanced degree it is described under the name of cascation. Two varieties are commonly depicted, dry and moist. Grossly a dry cheesy focus is usually defined, often encapsulated, whitish or yellowish in color, of a friable or crumbling consistence, reminding one much of dry "cottage cheese"; and under the microscope appears as a uniformly granular mass, occasionally exhibiting a few persisting fragments of the original cellular elements, scattered oil droplets and crystals, and when stained selects diffusely the acid stains like eosin. A moist cheesy focus (which may represent an area from which the moisture has not been absorbed or which may be determined from a dry focus by imbibition of lymph and by liquefaction of its own substance) is usually not so clearly defined as a dry caseated area, is paler in color, softer in consistence (pasty or mushy, like "cottage cheese" mixed with milk); and microscopically presents the same appearance as mentioned for the dry variety with the additional feature that usually the fat globules and crystals are more numerous. Caseation is especially common in tuberculous and syphilitic lesions, a form of coagulation (a variety of infectious hyalin degeneration) preceding the fatty change; it however may be met as a late change in a variety of lesions, as in atheroma, in the rnt?- 
tents of a "residual abscess," etc. The moist cheesy matter is the well known "pyoid" material of "cold abscesses." In dry caseation calcification is apt to occur as a terminal change.]

Necrosis with softening, liquefaction-necrosis. Necrosing tissues which are poor in coagulable albuminates but rich in fat and fatty substances, and which contain considerable fluid or are in position to obtain it from the entrance of lymph, usually break down into a soft pulpy mass or into a milk-like emulsion. The process may occur as a primary one, as in the brain and cord, because of the large amount of myelin contained by these parts (as also seen in infarcts and hæmorrhagic effusions); or it may occur as a secondary change where an originally coagulated necrotic substance becomes macerated after fatty degeneration or saturation with serous fluid, or is softened by the liquefying products of various bacteria (soft caseation, purulent softening). [The type of liquefaction here described is essentially the same mentioned by the editor under the name of moist caseation in the last note. There is a form of liquefaction however in which the fatty elements mentioned by the author are absent or at least not important, the necrotic tissues becoming converted into a clear, watery fluid. This resultant fluid may in a greater or less measure be the product of actual conversion of the solid substance into liquid; in part it is made up of lymph, which has penetrated the part. The disappearance of the solid substance may be a mere solution of the soluble portions in the absorbed fluid, but in addition the insoluble portions may be rendered soluble or changed into fluid by the influence of poisons (often bacterial), heat or cold or by enzymes originating in the necrotic tissue itself or generated by the surrounding living cells or by microorganisms present in the mass. Such material is apt to be removed from the affected part, usually by absorption, or may be retained within a capsule as a cyst.]

Necrosis with putrefaction, moist or putrid necrosis, gangrene

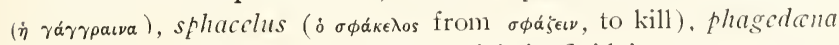
$(\dot{\eta} \phi a \gamma t \delta a \omega v a)$. When necrotic tissue rich in fluid is open to access of putrefactive bacteria a putrid decomposition sets in precisely as in case of a piece of meat or a cadaver. The gangrenous area is soft, pultaceous, filthy, dark brown to green or dark red in color, stinking and permeated by putrid gases. The putrefactive bacteria gain entrance from the surrounding air to the softened part through lesions (from wounds or ulcers) of its protective covering (epiderm, skin) or from the canals lined by mucous membrane, upon 
the surfaces of which there is apparently always present a rich variety of bacteria (pharynx, stomach and intestines). The putrefactive germs may be carried from such situations by the lymphatic and blood streams into the internal organs, where new foci of the putrefying process result from embolism. Tissues the seat of marked hæmorrhagic infiltration and those with large lymph spaces, are especially likely to become gangrenous, the stagnating blood and rich supply of moisture favoring the multiplication of the putrefactive organisms. The discolored liquid of the decomposing part is known as putrid ichorr.

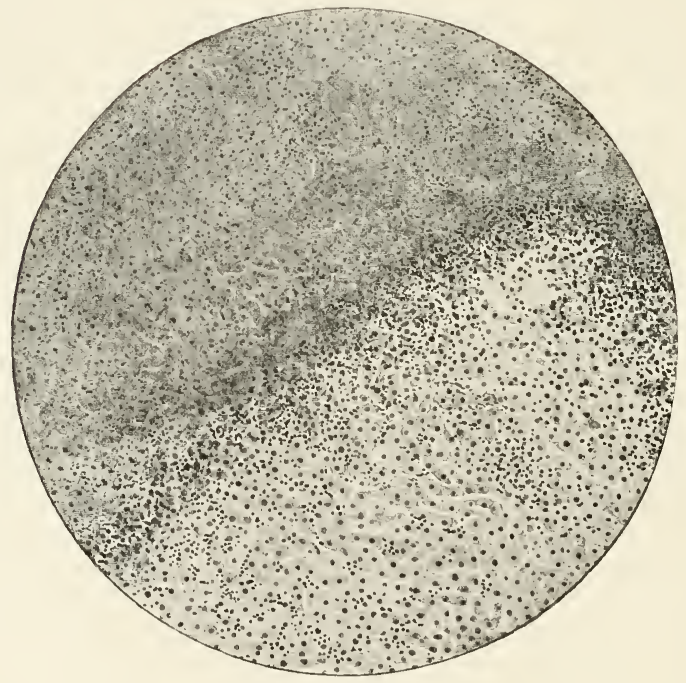

Fig. 20 .

Microscopic section through a necrotic area in liver of cow; the border of the coagulated neerotic material close to the no:mal tissue showing a zone of cellular infiltration.

Microscopically, the principal change exhibited by necrosing tissues is the disappearance of the nuclei, shown especially by their partial or complete loss of the staining quality with the ordinary nuclear staining reagents (hæmatoxylin, carmine stains). Sections of normal tissues present their cells and nuclei stained with clear definition and distinct color; necrotic portions look as though they were without nuclei, and either refuse to take the stain or only assume a faint diffuse coloration. The nuclear changes may consist of a loss of a definite outline, accumu- 
lation of the chromatin in fragments or clumps of irregular size and a variety of angular or globular shapes in the nuclear stroma along the periphery (karyorrhexis), or its escape into the cytoplasm (pyknosis of Schwann and Albrecht), or its complete dissolution (karyolysis of Klebs). Besides this fragmentation of the nuclei, displacement and solution of their chromatin, there are also to be observed changes in the cellular protoplasm and in the intercellular substance, resulting in the loss of specific structure. The cell protoplasm and supporting tissue are changed into a turbid, lumpy, more or less homogeneous mass, in which the individual cells can no longer be recognized. In case lymph diffusion in the necrosed tissue follows and coagulation occurs, the dead area seems to be filled with apparently swollen, shining, strongly refractile, fibrin-like masses of transudate (fibrinoid of Albrecht and Schwann) of lumpy, trabecular or reticulate appearance. In necrosis with softening the destructive change is usually recognized by the fat droplets (fatty detritus); and the ichor of gangrenous parts shows, in addition to shreds of the various tissues, the remnants of the liquefied red blood cells in the form of yellow and dark brown granules and clumps of blood pigment, and sometimes such solid decomposition products as leucin, tyrosin, margarin and triple phosphates, together with an enormous number of putrefactive microorganisms.

Symptoms of necrosis. Local death of tissues causes their complete loss of functional ability. When the necrosed focus is of small size and situated in the midst of healthy functionating tissue of the same type and having the same character of activities, there are often no symptoms, as in case of anæmic infarcts of the kidney and spleen. The distribution and production of heat ceases in the necrosed part with the cessation of the blood supply; gross areas of gangrene on the periphery of the body, as the skin, ears or extremities, feeling cold. Because of the coincident death of the sensory nerves within the gangrenous area the latter is itself analgesic, although the inflammatory reaction at the periphery causes sensations of pain. Gangrene in its inception is further recognizable in parts of the body exposed to view by the dark brown, dirty dark red to dark green discoloration, by the desiccation, or by its putrid odor. In this latter form, that is, gangrene, the parts assume a doughy, flabby consistence; and if the gases cannot easily escape and accumulate deep in the meshes of the tissue there inay be felt by light palpation a crackling (crepitation) of the breaking or displaced gas bubbles. The presence of the gases gives the putrefying tissue a bloated, spongy appearance, the cut surface full of small holes (gangrenous cmply'sema) ; together with the ichorous fluid these gases often accumulate under the epidermis in cutaneous gangrene and cause the formation of gangrenous blebs. 
Terminations. Dead tissue acts upon the surrounding healthy structures as a foreign body, causing both mechanical and chemical irritation: in consequence of which an inflammatory reaction is uniformly established in the adjacent healthy tissues. At the periphery of the necrotic part there may be observed an inflammatory zone marked by greater or less inflammatory hyperæmia and accumulation of lencocytes. From the action of the fluid exudates penetrating the dead tissue and of the immigrated cells removing the necrotic substance, partly by liquefaction and partly by phagocytosis, necrosed parts of small size may be gradually absorbed, especially foci softened down into detritus and small infarcts. At the site of the necrosis there is then left a space, a lefect. which is later filled in by proliferation of the surrounding tissue or by inflammatory scar formation. Larger necrotic areas, or such as apparently camnot be softened (nummified and coagulated portions) resist absorption; these may be circumscribed by the inflammation, encapsulated or completely separated from the rest of the body (demarcation, demarcating inflammation, scquestration or circumscribed necrosis). In this way while the invading mass of lencocytes and other exudates are softening down the tissue at its borders there is also proceeding an inflammatory proliferation of connective tissne and blood vessels (fibroblasts, angioplast: and blood vessel buds) to the formation of a protective wall. If the dead material be situated at the surface of the skin or mucous membrane it may be sloughed off (cutaneous slough), and the defect repaired either by subsequent proliferation of the adjacent tissue or by cicatrization. Necrotic parts situated deeply in the body are surrounded by the demarcating tissnes and come to be enclosed in a dense capsule of connective tissue. Dead fragments of bone separated from their circulatory supply (bone splinters) are enclosed by an osteoplastic proliferation of the bone marrow and periosteum and thus isolated as scqucstra.

In case the gangrenous foci contain substances of toxic nature the necrosis may assume a progressive character from the convection of the toxic products of disintegration and putrefactive hacteria by the invading lencocytes and lympl stream to other parts. When the process is reproduced in the immediate vicinity of the original focus it is spoken of as progressive necrosis or gangrene; when the putrefactive organisms are carried in the blood to distant parts, causing synchronously the same processes in the latter, these new foci are spoken of as sccondary, metastatic gan- 
grenous foci, cmbolic gangrene. A general putrid intoxication (sapremia. from oarpòs, decomposed, and aima, blood; mixed septic intoxication) may result from the toxines of putrefying bacteria in the blood and the products of decomposition absorbed from a gangrenous focus; this is likely to rum a fatal course with symptoms of fever, collapse, marked cerebral disturbances, myocardial degeneration, tendency to multiple hæmorrhage, etc. The poisons arising from putrefying matter or generated by special putrefactive bacteria have hæmolytic properties and are paralyzant to the cardiac ganglia or central nervous system.

Examples of this are frequently afforded in case of wounds of the skin which favor putrid suppuration of the underlying tissue (tooth bites, punctured wounds from stable forks), in putrefaction of the retained placenta and gangrene of the uterus, in intestinal strangulation, deglutition pneumonia and in escape of the contents of the stomach and intestine in consequence of perforation of the walls of these organs.

\section{Atrophy.}

The term atrophy is employed to indicate a diminution in volume of an organ or tissue without essential alteration in its structure and chemical composition. It involves a reduction in size of the cells and tissues (simple atrophy) : but this often proceeds to an extent that actual loss of the cells results from their complete regression (numerical atrophy). The name of the process, derived from $\tau \rho \dot{b} \phi o s$, nourishment, and a privative, means, precisely, loss of nutrition, and is used because atrophy in the main is due to insufficient nutrition. This latter factor may depend upon the fact that the cells actually do not receive sufficient nutriment (passive atrophy) or that they lack the ability to appropriate that which is offered them (active atrophy), both of which conditions may synchronously prevail and are impossible of definite separation in all cases.

Atrophy of organs and their elements may in some cases be a physiological phenomenon, a part of the cycle of their development and natural wearing out. Certain structures developed in embryonic life undergo retrogression even before birth or soon after, as the Wolffian bodies, the ductus Botalli and ductus Arantii, the umbilical vessels and the thymus gland. In the period of most active growth the milk teeth disappear after atrophy of the dental pulp. Throughout the entire lifetime there is a succession of atro- 
phy of worn out, aged cells with replacement by new and functionally capable cells, as the protective epithelial cells, glandular cells or the blood corpuscles. Dependent largely upon inherited capacity of length of life peculiar to the individual, sooner or later a retardation in the cytogenic energy makes its appearance as a characteristic of old age; this physiological loss of tissue-forming power leading to shrinkage of the affected parts is termed senile atrophy: This is particularly noticeable in the sexual glands. The ovaries dwindle after the discharge of the ova generated in early life; no more follicles mature: the connective tissue stroma and tunica albuginea become dense and the organs assume a cicatricial appearance. The testicular epithelium leaves off its spermatogenic activity, becomes lower and smaller. In the same way in the lymphatic structures, the spleen, lymph nodes and bone marrow, the ability for cellular proliferation is curtailed and hand in hand there occurs reduction in the formation of blood corpuscles. Even the skeletal system undergoes considerable atrophy in age. the bones becoming more porous and brittle and lose sensibly in weight; a progressive thinning of the bone trabecula is especially notable, probably caused by increase in a special group of cells (giant cells or osteo-

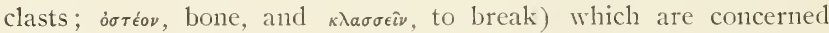
in the absorption of calcareous matter. The ganglion cells of the brain become reduced in size. At least in part senile atrophy is due to a physical wearing out, as when certain structures become dilated from mere loss of elastic resistive power (elastic fibres which have lost their tone like a worn out rubber band).

The following pathological conditions productive of atrophy are to be considered.

I. Lack of use of organs. Repeated physiological stimulation is essential for the preservation of all cells, keeping cellular function active by constant use. Cessation of this type of stimulation and the resultant failure of function are productive of atrophy (atrophy from inacticity). For instance, prolonged fixation of a limb by skillful bandaging or the immobility from ankylosis of a joint may be followed by atrophy of the muscles and bones of the part. As the stimuli are conveyed by the nerve tracts it must be evident that disturbances in the conductivity of the nerves or ioss of nervous impulse are especially apt to occasion atrophy (neuropathic atrophy). This may be observed in patsies, as those of the hind quarters (frequently seen in dogs from injuries to the spinal cord), in which the musculature of the hind legs may be reduced a 
third or half in volume from the important loss in size and number of the muscular fibres. Vice versa, if an organ to which they are distributed undergo atrophy or be destroyed, the nerves become atrophic; the optic nerve atrophies completely in protracted disease or destruction of its eye.

2. Compression. Continual pressure upon a tissue interferes with growth, hinders the blood supply to it and its lymph circulation, and directly causes diminution in the size of its cells. Pressure atrophy is conspicuous wherever unavoidable, protracted and gradually increasing compression of the tissues obtains. The renal parenchyma becomes atrophic and eventually as thin as paper from interference with the urinary discharge from the pelvis of the kidney; nodular tumors produce depressions in the tissues in contact with them; an iron cavesson makes a deep groove in the nasal bones: a hoof corn (growth of the horny hoof) causes a corresponding pressure wear on the hoof bone. Many facial malformations may be explained as the result of amniotic bands being wound about the jaws in the process of development, interfering with the further growth of the upper and lower processes of the first visceral arch. Cysticerci (echinococcus, coenurus) in their growth cause pressure atrophy of the substance of the organ in which they are lodged (conurus upon the brain and skull). Even the cellular and fibrillar connective tissue masses found in chronic inflammation as well as merely a marked engorgement of the blood vessels may serve as compressing influences upon the surrounding elements; the liver cells atrophy in chronic inflammation of the hepatic connective tissue and in chronic passive congestion.

3. Deficient Nutrition. A diminution of nutritive supply obviously must occasion atrophy (atrophy of inanition), as the tissues can only retain their dimensions and energy of growth when proper pabulum is provided them from the blood. Insufficient nutrition in diseases of the digestive organs which render difficult the ingestion of food or absorbtion of nutrition, or deficiency of local blood supply, allow the cells to waste away. In starvation, the fat of the body is lost (9I to 93 per cent. of the loss in weight) : and the musculature ( +2 per cent. according to Samuel) and the cells of various organs become smaller. According to Manawein in rabbits the cells of the liver, which average 22.3 micromillimeters in measurement, are reduced to 8.9 micromillimeters in diameter. The loss of nutrition is an associated factor in pressure atrophy and neuropathic atrophy. 
The loss of volume of the cells in atrophic conditions consists in part in decrease of their so-called paraplastic substances, partly in loss of their actively functionating constituents (Ribbert). The former, including the stored-up nutrient matter (fat, glycogen) and the cellular secretory products (mucin), are generally used up without chance of replacement from the blood supply or from the protoplasm; the above-mentioned diminution of size of the liver cells, for example, resulting. or of fat cells which after loss of their

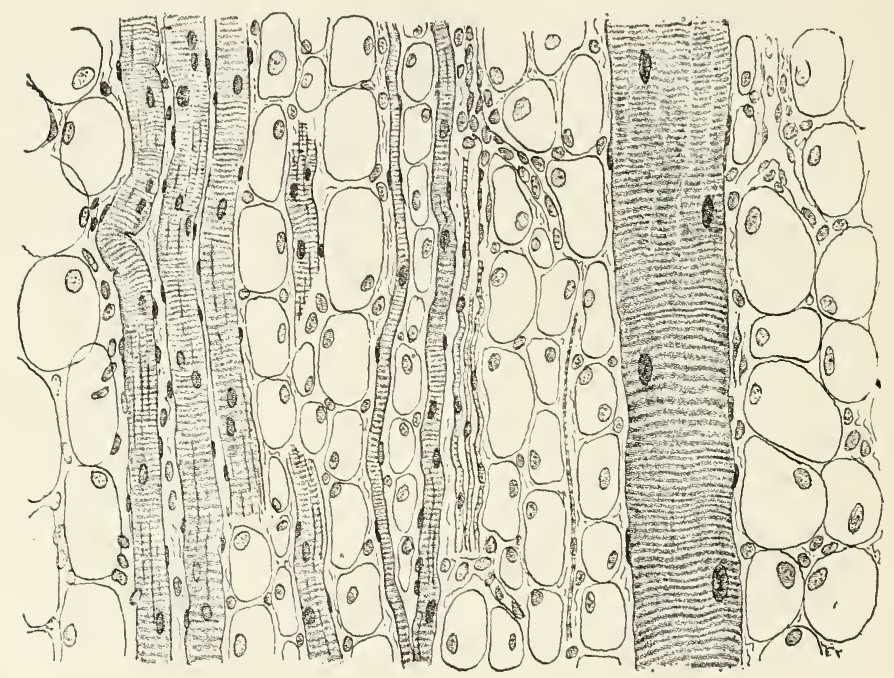

Fig. 21.

Fatty infiltation of the sural muscles in pseudohypertrophic muscular atrophy: $\times 20$. (After Thoma.)

fat are found shrunken into simple connective tissue spindle cells. The functional substance, as the contractile substance of muscle cells, undergoes metabolic alterations resulting in diminution of its bulk. It is difficult to make out how intercellular substances come to undergo the observed solution and removal, as it cannot be here assumed that it is simply carried off by the lymph, the appearance of giant cells indicating clearly that the processes operative are of complicated nature. In the bone marrow the presence and increase of these elements seem to be related with the removal 
of the hard bone substance, the calcified lamellæe being literally gnawed away by them. In addition muscle undergoing atrophy is apt to show proliferation of the muscle nuclei, the sarcolemma sheath often being full of nuclei and giving the impression of multiplication from lack of tissue pressure (lack of tissue tension) within the empty sheath, as an associated phenomenon. This same tendency to fill up spaces may also be observed in the fat cells, in which, after the fat has been used up, there often accumulates a - watery or mucinous fluid, giving the adipose tissue a loose gelatinous character (dropsical fat atroplyy, often met in old horses in the epicardium). Sometimes succeeding an atrophy in one tissue a hypertrophy may take place in another, adjacent tissue; as when in atrophy of muscular fibres the resulting space comes to be occupied by proliferation of fat cells (atrophia musculorum lipomatosa).

The deposition of the brownish pigment granules accompanying various atrophies (muscle, heart, liver, ganglionic nervous cells) is as yet not satisfactorily explained. [There is no doubt, however, that in most cases at least, this pigment is of hrmic derivation, although the substance has been in some degree modified by cellular metabolism, and may therefore be spoken of as autochthonous in conformity with its usual classification. It is a common feature of atrophic parts, as might well be expected, both from the importance of poor blood circulation as a cattsative factor and also from the diminished support afforded the capillary walls of the wasted tissue, that some degree of passive hyperæmia should prevail; and it may be noted that the degree of this pigmentary change is usually in direct relation with the degree of such congestion.]

Anatomically atrophic parts are ustlally characterized by diminution of volume, poor blood supply [poor circulation, not necessarily poverty of amount of blood present, however], loss of their adipose tissue, by a tough, dry appearance and by the assumption of a peculiar grayish tint sometimes approaching a brown color. The general bulk of the part may, however, be maintained by the compensatory occupation of the space caused by the wasting of its proper constituents by fat or connective tissue. Organs which, because of their situation or the density of their peripheral structure, are incapable of diminution may, however, manifest the presence of atrophy by loss of weight, as the bones and lungs (osteoporosis and emplysema*).

Atrophy invariably causes loss of function. Atrophic muscles eventually are incapable of motion; glands secrete less efficiently; bones become brittle: nervous structures lose their irritability and

* For details of these conditions ef. Kitt, Lehrb. d. pathol. Anatomie v. Haustiere, II. Aufl., Enke, Stuttgart, 1902. 
power of nervous conduction. Its consequence to the life of the individual varies with the importance of the affected organ. Atrophy of one of the bilaterally situated organs may be corrected (compensated) by hypertrophy of the other, as in atrophy of one kidney or of one lobe of the thyroid gland.

General atrophy of the organs involving the musculature, the general fat of the body and the glands, associated with anæmia and resulting from general disturbances of nutrition, is known as cacheria ( $\dot{\eta} \kappa a \chi \xi \xi \xi a$, a bad condition; from $\dot{\eta} \ddot{\xi} \xi \iota s$, condition, and какдs, bad), or when a phenomenon of old age, as marasmus

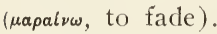

\section{Albumịnous Degeneration or Cloudy Swelling.}

Abnormal metabolic processes and lesions of the cellular protoplasm may often manifest themselves by increase of cellular volume and the appearance of densely packed fine dust-like albuminous particles in the cytoplasm, with consequent ebscuration of the nucleus and the production of a turbidity in the cell substance. By treatment with acetic acid (two per cent.) or potassimm hydrate (one per cent.) these granules are dissolved and the nucleus beconles clearly visible. Their solution in excess of acetic acid, brown coloration with iodine and acquirement of a bright yellow tint with nitric acid (xanthoprotein reaction) indicate the albuminous character of these granules. Various cells, particularly those of glandular type, are normally granular, but as a rule the nucleus is easily made out and the cells are of their fixed size and shape; while the pathological granulation is distinguished by the swelling and irregularity of outline of the cells and by the loss of their normal structural characteristics. In muscle fibres, which show the change particularly well, the transverse striations are lost and the myoplasm is filled with fine dust-like granules reminding one of particles of India ink; hepatic and renal epithelium looks swollen, expanded beyond the physiological limits, the former no longer arranged in columns (spoken of as dissociation by Browicz), the renal cells narrowing the uriniferous tubules to occlusion.

In sections this turbidity is usually not easily observed, being cleared up by the processes of preparation (alcohol, xylol, etc.). The structural lesion may also be inferred in cloudy swollen cells by the disappearance of Altmann's granules (bioblasts). (Altmann claimed that in the cells of the liver and kidneys there are fine granules around the nucleus arranged in regular rows, which could be uniformly demonstrated by certain staining methods. In cloudy swelling these granules are no longer recognizable.) 
This process, known as cloudy sielling, albuminous turbidity or albuminous degcneration, first described by Virchow, occurs principally as a result of toxic influences, both definite poisons (phosphorus, arsenic) and the toxines of infectious processes (diphtheria, septicæmia), and, too, from autointoxication from inflammatory changes. It may be regarded as an expression of disturbance of metabolism caused by such toxic action, with impair-

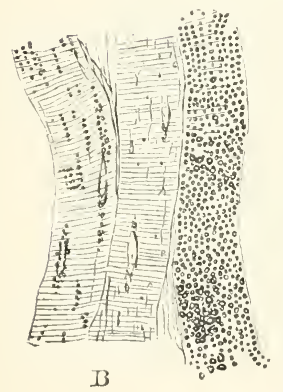

Fig. 22.

A, Cloudy swelling; B, Complete degeneration of striated muscle fobres. In $B$ the middle fibre is normal, that to the left in early, and that to the right in advanced degeration; $\times 250$. (After Perls.)

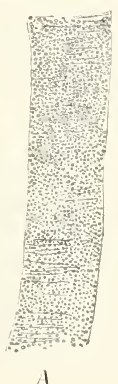

1

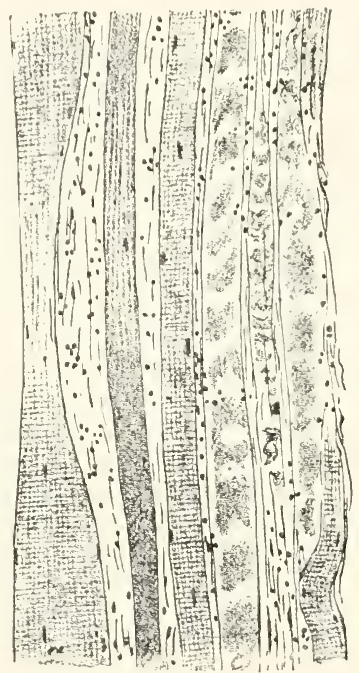

FIg 23 .

Iuscle of horse (himoglobinamia, 7 hours after the attack); a, normal fibre; b, turbid and granular fibre; $\mathrm{c}$, hyaline degenerated fibre. (After Zschokke.)

ment of the elaboration of the nutrient matter appropriated by the cells. Either the protoplasm is incapable of assimilating the albuminous bodies brought to it and they therefore accumulate in undissolved condition; or, as Virchow assumed, the cell is in a condition of metabolic irritation in which there occurs an excessive separation of its albumens; or it is a beginning cellular disintegration with formation of modified albumens in the tissue, which are with difficulty soluble. [To a certain extent it seems 
probable, too, that the swelling of the cells is referable to an excessive presence of fluid, a cellular dropsy.]

Functional disturbances are occasioned by clondy swelling, well seen in the diminished contractile power in muscle. It is a reparable process (recognizable in renal disease) provided the toxic substances which occasioned the protoplasmic fault are removed.

Anatomically organs which are affected by albuminous degeneration are usually less transparent than normally because of the turbicity of their cellular constituents, are more or less grayish or clay-colored, dry, friable, soft, and look as if they had been scalded or cooked.

The condition under discussion is very distinctly met in the psoas and thigh muscles of the horse in myopathic hæmoglobinuria, in the myocardium in various infections diseases (as pleuropnuemonia, tetanus), in the liver and kidneys in phosphorus poisoning.

\section{The Fatty Changes.}

The presence of fat in the tissues is a physiological condition depending in variable measure upon the nutrition brought about by storage of the fat directly derived from the food or formed within

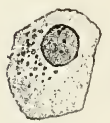

$\alpha$

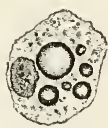

$b$

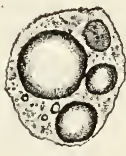

$c$

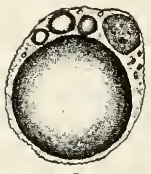

d

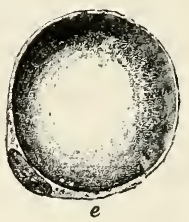

e

Fig. 24.

Fatty infiltration of liver cells: a, normal hepatic cell; b-e, various stages of development of fatty irfiltration; $\times 600$. (After Thoma.)

the cells from the carbohydrates and albumens. Deposition of fat is especially seen in the subcutaneous, submucous and subserous connective tissue, in intermuscular septa and in the glandular epithelium of the liver, mammary gland, sebaceous glands, and also in dogs and cats in the renal epithelium. The affected cells show the presence of fat as either large or small droplets; and may either have normal nuclei, with the cellular volume varying in size with the amount of fat present ( $f$ at cells, fatty infiltration), or structural changes are evident, which indicate a cellular destruction (fatty' degencration, fatty metamorphosis). In this latter case the fat is frequently found in innumerable minute, highly refractile globsles, 
the nuclei are obscured or destroyed, and the outlines of the cells irregular. The difference from albuminous granules :nay be recognized in teased preparations by the fact that the fat droplets are not affected by acetic acid and alkalies, and in microscopic sections, which have been carried through alcohol and ether. chloroform or xylol, by the removal of the fat by these reagents. the places where it was present in the protoplasm consequently appearing as vacuoles (small holes or spaces-foam structure of the

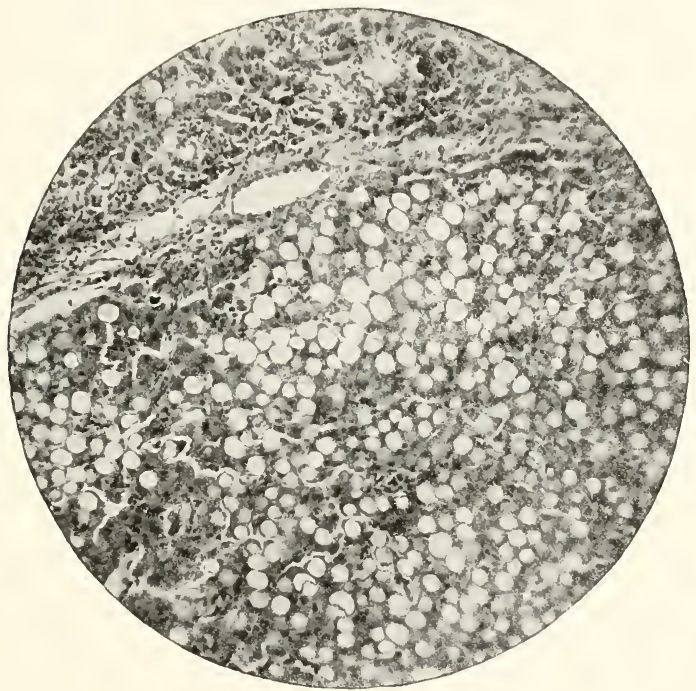

Fig. 25 .

Nodular fatty liver of dog; portion of a section under low magnification. After Rauscher.)

cells). An excellent method of demonstrating the fat microscopically is by staining with Sudan III (an aniline dye). which may be employed in teased tissue or frozen sections after hardening with formol, but not after the use of fat solvent reagents, the fat droplets being tinged an orange yellow color by the dye. Fat may also be fixed by osmic acid, which makes it black and renders it insoluble in alcohol, ether and chloroform (it is, however, still soluble in benzine, toluol and xylol-Dürk).

Fatty degeneration may be well seen in muscle fibres. in which 
the transverse striæ are lost and the substance found thickly beset by fine fat globules. Complete cellular destruction by fatty degeneration is shown by the fact that finally nothing of the cell
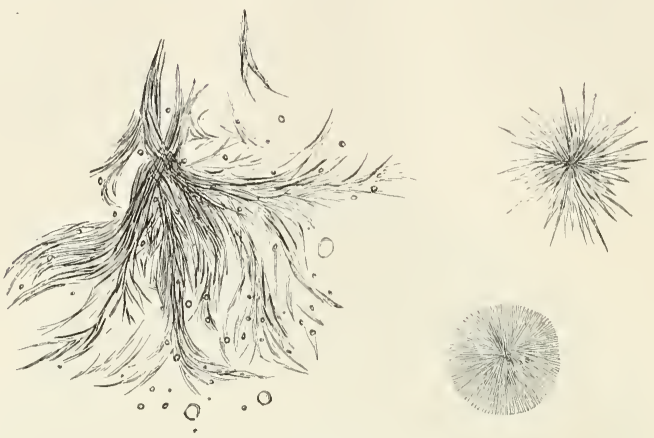

Fig. 26 .

Fat crystals (so-called margaric acid needles); $\times 250$. (After Perls.)

protoplasm remains but a mass of fat globules (fatty granular mass, fatty detritus, from deterere, to disintegrate; [also often known as compound gramule cells]). Where the fatty metamorphosis is further advanced crystallization of part of the fat in the

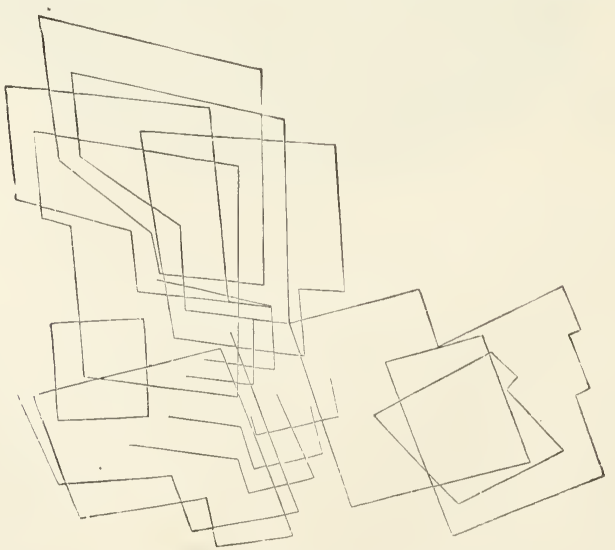

Fig. 27. 
pulpy fatty remnants of the tissue may take place: these fat crystals form clusters of needles, apparently not rigid, but seemingly easily bent (margaric acid crystals), or may appear in the form of thin plates with their corners broken ont and superimposed upon one another in a step-like fashion (cholestcrin plates, turning red with the addition of sulphuric acid, and upon further addition of iodine taking a violet or blue color).

The difference between fatty infiltration and fatty degeneration as well as their genesis (whether the fat present in the cells is derived from the fat brought to them or is produced by a local splitting process, is in most cases impossible of deternination; sometimes even the microscopic picture is confused and even in undoubted infiltration the cellular protoplasm may exhibit appearances of alteration. Moreover, it is often impossible to tell whether the destruction of the cell was brought about by the fat accumulation, or becanse of some special affection of the cell itself and the fat in the latter case merely an associated or resultant phenomenon of the destructive change.

Theoretically a difference may exist in the proportion of moisture retained in the tissue. In fatty infiltration the water is forced out of the tissue and may fall to below 5o per cent.: while in fatty degeneration the proportion of water in the tissue ( 75 to 78 per cent.) remains normal (Perls). In fatty infiltration the addition of fat to a liver may sometimes be so marked
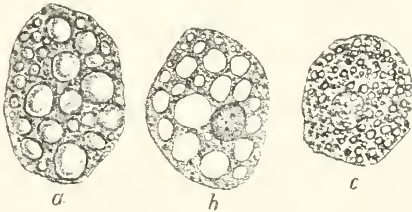

Fis. 25

Fatty degeneration of liver cells: a, liver cell of a man dead from acute phoswhorus poisoning, in phrsiological salt sulution: b, the same after removal of the fat by alcohol and oil of origanum: c, fattr degenerated liver cell of a woman dead from septicolyvamia, in physiolngical sa!t solution; $\times 600$. (After 'Thomit.) that it exceeds to per cent.;

while the fatty degenerated organ scarcely ever contains more t'man 8 per cent. (Perls).

It remains, therefore, a disputed question whether the fat is formed by cellular function or whether it is entirely derived from the food and is hence only transported fat. Cndoubtedly the supply. of fat or carbohydrates plays an important part both in physiological and pathological fatty degeneration. The studies of Rosenfeld indicate that in experimental fatty degeneration of the liver, the fat of this organ is not necessarily produced therein. but is 
brought to the liver (via blood or lymph) after removal from other parts of the body (subcutaneous tissue), because when sheep-fat was injected subcutaneously it later appeared in the liver and formed the principal part of the fat of this organ.

Outside the body fat may be produced from proteid, as shown by the investigations of Voit, Pettenkofer and Virchow. Examples are met with in the formation of a peculiar wax-like substance known as adipocere (adcps, fat, and cera, wax) in corpses, a fatty change taking place in those parts of the body lying in water or moist earth, as the result of which they acquire a spermaceti-like nature; in fatty transformation of fat-free pulmonary tissue preserved in water (E. Voit) : in the possibility of fattening animals on purely proteid diet (Voit, Pettenkofer, Cremer). Pflüger doubts, however, whether in the body fat is formed from proteid decomposition. [The prevailing opinion is that direct transformation of proteid into fat does not occur, and that when this is apparently the case, as in the author's examples, there are intervening stages or special causes. Thus glycogen may be formed from proteid by metabolic changes and the possibility of fat production from this polysaccharid must be apparent; or bacterial agency may be assumed in many instances. The substance of the bacteria may here be held a prosible source of fat, and, too, their enzymes seem capable of splitting the proteid molecule, with possible ultimate fat formation. In one of these indirect modes it may be accepted that fat may be produced from proteid: and to whatever degree these may apply to pathological changes in the living body it may be held that the fat in question may have had an original proteid origin.

There is another possibility, related, it is true, with the following paragraph, but not distinctly indicated by Professor Kitt, which should be held as a possible explanation of the appearance of fat in fatty degenerated cells. Fat combined in the general protoplasmic molecule may exist without being visible; in case of disintegration of the protoplasm this combined fat may be set free and become apparent.

And at last from the standpoint of application to the clinical importance of the case, the really important point is not so much the discovery of the fat in the cell as it is the fact that the cell is undergoing a disintegrating process-and it is this which should always be kept in mind. Whatever be the theoretical claims for considering fatty infiltration and fatty metamorphosis together. the student should, for practical purposes, hold them clearly sep- 
arated, infiltration as a relatively unimportant accumulation of fat from deposition, the fatty degeneration as a serious and eventually fatal process of disintegration with the appearance of fat, for one reason or another, in the degenerative protoplasm.]

Ribbert declares that the pathological essence of fatty metamorphosis is to be sought in the fact that the cells have undergone a retrograde change which renders them incapable of further dealing with the fat obtained from the blood, or that the cells actually synthesize the carbohydrates, and possibly the albumen appropriated from the blood, into fat, but cannot further deal with it, so that it is not oxidized and remains unchanged. According to this view the process is essentially one of disturbance of metabolism. The relation between pathological fatty degeneration and metabolic faults is apparent, moreover, in the fact that it is especially likely to occur whenever the processes of oxidation are impaired in the body; as in all disturbances accompanied by diminution in the red cells or in the hrmoglobin of the blood, the means of oxygen distribution; in general oligxenia caused by blood loss or affections of the alimentary tract; in parasitic anemias and in local anemias; in the fatty changes commonly resulting from imperfect vascularization and its accompanying local anæmia, particularly in rapidly growing tumors (carcinoma and sarcoma). Destruction of the erythrocytes and consequent diminution of the oxygen content of the blood, or, too, reduction in oxygen appropriation by the cells of a tissue because of some alteration, may result from various toxic causes; in these there arise, as important or associated lesions, fatty degeneration of the liver, kidneys, myocardium and other structures. It' sets in with especial intensity and rapidity in phosphorus poisoning, in which the liver is usually diffusely degenerated (phosphorus liver), in poisoning with arsenic, antimony, iodoform, phenol and in severe biliary intoxication of the blood (icterus). Bacterial toxines in the circulating blood in infectious diseases likewise occasion this metamorphosis.

In regard to cocess of fat in the tissue there does not obtain any sharp limit between the physiological and pathological grades. Marked general increase of adipose cells gives rise to a condition of corpulence or obesity (ob-cdere, to eat up), adipositas (adcps, fat) or lipomatosis ( $\lambda i m o s$, fat). Peculiarities of metabolism here, too, are essential basic factors, occurring in individuals both as congenital and acquired faults and arising after atrophy of the sexual glands or after castration. 
According to the experiments of Löwy and Richter,* there is considerable diminution in oxidation power connected with atrophy of the sexual glands. The authors found that in bitches, seven weeks after castration, the consumption of oxygen for each kilo. of body-weight was reduced about twenty per cent., and in spite of increase in body weight the total gas interchange decreased about nine per cent., a reduction followed up to about twelve months after operation; in castration of males the diminution of gas exchange set in within but a few days. Inasmuch as this loss is immediately removed by feeding ovariin to females and spermin and didymin to males after castration, and the same disturbances recur after stopping the administration of these substances, it may be assumed that the sexual glands possess a chemical function in connection with metabolism.

When obesity is referable to an especially rich supply of fat or fat-forming substances animals are said to be "fattened" (dietary obesity, obcsitas cralimentis). It is well known that this is favored by repeated venesection; the lowering of gaseous exchange from the removal of blood corpuscles may be a factor in this case.

Local accumulations of fat in the vicinity of atrophic tissues are found, filling in the spaces left through the atrophy, as in atrophy of the muscles and kidneys (fat hyportrophy c.r a'acuo).

[Anatomically a part, as the liver, which is the seat of fatty infiltration, is apt to be of a light yellowish color, enlarged, its capsule tense and smooth and the margins tending to be ronnded when the amount of fat deposited is great. It cuts with lessened resistance and the cut surface and the section knife are greasy.] A pathological obesity (which is progressive) is anatomically recognizable by the increase of the panniculus adiposus in the subcutaneous tissue, the peritonewn, mesentery, epicardium and as a diffuse extension through the tissues, as the muscles.

Fatty degcneration is characterized by a dull yellow, ochre or clay-colored appearance of the organs, sometimes by distinct increase of the volume [typically the reserve because of the cellular destruction which is taking place], a pasty, donghy consistence or friability, a greasy sensation given to the hand, and a greasy deposit on the knife used for section of the organ, and by indistinct structural marking of the part. Sometimes the discoloration is in streaks or flecks. The process may be associated with cloudy swelling, when the parts look as if they had been cooked [and are apt to be of increased volume from the latter degenerative change].

The pathological significance of fatty infiltration and of fatty degeneration depends upon the extent and intensity of the process

*Cited from IJerm. Hahn. Anat. u. phys, Folgeerschcin, d. Kastration. Sitzungsber. d. Gesellsch. f. Morph. u. Phys. in Mïnchen, 1902, XViII, Heft 1. 
and upon the relative importance of the affected tissues. Precisely in the same way that fat in the adipose connective tissue cells is a reserve and may be removed from these cells, there is possible a recovery from fatty infiltration in case of these or any other fat containing element, provided the cells are otherwise healthy: Even fatty degeneration is recoverable to the extent that replacement of loss in affected cells occurs from remaining normal cells or nuclei (mitotic nuclear figures have been found in fatty degenerated cells (Ribbert), and the ordinary recovery from the infectious diseases in which muscular and hepatic fatty metamorplosis is apt to occur would substantiate this view). The massive increase of adipose tissue in the body may be harmful to the rest of the tissue elements from the mechanical effects of diminishing space and compressing the cells against each other, as in the heart, where in case of excessive fatty infiltration the fat penetrates between the cardiac muscle fibers. Liver cells, which are the seat of marked fatty infiltration and consequent enlargement, compress the capillaries distributed between the cell columns, and in this way give rise to anæmia and its results. Both cells and tissues are rendered softer and more friable by fatty degeneration and this diminution of resistive power against mechanical influences may have serious consequences, as rupture of a degenerated liver or of arteries with degenerated walls from increased blood pressure. Advanced grades of fatty degeneration have, as might well be expected, a serious influence upon cellular function apart from the fact that this process is in itself an indication of pathological disturbance of the cellular protoplasm. For example, an intense fatty degeneration is capable of decidedly diminishing or entirely destroying the contractile power of muscular fibres (a cause of cardiac dilatation and paralysis of the heart). In exceptional circumstances the process may be distinctly favorable to efforts to effect recovery, facilitating the resorption or removal of injurious substances like tumors or foci of purulent softening in the tissues.

\section{Hyaline Degeneration.}

The transformation of a tissue into a microscopically homogeneous, glassy or transparent, colorless and highly refractile coagulum is known as hyaline degencration. There are produced as a result of this change one or more types of albuminates, separable chemically from each other with difficulty, the substance, 
hyaline ( $\mathrm{r}$. Recklinghausen), being characterized by its resistance to acids, by being uninfluenced by water, alcohol or ether, and by the fact that it is brilliantly stained with acid aniline dyes (eosin, orange) and with carmine.

This degeneration, or, as Dürk calls it, homogenization, occurs according to Ribbert as a local metabolic disturbance, in which the tissues to not properly use up the proteid supplied to them, do not assimilate it all or throw it off before it is completely converted. Such substance is then precipitated all through the tissue overlying the intercellilar substance and destroying the latter and the cells themseives (Ribbert). Hyaline formation is met principally in connective tissue and the vascular

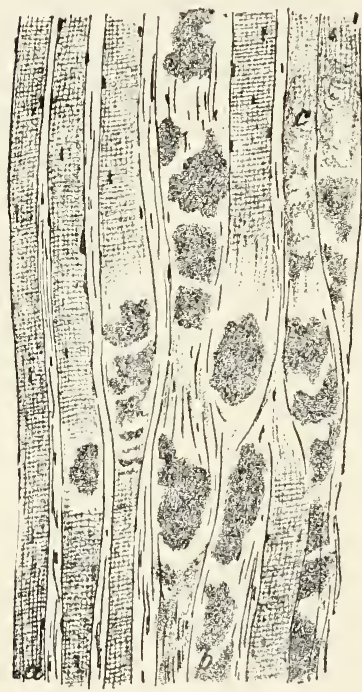

Fig. 29

Muscle of horse (hemoglobinmmia. ten days after attack): a, normal fibre; b, hraline lumps. (After Zscholke.) fibrinoid product referred to in the discussion of coagulation. Hyaline substance is, however, not entirely uniform, and the result of further study may be expected to further differentiate it into a num- 
ber of similar but not identical albuminates. There are minor differences recognized among the different examples in the staining reactions, which indicate this multiplicity, but as yet it is impossible to indicate a clear basis of separation. As a result of this uncertainty, however, there have arisen a number of types tentatively spoken of as forms of hyaline degeneration. Thus, the hyaline met in dense connective tissues as sears, walls of sclerotic blood vessels, etc., is usually regarded as the typical form, the fibrous tissue here forming minute or grossly visible masses of the glassy transparent matter, and grossly presenting an appearance very similar to hyaline cartilage, the material being of about the same consistency as the latter. Inother form is met in tuberculous and syphilitic lesions as a precursor of the later caseous change; it is spoken of as a "necrotic hyaline" and is apt to exist only in microscopically appreciable amounts, but under the microscope presents the same general appearances as the typical variety. In muscle a hyaline change minutely distinguishable by the uniform alteration of the muscle fibre into a homogeneous, glassy, cylindroid area or by the formation of lumps of such a material within the fibre, and oceasionally extensive enough to give the tissue, when grossly examined. a peculiar boiled appearance, is met in typhoid fever (Zenker's hyaline degeneration) in man, in the hæmoglobinxmia of horses. and in a variety of infectious diseases and local inflammatory changes in muscle in various forms of animal life. This variety closely resembles coagulation necrosis. In the blood a variety of hyaline thrombi, usually minute and representing the primary formation of the clot, is due to a fusion of blood plaques or of blood plaques and leucocytes; and in old clots a change often spoken of as "blood hyaline," leading to more or less homogenization of the cellular and fibrinous constituents of the clot into a transparent, structureless and yellow-colored (microscopically) or reddish (grossly) material. Definite fibrinous exudates, as that of pleurisy or pericarditis, and that of false membranes, as of diphtheria. sometimes undergo a similar change (exudative hyaline), losing all the reticular appearance of the earlier stages and presenting uniformly glass-like sheets or bands or clumps of hyaline matter. Within tumors, especially sarcomata, hyaline matter is often found: in some of the sarcomata mantles of the substance form over the blood vessels or actually involve the vessels and render them impervious (cylindromata). In various changes and in various situations in the body, rounded, spheroidal, minute masses, often show- 
a concentric marking in section, and reminding one of the wellknown corpora amylacea, but not responding to amyloid reagents, are occasionally met, especially along the blood ressels; these have the appearance of hyaline matter and are tentatively classed with this type of degeneration. Within the cells as well as between them, in a number of tumors small globular masses responding to the acid aniline dyes and having a hyaline appearance are not infrequently seen (Russel's acid fuchsin bodies); they were, when first noted, interpreted by some as of parasitic nature, but are now looked upon as a degenerated substance. The same bodies are not very uncommon within the various cells, as those of the liver or kidneys, in animals not subjects of neoplasms (intracellular hyaline).

From the fact that these and even other examples are all referred to this process the confusion in our prevailing ideas must be evident. The term "hyaloid" is often employed in order to evade somewhat this confusion, when it is sought to describe a clear, uniform, structureless substance suggesting true hyaline, but not giving perfect staining reactions and not corresponding chemically with amyloid, mucinous or colloid matter.

The cause of the change in a broat sense includes disturbances of nutrition and the influence of local intoxications, but the details of the action of these and of the development of hyaline and hyaloid substances are confused and largely wanting. The cliange is probably often that of a true coagulation necrosis; in some forms it involves further alterations of coagulated matter; in others it evidently does not mean the necrosis of the cells involved, but by its advance leads to their death, and is thus rather a true metamorphosis. When tissues are the seat of this change in its complete development they are entirely functionless and dead; and the hyaline material after a variable time becomes the seat of fatty degeneration, usually advancing to caseation and either softening or becoming the set of terminal calcification. There is no evidence that indiviclual cells containing small globules of hyaline substance are seriously incommoded by it, although the part of the cellular protoplasm thus affected is probably useless and if the cells be greatly involved it is probably eventually a serious lesion.]

\section{Mucoid Degeneration.}

The mucus or mucin which is secreted by the mucous glands of mucous membranes and is normally found in tendon sheaths, 
bursæ, synovial membranes and in notable quantities in fœtal connective tissues, is often produced in excessive amounts in pathological conditions and found in such quantities in the matrix of tissues that it becomes evidence of a degenerative process. Increased secretion of mucus from cells occurs for the most part as one of the phenomena of inflammatory irritation, the mucus appearing as an accumulation of a ropy fluid, resembling the albumen of an egg (mucous catarrh), sometimes more or less clouded from the admixture of desquamated and exuded cells. In connective tisste, cartilage and bone, the occurrence of mucus causes a gelatinous swelling, an appearance suggestive of head checse.

Lnder the microscope mucus may be recognized as transparent droplets coalescing into clumps (mucous spheroids), usually liaving fairly definite ontlines. The cells in which the mucus is formed generally swell up in one part, the mucin causing some nuclear deformation from pressure, flattening it and pushing it toward the base of the cell (transformation of cylindrical cells into goblet cells); the mucus seems to originate from the nucleus in the form of a chromatic substance thrown off in small globules (hyalosomes), which take a uniform blue color with hematoxylin and respond to the xanthroproteic test (yellow coloration with nitric acid).

[Nucoid connective tissues, under the microscope, are typified by the gelatinous structure of the umbilical cord, and present a delicate network of stellate cells, with mucin existing within the reticular spaces as a transparent structureless intercellular substance.]

Physically, mucin is characterized by swelling in water and the difficulty of passing it through a filter; chemically it is precipitated in white flocculi by the addition of acetic acid (mucoid softening), but is not precipitated by ferric chloride, mercuric chloride and nitrate of silver. It is insoluble in alcohol and ether. There are apparently a number of forms of mucin (Birch-Hirschfeld), among them some which are not thrown down by acetic acid (psendo-mucin, metalbumin). [Three types are commonly recognized, mucin, pseudo-mucin and paramucin. The first and secousd are usually found as products of epithelial cells; the third is more apt to be met in mucoid degeneration of connective tissue. Nucin and pseudo-mucin when heated with a mineral acid give origin to a substance capable of reducing Fehling's solution: paramucin will reduce it directly. They all, as a rule, take a blue stain with hæmatoxylin, but vary in their staining properties with aniline dyes. It should be noted here that the mucoid cells, really "goblet epithelium," of certain cancers were formerly regarded as colloid, and at present are often thus spoken of; but it should be definitely understood that these so-called colloid cancers are improperly named, and should be denominated mucoid cancers.] 
Mucoid degeneration of tissues should be considered as a destructive change; but the increased secretion of mucus may have a favorable influence in pathological conditions, by the envelopment of harmful corpuscular agencies and thus preventing inflammatory irritation (dust particles, bacteria from the bronchial tubes, larynx, pharynx and nose).

\section{Colloid Degeneration.}

Under the term colloid substances are included a number of products of a gelatinous, brownish and tenacious character, reminding one of half-set carpenter's glue, which, like mucus, originate from the cells in itroplets, appear under the microscope as hyaline balls and clumps and are entirely insoluble in alcolol, ether and both hot and cold water. The substance differs from mucus, however, in being completely soluble in acetic aciol after a preliminary swelling. This substance is normally found in the thyroid and prostatic glands. Hypersecretion of the material leads to the formation of cysts (goitre of the thyroid gland, adrenal cysts in the horse), which occasionally attain considerable dimensions (size of a fist). [The cause of the process is unknown, but there is reason to suspect that the product is the result of a change which follows the fusion of the blood coloring matter with some of the mucins. It involves epithelial structures, commonly the thyroid, but is not infrequently met in the kidneys, adrenals, ovaries, the cerebral hypophysis and elsewhere in the body. The colloid material is commonly found collected in spaces lined with epithelium (or spaces which probably were originally so lined), as cysts which range from a minute size to that of a walnut or rarely even that of a fist. The substance is an albuminate of uncertain and probably variable composition. Its stiff gelatinons consistency, brownish-yellow color and transparency, together with the other features above mentioned, grossly differentiate it from mucus and the hyaline substance. Nicroscopically the material is of a transparent, structureless appearance with yellowish tint, and typically takes the acid stains. When within the cells and before merging with the general colloid mass, the substance is colorless and appears in globules and small clumps, which, however, eventually fuse homogeneously with the mass of colloid in the acinus or cell-lined space. The cells thus affected are apparently not altered primarily and are affected mainly by the pressure of the collection of the colloid matter, which causes more or less atrophy.] 


\section{Glycogenic Infiltration.}

The glycogen which is plysiologically stored up in the liver and which is also met in muscle, kidneys, uterus, placenta, cartilage, squamous epithelium and in all of the organs of the foetus, may, when circulating in abnormal amount in the blood (diabetes mellitus) be found in still other cells, being present in considerable proportions in such circumstances in the leucocrtes and renal epithelial cells. It is also found in pus corpuscles in suppurative affections, and in tumors of embryonic derivation the cells contain glyengen. This condition can scarcely be regarded as a special degeneration, as the glycogen-bearing cells show no other features of alteration. The glycogen exists in the cells merely in the form of globules of larger or smaller size, clumps or grantules, staining browis with iodine and readily soluble in water (or saliva).

[The condition known as iodophilia, met typically in septic conditions, is due to the presence in the leucocytes of these iodine staining granules of glycogen. This condition is demonstrable by

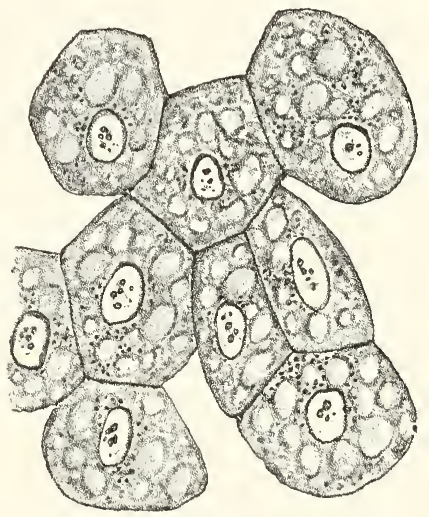

Fig. 30 .

(ilyongenic infiltration of the liver cells: 1,000 . (After Thoma.) making films of blocd from such cases; these, while still moist. being allowed to dry over iodine crystals.]

The condition is probably only an unusual metabolism, an exceptionally rich glycogen infiltration, in which, possibly, as suggested by Ehrlich, the glycogen was uniformly distributed in the living cells and separated in drops and lumps only as a postmortem phenomenon.

\section{Amyloid Degeneration.}

The name anyloid (amylum, starch; eldos, nature of) was applied by Virchow to a substance which gives a color reaction when treated with iodine and stuphuric acid somewhat similar 
to that of starch [when treated with iodine alone], and which is found in the tissues as the result of a special process of metamorphosis.

[The editor is accustomed to regard the process not as a metamorphosis or true degeneration as here indicated, but rather as an infiltrative process. The reasons for this view are, it is true, not absolutely convincing, but are suggestive. The material may in accordance with this idea be supposed to exist in the blood or elsewhere in a soluble pre-amyloid state and to be carried by the blood to be deposited in its favorite seat, the walls of arteries, where for some unknown local cause it is precipitated as amyloirl matter. Its common occurrence in the walls of arteries rather than those of veins, its appearance in masses in intercellular positions where normally there scarcely exists material which could by any known mode of transformation assume the size of the common amyloid masses (as between the bascment membrane and epithelium of the renal tubules), are the principal basis for this opinion.]

When unstained, amyloid substance is, like hyaline or colloid. of a glass-like, homogeneous appearance, and is principally found deposited in the connective tissue framework of the blood vessels in affected organs as lumpy, swollen-looking, trabecular or clumplike masses. While, however, the hyaline above discussed is tinged like the tissues themselves a yellow straw color by iodine: amyloid substance takes a mahogany-brown tint, sometimes with a violet tone, and stands ont conspicuously from the tissue. If dilute sulphuric acid or zinc chloride be applied to such a preparation, the amyloid substance takes on a deep violet to black color; its reaction being thus somewhat similar to that of starch granules or cellulose, these, however, becoming blue directly on the application of iodine alone. Other color reactions, characteristic of amyloid substance, are given by various aniline dyes: methyl-violet staining it a ruby red, methyl-green a redolish violet, in contrast to the bluish violet or green color given the normal tissues. Amyloid is colored yellow by nitric acid, indicating by this xanthoproteic reaction its albuminous nature.

According to the investigations of Krawkow this substance is a compound of an albuminate with chondritin-sulphuric acid. This latter substance, to which the jodine reaction is mainly due (Ribbert) is found normally in cartilage and elastic tissue. Amyloid material, as such, does not exist normally in any part 
of the body, but results from some disturbance of metabolism. It wotd appear that a hyaline, which either does not respond to the above reactions at all or only partially, is a precursor of anyloid.

Anyloid degeneration occurs especially after protracted suppurations: this, and the fact that its deposition mainly takes place in the connective tissue structures of blood ressels make it plausible that some unused albuminate passes from the local suppurative areas into the blood and is deposited by the latter in the tissues, where by the combination with the chondritin-sulphuric acid it is changed into amyloid matter (Ribbert). Experimental production of anyloid change lends some weight to this idea: abscesses have been produced in aninals by injection with progenic bacteria and predisposing chemicals (oil of turpentine), and amyloid matter found in varions organs after the abscesses have existed for a long time (Ribbert). However, not every suppurative process results in anyloid change: special nutritive disturbances are also required for its production.

The process is far more commonly seen in man than in animals. In man it is an ordinary accompaniment of adranced tuberculosis, asicle from its occurrence in chronic suppurations: but it is usually absent in this connection in animals, although it has been observed as a clegenerative accompaniment of tuberculosis in birds (Leisering. Röll, personal observations). In horses it has been encountered a number of times, especially in the liver by Rabe.

Organs, the seat of anyloid infiltration, become considerably enlarged: their consistence is changed, becoming dense and inelastic and more or less wax-like: in section the tissne is somewhat transparent and homogeneous, and is pale from an anæmia. In man amyloid livers are suggestive of such names as bacon-like lizers, wary lizers or a'ooden lizers; in the domestic animals amyloid livers have a somewhat different consistence, the decidedly enlarged organ (in the horse reaching eight to fourteen kilograms in weight) becoming friable, and therefore having a tendency to rupture. In the spleen, both in man and animals (hog. dog), the process occurs either as a diffuse firm enlargement of the organs with a translucent red or grayish red, homogeneous appearance of the whole substance suggesting an analogy to smoked salmon or bacon (ham spleen, bacon spleen). or as a change limiter to the splenic follicles. which in this case 
stand out like swollen sago-grains (sago-splecu). In the kidney the substance is likely to be deposited in the glomeruli, giving in these early cases the appearance of glassy granules; in more advanced cases the whole organ may, however, become waxy and firm. Microscopic study shows very clearly in these structures the deposition just outside the capillaries and along the conncetive tissue elements of the larger blood vessels. In the advanced stages of the process the substance is seen in thick homogeneous, bulging and lumpy strands. The glandular cells and connective tissue corpuscles generally only suffer passively by pressure atrophy, that is, they do not themselves become amyloid: Iohne, however, states that in the horse the liver cells also become enlarged, lobular and shining and lose their nuclei, this indicating. therefore, their participation in the process.

Amyloid infiltration may also occur as a purely local process, especially in the comnective tissue of tumors and inflammatory thickenings of mucous membranes having a substructure rich in elastic tissue, as in the growths in the nasal mucous membranes of horses known as narioblastomata.

Finally, amyloid may be met in the form of concrement-like deposits. The hyaline casts [waxy casts| forming in the urinary tubules in inflammations of the kidneys sometimes show amyloid reactions; so, too, the round granules made up of epithelial conglomerations and showing a concentric structure, which are found in the prostate in man and in old dogs and occasionally in the ependyma of the cerebral ventricles (Bruckmüller. Johne). These bodies are known as corpora amylacca or icrsicolorata in case they are colored brown, red and violet by tincture of iodine and sulphuric acid; and as corpora flac'a when they are merely turned yellow with iodine. Because of the lack of uniformity of these color reactions, and because anyloid substance in its earlier phases exhibits only the general characteristics of hyaline, it is reasonable to suppose that albumens, so modified as to appear hyaline, are precedents of the amyloid material.

The pathological significance of amyloid degeneration depends upon the extent to which it has proceeded. Small local deposits are merely incidental. In diffuse infiltration the progressive character of the process, the marked increase of the volume of the organ, the consequences of pressure atrophy on the parenchymatous cells, constitute the most important factors in production of immediate funtional disturbance of the organ. 


\section{Pigmentation.}

Autochthonus Melanosis.-Normally the epithelial cells of the cuticle, especially the deeper layers of the rete Malpighii, the hairs and horns, the epithelium of varions ingrowths from the skin (for example, the mouth in dogs), besides the pigmenterl epithelium of the retina and many ganglion cells, and in a number of sitnations the connective tissue elements (the cells of the choroid, sclera, pia, and also the cutis), contain in variable amounts coloring substance in the form of yellow, brown or black granules (the former homofuscin; the latter, mclanin).

The origin and formation of this pigment are as yet not entirely clear. The entire lack of similarity to blood pigment, especially the absence of iron as a constituent, the pigment granules being. however, rich in sulphur and containing nitrogen (v. Nenski, Sieber, Abel, Davids and Schmiedeberg), would indicate that these grantiles are transformed from the albuminates formed within the cells as products of a peculiar ("metabolic," from $\mu \epsilon \tau a \beta a ́ \lambda \lambda \epsilon \iota$, to transform, to change) cellular activity. It is not known whether the material emploved in the pigment production is originally derived from the blood or whether it represents an excretory substance of the system and its production is comparable to the formation of himuss: it can only be said that in the same way as in early fotal life and thence onward, formation of blood coloring matter takes place from the influence of cellular activity, or as, under the influence of light, chlorophyl formation is a form of cellular function, these pigments are developed within the cells.

Connective tissue cells have been noted in the human corium loaded with pigment and capable of movements which actually carry the pigment into the epithelial cells, and in some circumstances carry it away again (chromatophores). This has been observed particularly in connection with experimental transplantations; where a small bit of white skin has been grafted upon a black (negro), after healing it becomes as dark as the neighboring tissue from the penetration of chromatophores, and rice icrsa in transplanting black skin upon a white person, loss of color takes place in the graft by convection of the pigment through the agency of the wandering chromatophore cells to the lymph glands. (The existence of these wandering cells has been doubted by Kromayer.) According to C. Gessard, the black pigment, especially of melanotic tumors, is produced from oxidation of tyrosin (by an oxygenating diastase called tyrosinase)

An increased accumulation of autochthonous pigment, a hyperpigmentosis or hyperchromatosis, occurs in the human skin in the 
form of brown or black patches, as similarly colored papillary hypertrophies in the pigmented moles (nævi), freckles (ephelides), liver spots (chloasmata), lentigo; and is also met in increased pigmentation of the cardiac nuscle, of the enteric musculature and in a special type of cutaneous pigmentation occurring in a general disease (Morbus Addisonii). In some of the domestic animals the congenital, brown, lentiginous spots are, according to Schindelka, quite common; the condition known as mclanosis maculosa in calves is especially frequent, such an excess of pigment cells existing congenitally that spots of inky blackness may be found in large numbers beneath the skin, between the muscles, beneath the pleura and in the lungs, in the epicardium, in the liver, in the submucosa of various mucous membranes and in the membranes of the central nervous system. In foci of local overproduction there may often be observed in sheep an excess of the black pigment in the pia mater. There is also seen in calves and in adult cattle rather frequently a sepia-brown to ebony black color of the kidneys, which, from the studies of L. Roth, may be considered due to an infiltration of the epithelium of the convoluted tubules and Henle's loops (the thicker limb) with pigment granules which, in some cases, were identified as melanin, in others as biliverdin. Unquestionably the pigment of melanomata or melanosarcomata (cf. Tumors) is produced by metabolic activity; the proliferating cells contain within the protoplasm brown and black granules in thickest profusion. According to the investigations of Berdez and Nenski the coloring matter of the melanotic tumors is rich in sulphur; it is known as hippomclanin and phymatorhasin.

Haematogenous Pigmentation, Hamochromatosis.-The coloring matter of the blood, hemoglobin, in all conditions causing hæmolysis or destruction and washing out of red blood corpuscles, becomes freed from the cells; and is partly distributed in solution throughout the fluids and tissues, and may be in part precipitated, as is indicated by the pigmentation of the tissues. In the event of large numbers of red corpuscles undergoing solution (hæmolysis) within the blood passages, as in various intoxications and infections (blood poisons, piroplasmosis), the coloring matter (hæmoglobin or methæmoglobin) is transferred to the blood plasma. The blood becomes lake-tinged, and the serum obtained after venesection from the clot is stained, instead of limpid and yellowish, a deep red (hamoglobinamia). The 
dissolved coloring matter may pass into the urine, producing lacmoglobinuria and methamoglobinuria, in which conditions the urine assumes a bloody, dark-red or brownish black appearance. Precisely as in normal life, the dead blood cells and their remnants are taken up by lencocytes and carried to the lymph nodes, spleen, bone marrow and elsewhere: in cases of exaggerated destruction of the erythrocytes the same methods of transportation are carried on in greater measure, and the remnants of hæmoglobin are deposited in the various organs in proportion as this blood refuse fails of complete destruction. Some of these derivatives retain their iron (hamosiderin), as shown by microchemical reactions (blackened by ammonium sulphide; bluish green color on addition of yellow ferrocyanide of potassium); others contain no iron (hamofuscin and bilirubin) and may be seen as yellowish or brown granules and clumps or as a diffuse, rust-colored or yellowish impregnation of the tissues.

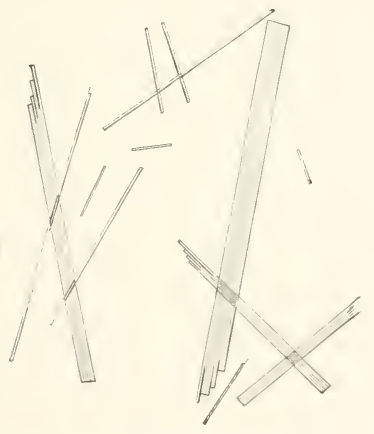

Fig. 31.

Hæmoglobin crystals from the blood of a dog killed by chloroform inhalation; $\times 250$. (After Thoma.)

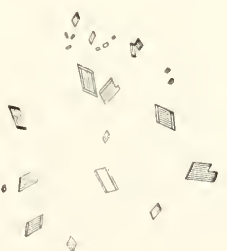

Fig. 32. IIrmatoidin crystals from a
large centralls softened peitoneal carity; $\times 2511$. (After Thoma.)

Hæmorrhagic foci give rise to very profuse hæmatogenous pigmentation, both the minute extravasations arising by diapedesis and the larger hæmorrhages; depending upon the amount of coloring matter deposited and the age of the lesion they cause a rusty, ochre-like to black or slate-colored discoloration of the tissues. The hæmoglobin at first diffuses from the escaped blood corpuscles and soaks through (is imbibed by) the tissues in the neighborhood of the hæmorrhagic focus; the washed out cor- 
puscles disintegrate and are carried off by other cells, especially lencocytes, by their phagocytic action. Just as in the above-mentioned intravascular liquefaction of the blood cells, the hæmoglobin in its freed condition is changed into amorphous masses and granules of a yellow or reddish-brown color, partly deposited in the intercellular substance of the tissue and partly taken up by leucocytes, endothelial and other cells. Blood extravasations may also contain crystalline deposits especially in the form of rhombic plates and needles of a ruby-red or yelowish-red tint, known as hamatoidin (identical with bilirubin). This is particularly seen where fluid blond remains stagnant for a long time.

Tissues discolored by hæmosiderin may become slate-colored or blackened (formation of iron sulphide, pseudomelanosis) in places where they come in contact with sulphuretted hydrogen (in the intestinal canal after death, or in ichorous cavities and putrefying hæmorrhagic exudates).

Icterus, Jaundice.-Staining of the tissues by biliary coloring matter is known as icterus (o ${ }^{\prime} \kappa \tau \epsilon \rho o s$ ); it occurs as the result of the entrance of bile or biliary pigment into the blood and fluids of the body. The common causes of such absorption of bile are diseases which occasion obstruction to its passage to the intestine, and therefore result in a biliary stasis (icterus from stasis), and all conditions which are accompanied by obstruction or narrowing of the biliary channels (obstruction from gall stones, compression by tumors, swelling of the mucous lining of the passages), providing the liver tissue meanwhile continues its production of bile. (In hepatic diseases which lead to destruction of the liver cells or to a lowered production of bile, of course obstruction of the ducts cannot occasion any jaundice or only minor grades of the symptom, as may be observed in distomiasis of the liver, in which condition this feature is usually absent.) In case the bile can no longer escape it first collects in the bile capillaries. This may be observed under the microscope as an engorgement of the intraäcinous biliary tubes which look as if actually injected with greenish bile substance; and the stasis may be further traced into the intercellular secretory alveol: and tubules. In case of rupture of these delicate capillaries the bile may gain entrance into the adjoining lynph-spaces, and be carried by way of the lymph channels into the blood (thoracic duct).

At other times it probably escapes directly from the liver cells into the blood capillaries; it is assumed that within the liver cells, in addition to the biliary secretary vacuoles, there is another canalicular system which carries urea and sugar into the blood capillaries. (Nauwerk, Browicz.) 
Biliary stasis may also depend upon an excessive production of the secretion, inasmuch as the profusely produced bile may also be sometimes too concentrated and thick and flow with difficulty, the cells in consequence becoming clogged. A hypercholia of this type occurs in conditions which cause a marked destruction of red blood cells, and the hæmoglobin set free en masse is then worked over in the liver and renders the bile particularly rich in its pigmentary matter. It is possible, too, that the bile may be thickened and less fluid or may diffuse directly into the blood ressels because of disease of the liver cells. This is met in a number of intoxications and infectious diseases. where, as the cause of the jaundice cannot be referred to a condition of biliary stasis, it is spoken of as a resorption jaundice or infectious or toxic paracholia. (Pick.)

It was formerly believed that the formation of biliary coloring matter in quantities sufficient to cause jaundice could also take place outside the liver, in the blood; and a hrematogenons jaundice was spoken of. From the studies of Minkowski and Naunyn, however, it may be considered established that, even in cases where a simple increase of destruction of erythrocytes causes this symptom, it is not produced except with the intervention of the liver, and that the transformation of the hrmoglobin into biliary pigment is accomplished in the liver. Birds jaundiced by arseniuretted hydrogen rapidly lost their icterus after extirpation of the liver, and where the liver had been previously ablated, jaundice could not be produced in any degree at all. [The term "local hematogenous jaundice" is sometimes applied to the yellowish discolorations of fading bruise marks; it is permissible only in a general way, and in precise discussions the pigment must be understood as not identical with the jaundice pigment. Although there may be more or less hæmatoidin present, there are also less closely allied blood pigments.]

The entrance of the bile into the blood is followed by an impregnation of all the tissues to which the blood vessels are distributed with the biliary coloring matter, this giving them a biliary tint of various shades. In the living body this is apt to be particularly conspicuous in the conjunctiva and sclera; in the dead animal the yellow color is principally apparent in the subcutaneous tissties, the fat, intermuscular connective tissue, the lungs, liver and kidneys, the mucous membranes and their muscular coats. Where the normal color of an organ was pale the discoloration may take on an intense citron-yellow hue; the dark liver and kidney tissue becomes saffron yellow to olive green or greenish-black. Even the bones as well as fibrinous blood clots and the blood serum assume a biliary tint. As the pigment gains 
access to all the glands their secretions also become yellowish; and the pigment is especially noticeable in the urine. [The saliva, gastric and pancreatic juice, tears and mucus are apt to be free from coloration.] Only cartilage, the cornea and the enamel of the tecth, and, too, the brain, take up but little of the color. Because of the diffuse uniformity of impregination of the intercellular substance and of the cells with the coloring matter, it at times is not noticeable under the microscope (perhaps washed away in the details of preparation of the specimen); but in other instances some of the cells, as those of the liver, are stained an intense yellow or yellowish-brown, and granular and coarser deposits of a yellow, brownish or green color are found in the protoplasm, especially in the hepatic and renal epithelial cells. The pigment is sometimes seen in crystalline form (rubyred rhombic plates of bilirubin) in the kidneys, spleen and bone marrow.

Jaundice may be a transitory condition; the liver after removal of the interference to the passage of the bile resuming its normal functions and the resorbed bile being eliminated by the urine, etc. In case of more prolonged duration faults of the organism become apparent. In the first place the absence of a sufficient amount of bile in the intestine may interfere with digestion; in the second place the bile stasis in the liver may cause pressure upon the liver cells and can give rise to degenerative and necrotic changes; thirdly the mingling of the bile with the blood brings into the latter and into the different organs substances which are hæmolytic and have other toxic influences. The biliary coloring matter and, too, the biliary acids are to be included among such toxic substances, the latter undoubtedly being toxic to the nervous system. [It is supposed that the itching complained of by jaundiced human beings, and to some extent present in animals, is due to an irritation of the skin by the biliary pigment; and the slowing of the cardiac rate in jaundice is directly referable to the action of the biliary acids or their salts upon the vagus, or in marked instances upon the heart itself.] Moreover, the influence of substances produced in intestinal putrefaction should be kept in mind, these materials being absorbed from the intestines and passed through the diseased liver which is no longer able to neutralize their poisonous properties. Severe cerebral symptoms developing in cases of biliary obstruction and sometimes fatal (convulsions, coma, delirium), accompanied by 
high fever, indicate the existence of such an autointoxication, which is designated as ictcrus gravis and cholamia.

Exogenous Pigmentation.-When the tissues are impregnated with pigmentary substances which gain access to the body from the exterior the process is known as exogenous pigmentation. The most common example is the deposition of carbonaceous material (anthracosis) in the lung, practically always found in man from inhalation of the particles; it also occurs in dogs which are compelled to pass much of their time in smoky places, and in horses kept for a long time in coal mines. The inhaled dust, except that which is removed by the bronchial mucus and by the ciliary activity of the tracheal cells, remains suspended in the alveoli [and smaller tubes], is taken up by wandering cells (dust cells); part being carried in the pulmonary lymph channels and reaching the lymph glands of the lungs and bronchial tree, part being deposited in the lymph spaces of the lungs. Varying with the quantity of carbon thus deposited, the lungs and lymph glands become mottled and of a gray slaty appearance or uniformly and completely blackened.*

Dark pigmentations may also be caused by the deposition of mercury, lead and silver, observed sometimes after medicinal administration of preparations of these metals, as in case of the black color of the intestinal villi in the horse after the administration of calomel. It is usually the result of the formation of mercuric and plumbic sulphides from contact with the sulphuretted hydrogen of the gastro-intestinal contents. In employing nitrate of silver in internal medication it is absorbed from the alimentary tract and deposited in extremely fine granules (reduced to metallic silver) in all the different organs, skin, conjunctiva, kidneys, etc., and gives them a grayish pigmentation. The condition is known as argyria.

By means of subcutaneous and intravenous injections of staining solutions (carmine, indigo-carmine, methylene blue) the tissues of the body may be colored blue or red. The protoplasm of many cells takes up the stain diffusely in part; sometimes instead a granular deposition takes place from combination of the pigment with certain granular constituents of the cells (Arnold, Ribbert).

[The effect of external or exogenous pigmentation is usually trivial aside from the mere discoloration and is only important when intense or as a predisposing cause to more serious dis-

*For details cf. Path. Anatomie a. Haustiere. II. Aut. Stuttgart, Ferd. Enke. 
turbances. Each pigment particle is essentially a foreign body and induces a certain amount of inflammatory reaction, usually of low grade and productive of an increased amount of fibrous tissue. Parts the seat of marked pigmentation, as the lungs and bronchial glands, become more or less indurated. The possibility of infection by tuberculosis, especially noted in man, becomes more easy when previous dust inhalation has induced a chronic bronchitis and has induced the secondary faults of pulmonary blood and lymph drainage and has lowered the general vital resistance of their tissue by a prolonged chronic interstitial inflammation. In less important organs than the lungs, of course, the changes occasioned by external pigmentary deposit are proportionately less serious.]

\section{Calcification and the Formation of Calculi.}

Calcium salts are normally present in solution in the body fluids (calcium glycerin-phosphate, carbonate, lactate, oxalate, etc.). Deposition of lime in solid form takes place normally in the bones and teeth of man and the animals; and in herbivora there is normally excreted in the urine such large amounts of calcium salts that by mere cooling they precipitate and the urine at time of voidance nuy even be turbid from their presence (horse). Calcium impregnates the matrix of bone and teeth with so much uniformity of distribution that it is not apparent in definite masses, being recognized only by the solidity of the structure and by chemical analysis; in the urine it separates as amorphous granular and crystalline forms.

The deposition of calcium salts in other parts than those mentioned is pathological and is known under the names calcification, cretaceous infiltration, petrification and incrustation.

The basis for this deposition is probably to be sought in the removal of the substances favoring the maintenance of the lime in solution, as free carbonic acid, and the transformation of earthy salts, which are soluble in water, into insoluble calcium compounds, and, too, in increased supply of lime. Thus, pathologically, calcification is apt to occur in dead or altered tissues in which free carbonic acid exists in lowered amount and in which gaseous interchange by the cells is essentially impaired, or may appear in secretions and excretions where it leads to the formation of concretions. [Klotz (Jour. of Exper. Med., I905, vii, p. 633) has recently attempted to explain pathological calcification in necrotic degenerative areas 
upon the assumption of a previous fatty degeneration of these structures, soaps of lime being formed primarily by combination of lime brought in solution by the juices permeating the altered tissues with the fatty acids. These soaps are later further changed by substitution of the fatty acids by carbonic acid and phosphoric acid. Wells (Jour. of Med. Research, I906. N. S. ix, p. 49I) cannot find evidence of constant and important occurrence of these soaps as a stage in the process, although in traces he finds such soaps to exist in areas of calcification. Fischler and Gross (Ziegler's Beiträge, igo5. Festschrift für Arnold, p. 326) have also found evidence of the presence of such soaps in atheroma and in the margins of infarcts, but not in caseous areas, and are unable to definitely declare that soap formation is an essential stage in calcification. Wells regards pathological calcification as probably essentially similar to normal deposition, and inclines to the idea that there exists or is produced in the area some substance having particular affinity for calcium, although he is unable to indicate its nature; it is not, however. dependent upon the vital state, as in cartilage it exists both when the cartilage is living and after it is boiled.]

In distinction from physological calcification, in which a perfectly homogeneous combination of the lime with the matrix obtains, pathological calcification is manifested on microscopic examination by the presence of fine, highly refractile granules, looking like particles of fat and pigments, which, with direct light (complete closure of the substage diaphragm), have a dull, glistening, whitish appearance, and are found both within and between the cells. On the addition of acids (especially hydrochloric acid) the calcium salts are dissolved and the presence of calcium car onate is indicated by the immediate effervescence (carbonic acid). With pure sulphuric acid great numbers of crystals of gypstum (calcium sulphate) separate in delicate pointed crystals, seattered all through the tissue. A calcified tissue (the same in case of partial calcification) takes a very intense dark blue stain with hamatoxylin.

Ossified tissue differs from merely calcified structures by the formation of lamelle of the matrix (Haversian lamella) and the inclusion of the bone cells in the intricately branched lacunæ.

Petrification of tissues renders them as hard as bone and gives them a dirty white appearance. As a senile change this is occasionally met as spots isolated in the cartilaginous nasal septum in cattle. in the laryngeal cartilages of logs, and quite uniformly in the costal cartilages of old animals. (These foci become easily visible as opaque points on drying the cartilage.) With much less frequence than in man, calcification of the vessel walls is observed in animals 
as a rather rare occurrence, transforming the intima of the vessel, as the aorta, into a rough, scaly surface, or appearing as flat plaquelike or disc-like areas, making the vessel at such places rigid and inelastic. The greatest tendency to this change is found in the large verminous aneurisms in horses, which form brittle, hard enlargements of the arteries. The intima and the media in these lesions are the especial seat of the calcification, preceded by hyaline degeneration.

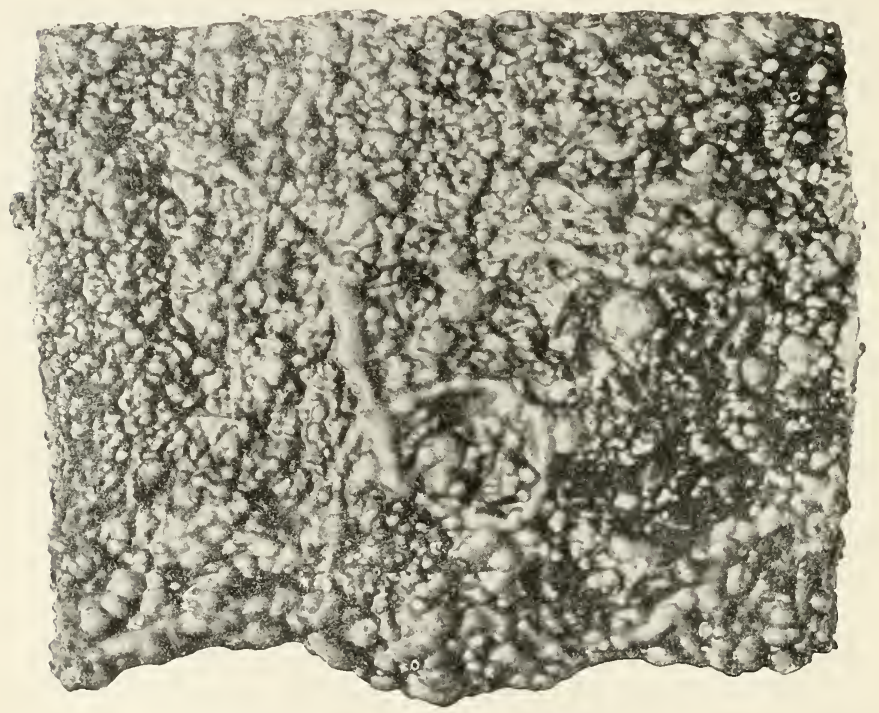

Fig. 33 .

Portion of liver of a horse with calcification of necrotic parasitic foci (After Trolldenier.)

In greater measure newly-formed inflammatory connective tissue is rather often affected; thus the cicatrized castration wound of the abdominal wall in hogs may be observed changed into a calcified plate as broad as a hand, and the opening into a hernial sac in umbilical hernias in the same animals is sometimes changed into a calcified ring of connective tissue. Calcareous plates in the connective tissue of the cow's udder are sometimes found; and even the connective tissue of the lungs is in rare instances (in cattle) trans- 
formed into a branched, rigid and corset-like enclosing structure. In the dense membranes of the spinal cord, in logs, calcareous infiltration gives rise to rigid thickenings which feel like splinters of wood. Bursæ and tendon sheaths in horses may become extensively calcified and ossified. The colloid matter often met in the thyroid gland and sometimes involving the connective tissue as well as the acini, may occasionally terminate in calcification of the organ (stone goitre, struma petrificans, in dogs). The herbivora and also hogs are with special frequence subject to calcareous infiltration of tuberculous caseated tissues. The yellow, cheesy foci produced by coagulation necrosis are frequently full of sandy, gritty particles and are very hard. In the end dead parasites and the connective tissue capsules which are formed about such foreign bodies in the parenchymatous organs, as echinococci and round worms in the livers of horses, become so permeated with lime salts that they form stony, bone-like or mortar-like, chalky masses.

In women, dead fotuses long retained in the uterus or peritoneal cavity may become so completely calcified as to be of stone-like hardness (pctrificd fotus, lithopadion): in our domestic animals the dead embryos, which are often found, usually remain more or less leathery, pliable and merely mummified. [In the musemm of the Medical Department of the University of Pennsylvania there is a thoroughly calcified excellent example of a lithopadion taken, after death, from the uterus of a mare, and presented to Dr. James of the above-named institution in I 85 I by Mr. Kearney of Gloncester County. New Jersey.]

Zschokke (Schw'ciz. Archiz'. 1902) has described a peculiar crystalline deposit observed in the liver of a cow confined to the interstitial spaces as white foci, scattered here and there. The abundant precipitate was made up of rounded and rhombohedral crystals (about the size of white blood corpuscles), the chemical nature of which was not precisely deternined, although with the iodine-sulphuric acid test they showed some relation to cholesterin.

Solicl 1!norganizer bodies which are formed in the secretions and cavities of the body are called concretions. They are formed by the precipitation of salts which have become insoluble; their chemical composition depending upon the ingredients of the normal secretions and its existing changes, and therefore not uniform. Sometimes they contain organic matter mingled with the saline constituents; or they may be foreign bodies or exudates which have become encrusted with salts. The fundamental conditions for the formation of these bodies include the following:

I. Supcrsaturation of the saline solution (Klimmer) and diminution of the medium of solution. Just as the crystalline salt 
separates from a saturated solution of copper sulphate or from Carlsbad-Sprudel water, in the same manner the salts of a secretion, like the urine, may precipitate, especially should there occur a loss of its fluid or of substances which keep the salts in solution.

2. Retention of secretions or excretions in their storage reservoirs.

3. Chemical decomposition of the secretion, with the formation of insoluble products, as may result from the presence of bacteria (ammoniacal fermentation).

4. Presence of a foreign body or any organic material which serves as a nucleus for the deposition of crystals, or as a supporting

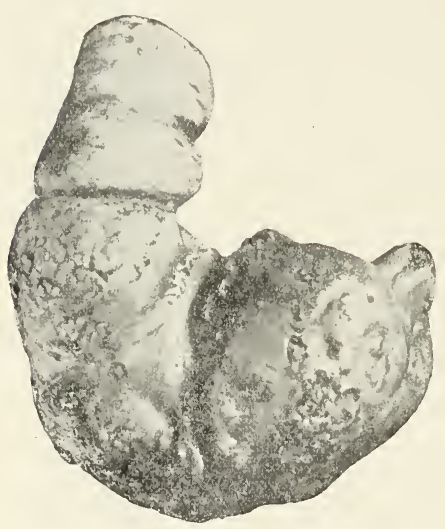

Fig. 34 .

Irinary calenlus from renal pelvis of framework for the precipitated salts, as bits of wood, particles of food, or viscid degenerative products.

\section{Urinary Calculi (Uro-} lithiasis, gravel, concrementa urinaria).-The saline constituents of the urine may be precipitated in the kidneys, in the renal pelvis and in the urinary bladder and give rise to the formation of urinary grazel, urinary sand, and larger urinary calculi. Even normally in the horse, calcium carbonate separates in the pelves of the kidneys and in the bladder from the al-

kaline urine, the latter being turbid even when freshly passed in consequence; in the other herbivora it becones clondy soon after it is voided because of loss of the solvent power of its fluid on cooling. The acid urine of carnivora and omnivora is, on the contrary, clear and permits sedimentation only after decomposition and long standing. The causes of urinary calculus formation may include such structural elements as parasites, blood clots and coagulated fibrin, shreds of necrotic tissue, tube casts and epithelial cells, these substances affording a place of attachment for deposition of the inorganic substances. The presence of living or dead bacteria is especially apt to cause precipitation, these at times forming small 
masses and favoring, as do protein clumps, the adhesion of any precipitate, and, too, perhaps causing ammoniacal fermentation. A high proportion of salines in the urine is, as pointed out by Klimmer, an important feature. This authority questions whether the presence of albuminoid or mucoid substances, such as are occasioned in the urinary tract by inflammation, provides any means of cohesion, as a sort of paste. Some such organic stroma is uniformly found, especially because the concretions determine a catarrhal process at times, as a result of which layers of mucus and albuminous material are found between the mineral deposits; such viscid matter is, however, also found in loose sediments and perhaps may in reality interfere with calculus formation by preventing [approxination and] cohesion of the particles. Such organic framework is not, as pointed out by Moritz, peculiar to calculi, but is, too, an important feature in any urinary crystal, whatever it may be. The size and shape of urinary concretions varies widely with the species of animal, the site of formation and the composition. Renal calculi are usually round, pearl-shaped, like writing sand, millet seed or hemp

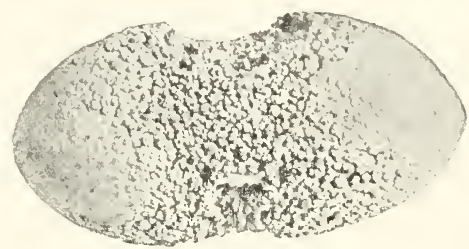

Fig. 35.

Vesical calculus from a horse. sced: those of the renal pelvis are irregular, warty, or correspond to the shape of the pelvic cavity, crescentic, and may reach a size nearly that of a fist. In the bladder the urine throws down a slimy, sandy material which forms a thick, soup-like urine or masses which may attain several pounds in weight and assume the shape of half of a pear (corresponding to the interior of the bladder and flattened on one side by the passage of the urine). When compact, vesical calculi are lentil shaped, oval, flattened or faceted from pressure, polished or sometimes rough and mulberry-like. gland shaped, and range in size from that of a millet seed to the size of a double fist. Small concretions met with in the ureters or urethra, and often impacted in these passages, are not formed in this situation, but are swept from the kidneys or bladder. The color of urinary calculi is usually brown, metallic, bronze-like: some concretions are, however, gray, yellowish or pure white, and occasionally reddish ones are met. 
In construction and chemical composition of urinary calculi a large variety of substances take part; and the concrement is rarely made up of but a single chemical constituent, but usually contains a number of ingredients. In horses these calculi generally contain carbonates and phosphates of lime, carbonate of magnesia, traces of iron and occasionally silicates; those of the cow, sheep and goat are at times rich in silicates or magnesium phosphate, but also contain calcium carbonate or triple phosphate. In swine they are made up either of triple phosphate or calcium and magnesium carbonate and phosphate; and oxylate concretions also occur. The uroliths of carnivora are a mixture of calcium carbonate, phosphate and urate, or are composed in other cases of uric acid or sodium

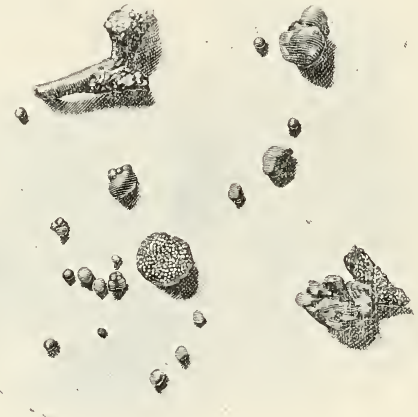

Fig. 36.

Renal calculi from cow.

and ammonium urates or of calcium-ammonio-magnesium-phosphate. The tabular forms, when fresh of soft consistence and of greasy, slimy appearance, are mainly made up of cystin. The color of urinary concretions is given by the pigment of the urine or biliary pigment; occasionally hæmatin has been found to explain the existence of a black color, or carbonate of iron that of a metallic bronze luster (Fürstenberg, Pflug, Müller, Dammann, Klimmer).

The especial causes of danger in connection with urinary calculi are to be found in the disturbances of urinary flow which they occasion, giving rise perhaps to urinary retention. In addition these foreign bodies act as irritants to the mucous membranes in 
varying grade; may cause dysuria (tenesmus) and pain of intense severity (spasm of the involuntary musculature of the ureters, bladder and urethra), local inflammation and erosion, obstruction of urine, dilatation of the bladder and possibly rupture, hydronephrosis, and submucous urinary infiltration and urinary intoxication of the blood. (For details cf. Spericlle pathol. Anatomie d. Haustiere, II. Bd.).

Gall Stones (Cholclithiasis).-Although in man gall stones are comparatively common ( 25 per cent. of women suffer from them), they are rather rare in animals. They are found as soft or half solid, slippery bodies in the gall ducts and gall bladder, about the size of a hazelnut but sometimes even larger than a fist, usually multiple in numbers and often occurring in hundreds. Their color is either a fine saffron or ochre yellow, brick red or dark brown, or they are whitish and chalky, with a yellowish or a greenish color on the outside or in layers internally. The larger ones are rounded or oval in form and in section they show a laminated structure; or they are from mutual pressure faceted and polyhedral because of their softness.

Their composition varies. From analyses made by Maly, Phipson, Hermann, of the gall stones of cattle, these concretions were found peculiarly rich in bilirubin or the compound of bilirubin with calcium (28-6I per cent.) and poor in cholesterin (only I.35 per cent. ; according to Zschokke, IO-I5 per cent.). E. T'oit found in a gall stone of a horse but little biliary pigment, no cholesterin, large amounts of biliary acids, and in the ash chiefly calcium phosphate. In the large examples not infrequently food particles (bits of cereals or of hay or straw) are present, apparently forced from the intestine into the widely dilated and relaxed biliary canal (peristalsis).*

The origin of gall stones is not as yet entirely clear. According to Naunyn the mucous membrane of the gall passages in catarrhal conditions produces a secretion rich in calcium salts, and at the same time albumen is exuded into the bile. The presence of the albumen favors the precipitation of the lime, and the increased proportion of bilirubin may be explained by the thickening of the bile from retardation of its flow. [The solid pigmentary substance in gall stones is a definite chemical combination between calcium and bilirubin, the latter having feeble acid combining powers.]

* Occasionally in swine and deer accumulations of ordinary sand (silicate and quartz fragments) have been found, which could not possibly get into the biliary passages except from duodenal contractions. 
Cholesterin gall stones are apparently the result of a pathological secretion from the biliary mucous membrane; according to Gamgee, in cholecystitis there may be observed myeline-like masses in the epithelial cells, a lumpy, glistening substance, which floats on bile and which, on the addition of acetic acid, crystallizes as cholesterin. These cholesterin clumps are regarded as the stage preceding the deposition of cholesterin for the formation of calculi (Naunyn).

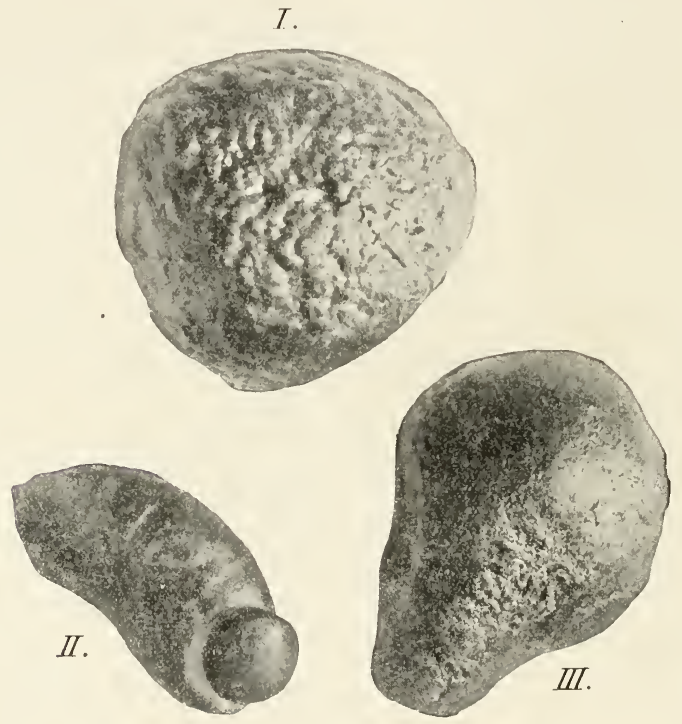

Fig. 37 .

Gallstones from cow. (After Trolldenier.)

It has been pointed out, too, that colon bacteria or a mixed infection penetrating from the intestine may set up a cholangitis and cause disintegration of the secretion and exudate and in this way give origin to calculus formation (Halia, Naunyn).

The presence of biliary concretions may produce no symptoms as long as the bile is able to flow without interference; but at times by obstructing the biliary passages they cause biliary stasis and jaundice or, by involvement of the cystic duct, retention of the mucus in the gall bladder with marked dilatation of the latter (hydrops iesica fellec). These calculi with theị bacteria and 
particles of food within them may excite inflammatory changes, and give origin to biliary colic from the spasm and inflammation of the muscular wall of the bladiler; and it is possible that rupture of the latter may occur.

Salivary Calculi (calculi salizales). - Small concretions and calculi are formed from the calcium salts of the secretion (calcium
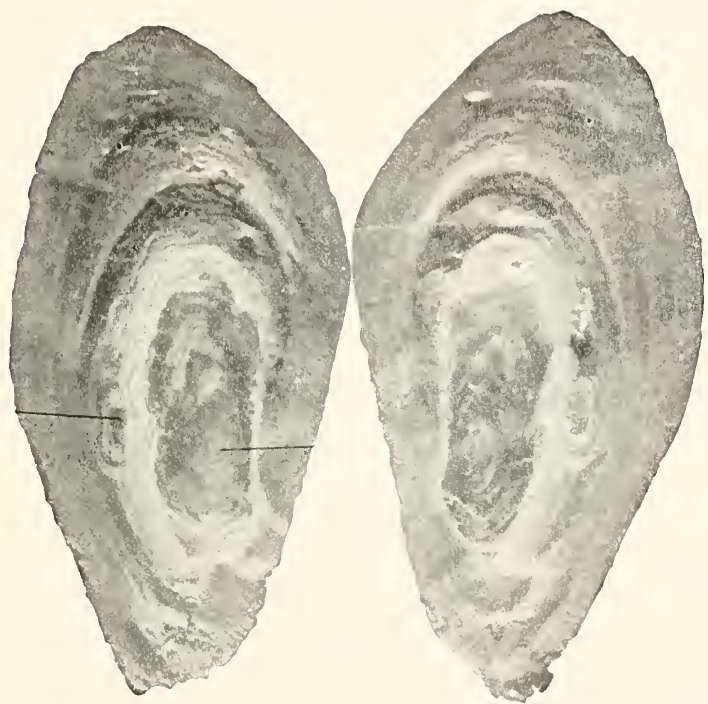

Fig. 38 .

Sectioned surface of a gall stone of a row. (After Trolldenier.)

carbonate and phosphate) and from carbonate of magnesium, potassium and sodium in the ducts of the oral salivary glands (parotid, sublingual and submaxillary) and of the pancreas (abdominal salivary gland). These concretions are usually chalky. In the horse salivary calculi sometimes form in Steno's duct to the size of a goose egg (200 to 600 grams in weight), often having as a nucleus a bit of chaff, a piece of straw or an oat seed, which has in some way gotten in from the mouth. According to Galippe bacteria by inducing fermentative changes in the saliva seem to be the occasion for the separation of the salts. 
The larger calculi cause distension of the passages and retention of the secretion. The earthy salts of the saliva may also precipitate in the month cavity. Nixed with desquanated epithelium and oral vegetable organisms they accumulate as hard, brittle, dirtywhite deposits, particularly about the neck of the teeth, forming the so-called tartar (common in dogs, rare in horses).

Intestinal Calculi. Fecal Concretions-Indigestible parts of the foor and earthy substances with them sometimes accumulate in the large intestine in more or less compact masses, which may have important consequences upon and menace the

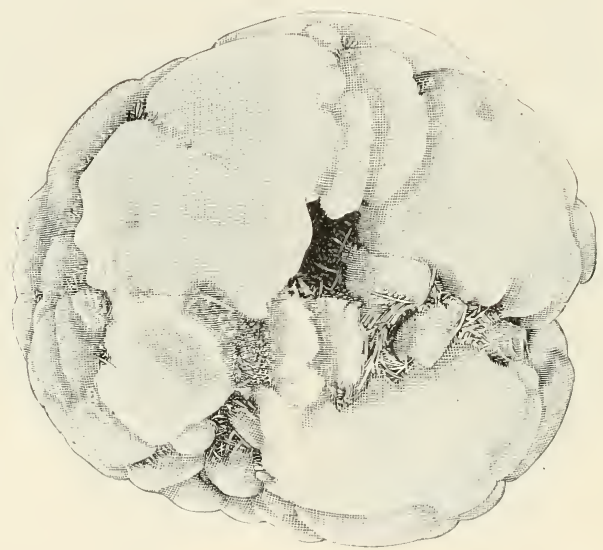

Fig. 39 .

Vegetable concretion from the colon of a horse.

health of the animal. In the dog, more rarely in the cat, bone fragments in the fecal material, forming in hard, dirty brown to black sausage-shaped masses, and perhaps reaching the thickness of the human arm, are quite likely to block up the rectum and colon and catuse erosion and necrotic ulcers of the mucous membrane, obstructing all the rest of the intestinal contents, and producing fecal fistulæ and rupture of the intestine. In horses concretions made up of vegetable fibres are often formed in the cæcum and colon, compressed into ball-like clumps, sometimes attaining the size of a human head, and more or less encrusted with mineral matter (phytoconcrements, mixed concrements). 
In the parts of the large intestine just mentioned there are also found not infrequently hard, stony concretions (intestinal calculi, enteroliths), ranging in weight up to eleven kilograms, looking not unlike billiard balls or bowling balls, or sometimes of pyramiclal shape from being worn off on the sides. The main constituent of these dense calculi is, according to Fürstenberg and (iurlt, ammoniomagnesium phosphate (over ninety per cent.), the precipitation of which occurs especially in feeding wheat and rye bran (the horses belonging to millers and bakers), such a food containing a large amount of magnesium phosphate. This is dissolved in the acid intestinal juice and in case of the development of anmoniacal compounds unites with the ammonia to form the almost insoluble triple

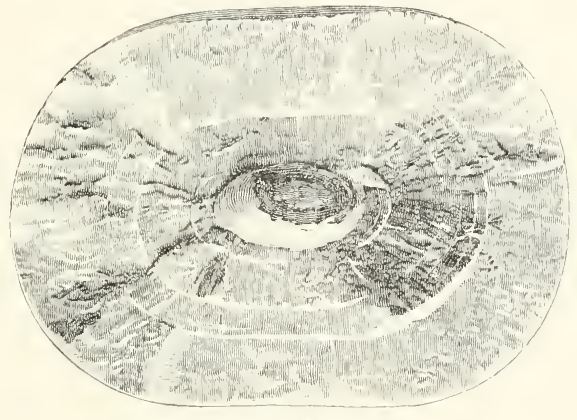

Fig. 40.

IIalf of a fractured lamellated enterolith from a horse.

phosphate. The marked sluggishness of peristalsis which obtains with this diet, and ammoniacal fermentation of the intestinal contents by bacteria, aid in the formation of the calculi. Usually the larger examples are single: but of the smaller sizes there may be dozens or hundreds in one intestine. In addition to pressure erosions caused in the nucous membrane by these calculi, they may produce fatal results, especially by accirlental impaction and obstruction at some narrow part of the intestinal tube. (For details cf. Spericlle pathol. Anatomic d. Hausticre, II. Aufl. 1902, Stuttgart, Enke's Verlag.) Horses which take in sand and mud in drinking from pools (and the same is true of hogs from swallowing ground while rooting) occasionally get large masses of such material into the intestine and may as a result suffer from the formation of diverticula. 
In pigs bristles which are swallowed may be massed together in the intestine by the peristaltic movements into cylindrical bristle balls; in ruminants hair which has been swallowed may form in the abomasum one or more spherical, felt-like bunches, and in the same way remnants of plant fibres may also be compressed into similar balls here (piliconcrements, phytoconcrements, bezoars).

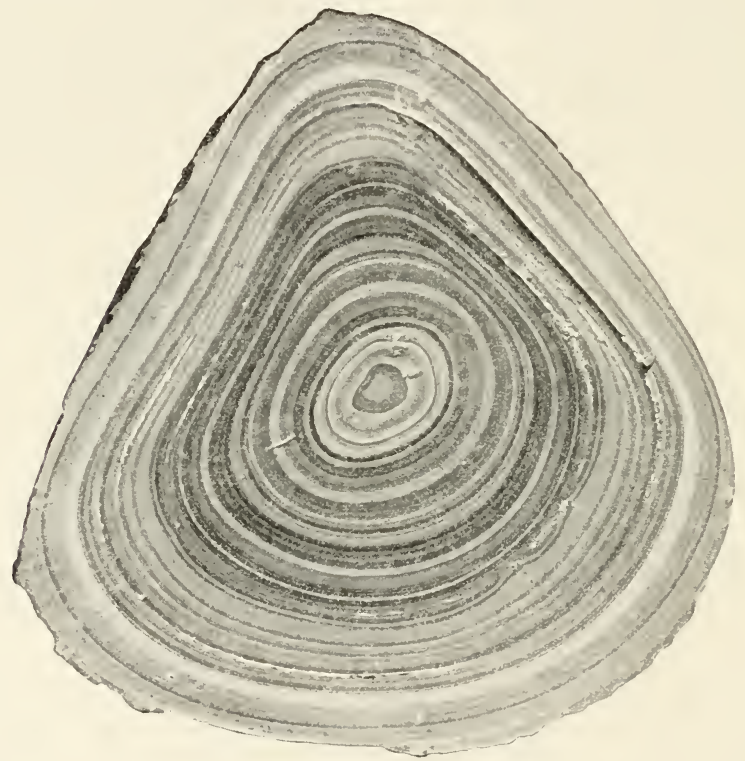

Fig. 41.

Cut surface of half of an enterolith from horse (one-half reduced).

Pus concretions result from the inspissation of pus retained in old abscesses and other places, possibly with more or less calcification. In the air chambers of horses they are met quite commonly, supposed to be due to the compression of thick streptococcus pus by muscular pressure (movements of chewing and swallowing), often into chestnut-like or potato-like masses. In cattle tuberculous caseated masses in bronchiectatic cavities may form hard lumps, perhaps reaching the size of a fist. 


\section{Gout.}

This name is applied to a disturbance of metabolism characterized by accumulation and deposition of urates of the alkalies in the tissues, the joints being special seats of uratic deposits, and developing painful nodular swellings (gouty nodes; tophi, from $\tau 6 \phi$ os, cretaceous stone). In man this is a common affection, but a rarity among mammalian animals. Only one case is on record (Bruckmüller) in a hunting dog. (In two published cases in swine [Virchow, Mendelsohn] there was not a deposi-

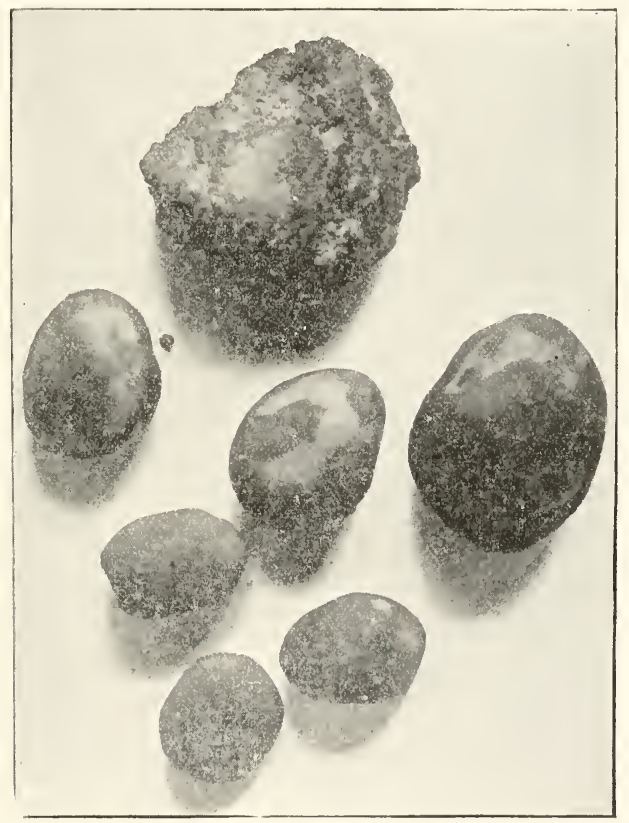

Fig. 42.

Pus concretions from an air chamber of hotse (reduced one-half).

tion of urates, but it would seem that the whitish, chalky masses found in the joints were composed of guanin, or, according to Voit, of tyrosin.) Genuine gout, however, is common in pigeons, chickens, parrots, ostriches, and is quite frequent in birds of prey (Kionka); it has been observed, too, in reptiles (alligators, snakes).

The symptoms of avian gout manifest themselves by progressive weakness of the bird, with loss of appetite and emaciation. The legs present 
more or less prominent arthritic swelling and are unfit either for standing or walking. Sometimes there is also a swelling of the wing joints, and in case of chickens, a strong bluish-black discoloration of the comb becomes apparent. At autopsy of birds dead from the affection after a course of some weeks or months, there is at times found within and about the joints a deposit of white, chalky, mortar-like material; and the serous membranes (membranes of thoracic and peritoneal air chambers and pericardium), the epicardium and the liver are apt to be found the seat of similar deposits, as though powdered with plaster of Paris. The kidneys are thickly beset with white points and sometimes the ureter is completely filled with white plugs. Microscopic examination shows the condition to consist of a deposit of and infiltration with crystals of sodium urate (plates and needle-shaped crystals grouped in stellate bunches).

As far as the causative conditions and nature of the metabolic disturbances, fundamental to the affection, are concerned, but little has been definitely established in case of human beings. Practically all that is known is that certain kinds of food (large amounts of albuminates, lighly seasoned meats [meats rich in nucleo-proteids], alcohol), lead-poisoning and a predisposition of the individual play a part. It has been established in case of avian gout, by the highly interesting investigations of Ebstein, Kionka and Kossa, that a number of poisons which injure the renal tissue and, too, exclusive meat diet are capable of inducing in experimental cases the characteristic features of gout. As early as 1766 Galvani pointed out that chickens became gouty after ligature of both ureters, an observation which has been repeated by Zaleski, Colasanti, Schröder and others. Ebstein injected subcutaneously in chickens neutral chromate of potassium in separate doses of 0.32 gram, with the idea of impairing the excretion of uric acid by producing serious renal lesions; with this dosage the birds lived for some weeks, and, as a matter of fact, Ebstein established that they undoubtedly became gouty. The same results were obtained by v. Kossa in chickens by intramuscular injections of solutions of oxalic acid, phenol, acetone, aloin, corrosive sublimate and, too, cane sugar. Kionka drew attention to the fact that carnivorous birds are the ones which are especially subject to gout, and from a series of studies established the fact that chickens on exclusive meat diet (I60 grams a day), to which they may become accustomed, may become gouty in the course of a few weeks or several months and then die of the affection. The large, heavy breeds showed greater tendency in this direction than the commoner small chickens. There were noted a great increase in the production of uric acid with this diet and a correspondingly high discharge of uric acid (8-9.5 grams each day) and of nitrogen (3-4-5 grams a day) in the excreta. Since M. Kochmann has shown that in dogs, also, when fed exclusively with horse flesh, the renal and hepatic parenchyma suffers a change (parenchymatous nephritis and hepatitis), and the poisons alove mentioned give similar results, v. Kossa looks upon the nephritis as the cause of the toxic gout interfering with the elinination of urates just as if the ureters were ligated. Under natural conditions almost every chronic nephritis in birds may be followed by gout. Zürn once observed the affection after feeding ustilago maidis to chickens. The common 
occurrence of the disease in birds of prey kept in captivity and having insufficient exercise, favors the view that muscular activity lowers the disposition, or, in other words, uric acid is destroyed in the working muscles. The difference, not merely in diet, but in the possibility of destruction of uric acid in the muscles, liver, kidneys and spleen are probably reasons for the rare occurrence of gout in mammals and the fact that it is never seen in herbivora.

[In man the affection, cliaracterized not only by the joint changes, but also by widespread sclerotic lesions, especially of the arterial system, is reforred to uric acid and certain precursors of this substance, the purin bodies. These are particularly the products of metabolism of nucleoalbumens, and much importance is ascribed to diet rich in these albuminates, young meats; pancreas, liver, etc. Other factors, as insufficient exercise, etc., are also deemed important.] 


\section{PROCESSES OF REPAIR AND NEW FORMATION}

\section{Regeneration.}

By the term regeneration is meant a process of new formation of tissue which occurs in connection with tissue loss, replacing such loss and then ceasing.

Renewal by metabolism of the functional capability of cells remaining alive after fatigue and exhausting diseases is known as recozery or reconstitution. In the living organism cellular material is being continually used up and in consequence there are always some cells being destroyed and new ones growing to take their place. The cellular covering of those surfaces which are in relation with the external world and from which the cells are loosened by mechanical influences (epithelium of the skin and mucous membranes), is especially subject to continual losses and compensation for such losses by a succession of new cells. In the glands, too, there is apparently a continual change of old and new cells, as the function of the gland cells cannot but occasion in them aging and death; this exchange is an active one in the tissues of the testicles during the period of seminal production, and in the ovary, too, the formation of ova is in a measure a regenerative production. In some glands which form their secretions without particular destruction of their cells (mammary glands, kidneys, sweat glands), renewal in this sense is, however, not recognizable; and in case of the important elements of the central nervous system, the ganglionic cells, it is a question whether they are at all subject to change or persist throughout the whole life of the individual.

The cellular constituents of the blood and lymph are very perishable, and in the spleen, bone marrow and lymph nodes compensatory production of these corpuscles is unceasingly going on.

A regenerative process may be regarded as pathological only in the sense that it occurs in connection with unusual losses of substance or pathological lesions of tissues, as after ruptures in continuity; it is in reality only an exacerbation of physiological histogenesis as the processes of growth in both are identical. 
The causes, or better, the reasons why, after tissue injuries and losses, regenerative growth occurs, are complex. Normal increase of cells depends upon inherent peculiarities of the cells themselves. The capacity for multiplication is characteristic of all cells for a certain time after they are formed; it is most active during embryonic life and in youth, diminishing and, within certain limits, ceasing after the full development of the body, as determined by the relative tension of the tissues and the inherited characteristics (phylogenetic). From those influences exerted by the tissues upon each other and by which mutual restraint is maintained so that an overgrowth of one cannot take place at the expense of the rest, the normal inter-resistance of tissues, the so-called tissue tension, ${ }^{*}$ takes its significance. (However, as Marchand has suggested, mechanical resistance to growth does not alone explain tissue equilibrium; there are other participating influences, as nutrition. The constancy of the shape and size of the organs and the cells is a fundamental peculiarity of the species.)

TVe may see in many cases where renoval of normal tissue resistance has taken place (where, for example, the tissue tension has been released by the formation of cavities) the parts bordcring on such situations assume active growth. This is very strikingly apparent where there is a cleft in the diaphragm next to the liver, as the result of which the liver tissue invariably grows through the round or oval diaphragmatic opening into the chest cavity (as a button goes through a buttonhole), the otherwise uniform opposition which the diaphragm exerts upon the anterior surface of the liver being interrupted at this position and lost. In any defect produced by trauma or analogous loss of continuity of the tissues the normal resistance is more or less lowered and the adjacent cells are able to penetrate into the vacant space and fill it (vacuum growth). As a result of the displacement of the cells thus penetrating the vacant area from their original station, space is afforded in the latter position as well, thus giving opportunity for further proliferation of the elements persisting there (Ribbert). According to Ribbert the displacement of cells even from distant positions may be explained by such a release of tension: thus in the regeneration of blood, leucocytes pass into the circulation and spaces are left in the bone marrow, which are filled by newly forming cells, and with the passage of these the process repeats itself indefi-

\footnotetext{
* Ribbert means by tissue tension not only pressure conditions, but all the opposing influences of the various tissues upon each other.
} 
nitely. Anything, even hyperemia, which tends to separate the tissues, has in Ribbert's opinion the same effect to decrease tension and produce space. Although objection may be made that the distending cause must also have a pressure effect which should interfere with growth, it must be granted that the vessels distended and engorged in hyperæmic conditions separate from each other the closely packed cells, and that similarly the filling up of the lymph spaces with exuded material forces the cells apart and in this sense has an influence to relieve tension. The release of mechanical tension is, however, not invariably the only cause of tissue growtli; there are other factors which are operative to directly stimulate cellular proliferation. There can be no doubt that increase of nutrition, provided the cells are in condition to assimilate it, must necessarily favor multiplication. Especially in inflammatory regeneration it may be appreciated that there is such a rich nutritive supply possible for the cells at the periphery of the inflammatory focus from the albuminous fluid exudates, fibrin and products of disintegration of leucocytes and blood coagula, that there cannot but coexist a condition of extra nutrition of these elements along with relaxation of and space formation in the tissue. The multiplication of tissue in chronic inflammations, protracted hyperemias and lymph congestions may also be partly explained by this idea.

In addition it is possible that the clevation of temperature attending inflammatory processes may play a part, as it is well known that warmth is an important stimulus to cellular activity and therefore to cellulai proliferation. Chemical agcuts should also be included among the important factors of stimulation of cell multiplication. Just as the mobile leucocytes are attracted by nutritive substances and other materials and assume a phagocrtic activity, the fixed cells respond to the chemotactic stimulus of nutrient matter. They do not merely manifest evidence of phagocytic action which underlies their hypernutrition and consequent cell division, but there may be distinctly recognized, as in the regeneration of nerves, that there exists some special substance exerting attraction, which determines the direction of growth of the proliferating cells. Fibrin especially seems to exert this twofold influence; in most cases where a layer of fibrin overlies the tissue, the latter is apt to soon proliferate into the fibrin (Marchand). The substances set free by the destruction of lencocytes are apparently capable of exerting a chemical stimulus 
in this sense. According to Marchand many cells have a tendency to adhere to their support with as broad a base as possible until forced off by other cells of the same type, as may be seen in the way young connective tissue cells force their way into the smallest cellulose meshes of elder pith, necessarily passing through its narrow pores and changing their shape to marked degrees. This tactile stimulus perhaps explains the tendency of connective tissue corpuscles to apply themselves about foreign bodies, fibrin fibrils and analogous objects.

In most cases the causes enumerated are combined to give origin to the stimulus for cellular proliferation ; for example, where granulation tissue grows up and out of a wound in a luxurious manner instead of ceasing to grow after filling the wound and then cicatrizing, the condition may be ascribed to the combined influence of a number of the conditions above indicated, as the absence of pressure opposition, excess of nutrition and the action of special irritants (saliva, medicaments).

Regenerative capacity is possessed by the cells and tissues of animals in varying degree. In invertebrates and the lower vertebrates the power of replacement or regeneration of important parts after loss is common to all the tissues. Both pieces of a divided earthworm grow into perfect creatures: salamanders and lizards can reproduce completely the loss of a tail, with bones, muscles, and even the portion of spinal cord belonging thereto; salamanders can form a new leg in place of one cut off, or an inferior maxilla (Samuel, Ribbert). This extensive regenerative capacity has been tentatively explained by the supposition that such low forms of animals are more commonly subjected to injuries of the type indicated and so their cells, naturally and because of their, less complicated structure, retain their power of adaptation better than those of the higher animals. In the latter the cellular construction is more complicated and more fully developed for special functional requirements, and possess a less marked tendency to divide in comparison with the simple cell forms, are less independent, and in some degree require the co-operation of other cells in their nutritive processes. The more a type of tissue or cell departs from its embryonic state and the higher differentiation it attains, the more difficulty it experiences in attempting regeneration. The epithelium of the skin and mucous membranes and connective tissue retain their power of adjustment in response to the numerous faults to which they are liable, and easily and quickly regenerate in the higher as well as the lower forms of life; but the ganglionic 
nervous cells and striated muscle, which can only exist, from a functional standpoint, in connection with nerves and which are of a decidedly complex structure, and in the same way glandular cells, regenerate only under special circumstances or not at all.

[The conditions, then, which underlie cellular regeneration may be summarized somewhat as follows: Only cells which from the simplicity of their structure and function or from the fact that they have not advanced far from their embryonic state are likely to multiply with any comparative ease. Any cell must be provided with sufficient nutrition to maintain its cellular activity and life if it be expected to divide, and extra n11trition as met in hyperæmia favors multiplication. Cells capable of division cannot, however, proliferate if there be important mechanical or physiological opposition exerted by the other cells and structures of the body, and release of such mutual tension (mechanical, nervous, chemical) must be afforded before proliferation is possible. But in addition a definite stimulus to division should exist. This stimulus may be of chemical nature, perhaps afforded by special chemical substances coming to the part from previous cellular disintegration (here the brilliant results of Jacques Loeb in producing multiplication and development of unfertilized ova of sea-urchins by immersion in special chemical solutions are strongly corroborative), electrical (here, too, the work of Loeb and his associates is illuminating), thermic or mechanical.]

In the adult organism of animals and birds the somatic cells reproduce in their process of division and multiplication only cells of their own type (omnis cellula e cellula ejusdem gencris); in other words, a newly formed tissue is invariably the product of tissue of the same kind. It was formerly believed the various types of cells could in their multiplication produce opposite types and that in the same way as the various organs arise by their differentiation from the three embryonic layers similar transformations of tissue conld take place in the restoration of post embryonic defects. A certain capacity for transformation (metaplasia) is possible in related types of cells and their matrix products. Thus the different varieties of epithelial cells may be changed by external influences (pressure); and within the group of connective tissues transformations occur, as the formation of bone and cartilage from fibrous connective tissue. But connective tissue is never changed into epithelium, or the latter into connective tissue, the four distinct groups of primary tissues (epithelium, connective tissue, nervous tissue and muscle) never interchanging. 
The nature and method of cellular proliferation, as far as nuclear and cellular division is concerned, is in general the same as in normal life, although there are pathological irregularities met in the chromatin network and detailed structure of the protoplasm. The replacement of lost tissue originates from the cells which happen to remain unchanged at the site of the defect or exist immediately about it. If there are elements present in such a position, of the same kind as those lost and capable of multiplication, their proliferation may completely restore the fault, the area of loss being filled up with identically similar elements with the same functional abilities (simple or complete rescneration). Should the proper cells be absent from the vicinity of the faulty area regeneration will be incomplete (false), and the space will be occupied by cicatricial tissue derived from the connective tissue structures everywhere present.

Regeneration of Connective Tissue and Bloodvessels. The connective tissue framework is the tissue which takes the principal part in repair in lesions of any kind, whether traumatic lesions of continuity or necrobiotic destruction of the parenchyma of organs; usually the first elements to multiply being the connective tissue cells, these taking the place of the various elements incapable of regeneration. For this purpose their power of movement and of taking up other cells and all sorts of disintegration products makes them of value to the organism in the removal of substance which has become nseless, and in the construction of a protective wall about foreign bodies. The greater part of the connective tissue seen in sections is made up of minute narrow spindle cells, almost completely occupied by dark elongated nuclei, and intervening bundles of fibrils. In case of proliferation of this tissue these spindle shaped elements multiply, the nuclei undergoing mitotic division and the protoplasm increasing in volume. The newly formed connective tissue corpuscles are thicker and polymorphous, sometimes rounded or polyhedral, and provided with a number of processes; they usually lie closely sitated, often in rows one after another, and show their active part in the proliferation by the presence of mitotic nuclear figures (one cell sometimes containing two or three nuclei). As they approach the type of embryonic mesoblastic cells and represent the young germinal elements of connective tissue they are

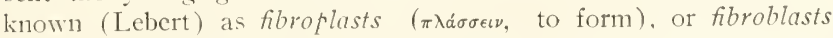

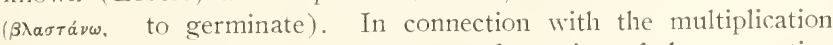
of these cells there also occurs a new formation of the connective 
tissue fibrils, the processes of the fibroblasts splitting up into fine threads, at first in a brush like fashion, the protoplasm of the cell gradually changing completely into a bundle of parallel fibrils (Dürk, Neumann, Ziegler). Thus by the splitting of the enlarged cell-body into fibrils the cell comes to resume the type of the fixed connective tissue corpuscles, and the mass of proliferated fibroplastic tissue assumes the character of adult connective tissue structure. In addition to the fibrils the protoplasm of the connective tissue cells gives off a greater or less amount of a soft mucoid intercellular substance (Marchand). The young fibroblasts are con-

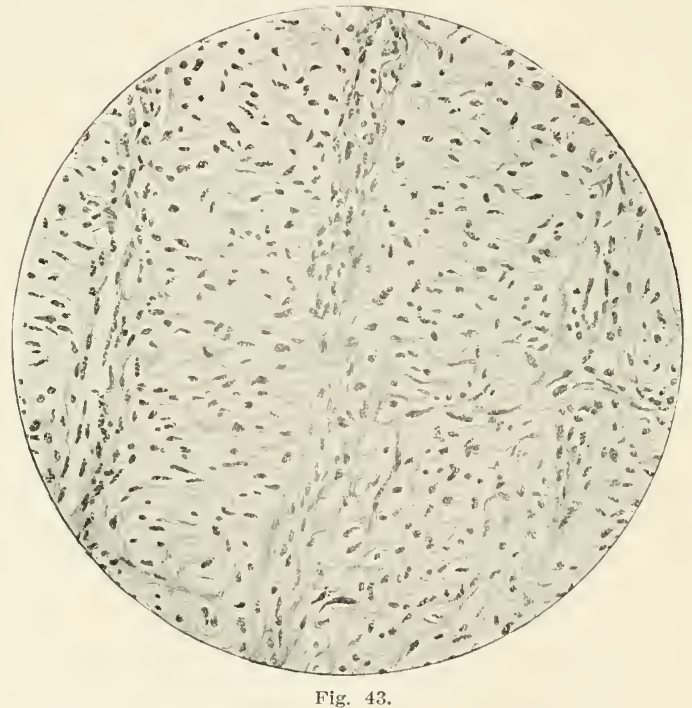

Fibroblasts and angioplasts in granulation tissue of herse.

tractile and consequently are inconstant in form; they can project processes of their substance and thus can surround other cells, shreds of tissues and nutrient substances and incorporate them like phagocytes. They can also change their position, this property explaining the fact already mentioned that foci of disintegration, areas of hæmorrhage and situations of other types of lesions are penetrated and occupied, and foreign bodies are encapsulated by these elements. Their motile power is so marked that if, for ex- 
ample, a bit of sponge or fragment of elder pith be introduced into a tissue or serous cavity it becomes completely penetrated by these fibroblasts.

Along with the connective tissue cells proper, the endothelial cells of the blood and lymphatic vessels take part in the new formation of connective tissue. In both forms of cells mitosis prevails profusely; the young endothelial elements resulting from the cell division are like the other fibroblasts, being distinguishable from those derived from the spindle cells only at their places of origin from the blood vessels, where they are forming capillary tubes. Whether endothelial cells ever form fibrils or solely develop into vessels is not fully determined (for details consult Marchand). Another long debated question, whether the lencocytes actually take part in the connective tissue formation and are transformed into connective tissue cells, may be answered on the basis of more recent studies (Marchand) that the white corpuscles which exude from the blood vessels are principally concemed in the removal of foreign matter and products of disintegration, that they furnish nutritive substance in case of their own destruction, but are incapable of forming intercellular material and therefore cannot be considered as actual constructive elements of the connective tissue. The presence of these leucocytes in every area of proliferating connective tissue is therefore dependent upon their entrance partly from the vessels, partly from points of lymphoid development to be found commonly along the bloodressels in any tissue. The whole cellular group more or less closely approaching leucocytes and cells from the lymph glands (lymphocytes), but because of their more conspicuous cytoplasm or a variety of granulations of their protoplasm given other names (plasma cclls, mast cells, leucocytoid cclls, polyblasts, klasmatocytes), are also found in the growing connective tissue. The origin of these cells and the question of their relationship is still rather uncertain; probably they are all immigrants originating from lymphocyte depots. Giant cclls (polykaryocytes) are also often observed in developing connective tissue, and multinucleated cells, especially when foreign bodies are present: they are formed partly from the multiplying spindle cells, partly from endothelial cells, and may be regarded as cells whose protoplasm has increased and whose nuclei have multiplied more rapidly than cell division could keep pace (proliferation giant cells). According to another view they are caused by coalescence of closely grouped embryonic cells (conglutination giant cells).

Opinions concerning the mode of origin of elastic fibres in the re- 
generation of connective tissue are not fully formulated ; their formation is, however, a slow process. It is only after four or six weeks or after several months after repair by the fibroplastic tissue that elastic fibrils appear in the intercellular substance; and even after years these do not attain the diameter of the original elastic elements.

Connective tissue, modified to form adipose tissue by fatty infiltration, is capable of proliferation to the same extent as ordinary connective tissue cells. The nucleated protoplasmic remnant of the fat cell undergoes division, and the new cells usually at first accumulate in the fat vacuole within the old cell membrane.

Formation of blood iesscls takes place by a process of budding and division of the endothelial cells from the existing capillaries. The protoplasm of the endothelial cells in the walls of the old capillaries which have remained intact send out pointed, conical processes, which lengthen out in thread like manner, and penetrate

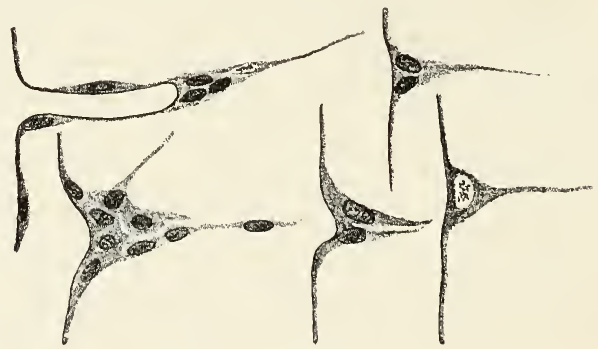

Fig. 44

Vascular sprouts, highly magnified; (after Thoma). The long thread-like ends of the sprouts are only partly reproduced.

between the proliferating fibroblasts. Coincidently mitosis of these endothelial cells takes place and at the base of the bud cellular division begins and the endothelial cells (angioplasts) proliferate. These new elements lie alongside of each other and fuse, with their often curved and reticular branches, into solid cords of cells. From these the capillary tubes are produced by the forcible penetration of the blood from the old vessel in between the dividing endothelial elements, thus making a space filled with blood. This proceeding at all places where these buds are forming, gradually the whole network becomes canalized. From some of the capillaries arteries are formed, from others veins, the lumen of the tubes 
being widened by blood pressure and growth of the wall (increase of surface by multiplication of the parietal endothelial cells); smooth muscle growing from the existing arteries into the newly formed vessels, and the surrounding fibroplastic tissue aiding in the formation of elastic fibres. (Regeneration of blood vessels has been especially studied by J. Arnold, Jos. Meyer, Billroth, C. Weil and Thoma.)

The development of angio-fibroplastic tissue is accompanied by more or less manifest phenomena of inflammation (hyperæmia. exudation of lencocytes) and is therefore in a sense a result of productive inflammation. Depending upon the number of blood ressels present and the relative anount of proliferated connective tissue the new structure presents a reddish-gray, grayish-white or pure white, swollen appearance, semitransparent and of a shape conforming to that of the lesion which it has followed. When it develops diffusely; as in parenchymatous organs, to replace destroyed glandular tissue, it gives rise to more or less marked induration of the part (indurative inflanmation), or forms translucent hard thickenings (in fascix, serous membranes), bands of adhesion (adhesire inflammation) and sometimes villus-like fringes. In focal development it forms white trabecular or nodular connective tissue masses (in infarcts of the kidney); as the result of a demarcating inflammation about some blood clot or foreign body it constitutes a connective tissue capsule. On the free surfaces of wounds the new formation is seen as a finely granular soft tissue, producing an exudate, with a deep red or reddish gray color from the abundance of capillary buds in its structure: it is known as granulation-tissue.

All fibro-angioplastic tissue is at first likely to form a greater bulk than the lesion which it replaces. There exists an excessive production of the young cells and these cause an excess of intercellular material. With restoration of normal tissue tension and maturation of the growing tissue, these originally enlarged cells shrink and the intercellular substance decreases; the fibrils are arranged in a parallel fashion from the tension and pressure of the neighboring structures infuencing them; some of the capillaries become obliterated, that is, narrowed, from pressure by the shrinking fibroplastic tissue. In this way, too, the whole tissue, sometimes, because elastic tisste is produced in deficient amount or not at all, becomes dense and hard and is changed into cicatricial tissue. This is apt to contract still further, thus forming in the surface of the skin and organs depressions or indentations of radiating stellate form. 
Regeneration of Epiderm and Epithelium of Mucous Membranes.-The epithelium on the surface of the skin and mucous membranes is capable of very rapid and complete regeneration. Because of the continual loss of cells from desquamation as they grow old the tissue is always prepared for and actively engaged in regeneration. Either because of cellular tactile irritability (v. p. 235) or because the removal of tissue resistance in defects mechanically causes it to undergo a regressive development, the new tissue is very apt to spread out upon the free surfaces and to some extent to increase in volume, a flat cell thus arising from a cylindrical cell at the border of the lesion. The new cells are always formed from existing epithelium and can only be produced providing vitally capable cells of the same tissue are present in the vicinity of the lesion.

The formerly accepted belief that epithelial cells could develop from connective tissue has been shown to be erroneous.

In case of superficial loss of epithelium (abrasions, epithelial necroses) the new surface cells grow over the denuded papillie from the margin of the surrounding epiderm. Within three-quarters of an hour after a wound has been inflicted, under the microscope there may be observed enlargement of nuclei and direct and indirect nuclear division in the cells of the stratum Malpighii, both in the margins of the lesion and at some distance from it: after eight or ten hours the epidermal border is visibly thickened; and small lesions only a few millimeters in circumference may be covered by several layers of epithelium in the course of forty-eight hours. The epithelium actually spreads over the surface of the wound, either from active motile power or because the superficial cells are dislodged by those growing back of them in the deeper layers. If the part deprived of epithelium is covered by fibrin, blood or pus, a granulating surface, the proliferating epithelium at the margin forces its way beneath the coagulated crust over the cicatrizing connective tissue; in these cases the epithelial layer is often riddled with leucocytes, and afterward in the clefts and spaces thus produced new cells may grow, forming branched plugs made up of concentrically laminated, keratosed, epidermal balls (horny pearls, atypical epithelial proliferation of Friedländer). Occasionally the epithelium may penetrate along fistulous passages; as in a perforating dental alveolar fistula (horse) the squamous epithelium may grow through the alveolus from the mouth into the nasal cavity.*

* Penetrative growth of epithelium at the border of ulcers and fistulæe may determine a free atypical new formation of this tissue and thus give rise to cancer. 
The advancing margin of the epiderm may be seen as a bluish white way zone, sometimes with a rosy tint from the hyperemic substructures showing through. Sometimes in addition to the epidermal growth at the margins of the lesion, islands of epithelium may be observed in the midst of the new surface. These may originate from the remnants of the ducts of sweat glands or hair follicles (the cells of which are practically identical with those of the surface) which were left intact because of the superficial extent of the loss of substance; or may arise from epitheliai cells separated from the border and caused to adhere to the surface of the wound by the application of a bandage.

The proliferation of the epithelium does not stop until the free surface of the lesion is completely covered. Epithelial scars are for a long time devoid of pigment and in a dark skin the cicatrix is conspicuous because of its whiteness.

The corneal cpithelium is very quickly reformed after destruction, a circular loss of three millimeters diameter being perhaps replaced in the course of from thirty-six to forty-eight hours. The cells at the margin, moving in by amoboid power and flattening out to form at first a protective single celled layer, spread out over the surface; these multiply, showing mitotic figures and multinuclear examples, and build up the layers of corneal epithelium; the cells assume a cuboidal shape and the lesion is completely repaired. In case of wounds of deeper extent or those perforating the whole thickness of the cornea and involving the corneal connective tissue and Descemet's membrane, the lesion is at first filled up by fibrin (from the aqueous humor). The rapidly growing epithelium extends over this and forces itself into the depth of the wound like a plug. The connective tissue is formed subsequently, and on the posterior surface the endothelium of Descemet's membrane regenerates its single layer of cubical cells. A milk white spot remains at the site, the result of cicatricial production by the newly formed connective tissue.

(Regeneration of the cornea has been exhaustively studied, especially from an experimental standpoint, in animals; for details cf. Natchand.)

Regencration of hair can occur only in case the hair follicles or portions of them remain. In all deep cutaneous lesions the cicatrix is permanently smooth and devoid of hair.

Regeneration of hormy tissue of the hoof. After injuries to the keratous envelope or after it has been ruptured from suppurations of the corium the horny layer may be regenerated by proliferation 
of the keratogenous cells over the surface of the granulating papillæ and fleshy laminæ of the persisting cutis (the latter the seat of compensatory and sometimes regenerative changes) and the formation of a new protective layer over the denuded papillary surface (Gutenäcker). The first formed cells are pushed forward by succeeding new growth and form keratous tubules by arranging themselves in circles about the papillae; later interpapillary keratous substance is deposited in the intervals between the tubules as a yellow, perhaps soft and incompletely keratosed, and usually very thick mass (cicatricial keratin). In parts where there are no papillæ the keratogenous cells are arranged in irregular, superimposed layers. Operative denudations of the corium of the hoof from five to ten square centimeters in area may be completely overgrown by new horny substance in the course of from four to six weeks.

(For details cf. Lchrb. d. Chirurgic, by Fröhner, and Hufkrankheiten, by Gutenäcker.)

Regeneration, compensatory for losses of cylindrical cpithelium upon mucous membranes, proceeds in the same way as the process in the epiderm. The epithelial cells growing in from the periphery over the surface of the lesion at first assume flat, then cubical and finally cylindrical shapes and in appropriate cells it is even possible that the ciliated border will be reformed. The surface epithelium of a wound of the gastric mucous membrane of a dog may be completely reformed in from four to ten days.

Regeneration of Glands.-Epithelium of glands is very irregular in its regenerative ability. The epithelium of glands of the mucous membranes corresponds histologically with that of the surface of the membrane, as in the intestine or uterus; and therefore in these parts epithelial repair over cicatrices, ulcers and wounds may, besides merely covering the surface of the lesion, sometimes also apparently attempt gland formation in points of depression. The epithelial cells formed in such cases grow from remnants of involved glandular structures or from the uninvolved adjacent tissue over the seat of the lesion. The depressions occur at the expense of the young growing connective tissue or granulation tissue which is being developed at the same time; but the more marked the retraction of the scar the less successful the glandular reformation. Ponfick, Gluck and v. Meister in their interesting experiments have pointed out in connection with regeneration of the liver that after operative removal of a portion of the hepatic tissue from rabbits, rats and dogs a remarkable regenerative activity 
occurs. After ablation of half of the bulk of the organ new liver tissue developed from the remaining portion by luxuriant proliferation of the hepatic cells, setting in as early as the third to the fifth day; after an average of from forty to sixty days the part lost was completely restored in volume and weight. It is conceivable that after other local lesions liver tissue may regenerate, yet after traumatic lesions or destruction of hepatic parenchyma by the penetration of parasites nothing but cicatricial connective tissue is seen at the site of the defects, always containing on microscopic examination decidedly proliferated bile ducts, but without evidence of formation of functionally capable liver cells. This probably is due to the fact that in the type of defects under consideration the connective tissue framework has also undergone pathological changes, and perfect restoration of functionating parenchymatous cells can only be expected when the connective tissue basic structures are in nomnal condition (Ribbert). The enormous enlargement of the liver sometimes seen in tuberculosis and echinococcus disease is apparently due not merely to the foreign elements and to connective tissue hypertrophy, but may possibly be dependent upon a vicarious hypertrophy or regeneration of the hepatic parenchyma.

It is not certain in case of the kidncy whether epithelial cells lost in consequence of inflammations and degenerations of the parenchyma can be restored completely. Lesions from infarction, suppuration or trauma always heal by cicatrization. There may, however, take place a compensatory hypertrophy of adjacent tubules and glomeruli to occupy the space caused by shrinkage of such scars, which in a measure may compensate for the lesion.

The tissue of the testicles and ovaries has but little regenerative power; space caused by trauma and degeneration is always filled in by cicatricial tissue. On the other hand the glandular epithelium of the mammary gland, thyroid, salivary and lachrymal glands is capable of extensive proliferation from remaining portions of the alveolar tissue, with formation of new ducts and glandular acini lined with secreting epithelium.

The spleen and lymph nodes although subject to continual change of their cellular constituents and capable of originating in pathological conditions a marked excess of their lymphoid tissue, do not regenerate after traumatic lesions, being invariably subject to cicatricial repair.

Striated muscle, when the fibres have been partially destroyed by degeneration of their substance, is able to regenerate in a certain 
measure; at least microscopically proliferation of remnants of nucleated sarcoplasm may be observed, whole chains or rows of new nuclei being produced by direct nuclear division, these in association with proliferation of the sarcoplasm by longitudinal division going to form the so-called muscle cylinders (Waldeyer) and lateral off-shoots known as muscle buds (Naumann). Apparently the sheath of the sarcolemma develops from the surrounding connective tissue, which is also proliferating; and the developing sarcoplasm takes on a fibrillar structure. In traumatic lesions connective tissue formation predominates, the healing of the wound usually giving rise not to the reconstruction of the contractile elements, but to the formation of dense scars.

Smooth muscle even in traumatic lesions exhibits active mitosis, and multiplication of the muscle of the blood ressel walls is to be observed in granulating tissue; but in lesions of muscular tunics, as of the stomach, intestine or uterus, complete restitutio ad integrum has never been observed, scar formation taking place instead.

Regeneration of tendon tissue (studies of this process being often afforded in the rather common performance of tenotomy) begins, according to Marchand, principally from the loose cellular tissue between the bundles of tendon fibrils. This formative material, composed principally of spindle cells, develops (by mitosis) into the cleft between the cut ends of the tendon, which is filled up with blood or exudate, and unites the tendon stumps as a soft grayish fibroplastic mass. Later the fibroblasts form a fibrillar matrix. the fibrils arranged parallel with each other, and the tendon scar gradually acquires great firmness. According to Paget a divided tendon of Achilles of a rabbit may be found united as early as from three to five days and in ten days may be of sufficient strength to require a tensile force of twenty-eight pounds to tear it apart.

Iesions of cartilage, as that of the air passages, the auricle, or ribs, are restored by connective tissue formation from the perichondrium, the cells of which give origin to a fibrillar, collagenons substance. The latter may become homogeneous and by a metaplastic process the connective tissue thickening be converted into hyaline cartilage; and it is possible that by an infiltration of calcareous salts ossification of the embryonic tissue occupying the space of the lesion may ensue. The cartilage itself takes no part in the process of restoration and connective tissue scar formation is usually but slight.

New formation of osseous tissue after fractures and operative lesions arises exclusively from the periosteum and bone marrow 
and ends by complete restitution of the most dense osseous substance. The special bone cells embedded in a dense solid calcified matrix are unable to take part in the construction of new tissue because they have no space for any activity. The osteoblasts of the periosteum and marrow, the bone forming cells, are connective tissue cells which possess the peculiar ability of giving origin to an intercellular material with an affinity for lime salts or of themselves producing calcification of the matrix and thus supplying the basic osseous material. They are, however, also capable of producing fibrillar connective tissue and may act precisely as fibroblasts; and finally may become transformed into cartilage cells, form a homogeneous inter-cellular material and remain in the lacunæ within the latter. In bone repair therefore there is first noted a fibroplastic callus which becomes partly changed into cartilage and later into calcified osseous tissue. Coincidently the blood vessels of the bone proliferate, complete analogy to the vascularized fibroplastic callus existing in the healing of lesions of the soft parts. The name callus is applied to the whole of the tissue arising in the osteogenetic process, its origin giving the basis for differentiating between periostcal and myelogenous callus; that portion which develops between the separated fragments is known as the intermediate callus.

In the neighborhood of the seat of fracture all of the periosteal and marrow cells and vascular endothelial cells become swollen, nuclear division actively proceeds, and cellular proliferation is so luxuriant that the capillaries are often found occluded by young endothelial cells and giving off profuse vascular buds; the whole cellular callus forming a growth decidedly exceeding the diameter of the bone itself (the wides the space between the bone fragments the greater this mass).

This callus, at first consisting of connective tissue and sometimes called the prozisional callus, has to do with the removal of the clotted blood occasioned by the fracture and other tissue remnants, even of small splinters of bone (the limy material of which is dissolved by the proliferating cells), through phagocytic cells originating from its newly forming constituents. It has a bacon-like, cartilaginous appearance, surrounds the ends of the bone as a thick girdle. occupies the medullary cavity at the level of the fracture and the whole space between the broken ends. The newly formed material is markedly in excess, and after calcification the osseous scar unites the fragments as a clumsy, thick enlargement of the shaft of more or less spindle shape. With calcification, which renders the callus 
hard like ivory and of the compactness of cortical bone, the osteoblasts in the provisional callus are gradually enclosed in the calcifying matrix and shrink into the spindle-shaped and stellate form of bone corpuscles; the newly formed vessels with their contained blood persist in the calcifying osteoplastic tissue, giving an analogy to the system of the Haversian canals of bone. There is, however, no lamellated structure in the tissue. After the osseous scar has become hard and the bone is again strong enough to sustain weight and muscular strain, a reduction of the excessive callus sets in, aplanation of the callus; the external bulging of the bone diminishes; the periosteum becomes closely adherent; the medullary callus becomes porous, the medullary canal opens and the medullary substance becomes full of fat. These changes in the callus are brought about by the growth of blood ressels into it from the marrow; and by the influence of multinucleated giant eells of the medulla known as osteoclasts ( $\kappa \lambda a \sigma \sigma \epsilon \hat{\imath}$, to break) because of their agency in breaking down osseous tissue (Rindfleisch). The shrinkage is explained by the biological law that lack of use causes structures to become atrophic, only functionating parts being maintained. Hence in conformity with this rule only those portions of the bony tissue which are required to sustain weight or to oppose strain persist as compact structures.

It is well known that the texture of any bone shows an arrangement of its osseous trabecula conforming to the mechanical forces acting upon it; this functional adaptation is operative as well as all pathological conditions which occasion new mechanical features. In the regenerated bone tissue the resumption of function not only assures the maintenance of the osseous scar, but in addition determines the precise arrangement of its osseous trabecula by further thickening of those portions which are most heavily taxed: the direction of the trabecular adjustment develops in correspondence with the lines of stress determined by their functional relations in supporting the body weight and opposing muscular strain.

Regeneration of Nerves.-Peripheral nerves severed from their connection with the central nervous system by section. contusion or other destructive lesion, degenerate from the point of the lesion centrifugally to their peripheral end fibres, their axis-cylinders and medullary sheaths being destroyed (broken down into myelin and fat droplets): but the neurolenma and its nuclei remain intact (Nasse, Waller, Lent, Benecke and others). The degeneration may also extend centrally in the divided nerve for the distance of one segment (to the first node of Ranvier). Conductivity ceases 
instantly when continuity is interrupted (provided there are no collateral paths). The space intervening between the central and peripheral portions is filled at first with fibroplastic cicatricial tissue; but later a complete regeneration of the nerve fibres is accomplished and in the course of two or more months the nervous function is fully reëstablished. This later development of the nerve fibres, as

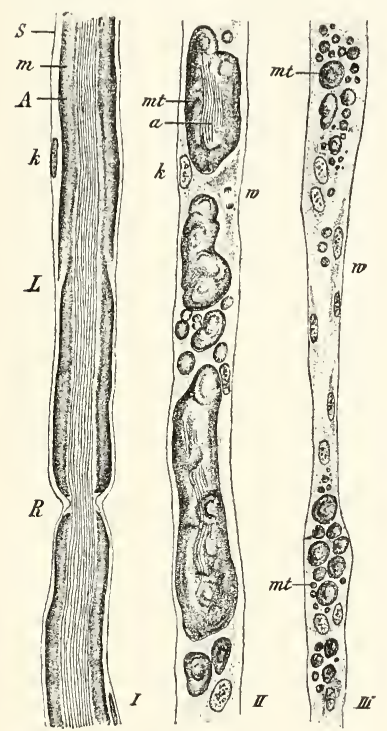

Fig. 45.

Degeneration of nerve fibres after sec. tion. 1 , normal nerve fibre. 11 and III. different degrees of destruction. s. neurolemma sheath of Schwann. $m$. medullary sheath. $A$, axis cylinder. $k$, nucleus of nemrolemma, $\dot{L}$, Lantermann's constriction of the medullary sheath. $m t$, myelin globules. $a$, remains of axis cylinder. $u$, proliferating cells of neurolemma. Partly schematic. $\times 476$. (After Thoma.)
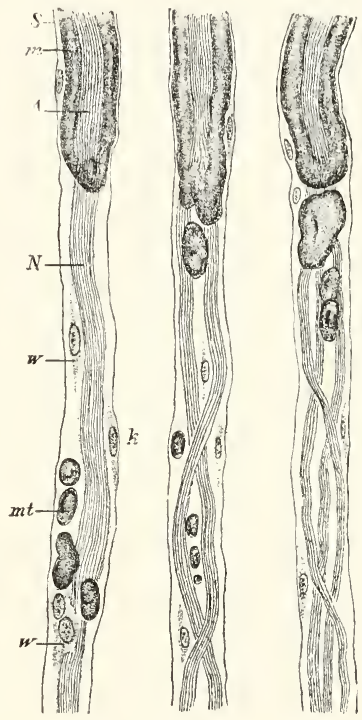

Fig. 46 .

Growths of the axis cylinder in the central stump of a divided nerve. $s$. neurolemma sheath of Schwann. $m$. medullary sheath. $t$, axis eylinder of undegenerated part of the nerve. $i$, newly formed axis cylinder. $k$, nucleus of neurolemma. $\quad w, w$, proliferated neurolemma cells, $m t$, myelin globules. schematic, based on an illustration from Eichhorst. $\times 476$. (After Thoma.)

indicated by the microscopic findings, which are demonstrable with much difficulty, may be explained in two ways. The majority of investigators adhere to the views advanced by WValler and Ströbe, according to which regeneration is the result of a growth of the axis cylinders of the central stump, these advancing into the young cicatricial mass and growing beyond it, the delicate fibres of the 
axis-cylinders meanwhile undergoing subdivision. It is a striking phenomenon in this process that the newly formed fibres of a divided nerve should grow into the original areas of distribution. The opinion most nearly explaining this assumes that the old paths of distribution persist as actually empty tubes, or as the neurolemma tubes of Schwann containing merely a softened fatty detritus, and that the sprouts of axis cylinder substance find here the line of least resistance in their penetration. It has, however, been shown that after resection the nerve-sprouts, in spite of the insertion of special interfering objects, follow along their original direction; and the interesting experiments of Forsmann show that the nerve stump will not invariably push its way into empty glass tubes which have been inserted but will on the contrary always force its sprouts into glass tubes containing nerve or brain substance, even if these be arranged in the wrong direction. It seems probable therefore that chemotactic force, exerted by the degenerating nerve matter of the peripheral portion of the nerve, determines the line of extension of the growing nerve fibres (neurotropism).

The force of growth of the central stump is usually very marked. Vanlair st:cceeded in enclosing calcareous tubes, made of bone, between the two ends of a divided nerve, and the growing sprouts not only found their way into the lumen of the tubes, but even into the Haversian canals (Thoma). Sometimes the budding axis cylinders form tumor-like masses in the scar tissue around the stump, the socalled amputation neuromata.

As the neurolemma with its nuclei remains intact in the degenerated peripheral fibres, it is possible that the formation of new medullary substance may arise from this source in case the new axis cylinders grow into these preformed paths. Proliferative phenomena in the cells of the neurolemma have been actually observed (Eichhorst, L. Mayer, v. Büngner), and consequently the view is held that this sheath, by differentiation of its protoplasm, may give rise in segments to the axis cylinder and medullary sheath; that in this way from the old sheath new nerve fibres may be formed. the segments then growing together to form continuous threads.

Ganglionic cells and the massize nervous tissue of the brain and cord are incapable of regeneration. It is well known from numerous experiments upon animals and studies in man in connection with inimries, partial ablations and other types of loss of substance, as from hrmorrhage, degeneration, etc., that even in case the usual 
fatal complications do not develop such lesions do not end in new formation of nervous elements but in fibroplastic cicatrization.

Regeneration of Blood Corpuscles and Lymph Cells.-The restoration of blood cells does not take place within the blood ressels, but originates in those organs in which normally the continual replacement of worn out cells of the blood and lymph is accomplished, particularly the bone marrow, spleen, lymph nodes and in lymphoid germinal foci scattered about in various tissues. The red blood corpuscles develop entirely from cells of the medulla of bones and are nucleated when first formed; by the time they have entered the circulation their nuclei have disappeared. Serious or repeated loss of blood cells calls forth so marked a proliferation of the hæmopoietic marrow tissue that the fatty marrow of the long bones comes to resume its embryonic character and is changed into red marrow. The colorless corpuscles are produced by proliferation of the leucocrtes and lymphocrtes in the structures mentioned and pass out into the bloot.

The fluid portion of the blood is renewed by the passage of water and salts from the tissues into the lymph and blood capillaries.

\section{Transplantation.}

Some cells and tissues can maintain their vitality for some time after their removal from the animal body and are capable of renewing their growth if artificially implanted upon another part of the same body or in another animal.* Experiments in transplanation have been performed frequently and sometimes successfully by surgeons ever since the fifteenth century, for the purpose of restoring denuded parts of the skin and other tissues in man; and, too, the same experiments have been repeatedly performed in animals in order to obtain theoretical grounds for operative procedures and explanations of pathological questions.

It is most easily and successfully performed in lower animals. Born has succeeded in causing adhesive growth in pieces cut $r f f$ from larval amphibians to take place in various ways, as of the head end of one larva to the tail cnd of another. Joest succecued in uniting the opposite ends of earthworms. As interesting trifles, after the castration of cocks the spurs used to be often cut off and

* Inorganic particles, as bits of ivory, wire or bullets, if they get into or are introduced into the tissues (implantation. insertion) may be encapsulated, that is, surrounded by connective tissue. Even living or dead tissues may be similarly surrounded. as an extracted tooth may be thus held in an alreolus in which it is implanted. In transplantation, also known as grafting (French, (reffe), there is not merely an encapsulation or adherence of the tissue to the structure, but in addition it multiplies in the site of implantation and forms part of the organic structure witr. the tissue about it. 
inserted into a split in the comb of the capon, becoming attached by growth in this situation (Aldrovandi, Worm, in the seventeenth century; Baronio in 1804 ). The spur, if engrafted when very young, may grow in this new and well vascularized base to become several inches in length; and sometimes the central bony core grows, carrying over it a horny epidermal covering, so as to produce two spurs growing alongside of each other. Embryonic tissue with undifferentiated cells shows a moderate capacity for growth when transplanted. Féré grafted bits of blastoderm (forty-eight hours) and the eyes of six to eight day chick embryos under the skin of young chickens and noted that the mesoblastic tissue underwent more or less further development to the production of small tumors (containing cartilage and in one case small feathers), which persisted for about a month. Such a result has an important bearing in explaining the conditions involved in the origin of tumors.

Transplantation of tissues is more difficult in mammals. The possibility is best realized when the part to be transplanted can retain connection with its neighboring structures by a bridge, as where a flap of skin is twisted around and stitched to an adjoining part, the nutrition of the flap being at least partially maintained (as in the operation of rhinoplasty in man). The result in transplanting unattached and completely separated bits of the corium and of the epiderm is not at all uniform. In this procedure, recommended by Reverdin and Thiersch for quickly obtaining an epidermal surface over large superficial wounds, which has been tested by many investigators, especially by Marchand and Enderlin experimentally, the transplanted fragments adhere by a layer of coagulated blood and lymph to the fresh or granulating wound. Later on there occurs an active vascular and cellular proliferation in the substructure, the fibroblasts and angioplasts of the latter growing up into and through the engrafted cuticular fragment, which is actually replaced by the new connective tissue. The epithelium desquamates and only to a very slight extent is the graft the starting point for the formation of the new layer of skin. In reality the new graft is more like a piece of plaster, beneath which the formation of new elements from the adjacent structures may take place with facility (Dürk). For success in such transplanations the size of the bit of tissue transplanted should be taken into consideration (even particles no larger than a pea may disintegrate in the central part because too much time is required to afford them proper nourishment), as well as the question whether the base of implantation affords thoroughly favorable conditions for nutrition. For example 
suppuration of the grantulating surface interferes with proper attachment; and, as the function of the tissue depends greatly upon nervous influences growth of a transplanted fragment is sometimes prevented because of the lack of any natural nervous communication. Again the portion to be transplanted ought to be kept as nearly as possible at its proper vital temperature (tissues have been successfully grafted, however, even after having been kept for from one to four days).

While transplantation of bits of skin to new positions in the same individual, as to the ear in a rabbit, has met with fair success, attempts to graft from one individual to another have not been so suecessful; and transplantation from the skin of an animal to man has failed in most instances. Efforts to transplant mueous membranes (that of the lip to the lid) and of keratous tissues (in the repair of corneal lesions), both in man and animals, have shown little uniformity. In transplanting cartilage, periosteal and bony tissue the graft as a rule persists for a long time and there may be noted a certain amount of increase of the cartilaginous and periosteal cells. However, this is not active enough to permit it to be said that there is an active part taken by the graft in the repair of an existing lesion; but rather that the fibroblasts, angioplasts and osteoplasts at the border of the lesion grow into the transplanted tissue and gradually take its place. Inasmuch as the transplantation of such material (small fragments or pieces of bone, the length of a finger, not necessarily fresh bone but with equally good results in the use of macerated or boiled bone or bone sterilized by heat) may materially aid and hasten the restoration of a given defect and the formation of a solidly ossified scar in it, the method has gained considerable importance in surgery.

Experiments by Ribbert, Lengemann, Lubarch and Enderlen have proved that transplantation of small pieces of salivary glands. sebaceous glands, mammary tissue, thyroid gland, epididymis, ovarian tissue, and liver tissue into the peritoneal cavity, anterior chamber of the eye, under the skin and within the lymph glands, is followed by more or less perfeet adhesive growth and actual proliferation of the glandular epithelium, with formation of new cellutar extensions with development of gland spaces and persistence of the vitality of the transported tissue. Ribbert's graft of mammary gland tissue into the ear of a young guinea pig has special interest from the fact that when the animal bore young the transplanted gland began to secrete milk. 
Operative grafting of thyroid tissue in case of loss of this organ (as in goitre and operation for the removal of goitre), which is apt to cause serious and even fatal disease, may successfully compensate for the loss and is therefore of value from a therapentic point of view.*

\section{Hypertrophy. Gigantism.}

By the term hypertroply is meant the increase of the essential tissue of a part of the body, leading to enlargement of that part.

Literally the term really means excess of nutrition ( $\dot{v} \pi \epsilon \rho$ and $\tau \rho \dot{\phi} \phi o s$, nutrition), and from ancient times it was used to express increase in volume of tissues and organs when it was supposed that this depended upon an increase of the nutritive supply to the structure iniolved. Theoretically and from the microscopic findings, two types of increase in volume may be conccived of: hypertrohly, in which the cellular elements are seen to be increased in size, and hyperplasia, where a numerical increase in the cells is the basis for the increase in the cinnensions of the organ. [These are often spoken of as simple hypertrophy and numerical hypertrophy.]

Where the increase of tissue involves the functionating elements of an organ or part, as the parenchyma of a gland or the muscle cells, it is spoken of as true hypertrophy; where, however, it consists in an increase of the supporting tissue or so-called interstitial substance alone (connective tissue, fat) it is a false hypertrophy.

True hypertrophies occur because of increased demands upon the activities of an organ and because of its increased functional effort. Such examples are termed work hypertrophics. This is most frequently seen in muscles, a very common example being met in the increase in the skeletal muscles in gymnasts and the arm muscles of wood choppers and smiths. The increased cardiac force in those conditions of life in man and animals requiring continuously special power of the heart is an expression of an hypertrophy. The heart possesses in a marked degree the power of accommodation to increased demands upon its ability; its muscular substance in case of permanent increase of labor increases (accommodatize hyportrophy) up to a certain degree, determined largely by the relation to the body weight. According to the investigations of Bollinger and Parrot all animals which are required to exert much muscular energy because of their mode of life possess a heart heavier in proportion to the body weight than those animals which lead a sluggish life, as the dog in comparison with the hog, the deer in

\footnotetext{
* In man thyroid preparations may he administered by the mouth.
} 
comparison with the sheep. There are marked differences in this connection between those birds which are good fliers and the domestic fowls which stay more on the ground. (For details cf. Kitt, Pathol. Anatomie der Hanstiere, II Bd. Chapter on the Heart. Ierl. v. F. Enke, Stuttgart. II Aufl. igor.)

All diseases which increase the work of emptying the ventricles of the heart in the period of systole and which force the heart to continuously increased pumping effort, give rise to cardiac hypertrophies, usually confined to one part of the organ according to the position of the opposition to the cardiac effort. Chronic valvular lesions, which interfere with the ready passage of the blood from the auricles into the ventricles, after a primary dilatation of the auricles cause hypertrophy of the auricular walls; stenosis of the semilunar aortic valves causes hypertrophy of the left ventricle, stenosis of the pulmonary valve a hypertrophy of the right ventricle: It is only by a heightened muscular force that the blood can be pressed forward in such conditions. If the cardiac hypertrophy is for a long time sufficient to accomplish the increased demands put upon its functional capability and thus prevents the serious results of the original disease or compensates for them, it is spoken of as a compcusatory hypertrophy.

Smooth muscles also become hypertrophied in case of increase of function, physiologically seen in the pregnant uterus, pathologically observed in marked degree in the intestinal wall where constrictions in the lumen gradually develop, over which the chyme can be driven only by increased peristaltic force. The same thing is seen in case of the urinary bladder, resulting from narrowing of the neck of the viscus by prostatic enlargements or from involvement by urinary calculi, etc. : and in the cesophagus in case of strictures. In all these cases there occurs both a multiplication and an increase in size of the muscle cells.

In the glands of the body true hypertrophy and the necessity for increased effort become apparent where smaller or larger portions of the glandular parenchyma are destroyed, and are restored or replaced by regeneration. In case this cannot fully obtain or does not occur at all, as in the liver tissue (after echinococcosis or chronic inflammations) the remaining portions of the organ become enlarged and the general volume of the liver may thus become greater than normal. Especially in case of bilateral organs where one becomes entirely incapable of function, the remaining one acts for the former, performs a correspondingly greater anlount of work and hypertrophies (compensatory or zicarious hypertrophy). Where 
one kidney is removed in an experiment, in the course of but a few days thereafter the other begins to enlarge; and it is not infrequent in slaughtered animals to find one kidney shrunken up and the other from one to two-thirds larger than the normal organ. The reason that the increase in volume does not reach double the normal is because all of the constituent elements do not hypertrophy in even proportion, the process involving particularly the secretory structures, the glomeruli and the convoluted tubules, the epithelial cells of which enlarge and the coils become more intricately convoluted and therefore longer. Of course compensation for a functional disturbance of a gland by hypertrophy can result only in case there exist analogous tissue or a glandular rest.* In this sense the lymph glands and the bone marrow, for example, might substitute for loss of the spleen, but not other kinds of glands.

As a reason why glandular organs are forced to higher secretory activity in case of removal of a portion of their parenchyma, it may be assumed that following the ablation of the organic parenchyma there accumulate in the blood certain secretory stimulants, substances which stimulate the gland cells. Whether these substances act directly on the protoplasm to stimulate it to productive activity, or whether the more active protoplasm takes up more nutritive material and is therefore enabled to throw off more products, is uncertain (Ribbert). The fact that muscles in a state of increased activity receive an augmented blood supply and in case of destructive changes in an organ a similar hyperæmia develops in its remaining tissue (due to the accumulation of materials no longer secreted) suggests according to Ribbert an explanation for the occurrence of hypertrophy, in that the persistent congestion distends the tissue, with the result of separating the individual cells from each other and thus gives origin to space for the increase of volume and for the multiplication of cells. Not only is it to be supposed that free spaces exist between the cells but the protoplasm itself is substantially distended, its individual molecules separated from each other and this intracellular increase of space affords opportunity for assumption of further elements which go to make up the protoplasm. Ribbert's theory of mechanical origin of tissue growth (cf. Regeneration) finds special applicability in the development of cardiac hypertrophy and the hypertrophy of muscular

* [What is meant here is the prosistence in undeveloped form of tissue capable of taking up the morphology and function of the adult tissue which bas been lost. Thus in the formation of an organ there may be embryonic elements in excess which are not emploved in the formation or subsequent development of the organ. remaining as "rests" and undifferentiated. In case of loss of substance of the organ such representatives may perhaps develop to maturity acd compensate for the destroyed portions.] 
canals (vessels, intestine, mrinary bladeler or gullet), the cause in such instance always being a retention of the contents (increase of blood pressurc, influence of calculi, obstruction to the movement of the chyme, etc.) causing primarily distension of the lumen of the part ancl stretching of the muscular wall.

That a diminution of the factors of resistance of growth, of tissue resistance, favors hypertrophy (zacumm hypertrophy) is apparent in the liver in case of the existence of a diaphragmatic cleft; the liver tissue invariably grows through such an opening, and, just as a button goes through a button hole, forms a new liver lobe protruding into the thorax, the soft and elastic lung tissue offering but little opposition. Such intrathoracic hepatic lobes may sometimes be found larger than a fist (in sheep, cattle, swine).

[The influence of nervous factors in some cases of hypertropluy can scarcely be doubted, althongh it is probable that for the most part such influence operates in ways conforming with the causes above mentioned. The ability to take up and assinilate nutrition is in relation with the trophic influence of the nervous systen, and the condition of the vessels supplying nutrition has even more apparent dependence upon the nervous functions; and so, too, the relative tone or the opposite condition of relaxation of tissues is regulated by nervous factors. Variations in functional activity are often determined by nervous influences. Leaving aside such influences in which perhaps the nervous factor may determine hypertrophy by evident involvement of such influences as increase of blood supply, the provision of mechanical relaxation of tissue or the stimulation of functional activity, there are more obscure examples (possibly in the end explicable on the same grounds but not clearly showing such reasons for growth) which for the time are properly spoken of as nerious hypertroplices, as the enlargements in acromegaly, for example, or the papillary enlargements of the skin in many warts.]

Connective tissue hypertrophies are usually the result of chronic inflammation and chronic passive hyperemia, in either of which there are features of tissue destruction and of tissue dilatation. Where the liver becomes enlarged and the seat of comnective tissue induration from chronic inflammation there occurs prinarily a destruction of the liver cells and a coincident growth of the supporting tissue: the termination of chronic lymphangitis of the cellular tissue of the foot in pachydermia (ruffle foot or bristle foot in the horse) may be explained by the idea that at first the connective tissue spaces are filled with lymph and exudate and later hecome oc- 
cupied by the proliferating connective tissue. The formation of thick, keratous epidermal layers (keratoses) over callosities of the skin or inflamed and distended bursæ is to be ascribed less to the direct influence of external pressure than to the pressure exerted from beneath by the hard and thickened cutis upon the epiderm (see also chapters on Regeneration and Inflammation).

The occurrence of congenital hypertrophics of certain parts of the body may be due to a number of causes. Some examples are compensatory hypertrophies, as when one kidney fails to develop the other grows to unusual size; others are due to chronic passive hyperæmia in the fotus caused by constrictions by amniotic bands, as the hypertrophy of the pinna of the ear or of the penis. (cf. Chapter on Malformations, Spezielle pathol. Anatomic der Hausticre, I Bd.).

\section{Inflammation.}

The term inflammation is applied to a condition of local irritation of the tissues, the seat of some type of injury, in which there occur, as phenomena of reaction to the irritant, changes in the blood current and lymphatic flow', exudation of the constituents of the blood, excessize secretion of glands, phagocytosis and proliferation of the tissues.

In dealing with inflammation we have to do with a series of processes which are nothing more than modifications and exaggerations of physiological procedures, in this case having for their object the defense of the organism against the harmful influences and the compensation for local disturbances of nutrition, and representing abilities of the animal organism acquired through phylogenetic adaptation. (Leber, Metschnikoff, Dürk, Marchand, Schmauss and others).

In the lowest unicellular organisms, as the amœbr, the phenomena of reaction to harmful influences are manifested by the properties of chemotaxis, phagocytosis and intracellular digestion. In the more highly organized forms of life, made up of differentiated cells, these defensive functions are assumed by certain groups of cells (Dürk); and in conformity with the complexity of structure and interdependence of tissues and organs and the variability of the injuries, these regulative and reparatory processes are made up of a number of separate phenomena which in their synchronous and consecutive operations are grouped together under the comprehensive term, inflammation.

In the class of harmful agents capable of inducing inflammation, the causes of inflammation or irritants, are included a great variety 
of foreign bodies, parasites, infectious substances, poisons and thermic influcuces which are spoken of as crternal canses. These do not act in reality as the immediate causes of the process but produce primarily some type or other of tissue change, as necroses, degenerations and injuries, and the inflammatory reaction is directly due to an effort to compensate for these latter. LIt is not the heat of the flame or the steel of the knife which actually causes the inflammation; these are probably at once withdrawn. They, however, alter the cells and tissues, and the inflammatory reaction arises in reality to eliminate or isolate these altered structures and prevent their acting as irritants, and to make repairs for their loss.] One and the same influence, depending upon the intensity and duration of its operation, may be responsible for a number of types of tissue alteration; an irritant poison, an acid, may in different degrees of concentration, heat or cold, give rise perhaps to a simple hyperemia, to a superficial inflammation or to deep ecshars. Strictly speaking tissue necrosis is always produced, sometimes only superficially, affecting only epithelium and vascular endothelium and essentially microscopic in extent, at other times extending more deeply or involving decidedly larger areas and appreciable to the unaided eye. In the first instance reparation is accomplished so quickly by phagocytosis and prompt regeneration that the full picture of inflammation is not developed, these two factors of inflammation alone occurring in the tissue; in case of the destruction of a larger amount of tissue, however, provided it is not fatal directly or from complicating infection and putrefaction, the inflammatory reaction with its entire group of phenomena results. A given infectious agent may in one case give rise to a rapid general infectious process with fatal termination or in another only to a typical local inflammation, as illustrated by the varying effects of bacillus aniscpticus in the virulent or attenuated state and in the particular state of individual predisposition of the subject. In reality the animal body reacts to all physical and chemical injuries by the production of an inflammatory process, provided time be afforded; that is, provided the injury does not involve a vital tissue or organ to such a degree as to immediately produce death from the functional disturbance. Where bacteria of extreme virulence, witl hæmolytic power and capable of rapid extension, invade the system, a bacteriæmia or toxic infection is said to exist; in such cases there is not enough time permitted for compensation by an inflammatory reaction for the disturbances occasioned. Were the microorganisms of lower virulence, did they multiply more slowly at the 
focus of infection so that their toxines might be restrained or rendered in some measure inert, or if the animal body be especially well provided with bactericidal substances, the course of the disease would be protracted and there would be time for the tissue to set into activity some or all of the reactions belonging to the inflammatory process. Inflammation may therefore, as the combined manifestation of a number of defensive efforts directed against harmful agencies, be looked upon as a curative process. When the forces brought into activity succeed in eliminating the source of harm the inflammation reaches in a relative measure its physiological termination (Dürk); when, however, the reaction directed to the removal of harmful influences is not successful in eliminating them, when. perhaps, the latter multiply in the system, the organism perishes in spite of the inflammation, not necessarily because of the inflammation but because of the infection or intoxication, or possibly actually because of the inflammatory reaction from the series of functional disturbances of the organs resulting from its onset.

The Phenomena of Inflammation,-From the earliest centuries of our era, when the Roman physicians Celsus and Galen attempted to formulate a conception of inflammation, there have been recognized as cardinal symptoms of the process in the grossly visible parts of the living body: increased heat, redness, sacelling and increased sensitiacness of the inflamed part (calor. rubor, tumor, dolor), to which may be added disturbance of function (functio lasa) as a fifth. These fundamental symptoms . are, it is true, clearly appreciable in most acute, that is, rapidly developed, inflammations of the skin and mucous membranes: but they are not invariably to be noted in the entire course of the process. The older the inflamnation becomes, the more likely are these features to be lost one after another: and a chronic inflammation beginning gradually and continuing in low grade may not show any of them. Noreover the supposition that internal organs in inflammation show the same intense redness, swelling, increase of temperature, and increased sensibility to pain, is only partly correct: and here too the chronic inflammations are especially likely not to be characterized by such phenomena.

In the effort to analyze the nature of inflammation from an anatomical standpoint modern studies have furnished definite explanations of the above mentioned symptoms of Galen and have given sufficient insight into the inflammatory process to afford a fairly precise knowledge of the character of the changes and the methods followed by the tissues in their inflammatory reactions to 
irritants. Thanks to the studies of Virchow, Recklinghausen, Cohnheim, Ziegler, Marchand and others, the various steps which develop and take part in the process in inflamed tissues have come to be well understood. Only in connection with relatively unimportant questions do there still exist differences of opinion and theoretical discussions, involving for instance such points as whether the proliferation attending inflammation is produced by excess of nutrition or lack of tissue tension, whether it may be assumed or not that there is a functional stimulation to excite cell formation, or

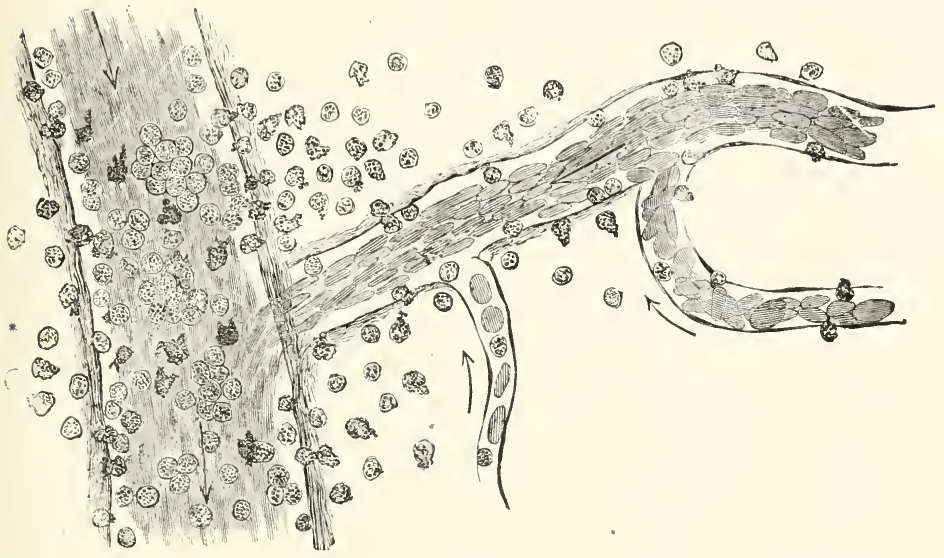

Fig. 47 .

Emigration of the leucocytes in frog's mesentery, six hours after exposure. The cells shaded with lines are the red corpuscles; the stippled ones are the leucocytes: $\times 25$. (Atter l'erls.)

whether certain granular changes of the cells should be spoken of as inflammatory or regarded as degenerative.

The inflammatory process may be directly traced (Cohnhein's experiment*) under the microscope in transparent inflamed living tissue, as the mesentery of a frog or rabbit, or in the expanded wing of a bat or the tongue of a frog drawn out from the mouth or in the web of a frog's foot, the inflammation being excited in such tissue by the application of a caustic or the production of a minute traumatic lesion.

The first step of inflammation in a vascular tissue consists of

*For detalls ef. Kitt, Bakterienkunde und pathol. mikroskopie fur Tierärzte. IV Aufl. Wien, M. Perles Verlag, 1903. 
distension of the blood i'essels, hyperæmia. In the exposed or irritated parts there may be first observed a dilatation of the arteries and later of the capillaries and veins. With this the blood current is first accelerated, but with the occurrence of dilatation of the veins of the tissue the circulation becomes slowed. Here or there it may stop for a variable period, and the corpuscles which previously were forced along in the central part of the stream become distributed uniformly throughout the lumen of the vessel in the stagnating blood. In addition the delicate capillaries, through which previously plasma mainly flowed with here and there a blood cell, are found engorged with blood corpuscles. With this slowing of the current the sluggishly moving white blood cells tend to collect
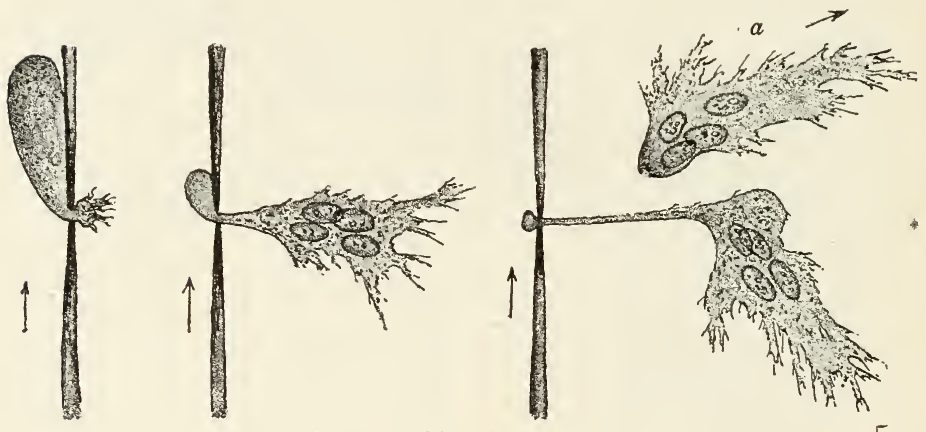

Fig. 48 .

Various steps in the passage of a white blood corpuscle through the wall of a vessel. On the left of the short lines which represent the ressel walls, is supposed to be the intravascular blood; on the right the tissue; $x 1$,ion. (After Thoma.)

more and more on the inner surface of the vessel wall, giving rise to the so-called marginal deposition of the white corpuscles, and as additional ones are continually added from the blood the capillaries may, from their increasing accumulation, be found completely occluded by leucocytes. There may next be observed a very characteristic feature, the crudation or emigration from the vessels of large numbers of the white corpuscles into the area of inflammation. These motile amœboid cells, which were more or less flattened against the internal surface of the vessel, penetrate the capillary or venous walls by protruding a pseudopod-like process of their protoplasm through it (mainly at the cement lines between the endothelial cells) the rest of the protoplasm following in a distinctly 
flowing manner until the whole cell is on the outside. In the living specimen all of the steps of the passage, so like a crceping movement, may be followed; and in sections of an inflamed tissue cells may be found partly outside and partly within the vessels, fixed in their constricted condition and reminding one very much of the appearance of a wasp with its abdomen constricted from the thorax. Sometimes these cells show a long stretched out protoplasmic process, swollen out into a button in case a part of the substance with the nucleus has moved forward. The leucocytes which have escaped

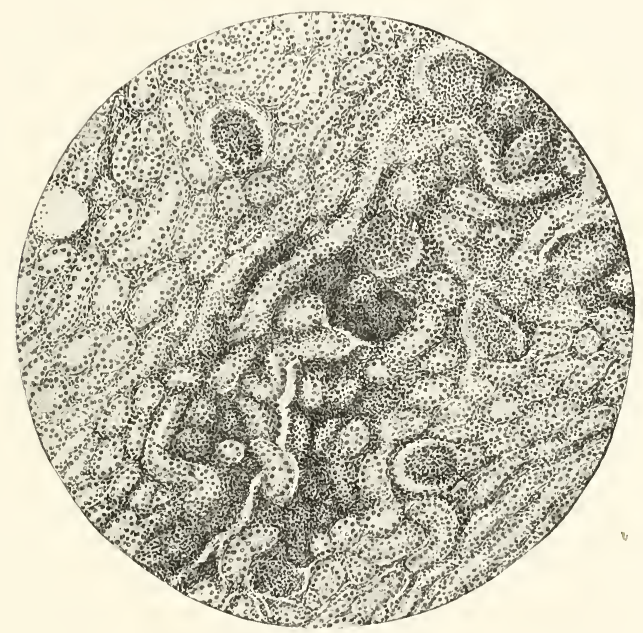

Fig. 49 .

An inflammatory focus the seat of cellular infiltration in the tissue of the kidney. (Section of the kidney of a cat.)

from the vessels continue their creeping movement and thus distribute themselves in the spaces and interstices of the tissue of the inflammatory area between the connective tissue cells and fibrillary bundles, and wandering on between the epithelial cells to the free surface of the part.

As new cells are constantly passing from the vessels the inflamed tissue becomes thickly studded with leucocytes, constituting a condition known as cellular infiltration. A number of authors believe that the leucocytes after their egress from the vessels multiply in the tissues by amitosis (direct division), and, too, that the lymphoid 
cells find their way into the part from the lymphatic foci scattered about in all organs.

Along with the leucocytes the fuid blood plasma also escapes in greater or less amount from the distended vessels, by a process of leaking through the walls through the openings made by the leucocytes and directly through the endothelial cells which have from injury become more readily penetrable; or, as some maintain, the fluid is given off to the tissues as a secretion of the endothelium. It is possible that red blood cells may also escape from the vessels, mixed with the fluid, being passively forced through any existing openings by the extra blood pressure. The transuded blood plasma with its cellular clements is known briefly as the erudate (from ersudare, to exude).

The histological events just detailed place us in a position to understand why and now the phenomena spoken of as the cardinal symptoms of inflammation are produced.

The redness (rubor) is the result of the hyperenia. In early stages of the process and in the peripheral zone of an inflammatory area, where the blood current is accelerated, this hyperemia has the characteristics of an arterial hyperæmia, the vessels showing up conspicuonsly with a scarlet red color as if injected (injection redness, branched redness). The more pronounced the slowing of the current and the more the stagnating blood gives off its oxygen to the inflamed tissue the more the color approximates that of the venous blood, becoming darlier and of a more violet hue and becoming more and more diffuse as the dilatation of all the capillaries develops. The heat (calor) depends upon the increased rapidity of flow and the volume of arterial blood; the more rapid the current, the less opportunity being afforded for loss of heat and the more nearly the temperature of the inflamed part approximates the temperature of the blood itself. The heat of an inflamed area is only noticeable on the exterior of the body, in comparison with the parts whose temperature is unchanged, and is not above the temperature of the general blood. The internal organs do not become essentially warmer than the blood, special increase of temperature from metabolic changes not taking place in inflamed parts (Perls, Ribbert). [It is claimed by some, however, on the basis of experiments carried on it is true with difficulty and open therefore to a chance of error, that the blood coming from an inflamed part is of higher temperature than that of the arterial blood going to such a part, and that increase of temperature is actually, therefore, produced in inflammation presumably from an increased metabolism which obtains.] 
The su'elling (tumor) of an inflamed tissue is easily comprehended. The exudate fills up the lymph spaces and stretches the structures apart; the ressels are dilated, and the lymph is prevented from escaping readily from the tissue because of loss of elasticity of the interstitial substance (Landerer). In consequence a turgescence, appreciable as an cedematous swelling in inflamed areas on the periphery of the body, occurs. In chronic inflammations the connective tissue proliferation gives to the organ or its connective tissue frame-work more or less increase of volume. The pain (dolor) is attributable to the pressure exerted by the exudate and swollen tissue upon the sensory nerve filaments. or to direct irritation of the latter. In addition this fault interferes with the transmission of nerrous impulses, in consequence of which arises functional disturbance, in addition to the disturbance occasioned by the primary influence of the cause of the inflammation (death of tissue) and the simple mechanical disturbances produced by the collection of exudate in carities (as the air spaces of the lung or the pleural cavities), the overgrowth of tissue, etc.

As already stated, the processes involved in inflammation are nothing but modifications of physiological processes. Exudation of plasma and leucocytes and their movement through the tissues are continually going on under normal conditions; the ordinary lymph formation is the result of a normal process which in inflammation is clearly only exaggerated. The reason for this heterometria, and more particularly for the circulatory disturbances, may be found in certain physical and chemical changes in the vessel wall. All inflammatory excitants apparently exert, directly or indirectly, chemical influences leading to changes of the endothelium of the vessels and thus making the vessel wall more permeable than normally. It can be shown for example that the endothelial cells may be caused to shrink, contract into spherical form, and thus give rise to imperfections in their interstitial cement. These openings produced pathologically facilitate the leakage of the plasma and the emigration of the leacocytes through the wall and make it possible for the red cells to undergo diapedesis. Changes in the tension of the vascular wall, relaxation of the arterial musculature, necessarily accompany lesions of the endothelial cells and the surrounding tissues, and this in turn occasions dilatation of the vessels. In every case, too, the nervous system, especially the network of vasodilators and vasoconstrictors distributed about the ressels, takes part in bringing about the vascular dilatation, these nerves being affected, irritated or paralyzed, by the original cause of the inflammation. 
The acceleration of the blood current which is seen in the early stages depends on the passage of the blood from narrow into wider channels [an explanation sufficient for the short period required for the filling of the additional space afforded by the dilated lumen of the vessel, but not clearly satisfying for its continuation even for the time it does prevail before stasis sets in, and for its longer persistence at the periphery of the inflamed area]; the slowing of the current upon the transudation of the plasma and the endothelial changes, both of which tend to cause a more concentrated condition of the blood. [Here, too, there should be considered the probability of relative inefficience in the outflow of the blood, loss of tone of the venous walls and loss of elasticity of the surrounding tissues, local compression of the walls of the capillaries and venules by exudate and proliferating or swollen and degenerate cells, all of which introduce elements of a relatively passive factor in the process for retardation of the escaping current.] The marginal deposition of the white corpuscles is explained by Hering and Schklarewski by the fact that when liquids containing light bodies in suspension are passed through tubes these bodies tend to move along the periphery as the rapidity of the current is slowed; they call attention to the lower specific gravity of the white in comparison with the red cells and their viscidity and tendency to adhere from their tactile irritability. The movement of these cells, physiologically determined everywhere by their tactile and chemotactic sensibility, is stimulated in inflamed tissues both by the causes of the inflammation and by the lesions occasioned by these causes. The studies of Pfeffer, Buchner and others have made us acquainted with a series of substances which possess marked power of attraction for leucocytes, especially certain proteins of the bacterial body; and in the destruction of tissue induced as a primary effect of the untoward. influences exerted by the causes of inflammation certain chemical substances, as the alkali albuminates, are set free which have a similar attractive influence upon the leucocytes. According to Thoma, even a variation in the amount of saline matter in a tissue will occasion a movement of the leucocytes.

The spaces produced between the endothelial cells are apparently not primarily due to perforative efforts by the leucocytes, but result from distension of the vessel or from shrinkage of the endothelial cells; and the leucocytes possessing tactile sensibility, closely adapting their substance to the surface and fitting into every variation, extend their processes, tentatively and uncertainly, through 
any existing openings. The presence of any substance with chemotactic power determines the direction taken in the further movements of these cells.

The majority (about seventy per cent.) of the motile cells collectively spoken of as leucocytes, which are found in an inflammatory focus, are characterized by the possession of multiple nuclei, or one polymorphous nucleus, and very fine granulations only seen in fresh specimens. These polymorphonuclear forms are regarded as the characteristic white blood cells and arise from the bone marrow (where they are mononuclear, acquiring biscuit and clover leaf types of nuclei only in passing into the blood as the result of nuclear division or fragmentation). Besides these, which are described by Ehrlich as neutrophilic leucocytes, there are met in inflamed tissues forms containing coarser granules which, because of the readiness of their granules to take up intensely "the acid aniline dyes, like eosin, are known as eosinophile leucocytes; and others with special tinctorial tendency of their coarse granules for the alkaline anilines (gentian violet), known as mast cells. Mononuclear (single nucleus) cells are also comparatively numerous in inflamed tissue, the nucleus round and rich in chromatin, and the cytoplasm showing as a very narrow margin about the small or large nucleus. These are regarded as lymphocytes, which have originated from the lymph glands or germinal lymphatic centres. As above mentioned, the great increase of these cells depends in part upon a chemical stimulation acting not only on the blood vessel walls but also upon the foci of lymphoid cells distributed in all the tissues and causing enlargement of these lymphcell forming centres; and in the second place, as many of these cells have come from the marrow, it may be inferred that these chemical substances, which act locally in the inflammatory area as chemotactic agents and stimulants to proliferation, are also present in the circulating blood and with it pass into the bone marrow and there exert the same influence, that is, stimulate this tissue to the formation of leucocytes. Often in inflammation there is not only a swelling of the lymph follicles, but even a hyperplasia or metaplasia (change of red marrow into lymphoid) of the bone marrow referable to its exaggerated function.

The causes of inflammation and the chemotactic substances set free in the affected tissue have, moreover, a stimulating influcnce upon the proliferation of the fixed cells. The endothelial cells of the blood vessels and lymphatics and the connective tissue cells take part in the proliferation in every inflammation, the beginning 
of proliferation being indicated by the appearance of mitotic changes. After conclusion of mitosis the cellular division takes place, the protoplasm also increasing in volume. The young cells arising from this proliferation persist in part in the inflamed tissue, and after the formation of an intercellular substance constitute an inflammatory cmbryonic tissue, made up of fibroblasts and angioplasts, which serves the purpose of restoration of the original lesion (cf. Regeneration, p. 237). In part these cells disappear, and they may perhaps exhibit motile activity. A number of the proliferating

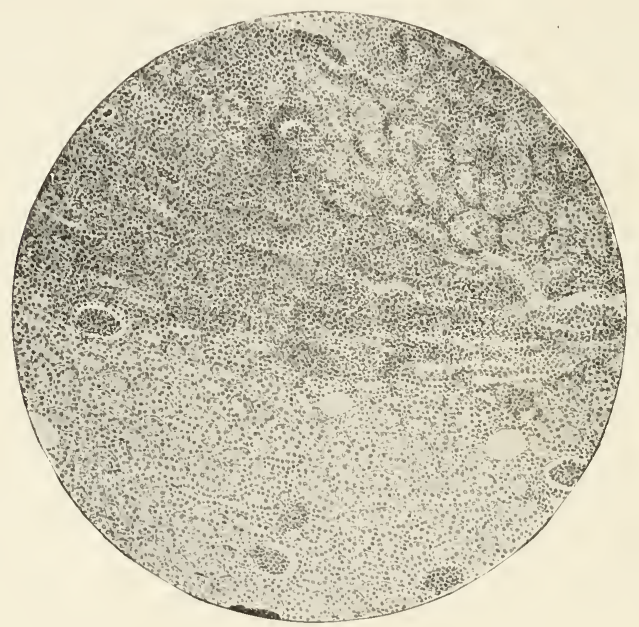

Fig. 50.

Inflammatory focus in kidney of calf, showing cellular infiltration.

connective tissue cells and endothelial cells are sometimes changed by direct nuclear division into multi-nucleated giant cells. The formation of these giant cells is by some supposed to be due to a chemotactic influence bringing a number of cells together and causing their fusion; another suggestion is that the cause of the inflammatory change has in some way disturbed the protoplasm of a cell so as to interfere with division of the cell substance, althongh nuclear division goes on. This type of cell is met especially in inflammation excited by foreign bodies, about which corpuscular elements the giant cells are found deposited in a manner explain- 
ing how the inflammatory reaction is able to carry out its protective purpose by removal or encapsulation of the harmful substances.

Demarcation and Phagocytosis are two important functions of the cells coming into consideration in the inflammatory process. The motile cells of leucocytic and lymphoid types, distinguished generally for their phagocytic properties even in normal circumstances, are attracted by the metabolic products of an injured tissue or by the chemical influences of the inflammatory agents. They take up all the substances in a disintegrating tissue which are acces-

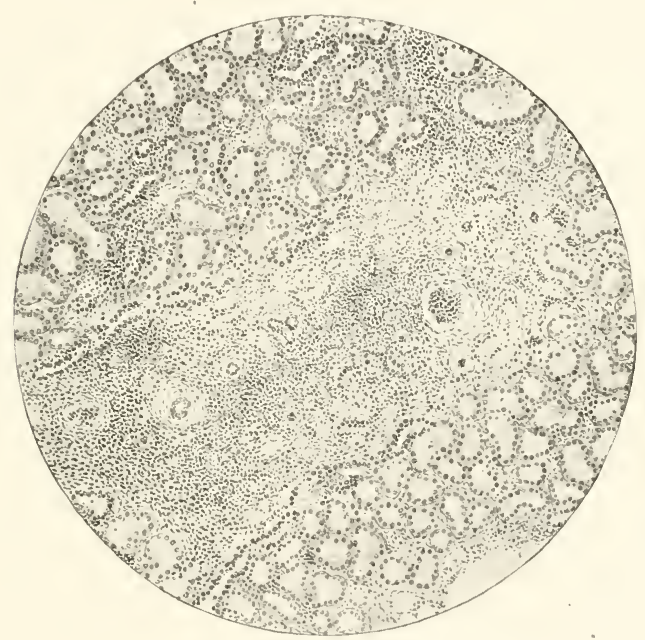

Fig. 51 .

Inflammatcry sear in the kidney of a log.

sible and with which they can deal, as albuminous granules, blood remnants, pigmentary granules and fat globules, and, indeed, all sorts of minnte particulate elements, as regetable and animal microorganisms; carrying these objects off or destroying and rendering them inert by the influence of the digestive power of their protoplasm. However, this power of phagocytosis also resides in the fixed cells. In the chemotactic accumulation of the cells, which takes place around foreign bodies or necrosed tissue, the lencocytes, fibroblasts and angioplasts and giant cells form a protective wall which either permanently imprisons the foreign body or from 
which fresh cells constantly move into the dead structure, grow all through it and break it down, bearing away particles of the disintegrated material or any minute foreign elements (bacteria, etc.), and in this way gradually removing the agents producing the inflammation.

These features have been studied experimentally in a variety of ways. They may be easily observed by injecting into a tissue an emulsion containing fine particles of carmine. An inflammation is determined in the course of which the leucocytes and fibroblasts become loaded with pigment granules, and giant cells are formed which also englobe the pigment particles. Some of the carmine is carried out of the area by the motile cells; when larger amounts have been introduced the parts which cannot be carried away are encapsulated by the connective tissue cells and the cicatricial tissue arising from the latter. When porous foreign bodies like bits of sponge or elder pith are introduced aseptically into the subcutaneous tissue or into the peritoneum, at first the exuding plasma and a large number of leucocytes occupy the spaces in the substance, but later the connective tissue corpuscles and vascular buds penetrate into them and take up all the available space (this reactive proliferation is seen as early as the second day).

After transformation into scar tissue (v. p. 24I) the connective tissue is found surrounding and filling all the spaces of the foreign substance and isolating it from the rest of the organism (Ribbert). Solid foreign material like silk thread, cat gut, silver wire, introduced aseptically as sutures by surgeons, or bullets, needles, bits of glass, wood splinters, or hairs which have gotten into wounds, provided they have no bacteria upon them and are truly aseptic foreign bodies in the tissue, are always surrounded by a wall of leucocytes and proliferating connective tissue and thus encapsulated.

The inflammatory reaction abont a foreign body always depends upon the soluble chemotactic substances which arise from the material. About some bodies which give rise to but little substance of this sort (aseptic cat-gut, silver wire) it may be very unimportant; inflammation ceases entirely after these substances are completely extracted from the foreign body, that is when it can no longer give off soluble chemotactic material. The functional activity and the movement of the cells mentioned toward the foreign matter can be beautifully demonstrated, as pointed out by Ribbert, by injecting liquefied agar or a blue-stained mass of gelatin into the subcutaneous tissue or anterior chamber of the eye. Soon after the jelly has hardened in the body it is surrounded by leucocytes and 
fibroblasts which approach; these cells push forward and into the jelly like a phalanx of soldiery, break it down and gradually carry it entirely away; and the local inflammatory condition disappears with the removal of the forcign matter, the lencocytes and other cells being able to return whence they came, or some perhaps dying and undergoing disintegration.

Hæmorrhagic effusions, blood clots, masses of fibrin, coagulated or completely necrosed tissue and loose bits of bone are all in the same way objects for phagocytic activity of the cells, making their appearance in the tissue reactions in inflammation. All dead tissue, in fact, is the same thing as a foreign body in relation to the functionating living tissue adjacent to it; it is a source of chemotactic substances and acts as an excitant of inflammation. So, too, just as non-vital foreign bodies, the living organisms which enter the body from the exterior, the animal parasites and all microbes, are to be looked upon as irritants and as causative of inflammation to a greater or less degree, according to the chemotactic substances which they contain.

Inflammation in non-z'ascular parts of the body, of which there are two which require special mention, the cornea and the cardiac valves (cartilage and the calcified bone substance, which are also avascular, have no marked inflammatory reactive power and are subject merely to retrograde metamorphoses), presents practically the same features as in the vascular tissues, save that emigration and exudation of course cannot take place where there are no vessels, but do occur from vessels at a distance. Numerous investigators, particularly His and Virchow, and besides these Cohnheim, Fuchs, Eberth, Ranvier, Güterbock. Ribbert and Marchand, have engaged in studies in connection with the process in question, and have thereby materially added to our appreciation of the nature of inflammation. Where the cornca has been injured by some tranmatic lesion, by a foreign body, or some type of infection, lencocytes actively penetrate to the focus from the blood vessels which encircle the margin of the cornea in the conjunctiva. Although these vessels are situated some distance from the focus of injury there are to be noted in them the same dilatation, slowing of the current and emigration as if they were immediately in the injured area. It is probable that these vessels are influenced by reflex nervous action, and it cannot be doubted that the injured corneal tissue exerts a strong chemical attraction for the leucocytes by means of substances dissolved in the corneal lymph and reaching the corneal margin in the latter or exerting a far-reaching influence from 
within the corneal mass. The leucocytes which escape from the blood vessels at the corneal border find their way along the spaces of the corneal tissue to the point of injury and there collect about it. This can be observed in the frog's cornea which has been touched with a corrosive, if the animal be killed a day or two later and the excised connea spread out on a glass slide, as the leucocytes of this cold-blooded animal retain their motility for a considerable time. The wandering cells are found elongated and conforming in shape to the narrow tissue spaces, their presence giving the cornea a milky cloudiness. At the same time the corneal cells are found proliferating; they undergo division and become slightly motile in response to the chemotactic agent connected with the inflammation. If the inflanmation continue for a comparatively long time, eventually the marginal vessels begin to send vascular buds into the cornea: these may penetrate so far that the cornea becomes covered or completely penetrated by a network of vessels, a pannus ( $\dot{\eta} \pi \dot{\eta} \nu \eta$ a cloth). This proliferation is also, according to Ribbert, probably due to a chemotactic stimulus affecting the endothelial cells. The opacity which involves the cornea in the course of the process mentioned may entirely disappear with the completion of repair; the transparency returns because the cells creep back and the fluid washes out the products of disintegration and the capillaries undergo regressive changes. In other cases a whitish scar remains as a remnant of the proliferated tissue.

Inflammation of the cardiac calces, according to Ribbert, is not attended by penetration of lencocytes into the tissue at all; aside from the formation of adhesive clots, which develop upon the roughened (from endothelial lesion) leaflet, the only evidence of reaction consists in a multiplication of the fixed cells of the endocardium and, in case the process continue for a time, of the penetration of the capillary vessels from the base of the valve into its substance.

Anatomical Types of Inflammation and Exudation.-Exudation of blood plasma, emigration of lencocytes and sometimes diapedesis of red cells occur in varying degree, depending upon the causes of the inflammation, its duration and local conditions: and give rise to special appearances which form the basis for differentiating a number of forms of inflammation and exudate.

The volume of exudate varies within wide limits; it may be so small that the inflammatory focus can be found practically only with the aid of the microscope, or there may be such a quantity of fluid that many liters may be removed (from the larger cavities of 
the body). The extulate may exist in the lymph spaces of the tissues, filling them completely (infiltration), or may escape to the surfaces, where it forms a deposit upon the latter; it may collect in the body cavities or may find free exit from the body. Even in normal conditions leucocytes find their way between epithelial cells, and when the epithelial tissues are loosened in inflammation or actually desquanated the exudate is given easy access to the free surface. The qualitative composition of the exudate is by no means uniform; sometimes the major portion is plasma and cells are but scantily met in it, sometimes the reverse is true. In addition admixtures of the secretions of organs may be found present, or products of tissue disintegration or changes due to coagulation, putrefaction or inspissation, all of which serve to give a very variable character to the exudation.

The terms serous c.rudate and serous inflammation are employed in connection with an aqueous product of inflammatory extravasation: the fluid is rich in albumen, like the serum of the blood, limpid or slightly clouded, of a yellow wine tint, or sometimes red from the presence of erythrocytes. When it soaks through a tissue. and gives it a juicy gelatinous appearance the structure is said to bc the seat of inflammatory adcma; collecting in the sacs of joints, tendon sheaths and the serous carities the expression inflammatory dropsy is applied. In the skin surfaces, which are covered with squamous epithelium, the tpper layers of which are but slightly permeable for fluids, it may collect in the more easily penetrated deeper lavers of the rete Malpighii and raise up the superior strata to the formation of blisters. In mucons membranes the fluid passes through the epithelial layer and mingles with the physiological secretion and the desquamated cells of the membrane, escaping with these: in this case the term catarhal exudate (катар) $\omega$. to flow away) is employed. Such catarrhal exudate may be very thin and water like, or may be viscid. The irritation of the inflammatory condition invariably induces an increase of secretion (increased flow of tears, increased secretion of nasal mucus).

It is said to be a fibrinous crudate in case the exuded fluid undergoes coagulation by precipitation of fibrin. This form is met particularly on the free surfaces of serous membranes and mucous membranes, within joints and in the lung, and to a less degree in the meshes of the tissue. Coagulation occurs in case the fibrin forming substance, which is always abundantly present in every exudate, comes in contact with fibrin ferment, the latter being supplied by necrosing cells, especially lencocytes and endothelial cells. 
The fibrinous exudate may form delicate or thick, more or. less adkerent, grayish white to yellow deposits or flocculent precipitates of a semi-transparent, elastic type. On the surfaces of serous membranes it may be observed as a frost-like deposit of thick patches of coagulated substance which can be torn off in shreds, look something like omelet and perhaps cover considerable areas of the serous surface (false membrane). The viscidity of the exudate is responsible for the fact that on opposed surfaces which move over

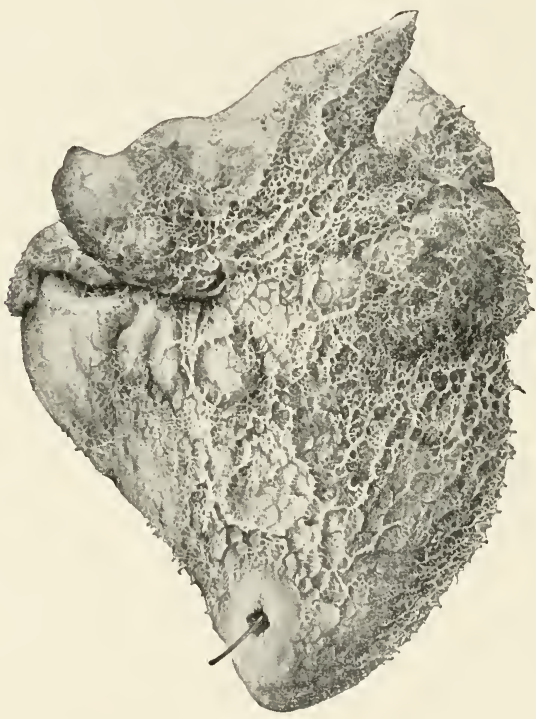

Fig. 52.

Fibrinous exudate on surface of heart of cow (traumatic pericarditis).

each other (pleura, diaphragm, peritoneum) the layers of exudate in rubbing upon each other leave the fibrinous material stretched and dragged into a reticular, villous appearance, reminding one of butter on two pieces of bread which have been pressed together and then pulled apart, or of the ridged appearance of tripe (dry or fibrinous erudation). In other instances the coagula of fibrin are so large as to appear as elastic rind-like layers, thick as one's hand, riddled with tiny holes which contain serum: or the serous exudate 
may predoninate and flakes of fibrin be found floating in it, in a state of fine division (scro-fibrinous erudation). On the mucous membranes also the fibrin may be encountered as membranous layers, which may be pulled off in sticky slureds, and which sometimes form distinct casts of the canal; these are spoken of usually as croupous exudates (according to Roth the word croupous has a Scottish origin). Croupous membranes may be tough or soft and grumous, depending upon the proportionate number of cells present

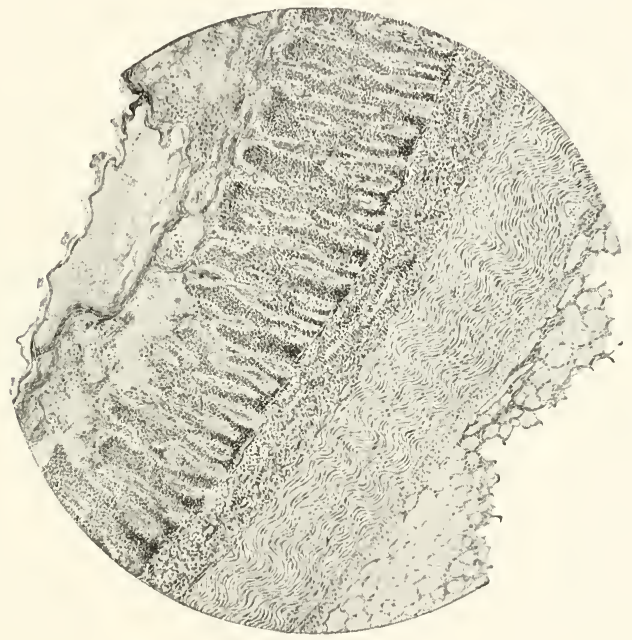

Fig. 53.

Enteritis membranacea of cat (section). To the left the pseudomembiane covering the intestinal villi, which are the seat of cellular infiltration and desIfumation; to the right the muscular layers and the serosa with its adipose tissue.

and the existence of some degree of degeneration; they vary from one-half to ten millimeters in thickness; when separated in the form of tubes they are full of the contents of the mucous canal, as the intestine, in which they develop; in other instances, as in the lungs, they form solid coagula. Under the microscope, at the beginning of fibrin separation the fibrin threads can often be seen arranged in a stellate, tuft-like manner about the cells which give origin to the fibrin ferment (Hauser); sometimes the fibrillar material is deposited in parallel layers or is formed in a reticulum, the meshes of 
which are occupied by leucocytes, desquamated epithelium and serum. By the cohesion of the fibrin, originally separated in the form of fine fibrils, thick homogeneous trabecula are sometimes produced (hyaline fibrin).

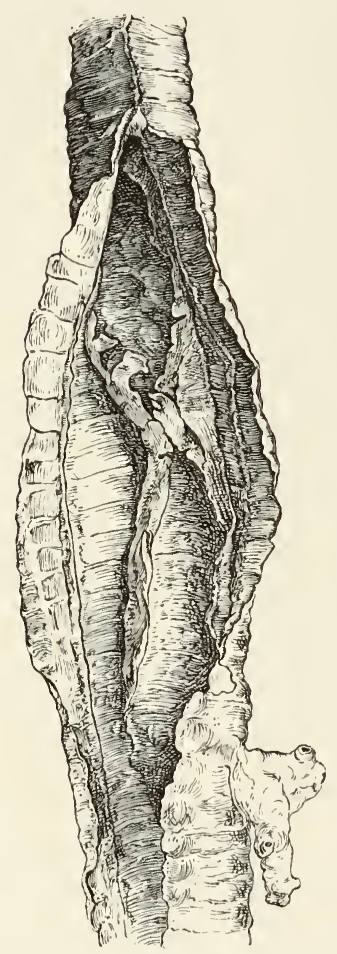

Fig. 54.

Croupous membrane in trachea (opened) of cow.
Fibrin may be well demonstrated in microscopic sections by Weigert's double staining method, the fibrin taking an intense blue tint, and the rest of the tissue a red contrast color.

Inflammations of mucous membranes, in which in addition to the formation of a fibrinous exudate there takes place a coagulation necrosis of the tissue of the mucous membrane, are spoken of as diphtheritic inflanmations ( $\delta ф \phi \theta t \rho a$, membrane). The coagulation-necrotic mass is here seen as a grayish-yellow to strawcolored or dirty gray, soft and pultaceous or drier, very opaque and usually fragmented material, which stands out sharply from the slightly transparent normal tissues as a thicker, somewhat swollen, prominent part. Here the deeper parts of the mucous membrane are permeated with the extrdate, and the substance cannot be as readily separated from the underlying tissue as the ordinary croupous exudate, the mucous membrane being torn when attempts are made to scrape it away.

This combined inflammation and necrosis in its typical form is met in the digestive tract of swine affected with swine plague, and is often seen

in calves in the pharynx and larynx following traumatic infectious influences, in birds in the mucous membranes of the head, and in horses in the larynx and trachea after drenching. There are a number of microorganisms (bacillus necrophorus, suipestifer, streptococci and others) which are capable of inducing coagulation necrosis of tissues and giving rise to the anatomical picture of a diphtheroid inflammation. 
(In man the name diphtheria is applied to a specific infectious disease caused by bacillus diphtheria, usually accompanied by deeply penetrating fibrinous inflammation of the pharyngeal mucous membrane. Anatomically similar conditions, due, however, to other causative agents, are spoken of as diphtheroid, to distinguish them from true diphtheria. This name diphtheria has not been happily chosen, however, as it really means inflammation of the membrane.)

Suppuratiz'e Inflammation with its product pus (Latin, pus; Greek, $\tau \dot{\partial} \pi i o v)$ is characterized by the liquefaction of the exudate and inflamed tissue (purulent softening) and by the important presence in the exudate of fatty degenerating leucocytes which have lost their power of movement; in addition it is always the result of infection with microorganisms known collectively as the pyogenic bacteria (pus bacteria). There are a number of these microorganisms occurring free in nature, mainly belonging to the groups of staphylococci, streptococci and colon bacilli, which are liable to gain access to the tissues through traumatic lesions or which may even penetrate from the unbroken surface through the pores of the skin

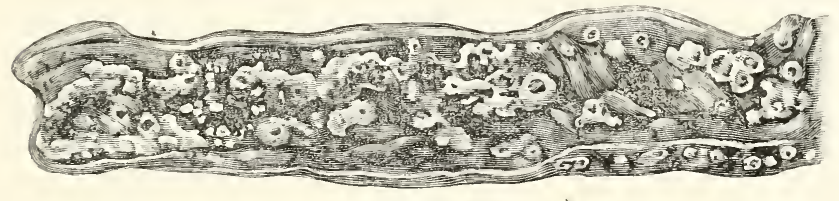

Fig. 55.

small intestine (laid open) of hog showing diphtheritic inflammation in swine plague.

or through lymph follicles. In addition to these specific pyogenic bacteria, whose most important influence is the production of pus, there are many other microphytes which are also capable of inducing suppuration, but which, because of special peculiarities of their toxic properties and the peculiarities of the diseases which they cause, receive special names, as the glanders bacillus, bacterium of chicken cholera, and actinomyces. Wherever suppuration occurs in a part an infection is to be suspected. This statement, first emphasized by Lister and Hunter, and of the utmost importance in surgery, is not impaired by the fact that suppuration may be artificially produced in experimental manner without the aid of bacteria by means of aseptic chemicals as by subcutaneous injection of turpentine, croton oil, chloride of zinc or corrosive sublimate, under conditions which thoroughly exclude the possibility of infection. Such an aseptic suppuration occurs under natural conditions only in the rarest instances, and differs from the ordinary bacterial suppuration 
20 ou dentoite local lumitation. Bacterial suppuration is progressive and is eccompanied by a moure co less setere ébrile gentral reaction on the pati of the subject.

As it is equally fossible to cbtam a suppurative infammation by means of sterile grem-iree cuivures of the progenic organisms or Eltrates which ondain coly the metabolic froducts of the bacteria. It may be accepted that the pyogenic action of bacteria is ancthe: instatoe of cherrical activity : an imprant factor. howerer, being the prestace of these lurng coganisms which multiply in the tissue. The ouncestion in spupuration is therefore an xtiological one. The pycetic organisms exer. apparently br means of their toxines which are diffused through the tissues, an extremely strong chemovectuc stimulus upm the levoocytes and in this way induce a greatly increased emigration of these cells: a: the same time ther have an importent harmf id infuence upon the walls of the resse's and nerves, th = causise bycerzomia and rotumisous exulation. and produce maitrials which destrcy and Bquefy the Lssuts which they involve. Their fowe of Iquefing animal alloumen and gelatine may be recognized in cultures of the orranism arown in solid gelatine and solidised birod se:um. thest media teing dissolved by peptonizing enzimes froluced tit the bactera. Letber has puinted out, bowere: thet diquefaction of the ussues is to srme degree due to the infuence

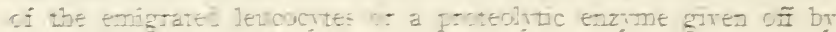
these cells. for examgle. the lutuefaction atrending streptococcus infections must of necessity be caused by the cells. as these organisms

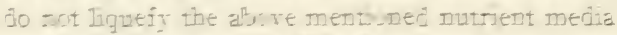

Pus appeers as a dutil yellsw. Tellowish-green or gravish-whice Juid. opaque and creamy thin and mikn onore thich like eggalbuten, accurdiser in the a ount of fiasma it contains. becoming reddish-tray of oi a caft-au-lait olich in case ci greater diapedesis of the red corpuscles It is citen mixed with fibrit in the form of small flatues of shreds. Which cires the pus a doughr consisience Iftrimo-fwre int crudare! and Fils the tissues with a Sne reticulated structure

Unde: the microscope pus shom great numbers of leucocytes. main'y of pripuruclear of polymorphonuclear type showing instead of one suncle round tucleus a number of nodular tbiscuit and clcrer-leaf furms and iragmented protions of nuclear sutbetasce. The crotutually frenetrating leucroctes, among which are also mononuciear examples. are similet to throse found in the birord In most of them, and evemtually in all mecnosis is recognizable because of noclear fragmentation and nore certainly br the presence of fatty 
degeneration. The cells become highly granular, the protoplasm full of fat vacuoles, and irregular, and finally the cells become nothing more than a bunch of fat globules held together by the protoplasmic remains (fatty granular cells) or falling apart into fatty detritus. The lencocytes, now known as pus corpuscles, and incapable of further motility, are suspended in the fluicl portion of the exuded plasma, here known as the

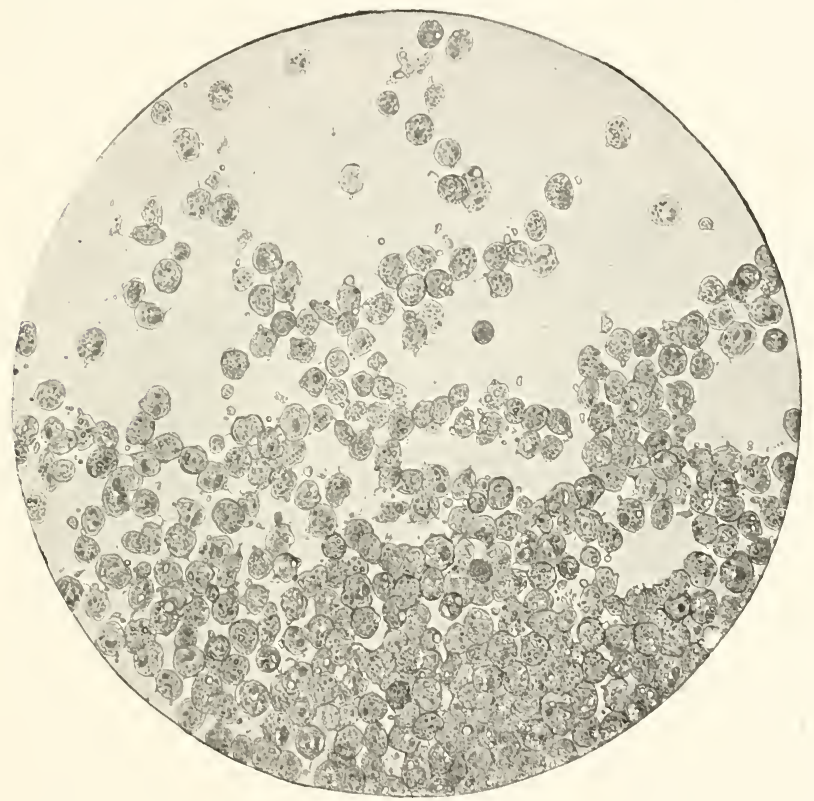

Fig. 56.

Pus from cow; unstained microscopic preparation of pus cells (highly magnified).

pus scrum [liquor puris]. or are deposited in the meshes of the tissue. The cells of the tissue in which the suppuration has taken place are also the seat of marked fatty degeneration; the connective tissue corpuscles and cells of the vessel walls often exhibit phagocytic appearances and are found with lencocytes englobed within their substance; and in chronic inflammations always multiply, thus leading to restoration of the lesion or its encapsulation. The pyogenic 
bacteria are usually found in comparatively large numbers in the pus serum and sometimes the leucocytes are loaded with them. According to the manner of commencement of the process a single variety may be found present as a perfectly pure culture, or there may be a number of forms associated together. As occasionally occurs in chronic cases, if no microorganisms can be discovered it may be assumed that the bacteria have gradually died in the pus and have undergone disintegration. If the suppuration occurs on the surface of a mucous membrane it is spoken of as a purulent catarrh,

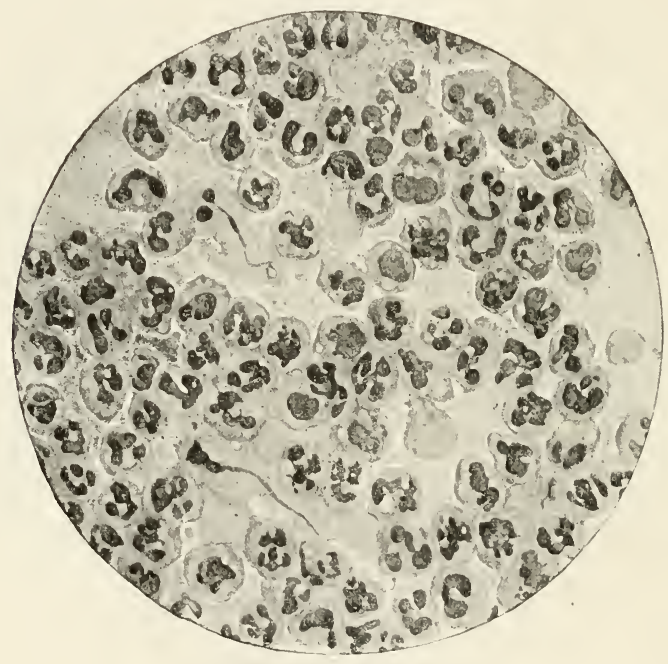

Fig. 57.

Film preparation of pus cells, stained with fuchsin (highly magnified).

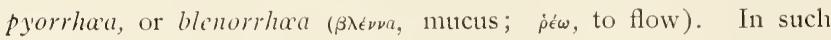
cases the blood vessels of the mucosa and submucosa are found dilated, containing large numbers of leucocytes, and the latter cells profusely accumulated in the meshes of the whole mucous membrane and penetrating between the epithelial cells of the surface. The epithelium is loosened, often hanging loosely in shreds. In addition the formation of mucus is increased, and many epithelial cells may be found transformed into goblet cells.

This viscid secretion, mixed with desquamated epithelium and 
emigrated leucocytes, constitutes the fluid catarhal secretion. On serous surfaces exudation is also attended with desquamation of the endothelial cells, and the exudate accumulates in the cavity lined by the serous membrane. A collection of pus in one of the large structural cavities of the body (pleura, joint, antrum of Highmore) is called an empyema ( $\mu_{\mu-\pi v \epsilon \hat{v}}$, to contain pus). When pus is distributed all through the spaces and meshes of tissue, it gives the latter a dull yellowish-white or gray color and can be expressed trom it in drops by squeezing between the fingers (purulent infil-

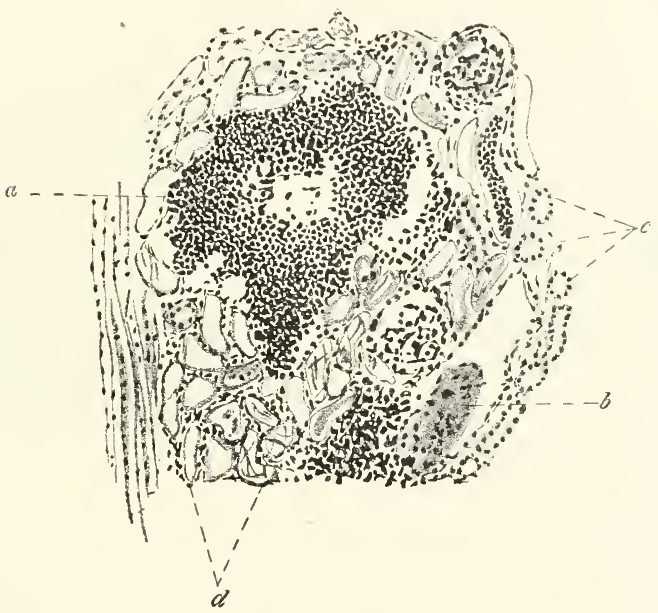

Fig. Is.

Purnlent nephritis of mixed type, from calf : $a$. microscopic alsseess : $b$, embolus : $c$. normal urixary tubules; $l$, nrinury tubules deroid of their epithelinm, containing coagulated material.

tration). Where it collects in larger lymph spaces or occurs in cavities formed by local liquefaction of the tissue, it constitutes an abscess (pus sac), that is, a hollow space filled with pus: from progressive tissue destruction and continued entrance of leucocytes such an abscess may attain considerable dimensions (size of a fist or head). A circumscribed collection of pus in the stratum Malpighii, elevating the keratous epiderm over it, constitutes a pustule (pus vesicle). When a purulent infiltration extends over a large area of subcutaneous, submucous or other loose cellular tis- 
sue, with production of spaces filled with pus and confluence of abscesses the condition is called a phlcgmon. Where the tissue softening or the gravitation of the pus into dependent positions allows the exudate to escape the abscess is said to break or perforate; and if in such instances there are formed narrow canals lined with pus and reaching to the surface these are called fistulc. A loss of substance upon the external surface or in mucous membranes caused by suppurative destruction of the tissue is known as an ulccr, the process as ulccration.

In chronic suppurations in the zone between the dead tissue breaking down into pus and the normal tissues, there occurs a fibro-

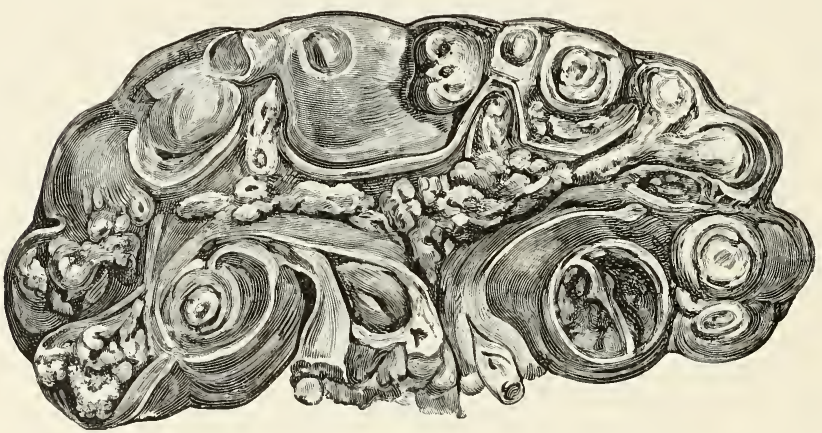

Fig. 59.

Section through a calf's kidney riddled with embolie abscesses; calyces and pelvis full of purulent masses.

angioplastic proliferation, as a bacony, light gray, firm tissue. The suppurating focus is encapsulated by this dcmarcating grocith, that is, it is enclosed in an absccss wall, which on its inner surface usually has a grimy, grayish-yellow or slate-gray color. As long as virulent pyogenic bacteria exist in the cavity this abscess membrane continues to give rise to more pus [only in the sense that the exudative processes which underlie the appearance of the lencocytes and fluid are largely going on within it, and not in the sense of a secretion as formerly believed] in the focus, for which reason it is sometimes known also as a pyogenic membrane. The involuntary muscle and elastic tissue of arteries exhibit marked resistance to purulent softening: in suppurative cavities, as of the lungs or udder or of a muscular tissue, vessels are often found as bridge-like 
bands running across the space, perhaps as thick as a lead pencil, their walls not destroyed by the suppurative process but hardened by cicatricial proliferation and resisting destruction.

The vascular connective tissue which develops upon exposed suppurating surfaces (ulcers, surfaces of wounds) presents a reddish-gray color and a granular or finely nodular, uneven appearance (gramulation tissue) ; purulent exudate arises from this also so long as the nicroorganismal canse of inflammation continues active. This tissue serves to restore the tissue loss, filling out the cavity into scar tisstue (cf. Regeneration).

With multiplication of the pyogenic organisms in the affected tissue not only does the local suppurative inflammation become correspondingly prolonged, but there arises the probability of its extension. The bacteria advancing along the lymph spaces give rise to fresh inflammatory reaction in a constantly expanding area. The fact that the leucocytes have wandered into the suppurating area, may take up some of the bacteria and carry them some distance away, until stopped by the occurrence of paralysis and death and their transporting function thus ended, gives an excellent opporttnity for the entrance of pus-producing germs into the lymph channels, lymph nodes and even the blood. Malignant and actively multiplying organisms, over which the bactericidal forces of the blood have no influence and the phagocytic cells no power, are thus likely to set up new foci of suppuration, so-called metastatic suppuration, in the lymph glands and any other places into which they have been carried as emboli.

We speak of hamorrhagic inflammation, where the exudate contains a notable admixture of red blood cells, and is consequently of a reddish, grayish-red to dark red, chocolate or café-au-lait appearance. Serous as well as fibrinous and purulent exudates may assume this character, which may be regarded as indicative of some especially severe disturbance of the vessel walls, which in turn has occasioned marked slowing of the blood current in the inflamed part, diapedesis of the erythrocytes or actual rupture of the ressels, and is therefore accompanied by stasis and hæmorrhage.

Putrefaction of the crudate and of the inflamed tissue (ichorous, gangrenous or putrid inflammation) is necessarily the result of the invasion of putrefying bacteria into the necrotic tissue and the masses of blood and exudate, dead material like the tissue itself. This combination of inflammation and moist gangrene, or, rather, the secondary changes produced by gangrene, breaks down the tissue or exudate into a dirty, grayish-green or dark green, malodorous 
material and indicates an unfavorable prognosis for the inflammation because of the almost unavoidable absorbtion of toxic matter into the fluids of the body.

All chronic inflammations, whatever the character of the exudate, result in the production of vascular connective tissue; the existence of a bacony, indurated, semitransparent or opaque milky tissue in or about an inflammatory area is a distinct evidence of its chronicity. This is said to be a productive inflammation. What factors are responsible for this tissue formation cannot be definitely determined. It may be assumed that the same chemotactic influences, which are at the bottom of the escape of the leucocytes from the vessels, stimulate the physiological formative energy of the cells, in other words exert a demand for growth. The young cells derived from these fixed cells are capable of some degree of motility and indeed do move; and the growing connective tissue cells and endothelial cells are forced to take the direction in which chemotactic and nutritive materials are located. The primary exudate, especially fibrin, possesses this power of attraction, the fibroblasts beneath a fibrinous covering being found actively proliferating and pushing into the fibrin. It is possible that the infiltration of a tissue with exudate directly occasions an excessive nutrition of the cells, enabling them to form more protoplasm and then divide; and again the formation of spaces and the loss of substance of the tissues should be considered, these factors favoring the development of inflammation, and having a tendency to increase physiological regeneration by release of tissue tension. The area of the proliferation depends upon the extent and duration of the inflammation. As already stated, the embryonic tissue appears in the form of granulations upon free surfaces (wounds, ulcers, fistulous passages, serous membranes). With a reddish-gray or fleshy red color, it presents a granular, uneven, undulating surface, from which in aseptic inflammation is given off a serous, reddish, viscid exudate, or when bacteria are present a purulent fluid. The granular appearance which gives to the tissue its name (granulum, a small grain) is due to the projection of the outward growing capillaries which extend out in loops and intertwine about the arteries like the top branches of the trees in a leafy wood. The precise tint of the granulation tissue, at times more deeply red or again more grayish red, depends upon the relative engorgement of the capillaries or the predominance of the gray color of the cells lying between the vessels. These intercapillary cells are leucocytes and fibroblasts, usually spindle shaped. On serous surfaces granulation tissue is apt 
to present a villous appearance, with finger-like, reddish and later whitish processes (filaments); growing in a fluid exudate this new tissue is found floating and waving back and forth with the movements of the viscera. When there is motionless contact with an opposed and similarly inflamed surface, the two surfaces become adherent, at first by the embryonic tissue, but later by capillary anastomoses and the thorough interlacing of the developing tissue. Thus adhesiz' inflammation, the formation of adhesive bands, often in the form of broad connective tissue cords, results. When

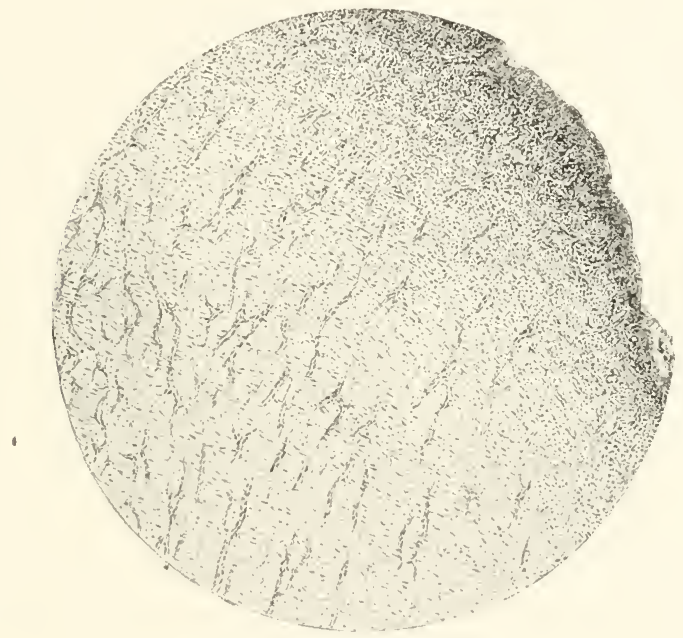

Fig. 60 .

Granulation tissue from skin of horse.

the embryonic tissue develops beneath a serous surface it may give rise to broad flat pale areas of thickening, the so-called milk patches. About abscesses it forms abscess walls; along fasciæ or extending along other connective tissue structures (stubmucous, subcutaneous tissue or about fistulous passages) it forms extensive indurated layers. When the process involves the connective tissue structures of an organ diffusely, as between the columns of liver cells or in the kidney, this interstitial inflammation occasions a widespread firm induration of the organ (induratize inflammation). The inflammatory embryonic tissue gradually becomes pale and firm and 
tough, the young fibroblastic elements like the cells from which they were derived giving rise to a fibrillar intercellular substance (cf. Regeneration). The bundles of fibrils continually growing thicker add firmness to the tissue, and with the cessation of inflammation the young tissue shrinks gradually to smaller bulk. By such changes it is transformed into scar tissue, here producing the depressed scars of surfaces, there constricting the lumen of tubes (stenosis, atresia), or again permanently and securely enclosing necrotic tissue or foreign bodies (encapsulation). Productive inflammation usually stops when the lesion which caused the inflammation has been finally dealt with, that is after elimination or encapsulation of the foreign body, necrotic tissue or other substance which originated the inflammatory process. Where, however, the irritation continues and final cicatrization is delayed the proliferative changes sometimes take on an exuberant character. This may be particularly well seen in superficial granulations, which may grow up out of the lesion as projecting tumor-like masses (zwild flesh, caro lnturians, granuloma) [proud flesh] perhaps as large as a human head. Indeed, interstitial proliferation of fibroblasts proceeding uninterruptedly for a long time may produce a mass of young inflammatory tissue far in excess of the original tissue it replaced and producing huge connective tissue enlargements (fibromatous inflammation, fibrous hypertrophy). On mucous membranes as that of the gall bladder, such proliferations meet but little resistance from the fluid in the cavity and often give rise to villous enlargements (polyps).

Specific Inflammations.-There are a number of parasitic microorganisms or infections which induce inflammatory changes but which give rise to tissue reactions of such special peculiarity that it is possible to conclude the agency of some one special infection from the nature of the inflammatory proliferation and the changes which take place in the products of the inflammation. Although the ordinary inflammatory irritants, the thermic, toxic and mechanical causes, act according to the intensity of their influence to caise now this now that type of exudate, there are some causative influences which give rise to inflammatory reactions invariably having the same appearance and constant characteristics. These infectious agents are said to act specifically (that is peculiarly). Usually these types of cisease are considered under special names, and will here be treated of in special chapters (v. Tuberculosis, Actinomycosis, Botryomycosis, Glanders). 
Parenchymatous, Degenerative Inflammation. - The inflammatory processes almost exclusively run their course in the rascular connective tissue of the organ; the other constituents, gland cells, muscles or nerves, take a more or less passive part. Inasmuch, however, as there may often be noted in the tissue elements constituting the organic parenchyma nutritive disturbances and regressive metamorphoses, either as primary changes produced by the cause of the inflammation, or as secondary alterations, due to the inflammatory process itself, it has become more or less customary to speak of such instances under the names parcnchymatous or degeneratic inflammation. Due to the action of irritants which primarily cause inflammatory changes in the interstitial tissue, there often occur such changes as cloudy swelling, fatty degeneration or necrosis in parenchymatous organs like the liver, kidneys or brain, of so marked a character that the real signs of inflammation in these cases almost entirely disappear, and perhaps can only be demonstrated by means of the microscope. The process and condition might well be summarily relegated, because of its principal features, to the degenerations. As the type of the degeneration is sometimes a complex one, made up of a number of forms of regressive changes, it is customary when some of the other features of inflammation coexist (hyperæmia, swelling, induration) and the gross anatomical picture does not necessitate their separation, to employ the term "degenerative inflammation" for the combination of the two processes.

[The editor feels that here too much stress is laid upon the passivity of the parenchymatous tissues in inflammation. They are, it is true, not the elements which strikingly react in the process, and their regressive changes are usually much more apparent than their active participation. Yet they do react and in practically the same way, as by proliferation, to some degree in this or that inflammatory process. A hepatitis is more apt, it is true, to exhibit the liver cells swollen and granular, yet occasionally there are found calls with karyokinetic figures in their nuclei; direct nuclear division is not infrequently seen in the nuclei of muscle fibres in the case of myositis; the axis cylinder buds in the inflammation caused by traumatic injury of a nerve, or the new growtl of muscle spindles in the inflammation about a similar lesion of muscular tissue. argue to the same end. Proliferation commonly takes place in the epithelium of an inflamed mucous membrane or in a gland, and while parenchymatous cells probably take no part in ordinary encapsulation of foreign bodies, they may take part in elimination. They probably exert no unimportant part in the destruction or dimunition of virulence of many toxic chemicals, as is a generally accepted function of the liver; and their aid in excretory elimination from the body of the same type of irritants, and in a minor measure of particulate elements, as unfit and perhaps harmful albuminous granules, cannot be overlooked. That they are in a way phagocytic is evinced by their assumption of pigment nuterial, changing it, it is true, in many cases by their metaholic activity. A microorganism may lodge upon some epithelial cell on a micous surface. on a duct, or even in an acinus of some gland, and fail to be dislodged. The cell may react to the irritant microbic influences by pouring out a volume of secretion, possibly mucus, in which the microorganism is 
caught and with which it is carried away. Or the microbe may destroy the cell, which itself then becomes a further source of irritation. 'The cell may, it is true, desquamate from mere necrotic loss of adhesion to its basement membrane; but at times-and too soon to permit of classing the phenomenon as anything but the result of an inflammatory proliferation-one or more young cells grow up at its base and dislodge the cell with its bacterial burden, both being carried off by the fluid on the surface (as in a proliferative catarrh). As far as the proliferative feature of inflammation is concerned, it is essentially the same as the atthor has detailed in the chapter on Regeneration; regeneration is. one part of inflammation. Therefore it may be probably claimed that in some measure, often insignificant, it is true, but in some cases clear and well marked, all the capabilities detailed as regenerative for parenchymatous cells are possible for inflammation. One should therefore be willing to accept a prolifcrative parenchymatous inflammation as well as the more striking degencratic inflammation, which alone may be inferred from the author's paragraph as characterizing parenchymatous structures in the inflammatory process. Moreover, from a theoretical standpoint, what has just been said may be understood as indicating that parenchymatous cells are capable of participating in all the ways ascribed to other fixed cells; practically they do not manifest such ability in a degree comparable to that exhibited by the connective tissues. These features are considered further on by the author, who would exclude as normal some of these minor reactions.]

The nomenclature and classification of inflammatory processes inclucle considerations of the retiology (traumatic, toric, thermic, infcctious inflammations), the duration of the process (acute, sub. acutc, chronic) and the form of exudate and other anatomical features (fibrinous, scrous, purulcut, ctc.).

The scientific name (tcrminus téchnicus) used to express an inflammation of an organ is constructed by adding to the root of the Latin or Greek wor 1 for the organ the termination itis (really from the feminine form of the major name ending in $\eta s$ ) ; for example, $\pi \lambda \epsilon v \rho a$, lining of chest-cavity, $\pi \lambda \epsilon \nu \rho \hat{\imath} \tau \eta$ s, one suffering in the pleura, $\pi \lambda \epsilon v \rho i \tau i s$ (supply vososs), inflammation of the pleura. In this way for example are built up such words as: gastritis, inflammation of the stomach; arthritis, inflammation of a joint; peritonitis, inflanmation of the peritoneum; ostcitis, inflammation of bone, etc. In case of inflammation of the serous covering of an organ the prefix "peri" is added to the name ( $\pi \epsilon \rho l$, about, around); in inflammation of the connective tissue alongside of an organ, the prefix "para"

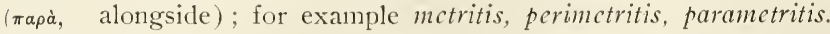
Special names are applied to some inflammations: pncmliznia (not pulmonitis) for pulmonary inflammation; angina for inflammation 
of the soft palate and neighboring structures; coryi a, for nasal catarrh; crysipclas for a special inflammation of the skin.

As above stated inflammation is a local reactive process in which the mechanisms of the body underlying its protection from harmful influences and its compensation for disturbances are aroused to an especially intense activity. This defensive and compensatory function exists even in normal conditions, as a vital cellular phenomenon of the animal body. Reactions of the same kind are almost all the time going on in the system; here and there cells are dying and are being made away with by phagocytosis, and are being replaced by regenerative proliferation. Microorganisms are deposited at many points on a mucous membrane in communicating relation with the outside world and are rendered harmless by phagocytosis; in the stomach and intestinal canal there often accumulate toxic substances, formed from the food, which cause a hyperæmia and are swept away by the increased secretion, or are taken up by the emigrated leucocytes (being quickly rendered inert in the liver) and only transiently excite some of the individual inflammatory phenomena. Insignificant and minor grades of inflammation are thus not infrequently induced, but these are not classed as inflammations. They are regarded as physiological phagocytosis, functional hyperæmia, physiological regeneration, each running its individual course; they are not considered as disturbances. We only speak of inflammation when these reactive phenomena occur together and in unusually marked degree and when the causative injury or the reactive process itself is productive of functional faults in the affected part.

Termination of Inflammations.-Since inflammation in many instances serves successfully to do away with useless and injurious materials and to restore the injured tissue or at least to replace faults with cicatricial tissue, it may be looked upon as a regulative and defensive effort of important use to the economy. In this adaptive effort, with much propriety of comparison, the advancing leucocytes may be likened to mobilized troops or to a body of street cleaners; but it cannot be said that the cells of the body pursue any fixed plan of procedure always under nervous control, but rather that the whole process is in reality the occurrence, it may be said the accidental occurrence, of phenomena of physiological motility and secretion of living protoplasm excited by external stimuli. The physiological component processes of inflammation are the same as the cellular functions of phagocytosis and secretion which take place in digestion, the result of mechanical and chemical excitation, 
peculiar, however, in that these cellular activities are not confined to any single organ but extend to all the organs, to the vascular connective tissue framework of all parts of the body.

The favorable or unfavorable outcome of inflammation depends upon accidental factors, upon the nature and mode of action of the causative agents, upon the location of the part injured and the degree of functional disturbance which the inflamed organ experiences. Of the individual reactions which in combination constitute inflammation, it can be accepted that phagocytosis removes structures killed by harmful foreign bodies, that the exudation exerts a digestive action upon and washes away injurious substances, that the blood plasma (and possibly too a secretion given off from the lencocytes) supplies antitoxic substances, and that the tissues are brought into a state of regenerative ability by the increased nutritive supply. All of these factors combine to bring about compensation for the influences of a variety of harmful agencies. When the irritant is removed or encapsulated the condition of special stimulation ceases and the inflammation ends in resolution, because no more fresh cells advance into the area and those which have previonsly escaped from the vessels either break down and are reabsorbed as detritus with the other parts of the exudate by the veins and lymphatics, or, in case they retain their power of movement, pass off to other places toward which they are attracted by nutrient matter.

The inflammatory process cannot invariably bring about complete regeneration of destroyed tissue; as a rule only the connective tissue is fully replaced, the specific elements which are lost, as glandular or ganglionic cells, not being reformed. It is in this way, by the formation of connective tissue to occupy the space of a lesion. that inflammation brings about healing of the lesion. The inflammatory connective tissue growth which paves the way for encapsulation of foreign bodies and demarcation of dead tissue is in the same way to be regarded as an attempt at healing.

However, there are also disadvantageous features connected with inflammation. Adhesions between organs, the occupation of cavities by masses of exudate, as in the lung or pletura, exudative effusions in the brain or in the kidneys, occasion functional disturbances, which, depending on the importance of the affected organ, may threaten the life of the subject; and inflammation may terminate in death. This does not, however, essentially alter the conception of inflammation as a defensive effort. Ribbert compares it to an army, which, it is true, is in the main a valuable organization, but which may fail at times to overpower a stronger enemy or which 
nay from the cost of organization and armament, financially ruin a land. The fatal termination and the local disadvantages of the disease are, strictly speaking, not attributable to the inflammation but to the causes of the inflammation.

\section{Tuberculosis.}

Tuberculosis is a contagious infcctious disease having a very wide distribution among man and the domestic animals, caused by the tubercle bacillus discovered by Robert Koch in 1882 , and characterized anatomically by the formation of minute nodular inflammatory foci (tubcrculum, a small node) which uniformly undergo necrotic disintegration and by their progressive increase destroy the tissue involved by them.

This malady, the most common of all diseases, manifests itself in man generally as a pulmonary affection of years' duration, causing pulmonary wasting and consumption (phthisis, from $\phi \theta t \omega$, to waste, consume), but also producing painful destruction of bones and joints, ulceration of the intestines, and tuberculous disease of the lymph nodes, brain and other structures. Year after year tuberculosis carries off over a million people in Europe, or nearly three thousand every day, and must therefore be regarded as the most deadly of all plagues, decimating the populace, and hurrying to death the efficient youth and destroying the earning capacity of families by the tedious course of the affection and death of their members.

The disease is no less a calamity to the cattle industry. Among domestic animals cattle and swine are most frequently affected; ten, twenty, yes eighty per cent. of the cattle brought to the larger stock yards in some districts are diseased. The financial losses occasioned by the affection of so many animals may be estimated by hundreds of thousands of dollars annually, for these include such varied elements as loss of weight and forced slaughter, impairment of milk production and of edibility of the meat, and sterility or transmission of the disease to offspring. Tuberculosis in cattle becomes doubly important when the danger to human health is contemplated, because the milk of animals with tuberculosis of the udder can transmit the disease to children and adults.

The effort to overcome this infectious disease, which has been continually on the increase during recent decades in man and in animals, has therefore come to be one of the most important aims of medicine, an object of municipal and national thought, and a matter of interest both to the community and to the individual. 
The communicability of pulmonary consumption was suspected even by the physicians of antiquity (Hippocrates, Isocrates) and had obtained some credence among the laity. More or less energetic measures (burning of beds and linen used by consumptives, disinfection of furniture and dwellings by means of smoke, etc.) were practiced as early as 1750 at Nancy, I782 at Naples and about the beginning of the nineteenth century were ordered and carried out by authority of court, but were without result because of their insufficiency to remove all the factors of infection.

According to Nocard* phthisis is said to have been declared a contagious disease in one of the Gothic laws.

Under the influence of the erroneous teaching of Broussais that phthisis arose spontaneously as a result of meteorological conditions, social misery, etc., and the widely spread mistaken conception which prevailed after the discovery of the tubercle bacillus to the effect that this disease germ is omnipresent (ubiquitous). efforts toward prophylaxis remained restricted. With the idea that predisposition was the most important fault it was considered impossible to root out the evil; or it was held that the difficulties involved in the campaign were insurmountable because of the impracticability of enforcing the necessary rules of procedure. The old suspicion of the contagious character of the disease received the first important confirmation in the studies of Klencke (1843) and Villemin (I865-r868), the latter proving by a series of positive experiments the inoculability of human tuberculosis and pearl disease of cattle into rabbits, etc., and establishing the identity of animal and human tuberculosis. Thereafter numerous investigators interested themselves in Villemin's teaching and repeatedly carried out similar experiments, sometimes confirming, sometimes opposing his results. (For details cf. writings of Johne and Nocard.) With the discovery of the tubercule bacillus by Robert Koch and the ingenious labors of this German investigator, which have completely unraveled the rtiology of tuberculosis of man and animals, all doubts as to the nature of the affection disappeared. Koch succeeded in discovering and demonstrating the tubercle bacillus by a special method of staining devised by him; and it is always possible by means of this method in case of tuberculosis to recognize the tubercle bacillus in diseased parts and material discharged from them both in man and in animals. This microorganism presents itself as a rod-shaped microphyte, from two to four micromillimeters in length and from three to five-tenths

* E. Nncard, Les Tuberculoses Animales; Encyclopedie Scientifique des Alde Mémoire. Paris: Masson. 
micromillimeters in width (about one-fourth to three-fourths the diameter of a red blood corpuscle). Koch also pointed out the possibility and methods of growing the tubercle bacillus in artificial nutritive media outside the animal body; and thousands of experiments have contributed to the proof that by inoculation of artificially cultivated germs tuberculosis may be reproduced in all its forms in any of the warm-blooded animals.

The tubercle bacillus does not grow free in nature; occurring only where it is deposited with tuberculous discharges. It multiplies in this climate but not outside the animal body and only within the organs of the animal or human economy. In the free state the ger'n of tuberculosis may retain its vitality and virulence when dried and in the dark for a long time (more than a year); but a variety of conditions may serve to render the infectious material harmless or destroy it. Sunlight, for ex-

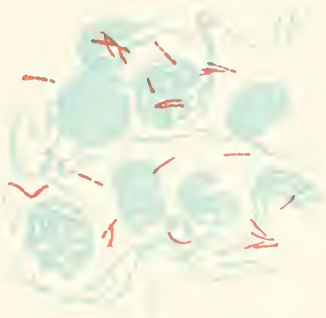

Fig. 61.

Tubercle bacilli in bronchial
mucus of cow; $\times$ about 1,000 . ample, will kill the organism in as short a time as two to four hours and even diffuse daylight will destroy it. Rain has a favorable influence in washing away the vehicle of the contagion and thus exposing it to light; a temperature of $50^{\circ}-70^{\circ} \mathrm{C}$. developing in manure will also kill it.

For such reasons tuberculosis must be regarded as a true contagious affection. Its transmission from one human being to another occurs chiefly from the expectorated sputum, which, dried and blown about as dust, gains access to the pharynx and air passages by inhalation, sometimes a portion being swallowed and finding its way from the intestines into the,tissues or sometimes infecting the pharyngeal tissues and air passages. Tiny drops of saliva or mucus containing the organism may sometimes be discharged in talking or sneezing and in case these are directly inhaled by a second individual, or if they fall upon the food and are ingested with it, they may give occasion for development of tuberculosis. The number of tubercle bacilli in such expectorated matter may be enormous; in a single drop of microscopic size there may be hundreds and it has been estimated that one consumptive human being may give off seventy-two hundred millions of tubercle bacilli in the course of a single day (Heller). 
The contagion is carried from animal to animal in the same way. Cattle and hogs affected with pulmonary tuberculosis discharge bronchial mucus loaded with tubercle bacilli by their coughing; and this is inhaled as fine spray-like droplets by other animals standing near by, or falls upon the fodder or into the water and with these substances may gain entrance to a new animal body. One of the most important sources of tuberculous infection both for man and animals is the milk of cattle affected by tuberculosis of the udder; milk from such a source may, as shown by Bangs, remain apparently normal even for weeks though it contain millions of tubercle bacilli. If swallowed in raw state, as used in the feeding of calves and pigs. such milk gives rise to infection of the body by way of the alimentary canal and chyle vessels, as proved by experimental feeding. The general milk from a dairy may become infected if there ?e but one cow in the stalls with tuberculosis of the udder, provided that cow be milked with the rest and her milk mixed with that from the other cows.

Again the intestinal discharges of consumptive animals contain the infection, either because these animals, as is often the case, have tuberculous ulcers of the irtestine, the bacilli from which become mixed with the excrement, or because the animals swallow their pulmonary expectorate and the bacilli pass through the bowel without being all destroyed. The vaginal discharge from cows with uterine tuberculosis furnishes another source of infection. Th? straw saturated with these infectious discharges, should it happen to be eaten by other cattle with their fodder, may serve as a means of transmission of the disease. Occasionally infection occurs in coition, as the vaginal secretion may be carried from one animal to another by the male, or the latter, when tuberculous, may transmit his own bacilli in the spermatic fluid. Tuberculous infection of cutaneous wounds is rare among animals, although occasionally in man post mortem section of tuberculous organs gives opportunity for this mode of infection.

The horse is usually infected with tuberculosis by eating straw (soiled with the tuberculous discharges of cattle); the dog often by licking up human tuberculous sputum; the cat by drinking tuberculous milk. The disease develops in fowls where the latter have opportunities to pick up human sputum or tuberculous discharges from horses, cattle or hogs; the excrement of chickens already affected with intestinal tuberculosis, however, affords the greatest chance for the infection of the other feathered inhabitants of the premises (soiling of food, ground, etc.). 
Tuberculous human beings and animals are sources of further infection only as they throw off materials containing tubercle bacilli. The foci of the disease which exist within the animal, shut off from the exterior, as tuberculous lymph glands, do not give off bacilli in such a way that they may be transmitted to another animal. For example, the milk of cows which do not have tuberculosis of the udder is harmless even though there are tuberculous lymph glands and serous membranes in the animals; the meat of a tuberculous animal is free from danger as long as it does not contain actual tubercles or as long as the lymph nodes situated in the flesh remain free from tubercle bacilli. (For further details consult Ostertag: Zcitschr. für Fleisch-und Wilchhygiene, I89o-1903.)

Concerning the question of inheritance of tuberculosis, observations running over a number of years upon the condition of calves born of tuberculous cows, with control by tuberculin injections and slaughtering (Bang), as well as a series of experiments upon pregnant tuberculous guinea pigs (Gärtner), give positive information. Tuberculosis may be acquired through the placenta. Nany examples of this have been found in newly born calves and children, and occasionally too in the miscarried foetus. In such cases the liver and periportal lymph nodes are invariably involved and the disease is apt to be more or less distributed to other lympl glands and organs. When it has been possible to investigate the mother of the tuberculous offspring, invariably a tuberculous affection of the uterus and sometimes of the chorion were found. The tubercle bacillus is scarcely likely to pass directly from the blood of the mother to the fotus (the blood is separate in the two parts of the placenta); but if during pregnancy the uterus should become involved by tuberculosis (focal, as in one cornu) the tubercle bacilli find their way into the placental milky humor and penetrate the fotal placenta, and then pass with the blood of the umbilical vein to the liver of the fotus. In this way the latter is born into the world a subject of tuberculosis; but it is possible that after birth the disease may progress very slowly and remain latent for months.

A conceptional or germinal infection, that is through the ovum or spermatozoon, is so improbable that it may be said to be entirely inpossible. Supposing the possibility that a fertilized ovum could be infected by tubercle bacilli, it would die from the caseating influence of these germs long before it could develop into an embryo, or else the embryo in its earliest stages of germ vesicle and formation of the blastodermic layers would already be but a degenerating malformation. When it is remembered that of the millions of sperma- 
tozoa which enter the female genital canal in coition only a single one passes through the micropyle of the ovum, it cannot but be a strange coincidence that just this very one should carry with it a tubercle bacillus. (Tubercle bacilli in the sperm are scarcely likely to adhere to the spermatozoa but are more likely to remain in the fluid.) And, too, if the fertilizing spermatozoon accompanied by a bacillus should penetrate into an ovum the bacillus as it multiplied would at once seriously interfere with the process of multiplication of the copulated cells and quickly put an end to the embryonic germ by coagulation necrosis. The assumption that tubercle bacilli may somehow lie dormant in an ovum in course of embryonic development, and later when the fœetus has been formed start to multiply, is without foundation and is in contradiction to all our knowledge of the growth of vegetable microörganisms in the animal body. [Mafucci's experiments in chicken eggs (Contralbl. f. Bakt. $u$. Parasitenk., I889, p. 237.) are usually offered as contradicting this contention. He inoculated eggs with tubercle bacilli and then incubated them. Out of eighteen eggs he obtained one dead and eight living chicks. He was unable to find tubercle bacilli in the dead chick, and examining the unfertile and rotten eggs which failed to hatch he was unable to be satisfied of any growth of the tubercle bacilli in them. His chicks were at first apparently healthy, but later died with well defined tuberculous lesions. That latency of infections is possible in relation with embryonic development is also indicated by the transmission of the psorosperms of pebrine, pointed out by Pasteur, through the ova of silk worm moths to the silk worms, which at first seem healthy but soon die from the disease.]

Attempts to prove from genealogical tables and statistics that there is a true hereditary transmission of tuberculosis fro:n parents to offspring must always meet with the objection that children or calves may be born free from the disease and may acquire the tuberculosis in the first years of their lives from living in the presence of their tuberculous parents. The above principal modes of transmission of the tuberculous virus have been proved by great numbers of experiments. With human tuberculous sputum as well as material obtained from tuberculous foci of the lungs, liver, lymph nodes, intestine, etc., human or animal, and, too, with pure cultures of tubercle bacilli, typical tuberculous disease can be caused either by feeding, inhalation, or by subcutaneous, intraperitoneal or intravenous injection in all susceptible species of animals. There are, however, certain differences, or rather dissimilarities, in pathogenic 
ability in tubercle bacilli derived from human beings and from various types of animals, referable to the source of the virus. For example, as shown by $\mathrm{R}$. Koch and Schütz in a long series of experiments, and even earlier by Putz (I882) and Sinith (I896), it is not as a rule easy to render cattle, sheep and swine tuberculous by using tubercle bacilli from a human source (even in case of inoculation of large quantities); and it is also difficult to infect birds with tuberculous material derived from man or mammals. These negative results at first caused doubt as to the identity of the tuberculosis of all individuals. However, it was recognized that no distinct line of difference could be drawn between the organisms, for even a few positive results were sufficient to show the identity of the affection in man and in animals. Positive cases of the kind required have been obtained in such abundant number and from experiments of indisputable accuracy that the possibility of infecting man with bovine tuberculosis and of the transmission of human tuberculosis to the domestic animals cannot be denied. Besides the older experiments of Bollinger, Klebs, Chanveau and the later ones of Sidney Martin, Frothingham, Arloing, de Jong, Stuurmann, Thomassen, Prettner. Klebs and Rievel who were successful in inoculating cattle with tuberculous virus from man, the examples collected by Johne. Ostertag and Nocard and Leclainche of wound inoculation in man, occasionally actually occurring in the course of slaughtering operations and meat inspection, speak strongly for the idea that bovine tuberculosis is an infection of no litthe importance to man. Important contributions have also been made in this connection by Johannes Fibiger and C. O. Jensen, going to show that tubercle bacilli virulent to man may also be highly virulent to cattle, and confirming the belief that many cases of intestinal tuberculosis in children are properly attributed to the ingestion of cow's nilk containing tubercle bacilli. von Behring arrives at the same conclusion. finding that some strains of tubercle bacilli obtained by culture from human source possess high grade of virulence for cattle while other strains have no pathogenic influence for cattle at all. Further the observations made by Eberlein and Cadiot, indicating the acquirement by man of the infection from tuberculous parrots and the rather common transmission of the disease to parrots from consumptive human beings, as well as the transmissibility by inoculation of bovine tuberculosis to apes, proved by Nocard, force the assumption of an xtiological relationship of the disease or of a family identity of the tubercle bacilli of heterogeneous derivation. [Recently Koch has brought forward anew the idea of specific dif- 
ference between the bacilli of human and bovine tuberculosis, but has met strong opposition at the hands of a large number of students. In this country Ravenel and Pearson have shown distinctly the identity of the microörganisms; and the work of these investigators in inducing immunity in cattle against bovine tuberculosis by vaccination with cultures of human tubercle bacilli of low virulence is of extreme significance.]

The variations in pathogenic power exhibited by the bacilli obtained from different sources find their explanation in the adaptation of the organisms to the different animal bodies. It is obvious and reasonable that, in case of transmission of consumption which has been going on for centuries from one human being to another, in which the tubercle bacilli of every generation always grow exclusively in the human body, the organisms may come to adapt themselves to the conditions of nutrition which the human system supplies to them, and that they attain their greatest infectious power for it and find more difficulty of growth in another type of animal body. In the same way tubercle bacilli which have passed through many generations in the bodies of herbivora become as it were acclimated, and are best able to infect again an animal of the same species. The tubercle bacilli of birds (whose normal body temperature is known to be $41^{\circ}-42^{\circ} \mathrm{C}$., and whose tissues and fluids from their known resistance to tetanus virus, etc., must have totally different affinities from those of mammals) are particularly changed in their biological characteristics so that they seem to be of a totally different stock; and, too, it is difficult to successfully induce a growth of tubercle bacilli from mammals in the avian body. However, now and again birds have been rendered typically tuberculous by inoculation with mammalian tuberculosis, and conversely horses (Nocard) have been infected with avian tuberculosis; and therefore the differences may be considered as merely expressions of a variability of biological properties. We must consider tuberculosis of man and the animals as cansed by one and the sane infections agency, which from long transmission through one particular animad species may dez'clop definite z'arietics and strains of unez'en pathogenic power. (For further details consult Nocard-Leclainche: Les Maladies Microbiennes des Animaux. III. Ed., Paris, I903.)

The anatomical lesions determined by the tubercle bacillus are as follows: After being conveyed into the body of a man or animal the tubercle bacillus multiplies into masses, provided it finds a suitable soil at the temperature of the animal : penetrates by growth into the tissues and is carried more widely by the wandering 
cells. Its deposition, along with the toxine which it produces, causes a reaction on the part of the tissue as about a foreign body, in which, however, the special toxic action of the microorganism takes part and gives rise to coagulation necrosis. The first evidence of reaction as shown by the studies of

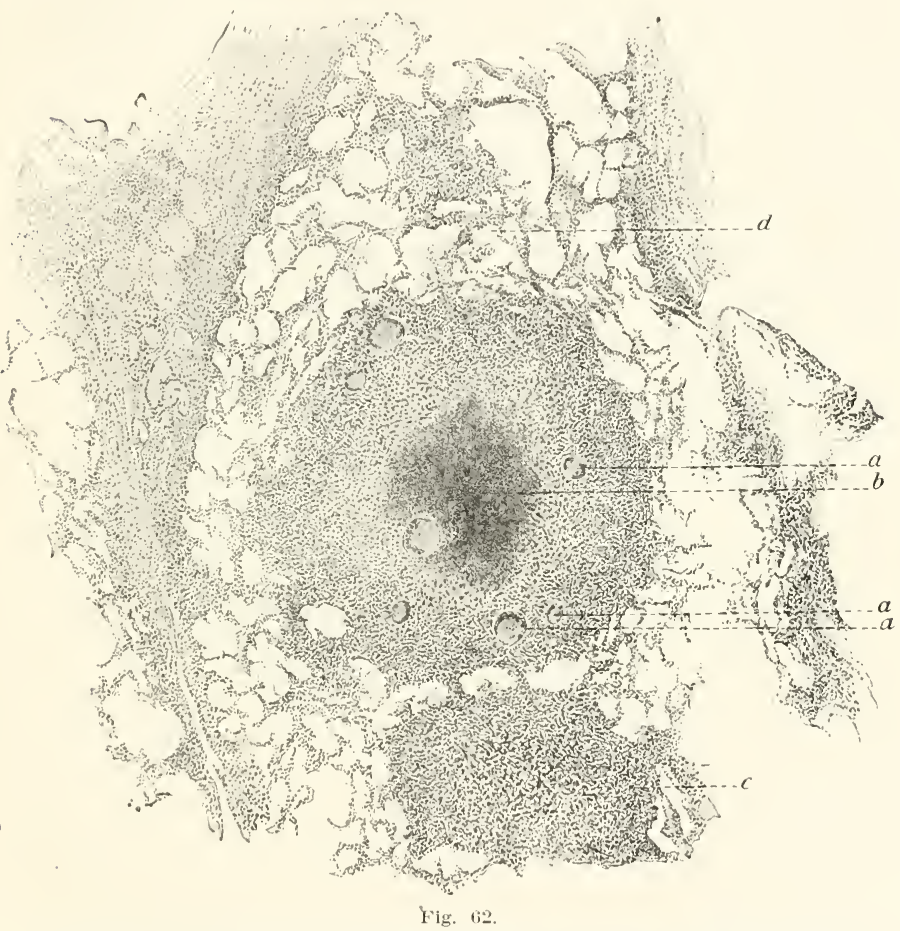

Embolic miliary tubercle from lung of horse (after Johne); $a$. giant cells: $b$, cheesy centre: $r$, small as ret not caseated tubercle; $d$, surrounding pulmonary tissue; $\times 90$.

baumgarten, Jolne and others is the appearance of karyokinetic figures in the fixed connective tissue cells and endothelial cells, and side by side with this an increase of the latter. The proliferating fibroblasts, among which usually giant cells occur, surround the tubercle bacilli; and further out immigrating leucocytes 
or lymphocytes collect about the periphery of the focus occupied by the bacilli. In this manner a rounded cellular nodule is formed, at first microscopic in size, but later increasing in bulk to that of the head of a pin or millet seed, and known when visible to the naked eye as a submiliary and miliary tubercle (milium, a millet seed). It is of a gray, opal-glass-like color, with a dead-white to yellowish point in the centre which indicates the early stage of coagulation necrosis (caseation). [The central cells of the unde-

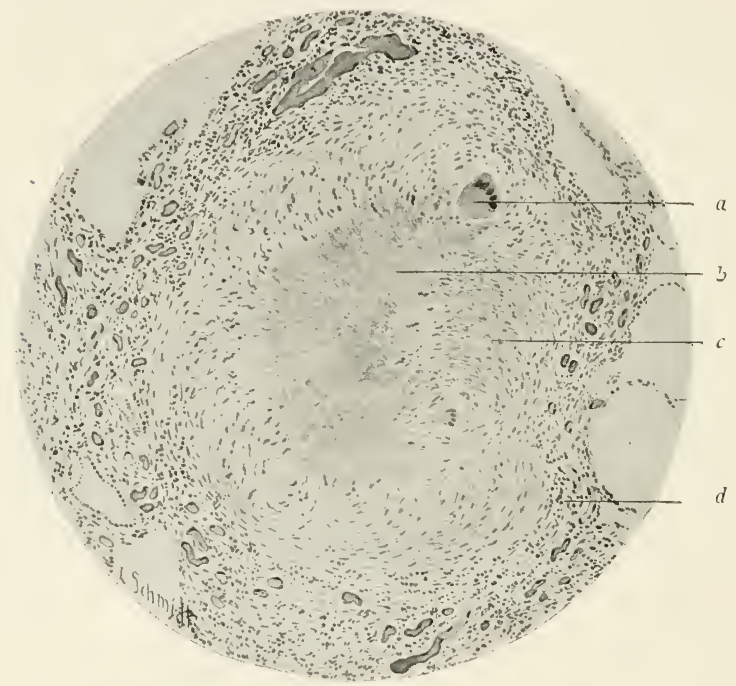

Fig. 63 .

Miliary tuberele (higher magnifieation): a. giant cell: b. caseated centre; c, zone of epithelioid cells; $d$, zone of lymphoid cells.

generated miliary tubercle, in and among which the tubercle bacilli are found, are of uncertain origin. They are commonly spoken of as endothelioid or epithelioid from the comparatively large amount of protoplasm and the rather pale and vesicular nucleus, and are distinguishable by these features from the ordinary fibroblasts. They vary in shape but are more commonly of a rather flattened spindle form than otherwise. The author's view that these are essentially the same as young connective tissue corpuscles and the product of proliferation of the fixed connective tissue cells is accepted by some; 
others believe, as the name endothelioid would suggest (epithelioid synonymous and used rather to indicate the general epithelial-like appearance than referring to the source of these elements), that they are derived from the endothelial cells, mainly those of the lymph spaces in which the bacilli are lodged. The true lymphocytic nature and origin of the peripheral zone of small mononuclear cells is commonly accepted. There is considerable uncertainty as to the mode of formation of the giant cells. According to some they are the result of the fusion of cells undergoing hyaline or coagulative change: and are therefore looked upon by these persons as evidence of beginning or advancing degeneration in the focus. From this standpoint a miliary tubercle when exhibiting one or more giant cells in its structure is essentially a degenerating tubercle. Others believe that they are the result of inefficient protoplasmic division of growing cells, in other words are of a proliferative type of development and not of the significance just indicated. Tubercles of fresh and undegenerated structure are commonly called gray tubercles; older and degenerated ones, yellow tubercles. I

The site of formation of the tubercle is in the connective tissue framework of the affected part or in the walls of the blood vessels and in the lymphatic foci of the tissue. This gives the cellular proliferation a connective tissue frame work or reticulum as a skeleton, which may, however, be in part, too, a product of the fibroblasts and giant cells, which of course also supply an intercellular substance. Although endothelial cells also take part in the formation of the cellular nodule these do not develop into vascular buds, and the miliary tubercle is avascular. The endothelial cells in their proliferation may occlude the lumen of the capillary vessels; and moreover by the growth of the cellular mass the vessels may be compressed. In contrast to these factors the tuberculous reaction may also present itself as a diffuse proliferation, without production of sharply defined nodules and with the formation of new blood vessels. In this case there is found a soft, gray or grayish red layer, especially seen in serous surfaces (bovine heart), which is not likely to undergo more than a slight caseation, or, if it does, only after considerable time. This, too, always has a distinct granular appearance looking as if caused by fusion of closely set miliary tubercles. This diffuse growth is due to the fact that separate groups of tubercle bacilli do not here oecur as the centres of nodular circumscribed cellular collections, but that large numbers of the organisms are scattered all through the tissue. 
This inflammatory reaction is not characterized merely by the marked collection of wandering cells in the tuberculous tissue, but also by actual exudation upon the surfaces of the organs. Especially in the pleural cavity of dogs this may be seen as a marked sero-cellular collection of fluid, the result of a purely tuberculous granulation; and in various tuberculous tissues microscopic examination shows the appearance of thread-like (fibrinoid. Schmauss and Al-

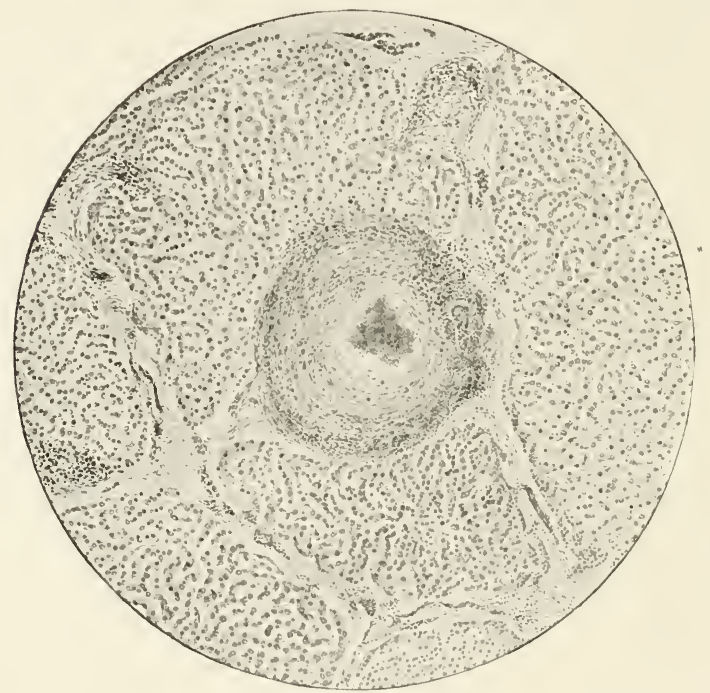

Fig. 64 .

Miliary tubercle in the liver of a dog (slightly magnified).

brecht) coagulation masses lying between the proliferating and degenerating cells and suggesting an exudative origin.

With the proliferation of the tubercle bacilli and their extension into the adjacent tissues the amount of toxic material given off by them and taken up by the lymph becomes increased. The caseation is to be ascribed to the influence of this substance; and in proportion to the latter the caseous areas grow larger as the reactive cell proliferation increases the size of the nodule. At first punctiform or streaked cloudy specks appear and the necrotic foci fuse into progressively larger and larger, dry, anæmic or (after a fatty 
(lisintegration) into soft, partly purulent, partly cascous areas of very variable size. Such necrosed parts may become impregnated with lime salts (especially in cattle) and eventually assume a chalky, plaster-like or sandy appearance.

Just as in chronic productive inflammation the fibroplastic tissue is changed into a firm cicatricial mass, the proliferated connective tissue cells of a tubercle are also able to assume a genuine fibro-

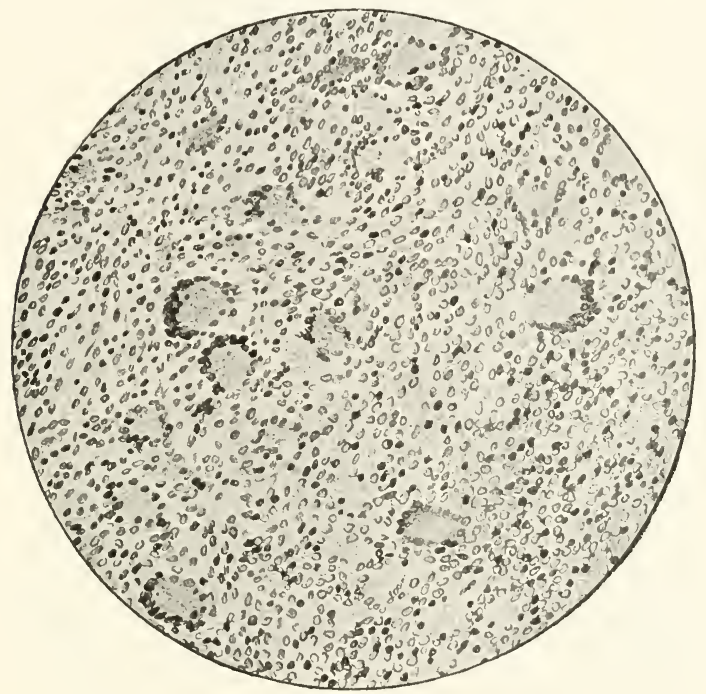

Fig. 65 .

Fungous tubercle with giant cells (without caseation) from the largnx of a cow.

plastic function, particularly in the formation of a fibrillar matrix and may thus give to the tubercle a firm fibrous character.

It may therefore be appreciated that the anatomical forms of tuberculosis vary considerably according to the tissue predisposition in the different animal species, and the extent and duration of the process. The principal forms are: miliary tubcrculosis, tubcrculous ulccration, tuberculous checsy infiltration, tubcrculous cazities, fungous granulomatous tubcrculosis (or tubcrculoma).

The miliary tubercle, the earliest product of the reactive process of the disease, as already stated, appears as a gray or yellowish-red 
nodule of the size of a pin head or millet seed; these may be single or multiple, may be scattered widely over a large surface and disseminated in the same way throughout the organic substance, par-

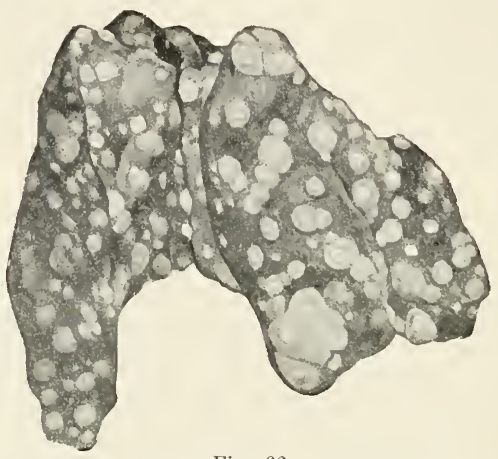

Fig. 66.

Tuberculosis of liver of a pheasant. The tubereles show a sharp limitation of the cheesy centres.

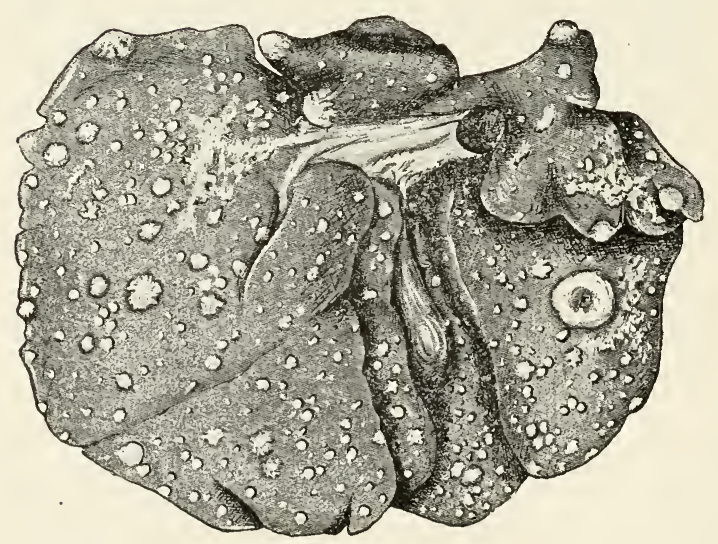

Fig. 67 .

Tuberculosis of liver of dog. (After Cadiot.)

ticularly on serous surfaces and in the lungs. They develop to visible size within from fourteen to thirty days after infection; the microscopic stages at the very beginning may be made out as early as from five to ten days. If the organ is the seat of submiliary or 
miliary nodules alone, all of the same age, that is of uniform size, it is said to be involved by acute miliary tuberculosis. With local multiplication of the tubercle bacilli, the nodules enlarge to the size of a lentil, pea or nut, these larger forms usually showing their origin from fusion or conglomeration of smaller miliary tubercles. This condition constitutes what is known as chronic miliary tuberculosis. On serous surfaces the formation of nodular masses gives rise to the so-called pearl discase.

The tubcrculous fungous granuloma is the result of a progressive formation of cellular nodules and their profuse growth on free surfaces; it is chiefly met with in the thoracic serous membranes and peritoneum and sometimes, too, in the intestinal mucous membrane. By the synchronous development of the nodules and of a vascular connective tissue growth, there may be produced grayish-white, gravish-red and yellowish-red tumor-like masses from the size of an egg to that of a fist, which are piled up about and over each other as large excrescences, covering considerable surface areas and reaching perhaps a weight of from twenty to forty kilogrammes. Caseation of these tuberculomata, which also occasion adhesions of the serous surfaces, at first appears in punctiform foci and iu small spots in the granulation tissue, as yellow or light yellow opacities, and can advance to complete transformation of the whole bunch of nodules into

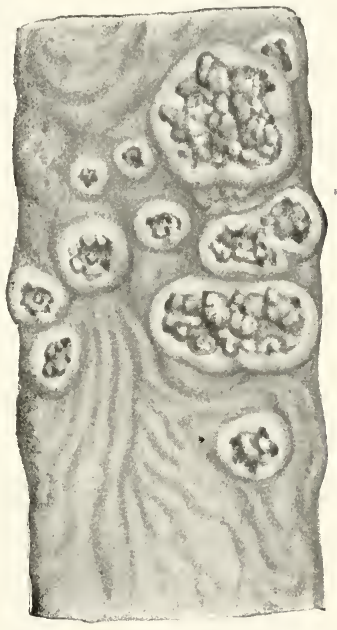

Fig. 68

Ulcerative tubereulosis of intestiue of cow. (Mucous surface of small intestine.) a mass which on section looks like corn-bread, yellow, hard, gritty and partly calcified.

Tuberculous ulcers, especially in the bowel, larynx and bronchial tree, result from the maceration and disintegration of the caseated parts as well as from a purulent softening of the tubercles developing in the mucous surfaces (lymph follicles). These areas of tissue destruction are circular or oblong, shallow or sometimes a millimeter in depth, as large as a lentil to a little over a centimeter in diameter, or in the bowel reaching perhaps the length of a finger and two fingers in breadth. The base of the eroded spot 
is mottled grayish-red and yellowish, and minute tubercles can be distinguished growing on the base and margins of the ulcer. The base looks granulated; the bordering wall of mucous membrane is thickened and sometimes a marginal circlet of projecting miliary tubercles can be seen about it.

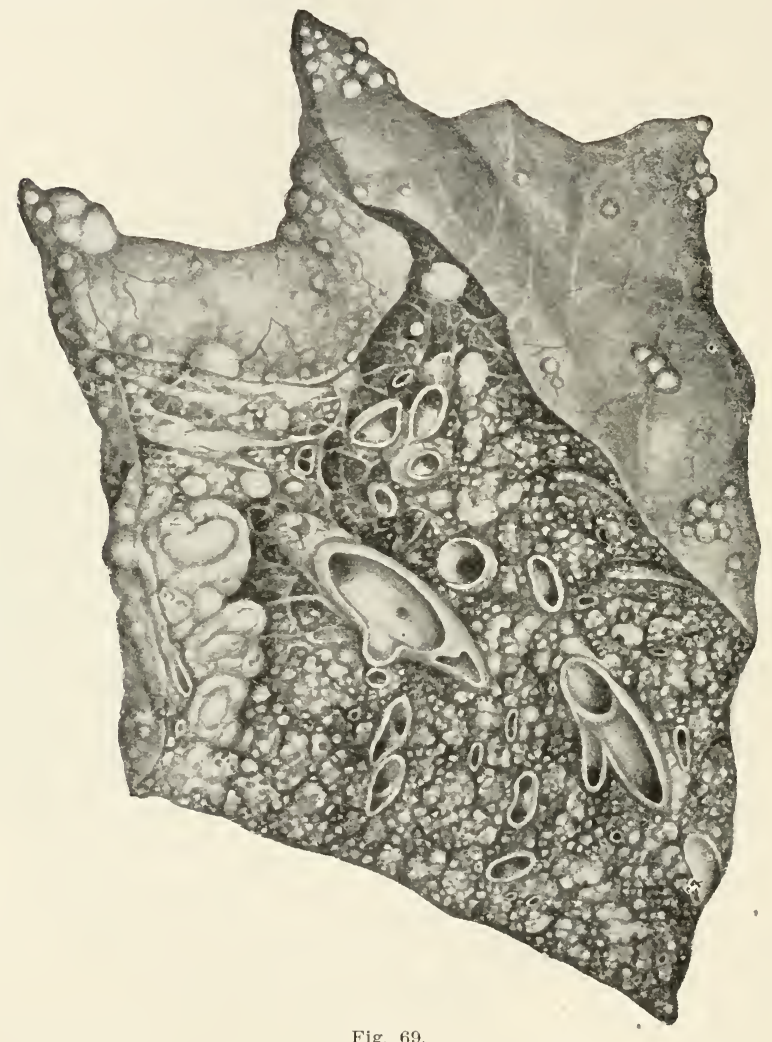

Fig. 69 .

Chronic miliary tuberculosis and lobular tuberculosis of lung of horse.

Cheesy tuberculous infiltration, apt to be found involving lymph nodes or lungs, changes the affected area of the organ into a very large, hard, densely caseated mass of a yellowish white to chrome yellow color, reminding one much of the boiled yolk of an egg. This change is apt to be very diffuse and is generally accompanied 
by the formation of a firm bacony connective tissue of the nature of a confluence of fibrous tubercles, or in other words a chronic tuberculous inflammation. Lymph glands, the seat of tuberculous infiltration, may grow to enormous masses as thick as an arm, or the size of loaves of bread. Bunches of tuberculous pulmonary lobules are converted into dry necrotic masses as big as a fist and comparable in appearance to Edam clieese or Swiss cheese; or sometimes whole lobes are changed into large, lumpy masses, hard, weighing over a kilogram, and riddled with points of cheesy softening.

Tubcrculous carities, developing in the areas of cheesy infiltration, are cavities varying from the size of a nut or fist to a span [a span is nine inches] and a half in diameter. They contain a greasy, caseous and slimy mass of detritus and are limited by a wall of pale, indurated fibrous tissue or by the tissue of the organ modified by the different color shades given by the tuberculous inflanmation, and giving rise to the cavitics by degenerative changes affecting it.

These more important types of tuberculous changes* may combine in various ways and there are apt to be, too, special modifications due to peculiarities of the tissues in the various types of animals. While cheesy areas in cattle usually have an intense yellow color, in horses and carnivora they are apt to be whiter and sometimes quite milky. They are not at all uniform in consistence, sometimes dry and cheesy, sometimes soft and pultaceous or possibly broken down into a pus-like fluid. Caseation may in some instances be absent and the tubercles may be of a more cellular character, looking like the tissue of lymph glands; or they may become hard and fibrous (in horse).

The entrance of tubercle bacilli into a tissue generally occurring at places where lymph nodes lie exposed (pharynx, intestine) at first causes a primary local tubcrculosis at the point of infection. How-

*For detailed examples of. Kitt, Lehrbuch d. spez. path. Inat., II. Aufl., Stuttgart, 1903. F. Enke, 
ever the point of infection may escape involvement provided the tubercle bacilli are rapidly carried onward by the lymph, under which circumstances the tuberculous inflammation may first show itself in the lymph glands. After feeding tuberculous material to hogs the mucous membrane of the intestine is uniformly unaffected and the disease primarily occurs in the mesenteric lymph nodes. [Ravenel has produced a similar primary involvement of the lymph nodes of the mesentery in dogs by feeding the tubercle bacilli rubbed up in butter, having previously starved the experiment animal for a time.] After being taken up by the chylous vessels the tuberculous virus passes into the blood by way of the thoracic duct and thence into the lungs, and after traversing the lesser circulation is carried to all of the organs. The different organs do not all show

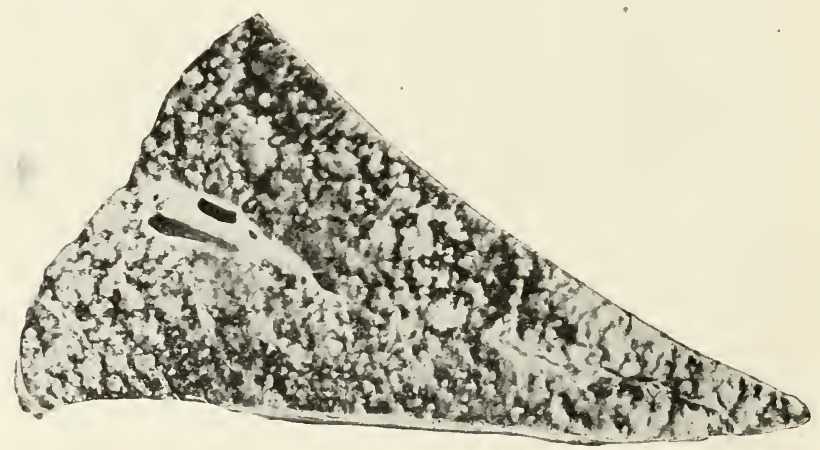

Fig. 71.

Portion of lung of horse showing miliary tubercles and tuberculous infiltration.

a uniform predisposition to the influences of the bacilli, that is, they do not all offer equally favorable conditions for the nutrition of the microörganisms. The lungs, lymph glands and especially the general lymphatic tissues (bone-marrow, spleen), are places where the bacillus most readily lodges. For this reason the lungs may become the seat of the primary involvement after feeding tubercle bacilli to an animal, and the existence of the pulmonary affection is by no means to be looked upon as indicating that infection took place by inhalation.

The primary, and in fact the only tuberculous focus may be encountered in the interior of bones, or in lymph glands or the testicle. In such instances isolated tubercle bacilli which have gained access to the blood may have been caught and lodged in any such 
situation and there caused the disease. Cienerally the whole path traversed by the bacilli is mapped out by foci of the disease, first the primary local focus and after it the neighboring regional infection, then the secondary tuberculosis of the lymph glands which receive the lymph from the affected part. From the latter along the lymphatics or, as above mentioned, by the chylous route, the germs find their way into the blood or may directly penetrate through the walls of the blood vessels in the course of the t11berculous proliferation; and then, depending upon whether many or only isolated bacilli are carried to the various organs, there is pro-

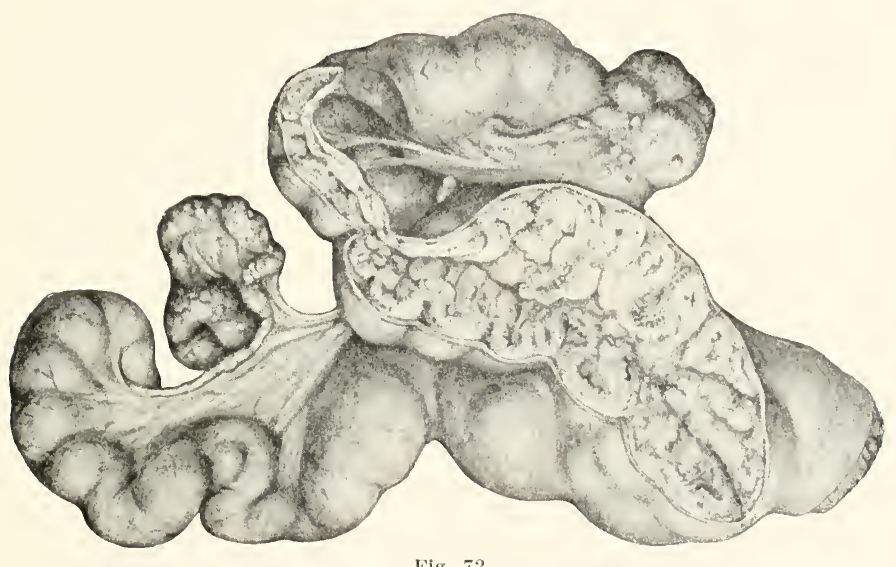

Fig. 72 .

Eterine tuberulosis, from cow.

duced a metastatic, hamatogenous, cmbolic, multiple or disscminated miliary tuberculosis of the lungs, brain, liver, kidneys, etc. Muscle (meat) shows the greatest degree of resistance, usually only the intermuscular lymph nodes being affected. This general invasion of the tuberculous virus with its resulting tuberculous foci is spoken of as gencral tubcrulosis. The metastatic miliary nodules of course increase in size, and by confluence and advancing caseation cause progressive extension of the tuberculous destructive changes. Finally, too, contact infection along mucous membranes occurs from the convection of the germs over the surface with its fluid secretion, as infection of the larynx from the lungs, of the vulva from the uterus, etc. 
The symptoms of tuberculosis appreciable during the life of the affected animal vary widely with the different situations of the primary disease. Fuller details than space here permits are contained in the publications of Johne, Siedamgrotzky, Albrecht, Lydtin and other authors and particularly the text books of Friedberger and Frölner, Dieckerhoff, Csokor, Nocard-Leclainche and Moussu. Pulmonary tubcrculosis manifests itself by a remittent fever, impaired condition of nutrition in spite of good appetite, induration of the skin (hide binding) associated with loss of subcutaneous fat, rapid and difficult breathing, a feeble dry cough, impairment of percussion resonance and the presence of rales. Chronic Hatulence, as pointed out by Johne, is a frequent evidence of the existence of greatly enlarged tuberculous mediastinat lymph glands from pressure on the gullet, interfering with belching. Tubcrculosis of the mammary gland may be recognized by a gradually increasing induration and diffuse painless swelling in one, two or all parts of the udder, with synchronous enlargement of the supramammary lymph nodes. In this condition the milk may be quite normal in appearance for weeks or at least only changed in that it is more watery, thinner (in the last stages only may one notice the admixture of small flakes of a pus-like appearance). Superficial lymphatic glands, as the retropharyngeal, often become very large in tuberculosis, hard and nodular. Tubcrculosis of the brain commonly shows the violent symptoms of meningitis (excitement, $11 n-$ consciousness). In tubcrulosis of the orary symptoms of sexual desire are sometimes observed. Tuberculosis of serous membranes can in some cases only be made out by the detection of friction sounds or by palpation of the peritoneum through the rectum; it may exist extensively without the occurrence of any emaciation of the animal (so-called "fat Frenchmen"), but on the contrary with preservation of an excellent state of nutrition. In the same way involvement of the deeper lymph glands, liver and intestine is apt to escape diagnosis during life.

Diagnosis can be positively confirmed by the microscopic examination of the various emanations (milk, vaginal discharge, bronchial mucus, etc.) by determination of the tubercle bacilli therein.

As a valuable aid to diagnosis especially in the latent forms of the disease, the use of fubcrculin may be approved; this is a decoction of cultures of the tubercle bacillus [sterilized by filtration through porcelain] which has the effect of causing a febrile reaction in tuberculous subjects, non-tuberculous individuals, however, manifesting no reaction to similar doses of the material. 
Tuberculosis can be successfully combated as purely contagious-infectious disease by regulations contemplating required disinfection of all effluvia containing tubercle bacilli. Recently a method of protective inoculation of cattle against tuberculosis has been discovered by v. Behring, immunizing the animals by means of strains of tubercle bacilli of low virulence. [Dixon in 1889 - found that he obtained resistance in rabbits against virulent bovine bacilli after having previously inoculated them with material from an old laboratory culture containing club-shaped and branching forms. It is of interest, too, that successful vaccination of cattle against virulent bovine tuberculosis has been practiced by Pearson in this country, using cultures of human tubercle bacilli of low virulence.] Cows which have obtained a high degree of immunization produce milk which possesses a certain amount of immunizing bodies; and it may be hoped that either by using such milk or the serum of highly immunized animals there may be obtained also a curative method for consumption in man.

\section{Glanders.}

Glanders (malleosis) is a contagious disease caused by the glanders bacillus (bacillus mallci), occurring in the horse, ass and mule, but very infectious and dangerous to man and also transmissible to carnivora, sheep, goat, guinea-pig, hẻdge-hog, field-mouse, rabbit and camel. The cow is entirely immune even after every effort at inoculation; and the hog can be infected only with considerable difficulty; domestic fowls are also immune.

This infectious malady causes both in its acute and chronic course (the latter perhaps persisting for years) a purulent fibroplastic inflammatory change, developing mainly in the skin and respiratory mucous membranes. Pathological changes also invariably occur in the lymph vessels and lymphatic glands; and in addition metastatic foci may follow in the spleen, liver, testicles, kidneys, sometimes in the flesh, and finally a general blood infection results.

The microörganism of the disease, the glanders bacillus, discovered by Schütz and Loeffler, is always present in all tissues the seat of glanders changes, in the discharges of the subject mixed with glanders pus, and is sometimes found in the blood. It is a short rodshaped organism, measuring from two to five micromillimeters in length and from one-half to one micromillimeter in widtll. It does not exist free in nature, but multiplies exclusively in the animal body (cf. contagious diseases, p. 7r) : only under special conditions 
can it be grown* in the incubator on nutrient media (glycerine boutillon, serum, agar, potatoes).

Transmission of the infectious agents from one animal to another or to man is the result of a close relationship (living together) or actual contact of the diseased animals with others; and may take place when the vehicle of the contagion (pus, nasal discharge, etc.) gets upon the fodder and in the water, or when discharged in coughing or blown out in sprays of fine drop-

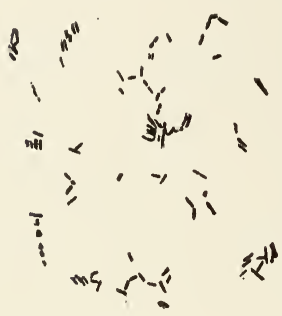

Fig. 73.

Glanders bacilli (from cul-
ture). lets and inlaled by other animals. If the material should lodge on the skin and mucous membranes it may pene- trate even through apparently intact pores (transmission by blankets, saddles and harness soiled with the pus). Artificially the disease may be reproduced by cutaneous, subcutaneous, intraperitoneal and other forms of inoculation, and by feeding the glandiers germs to the susceptible animals. From the experimental studies of Nocard and Leclainche, Galtier, Conte, Renault, Cadear and Malet, the smallest lesion of the epithelium in mucous membranes may serve as a point of entrance for the germ, as the trivial lesion which is produced by rubbing with a piece of linen cloth or the tiny epithelial abrasions about the nostrils caused by bits of foodstuff. In guinea-pigs the infection may be successfully produced by dropping the glanders bacilli into the conjunctival sac upon the intact mucous surface, probably through the lymph follicles and tear ducts. In feeding experiments the lymph-follicles of the pharnyx and intestine are the points of entrance of the infection. Inhalation of the germs distributed by spraying also occasions infection of the pharyngeal mucous membrane. The infection develops most rapidly after intravenous and intracranial injection.

The bacilli multiply at first locally upon and within the tissue (lymph spaces, lymph follicles) to which they have gained access, and produce by the influence of their metabolic products a progressive cellular necrosis with destruction of the nuclei (formation of granules and globules, fragmentation of chromatin) and transformation of the cells into finely granular detritus. Coincidently there is seen a continual emigration of the white blood corpuscles,

* For details ef. Kitt, Bakterienkunde fur Tierärzte. IV Aufl, Wien., 1903. 
that is, a local inflammation, which in the chronic form of the affection also produces a wall of demarcation by fibroblasts. With the persistent and progressive multiplication of the bacilli in the

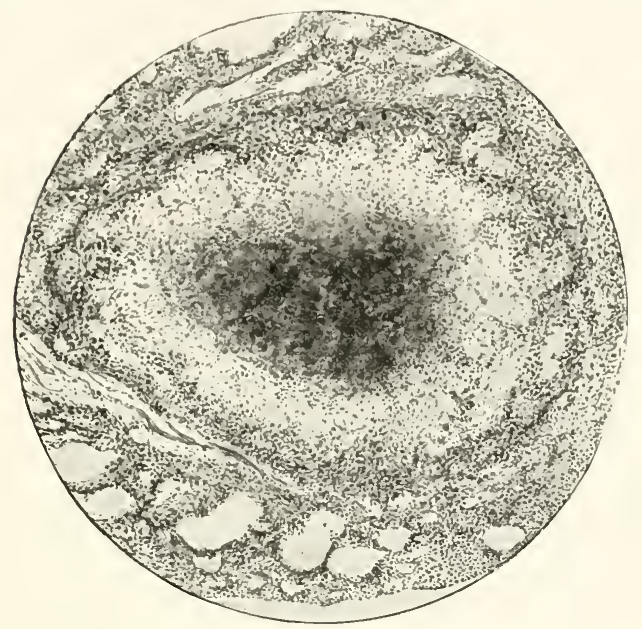

Fig. 74 .

Vicrosenpic section through a glanders nodule in lung of horse.

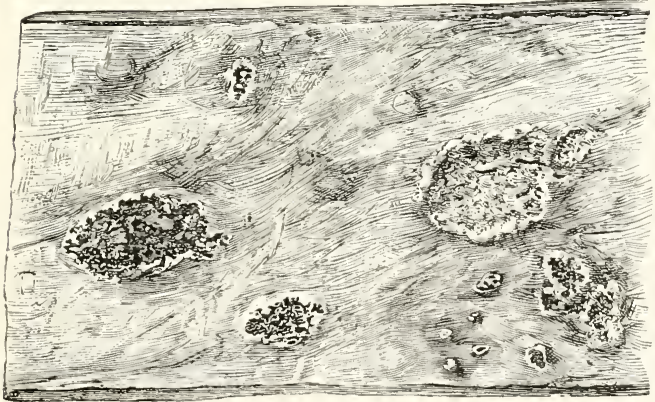

Fig 7.5

Glanders nodules, erosions and ulcers of the nasal septum of horse

inflamed and necrobiotic tissue, this limiting new tissue is, however, continually being broken down and the area of destruction is widened. The virus being taken up by the leucocytes, multiplying 
in the lymph spaces and therefore open to lymph convection, is disseminated in the vicinity of the primary focus, passes along the lymph vessels and into the blood; and new points of deposition and new foci of the disease occur locally and metastatically. The bacilli are also carried by currents of secretion and may lodge in a fresh position on the mucous membrane, here again to give rise to new colonies, as where they are carried from the lungs to the larynx

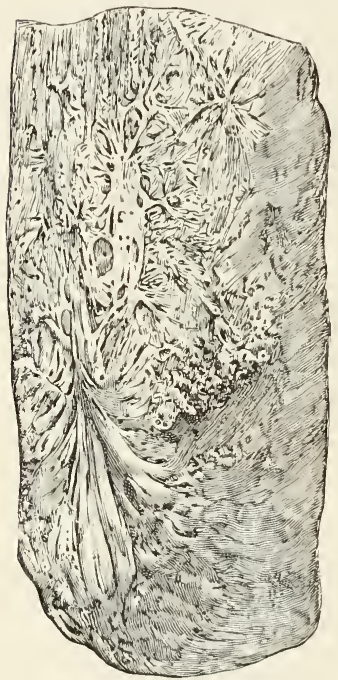

Fig. 76 .

Glanders induration of the nasal mucous membrane of the
horse. and pharynx. The metabolic products of the organisms, developing in the system, by their toxic influences occasion fever and wasting.

The visible anatomical changes of the disease vary considerably with the virulence of the affection and the tissue predisposition of the particular kind of animal. The principal types are the glanders nodnles, glanders abscesses, glanders indurations and glanders infiltrations.

Gl anders nodules, developing mainly in the lungs and parenchymatous organs, appear as grayish-white, semitransparent, opalescent, rounded prominences or deposits ranging from the size of a grain of sand to that of a pea, their central portion breaking down into a yellowish-gray, purulent or dry cheesy material. In the ling they are often surrounded by a dark red area. The glanders ulccrs present themselves on the mucous membranes of the respiratory tract as shallow or deep lesions, varying from the size of a lentil to that of a silver dollar; the loss of substance giving the mucous membrane an appearance of being eaten or gnawed. The larger ones have thick, wall-like, eroded margins. These ulcers have a bacon-like gray color, with the bordering mucous membrane often dark red and hyperæmic, bleeding on the least provocation. There are usually large numbers upon an affected mucous membrane. The glanders ulcers of the skin range from the size of a lentil to that of a half dollar, are of a dirty gravish-red color, covered with 
pus and half dried crusts, and the surrounding hairs are soiled with pus. These uicers often undermine the skin producing crater-like openings (like the anus of a chicken) and are apt to be found in rows one after another and alongside of each other.

Glanders absesses are commonly. found in the skin as somewhat rounded boils of the size of a hazel nut or a walnut, with the hair lost from over their surface. They fluctuate and contain a thin, oily, yellow or yellowish-red pus, sometimes a rather thick and mushy detritus, and their wall is made up of bacony, eroded cutis

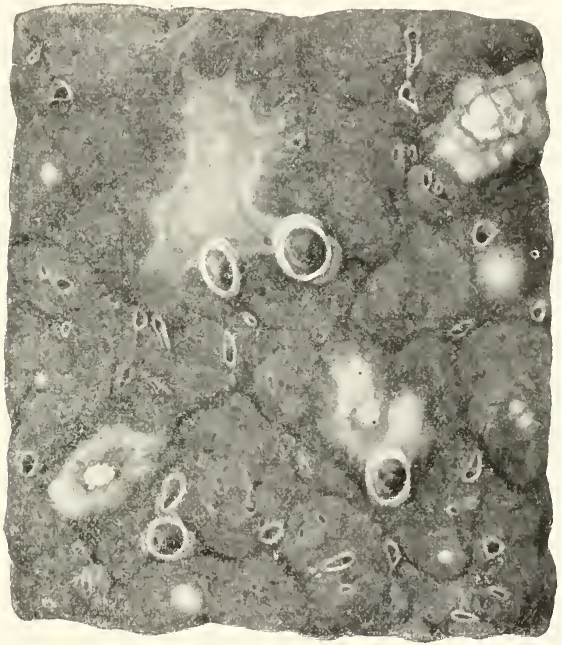

Fig. 77 .

Glanders nodules and indurations of lung of horse. Natural size.

and induration tissue. They are apt to be found in rows enclosed in the swollen, dense lymphatic cords; that is they form foci of suppuration interrupting the lymph vessels like a rosary.

Glanders indurations occur on mucous membranes as the result of a demarcating fibroplastic proliferation in the form of solid, nodular or ridge-like, thick prominences of the mucous membrane, with a yellowish-gray to reddish-yellow color, and a smooth slippery surface. These thickenings may appear as isolated prominences of the size of a hemp seed or oat seed, or may be fused so as to form hard stellate and radiating scars like frost-crystals, not extending deeply, 
and projecting somewhat above the surface. They are scattered all over the surface of the mucous membrane, often in large numbers, and may sometimes occur in rows. Sometimes this chronic indurative inflammation may form patches of thickening a finger's length in extent and several centimeters broad, standing out like cushions in marked relief, sometimes of dull grayish-yellow color or mottled with bright red points, and their smooth surface covered with a viscid pus. Occasionally warty, soft, raspberry-colored, projecting granulations are found growing from the mucous membranes.

In the lungs indurative patches are met sometimes about suppu-

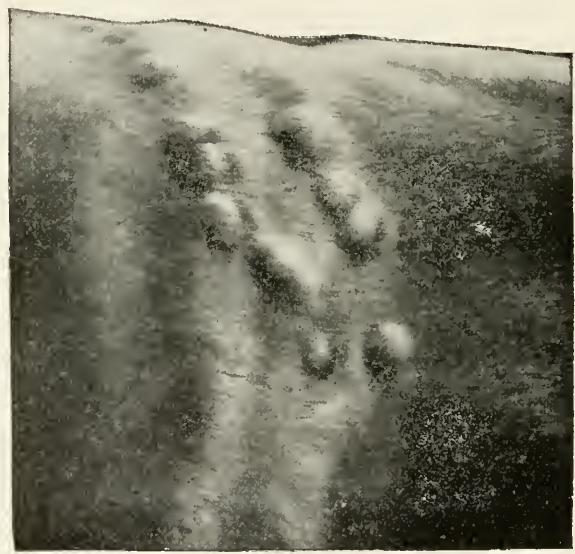

Fig. 78 .

Glanders abscesses of the skin of horse. (Photographed by Ir. Jakob.)

rating and caseating glanders nodules as bacony, grayish-white zones of demarcating tissue, sending radiations into the pulmonary tissue; sometimes as broad yellowish-white, poorly defined areas of connective tissue, merging into the pulmonary structure, involving numerous lobules and changing them into a dense mass, and cutting with a tough, leathery resistance. On the inside of such areas are met dull yellow, ochre-colored, moist cheesy or dry plaster-like deposits.

Glanders infiltrations develop in the lung as true pneumonias of catarrhal, suppurative or croupous types, and as a sclerosing form; the former in acute, the latter in chronic glanders. Especially in 
the cat family the lungs are involved just as in croupous pneumonia and show stages of red and gray hepatization. In the horse the lungs are apt to show a confused mixture of inflammatory changes; groups of lobules are found all through the lungs forming wedgeshaped, swollen areas of a densely elastic consistence cutting like bacon, mottled reddish-yellow or grayish-yellow and leaving on the knife blade a turbid muco-purulent juice. In the chronic type the pulmonary tissue is changed into an extremely tough, light grayishyellow mass of connective tissue riddled with confluent cavities about as large as a thumb's breadth, filled with a slimy, oily pus and having a deeply reddened inner surface. In this type of change the lymph

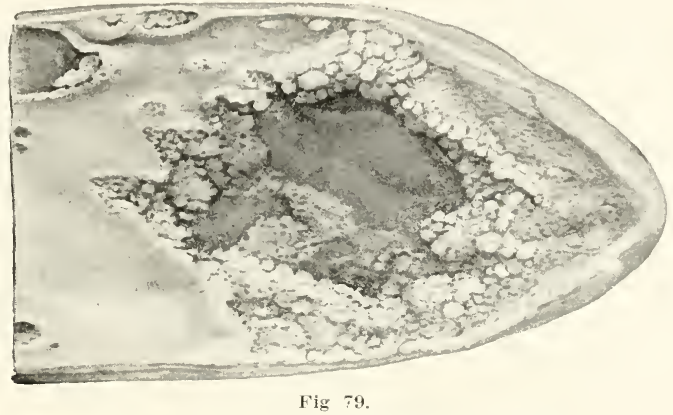

Large glanders ulcer of nasal septum of horse.

glands become very large and swollen, soft like marrow; afterwaris densely indurated and changed into a tough white connective tissue, forming nodular bunches as large as a goose egg which become adherent to the surrounding structures by simiiar fibrous bands. In the interior of these hyperplastic and inflamed lymph glands is encountered either a purulent softening in isolated spots or extensive purulent liquefaction with cavity formation, or an indurated, dry cheesy necrotic focus. The multiplication of the glanders bacilli in the lymph paths, besides causing them to swell up like cords, also gives rise to a diffuse gelatinous infiltration (especially because of the lymph stagnation), and, if the inflammation beconte chronic, to broad connective tissue thickenings; so that, for example, the loose cellular tissue of the skin becomes the seat of gelatinous thickening with extensive indurations all through it (pachydermia, elephantiasis) with foci of suppurative necrotic destruction runniner all throngh the skin and subcutaneous tissue. 
These more important anatomical. changes of glanders are met associated with each other and succeeding one another; so that along with the pneumonic infiltration of the pulmonary lobules glanders nodules are apt to be met in the same case; along with the abscesses, ulcers also are encountered, the latter developing from the former, and the diffuse infiltrations occurring as a terminal change.

Glanders generally may be considered as an irrecoverable infectious disease; exceptions have been met occasionally in man, guineapigs and horses, but are so rare that attempts to cure are hardly worth the effort in the case of animals, and seem to be injudicious, when the daily danger is considered of the transmission of the infection to man and other animals from the subjects dragging out the cotrse of their disease for months and years. The only rational method of exterminating the disease consists in killing the animals and carefully doing away with every chance of harm from any animal known to be afflicted with glanders : and these provisions should be made binding by law.

For details concerning the xtiology, symptoms and prophylaxis, reference may be made to Friedberger and Fröhner, I ctcrinary Pathology. Amer. Ed.. W. T. Keener \& Co., Chicago, 1904: Nocard-Leclainche, Les maladies microbicnnes des animaut. III edition, Paris, 1903.

\section{Actinomycosis.}

Actinomy'cosis (åктis, ray; ;úкns, fungus), or Ray'-fungus Discasc, is a form of wound infection manifesting itself by the anatomical changes of a suppurating granulonatous inflammation; it may be caused by several varieties of a group of fungi known as the ray fungi or actinomycetes. It occurs not infrequently in man, cattle and swine and has been exceptionally observed also in sheep, hart and roe deer, dogs, cats and elephants.

The fungi which cause the affection were first discovered by Langenbeck in $18+5$ in the carions lumbar vertebræ of a man, later by Rivolta in 1868 in tumors of the jaw in cattle and by C. Hahn in 1870 in the tongue in cattle; and have been more fully investigated by Bollinger, Harz, Johne. Israel, Ponfick, Gasperini, Berestnew. Boström and others. They have wide distribution in nature, existing especially in field soil and the beards of cereals. The ustral mode of infection by the ray fungi is through wounds made by small foreign bodies penetrating into the skin or mucous membranes, as sharp 
spicules of grain or other stiff particles of food to which the fungi are adherent; or they may gain access to the tissues through scratches and similar lesions of the skin. 'Examples of such mode of infection are common. Persons who put heads of grain into their mouths or who accidentally swallow portions of such heads or who have been injured while harvesting, have been known to develop at the points of injury (gums, throat, hands) actinomycotic abscesses and growths. Swine pastured in stubble land where their teats are casily wounded by the stiff straw stubble are not infre-

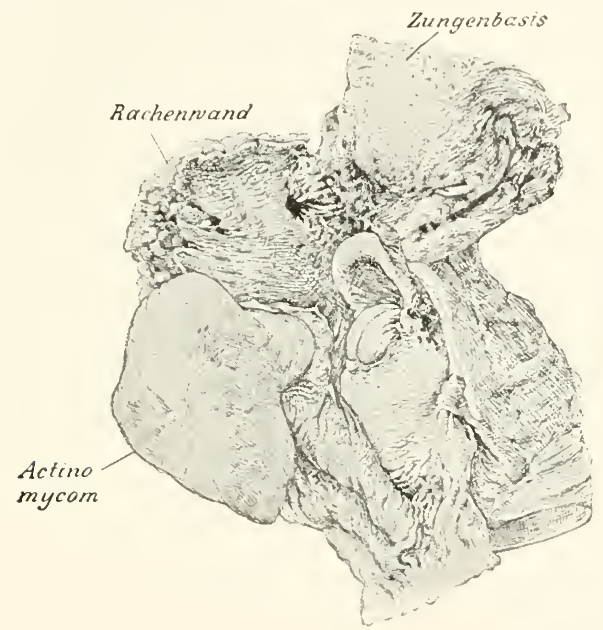

Fig. 80 .

Fungous actinomrensis in pharynx of cow. Artinomyeom, actinomycotic tumor Ruchenuand, wall of pharyox. Zungenbusis, base of tongue.

quently known to develop actinomycosis of a manmary gland. Cattle frequently show actinomycosis of the tengue, gums or lips along with the presence in the tissues of spicules of grain or other food materials which have been forcibly lodged in between the teeth. in the pharynx, etc. Occasionally in castration the fungi from the straw of the bedding get into the operation wound and set up actinomycosis of the stump of the seminal cord and scrotum (for details cf. Schlegel and Kitt*). The occurrence of actinomycosis occasioned in some such accidental way (pasturing in stubble fields)

*Handb. der pathog. Vikroorg.. v. kiolle and Wasseminnn: Gustar Fischer.

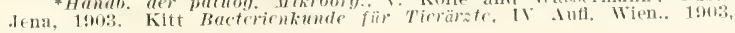


has more frequently been observed than an actual epidemic affection (Bang, C. O. Jensen, Preusse).

Transmission of actinomycosis from one animal to another or to man is very improbable; the few alleged cases of such an occurrence which have been recorded in literature might equally well have been caused by traumatic infection. Artificial transmission by inoculation has been successful only in a few cases (Johne, Wolff and Israel, Ponfick and others), and in these the experiment animal had to receive rather deep inoculation with comparatively large amounts of the infectious material (intraperitoneal, subcutaneous injection); while in ordinary wound infection and feeding transmission failed completely.

The anatomical changes brought about by the actinomycotic infection include the formation of actinomycotic nodules, abscesses and fungous granulomatous proliferations, sometimes accompanied by induratice connective tissue prolifcrations.

The actinomycotic nodules (actinomycosis nodularis) are small inflammatory foci of the size of a millet seed to that of a pea, yellowish-red or grayish-yellow in color, composed of a soft granulation tissue, which is developed as the product of a demarcating inflammation about the fungus which acts as a foreign body. They show one or a number of opaque yellow punctiform spots from the presence in minute foci within them of the yellow colored clumps of fungi and pus cells. When they are in the parenchyma of an organ they are surrounded by a connective tissue

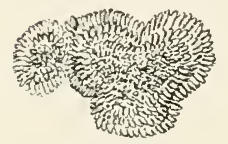

Fig. 81 .

Actinomycotic elump. (After Ostertag.) zone of induration; when on mucous surfaces they break through and project somewhat.

Actinomycotic abscesses (actinomycosis purulcuta, apostematosa) are areas of softening from the size of a plum stone to that of a human head, with purulent content which either appears as a thin mushy fluid, creamy and of a sulphur yellow color, enclosed in a white indurated abscess wall, or the areas are made up of a flabby, grayish-yellow to reddish-yellow matrix, which contains the infiltrating pus, not in separate foci large enough to allow it to be taken out in a spoon, but just as though it were in a fine sponge. In the latter case there is no real abscess membrane present, although the surrounding tissue for some distance is converted into an indurated, dense white connective tissue.

The fungous actinomycotic growths (actinomycosis fungosa) look like mushrooms or soft cushions, projecting above the surface 
of the skin or nucous membrane. They are more or less pedunculated growths, varying in size from that of a nut to that of a fist, covered with blood and pus and crusts, with an elastic, soft consistence, on section looking like bacon, grayish-white or grayish-red, and thickly beset with points of suppuration or the described flabby yellow patches of softening.

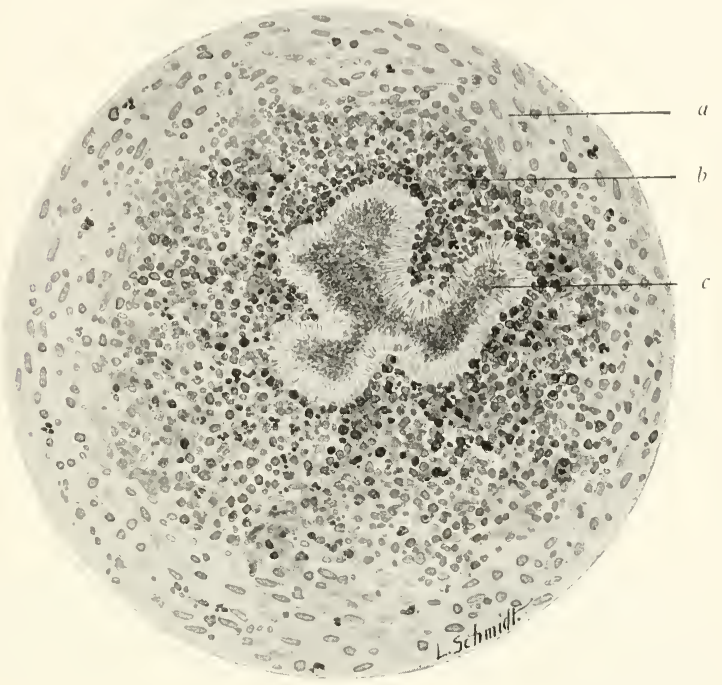

Fig. 82.

Vodule of actinomscosix thigh wagnification): a, fibloblastic and epitheliobl ber der. iencocric antral portion: r, actinomyces.

All three of these forms may occur together and pass into each other. For example, after rupture of an abscess the granulomatous proliferation springs up and grows out as a fungous mass; or the small nodules, because of the progressive multiplication of the fungi, become confluent and form the larger flabby areas of softening; or the bacon-like connective tissue growth prevails, and this causes more or less marked induration of the organ.

The most characteristic thing about the actinomicotic growth is the fungus. This may be recognized even by the unaided eye as minute granules the size of a sand grain, soft like tallow or sometimes of a chalky consistence, of a sulphur-yellow color or white; 
they may often be present in large numbers in the softened areas and the pus from this may sometimes have an almost sand-like, gritty consistence. Under the microscope the fungi may be discerned in

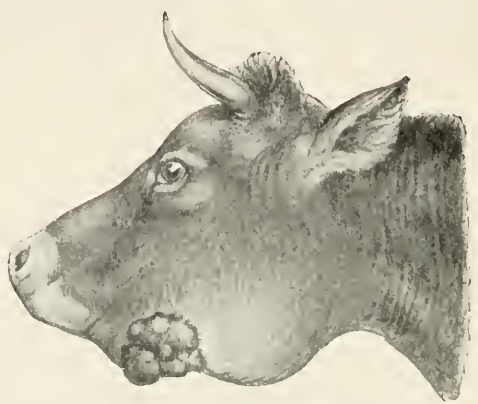

Fig. 83 .

Head of cow with actinomycosis of jaw. (After Moussu.)

unstained preparations as strongly refractive, gray or shining-yellow clumps of club-shaped filaments arranged in the form of a rosette (fungus glands): in stained sections the filamentous, intricately

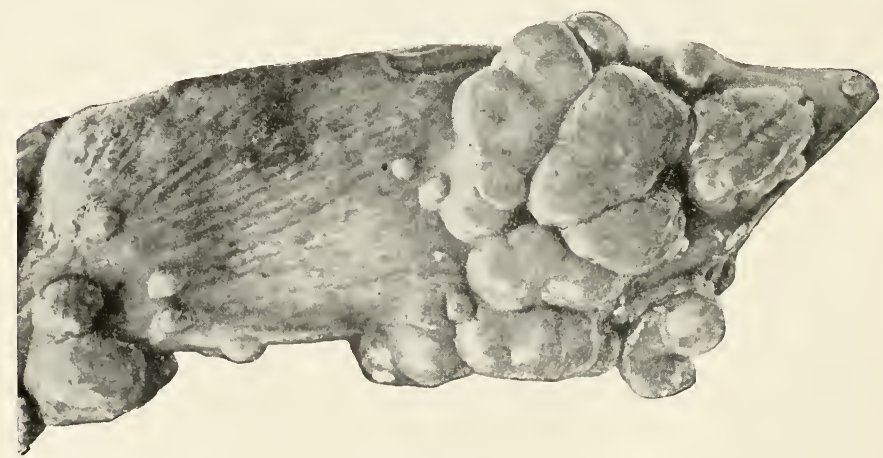

Fig. 84 .

Actinomycosis of the nasal mucous membrane of cow.

branched mycelium forming the matrix may be seen, and the clump, with its budding elements swollen into club shape and growing out from the periphery in radiating fashion.* The

* For fuller description ef. Kitt, Bakterienkunde f. Tierärzte, IV Aufl., Wien, M. Perles Verl. 1903. 
clumps of fungi are always surrounded by leucocytes in the fatty and granular detritus, sometinses, too, with here and there a giant cell. About this central, softened focus proliferating fibroplastic tissue is formed, vascular and full of emigrated leucocytes, as a zone of varying width.

Actinomycosis is primarily a local affection, running a course of months or years in duration. As the fungi penetrate the lymph spaces and are carried to new positions fresh eruptions in multiple foci of inflammation along the lymph ressels and in the lymph glands arise, with purulent softening and coincident production of new tissue in the soft parts and in the bones. liy hemic convection also the process may become a general one, a number of organs, bones, etc., becoming synchronously or one after another involved. [In cattle one of the most common and characteristic results of actinonycotic infection is that seen in involvement of the jaw, which may well illustrate many of the features of the disease. The infection here is supposed to take place by the penetration of a small spicule of grass or beard of grain into the gum along the root of the tooth, such a foreign element having upon it the actinomyentic fungus. In unknown way the fungus penetrates along the root well into the alveolar process of the jaw, and there produces the small nodules above described, each undergoing central softening and disintegration, and being surrounded by a zone of new tissue formation at its periphery. Gradually the process loosens the teeth: and as they are elevated in their sockets by the inflammatory tissue, and chewing becomes painful, the animal stops eating. Sometimes the teeth are forced up so that the animal is unable to close the mouth without pain, and the teeth may even be lost. The process gradually spreads throughout the alveolar bone and into and through the whole thickness of the jaw, the inflammatory change about each nodule at first causing absorbtion of the calcareous matter, and thus giving the fungi a chance to spread in this softened tissue. As each nodule grows older, the formative tissue at its periphery produces new bone: and from the coincident operation of the two factors of bone destruction and bone formation the jaw becomes enormously enlarged ("big-jaw"), riddled with the small points of softening representing the different actinomycotic foci and with fistulous paths rumning all through the mass, connecting these points of disintegration. The jaw may thus have developed within and upon it a tumor-like mass the size of a double fist or much larger, composed of a coarse framework of 
newly formed bone like a coarse calcareous sponge, the meshes of which are occupied by the actinomycotic nodules and their purulent matter. Fistulous sinuses discharge upon the surface and into the mouth; and in the purulent material are to be seen the tiny yellow sand-like grains known as "sulphur grains," consisting of the fungi themselves. The animal may die from starvation; the process may extend along the ramus of the jaw to the base of the skull and gradually advance by the same changes through the latter and cause death from a purulent meningitis; or the fungi may be carried along the lymphatics of the neck to the thorax, producing an actinomycotic pleurisy and entering the lung to cause fibrosis and purulent destruction of these organs. Occasionally the fungi swallowed with the discharge into the mouth give rise to alimentary actinomycotic abscesses.]

Ligniéres and Spitz have described an epidemic disease of cattle similar to actinomycosis occurring in Argentina, in which the skin and lymph glands are principally involved (rarely the bones of the face and tongue). The yellow fungus grains are not present, and another species of microphyte which does not stain by Gram's method is regarded as the cause (actinobacillosis).

\section{Botryomycosis.}

By the term botryomycosis is meant a productive inflammation which leads to the formation of fibrous growths or cicatricial indurations attended invariably with suppuration and fistula formation or the production of brownish to yellowish-red foci of softening, and which are caused by infection with botryomyces ascoformans. The disease is quite common in the horse; and has been met a very few times also in the cow and hog (Csokor, Wilbrandt).

The fungus which is the productive agent of the disease, discorered independently by Bollinger in 1870, Rivolta and Micellone in 1879 , and by Rabe and Johne, presents itself in the diseased tissue as a deposit of fine sand-like granules, visible to the naked eye. Nicroscopically it is characterized by the occurrence of the fungi in blackberry-like masses (fifty to one hundred micromillimeters in size) made ip of conglomerated round, disclike masses of cocci grouped together in zooglœæ.*

This fungus gains entrance to the tissue by infection of wounds: apparently it may find its way through small excoria-

\footnotetext{
* For fuller description cf. Kitt, Bakterienkunde für Tierärzte. IV Aufl.,
} Wien, 1903. 
tions in the epiderm or through the glands of the skin, as botryomycotic growths derelop especially at places in the skin which are frequently subject to friction (places worn by harness and saddle.) Very frequently the growth involves the stump of the spermatic cord after castration, or the udder and penis sheath, as well as the burse and fascix of the withers, elbow and shoulder. The tissue invaded by these microorganisms becomes the seat of a chronic inflammation. At first it has a grayish-red color, later becoming pale and translucent (like bacon); and eventually a white cicatricial connective tissue develops in the mass, spreading through the loose cellular tissue and over the surfaces of the fascixe and producing enormous induration and thickening* of the part. Or the process may be more definitely limited to spheroidal nodular masses which project from the surface as fibroma-like tumors, ranging in size from that of a fist to that of a human head or larger. The growth of the clumps of

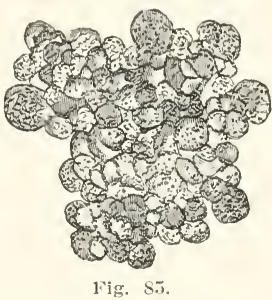

Botryomyces ascoformans (low power). microorganisms in this fibroplastic tissue brings about foci of softening of a viscid purulent type, sometimes of a distinct yellow color like ordinary pus, but commonly of a saffron or orange tint, with formation of fistule to the surface and paths of confluence in the mass. As the result of multiple coincident or successive infection of the skin there often are met dozens of these botryomycomata in one subject. Convection of the microbic agents by the lymph and blood learls to metastatic growths, especially in the lungs.

For fuller account cf. Kitt, Spesielle pathol. Anatomic der Hauticre, II. Aufl. F. Enke, Stuttgart, Igor.

\section{New Growths, Tumors, Autoblastomata.}

By the terms new growths, tumors, new formations, neoplasms, autoblastomata (Klebs), are meant tissue proliferations of persistently progressize character, which alter the conformation of the part and disturb its function. These growths appear as excessive tissue accumulations caused by cells of the animal body which have become parasitic in nature and which invade the soft structures or skeleton of the animal as more or less isolated and independent bodies. Enlarging sometimes slowly, 
sometimes rapidly, and never undergoing diminution, they interfere with the other tissues, perhaps by robbing them of their nutrition, perhaps by some of the products of their metabolic changes, but particularly in a mechanical way by their pressure influences.

It has been only within the last sixty years, since Rudolf Virchow by comprehensive histological studies and critical examination of the older literature established a new basis for the systematic consideration of the different types of tumors, that a scientific conception of the origin and nature of new growths has entered into their study (oncology; o brкos, node). Previous to this period tumors were classified mainly from their external appearances and their gross anatomical characteristics, and were named frequently from incidental features often of merely accidental prominence; and no real knowledge was had of the developmental history of these growths. Althongh in our present knowledge of the development of tumors there are many missing links, we nevertheless possess from microscopic and experimental investigations sufficient information in regard to their nature to permit us to clearly recognize their derivation from the cells of the body, the mode of their extension and their actual method of growth, and the significance of the pathological process in its bearing upon the life of the subject.

Tumors may be classified from their histogenesis, that is, from their structure and from their origin and construction from the cells and tissues of the body. There are no foreign tumor elements brought into the body from without; tumors are offsprings of the body cells, and are accordingly found to consist of these and of the intercellular substances of the various tissues. They develop according to the same laws which govern the embryonic development and later growth of the individual and the processes of regeneration; and they arise from the four primary types of body tissue (connective tissue in all its forms, epithelium, muscle and nervous tissue.)

Just as the tumor cells present the same form and the same mode of multiplication as the mother cells from which they are derived, the intercellular substances, as products of the cells, are the same as are produced by the original cells. Generally, therefore, the source of a tumor can be determined from the character of its tissue; although because of the fact that they grow more or less independently, as isolated masses of tissue, it is quite reasonable to expect more or less modifications in the structure 
and adaptation of the cells and matrix of tumors. The cells frequently do not attain the same size as the mother cells or may be larger than the latter; cellular division takes place sometimes as a direct process, sometimes indirectly with mitotic changes. The mitotic figures, which are likely to be found in great numbers, especially in rapidly growing tumors, and the process of cellular division often show irregularities pointing to a pathological disturbance of the process. The chromatin filaments derived from the cliromatin skein (chromatosomes) may be too numerous or asymmetrical; or there may be found giant mitoses, karyokinetic figures in multipolar arrangement, arranged about a number of attraction centres (centrosomes), or giant cells. The cells may also, as stated by Ribbert, exhibit alterations which may be regarded as regressive in character, as a return to an embryonic state in which there is but little evidence of differentiation: or they may really be embryonic cells whose power of forming normally functionating organs has been lost (a change designated by Hansenann by the term Anaplasia).

The cellular type and the character of the tissue of the growth furnish the basis for nomenclature of tumors. Many tumors are made up of but a single kind of tissue, as fibrous connective tissue, fat tissue or cartilage, or at least mainly of one type (the accompanying bloodvessels are not regarded as a special form of tissue but as essential constituents of any form): such growths are called simple autoblastomata. Others, no less numerous, contain two or more tissues, as is obviously required in epithelial new growths because epithelium always requires a connective tissue substructure, and as is likely to occur, too, in other tumors from the mixture of the various forms of connective tissue, as cartilage, bone or fat; we therefore recognize as a second group, the compound autoblastomata or mixed tumors.

It should be recognized, too, that tumors as independent developments, although relying on the animal body for their nutrition, yet having their own blood vessels and carrying on a separate metabolism, are subject to all the pathological processes which may affect the normal tissues of the body. A tumor may become the seat of passive hyperæmia, of inflammatory reaction: and any kind of degeneration and necrosis may occur in the tumor structure. Tumors are sometimes subject to traumatic influences from without (strangulation, wrenching, penetration by foreign bodies or bacteria) which may occasion tissue changes; sometimes the conditions of nutrition become so un- 
favorable from irregularities of growth of the individual constituents of the tumor. (as the imperfect development of blood vessels) or from the absence of ducts for the escape of secretions, or from the retention of metabolic products, that regressive metamorphoses, as fatty degeneration, calcification, mucoid degeneration, etc., necessarily take place. When such conditions prevail and when the structure of the growth is modified by this or that change there is often considerable difficulty in determining the derivation of the tumor and there may be much uncertainty under which group of tumors a given neoplasm should be classed. In such cases we follow the proverb: a potiori fiat denominatio; and the anatomical term is given from the predominating type of tissue present.

Growth of Tumors.-Deriverl from one or more tissues, tumors at first form cellular masses (or a tissue complex). These increase in size as the internal portions of the new formation multiply, that is, by contral growth; or they enlarge by progressive multiplication of the outer portions, that is, by peripheral or appositional growth. The increase in size and extension of the tumor therefore may take place by one or other of two ways; either by the tumor growing "as a bulb, lying in the soil and developing, presses aside the ground" (Dürk). "as a rubber balloon which one inflates" (Ribbert) (cxpansile growith), or by the tumor tissue sending out processes into the neigluboring structures as a plant forces its roots into the earth (infiltration growth). It was formerly believed that the enlargement took place by the surrounding tissues beconing gradually involved in the same proliferative process, being actually infected (local infection); but this is not probable, as we may usually recognize under the microscope that the proliferating cells of the neoplastic primary focus, that is, the first elements of the tumor, are extending into the surrounding tissue, growing into the interior of the tissue or toward the surface of the part. The surrounding tissue supplies only the nutrition for growth and takes part only in this sense that its blood vessels and supporting tissue may be appropriated by the new growth, and that it may also multiply as the result of the changes in tissue tension which may be produced ( $\mathrm{v}$. chapter on Regeneration); otherwise, however, the surrounding tissue plays but a passive part, like the soil in relation to the seed of grain.

External Shape of Tumors.-In correspondence with their mode of growth autoblastomata form usually circumscribed 
tumors well defined from the normal tissues. They may develop as nodes (tuberous growths) which lie embedled in the tissue like the yolk within an egg, or may protrude as tuberositics; they may grow out from the surface of an organ as pedunculated protuberances, with thick pedicle like a mushroom (fungous form), or with slender pedicle and provided with free offshoots like a polyp with its tentacles (polypous shape), or like a tree (dentritic), like a coral stalk or like cauliflower; or, in the infiltrating varieties they may accasion a uniform saielling of the affected organ. [Any tumor growing from a surface and provided with a slender pedicle may be spoken of as a polyp or a polypoid grouth, whether branched or smooth. A form known as a flat tabular swelling is often encountered where upon a surface the growth has produced a more or less uniform swelling with a flattened surface, as is often seen where the surface of a growth is opposed by a second surface causing pressure. The causes determining the various shapes of tumors include such factors as the mode of growth (central growth producing a regular ap. pearance of the periphery, while infiltrative growths are apt to be irregular and ragged in outline), pressure, (as just suggested in connection with the flat tabular swelling, or the preservation of the spheroidal shape of a node by the pressure from all sides by the surrounding tissues, or where an analogons growth appears as a tuberosity or polyp if developing close to a surface, being thus relieved of pressure on the surface side), and the original structure from which the tumor arises (as where a surface growth, a papilloma, takes on a villous type of branching following the natural formation of villi from the original tissue, as in the intestine.) ]

The Pathological Significance of Tumors is determined primarily by their location, their size and the mechanical pressure which they exert upon the adjacent tissues. By pressure upon the peripheral tissues a tumor as it grows in size occasions sometimes trifling and at other times serious disturbance of function, varying with the particular organ affected. A small tumor in the brain or in the cranial cavity may by compression of the former or of the cerebral vessels bring about most intense nervous troubles and terminate fatally; and by obstructing the larynx, gullet or intestinal canal a comparatively small growth may endanger life. Pressure upon bloodvessels may occasion a great variety of circulatory disturbances. But, on the other hand, even 
large growths of the skin or subcutaneous tissue often produce only the most trifling effects.

In the second place the pathological significance depends upon the degree of special power which the tumor possesses to disseminate through the system. The less a tumor involves strictly the surface of an organ or the less it remains closely confined to its point of origin as a single isolated growth, and the more it sends out offshoots which extend into the lymphpassages and bloodvessels and thus obtains opportunity for its cells to be loosened and carried into new locations in the body, the more the derelopment of the neoplasm acquires specially dangerous characteristics. The outshoots of a tumor, made up of its proliferating cells, force their way first in the surrounding tissue, especially the connective tissue lymph spaces, which represent the beginnings of the lymph passages, causing a continually increasing portion of the organ in which the growth occurs to be destroyed by the infiltration (the tumor therefore spoken of as a destructive growth or locally penetrating growth). In such new formations the external, visible outlines are usually not its actual limits; the apparently healthy adjacent tissue may conceal the roots of the tumor only to be found microscopically. The surgeon, whose skill can in many cases afford permanent recovery by removal of the growth, is unable occasionally (for technical reasons or because the precise limits of the individual roots cannot be recognized by the unaided eye) to remove the entire tumor in the operation performed for its ablation; from the portions thus left, earlier or later the same tumor may return in the original site or close by (recurrence of tumor). The tumor cells grow into the lymph passages, sometimes as continuous cords or roots; or the cells may become loosened or be carried away by the lymph current or perhaps by leucocytes. The same thing occurs where the roots of the tumor penetrate through the wall of a blood ressel, sometimes developing as long processes within its lumen; and particles of the growth may be swept off by the blood current. Under such circumstances, at a shorter or longer distance from the (primary) autoblastoma (the principal tumor) arise sccondary tumors, daughter tumors or metastatic tumors. The lymphogenous metastases are carried into the immediately adjacent tissues and into the lymphatic glands draining the site of the original growth; the embolic (hæmatogenous) ones pass into the lungs or the organs of the greater circulation ( $\mathrm{v}$. chapter on Embolism). When in this 
manner the tumor is disseminated widely to the various organs, we speak of the generalization of the tumor. This lodgment and development of daughter nodules from the cells which have become freed from a tumor, an actual form of seeding by transplantation, is met also at contact points on mucous and serous surfaces. For example, an ovarian tumor may become disseminated all over the peritoneum by having its cells loosened and scattered over the serous membrane; a tumor of the costal pleura may become transplanted to the visceral pleura; one of the upper lip to the lower lip. The cellular elements of the growth in such instances are freed and distributed by the movements of the viscera, or by the friction of contiguous surfaces.

All secondary growths correspond closely with the primary tumor from which they arose in their histological structure; where a cancer of the gum metastasizes to the retropharyngeal lymph glands and lungs the metastatic formations are composed of squamous epithelial cells just as is the primary tumor. They need not necessarily be smaller than the original giowth, and are often of larger size than the latter.

Tumors which can be completely removed, which are not metastatic and do not recur, are ordinarily called, from the clinical point of view, benign (innocent); those which penetrate into the adjacent tissues and are removed in their entirety with difficulty, or are recurrent and give rise to metastases, are called malignant (harmful). To the extent that experience shows that one or other of these results may generally be expected in operations for removal, the classification into these two types may be accepted; but the differences are not to be depended upon, as some tumors which do not form metastases recur if not completely removed, and tumors which are ordinarily regarded as benign may occasionally produce metastases; and, again, well-known malignant growths often remain strictly localized and harmless for a long time, and if they are removed early enough will not recur.

As a further factor by which tumors may affect the system, it should be recalled that within these neoplasms all sorts of metabolic products and decomposition substances are being freed, and these may by absorption have toxic influences upon the possessor of the growth. It is quite common to meet with inflammatory changes in the tissue about a tumor and the growth itself may be found richly infiltrated with immigrating leucocytes, or may be found undergoing suppurative or gangrenous changes. This may be due to the fact that tumors which happen to be situated on exposed surfaces are invaded by bacteria, and the toxines of the latter excite inflammation: but there is little doubt that 
some tumors give off into the surrounding tissues metabolic products having chemotactic power and thus stimulate leucocytes to escape from the vessels. To all appearances we may ascribe to the products of disintegration occasioned by bacterial contaminations and occurring in connection with the various degenerations in tumors the fact that in a number of these growths there occur general nutritive disturbances (oligæmia, loss of appetite, marked loss of strength, emaciation), which look like the result of a chronic intoxication-a condition known as tumor cacheria.

Just as the specifically differentiated cells of the body give off specific products, in the same way the different kinds of tumor cells, as the offspring of this or that variety of physiological cells, produce in more or less similar manner the substances characteristic of the latter; it may be claimed that they too perform physiological functions.* This function, however, is only in the rarest instances of any service to the general system; being without any relation to the co-ordinated activities of the rest of the organs, it is merely a vital phenomenon of the independent cellular complex of the tumor. The formation of intercellular substances by the tumor cells, as the formation of osteoid callus in tumors arising from periosteum, is an example of such function; but tumors originating from glandular cells and having gland-like structure show this power especially, and are able to produce the same secretory substances as the corresponding glands. Thus cancers of the intestinal mucous membrane secrete mucus from goblet cells; thyroid cancers produce colloid; tumors of the mammary gland form a secretion analogous to milk (but pathologically altered), and tumors arising from liver cells often secrete so much bile that the growth becomes thoroughly infiltrated with it (icteric). E. Albrecht has observed that the formation of red blood corpuscles normally performed by the bone marrow may also be carried on by a tumor of the dura mater made up of endothelial cells and erythroblasts.

As all offsprings of given cells manifest an ability given them by heredity to perform the same functions as their predecessors, it may be expected that the metastatic nodules also show the same phenomenon. For example, one of a number of metastatic nodes which had formed in the lung from a tumor originating in

*E. Albrecht, Sitzungsbericht d. morph. phys. Gesellsehaft in rïnehen, 1901. Heft II. 
the liver produced bile in this position. Generally the secretions of tumors are apt to be the seat of pathological ehanges, as ordinarily there are no channels for discharge in the growth and the secretion therefore is stagnant; and moreover the function would be more or less abnormal, because of the abnormal topographical relations of the proliferated glandular epithelium, the failure of complete differentiation of the cells, or the reversion of tumor cells to embryonic type and the lack of relation with the nervous system of the host of the tumor.

There are conditions however under which the secretory products of a tumor are not devoid of value to the body, particularly tumors of glands which produce internal secretions of importance to metabolism and whose destruction would be ominous to the well-being of the body, as the thyroid gland and pancreas. The fact that growths from these glands may also produce internal secretions may in some measure compensate for the death or destruction of the organ by the tumor.

[Of all the body functions, that of growth (and that is rather a mere vital phenomenon and not a differentiated function) is the one which is best and most completely manifested, being in line with the embryonic characteristics which tumors as a class possess. The example of the performance of a function of value to the economy selected by the author in the preceding paragraph is practically the only one which may be named. The fat which accumulates in a lipoma is of no service to the host of the tumor even if he be starving; the connective tissue of a fibroma is of no connective or supportive value, but rather a continual source of annoyance from weight and pressure. Even the function of growth, most nearly typifying natural energy, is, as the author points out, irregular in the manner of its occurrence, is without efficient organogenic power and especially abnormal in that it has no definite period of cessation.]

Aetiology of Tumors.- We possess but few facts and have but a meagre idea about the mode of origin of tumors. Nany theories have been framed to explain the luxuriant growth of the cells, but the difficulty of positive proof leaves us in uncertainty between probabilities and theoretical dicta. Mere suppositions that as the result of the action of a given stimulus this kind or that kind of cells are excited into irrepressible multiplication. revert to their embryonic characteristics or undergo biological changes as expressed by the terms metaplasia or anaplasia, are in no sense satisfying, but merely serve to conceal our igno- 
rance of the origin of these growths and to bring into the problem new difficulties.

Among the more prominently urged hypotheses may be mentioned the idea that an infcctious cause underlies the cellular proliferation, a view which has had numerous adherents in recent years. At first bacteria were sought as causative factors, some of which as the causes of productive inflammation had manifested functions suggesting analogies with tumor formation; but nothing has been shown beyond the mere secondary presence in tumors of various saprophytes, pyogenic and other bacteria; and no microphytes have been encountered which can be regarded as responsible for the proliferation of cells into tumors. In time the line of investigation was directed to the protozoa. The inclusions, often shown by a variety of methods of staining in many tumor cells, and apparently of foreign character (that is, intracellular and nuclear structures not conforming to normal parts of cells), led a number of microscopists to regard them as of some type of protozoan character and as the parasitic causes of cancer and malignant tumors generally. However subsequent investigations directed to the final proof of this discovery have shown that these supposedly foreign elements are nothing more than altered blood corpuscles, lymphocytes, nuclei of the tumor cells, degeneration products or artefacts, produced by stains and reagents in the tissues. In the same way the discovery of yeast fungi having pathogenic properties capable of causing inflammatory granulomatous formations by inoculations in small experiment animals, resulted in no definite or generally applicable conclusions; because these blastomycetes were not to be found in trie tumors. or if occasionally found occurred only on the surface of the growth and failed to give rise to characteristic tumors. With the demonstration of parasites as causative agents left in this unsatisfactory condition, consideration should be given to the so-called invisible microorganisms; or we should remember that perhaps with our present technique the infectious agents in question cannot be rendered visible.

A number of investigators insist upon the possibility that an infectious factor underlies some types of tumors (Sticker), because as a matter of fact there have been noted a number of peculiar circumstances which point to the existence of a general extraneous (not individual) cause; as the endemic occurrence of cancer, its occurrence in connection with dermal excoriations, or 
the occurrence of dermal proliferations (papillomata) in a number of horses in the same stable.

However, a great number of objections have been raised against the parasitic hypothesis, especially by Ribbert, which leave this method of causation in extreme doubt. In the first place the various forms of tumors always have their fixed tissue-characteristics, and the cells of the metastatic nodules invariably conform with those of the original growth; if an infection were the cause of the tumors it would always have to cause the production of the same kind of cells in the various organs (that is, the cause of a cylindrical cell cancer in the intestine would have to be able to form cylindrical intestinal epithelimm in the interior of the liver; the cause of a squamous epithelioma would have to form keratous flat epithelial cells in the lymph glands). How that could occur cannot be understood: it would have to be assumed that the cells were so intimately associated with the parasites that both should always go together and grow together. If such symbiosis be claimed to actually exist, then,. as Ribbert logically argues, it must be assumed that the microorganism invariably divides at the same time as the tumor cells so that one of its offspring may be present in each of the two new cells. "If this be not essential, it may be asked low the cells which do not contain parasites come to proliferate any further. If they are able to multiply, however, without the existence of their parasites, why was it of essential importance that the latter should have ever been present?" (Ribbert). Then there is the established fact that every tumor grows from its own substance under direct pressure contact from the contiguous parts, and the surrounding tissue does not become infected; therefore the parasite would have to confine its multiplication strictly within the tumor cells. This would be strange; and the question naturally arises, why, for example, the neighboring epithelia which are quite like those of a skin cancer do not furnish equally favorable soil for the development of the microorganisms. In the end, as Ribbert emphasizes, we should have to assume that for every new growth, for every type of cell of malignant tumors, there exists a special type of infectious agent incapable of infecting other cells, as one special form for cylindrical cell cancers, another for squamous cell cancers, etc. The genesis of tumors would become progressively more and more complicated if such ideas were to prevail. 
The explanatory theories of Cohnheim and Ribbert based upon considerations of cmbryology and mechanical conditions for growth are far more simple. Tumors have been repeatedly met in the new born; for example a melano-sarcoma in a calf, a papilloma in a foal, and a number of growths, which although first noted in young animals were of a composition necessitating an embryonal origin for their development, as dermoid cysts, dental teratomata, branchial cleft teratomata. In the earliest stages of embryonic development when in a great variety of ways there occur formation of folds and of buds, pressure displacements and separations of the cells growing with unequal rapidity, it is possible that cells and groups of cells may be cut off from the rest, misplaced or isolated. Such misplaced cells will for the most part perish, because of the necessarily occurring nutritive disturbances; but where according to circumstances the nutrition remains sufficient the misplaced cells will continue to grow and multiply, and there is necessarily produced from them a structure which is apparently a foreign growth and not normally belonging to the structure of the tissues and organs. The formation of blood vessels probably proceeds in a more or less atypical manner in such separated and misplaced groups of cells, as a resuit of which their growth is in one instance a very slow one, in another an exuberant cellular proliferation. The experimental studies of Roux have shown that by artificial isolation and misplacement of segmentation cells each one is capable of growing into an embryo; and Barfurth has observed that aftẹr needling an egg in the gastrula stage tumor-like formations (deriroid-like) develop from the misplaced cells. Noreover Ribbert has succeeded, by transplantation of fragments of the auricle of a rabbit, in obtaining polypoid nodules persisting for more than a year, consisting of skin, fibrous tissue, cartilage and bone; and in other experiments tumor-like structures from bits of the notochord, by transplantation to the anterior surface of the intervertebral tissues. Occasionally the accidental or intentional cperative transplantation of epithelium or bits of skin into a wound has resulted in the formation of a small epithelial cyst or dermoid cyst from the engrafted tissue, which may be regarded as evidence in the same line with the larger congenital growths of this type. The fact that experiments of this sort have thus far fiilied to produce larger tumors of progressive and persistent character of growth is not an argument against this theory; we must remember, as Ribbert insists, that our technique in artificial 
isolation and transplantation of tissues is as yet not delicate enough to prevent damaging the tissues and thus causing the failure of the desired results.

Cohnheim extended his theory of the embryonic origin of tumors to a wider application by advancing the hypothesis that at an early period of embryonal development more cells may be formed than are needed in the construction of the organs and that some of these cells may remain unused as "rests," retaining, becanse of their embryonal nature, a marked capacity for proliferation; and that from such excesses of cellular material tumors may develop. The views advanced by Cohnheim, to whom we are indebted for first pointing ont the important significance of foetal isolation of cells in connection with tumor formation, are coming to be recognized as valid; that from isolated segmentation cells, which have actually been observed, tissues and organs may originate, and in all probability, too, independent tissue formations and therefore autoblastomata.

In a somewhat different form practically the same hypothesis has been recently advanced by J. Beard. Beard suggests that from every fertilized ovum a number of germ cells originate (?), from which, however, only one goes to form the embryo, the remaining wandering into the embryonic mass and entering into the formation of the chorion (or the sexual cells?). It may be possible that a tumor might develop from such wandering germ cell if it stray to an improper part, and that such a tumor may be absolutely nothing but an incompletely developed (rudimentary) embryo (which, of course, is applicable for a number of tumors, especially the true teratomata).

As a further application of Cohnheim's theory, which it is true affords a satisfactory explanation, not for all but for a number of tumors, Ribbert has suggested that cells may escape from their normal connection also in extra-11terine life and thus become misplaced and in consequence provide the nucleus for a tumor formation. It has been repeatedly noticed that tumors have developed after a traumatic lesion, at the site of injury by foreign bodies, after contusion, etc., as osteomata of the jaw after contusion, large chondromata in animals and man after fracture of the ribs at the seat of the fracture, cancer of the cheek in man after being hit by a stone, cancer of the lip at the place where the mouthpiece of a tobacco pipe has been frequently rubbing. As it is very difficult to think of such cases being due to any specific irritative influence of the foreign body which cansed the injury, we are forced to consider changes in the positions of the cells, 
displacements and isolations of osteoblasts, cartilage tissue or epithelium, as causative of the autoblastomatous new formations. It is not rare to see connective tissue proliferations of considerable extent, completely reproducing the characteristics of fibromata, developing from injuries of the tendon sheaths (horse and cow) or of the tongue (cow); the growth is apparently the product of a chronic inflammatory process and the result of an excessive growth of granulation tissue. Whether some special irritant, perhaps bacteria, bring about such tumor-like formations. whether absence of tissue tension occasioned by the trauma is the single or principal reason for its occurrence, remains an open question. At all events it is recognized that there are no sharp lines of difference between tumors and regenerative proliferations, and that the hypertrophies, which are regarded as simple proliferations in vacuo (as of liver) may pass over into tumors.

Objections have been urged from various sides (Hansemann, Hanat, Ziegler) to the opinions of Ribbert (who also accepts inflammatory processes as possible causes for the inception of cellular proliferations, as in case of epithelial cells). It has been urged that if the above-mentioned displacements and isolations of cells of epithelium were sufficient to call a cancer into existence, there necessarily should develop numberless cancers as results of chronic inflammatory phlegmons; that the cells of the tumors ought not to appear merely as normal elements in luxuriant multiplication, but the general rule should be that there exist a special change, a differentiation of the cells (even though the causes of cellular differentiation be entirely unknown).

Although we still await the solution of questions regarding the conditions which underlie the origin of tumors, and concerning the transformation of this or that kind of cell into tumor cells, it is at least certain that tumor cells are tissue elements which hav'e become independent and may be looked upon as cells which have actually become parasitic. This conception of the nature of tumors (for which from this point of view the name autoblastomata, proposed by Klebs, is most appropriate) has received important support in the extremely interesting experimental studies of a veterinarian, C. O. Jensen. This investigator succeeded in carrying a carcinomatous tumor of a mouse through nineteen generations in mice by transplantation, and in demonstrating that infection played no part in the process but that the transplanted tumor cells, themselves parasitic upon the individuals in whom 
they were transplanted, continued to grow and form tumors of the size of walnuts, growths of considerable size when that of the experimental animal is taken into consideration.* Earlier than this Hanau had succeeded in transferring an unquestionable skin cancer of one rat to other rats with similar results (in peritoneal cavity); and L. Loeb and Velich have successfully transplanted rat sarcomas, and Moran has transplanted adenocarcinomata of a white mouse a number of times. [Still more recently L. Loeb has carried an adenocarcinomatous tumor of a Japanese dancing mouse through a number of generations, eventually finding a change in the nature of the tumor which took on the histological characteristics of a spindle cell sarcoma. Other investigators have described similar success in transplantation; and Loeb's instance of change in the tumor characteristics is not an isolated one.] (Attempts to transplant sarcomas and cancers in dogs undertaken as much as ten years ago by Pütz and other investigators have thus far failed.) The point of special interest in Jensen's studies is the discovery that it is possible, by special methods of implanting the tumor cells, to render the diseased mice immume so that the tumors, which are present in them and which are ordinarily fatal, disappear and the subjects recover. Jensen brought about this result in two ways, first by treating the diseased mouse with its own tumor cells, substances developing in consequence in the blood (crtotoxins), which inhibited further extension of the tumor cells at the original site (v. Chapter on Immunity); and second by repeated introduction of the tumor cells into rabbits (not susceptible to the mouse carcinoma) he obtained a serum which manifested specific cytotoxic action. [In a similar set of transplanted adenocarcinomata of mice Gaylord found that some of the affected individuals recovered naturally, their tumors after more or less growth undergoing atrophy and disappearing. From such individuals he obtained a serum which, when introduced into mice with marked tumor growths, uniformly caused their disappearance by its cytolytic power.]

The tissue of tumors isolated from the body will retain its vitality at a temperature of from $I^{\circ}-3^{\circ} \mathrm{C}$. for about eighteen days, at room temperature for about twelve days, but at body temperature for scarcely twentyfour hours. This difference is supposed to be due to the cells passing into a sort of resting condition at the lower temperatures, while at the body temperature they cannot avoid metabolism. Bd.

*For details v. rentralbl, f. Bakteriol. u. Parasitenkunde, 1903, No. 1, Ixx15 
Statistics of Tumors in Animals; Influence "of Age and Sex.-It has only been within very recent times that any attempt has been undertaken to systematically collect the material relating to the frequence of tumor occurrence in animals (Johne, Fröhner, Casper, Sticker). It is very difficult at the present time to draw any comparison of these data with those of man whose tumor involvements have for many years been subjects of careful, conscientious and extensive statistics in literature. The early slatightering of the food animals is partly at fault, as many tumors are not apt to appear until advancing age. This is especially true of cancer, according to Fröhner; which in dogs is not encountered at all for the first two years, and is met with any degree of frequency only in old dogs; eighty-seven per cent. of his animals affected with cancers were over five years of age, fifty-four per cent. over seven years old (in man cancer is most frequent between the fortieth and seventieth year-about seventy per cent.). While in man cancer is of special frequence on the lips, stomach and uterus, in these sites the tumors are very rare in animals (gastric cancer has been observed but one time in the dog-Eberlein). Sarcomata, however, are mainly met in young animals.

As far as sex may have influence upon tumor formation, it can merely be said that the female sexual apparatus in animals is far less frequently involved by nualignant tumors than in mankind; only in the mammary glands of bitches are various tumors to be met with any frequence, while cancers of the genital tract occur only as great rarities.

As indicative of an influence exerted by the species upon the development of tumors, it may be stated that cancer is relatively frequent in dogs, melanosarcoma and cancer in horses, and sarcomas in cattle and swine (Casper).

Resume of Tumors.-The nomenclature and classification of tumors is, as explained on p. 326, mainly dependent upon their histogenesis, origin and tissue composition. The existence of many types of component tissues, together with the fact that cells of multiplying tissue may present all sorts of shapes, and that sometimes unusual tissues take part in the formation and that it is often impossible to determine from what normal type of tissue the tumor had its origin, make it difficult to carry out the classification strictly upon this basis. A certain number of tumors present themselves always in the same forms and these can be sharply defined; but there are others which are of mixed structure and of intermediate types.

The principal types of tumors may be distinguished as follows :

I-Fibroma, simple fibrous connective tissue tumor or fibrous tumor.

2-Lipoma, fatty tumor.

3-Myxoma, mucoid tumor. 
4-Chondroma, cartilaginous tumor.

5-Ostcoma, bone tumor.

6-Myoma, muscular tumor.

7-Neuroma and Glioma, nerve tumors.

8-Hamangioma, Lymphangioma, vascular tumors.

9-Sarcoma, atypical connective tissue tumor.

Io-Lymphoma, lymph gland tumor.

I I-Melanoma, pigmented tumor.

12-Endothelioma, Perithclioma, Cholesteatoma.

I3-Papilloma.

14-Adenoma, epithelial glandular tumor.

15-Carcinoma, atypical epithelial cancer growth.

r6-Epithelial cysts, Dermoids (skin tumors), Odontoma, Compound Teratomata.

\section{The Indizidual Types of Tumors.}

\section{Fibromata.}

A fibroma, or fibrous connective tissue tumor, is a growth made up of adult fibrous connective tissue, that is, of connective tissue cells, fibrils and bloodvessels.

Fibromata vary in consistence with the degree of predominance of their fibrillar basic substance, with the density or looseness of the bundles in which the fibres are united and the relative presence of blood vessels and lymph vessels. From this point of view two types of these tumors are distinguished, the hard and the soft fibroma.

The hard fibroma (fibroma durum) is met as rounded nodes of very dense consistence, usually presenting the firmness and appearance of tendon tissue, cutting with a tough resistance and creaking on section. The cut surface which shows but little moisture and is nearly dry, is of a white or light yellow color. with intermingled shining and dead white markings from the intricately interwoven bundles of fibres (depending on whether these are cut in longitudinal or transverse planes of section), and with a fibrous appearance. The tumor as it expands in growth is usually sharply defined, presses upon the adjacent structures and has no tendency to penetrate them: the surrounding tissue, however, being thinned more and more as the layer over the growth is stretched. The stretching and loss of tissue tension may cause coincident proliferation of these adjacent structures, as epithelium, over the tumor. Under the microscope the bundles 
of fibres, compactly arranged and thick, may be seen running in a wavy course, usually arranged concentrically about the bloodvessels and often of considerable length. Between the fibres are spindle cells with spindle shaped nuclei, and occasionally elastic fibres are intermingled.

Sometimes the fibroma develops as a diffuse growth, giving rise to large wooden-like thickenings of the subcutaneous tissue, with radiating extensions running into the surrounding structures.

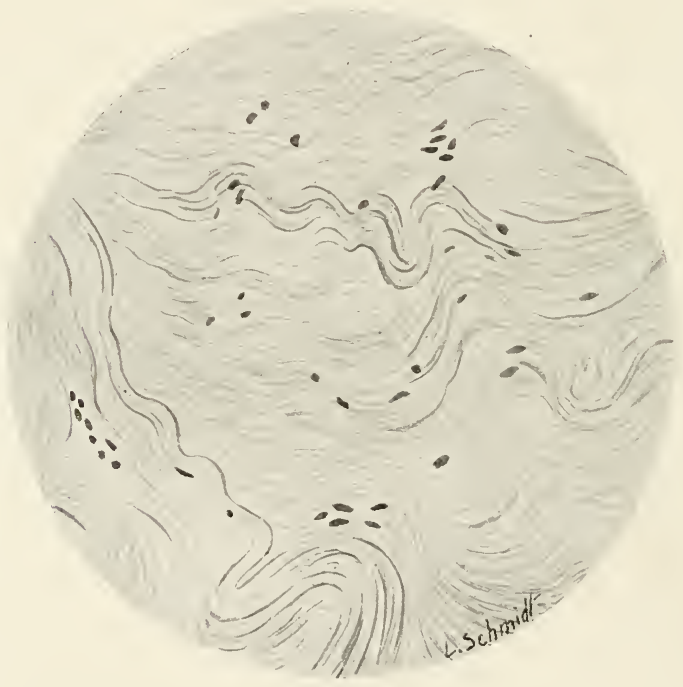

Fig. S6.

Hard fibroma: subeutaneous nodule, from herse.

The soft fibroma (fibroma molle) is characterized by its softer consistence, and the greater proportion of bloodvessels and lymphatics in its construction.: It is in consequence of a more reddishgray color, is sometimes gelatinous: and its shape is sometimes nodular or lobulated, sometimes with a villous papillary surface. Under the microscope it contains in addition to the loosely arranged bundles of fibres numerous spindle and rounded connective tissue cells and wandering corpuscles, and is quite rich in 
blood vessels. The soft character of the growth is due partly to the numerous cells it contains, partly to the presence of lymph fluid, especially where the latter is practically stagnant because of twisting of the pedicle of a pedunculated tumor. In such cases the bundles of fibres and cellular groups are separated from

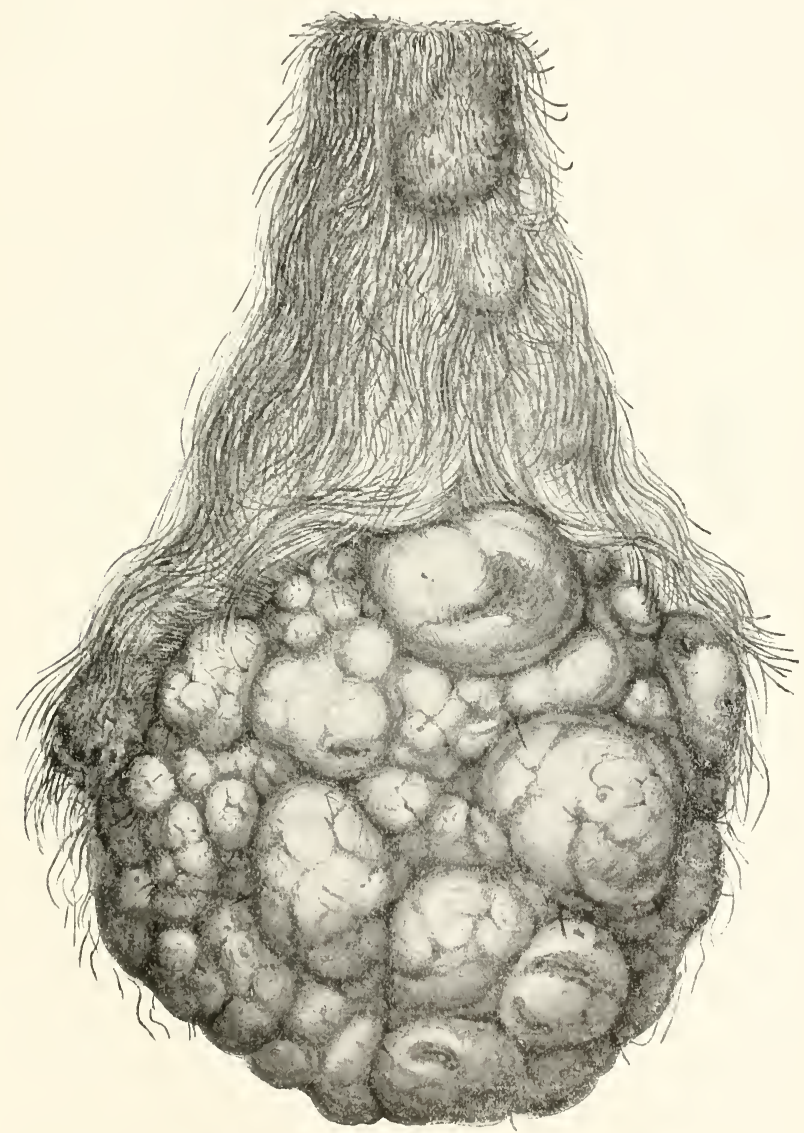

Fig. 87 .

Fibroma (mrxomatous) of the end of cow's tail. 
each other and meshes are formed and become more or less distended, and the cells take on branched, stellate shapes. Such a growth is also known as fibroma mytromatodes or fibromy.roma from the gelatinous change present in it; the more vascular reddish ones as raspberry polyps, fibroma angiomatodes, cazernosum or teleangiectaticum.

Occasionally calcification or even ossification takes place in

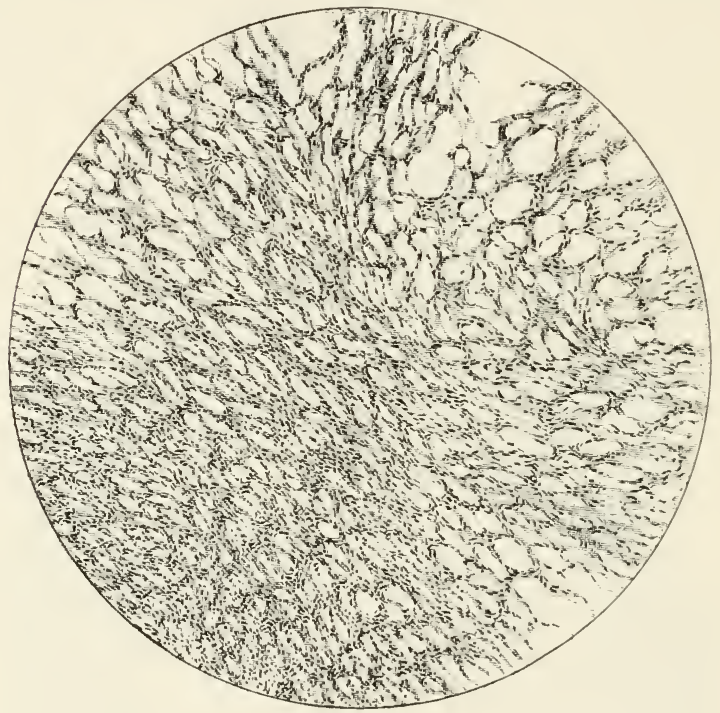

Fig. SS,

Section of a fibroma from a horse.

the tissue of fibromata, producing dull white foci of bony hardness (fibroma petrificans, ossificans).

Sometimes the fibrous tissue formation is found about the bloodvessels or about the glandular ducts or nerves, arranged in circular concentric whorls and systems of bundles about these structures; such growths being described under the terms plexiform or pericanalicular fibromata.

Fibromata growing from scars or imperfectly cicatrized granulation tissue from wounds are apt to become especially dense, consisting of thick, closely packed, interwoven bundles of fine fibrils, are known in human 
medicine as keloids ( $\chi^{\epsilon} \lambda \eta$, the claws of a crab), because of the resemblance of the rounded or finger-like nodes to the legs of a crab; in man spontaneous keloids are encountered as tumor formations, apparently hereditary in families.

The fibromatous tissue may combine with fat tissue, bone or muscle giving rise to fibrolipomata, fibro-osteomata or fibromyomata, or, when the second type of tissue predominates, to lipofibromata, osteofibromata or myofibromata. [It is not a matter of special importance, but generally the name of the tissue predominating is given as the basic name of the combined tumor, contrary to the above statement. Thus where in a tumor composed of fibres and adipose tissue the former predominates the growth is known usually as a lipofibroma: where the latter is the more important constituent, the term fibrolipoma is used.] In case of an associated proliferation of the epithelium covering a fibroma, or of glandular tissue the resultant combinations are known as fibropapillomata and fibroadenomata. Soft fibromata, more particularly because of active proliferation of their cells and from the fact that these young cells are not apt to maintain uniformity of shape but assume in varying measure embryonal characteristics in size and shape, occasionally acquire features which may justify the name fibrosarcoma.

Fibromata are of slow growth, usually single tumors, but sometimes primarily multiple, ranging in size from that of a nut to that of a human head or even larger, being known to weigh as much as one hundred, and even one hundred and seventy-eight kilograns (Wehenkel, Siedangrotzky). Because of the wide distribution of fibrous connective tissue structures their development may take place in practically any part of the body, although there are certain parts in which they appear with especial frequence. The places of election are the skin and subcutaneous tissue, especially of the front of the breast (horse), the dewlap (cow), the elbows ( $\log$ ), and the end of the tail (cow). In these situations they form hard nodular swellings or nodes covered with epidermis, or in other instances lobulated pedunculated growths (fibroma pendulans). As multiple primary formations Siedamgrotzky once counted in a thirteen-yearold stallion more than a hundred fibromata in the skin, which had grown in the course of three months in the breast, abdomen, neck and face, to sizes varying from that of a hazel nut to that of the palm of the hand. Similar multiplicity, warranting the term fibromatosis, has been observed in dogs by Fröhner and Cadiot 
(cited by Casper). Fibromata are frequently found in the tongue of the cow, as well as in the ragina, in the latter situation as pedunculated growths of large size projecting into the vaginal canal. Those fibromata which are found in horses and cattle after injury of the skin and tendon sheaths at the joints of the feet may form enormous masses; they are apt to have the surfaces covered with granulation tissue. Fibromyxomata are especially common at the end of the tail and in the heart (epicardium and endocardium) as pelunculated growths, as well as in the naso-pharnyx (where they are found hanging from the wall covered with mucous membrane and are known as nasal polyps). The author has observed a perfect string of hard rounded fibromata in the lung of a horse (for details v. Kitt, Lehrbuch $d$. speriellen pathol. Anatomie d. Hausticre, Terl. v. Enke, Stuttgart, II. Aufl., I90I).

A fibroma may sometimes be harmful because of its size and location (occlusion of passages, pressure upon vital organs), but does not give rise to metastasis and usually does not recur after cxtirpation.

The hard fibromata of the skin and tongue undoubtedly are often results of trannatic lesions. The connective tissue scleroses resulting from injuries by foreign bodies, or that arising after healing of an actinomycotic area, a chronic botryomycosis or even a malignant nedematous infection, may be sometimes such hard, definitely outlined, biscuit-shaped or nodular deposits and prominences that an anatomical distinction between these sequels of chronic productive inflammation and true tumors cannot be made.

\section{Lipomata.}

The lipoma or fatty tumor is made up of adipose tissue, of clusters of fat cells in various stages of development, along with bloodvessels and fibrous connective tissue. The fat cells are apt to be larger than those of normal adipose tissue. These tumors arise from situations normally rich in adipose tissue, as the mesentery or subserous peritoneal fat, where they frequently occur in the horse. The peritoneal adipose tissue often forms lobular appendages, which when much overgrown and stretched by the intestinal movements become pedunculated and present themselves as pearshaped masses or tumors of the size of a fist. They may give rise to fatal intestinal entanglements. In case 


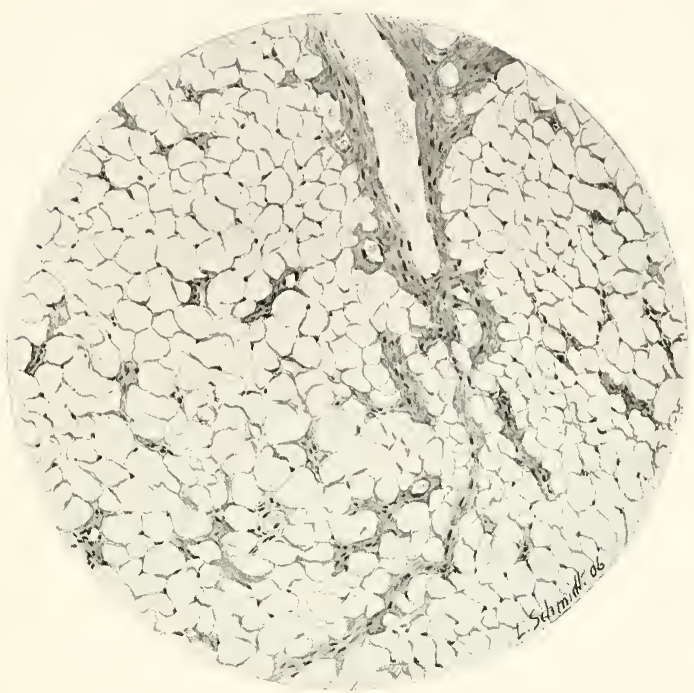

Fig. 89 .

Lipoma.

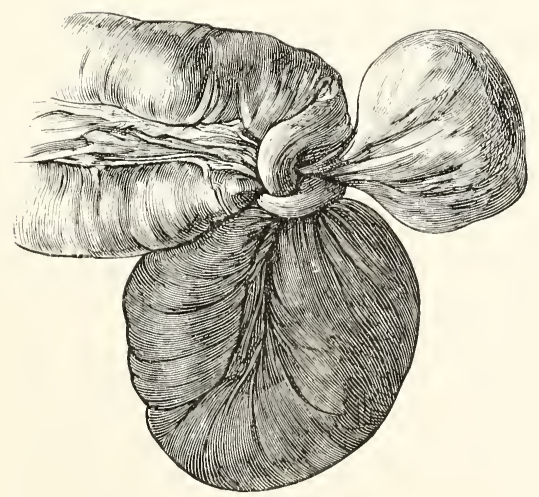

Fig. 90 .

Loop of intestine of horse strangulated from the entanglement of a lipoma. 
of rupture of the pedicle such lipomata become loose in the peritoneal cavity and may be found at autopsy as compressed free bodies. These peritoneal lipomata present a smooth surface, are semitransparent, yellowish or white. Lobulated lipomata are found also in the cow and hog in the omentum, and in the intestine and perirenal fat; in these animals the fat is firm and white. The subcutaneous tissue is a frequent site in the horse, especially in the neighborhood of the knees where they may attain very large size (twenty-six and a half kilograms in a case reported by Möller); and in the dog especially on the inner surface of the thigh, in the fold of the knee (Fröhner), and in the breast (Stockfleth). As submucous growths they have been found in the uterus (a lipoma of a cow recorded by Lund reaching a weight of seventy-five kilograms), and in the membrana nictitans in the horse and dog (Fröhner). Here, too, their shape is nodular or lobular and the growth is usually well circumscribed and therefore easily shelled out.

Sometimes lipomata appear in positions where adipose tissue does not normally exist; thus two lipomata of the size of a child's head have been found in a dog's liver by Trasbot, small lipomata in the kidneys of the same animal (Bruckmüller), and Kühnau encountered a rare instance of a lipoma of the brain in a three-year-old beef (four centimeters in diameter, rounded and arising from one of the bloodvessels of the pia mater). This last instance was congenital. According to Boström it is possible that a bit of germinal fat tissue of the skin of the embryo might have been separated and been misplaced in the cranial area. Lubarsch suggests that intraparenchymatous renal lipomata take their origin from portions of the renal capsule.

Lipomata occur as either single or multiple primary growths. They may occasionally undergo mucoid degeneration (lipoma myxomatodes), calcification (lipoma petrificans) or dry anaemic necrosis. When the combined fibrous connective tissue is especially abundant they may be very firm (lipofibroma). They are slow in growth, but as above stated may attain considerable size. Those which are situated subcutaneously may be easily extirpated: they are not recurrent and do not give rise to metastases. 


\section{Myxomata.}

A myxoma, a mucous connective tissue tumor, is a ncoplasm which consists either entirely or in part of gelatinous embryonic connective tissue; and therefore its tissue closely simulates the Wharton's jelly of the umbilical cord in consistence, transparency and texture. It is characterized microscopically by an intercellular substance rich in mucin, in which are sparsely scattered cells of spindle and highly branched steliate forms with long

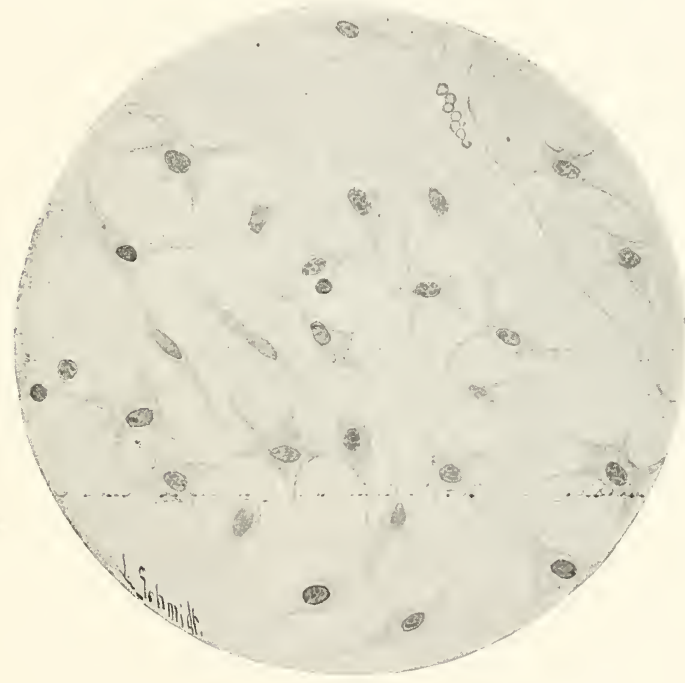

Fig. 91.

Myxoma.

slender prolongations; while here and there are to be seen blood vessels and a small amount of fibrillar connective tissue. In fresh material the mucinous substance may be precipitater by the addition of acetic acid. Often only individual portions of the tumor are conspicuously myxomatous, the remainder being made up of fat tissue or dense fibrous tissue (myxolipoma, myxofibroma); and many luxuriantly growing types show features of transition to sarcoma and mixed tumors (mytrosarcomata). Occasionally fibromata and lipomata of pedunculated shape, 
which have become œdematous from lymphstasis, simulate myxomata (fibroma my.romatodes), the absence of mucin or its scanty proportion in the fluid content distinguishing them from the true myxoma.

Myxomata develop as single or more or less multiple primary growths according to their situation, and as sarcomatous mixed

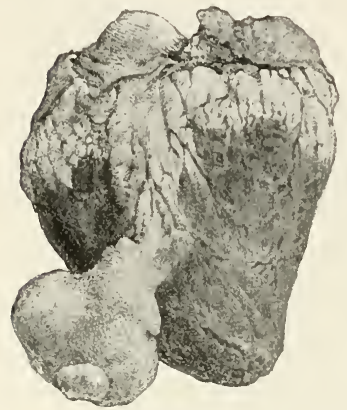

Fig. 92 .

Myxofibroma of the heart, from cow. tumors capable of metastasis and of embryonic appearance. They are especially met upon and in the heart of cattle (when the subpericardial and subendocardial foetal mucous tissue acts as their developmental substance) as rounded, lobulated growths ranging in size from that of a nut to that of a fist; they also occur in the nose in the cow and horse, in the parotid, and in one case in the dog around the spinal cord (Holzmann). and, too, in cattle along the nerve trunks (neuromyxoma). The gelatinous, glassy, swollen, yellowish (sometimes like flesh of the codfish) appearance, their softness and the ropy mucoid character of their substance, constitute their important distinguishing features.

\section{Chondromata.}

A chondroma or cartilaginous tumor has, in addition to a vascular fibrous connective tissue framework, as its main constituent cartilaginous tissue, usually of the hyaline type, and contains cartilaginous cells of very irregular size encapsulated in this substance without regularity of arrangement. The fibrous tissue forms a capsule and the trabecular framework about the different cartilaginous areas (which occur as islands and lobulated nodules in the tumor) and acts as their nutritive perichondrium.

Besides the pure chondromata there are met many mixed types, made up of fibrous tissue, bone and glandular tissue, in which cartilage enters as only one of the constituents: and modifications may also result from the rather marked tendency of the cartilage to metamorphosis, both regressive and progressive. Mucoid degeneration with production of gelatinous softened foci (cysts), and calcification, giving rise to white opaque patches 
(chondroma cysticum, mytromatodes, petrificans) are especially likely to be observed; and sometimes ossification (chondroma ossificans) takes place.

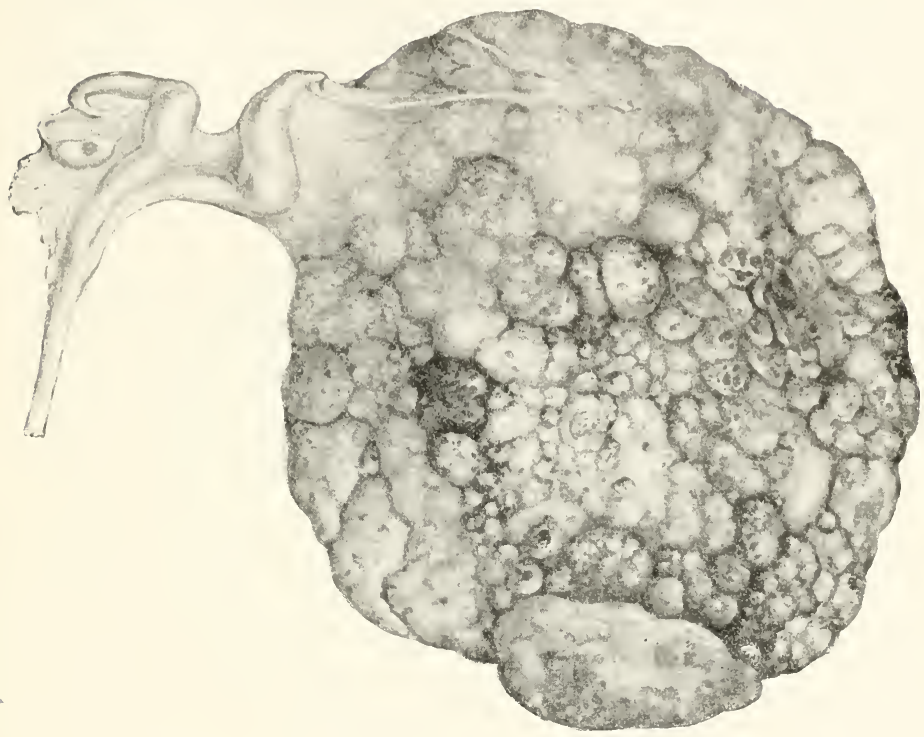

Fig. 93.

Chondrofibroma of ovary of sheep ithe uterus to the left).

Chondromata are nodular, nodose, lobulated, rounded tuniors of dense (in case of myxomatous change or when other tissues are combined they are softer) consistence; showing their cartilaginous substance as a milk-white, grayish and bluish white, semitransparent material. They may attain considerable dimensions, perhaps the size of a hmman head, and may weigh from ten to twenty-eight kilograms. According to their location the cartilaginous tumors may arise from previously existing cartilage (ccchondromata), or may develop within the bone marrow or in the soft tissues which do not contain cartilage (cnchondromata). The former originate from the perichondrimm, the cells of which are usually concerned in formation of cartilage; the latter arise probably from misplaced embryonic cartilaginous rudiments. 
Isolations of bits of tissue from either of these sources may occur in embryonic life from developmental faults, as in the formation of the branchial arches or the primitive vertebræ and embryonal elements of the ribs. The fact that chondromata may be congenital is an argument for this view. In the postfotal period cartilaginous isolations may take place from disturbance in growth in the skeleton (rachitis); and tranmatic lesions, as fractures of ribs, may give origin to separation of bits of perichondrium, and

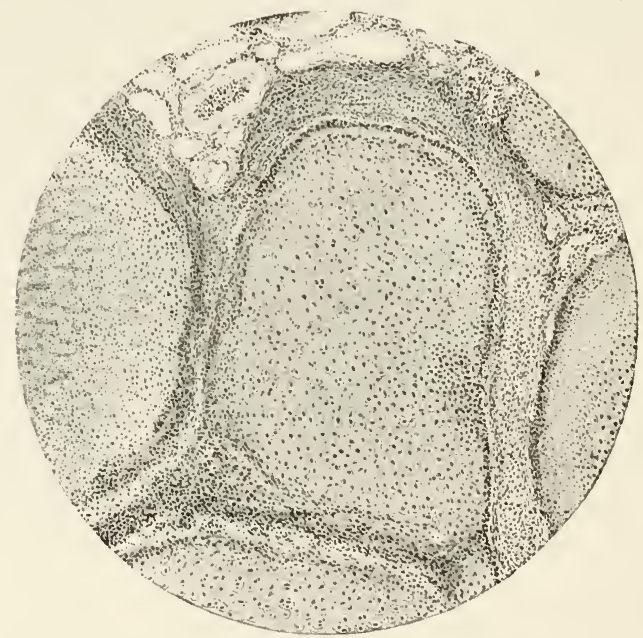

Fig. 94.

Section of a chondroma from a hen.

these in association with profuse callus formation might develop into chondromatous tumors.

Chondromata are most common in the bony framework of the chest as the result of fractures of the ribs, projecting into the chest cavity or beneath the skin as large tumor masses; they are also frequently met in the neighborhood of the jaw and thyroid gland, originating from remnants of the branchial cartilages, in goats, horses, dogs and cattle. As mixed growths (chondroadenoma) they have been seen a number of times in the lungs, mammary gland and in the testicles in animals. As exceptionally rare instances may be mentioned enchondromata of the vitreous 
humor of both eves in a congenitally blind colt (Renner), and a chondroma the size of a fist in the wall of a calf's stomach (first stomach).

A chondroma should be looked upon as a dangerons growth, at times because of the position it may occupy, but also because of its usually marked tendency to grow large, and further becanse it can give rise to metastasis. The cartilaginous masses happening to penetrate by growth into the veins sometimes develop long processes within the lumen of the vessels, which are of course obstructed: and any little portions which may be carried away by the blood may form metastatic nodes in other organs.

In man there are seen from time to time pea-sized growths of cartilaginous tissue. peculiar in the marked vacuolation of their cells and in their gelatinous consistence, at the base of the skull in the neighborhood of the spheno-occipital suture; these, because of the bladder-like, swolien character of the cells, were formerly known as ccchondromata plyysaliformia, but are recognized to be remnanis of the notochord and are called chordomata.

\section{0steomata.}

By the term osteoma or bone tmmor is meant a definite new formation presenting the structure of bone tissue and its marrow. According to the density of the calcified bone substance there are distinguished (I) the ibumated ostioma (osteoma (burncum) which is of ivory-like hardness like the lense cortical bone; (2) the spongy ostcoma (ostcoma spongiosum), of a more cancellous type, containing considerable marrow substance: and (3) the medullary osteoma (osteoma medullosum) composed principally of marrow. In most instances the structure of the bone (calcified matrix in lamellar arrangement about Haversian canals, bone corpuscles in lacunæ, bloodvessels, marrow cells and fat) is characteristic: but, often as far as the marrow is concerned, abnormalities are common, the medullary spaces being occupied perhaps rather by spindle cells and fibrous connective tissue (ostcofibroma) or showing special abundance of round cells and giant cells (osteosarcoma). Where the bone trabecula have no lamellated structure and the bone corpuscles do not show the usual projecting branches the specimen is spoken of as an osteoid sarcoma.

In many cases it is difficult and even impossible to determine whether a tumor-like osseous growth is really of autoblastomatous origin or is the product of inflammatory proliferation. because definite lines of separation between the two cannot be 
drawn. For this reason usually only the more massive, large and clearly defined bony growths are called osteomata, and the rest are known collectively as osteophytes. The small protuberances, of the size of a pea to that of a nut, projecting from the surface of bones, are also called exostoses; the flat superficial thickenings around bone shafts, periostoses; those which grow along bones as bony formations in fascie and conncctive tissue, parostoses; an ivory-like, hard, well-clefined formation in the interior of bones, an enostosis. Diffuse thickenings of the bones in the skeleton from osseous lyperplasia are known as hyperostoses; osteophytes with cartilaginous coverings, as cartilaginous osteophytes.

The osteophytes and osteomata growing from the skeleton develop principally from the osteoblastic layer of the periosteum or from cartilaginous developmental bases (anlage); some are congenital or from their position may be looked upon as caused by disturbances of embryonal derelopment and as referable to inclusive misplacement of bits of the embryonic bone tissue affected. This view is especially applicable to the rather common large osteomata of the head cavities (cattle, horses), arising from the sphenoid, ethmoid or the turbinate bones; in their gradual enlargement they press upon the surrounding bony walls, force the jaw and nose out of position, project into the maxillary sinus, nasal cavities or into the orbit or cranial cavity, and obstruct these spaces with continuous pressure 11 pon the respective soft parts. Other forms which are located on the ontside of bones, sometimes as broad expanded growths or arising from slender pedicles, probably are caused by trammatic influences; and the exostoses which are met commonly in the under jaw of the horse apparently arise from isolated osteoplastic foci; and the large osteomata on the horn process in cattle are probably due to loss of tissue tension caused by injury to the horn capsule.

A remarkable variety of pathological bone proliferation is the progressice ostcitosis met with in young dogs. It consists in the occurrence of bone formation at the places of attachnent of the tendons and muscles of the lower jaw and extremities, causing as it progressively advances an atroply of the muscles and giving the animal a stiff awkward gait; it is accompanied by marked thickening of all the bones of the skeleton. The disease is similar to the affection in man known as myositis ossificans progressiz $a$, and is apparently an abnormality of development, in which as Ribbert supposes the intermuscular con- 
nective tissue acts as a derivative of the periosteum. In fresh specimens the periosteum and muscular connective tissue of these cases appear to be merged into one mass of bacony, cartilaginous and rigid character; after maceration, brittle, papyraceous

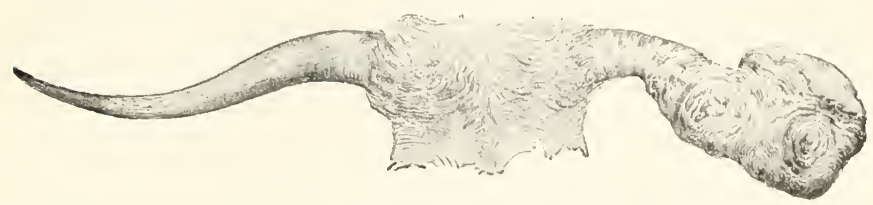

Fig. 95.

Osteoma on the horn process of a cow.

and gland-like plaques and nodosities of bone are found along the muscular attachments.

Osseous growths of irregular and flat shape (ossificatio raccmosa, plana) and intermediate types approaching compact osteomata have been encountered as isolated deposits and in combination with cartilage and fibrous tissue occasionally in the lungs (cow), peritoneum and mesentery (cow, hog, dog), upon and within the udder (cow, dog), testicles (horse), in the vitreous humor of the eye (horse), and in the right cardiac auricle of the horse. The attempt to explain the origin of these bone forma-

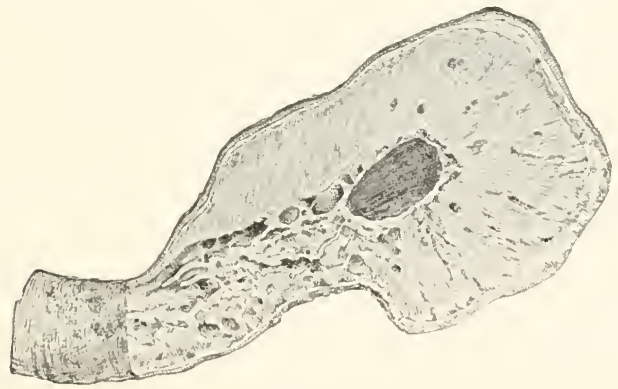

Fig. 96.

Section of above osteoma of horn process.

tions must rest with the assumption of the possibility of a metaplasia of fibrillar connective tissue into a calcified matrix, with transformation of the connective tissue corpuscles into bone corpuscles. 


\section{Myomata.}

A myoma or muscle tissue tumor is composed of muscle fibres, together with an accompanying vascular connective tissue. Corresponding with the two varieties of muscle, these tumors occur in two forms, one, the lciomyoma (myoma lavicollulare). composed of smooth muscle cells, and the other, rhabdomyoma (myoma strioccllulare), a tumor macle up of transversely striatęd fibres.

The latter (rhabdomyoma) is a very rare tumor; and never shows the muscle tissue in a state of perfect development like that of the muscles of the body. The muscle fibres are rather of an embryonic type and are usually mingled with embryonic connective tissue (rhabdomyosarcoma, rhabdomyoma sarcomatodes) or combined with glandular tissue in addition (rhabdomyoadenosarcoma). The muscle fibres are thinner than normally, ribbon shaped; their transverse strixe are not complete and are only apparent along the edges of the fibres, a richly nucleated sarcoplasm occupying the interior of the fibres. Numerous spindle cells with longitudinal striations may be noticed in addition, which may be looked upon as early stages or as muscle fibres persisting in undeveloped condition. These nuscle elenents are not arranged in compact parallel bundles but are scattered about in irregular manner. They are therefore not of as deep red a color as normal striated muscle, having rather a pale reddish-gray tint. They occur as more or less well defined nodes: and rarely occur in situations where striated muscle tissue exists normally but rather in complete heterotopism, in organs which have no such basic elements. This would indicate that rhabdomyomata originate in muscle germinal tissue misplaced in embryonal life. This is doubtless true of the tumors in question which develop from the kidney, the muscular elements in their structure apparently originating from misplaced portions of the myotomes of the primitive vertebral column. Johne has recorded an adenosarco-rhabdomyoma of this type from the kidney (hog) which weighed fourteen kilograms. (In man rhabdomyomata have been recognized frequently in the kidneys, testis, uterine wall, urinary bladder and the œophagus.) Besides the case above mentioned, contributed by Johne, there are only two instances of rhabdomyomata in animals recorded in literature (Casper). One was observed by Gratia in an old horse in a dissecting room, a fusiform tumor about eight or ten centimeters in length, in the 
vagus nerve trunk about the middle of the neck; the other was found by Kolessnikow, growing in the tail and perianal connective tissue of a twenty-year-old stallion, a tumor containing pigment cells (rhabdomyosarcoma melanodes), which had given rise to numerous metastases in the liver, spleen, peritoneum and pleura.

The lciomyoma is found more frequently and in easily recognized forms in animals: in case of extensive combination with

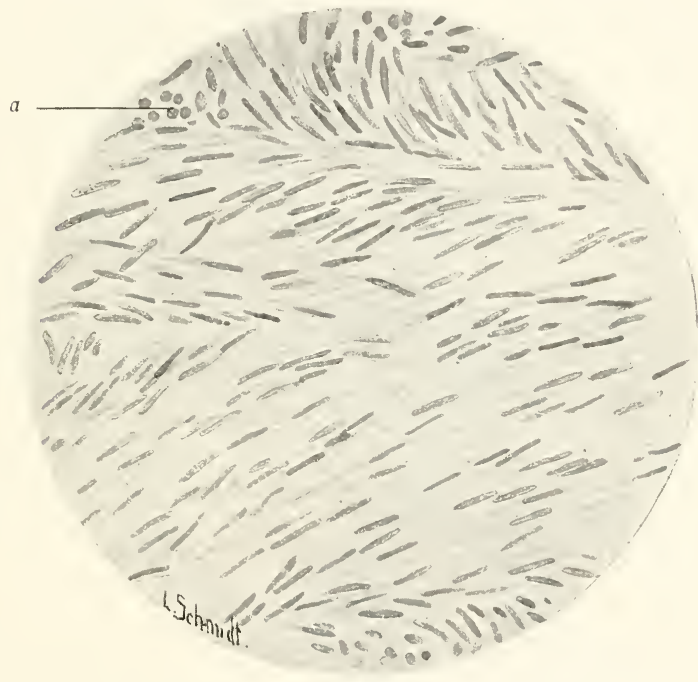

Fig. 97 .

Leiomyoma (from uterus): $a$, place showing buidle of muscle fibres cut transversely.

fibrous connective tissue, as may sometimes be seen, the growth is spoken of as a myofibroma. These tumors always derelop in a structure rich in smooth muscle, and are consequently met principally in the muscular wall of the uterus and vagina (in cow and hog, less frequently in the dog), of the intestine and stomach (horse), urinary bladder (dog), now and again in the testis (horse), and in the subcutaneous tissue (horse). They are rounded or globular, firm tumor masses, which are sometines found projecting from their places of origin, covered over smoothly with mucous or serous membrane, presenting on their 
cut surface a fibrous or striated appearance, of a reddish-gray or pale gray color. Especially the leiomyomata growing from the uterus and hanging to this organ in the peritoneal sac, may attain enormous size, possibly as large as a well filled horse's stomach, weighing from thirteen to thirty-six, to one hundred kilograms.

The smooth muscle fibres, easily recognized by their long fusiform shape and rod-shaped nuclei, are arranged parallel to one another in bundles interlacing in every direction. with each other in confused manner. In sections the fascicles are found cut longitudinally, obliquely and transversely, and between these the vascular connective tissue may be observed in variable amount as clearer threads. In pedunculated examples where the myomata have in consequence been subject to twisting, circulatory disturbances are often determined, as results of which congestive hæmorrhages, œdematous appearance, anæmic necroses and fatty degeneration may take place in the tumor mass.

In man epithelial inclusions and hollow tubules have been noted in the myomata of the genital organs, this histological feature giving support to the view that the myoma in this situation is related to embryonal disturbances which have occasioned misplacement and isolation of portions of the Wolffian body or of the Müllerian ducts (adenomyomata).

Leiomyomata are usually single primary growths [in human beings they are often multiple], and give rise to harm only by their volume. They are not recurrent tumors [and are not metastatic.]

\section{Neuromata and Gliomata.}

A neuroma, or nervous tissue tumor, strictly speaking must be made up of nerve fibres or gangliomic cells in luxuriant proliferation, accompanied by a supporting framework and by bloodvessels. If we exclude the terminal thickenings of nerve stumps occasionally occurring after neurotomy, the so-called amputation neuromata (v., p. 250), from the list of true tumors (because their growth ceases after a time), it may be said that the formation of true neuromata in animals has as yet never been certainly proved to occur. Even in man but few instances of this type of neoplasm are known, situated in the sympathetic area (especially cœliac ganglion and pelvic plexus), as nodes ranging up to the size of a child's head.

The growths summarily designated by the name neuroma in 
the literature are neurofibromata and neuromy'tomata, tumors which develop in corded, fusiform or cylindrical thickenings up to the size of a finger, or in nodular shape up to the size of a

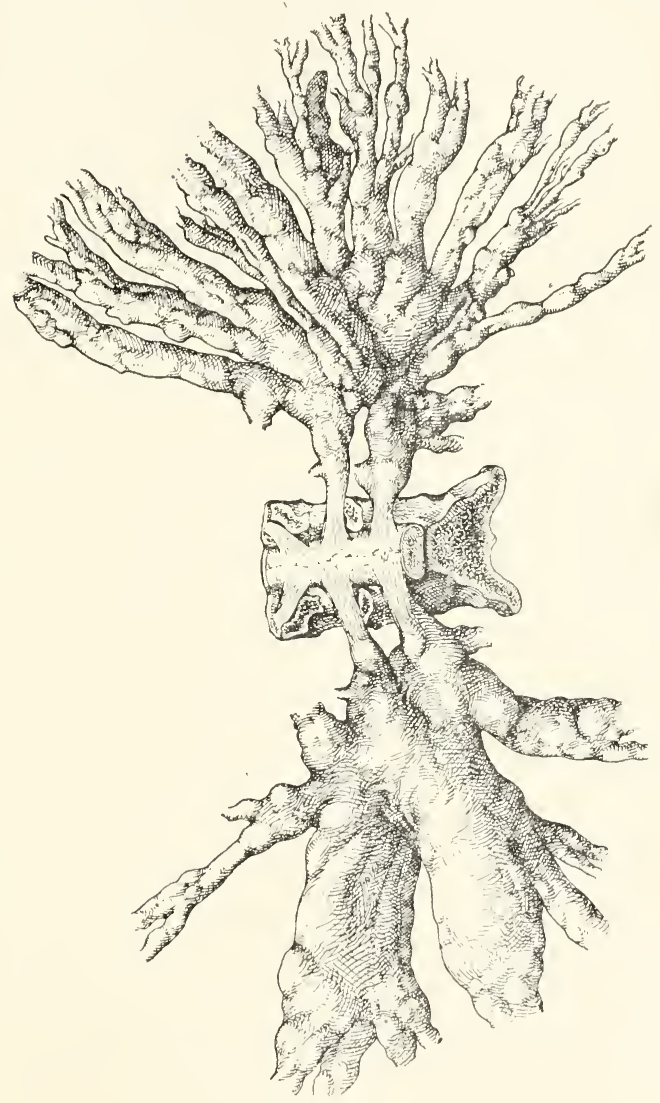

Fig. 98 .

Neurofibromatosis of brachial plexus of cow.

potato, on the nerve trunks. Their formation results from a marked proliferation of the perineural connective tissue and nerve sheath about the nerve fibres. The proliferated mass has 
a grayish-red, semitransparent appearance and usually extends along many adjacent nerves as a nerve plexus, giving rise to an extensively intertwining growth (plexiform or vine-like neuro= fibroma). Tumors of this kind have been observed, especially in cattle, involving the brachial plexus and the dorsal, costal and sternal nerves (Ostertag, Morot, Blanc, author's personal observation), and producing in these structures hundreds of nodular thickenings. Morot counted in one case thirteen hundred and fifteen neuromyxomata in an old cow and traced them even to the nerves of the pharynx and heart. Leisering observed similar growths in a horse with extensive involvement of a number of nerve trunks.

An embryonic developmental disturbance of unknown nature has been regarded as the basic factor for the neurofibromata and neuromata observed in man, because the growths have been met as congenital and inherited faults.

Glioma or ncuroglioma, the tumor of nervous connective tissue substance, is produced by proliferation of the glia cells of the central nervous system or the granular layer of the retina of the eye, and occurs primarily only in these two positions. In animals it has been recognized with certainty but twice, once by Gratia in a dog's brain (ganglion Gasseri) and again by Piana in the spinal cord of a dog, in both instances causing palsies from pressure upon the central nervous substance and the emerging trunks.

The gliomata of the human brain are usually embedded in the brain tissue without sharp definition from the latter and are so merged with it that they are only recognizable as diffuse grayish or reddish firm areas. They are made up of protoplasmic cells with numerous fibrils projecting in a radiating fashion from all sides of the cell (spider cells, astrocytes); and in this fibrillar meshwork there are in some instances also present polymorphous sarcomatous or ganglionic cells, and epithelial inclusions may be observed (spaces lined with cylindrical epithelium). This last feature and the occurrence of these growths in newly born children confirm the belief that disturbances of development of the cerebral vesicles are the basis of these neuroglia growths, for example isolation of offshoots from parts covered by ependyma (perhaps, too, transformation of the glia cells to embryonic cellular type, the glia tissue being originally an epithelial tissue differentiated into a supporting material). 
Retinal gliomata of man spread out as grayish nodular tumors over the inner surface of the globe of the eye, but are also met perforating the sclera, growing along the optic nerve and perhaps escaping into the orbit; they occur as congenital growths sometimes involving both eyes, and have been observed in a few instances in several children of the same parents. For these reasons and because of their structure they may invariably be referred for their origin to developmental faults. They are only in part composed of glia tissue, being principally made up of neural epithelium of the retina reverted to embryonal type, arranged in thick sheathlike layers around the synchronously proliferating bloodvessels. [These so-called retinal gliomata are the subject of considerable controversy. The presence of any true glia tissue comparable to that of the brain is in itself questionable, and the evident part taken by the retinal neuroepithelial tissues has led to their being denominated neuroepitheliomata. These neuroepithelial cells are frequently found in a so-called rosette arrangement, as the cylindrical epithelium seen in sections of small ducts. The tumor in its infiltrating and metastatic character is a very different growth from the glioma of the brain; the former is rapidly fatal, while the latter shows little tendency beyond a slow peripheral infiltrative enlargement to extend, and persons may have harbored these growths for years with little definite evidence of their presence. The more separate arrangement of the cells of the true glioma, the branching fibrils of the cells preventing close adjustment, is not seen in the retinal glioma, whose cells are very closely packed and evidently have but few and insignificant projections upon them (in fact one must examine with great care even after special staining to think that these projections exist in any comparable (egree). The editor would regard favorably the separation of these growths from the class of true gliomata, preferring the more distinctive and well justified term of neuroepithelioma.

The true glioma of the brain is usually single: sometimes several have been found, usually in close relation and probably in reality representing the same growth. When pure and free from sarcomatous combination the tumor should be regardeit as relatively benign save for its pressure effects. Distant metastasis is rare; although local metastasis from peripheral infiltration is the rule.] 


\section{Haemangiomata and Lymphangiomata.}

The hamangioma, or blood vessel tumor, is made up of distended and proliferated vascular tubes filled with blood, together with the connective tissue supporting these vessels. Strictly speaking, a true vessel tumor must consist for the most part at least of vessels which have been newly formed, and should not be a tumorlike enlargement made up simply of pre-existing vessels which have become enlarged from some pathological cause. It is, however, difficult to make a distinction between these two conditions, partly possible only by examination of microscopic sections. Therefore in a gross anatomical sense there is usually included under the term hæmangioma, in a tentative way, a second variety which is made up of blood lacunæ, which give rise to a nodular irregularity in shape and a spongy consistence of the part affected [cazernous hamangioma].

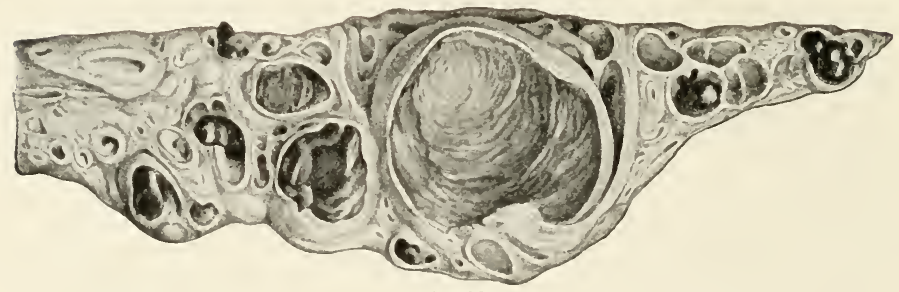

Fig. 99 .

Portion of cavernous angioma of cow, seat of multiple thrombi.

In man hæmangeiomata are represented first by the invariably congenital blood-red or bluish-red, sharply outlined areas of skin, in which the tissue of the cutis, often to the depth of the subcutaneous fat, is filled completely with thin-walled bloodvessels in a definitely limited area (Hamangioma teleangiectaticum, ncs'us vasculosus, flammous, vinosus, wine mark).

Such independent vascular tumors, caused by a disturbance of tissue development, are, it appears, but rarely met in animals, probably because the pigment and hair of the skin conceals their presence. However, Leisering observed an angioma as large as a hen's egg in a dog, in the subcutaneous tissue (under surface of the tail); Siedamgrotzky a similar one in a poodle, showing as a bluish-black area through the skin; Bonnet a fibroangioma as large as a child's head with a strong connective tissue stroma in a horse, in the papil- 
lary bodies of the root of the tail. Similar teleangiectases are more frequently recorded as occurring in the nasal septum in the lorse (Liipke, Hofmann, Diegendesch, et al.), appearing as superficial red or bluish-red, smooth, easily bleeding arcas.

The very common capillary teleangicctases in the bovine liver. multiple purplish-red or bluish-red spots showing through the Glissonian capsule, and appearing as finely spongy, bloody areas in section, are to be regarded according to the studies of Stroh as due to passive hyperaemia (pressure by the paunch in tympanites).

The second type of angioma above mentioned is the carcrnous angioma of the human liver, occurring in similar forms in the cow and horse. A system of blood spaces is formed in the liver, com-

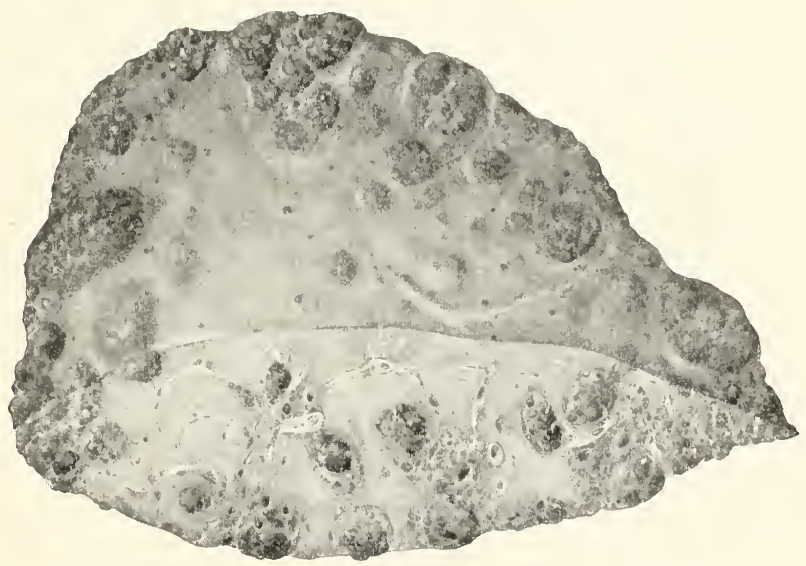

Fig. 100.

Fortions of an angiomatous liver of a horse.

parable to the erectile tissue of the penis or to coarse sponge, with cavernous passages of very irregular width and varicose, interrupted by projecting ledge-like bands of connective tissue. The area, rccognizable from the external surface by the superficial dark red elevations and the increased volume of the involved lobe of the liver, is not sharply outlined, and contains quantities of tarry blood and frequently also firm laminated clots. Between the connective tissue septa islets of liver structure sometimes persist. The whole appearance gives one the impression of a bunch of distended veins, dilated 
from passive congestion, or a nodular hæmorrhagic focus, rather than of a neoplasm. In man, however, such foci have been observed in congenital occurrences; and Ribbert suspects as the fundamental cause some developmental disturbances of the affected part of the liver.

The name lymphangioma, or lymph vessel tumor, is applied to congenital tumors in man, which occur as independent bunches of lymph vessels (that is, not in direct relation with the lymph vessels

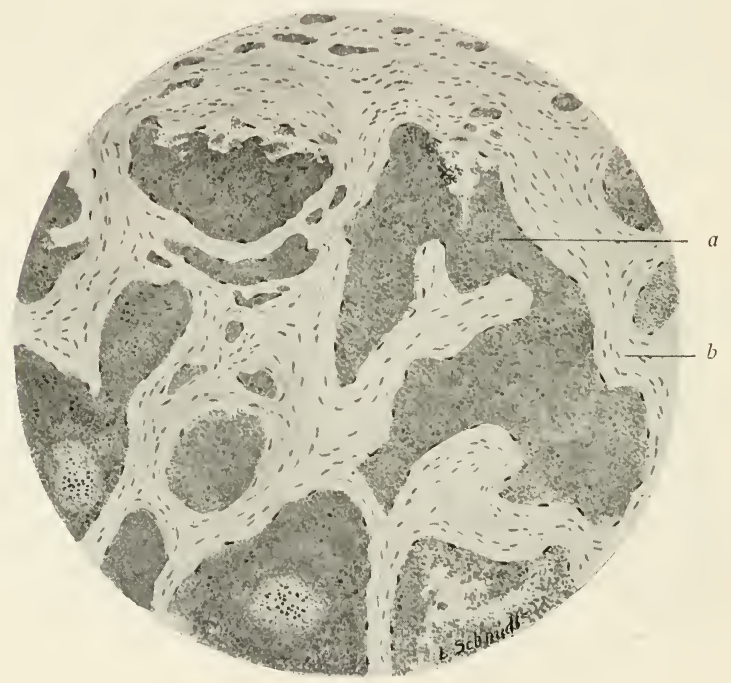

Fig. 101.

Cavernous angioma: $a$, lacuna filled with blood: $b$, indurated intervascular connective tissue.

of the adjacent tissues). They are especially seen in the neck, in the mesentery, the lips and the genital organs, sometimes not well defined and causing rather a diffuse swelling of the part. They are made up of channel-like, anastomosing, multiloculated passages filled with lymph, their walls of fibrous tissue lined with endothelium.

There is only one definite and certain record of the occurrence of this type of tumor in animals, published by H. Markus; ; on the

* Honatsheft f. pr. Tierheilk. Stuttgart, 1902, p. 185. 
costal, pulmonary and pericardial pleura of a horse there were found numerous superficial, vesicular growths from the size of a pin's head to that of a nut or pigeon's egg, some with broad surface, others pedunculated, exuding a serous fluid on section from the spongy tissue. Microscopically, they consisted of a loculated connective tissue framework, the spaces lined with endothelium and containing scattered lymph cells. The growth was entirely local, the lymph glands not affected.

Schindelka observed in a cat as an anomaly, perhaps of thi, same category, a number of tumors abont all the nipples, ranging in size from that of a hazel nut to that of a hen's egg, encircling the nipples and hanging loosely, filled with fluid and fluctuating like a leather bottle when handled; firm lympli cords, of the thickness of the little finger and varicose, branched, out from these formations into the skin.

Now and then large, tortuous lymph vessels filled with lymph are found in the horse in the heart or mesentery, which are, however, merely lymphangiectases.

\section{Sarcomata.}

The name sarcoma ( $\sigma \alpha \dot{\alpha} \xi$, flesh; fleshy tumor, from its similarity to proud flesh or exuberant granulation tissue) was applied hy Virchow to those connective tissue tumors which do not possess the definitive characteristics of at fibroma, chondroma, lipoma, etc., that is, which are not composed entirely of mature fibrous tissue, cartilage, bone, etc, but throughout their continuous growth are made up of cells of the connective tissue group persisting in their embryonic characters. They are $11 \mathrm{ew}$ growths whose tissue shows no tendency to maturation and is composed principally just as in embryonic life of undifferentiated mesoblastic cells and scanty intercellular substance, and which are distingtished from the simple connective tissue tumors particularly by their malignant character, their rapid and destructive growth, their tendency to recurrence after removal and their formation of metastases.

The sarcomata are classified from two standpoints: first, from the predominating type of the cells of the growth: and second, from the admixture of mature tissue with the embryonic cells.

Many sarcomata are characterized by a special predominating type of cell; and although the shape of a cell does not alone determine the character of a tumor, the tissues of origin and the nature of growth being also taken into consideration, it gives the microscopic picture of a tumor an individuality of importance in nomenclature, and characteristic because of the deficiency of fully matured trpes of 
tissue. Those forms of sarcoma whose cells do not grow with any special uniformity, but rather as a motley mass of various forms in atypical embryonal proliferation, cannot of course be named from the cellular character. These instances, as a rule, represent the malignant form of fibroma, chondroma, osteoma or melanoma, or are mixed tumors; in these cases a combined expression is used. The completely developed tissue forms, as it were, the framework or stroma, and the cells the parenchyma.

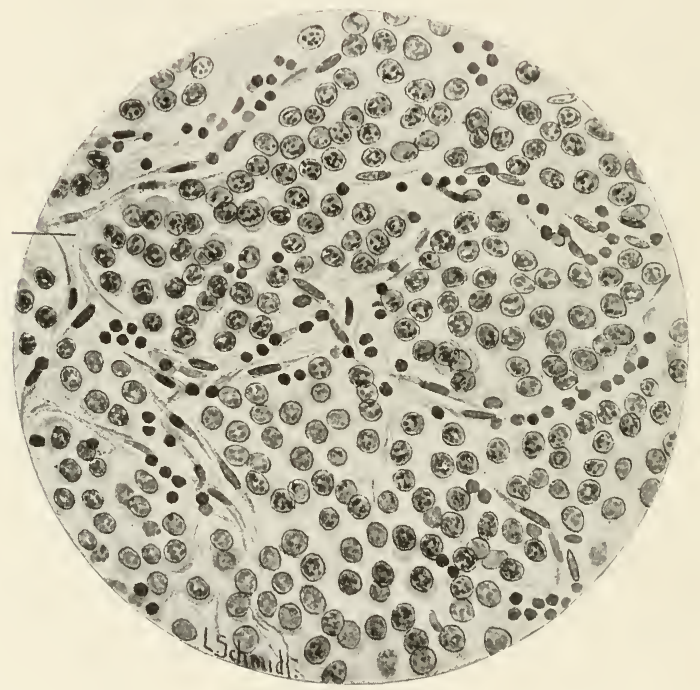

Fig. 102.

Alveolar round cell sarcoma, from testicle (human).

The principal types of sarcoma may be divided as follows:

A-Ccllular Sarcomata: the round cell sarcoma, spindle cell sarcoma, giant cell sarcoma, stellate cell sarcoma, polymorphocellular sarcoma.

B-Combined Sarcomata: the fibrosarcoma, liposarcoma, chondrosarcoma, osteosarcoma, myxosarcoma, myosarcoma, gliosarcoma, lymphosarcoma, melanosarcoma.

In stricter application it would be well to determine precisely, as Borst and Dürk have urged, whether the embryonal cells were present from the 
beginning, and their proliferation and that of the mature types of cells have from the first developed side by side, or whether a simple tumor originally existed and later assumed a typical growtl from clianges in the cellular proliferate. In the former case the combined types of tissue may be indicated by the use of two nouns (as fibro-sarcoma); in the latter by the adjective prefix "sarcomatous" [the change into sarcomatous type may be expressed]. In the course of clinical observations, where the succession of various features is recognizable, such distinctions may be occasionally made; but in purely anatomical considerations they cannot be made.

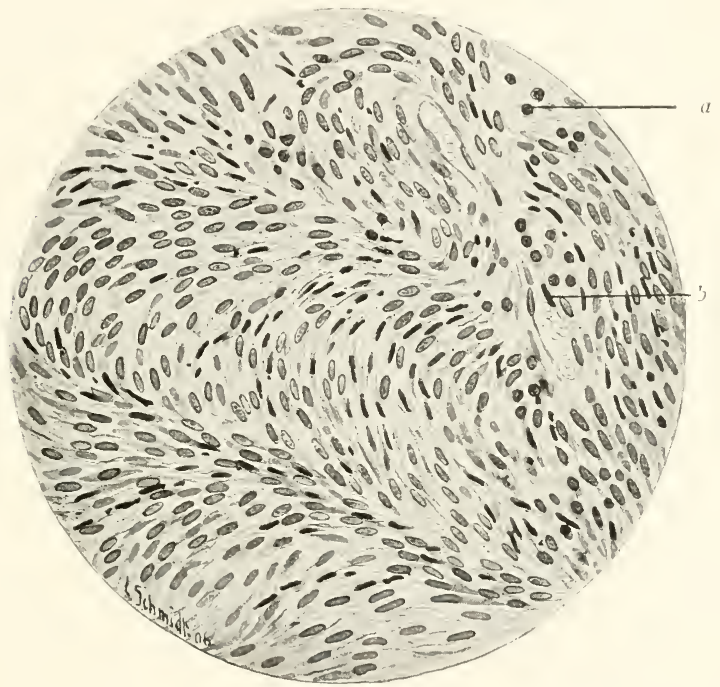

Fig. 103.

spindle cell sarcoma, from periosteal orisin: $a$, transverse section of bundle of spindle cells ; b, capillary blood vessel.

The round coll sarcoma (sarcoma globocellulare) consists either of cells of the appearance of lymphocytes, small round elements with narrow cytoplasm and granular nucleus ( small cell sarcoma), or of large round and polymorphous cells (large cell sarcoma), closely packed together and with such a small amount of fibrillar intercellular substance that the cells are but loosely attached. The tumor has a very soft consistence and is of a grayish to a grayish-red color. When the supportive tissue enclosing the round cells is arranged in a reticular manner, forming a fine fibrillar meshwork, and the tumor has originated from one of the lymphatic structures 
(spleen, lymph glands, bone marrow), it is known as a lymphosarcoma.

The spindle cell sarcoma (sarcoma fusicellulare) also presents both small and large cellular elements and originates from ordinary connective tissue. Its spindle shaped, more or less elongated cells contain one or more round to fusiform nuclei, are placed parallel to each other, arranged along the bloodvessels in bundles intertwining in different lirections, and are comparable to the fibro-

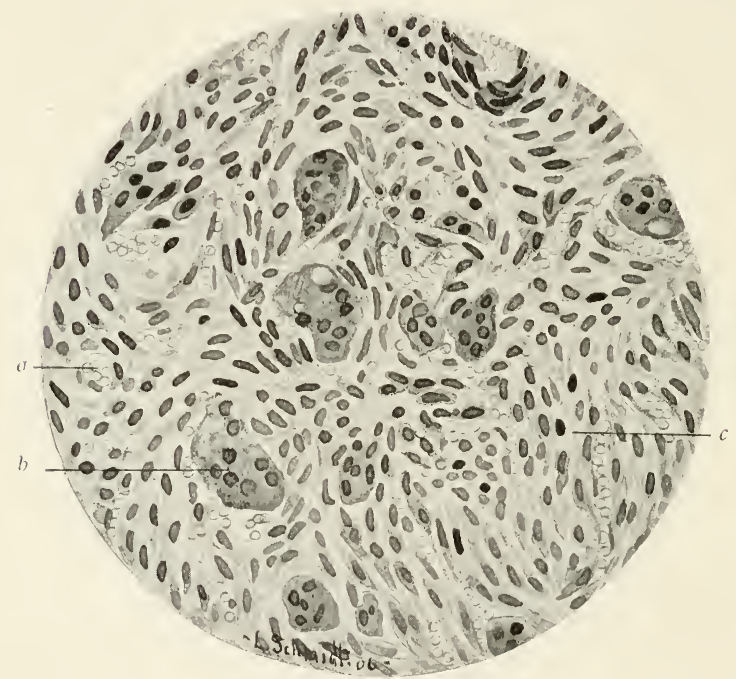

Fig. 104.

Giant cell sarcoma, from human jaw : $a$, capillary blood channel: $b$, aiant cell: $c$. salcomatous cells, in this specimen of spindle type.

blasts of granulation tissue. The bloodvessels are also of embryonic type, persisting as simple endothelial channels, often distinguishable from lymph spaces merely by the fact that they contain red blood cells. Where the intercellular fibrillar substance is more prominently developed the spindle cell sarcoma becomes a fibrosarcoma.

The giant cell sarcoma (sarcoma gigantocellulare). The presence of numerous giant cells in a connective tissue tumior, always a striking feature of the microscopic sections, gives origin to the term giant cell sarcoma. The large polynucleated cells thus known 
are usually derivatives of the marrow or periostem where they occur normally (known as myeloplaxes). They differ from the (legencrative types oi giant cells (formed in tubercles and about foreign bodies) in the fact that their nuclei are not located about the periphery but are scattered well in the interior and all through the cell protoplasm; but all sorts of variations may be found (completely divided or wreath-like clumping of the nuclei, giant nuclei. eccentric dividing forms, budding nuclei). The number of nuclei in one cell may reach not merely a dozen or two, but hundreds. The cells, whose dimensions may exceed ten to thirty times that of a white blood corpuscle, are irregularly rounded, their margins jagged, are flattened out like large fibroblasts and are often vacuolated with fat droplets. In addition to the giant cells there are tistally numerous round and spindle shaped cells in the tumor, and a rich supply of capillary ressels from which blood extravasations are frequently found in the tissue, and which give the tumor a dark-red or brownish-red color. From its medullary origin the giant cell sarcoma is often called a myclogenous sarcoma or myeloma; and when a homogeneous intercellular strbstance is present. becoming calcified and forming cartilaginons islands or bony trabectla, the combined appearances are denoted by the terms ostcoid sarcoma and ostcochondrosarcoma.

The stcllate or reticular cell sarcoma (sarcoma stellocellulare) is characterized by highly branched cells forming an intricate meshwork, usually enclosing between their projections a mucoid, glasslike intercellular material, giving to the growth characteristics commonly indicated by the term my rosarcoma.

The polymorphocellular sarcomia (sarcoma mirtoccllularc) is a name given to those connective tissue tumors whose cellular constituents follow no one cellular form, but are made up of a confused mixture of round, spindle, stellate and giant cells, or the bulk of whose cells are elements of variable form, irregularly polygonal, enclosed in a comparatively small amount of intercellular matrix.

The morphological appearance of sarcoma cells, the size and polygonal shape of their cellular substance and their alveolated arrangement make it often very difficult to determine to which of the classes of sarcomata described and specially named by different authors a given sarcomatous neoplasm should be referred. This difficulty and the lack of finality in our histogenic basis of classification are apparent in the frequent emplorment of combined nomes indicating the mental uncertainty of the pathologist. as sarcoma carcinomatodes, or carcinoma sarcomatodes: and it is not an 
uncommon thing for good histologists to make use of different diagnostic terms to describe one and the same tumor, one perhaps calling it sarcoma, others endothelioma, perithelioma or alveolar sarcoma. This is but natural when one considers the number and variability of connective tissue tumors and their combinations, the differences of composition which exist in different parts of one individual growth and the changes and metaplasias which are possible, these features preventing any schematic and clearly defined classification.

The macroscopic appearances of sarcomata are naturally very variable on account of the many types of histological structure which they may present. Some are soft, others hard; their color is generally a grayish-white, similar to the tissue of lymph glands or fotal brain tissue (hence the old terms medullary sarcoma and encephaloid sarcoma). They are usually rapidly growing tumors, some growing in nodular, nodal or lobulated forms, others as infiltrative growths. Their expansion not only occasions considerable pressure upon the surrounding tissues and organs, but by stretching the tissues they approach the surface of the organ, hreak through the mucous membrane or external skin, or destroy the part from which they arise by their deeper growth. By their infiltrative growth and penetration between the tissues sarcomatous formations occasion marked enlargement in bulk and expansion of the part affected. Isolated tumors, reaching from five to seventeen kilograms in weight, and enlargennents of organs of perlaps twice or three times the normal size, are not uncommon in sarcomatosis of the kidneys, liver, rectum, spleen and lymph glands. The great tendency to infiltration occasions daughter nodes in the vicinity of the original growth and leads to metastases, which are especially likely to follow the blood current. Penetration into blood vessels is often apparent to the unaided eye in case of large veins, as those of the liver. The tissue increase, both in the primary and secondary tumors, is always due to the independent proliferation of the sarcoma cells, the formation of blood ressels (associated proliferation), however, proceeding directly from the organ in which the sarcoma cells are distributed, apparently from a peculiar influence exerted by the tumor cells upon the endothelium (Ribbert). These vessels, proliferating in association with the tumor cells, for the most part maintain their capillary type, often, of course, as wide endothelial tubes; the frequence of hæmorrhage in the sarcoma substance being due to the thinness of their walls. Because of this abnormality in the blood stupply, which may not keep pace 
with the rapid exuberance of the cellular growth, various retrograde metamorphoses are possible, of which fatty degeneration is the most common.

Nothing certain is known of the development and cause of origin of sarcomata. Now and again sarcomata and their combined growths are met following traumatism (fractures of bone, contusions and injuries to the soft parts); from the analogy shown by luxuriant traumatic granulation tissue growth to the sarcomata, it may be supposed that some instances are due to lesions causing loss of tissue tension, and further investigation of convincing examples would be of much interest in this line. In the second place sarcomata may be thought of as perhaps related in their origin with faults of development, both because they are common in man and animals in early life and because their cellular character is suggestive of embryonal conditions.

The principal seats of primary sarcoma formation in animals are the bones (jaw in dogs and horses, cranial cavity in cattle), the lymphatic glands and spleen (dogs, cattle), and the intestine (cattle) (Fröhner, Leisering, Dieckerhoff, Siedamgrotzky, Casper).

[Of the sarcomas it may be said in a general way, to which exceptions are not infrequent, that the spindle cell varieties more commonly arise from the denser types of connective tissue as the periosteum, fasciæ, intermuscular septa and capsules or framework of organs; and that the round cell forms more commonly originate from the softer and more cellular types of connective tissue, as the lymphoid structures and the intraparenchymatous intercellular connective tissue of organs. The giant cell sarcoma is practically limited to the bone marrow, usually growing toward the ends of the shafts of the long bones.

In the same comprehensive way, again with many exceptions, it may be expected that the round cell types are the larger, more irregular and more infiltrative as primary growths, and of a soft fleshy consistence, highly vascular, and, before the blood has been washed out, of a red, translucent, soft fleshy appearance (paler, as above described, after the blood has been removed or if the growth when obtained from the subject is relatively freed from its blood contents); the spindle cell form tending in the same general and unfixed manner to be smaller in size, less infiltrative, but rarely encapsulated, more regular in outline and nodular or lobulated, somewhat firmer in consistence and of a lighter color than the round cell varieties. The giant cell sarcoma ustually causes an expansion of the bone shaft, is covered with a thin and generally imperfect shell of 
bone, the interior sarcomatous mass soft (sometimes almost mushy), deep red and hæmorrhagic and bleeding freely when cut into. The hæmorrhagic tendency is often so pronounced that the term aneurismal sarcoma has been applied to describe this feature of marked vascularity.

Of the sarcomata above described by the author, the highest grade of malignancy from metastasis is met among the round cell varieties, especially the small round cell sarcoma and the alveolar round cell sarcoma; spindle cell sarcomata are also decidedly metastatic in the small cell form; the large spindle cell sarcoma being sometimes metastatic, but especially showing its power in this direction by recurrence after removal (hence sometimes called recurrent fibroid tumor). The giant cell sarcoma is often said not to give rise to metastasis; but this not strictly the case, as in man the editor has encountered two well-established instances of metastatic formations of this type, once from a myeloid sarcoma of the upper end of the tibia to one of the tarsal bones, and again from a similar growth in the upper end of the shaft of the femur to the pelvis].

\section{Lymphomata.}

By the names lymphomata or lympho-sarcomata are meant progressive proliferations of a tissue of the type of that of the lymphatic glands, originating from the lymphatic structures of the body. Lymphatic tissue is found in the lymph glands, bone marrow, spleen and thymus gland and is represented, too, by microscopically small developmental areas of lymphocytes widely distributed through the connective tissue of the system. The number and size of these depots and developmental foci of the leucocytes and lymphocytes vary considerably in different individuals, even physiologically. For example, the thymus gland is often found double the size attributed to it as normal, and in some calves the intestinal lymphatic glands are so much enlarged and so confluent that the mucous membrane in its entire extent may approach a centimeter in thickness, without the possibility of speaking of this so-called lymphatismus [status lymphaticus] directly as a pathological condition (Johne). So, too, in calves and also in goats, hogs and dogs, enlargements of the lymph glands are found which are due to proliferation of their lymphoid tissue, and from one standpoint may be regarded as hyperplasias, but which assume the character of tumors in their progressive infiltrative extension and are very doubtful so far as the history of their causation is concerned. These 
growths are spoken of as lymphomatosis, progressize lymphomata, Hodgkin's Disease or malignant lymphosarcomatosis. The affection manifests itself by a gradual enlargement of one group of lymph glands in excess of the rest. In all parts where the glands are palpable, as in the flexures of joints, in front and back of joints, along the neck, in the throat, they may be recognized as rounded

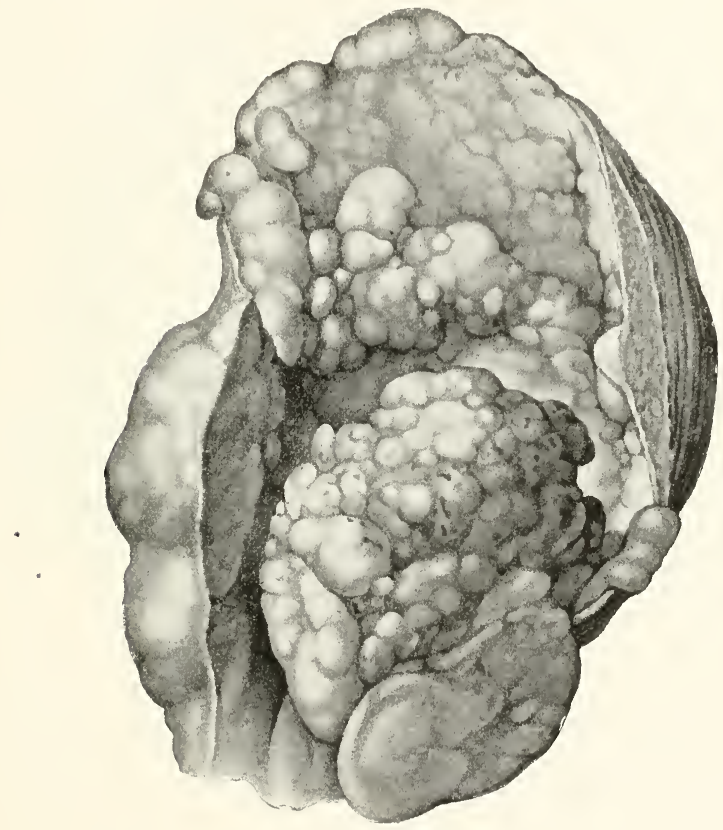

Fig. 105.

Urinary bladder of cow beset with lymphemata (laid open).

oval movable bodies, enlarged to the size of a nut or that of a fist. At autopsy the lymph glands of the interior of the body are also found changed into large nodulated masses, sometimes reaching the size of a human head. At first the capsules of the glands are retained and, as a consequence, the nodular enlargements are well defined; their consistence varies, sometimes soft, sometimes firm. and the surface of section has throughout a uniform grayish-white 
color. The minute structure of these enlarged glands corresponds more or less closely to that of normal ones, but differentiation into follicles, lymph cords and lymph sinuses is less marked, and the tissue is more uniform. The tumor mass is made up of a finely fibrillar or more coarsely trabecular network of connective tissue and blood vessels in which are uniformly distributed, in close apposition to each other, cells of the type of lymphocytes. In addition to the small rounded lymphocytes with round nuclei rich in chromatin, there may be found larger cells like those often met as early or provisional forms in the germinal centers of the follicles, and once and a while a few giant cells (Ribbert). Besides the proper lymph nodes the follicles of the intestine, pharnyx, and especially the lymphatic foci of the spleen, also undergo enlargement. The last-

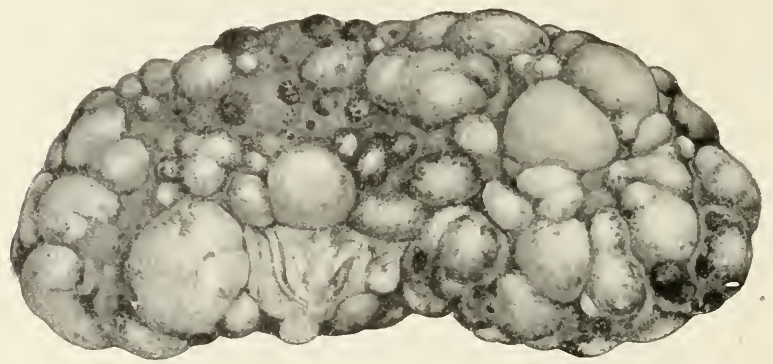

Fig. 106.

Malignant lymphoma in kidney of hog.

named organ may reach very marked excess of volume, either showing its Malpighian bodies increased to the size of a millet seed or a pea (follicular hyperplasia of the spleen), or the whole pulp as well evincing great increase of its lymphatic constituents as a grayish-red swollen mass (diffuse splenic hyperplasia).

In the course of the progressive proliferation the lymphatic tissue passes beyond its original limits, the capsules of the lymph nodes are broken through and the bunches of glands coalesce into shapeless masses. Diffuse extension takes place, the adjacent soft parts becoming occupied by the penetrating lymphoma, and an infiltrative type of enlargement is assumed from which the glandular parenchymatous structures, like the liver and kidneys, may also become diffusely and very markedly enlarged. The infiltrating lymphoid tissue gives to the parenchyma of such parts a pale gray 
color, which may be either diffuse or present an irregular mottling. The infiltration follows along the lymph spaces of the connective tissue, especially along the perivascular lymph channels, and may lead by special local accummlations of the tumor cells to the production of new tumor-like nodes as well. The lymphosarcomatous cells, however, also penetrate into the blood vessels, and by con-

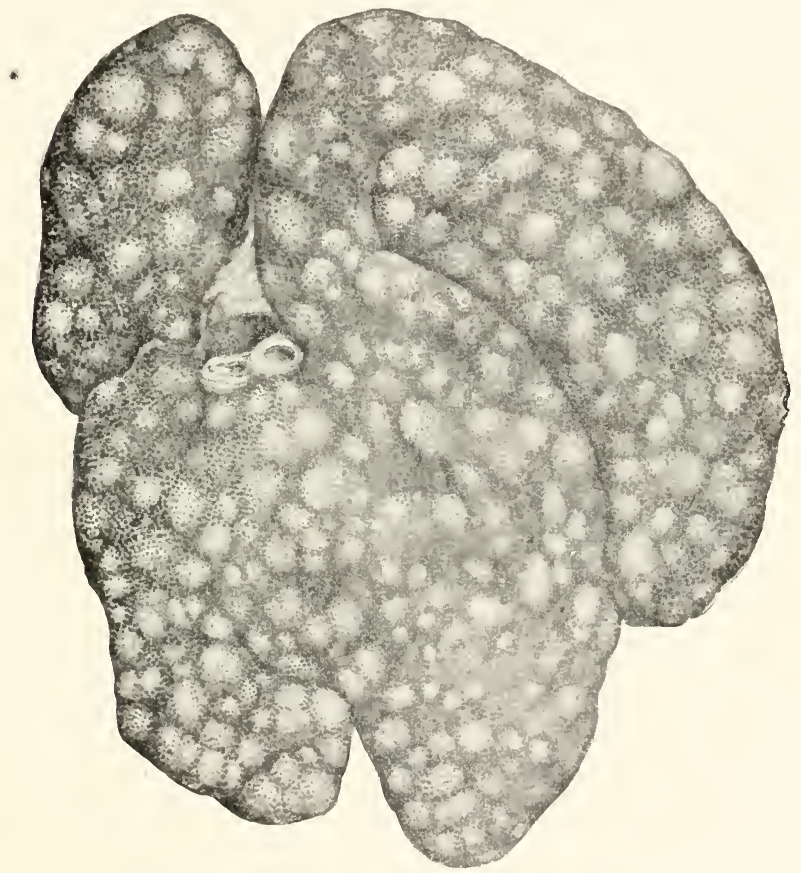

Fig. 107.

Nultiple lymphomata in liver of hog (yeneral progressice lymphomatosis).

vection may occasion metastatic nodules in various organs, especially in their lymphatic structures. The fact that the lymph glands in order of position undergo enlargement one after another, and that the metastases are principally located in the lymphatic structures, may, it is thought, be due to chemotactic influences of related types of cells (Ribbert). It may be that the lymphosarcoma cells 
are lodged in the lymph centres having passed through the capillaries, because the lymph cells present in these foci have an attraction for them; besides, apart from such a mode, the tumor cells carried in the lymph stream are bound to enter the lymph glands, where they are filtered from the lymph and remain. According to Ribbert's views, progressive lymphomatosis is a true tumor formation. The successively involved glands show the inception of the new formation often by well defined foci, sometimes only to be recognized in the peripheral sinus of the node, this arguing for

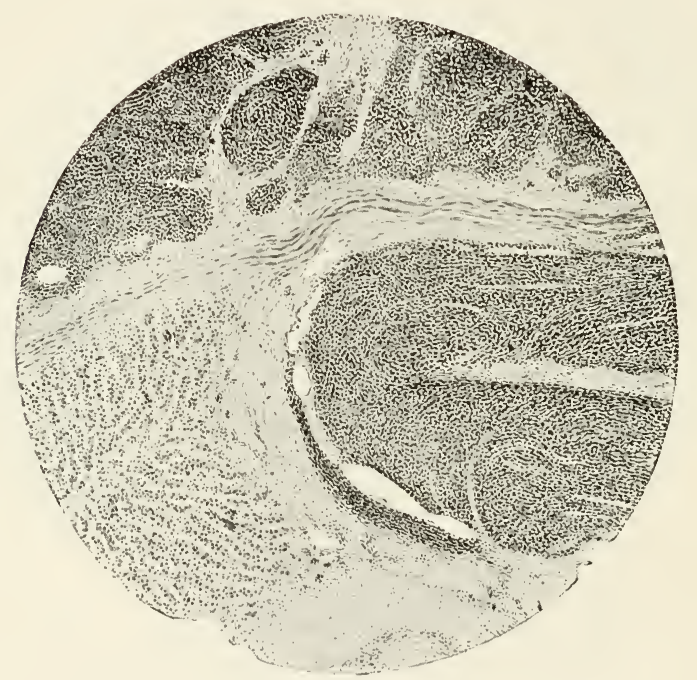

Lymphosarcomatosis of liver of ecw (tow power).

Fig. $10 \mathrm{~s}$.

transference of the tumor element from a primary focus. By others it is believed that some infectious process, bacterial in nature, is connected, and that the microorganisms gain access to the lymph glands in the lymph circulation and set up proliferative processes in the nodes; but as yet moculation experiments have given no positive evidence. [The author's statement is true; yet glandular enlargements, clinically regarded as belonging to Hodgkin's disease, have repeatedly been found to be tuberculous in nature. The lymphomatoses probably should be looked upon as of two types, one 
of which is best regarded as merely a lymphatic hyperplasia occassioned by microorganisms or toxic substances which are in some way brought to the lymph nodes (and some of these are tuberculous, the remainder probably being products of a variety of microbic and toxic influences). The second group may be properly referred to the class of the sarcomas and spoken of as lymphosarcoma and lymphadenoid sarcoma. The term lymphoma may be provisionally retained to cover the group of uncertain or indistinguishable examples, but has no other place properly in tumor nomenclature. If we limit ourselves to that series of cases, to which, from their higher infiltrative and metastatic tendency (especially where we can recognize that the secondary nodules are true metastases and not mere hyperplasias of previously existing lymphoid foci which may have been induced by an original microbic or toxic cause), the name sarcoma may be reasonably applied. It should be added that lymphosarcoma is a highly malignant type of sarcoma, ranking with the ordinary small round cell variety in the severity of malignancy from metastasis. It is, of course, difficult to make the separation in a large gromp of cases, but as far as possible we should endeavor to exclude from the term lymphosarcoma those cases in which enlargements manifest any appearances of infections origin. Where among these there is evidence of tuberculosis of course the case is promptly referred to as tuberculous lymphatic hyperplasia. For the rest of the cases the non-distinctive term Hodgkin's disease may be employed if desired; and it is well to keep clearly in mind that the affection known as lymphatic lenkemia. both acute and chronic, bears close relationship with the latter group.]

\section{Melanomata.}

A melanoma, melanosarcoma (chromatophoroma), or pigmented tumor, is characterized by a black-brown or slate-gray color produced by the pigment cells (chromatophores) which constitute the growth. Normally pigment cells are found as specially differentiated connective tissue elements in the skin and choroid coat of the eye, and in some animals, as sheep, also in the pia mater of the brain. The melanomata take their origin from these cells and are therefore found most frequently in the skin, being especially common in horses. It is peculiar that gray and light colored horses, which as foals had a dark coat of hair, should be especially prone to these tumors; and it may be 
presumed that irregularities of their loss of pigment underlies the anomaly. Melanoma may, however, also occur in brown horses and has been seen once in a black (Hall). In most instances these growths develop in horses in the region of the root of the tail, the anus and external genitalia. In these positions they form nodes which protrude like boils beneath the skin, attaining the size of several fists and several kilograms in weight. Secondary nodes are formed along the lymph passages, so that in the cellular tissue of the pelvis there may be found whole chains of tumor nodes; and by infiltrative growth the tumor

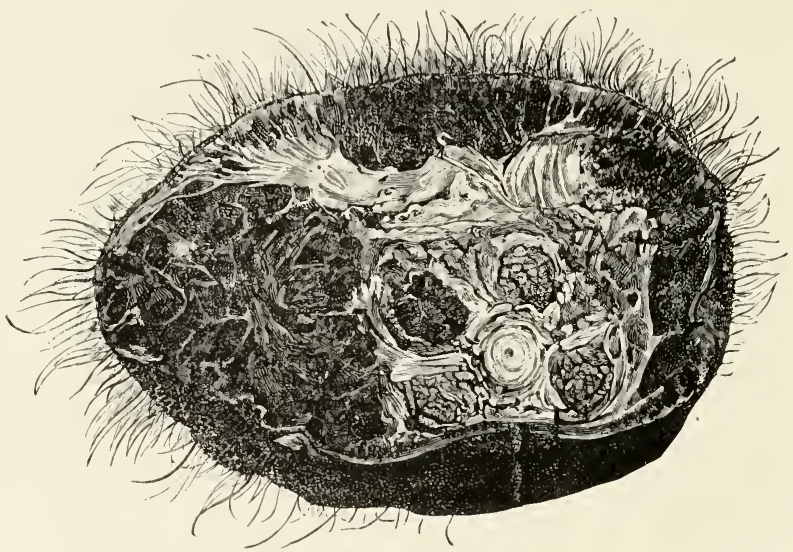

Fig. 109.

Section through horse's tail involved by melanoma.

may completely invade the cutis and subcutaneous tissue, and cause widespread thickening and swelling. The growth is usually slow; it may continue for several years as a circumscribed and strictly local process, at other times being highly metastatic and causing an extensive generalized melanosarcomatosis, with secondary nodes in the lung, pleura, spleen, liver, kidneys, bones, musculature and elsewhere. Melanomata of the same nature as the above have been met also as primary growths in the cellular tissue near the parotid gland, in the pancreas (Käsewurm), intestine (Csokor), at the base of the heart (Dexler), and in the spleen. 
In cattle melanomata are of less frequent occurrence. Metz saw two cases in white animals; in one the tumor was situated in the dewlap, in the second in the pelvic cavity near the uterus. Hamburger found one tumor of this kind in the diaphragm; Hoare removed a melanoma weighing fifteen kilograms from the temporal region of a brown ox. Bollinger has recorded the occurrence of a congenital melanoma of the size of a fist in the cranial cavity of a thirty-day-old calf; Wulf, a similar growth in the subcutaneous tissue of the ankle of a calf. In sheep Worseley has reported a case of melanosarcomatosis involving

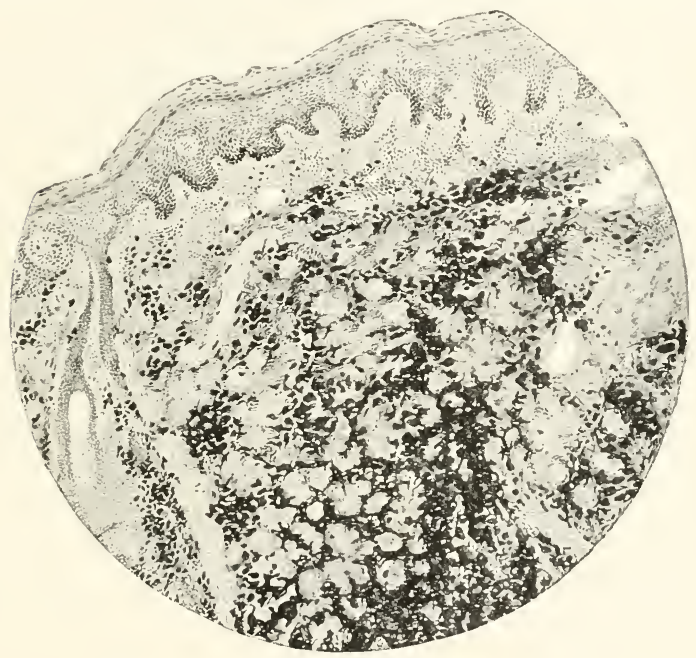

Fig. 110 .

Microscopic section of skin of horse infiltrated with melanoma.

the lumbar region, mammary glands, lungs and liver. In the dog the author has observed a melanoma.springing from the gums, and Bruckmüller one at the base of the brain.

Some melanomata have a firm, rather dry appearance; others are found of soft consistence. The cut surface is uniformly dark, or sepia-brown or slaty; when handled the fingers are soiled as if with shoe-blacking.

Microscopically the pigment is found in the form of brownish black granules, which are distributed in the fluids of the 
tumor and within the cells. The latter elements are seen as variably shaped, irregularly rounded, fusiform and branched cells, their cytoplasm usually so thickly loaded with the dark brown granules that the nucleus is obscured; at places, however, they may be found entirely free from pigment. The cells are usually so closely packed together that their outlines cannot always be differentiated. The tumor tissue is very clearly defined from the rest of the tissues, into the lymph spaces of which the neoplasm can be seen to be extending its way, affording an excellent demonstration under the microscope of the mode of propagation of these growths. The cells are so easily loosened in the softer specinens that the tumor mass may stand out like thick pultaceous material; Csokor has observed in the case of a horse with the intestinal mucous membrane (colcn) the seat of melanoma, the discharge of black fecal matter; and Bollinger, Bruckmüller and Senmer have found the pigment granules in the blood (melanxmia).

The pigment material known as melanin, certainly does not come from the blood (iron free), but is formed by a specific activity of the chromatophorous cells, as Berdez and Nencki lave shown; the important proportion of sulphur in its composition suggests that it is a derivative of albuminous bodies.

The origin of melanomata is necessarily in some way related to developmental disturbances which give rise to excessive production or misplacement of pigment cells; their occurrence as congenital tumors and the fact that in man the growths are known to frequently take their start from congenital pigment moles of the skin, are evidence in favor of such a view.

The malignancy of these growths is not miform; as above stated, some may remain in their primary situation for years without appreciable enlargement, and may not recur when removed, but others, especially the softer forms, spread rapidly and widely and give rise to great numbers of secondary nodes. [The melanotic sarcoma of man, most commonly met in the eye, is extremely malignant, ranking probably first of all in the list of sarcomata in this respect.]

\section{Endotheliomata, Peritheliomata, Cholesteatomata.}

By the term cndothclioma is meant a tumor which is composed of endothelial cells of connective tissue derivation, especially those of blood vessels and lymph vessels, as well as those 
of the dura mater of the brain and spinal cord. Endotheliomata in their structure stand relatively midway between the atypical connective tissue growths and epithelial tumors, and resemble now one and now the other of these so closely as to make their distinction from sarcomata or carcinomata often practically impossible where only single bits of the growths are subjected to microscopic examination. They differ from cancers in the history of their development, the latter taking their origin from epithelium,

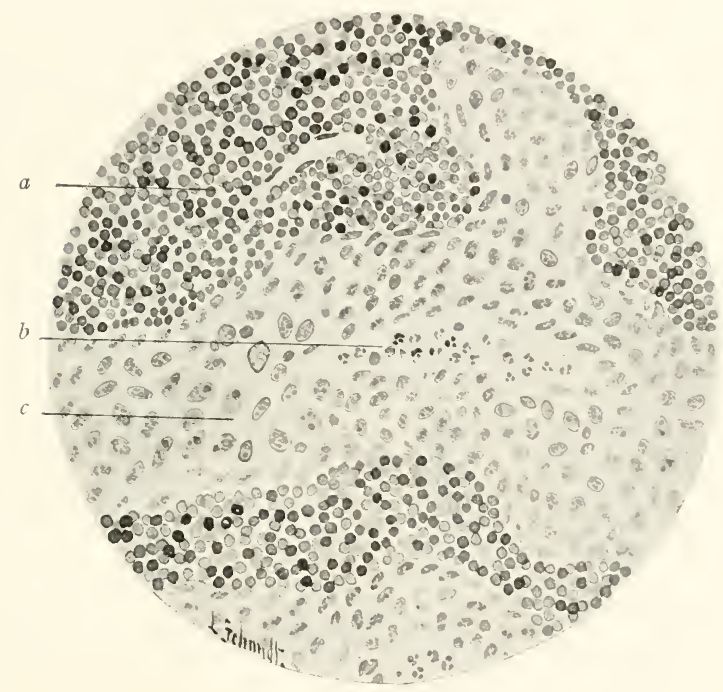

Fig. 111.

Endothelioma of 1rmphgland (human): $a$, lymphoid tissue of gland: $b$, leucocytes in nearly obliterated lymph-sinns; $c$, endothelial cells proliferated from lining of $1 \mathrm{ymph}$-sinns.

either ectodermal or entodermal, while the true endotheliomata are of mesoblastic origin. It is more difficult to define them from sarcomata, the cells of the latter being also derived from the middle germinal layer; and for this reason some authors speak of them as endothelial sarcomata. (The layer of so-called endothelial cells lining the large serous carities of the body, the coelomatous lining, is to be regarded as epithelial and the tumors which arise from these cells are therefore to be classed as cancers.) 
The cells of an endothelioma present the same polygonal, flattened, fusiform and polyhedral forms as the fibroblasts and angioblasts, the cells of the blood and lymph vessels being, in fact, formed originally of the same material. In the tumors they are arranged as scales, or in cylindrical cords or in hollow tubular manner so as to form a network with thickened nodal points, and are supported by a vascular stroma. The microscopic appearance is therefore very like that of an alveolar sarcoma or carcinoma; sometimes the arrangement of the cells in lamellæ may" be concentric like the tunic of an onion.

Those endotheliomata which take their origin from the outer coat (adventitia) of the bloodvessels (from the perithelial cells) and are recognized as forming mantles of tumor cells about the blood vessel and its branches, are generally spoken of as peritheliomata.

Retrograde changes which occur in endotheliomata may give an unusual appearance to some forms, in consequence of which special names are applied. As an example the cylindrical cords may undergo, hyaline degeneration of their endothelial cells, be transformed into shining homogeneous structures, standing out conspicuously in transverse section like hyaline, concentrically laminated balls or masses; this appearance has led to their being called cylindromata. In other instances calcareous deposits (in the form of globules, needles and club-shaped masses) occur in the structure of the growth, giving a grittiness to the tumor in section and making the cut surface rough and sandy; these are especially met in the brain and are known as psammomata (acerz'uloma, sand tumor). The endotheliomata which develop from the choroid plexus of the brain are always richly infiltrated with cholesterin deposits, and are called in a superficial way cholesteatomata.

However these changes or depositions which give occasion for the special names indicated are not absolutely restricted to one particular type of tumor. In the collection of lime and cholesterin it must be kept in mind that the cells of the neoplasm are preserving qualities of the cells from which they had their derivation, for brain sand and cholesterin are found normally also in the pineal gland and in the choroid tissue. True epithelial tumors, however, which occur in the cranial cavity and are referable to inclusions of epidermoidal rests usually contain cholesterin, and it is also met in dermoid cysts or epidermoid cysts of the subcutaneous tissue; these types requiring the acceptance of a further class, the epithelial cholesteatomata.

Contributions recording the occurrence of endotheliomata in animals are very meagrely found in literature. Dexler met in a 
dog an endotheliona originating from the spinal dura mater; Künnemann a psammoma of the cranial dura mater about the size of a duck's egg, overlying the anterior part of the brain in a cow. Cholesteatomata have been found rather frequently in the lateral ventricles in the horse; met in this location they are rounded, reniform nodes, varying in size from that of a lentil to that of an egg, usually occurring bilaterally, a tumor suspended from the choroid plexus in each ventricle. The surface is smooth and shining, sometimes granular; the color white, gray or grayishyellow; the consistence about that of the liver, the cut surface slightly gritty and the cholesterin recognizable in tiny opalescent, shiny spots. Microscopically these tumors are made up of bloodvessels, a loose connective tissue stroma and the endothelial elements, which have grown from the bloodvessel wall; between these cells, in the vessel walls and in the widened lumina of the latter the cholesterin may be seen in the form of superimposed, step-like plates with the borders broken out. In addition round cells and pigmentary deposits may also be found. (Cornil and Ranvier speak of the tumors in question as angiolithic sarcomata: Casper employs the term hæmangiosarcoma perivasculosum).

[The position taken by the author in excluding from the endotheliomata tumors originating from endothelium of the large serous sacs, is not generally accepted; and endotheliomata of the pleura especially have been frequently described.

Endotheliomata as a rule are slowly growing tumors and although capable of metastasis are generally not marker by many secondary formations. This, however, in some individual cxamples is quite reversed and high metastatic malignancy is manifested. So, too, in most cases they are not recurrent after extirpation, but here again exceptions nust be recognized. They may occur as multiple primary growths. The subdivisions above mentioned, cylindroma, cholesteatoma and psammoma, are as a rule merely local growths, and arc among the least metastatic forms of endotheliomata.]

\section{Papillomata.}

The name papilloma is applied as a comprehensive, even if not well chosen, term for fibroepithelial neoplasins which occur on the surface of the skin and mucous membranes in the form of warty, conical, coralline, villous or nodular proliferations of 
the papillæ and their overlying epithelium. The physiological prototype of this combination of tissue and its source of development are in fact the epithelial-covered papillæ of the skin. but in their pathological proliferation the quantitive relations between the two forms of tissue are very irregular. In some tumors the connective tissue growth is so predominant that the tumor practically consists of thick clumps of this tissue covered only by a thin layer of epithelium rather stretched over the mass

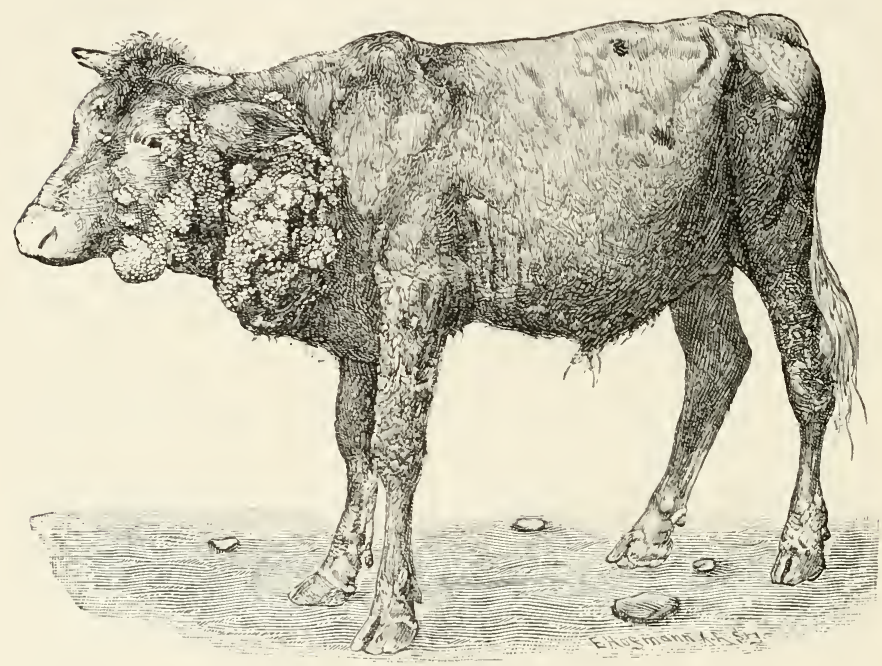

Fig. 112.

cutaneous papillomatosis of beef.

than growing with it (for which reason some authors prefer to speak of such a specimen as fibroma papillare). the superficial papillary structure being only recognizable under the microscope. In other instances the fibrous tissue of the base of the tumor sends out long branched villous processes, or may undergo changes which give it a soft, œdematous or very vascular appearance; and again the keratous epithelium may form so thick a layer as to throw the connective tissue substructure into insignificance. Different types of these fibroepithelial autoblastomata may be distinguished by taking such features into considerațion. 
On the skin and mucons membranes covered with squamous epithelium they occur as rounded projections, sometimes only as large as a pea or nut (warts, verruca), simple local hyperplasias of the papillary body with smooth epidermal covering; or they sometimes occur in clumps, like grapes, as cauliflower tumors of the size of a fist (papilloma tuberosum, polyposum, coralliformc), with superficial subdivisions, provided with broad

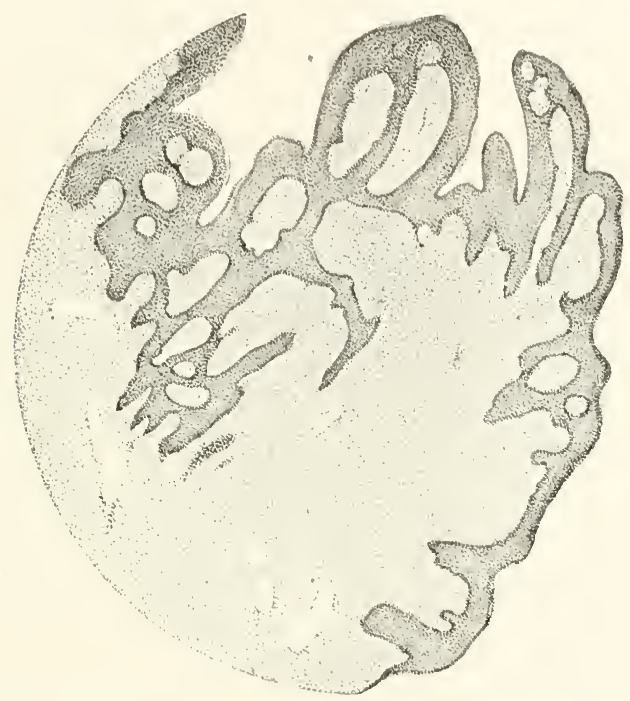

Fig. 113.

Section of papilloma of cow.

pedunckes or hanging suspended by a thin stretched-out pedicle; or again as thread-like or bristle-like projections (papilloma filiforme).

Under the microscope, varying with these external shapes of the growth, the connective tissue, which is made up merely of spindle cells and fibrils with bloodvessels, may be seen as papillæ of varying length and thickness and of all sorts of shapes, with knotted and clubbed branches and secondary pointed projections; and the epiderm in a single* or multiple layer extends over this, following the depressions and clefts, in which 
the epithelium may become keratosed and formed into concentrically laminated arrangements (horny pearls).

Sometimes these papillomata occur congenitally (two cases of the kind in question fully described by Pirl and Trolldenier*

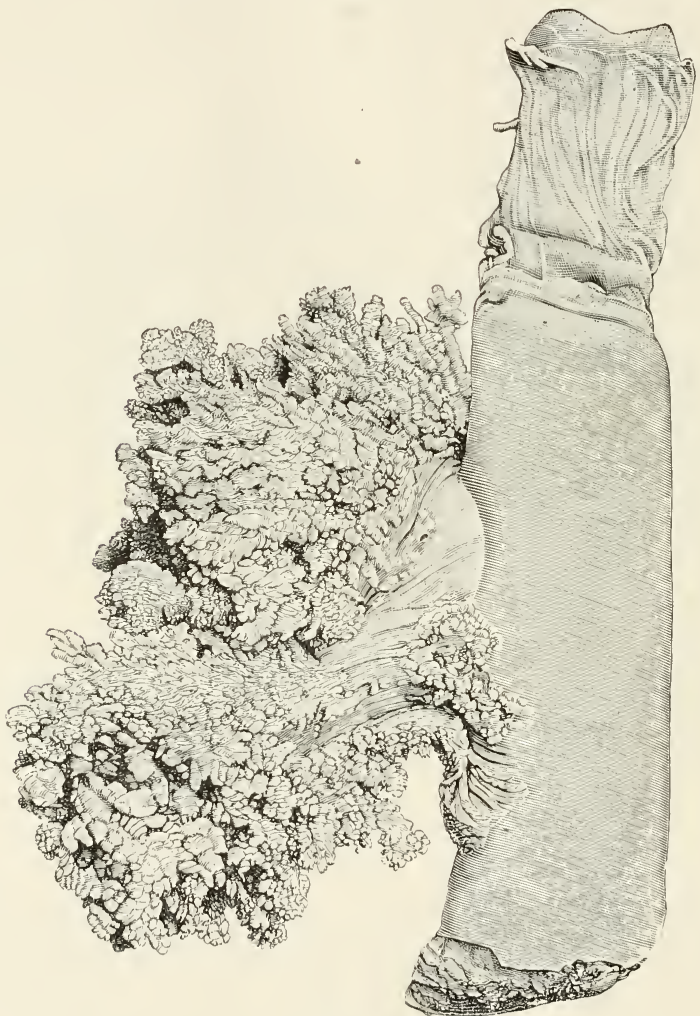

Fig. 114.

Papilloma coralliforme in gullet of cow. (The œesophagus has been everted and the mucous surface exposed: the partly smooth appearance is due to the fact that a piece of wood has been forced into the lumen of the tube turned inside out.) (Fhotograph.)

in foals), probably due to traumatic intrauterine lesions of the skin caused by amniotic threads. By some authors an infec-

* Monatsheft f, $p$, Tierheilk, 1903. 
tious catise is suspected for certain forms of papillomata because the affection often appears in a number of animals at the same time in one stable in wide distribution over the cutaneous surface; development after chronic inflammatory irritation of the skin has also been observed. Especially in the skin of the udder of the cow and in roung cattle, but also in other positions in this species there often develop great numbers of warty and nodular papillomata which may weigh $c h$ masse many pounds. These superficially divided growths, which are of course exposed to trammatic influences and contamination with foul pus, are apt to become malodorous from maceration of the desquitmated epiderm retained in the depressions; they often become oedematous and swollen, and may become more or less suppurative and putrefactive from the influence of microorganisms, which may have gained access into these parts. In similar manner the papillomata frequently developing in the rog of the horse's hoof (known as hoof cancer or frog cancer) are apt to break down into a foul-smelling mass because of incomplete keratinization of the epithelial surface layer and contact with all sorts of germs and putrid material (dung, foul pus).

A favorite location for these tumors is the œsophagus of cattle, where the papilla project into the lumen of the tube either as thorn-like, bristle-like or

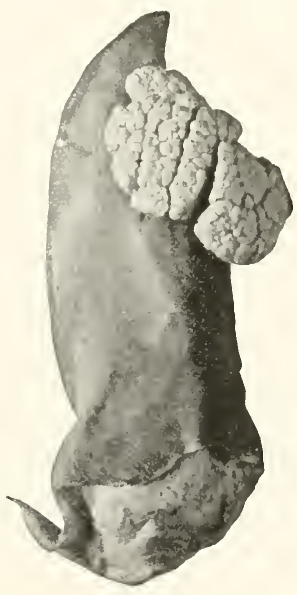

Fis. 115.

Congenital papilloma of pinna of colt. IAfter Trolldenier.)

brush-like prominences, or as circumscribed villous or coralline growths, or as firm nodular bunches; the passage of food, especially the return of the cud (in ruminants) being more or less interfered with. Papillomata often occur also in the psalter of cattle as berry-like, slightly cedematous, swollen-looking growths, ranging in size from the millet seed size normal to the papillæ of the part, to pendant rosette-like bunches of projections half the length of the finger, club-shaped and covered smoothly with epithelium. In dogs the lips, buccal cavity, foreskin and anus are the principal sites of papillomata. 
In the urinary and gall bladders of cattle, in the former also of horses, dogs and swine, papillary mucous membrane proliferations occur, usually projecting as long villous, highly branched growths with a common pedicle into the cavity of the viscus, like the tentacles of a polyp (papilloma polyposum); they are usually very soft, succulent, their comnective tissue wedematous and perhaps actually myxomatous (papilloma polyposum my.romatodes), or in other cases the seat of a cellular infiltration and presenting evidence of inflammatory involvement. Inflammatory changes in the last named situations are so frequently accompanied by mucous membrane proliferations that some causal relationship may be assumed and the hyperplasia of the mucous membrane regarded as a sequel to a productive inflammation (Zellhuber).

Where the epithelium of the papillary growth of the skin is not desquamated in scales, but in its active proliferation forms thicker and thicker horny layers, there is produced a hard body which continually grows more and more prominent and acquires the form of a horn-like outgrowth, a cutaneous horn (comu cuta$n c u m)$. These structures are very frequently seen in cattle, especially in range cattle, occasionally attaining a length of half a meter, growing from the forehead or neck, or as a short conical horn from the skin of the udder. Now and then large spirally curved cutaneons horns are seen in goats in the thoracic and abdominal walls; in sheep in the ear and back; in horses and dogs as small claw-like horns in the ear and in other parts of the body. (For fuller description see Casper, Pathol. d. Geschwiilstc b. Ticren, Wiesbaden, I899; Kitt, Spes. pathol. Anatomic, II. Aufl., Stuttgart, Igor.)

Papillomata and cutaneous horns are regarded as benign, nonrecurrent, persistently local new growths; they manifest an unfavorable tendency only from their size and situation.

\section{Adenoma.}

An adcnoma, or [epithelial] glandular tumor, is a proliferation of glandular tissue, with reproduction of its structural type, in the form of a tumor. Epithelium and a vascular connective tissue stroma in definite structural relations constitute glands, and the two forms of tissue in common contribute to the formation of the adenoma. Whether the epithelium was the primary proliferating element and, as Borst believes, the connective tissue cells took a relatively secondary part because 
of their tactile sensibility, is an open-question. In some adenomata the reverse is apparently the case, the connective tissue proliferation apparently being the major feature, in its proliferation causing expansion of the surfaces of the gland lumina and thus affording space for the epithelial gland lining to develop. Both tissues proliferate side by side in mutual interdependence in the production of gland tissue.

There are as many varieties of adenoma as there are different glands in the animal body, and as the tumor invariably takes its origin from a gland it always represents a histological copy of the particular gland in question. The growth has, too, in all instances the same type of epithelium which characterized the original gland, and the same kind of connective tissue stroma is

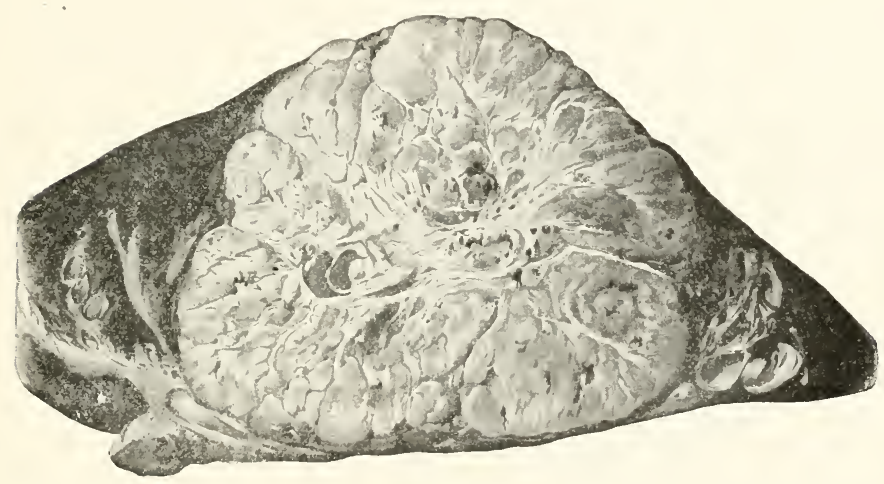

Fig 116.

Adenoma, of size of human head, of the gall ducts in the lirel of cow; (cut surface).

preserved in more or less precise simulation of the structure peculiar to the parent gland. Corresponding to the different glands these tumors may be divided into major types of tubular, alieolar and follicular adenomata; and depending upon their origin it is customary to speak of them as sweat-gland, sebaceous, hepatic, salivary, renal and other adenomata.

In this pathological proliferation of the glandular tissue there occur, however, manifold modifications and abnormal features. The epithelium, although generally exhibiting the same structure and eren secretory function as the original type, is often increased 
in size or may be present in a greater number of layers, and its secretion may be of a degenerative character (for example, from the assumption of mucoid change by the adenomatons cells or their destruction by excessive fat production). The connective tissue, which in direct limiting relations to the epithelium forms its tunica propria and in lobulated types of gland tissue forms a meshwork of septa, is often developed in special excess, forming densely fibrous sclerotic trabecula which embrace the glandular ducts in compact circular investing bands (adenofibroma pericanaliculare) or form club-shaped or finger-like projections, stretching the adenomatous lumina, increasing the surface of the glandular canals and extending into the latter as villous papillæ (adenofibroma intracanaliculare, adcnoma papilliferum).

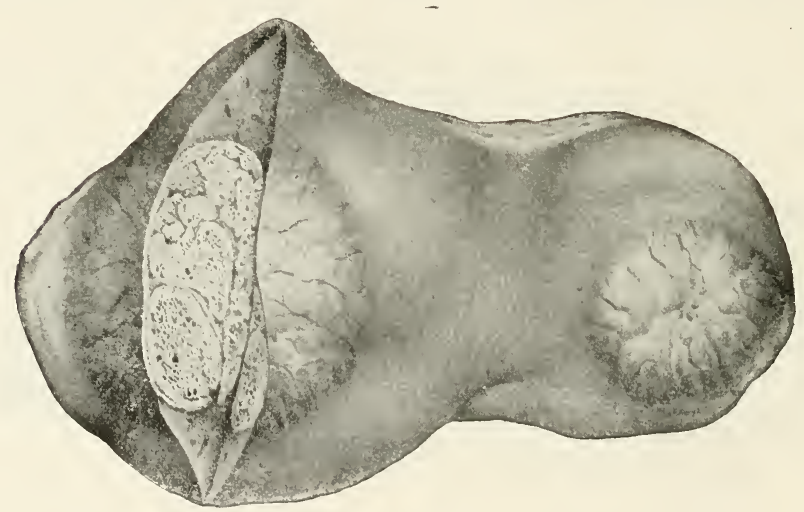

Fig. 117.

Adenoma flavum of liver of cow.

The tubular or vesicular spaces of the adenoma, by continuous and progressive formation of hollow buds lined with epithelium, become more and more branched and more irregular than in the normal gland; and by this intricate projection of epithelialcovered connective tissue processes into their interior become changed into torttous passages and openings, which may become quite large. The cut surface of an adenoma may in this way come to closely resemble a section through a head of cabbage, in which spaces are to be seen between the layers of leaves. As these adenomata are completely isolated new growths and their 
canalicular systems are in no way in communication with the emerging ducts of the nommal glands, an accumulation of secretory material and desquamated epithelial cells is likely to be retained in their passages and spaces; thus in an adenoma of the mammary gland milk is found as a pathological product; in follicular adenomata of the thyroid, masses of colloid matter; in adenomata of mucous glands, mucin. As a result of the retention of such material the walls of the canals and alveoli become stretched, and this feature, together with continuous enlargement of their surfaces from increase of the epithelial lining and the connective tissue framework, may make the distended cavities visible to the naked eye. To tumors thus altered and containing cavities filled with fluid the term cystadenoma is applied; and where villous projections of the lining membranes extend into the carities the growth is known as cystadenoma papilliferum phylloides.

The growing vascular connective tissue of the adenomatous structure is sometimes the seat of hremorrhagic effusions or cedematous swelling, due to passive congestion and transudation caused by kinking of the folds and villi projecting into the cysts. Other regressive changes, as fatty degeneration or coagulation necrosis, may also be observed. In sone instances the stroma of the tumor is a tissue very rich in cells and of a reticular structure resembling a sarcoma or myxoma (adenosarcoma, adenomy.roma, adenoma sarcomatodes, myomatodes).

Finally glandular tumors, which in typical cases are usually distinctly limited from the organ in which they are situated, may become atypical and send out infiltrating processes into the surrounding tissue, penetrating into the lymph vessels and bloodvessels and thus assume the character of a malignant fibroepithelial growth. These forms are known by the names adenoma destruens, adenocarcinoma [malignant adenoma].

Two points may be considered in attemptning an explanation of the xtiology of adenomata. The definitely circumscribed nodular adenomata which develop in or close to parenchymatous organs are doubtless of foetal origin, coming from misplaced, independently developing parts of a gland. Those growing as single or multiple tumors from mucous surfaces, sometimes occupying extensive areas of the mucous membrane, often show in themselves, just as the neighboring parts of the membrane may also show, evidences of chronic inflammatory change, recognizable by cellular infiltration and by giving rise to a mixed secre- 
tory and exudative type of product. These peculiarities as well as the clinical course of such cases, suggestive of their origin as direct sequences of chronic inflammatory processes, force the conclusion that injuries capable of causing inflammation may either directly or indirectly (disturbance of tissue tension) give origin to adenoma production. Mucous membrane and glandular vegetations of this type are usually spoken of as glandular hyperplasias or mucous membrane polyps. It should be recognized, however, that such superficially situated adenomata may become inflamed secondarily; from their coming in contact with irritants.

The most frequent type of adenomata occurring in animals are those of the sebaceous and sweat glands (adenoma sebac'um, sudoriparum) in the skin of the dog; among them particularly growths originating from the perianal glands, presenting rounded, nodular and lobulated forms, of a reddish yellow or light yellow color and giving off a fatty, greasy fluid (a number of descriptions of Siedamgrotzky, Lienaux, Viverner). True adenomata of the liver are also comparatively common in cattle and sheep (Bollinger, Martin, Siedamgrotzky, Johne, personal observations) as sharply defined tumors, surrounded by a connective tissue capsule, situated in the midst of the hepatic tissue. These may sometimes be found as large as a human head, composed of liver cells and delicate cob-web like connective tissue septa. They are usually very striking because of their bile-stained yellow to green hue. Sinilar to these there also occur in the liver gall-duct tumors, with dense fibrous stroma, having, in consequence, a firm consistency and appearing as light yellow nodular deposits ranging in size from that of a potato to that of a human head. The mammary gland in dogs is another frequent site for cystadenomata and combined adenofibromata or for transitional forms becoming cancerous. In the lungs adenomata of nodular form may originate from the bronchial mucous glands, observed

- in sheep (A. Eber) and horse.

Although in man mucous membrane polyps occur quite frequently in the nose, such growths are more uncommon in animals: in the horse they are met hanging into the naso-pharynx, hyperplasias perhaps as large as a fist, lobulated, elongated, slippery and of mucoid consistence, composed of soft mucous membrane tissue. There is difficulty in trying to accurately define thyroid adenomata. The colloid secreting epithelium of this organ in its proliferation sometimes gives rise to a general enlargement of the gland, the con- 
dition known as goitre (struma); and sometimes leads to nodular formations which originate from isolated and independently growing cellular foci and are called adenomata, cystadenomata, or adenocarcinomata, but which are usually accompanied by diffuse enlargement of the rest of the parenchyma of the organ. In the same way proliferation of the glandular tissue of the ovary and testicle is usually diffuse, and the distinction detween hypertrophy and true tumor formation is difficult here as well as in the previous example. The cells are usually of an indifferent type and can scarcely be distinguished from sarcoma cells. In these cases the terms orarioblastoma or orchidoblastoma are convenient names, according to the organ involved. They may be seen as solid growths or with cyst formation associated, the whole organ involved, the tumors reaching enormons size, weighing perhaps ten or fifteen kilograms and often attaining the dimensions of a human head or even twice that of the full stomach of a horse (in horses, cattle, sheep).

In the kidneys true adenomata of the uriniferous tubules have never been observed. The tissue proliferations which develop here in the form of tumors, as a rule are of the destructive adenocarcinomatous type and convert the organ into a shapeless, soft material which may break through the capsule. This substance is sometimes as soft as marrow, gray to brownish-red in color, and is made up of large canals lined by cylindrical epithelium. In other cases the growth is combined with sarcoma and possibly also with muscle fibres and constitutes an adeno-rhabdomyomatous sarcoma (Johne). [Some of the tumors described as renal adenomata are in reality hypernephromata (v. p. 397) ; although the latter growths, which occur in the kidney as their most frequent seat, are ordinarily easily distinguishable. In man there occasionally are seen in sections of kidneys small isolated foci of tubular structure, with the tubules either small and compressed or at places distended into cystic spaces. In the latter papillary proliferations may be found projecting; the whole appearance corresponding with the adenoma papilliferum above described. In these cases as a rule the foci are small, perhaps microscopic, and the general picture of the structures suggests their isotation from the rest of the tissue by an inflammatory change. Rarely larger nodules of this character are met, to which we cannot refrain from applying the term adenoma.

The prognosis of the tumors of the adenomatous group is fundamentally favorable; the true adenomata being entirely free from any tendency to infiltrate or to give rise to metastasis. Such growths are harmful merely from their local influences. The variety above 
mentioned as destructive or malignant adenoma or adenocarcinoma is essentially cancerous in its nature and is malignant both by local extension and by metastasis. Any adenoma may be regarded with suspicion and dealt with accordingly, when it is recalled that it is not very uncommon for these growths to lose their purely adenomatous character and become changed into adenocarcinomata.]

\section{Carcinomata.}

A carcinoma or cancer is a malignant new growth composed of epithelium and connective tissue, characterized by a persistent and progressive penetration of its epithelial elements into the underlying tissues. The cancers always originate from epithelium, either surface epithelium or the glandular epithelial cells of functionating organs, or from islets of epithelial cells formed in fotal life and occurring in unusual situations. They may also take their origin from previously existing papillomata, adenomata and cystomata, these having from unknown cause assumed a proliferation of their epithelial cells with departure from their ordinary forms and boundaries.

The proliferating epithelium forming the cancerous parenchyma or body of the cancer behaves as a foreign intruder; it assumes an independent status by no longer remaining confined to the surface which it previously covered, breaking through its normal limiting structures and playing the part of epithelial cells which have become practically parasitic. These cells, derivatives of previously existing epithelium, by their penetrative growth and active multiplication usually form in the deeper structures solid cords or ductlike processes which branch like the roots of a tree and are continually sending out new projections into the tissue. These retaining their continuity with each other result in forming a network or meshwork [of epithelial character throughout the invaded tissue] varying in its density and with irregular nodal points (Ribbert). This net-like and corded arrangement of the epithelial roots is due to the fact that the cells in their penetration follow the lines of least resistance and therefore penetrate the lumen of the lymph passages, come to completely fill them with their growth and therefore to assume their shape. After occupying all the lymph spaces of the connective tissue the epithelial roots may penetrate into the tissue itself in such numbers as to practically constitute a diffuse infiltration (Ribbert).

In microscopic sections the continuity of the cords is not entirely apprecrated, as each sectional layer, of course, contains only segments of them. 
In serial sections or in plastic reconstruction of the general tumor-picture by means of the methods of planar modeling, one may always convince himself of the interconnection of all the epithelial cords (Durk).

The connective tissue of the organ in which the epithelial cells are in active cancerous proliferation, does not, however, act in a purely passive manner, as a tissue shoved aside and compressed by the epithelial growth; but reacts by an inflammatory change which in its chronic course leads to the production of a connective tissue hyperplasia. The epithelial masses act precisely like foreign bodies, attract leucocytes to them by chemotaxis, the latter cells collecting about the margins of the epithelium just as in a demarcating inflammation and sometimes producing a marked cellular infiltration of the connective tissue. At the same time the fixed connective tissue cells proliferate and form a fibrous stroma (framework of the cancer) of varying texture, containing young bloodvessels and enclosing epithelial nests.

The distinction from the typical fibroepithelial tumors [adenoma, [apilloma] lies in the fact that in the latter the epithelium and connective tissue are united in common growth to form a tissue complex, a compound tumor basis; while in cancer in reality only the epithelium is the basic tumor element, and the connective tissue growing along with it is the result of a productive inflammation and belongs properly to the organ in which the cancer is located. The cancer stroma therefore is but the interstitial tissue of the affected organ, involved by inflammation. The epithelial elements of the cancer develop in the organ in which the tumor originally (primarily) grew, from cells, it is true, already present therein: but the epithelial cells of the organ are not uniformly changed into cancer cells, do not all take part in the tumor construction. The growth and extension of the latter involves invariably a fixed group of cells acting as the germinal elements of the tumor, toward the multiplication of which the other epithelial cells remain passive.

The microscopic studies of Ribbert have furnished a number of considerations which concern the histological production of the primary stage of this type of tumor, the start of the cancer formation. Ribbert refers the origin of the growth to an isolation from the cellular union in an organ of individual epithelial cells or a group of cells, as an epithelial ingrowth, a separated bit of epiderm, or a lobule of a gland; this subsequently assuming independent proliferation. This isolation from physiological comnection is usually brought about, as Ribbert recognized in microscopic sections in the 
initial stages of skin eancers, by the intrusion of connective tissue, stimulated to proliferation by inflammation, between the epithelial cells. In other words, according to Ribbert cancers always start on a base of chronically inflamed tissue. The epithelial cells do not make the initial penetration into unaltered connective tissue; the primary change being the inception of the inflammatory proliferation of the subepithelial connective tissue, which raises the epithelium and, as the proliferating connective tissue elements invade the deeper epithelial layers and destroy the intercellular cement, separates isolated cells from their proper relations and thus misplaces them. The isolated epithelium, now situated in the midst of the connective tissue and sufficiently nourished by it, begins to multiply; and, being prevented from growing upward toward the surface of the skin or mucous membrane, and its relations with the general epithelial tissue, its nerves and normal substratum (papillary layer) being destroyed, it must necessarily penetrate irregularly into the tissues along the course of the lymph spaces. Thus it comes to force its radicles and cords in every direction and to form the beginning of the cancer.

As a matter of fact cancers do occur with frequence in places where for some time previously inflammatory irritation has existed. In man, for example, cancer of the lip is especially likely to be met in persons who smoke pipes habitually, and, too, just in the corner of the mouth which is always exposed to irritation from the juices from the pipe. The influence of soot as an irritant capable of inducing cancer is well seen in chimney sweeps (chimney sweep's cancer); and that of paraffine has become well known among the employees of paraffine factories as causing epidermoidal proliferations (paraffine cancer of the hands). A. Sticker has called attention to several examples which indicate a causative relation of external influences upon the establishment of cancer, as the occurrence of skin cancer in cattle after branding (Mac Fadyan), and beneath the chin in swine from chaffing (Eggeling). Possibly the frequence of cancer of the anus of dogs has some connection with external injuries (from the animal scraping the anal region along the ground, socalled "sleigh-driving").

The origin of cancer should not, therefore, be attributed to any one cause, as a definite cancer parasite or an infectious agent, but may be determined from any influence (traumatic, chemical, infectious) which occasions chronic inflammation and because of which, as a result of the inflammatory process, an isolation of the living 
epithelial cells, retaining their power of proliferation, occurs. It is of course true that inflammations of surfaces covered by epithelium and epithelial organs are frequently seen without resultant cancer production, even though it may be presumed that isolations of epithelial cells may often obtain in connection with these processes. But, as Ribbert insists in case of such exceptions, much depends upon the length of time over which the inflammation extends. In acute inflammations the epithelium is apt to be injured, and even if isolated cells be nisplaced in the subjacent tissues the process is of too short duration to allow the cells to accommodate themselves to their new surroundings; they therefore perish (Ribbert). In the same way as an inflammatory connective tissue production, epithelial cells may in papillary and polypous new growths be cut off and isolated by the proliferation of the stroma, or whole cores or bunches of them; and thus a primarily benign tumor may be converted into a malignant one by the progressive growth of such cells into the underlving tissues. The change of fibroepithelial growths which for years have been of simple type into destructive cancers is a well authenticated observation in human medicine. Finally, epithelial misplacements, which may accidentally be caused in fotal life in the processes of arrangement of the various tissues. in the formation of folds and cavities which are taking place among the tissues in the construction of the various organs, may furnish the original stibstance for a subsequent cancer.

A good example of this is afforded by the nodular growths met in the human kidney cortex, originating from misplaced adrenal tissue. They are met as single or multiple growths, well defined; and sometimes destroy the kidney, penetrate into the blood vessels, and give rise to fatal metastasis. These nodes are of a yellowish color from the fat which they contain, and under the microscope are seen to be made up of cords of cells arranged in parallel lines along the blood vessels, and resembling in their appearance the epithelium of the suprarenal bodies (hypernephroma, adrenal cancer). LThese growths are also met with not very infrequently in the kidneys of cattle and occasionally in hogs; they are not confined to the kidney, but may be found in any of the structures near the adrenals or in the adrenals themselves, where they may either represent fœtal rests, or may have started from isolated parts or inclusions of the tissue of the organ caused by inflammatory separations.]

Ribbert's attempted explanation of the genesis of cancer is not as ret accepted by many pathologists. Inflammatory changes are often not demonstrable at the places of origin of cancers; and some authors do not believe that the atypical proliferation of the epithelium is a result of the invasive growth of the connective tissue but 
that some other as yet unknown causes underlie the irregular progressive proliferation which these cells manifest (in other words, that the epithelial multiplication is the primary process) : and search for some kind of infectious agent is not as yet abandoned. The occasional endenic occurrence of cancer (also observed by Eggeling in hogs in a certain locality) and isolated cases which suggest the transmissibility of certain forms of cancer continue to keep the question of the infectionsness of cancer in discussion. A. Sticker, for example, has called attention to a dog which for a long time had been in the habit of lying by the bed of a man suffering from cancer of the stomach and ate all sorts of material which the man had romited, and which became affected by a general carcinomatosis (lungs, liver, omental sac, but not the almentary canal). This may have been a mere coincidence, but the possibility of an retiological relationship between the two cases cannot be entirely ignored. (Cf. p. 33+) Experimental attempts to prove an infectious nature as existing in these growths (inoculation and feeding experiments) have hitherto always failed. Trasbot obtained no positive result from hundreds of such attempts; Duplay and Cazin failed in over one hundred and twenty experiments in dogs and rats; Gratia and Liènaux, Cadiot and Gilbert had no more success in numerous attempts to transmit these tumors, employing all sorts of methods of inoculation (from dog to dog, from man to dog). The transplantation of certain forms of cancer in rats and mice has alone succeeded (Hanau, Moraus, C. O. Jensen); but the methods employed do not indicate that an infectious agent was operative, but rather that the cells of the cancer used in the experiment were capable of multiplying if placed in uninjured condition in a new specifically similar soil (i. e., transplanted to the same species of animal as that from which derived), and of continuous development into an independently growing tumor tissue in their practically parasitic colonization and multiplication. (cf. Definition of tumors p. 325.) [Transplantation of cancers in animals has invariably shown that success is to be expected only when the animal to which the tumor is transplanted is of the same species as that from which it was derived, even varieties of the same species making the result doubtful. In this country Leo Loeb, Gaylord, Herzog and others have carried various cancerous growths or mixed cancers through a number of generations in rats and mice. In these experiments actual portions of the living growth (bits may retain life for some hours when kept in moderate refrigeration after removal from the original animal) must be introduced into the experiment animals and 
filtered extracts are not followed by success. Ehrlich (Berlin. Klin. Woehenschr., No. 28, 1905: No. 2, 1go6) and Loeb (Univ. of Penna. MICd. Bull., July, I905) have both recorded the occurrence of sarcoma succeeding the original carcinomatous tumors after some generations of transmissions. This would not necessarily suggest the direct transformation of the cancer into sarcoma, as much as that in the course of growth the proliferating epithelium had induced such changes in the connective tissue portions of the tumor as to cause them to acquire an analogous energy of atypical proliferation, and that perhaps this latter tissue or, what perhaps is more probable, immune bodies, reactively developing to the cancer, have caused the disappearance of the epithelium itself. The known spontaneous disappearance of cancers (cf. Gaylord and Clowes: Surgery, Gynecology and Obstetrics, June, 1906) speaks in favor of the existence of such cytolytic factors. Gaylord's success in obtaining a serum from mice spontaneously recovering from cancers, which on injection into mice having in their bodies actively growing tumors of the same strain causes them to recover, speaks in the same line; and the fact that an extract of a tumor (v. p. 339) injected into the body of the original animal having other nodes of the same growth in its body, strongly supports the same idea. Tentatively then it may be held that in case of cancers at least the present tendency is not so much to accept the existence of a specific parasitic cause for the growth as to believe that once established as an independent focus of growth by some such method as is suggested in Ribbert's isolation theory, the epithelial cells become themselves, as it were, practically parasitic in the organism of which they were once an integral part; that they grow as parasites and act as parasites; and that the body reacts to their presence as it does to other parasitic organisms, and endeavors (sometimes successfully) to produce cytolytic or other protective reactions which will tend to destroy the cancer cells. This view opens an attractive and wide field for application in many lines, not merely therapeutic, and suggests reasons which may explain the special prevalence or special failure of secondary growths in different systems and organs of the body, as it may be supposed that all parts are more or less open by the lymph or blood streams to the reception of secondary tumor emboli.]

All other contributions dealing with cancer parasites (cancer bacilli, blastomycetes, etc.) recognized by microscopic methods have originated from mistaken interpretations of the microscopic pictures presented in various examples. 
The disscmination of the cancer into the tissues, as above indicated, occurs primarily from the penetration of the multiplying epithelium into the lymph spaces. They here form tubular or solid laminated cords, push aside the endothelium and other connective tissue elements, and by continuous progression of growth force their way deeper into the tissues, between the muscle fibres and beyond. In this way, for example, a growth on the surface of a mucous

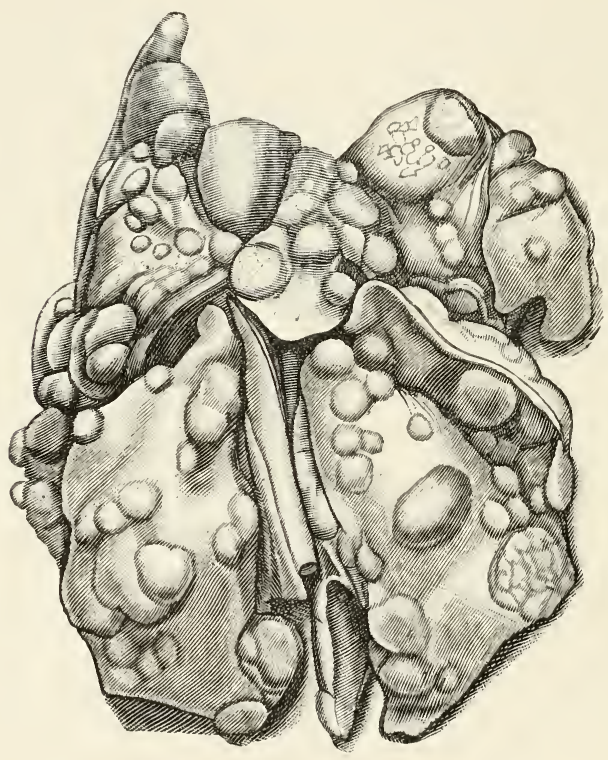

Fig. 118 .

Lung of dog with metastatic cancer nodes (thyroid cancer).

membrane forces its way through the muscularis mucosa and onward into the muscular tunics and serous coat of the tube. In the lymph passages, by continuity of growth the cords of cancer cells may extend as far as the nearest lymph glands; or individual cells loosened in some way may be carried by the lymph current to the latter and to more distant portions of the lymphatic system. Multiplication of the tumor cells in the tissue of the lymph nodes, and the reactive inflammatory proliferation of the connective tissue of these 
structures, lead to marked chronic enlargement of the glands (lymplogenous cancer metastases), one of the clinical characteristics of the cancerous affection. Passing from the lymph glands the cells of the growth may by following the course of the efferent lymph vessels, pass into the blood (anterior vena cava); or they may gain entrance to the blood by direct penetration of the blood vessel wall in their growth. In either cvent the cells are carried onward with the blood stream and form new foci wherever they may lodge in the capillaries and give rise to the formation of nodes (hamatogenous, embolic cancer metastases).

Metastatic nodes are as a rule round, and nutultiple or disseninated because the cells have been widely scattered through an extensive area of vascular distribution. Is a rule they are first sitwated in the lungs, because the cells are rery likely to penetrate into a vein and be carried through the right heart and into the lungs; in case of primary cancer of the stomach, intestine or pancreas the liver is apt to be involved, by convection through the portal vein. Should isolated cells pass through the pulmonary capillaries and get into the general circulation by way of the left heart, other portions of the body; as the spleen, kidneys or bones, may be involved by dissemination of the metastatic cells. Sometimes. too. when these cells penetrate into bloodressels they grow into cordlike processes along the vascular lumen: solid plugs of cancer cells as thick as lead pencils may occasionally be found extending in the thyroid veins from a cancer of the thyroid gland, even down into the thoracic cavity. The vascular lumen becomes more or less obstructed and the secondary thrombi formed are very likely to be invaded by the cancer cells.

As the tumor enlarges it compresses and puslies asicle all the elements of the organ with which it comes in contact; in their penetration into the deeper tissues and by their lateral extensions the cellular roots of the cancer distend and occupy the lymph spaces. pushing aside whatever gland-tissue, muscle or nerve may be present, spreading out beneath the epithelial covering of mucous membranes or integument and perforating layers of epithelium which they may encounter. Pressure atrophy and necmsis of the surrounding tissue and tissue-destruction from the accompanying inflammation result from the cancerous infiltration. even hard bone and cartilage, perhaps, being broken down by these changes. Is the tumor tissue multiplies beneath the epithelial layer of a surface it raises it up, spreads out in larger and larger arca close to the surface 
and develops in nodular prominences. By cropping out on the surface, by perforation at one or more places of such a protuberance, it may become a direct surface growth with nodulated, uneven, undulating elevations and more or less fissured surface. The inflammatory infiltration of the stroma, exaggerated by the entrance of extraneous irritants into the exposed growth (contact with air, intestinal contents, dirt or bacteria), is likely in such event to bring about an ulcerative, foully suppurating destruction of the surface of the cancer (cancerous ulcer). In case of penetration into aseptic cavities, especially the peritoneal (gastric cancer, cancer of the

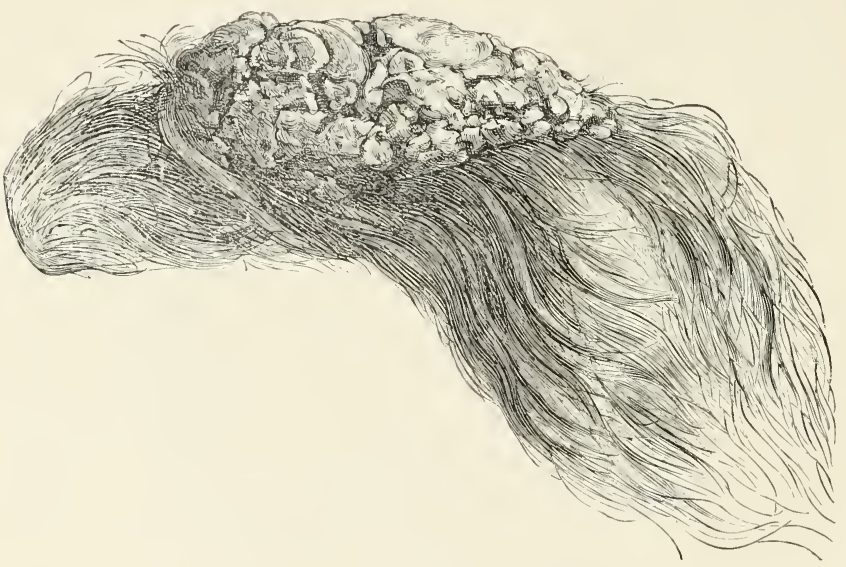

Fig. 119.

Cancer of tail of cow

intestine, cancer of kidney), transplantation of epithelial cells loosened from the growth nay be brought about by the intestinal peristalsis (by movements of the lungs, diaphragm and heart in the chest) and give rise to extensive secondary carcinomatosis in the serous membranes by their growth. The secondary formations are at first small grayish-white nodules (miliary carcinomatosis), but later, by confluence of the enlarging cancerous foci and the accompanying inflammatory changes, a diffuse cancerous peritonitis or pleuritis may be established.

The morphology of the cpithclial cells of which a cancer is composed corresponds in a general way with that of the epithelium 
from which the tumor took its origin; in a greater or less measure the cancer cells preserve the peculiarities of the epithelium of the place of inception, as a tendency to keratinization, to formation of mucin and other secretory substances. This retention of original characteristics, which is well seen in the metastatic nodles, often makes it possible to determine from microscopic examination the primary source or origin of a cancer. This is, however, often subject to modification. The growth of epithelial cells in unfamiliar positions, their separation from their normal substructures, must directly, because of the altered nutritional conditions and the various factors of tissue resistance, occasion variation in their shape and size. In consequence we often meet with morphological variations and anomalies which differ so widely from the original

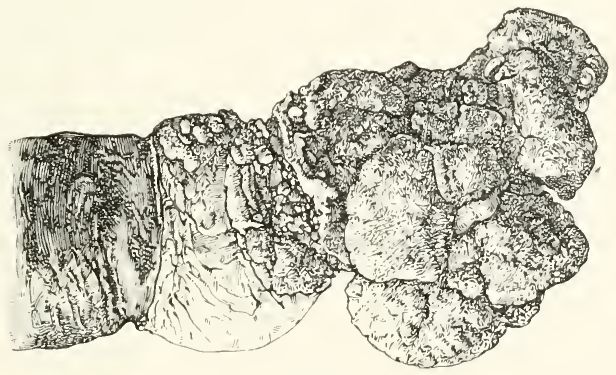

Fig. 120 .

Cancer of glans penis of iorse.

characteristics of the mother cells, that it may be quite impossible to come to any conclusion as to the source and type of the tumor from the morphology of its individual cells. In addition to this polymorphism of the cancer cells there may also occur one or other of a variety of retrograde metamorphoses in the luxuriantly growing and irregularly nourished cells, rendering them completely unrecognizable; so that at times the real character of the cellular elements can be made out only in the more recent parts of the tumor.

Corresponding to the major types of cutaneons, mucous membrane and glandular epithelium, three classes of cancers may with Dürk be recognized:

I-Surface cell cancers of the skin and mucous membranes corcred aith flat cpithclium (called briefly by most authors squamous epitheliomata.) 
2-Surface cell cancers of mucous membranes coiered with cylindrical epithelium (cylindrical cell cancers).

3-Glandular cell canccrs [carcinomata].

Squamous cpitheliomata, originating from the skin and mucous membranes covered with squanous epithelium, manifest their epithelial proliferation by forming solid cords of cells penetrating the deeper tissues, these cords for the most part made up of cells of the type found in the stratum Malpighii. The prickle and ridged borders of the cells, the protoplasmic processes extending through the in-

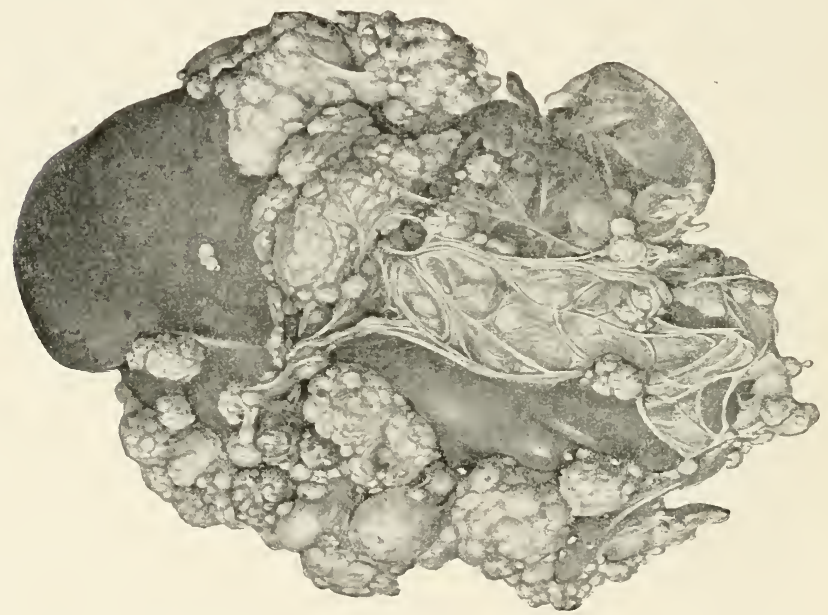

Fig. 121.

Secondary carcinomatosis of serous surface of liver and omentum of horse: primary growth of cancer in the ovary.

tercellular cement (so-called intercellular bridges) are usually well marked, and the cells are arranged in layers as over the papillary layer of the cutis; and in consequence we usually find the cells next to the stroma of the tumor of a cylindrical form, the next layers cubical, and the inner parts of the cord occupied by squamous cells. The epithelium in its growth has no opportunity for desquamation in these connective tissue spaces, and the old and first formed layers must necessarily be found in the central part of the plug-like cord; being derivatives of epithelium subject normally to keratous change, the cells of a squamous epithelioma usually also undergo 
keratinization, and here, too, the change affects the older cellular clements. The interior of the cancer roots show these flat cells, homogeneous and staining diffusely with eosin or fuchsine, with their nuclei poorly shown or entirely lost, precisely as those of the surface of the epiderm. In consequence on cross section an onionlike concentric lamination of the cells is apparent, with the cells hent in crescentic manner and applied upon each other like flat

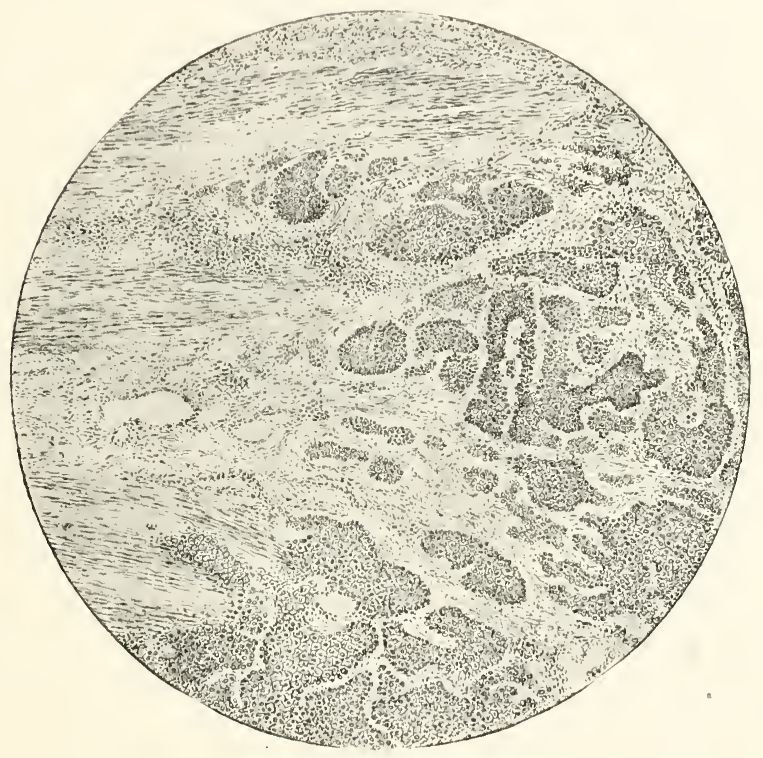

Fig. 122.

Microsfopic section of a squamous epithelioma of urinary bladder of cow (low power). Epithelial cords have grown in between the sinouth muscle fibres.

scales. These horny plugs can sometimes be picked out entire with the knife and are called cancer pearls ["pearly bodies," "squamous cell or concentric nests" |. The arrangement of layers otherwise is apt to be quite irregular, the composite network of epithelial roots forming at places broad, thick masses with wavy outline, at others delicate, line-like threads.

The stroma of the squamous cell cancer, present in varying proportions in different cases, is nsually the seat of a marked round 
cell infiltration, a true inflammatory infiltration; and the wandering cells may be found penetrating even into the epithelial plugs.

In cylindrical cell cancers the cylindrical epithelium, extending in its proliferation into the deeper tissues, laterally and, together with its connective tissue, also superficially, usually forms glandlike tubes which branch out in different directions as narrow or wide hollow offshoots. The proliferating columnar cells may re-

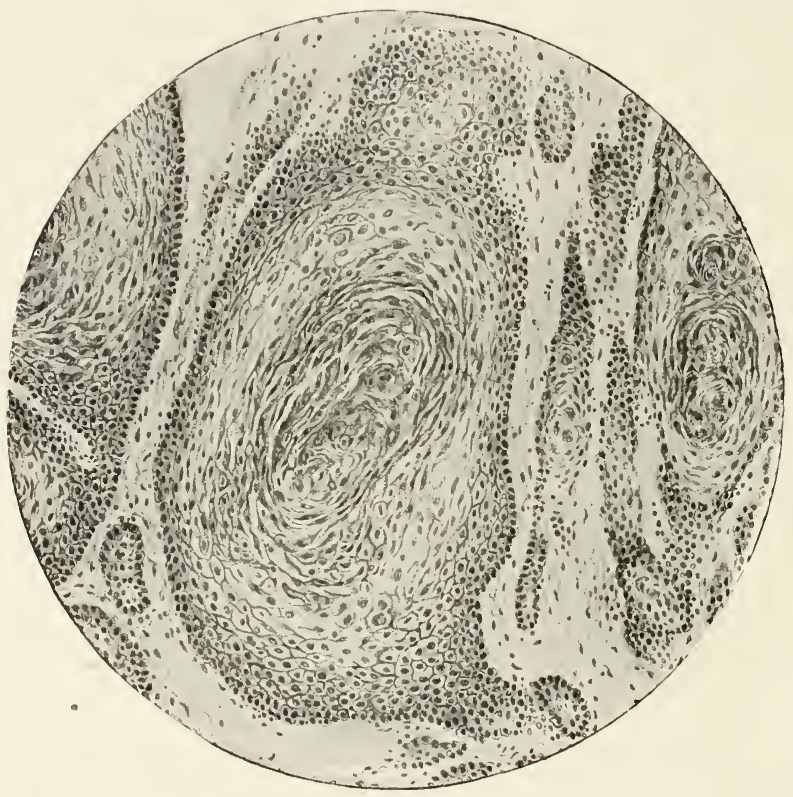

Fig. 123.

Iicroscopic section of a squamous epithelioma of glans penis of horse.

main in a single layer or become stratified, in the latter case undergoing various clanges in shape corresponding with the prevailing pressure conditions, goblet cells peculiar to the mucous membrane often being met in great numbers.

Glandular cell canccrs, as far as the morphology of their cells is concerned, are as varied as the types of cells in the different glands of the body. As in adenomata, in these growths the specific gland tissue repeats itself in tumor form. The retention of the 
tubular arrangement of the cells, and the simple (one layer) lining of these tubes nay so well preserve the gland pattern that the name adeno-carcinoma has been applied to these and to the tubule-forming cylindrical cell cancers. The resemblance to the original gland may be so great that not merely the morphology of the most of the tumor cells remains unchanged, but even the production of secretory substances in the tumor tissue is distinctly analogous. Thus hepatic cancers have epithelial cells of precisely the same type as the polygo-

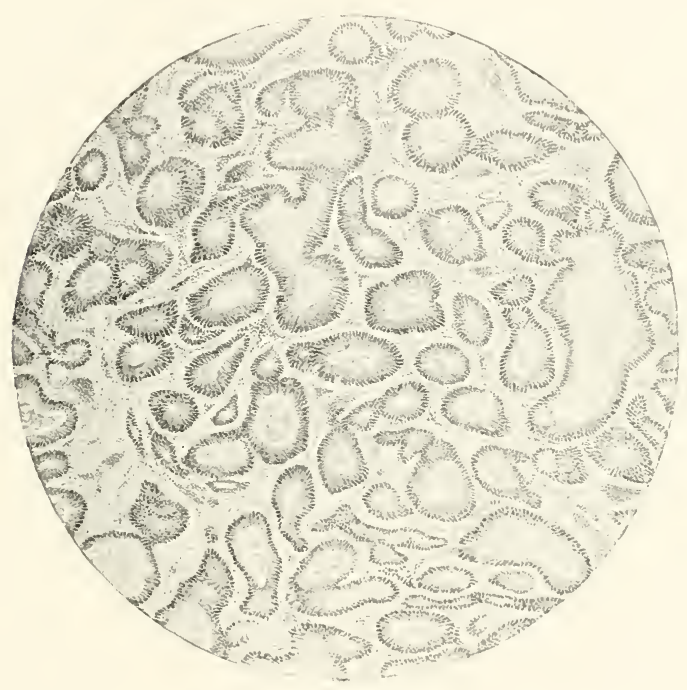

Fig. 124 .

Microscopic section of adenocarcinuma of kidney of horse.

nal hepatic cells, arranged in anastomosing trabecular columns, with capillaries and scanty connective tissue extending between them: and these epithelial cells secrete bile, the tumor sometimes being stained an intense yellow or green color from the latter. Thyroicl cancers, made up of low, cubical, thyroid epithelial cells and a vascular stroma, show as a rule a practical cluplication of the structure of the thyroid gland in the production of colloid material ant in the alveolar distension of their epithelial offshoots. Cancers of the intestinal glands repeat the tubular invaginations of the Lieber- 
kuihn's crypts with of course considerable enlargement of the lumina, and are characterized by marked production of mucin and by the presence of numerous goblet cells among the lining epithelium. Mammary cancers have so marked a tendency to 'fat formation from their cells arranged in alveoli, that milk-like fluid is usually found filling the tubular cancerous spaces; and ovarian cancers undergo the formation of cysts which might well be mistaken for the follicles

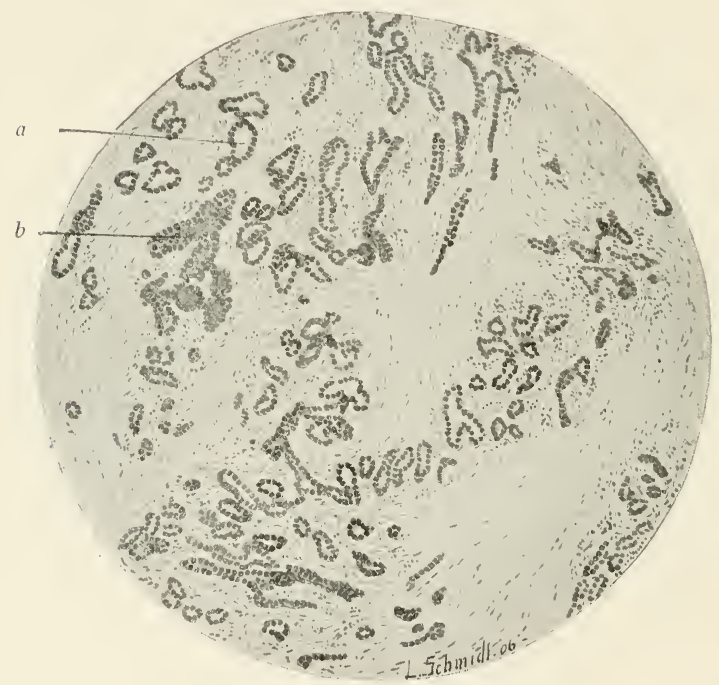

Fig. 125.

Hard adenocarcinoma of mammary gland of a bitch: shows the predominance of the connective tissue strema, the preservation of duct and acinons arrangement of epithelium (as at a). but also the irregular and excessive epithelial formation of solid cords and infiltration of tissues by their penetrating anastomoses (as at b).

The same structure, too, is repeated in the metastatic nodes in the lungs, peritoneum and elsewhere, proving their derivation from the primary tumor and its original source.

This correspondence in cellular character between the carcinoma and the gland from which it originated is, however, not always apparent, the atypical nature of the growth manifesting itself in the extension of the cells beyond their normal limiting membranes, 
their dissemination in the surrounding tissue and their metastasis, and in the fact that the epithelial outgrowths do not form completely hollow passages, but are seen in massive proliferation as solid cellular roots. The ducts of the involved structure are filled with epithelium, and the cells show a great variety of altered shapes depending upon the pressure conditions prevailing, fusiform, rounded, irregular, etc. The connective stroma varies much in its (legree of development, and is sometimes found in villous masses, in folds and laminated processes just as in adenomata, especially when there are spaces and large cavities in the tumor (cystocarcinoma papillare).

[The essence of the histological fault of any cancer lies in the fact that in its proliferation the epithelium of the tumor does not maintain its normal topographical relations, but grows through its limiting membrane and is found infiltrating to a greater or less degree the tissue about it, either as isolated cells or as roots or cordlike groups of cells. In most cases, too, increase in the amount of epithelium is recognizable not only by this infiltrative or metastatic excess but by an excessive number of cells upon the surface involved, or within the tubular spaces or the alveoli of the affected portion of the gland; thus instead of a single layer of cells lining the tubular canals of a cancerous area of endometrium, it is common to find a number of layers and the tubules distended and branched as the author above indicates. This excess, however, is by no means an essential feature, many scirrhous carcinomata showing a manifest reduction in the epithelial elements below that realized as normal to the original gland and to the ordinary cancers of the same organ; and in such cases one finds not only relatively few epithelial areas but also no excess of layers in the tubular lumina, the cells which do line such spaces being in fact themselves small, atrophic or degenerative. It will be found, however, that many of the cells in these have no relations to tubes or alveoli and are clearly free in the lymph spaces of the connective tissue stroma; and careful examination of the gland-like spaces or tubes will somewhere show the direct growth of the epithelium into the surrounding tissue beyond the proper membrane. The existence of karyokinetic nuclear figures is of some value as indicating the active proliferating state of the epithelium.

The editor believes that for practical purposes in microscopic diagnosis of cancers of the author's two varieties, cylindrical cell and glandular cell cancer, it is appropriate to recognize two types according to the existence or non-existence of marked resemblance 
to glands. Those cancers which exhibit a distinct tubular or acinous structure (from which, however, the deviations just indicated are evident) are adcnocarcinomata. All of the types of cancer here under discussion may be said, at least in their early period, to be adenocarcinomata. Those in which the proliferative and infiltrative processes have so advanced as to destroy the original gland resemblance, so that definite tubes or acini are not well or numerously recognizable, may well be called carcinoma simplex. These merely represent more advanced stages of the first, and, are more common as actively growing and extending cancers.

The stroma of true cancer, as the author remarks, is exceedingly variable and is often the basis for special sub-divisions of the growths. Thus it may be relatively small in amount, the epithelial elements predominating; this condition underlies the group of soft cancers (medullary cancers). Or it may be greatly in excess, markedly predominating over the epithelium of the growth, dense and fibrous; such growths are the well known hard cancers or scirrhous cancers (substantive scirrlins). The connective tissue stroma may exceptionally be found largely or totally of a gelatinous type (carcinoma my.xomatodes); or highly cellular and evidently of a sarcomatous type (carcinoma sarcomatodes). Other types of connective tissue may also be encountered in varying amounts in the more highly mixed cancers.]

The external shape and other macroscopic characters of cancer vary with the location of the original growth, the structural constituents of the tumor and the age of the neoplasm. Cancers developing on the free surfaces of the skin and mucous membranes [squamous cpithcliomata, surface adcnocarcinomata] often appear as cancerous ulcers. The infiltrative growth causes a thickening of the membrane, with the [ulcerated] exposed surface uneven with nodular prominences, suppurating, red or dirty dark brown, and perhaps covered with crusts, with the base and wall shown in cross sections as a more or less defined growth and as a light gray bacony zone often penetrating by root-like extensions into the deeper tissue. The normal epiderm is usually sharply defined along the border of the ulcerating exposed tumor surface. In mucous membranes, as the urinary bladder, cancer often forms merely superficial, undulating, slightly elevated thickenings [flat tabular swellings], recognizable from simple inflammatory changes by the accompanying enlargement of the lymph glands, the infiltration of the growth into the muscular layers and by the definition of the 
grayish-white medullary tumor tissue seen in cross section. Of course the microscope affords the conclusive diagnosis. These surface cancers may also develop into cauliflower forms

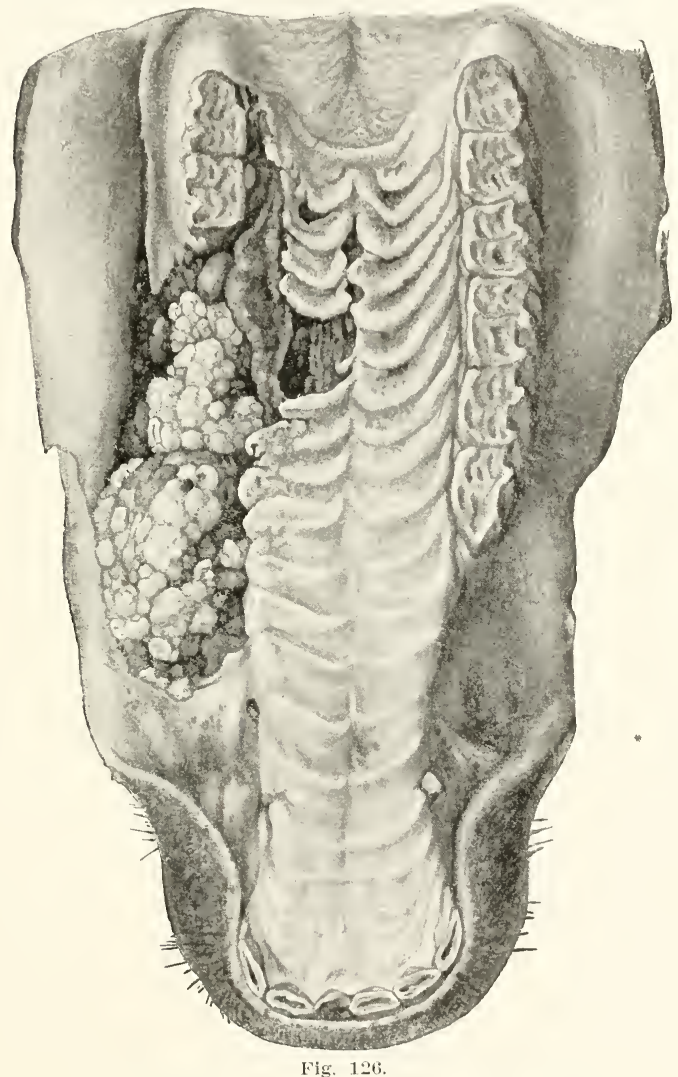

Cancer of gum (squamons epithelioma) of horse.

(carviol), masses of a light gray to more reddish hue, growing from the surface of the skin or mucous membrane, the surface subdivided and overlaid by a suppurating, ichorous, pulpy mass of disintegrating cancer tissue. 
In the interior organs cancers form nodes of widely varying dimensions, sometimes sharply circumscribed and rounded, or with their tissue gradually merging by infiltrative extension with that of the synchronously enlarging organ. The whole organ, as a kidney, may be incorporated into the tumor tissue, and in its stead a shapeless mass of cancer is met weighing perhaps from one to fifteen kilograms. The tendency to perforate to the surface of the involved organ is a marked characteristic of cancers. Another peculiar feature is the production of cancer umbilication, seen rather irregularly in the smaller nodes [secondary nodes] about to perforate through serous surfaces; it is a central depression seen in these nodes due to the prominence of the marginal growth and destruction of the interior of the node. On section of cancers a milky fluid or juice, briefly known as "cancer milk," may frequently be obtained by scraping the cut surface or may be expressed in drops by compression with the fingers; upon microscopic examination it is apparently almost entirely composed of the epithelial cells (with fatty degenerative changes) of which the cancer is constructed.

The secretion of mucus and of colloid substance, as seen in adenocarcinomata of mucous membranes and follicular glands (ovary, thyroid) and the retention of these materials in the tumor tissue sometimes gives an unusual softness and transparency to the cancerous growth, the name gclatinous cancor being often used in connection with such examples. [The term colloid cancer has, from confusion, been employed indiscriminately in these cases. Colloid cancer, using the term in its strict sense, is practically confined to the thyroid gland, and even there much of the gelatinous material contained in it is not definitely colloid, but rather mucoid. The use of the term colloid for these gelatinous cancers in other situations of the body (stomach, intestine, ovary, etc.) is almost sure to be wrong; the change really being a collection of some type of mucin within the epithelial cells and perhaps also in the tissue. The cells which in older text books are described as "colloid sealring cells" are really nothing but mucoid goblet cells, quite like those found in a catarrhal mucous surface, but modified by pressure so as to have a rounded shape, the mucin in their interior pressing the nucleus and a part of the cytoplasm to one side so as to form the prominence compared to the seal of a ring.] On the other hand some cancers come to be very dense and cicatricial in consistence in case the associated growth of their connective tissue should be unusually marked in quantity, in density of fibrillation and in- 
durative. The epithelial elements in such examples may become very unimportant structural factors: these hard specimens are known by the names scirrhus, scirrhous cancer.

According to statistics compiled by Casper, Fröhner and Sticker, cancers [among animals] are most frequent among dogs (three per cent.): horses rank next in order, and after then cats. Just as in man cancer in animals is a disease developing principally in middle and later life. This is probably the reason for the infrequence of these tumors in cattle, sheep, goats and swine, such animals not attaining an advanced age because of their use for meat supply. The skin and its junctions with the mucous membranes are the most frequent seat of growth [for squamous epitheliomata], anal cancer in the $\operatorname{dog}$ and cancer of the glans penis, gums and lips in the horse being frequently met with. The mammary glands and thyroid gland of the dog are comparatively frequent points of primary involvement [adenocarcinoma or carcinoma simplex according to the degree of atypical structure to which the growth is advanced].

In human beings cancer of the stomach and of the female urogenital organs constitute a high proportion of these growths; in animals these organs are affected by cancer to a far less degree.* As far as the rest of the body is concerned it may be said that primary cancer has been observed in all the epithelial organs. In structures which do not possess epithelial cells (lymphglands, marrow, spleen, muscles, etc.) cancer can occur only in metastases (in rare exceptions also from embryonal germinal misplacements). Such metastatic growths sometimes become of greater bulk than the primary tumor, especially those of lymph glands. $\dagger$

The general peculiarities of cancerous growths, their irrepressible penetration into the tissues in every direction, the difficulty of thorough operative removal of all their roots which is only occasionally successful in the early stages of the cancer and if incompletely accomplished invariably results in recurrence, the destruction of the affected organ and the certainty of metastasis, stamp these tumors as highly malignant autoblastomata. In the course of the cancerous affection, the severity of which is determined by the rapidity of dissemination and the location of the growth and the importance of the tissue involved, there usually develops a condition of

* For details see espoclally the statisties of sticker, Archiv. f. Klinisch. Chir. urgie, Bd. 65, Berlin, 1902.

+ If the primary growth has been removed by operation and thls fact be unknown to the examiner, or if the scar at the affected place be overlooked. the secondary formations may give the erroneous impression of being primary tumors. 
general emaciation, anæmia and bodily weakness, known as cancerous cachexia, due at least in part to the reaction upon the system by toxic products formed in the cancerous tissue.

[The cachexia of cancer is of course induced the more early and markedly in case the cancer involve some of the organs of nutritional importance to the animal, as the alimentary tube or pancreas: the faults of nutrition being important contributors to the production of oligamia and loss of flesh and strength. The idea of toxic factors, although usually not urged by writers because of our ignorance, is, however, probably an important one, these toxines arising from the secretory activity of the tumor tissue or as products of tissue metabolism or destruction. The metastatic tendency, involving the progressive affection of more and more of the body by cancerous foci, must of course be an important factor of malignance. The occurrence of metastases varies greatly with the type of the tumor and with the definite resistive power which the body exerts against these practically parasitic cells, this resistance being apparently not at all uniformly possessed by the various organic systems or by different individuals. In a general way it may be said that the squamons epitheliomata are likely to disseminate more slowly than the other varieties, progressing more regularly by a continuous invasion of the lymph spaces and channels, and therefore showing a greater tendency to local and neighboring infiltration and node production. Distant metastases are to be met with but are not as frequent as in the cylindrical cell and glandular cell cancers. Of the latter groups in a rough way, to which exceptions are common, it may be said that those that manifest in their structure the greatest departure from the tubular and acinous types (carcinoma simplex) are those showing the more rapid and extensive metastatic tendency; the adenocarcinonatous forms the less. Both are, however, more apt to give distant metastasis than the squamous cell form. The condition of the stroma makes a difference as well, the soft medullary types with a small amount of stroma being more metastatic and the hard scirrhous varieties tending to remain local for a longer period. It is said that cancers as a group are characteristically generalized by way of the lymphatic system, in contrast to sarcomata which are more frequently conveyed by the blood current. This is, in the main, true, and the usual rule is to find the lymph glands into which the lymph drainage of the cancerous part passes the seat of secondary cancers. Yet it is by no means necessary. The route of metastasis and in a great measure the tendency 
to give rise to metastasis are explicable on mechanical grounds. The cells of squamous epitheliomata are usually large cells and are less easily displaced from their site of formation than many of the smaller cells of the cylindrical cell and glandular types of cancers; and as a group all epithelial cells are larger and less easily displaced than the small round cells of small round cell sarcoma. These last mentioned growths have cells comparable to the small lymphocytes which are always and readily passing with the blood throughout its entire capillary system. It amoints therefore to the general rule that, whatever tumor cells may be able to gain entrance to some small capillary blood vessels in the tissue in which they are growing, the sarcoma cells have a better chance of convection than do the epithelial cells. On the other hand sarcomas in their growth, directly from and in the connective tissue structures in which the bloodvessels are distributed, are more favorably situated as early tumors to gain access to these vessels than are the epithelial cells, which primarily are separated from the blood stream not alone by the vessel walls but by more or less connective tissue and by remnants, at least, of a membrana propria. In their mode of growth cancers primarily are more likely to gain entrance to the lymph spaces an $i$ channels, and are therefore from the first more favorably situated to follow this system provided the spaces and channels are of sufficient calibre to allow the convection of the epithelial cells (as they usually are, in comparison with the minute blood capillaries). Yet granting such factors as these, as determining the usual course and occurrence of metastasis, exceptions must arise and are constant'y being seen. If a sarcoma grow in lymphatic tissue (a lymphosarcoma for example) it is very likely, whatever the size of its cells, to follow the lymph current to the neighboring nodes: and if in some situation (as in stomach or intestinal wall where the larger radicles of the portal vein are close to the cancerous growth starting in the mucosa) the cancer cells be afforded easy access to bloodvessels of sufficient calibre to accommodate their size they wi' be found to give rise to hrmatogenous metastasis (as the portal convection of gastric cancer to the liver).]

\section{Epithelial Cysts, Dermoid Cysts, Odontomata, Mixed Teratomata.}

There are a number of tumor-like formations which, as hollow epithelial lined sacs, limited to their place of development, originate from embryonic cellular misplacements. These are cysts ( $\dot{\eta}$ kóotis. vesicle) which are not produced from mature glandular organs 
from simple retention of their contents and dilatation of their canals and are not formed in the loose structufes of the body from exudation into them or from liquefaction of the tissues themselves, but take their inception in early embryonic life from displaced and isolated portions of organs, organic rests and hollow offshoots of such structures, and acquire an independence of growth.

The simplest type of these cysts is the chidcrmoid cystoma or epithelioma cy'sticum, a sac of the size of a nut, a hen's egg or even of a fist, which is completely filled with loosened squamous epider mal cells almost all in a keratous condition; sometimes cholesterin is found mixed in with these. Bonnet met a genuine example of this type in the cranial cavity of a horse. All these cysts containing squamous epithelium doubtless originate from ectodermal cellular inclusions in fotal life, conceivable as occurring from friction of the skin by amniotic strands. Their occurrence in the cranial vault, near the aural and temporal region, suggests that displaced portions of the epithelium of the branchial arches constituted their original substance, and in case of those developing in the brain, from the medullary folds, the ectoderm must certainly play a part in their origin. Experimental studies by Schweninger, E. Kaufmann and Ribbert show that epithelial cysts can be readily produced artificially by insertion of a bit of epiderm, conjunctiva or tracheal mucous membrane into the peritoneum or under the skin. When the epithelial cells are supported by a little connective tissue to preserve their nutrition they grow over the interior surfaces of the wound with which they have been placed in contact, and form the lining of the hollow space, the latter being occupied by the wound secretions and desquamated epithelium. Epithelial lined cysts may also be produced, as pointed out by these authors, by suturing over a circumscribed bit of epiderm the adjacent loosened borders of skin: the bit of epiderm beneath the sutured parts grows and fills in the space beneath the elevated cutis (Ribbert). In man as the result of accidental misplacement of epithelium in operations, or of cutaneous lacerations, similar cyst formations are well known to occur. Such results are known as traumatic epithelial cyists.

As derivatives of the skin in its entirety, of the cutis with its glands, of hairs and epiderm, may be mentioned the dermoid cy'sts or hair follicle tumors (dermatocystis congcnita or cystoma dermoides), which occur comparatively frequently in cattle and horses, situated in the subcutaneons connective tissue. They appear from the exterior as flat cutaneous swellings or may be found accidentally in skinning or eating beef. The cyst varies from the size 
of a nut to that of a fist, is rounderl and filled with a bunch of tangled hair and a dirty brown fluid consisting of secretion from the sweat-glands, fat and desquamated epithelial cells. The wall of the sac, which may be easily shelled out of its berl, is thin, quite like the fibrous structure of the cutis, and lined on the inside with epiderm and attached hairs, loosened hairs recurring in the contents as free bunches. Microseopically the wall hats all the appearances of the cutaneous structure. These dermoid cysts are met particularly in the region of the neck and jaw, generally developing in these situations from tissue derived from the branchial arches (branchiogenous cysts) ; they are also met about the shoulder or elbows, in which eases they may be referred to faults in the development of the anterior limb bud (Leisering. Johne, Grams and others), in the umbilical region, and in the testicles and ovaries.

As early as I $85+$ Remak explained the origin of dermoid cysts by conceiving an abnormal development of the arches and resultant enclosure of a bit of the germinal epidermal layer as their basis: and it may also be possible that injuries eaused to the skin in the region of fissures may give rise to them, provided the borders of the fissures become the seat of a cicatrizing proliferation and. further, provided the base of the skin fissure becomes isolated and brought to lie underneath the scar.

The dermoid eysts of birds contain feathers (fiather-follicle cysts, cystoma pennifermm) and are found from the size of an ege to that of a fist within the abdominal cavity of geese, more rarely in ducks and chickens. The cyst, surrounded by a thick layer of fat. is sometimes free in the peritonemm or may be attached by a peritoneal fold to the ovary and lumbar region. The number of feathers (the plume not infolderl because wet with fluid) may reach several hundred.

Analogous to the dermoid cyst but not growing in cystic form, and appearing as protruding islands of hairy skin, are the dermoid teratomata of the cornea (also of palpchra tertia), which are met comparatively conmonly in cattle and dogs. They are congenital anomalies and originate from a misplacement or transplantation in the formation of the palpebral fissure.

In the same way as from the epiderm and cutis cystic foci may arise from separation or misplacement of their cells, epithelial lined eysts may also develop from the mucous membranc tubules and glandular duets in embryonic life from budding or other anomalies of development, particularly from "rests" of 
gland tissue (adenocystoma). These entodermal cysts are lined by columnar epithelium, sometimes ciliated, and in man are found in the liver, intestine or peritoneum, in the neighborhood of the trachea and bronchi, and in the neck, varying in size from that of a pin-head to that of an adult human head. In animals they most usually arise from rests of the original urinary structures,

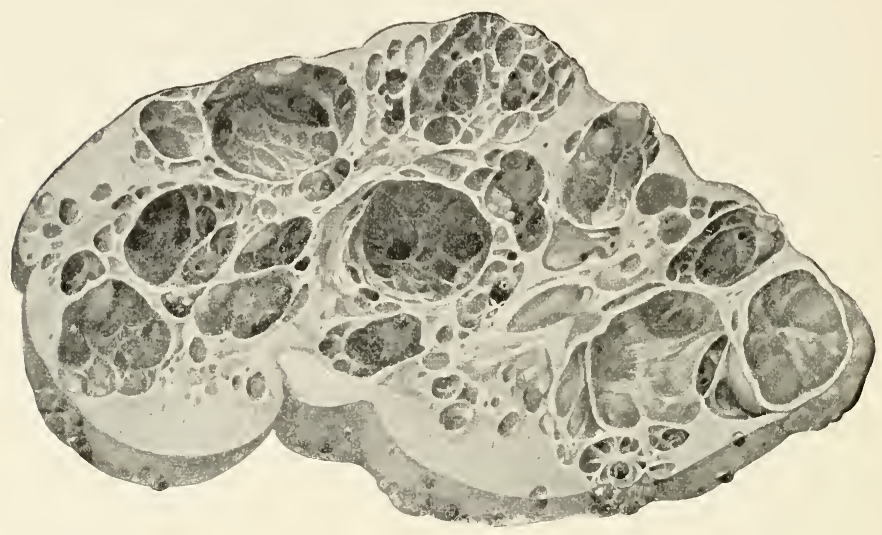

Fig. 127.

Cystic kidney of hog (section).

the Müllerian or Wolffian ducts, and are found as transparent vesicles of the size of a millet seed or pea or sometimes larger, along the broad ligaments of the uterus, in the fimbrix of the oviduct, and in the epididymis. The congenital cysts of the nvary, occurring as single or multilocular and closely packed. cysts of this organ, and containing a thin mucoid and sometimes bloody material, are further examples of the same type. Congenital cysts of the kidney are also regarded as results of developmental disturbances, converting the kidneys, usually bilaterally; into a grape-like mass of vesicles with watery contents, between which an inconspicuous amount of parenchyma is found reduced mainly to thin connective tissue septa (cystic kidneys, vesiculated kidneys). This anomaly is supposed to be due to a failure of union between the uriniferous tubules and the developing glomeruli, these two parts growing separately (Ribbert).

The term odontoma and odontoblastoma are applied to mon- 
strous tooth-formations, which arise from faults in the cmbryonic development of dental suibstances and present themselves cither as excrescences on the teeth, of bone-like hardness and composed of cement and dentine, or as abnormities in which an entire tooth has been changed into a shapeless, nodular form with enlargement; or in which in combination with an accompanying proliferation of the soft connective tissues a componnd dental tumor has been produced. These neoplasms are always congenital, either becoming apparent at the time of the eruption of the tooth or the teeth fail to erupt and a tumor slowly develops in the alveolar portion of the jaw beneath the gum, bulging the latter and expanding the bone as it enlarges within the alveolus. The composition and form of these odontoblastomata vary considerably with the part taken by the different tissues concerned in embryonic tooth formation, the dentine and its covcring of enamel, the dental sac and the peripheral alveolar bone. The simple odontoma durum, now and then seen on single teeth in the cow and horse, occurs as a mass about the size of a nut, rounded, hard as bone, and usually. surrounding the crown or more rarely the roct of the tootli. In other instances, as observed by Wedl and Magitot, the whole tooth lying within the alveolar process is changed into a mass of bony hardness as large as an orange or possibly weigh-

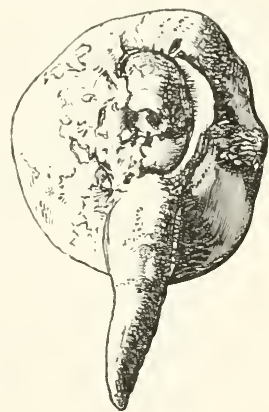

Fig. 12s.

Odontoma of incisol tooth of cow (natural size). ing as much as a kilogram. The name odontoma mirtum is applied to a growth composed partiy of fibrous tisste, bloodvessels, odontoblasts and agglomerated tooth papillæ, with the hard parts in rudimentary mshaped masses of dentine and enamel over the surface or mixed in the interior. Sutton has met with a mixed growth of this type weighing seven hundred grams in a horse. Similar tumors, sometimes only enclosing in their structure a single hard rudiment of a tooth, or sometimes containing a number, possibly several dozen, misshapen or fairly well formed teeth, occur as cysts or bunches of cysts. completely included by fibrogelatinous dental sacs and alveolar bone capsules (odontocystoma capsulare. cystoma dentifermm alceolare). In other instances the proliferation of the bony capsule is especially marked, surrounding the dental sac- 
cular tumor and the deformed tooth, and giving rise to the type known as ostcocystoma capsulare dentiferum. Occasionally in young horses at the base of the ear over the temporal bone cysts may be found which contain one or several imperfect teeth, usually opening by a fistulous sinus upon the skin; these are blind tubular invaginations from the skin, lined with mucous membrane and provided with a bony covering or with small bone platelets in simulation of the alveolar process of the jaw; they originate from the branchial arches and are known as

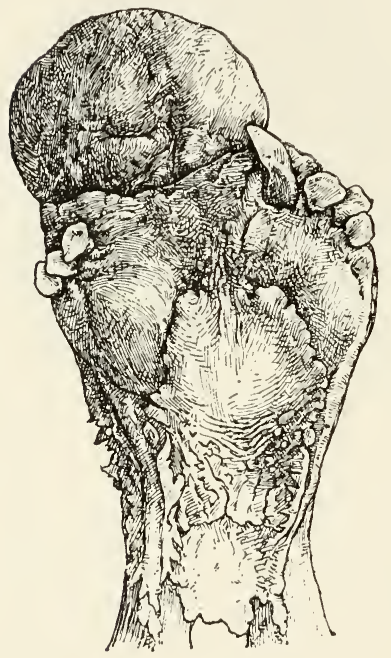

Fig. 129 ,

Odontocyst of lower jaw of cow.

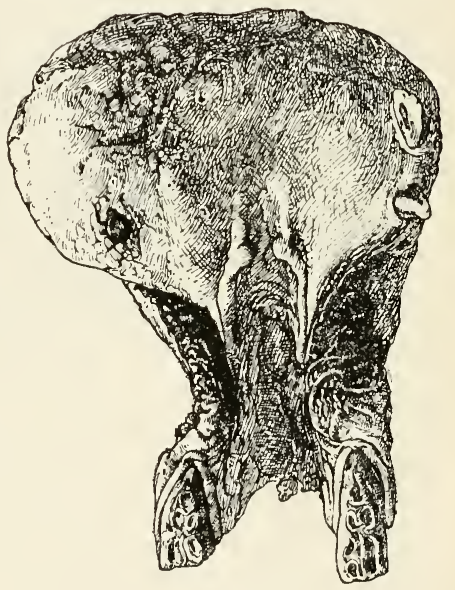

Fig. 130 .

Osteocystoma dentiferum of horse.

branchiogenous dental follicular cysts (odonto-teratoma branchiale, odontocystis branchiogenctica).* This type of cysts is only rarely met in other animals; Siedamgrotzky encomuterer two dental cysts in the ethmoidal turbinate in a hog; Verwey has recorded one in the petrous portion of the temporal bone in a dog; Hertwig met a dental cyst in an ox.

Teeth are sometimes fonnd in dermoid cysts and teratomata in other parts of the body, especially in this type of growth in

* For fuller description s. Kitt, Lehrbuch der pathol, Lnatomie der Hausticre, Stuttgart, F. Enke's Verl. II Aufl., 1901. 
the ovary and testicle (heterotopic odontoteratomata). Gurlt recorded a testicular tumor of a horse, about the size of a fist, of stony hardness, containing six molar teeth (three separate, three merged together), together with thin plates of bone representing a rudimentary alveolar process, and a fibrous capsule; in a second instance the egg-sized tumor contained, besides a single tooth, a lot of hair and a fluid material made up of a mixture of perspiratory and sebaceous material.

In addition to the above cysts and dentigerous tumors there occur, especially in the sexual glands of both sexes, a number of other tumors of complex composition, sometimes showing an irregular mixture of heterogeneous tissues of all three embryoblastic layers, sometimes a definite tissue arrangement representing clearly the structure of a number of organs. Because of their close relation to monsters the collective names teratoma or cmbryoma are used in connection with these peculiar formations, which undoubtedly originate from embryonal cells or tissues which have been segregated and become independent at an early developmental period.

The external tumors of this type, growing in the superficial tegumental tissue or in the mouth, are possibly the results of partial cleavage from amniotic threads, as it is known that indentation of an embryonic tissue can cause its division and double development; and even transplantation may be effected from injuries caused by amniotic bands. These external teratomata usually appear as tumor-like, pedunculated formations, of lobulated outline, and are composed of hairy skin, rudimentary jaws with teeth and mucous membrane covering, fat tissue, nerves, muscle and glandular structures. (For illustrations and details v. E. Sigl, Monatsheft f. pr. Tierheilkunde, Stuttgart, 1902.) The compound teratomata developing in the interior of the body, especially in the ovary and testicle, may sometimes originate from germinal segregations from the primitive segments of the vertebral column or from the indifferent cells of the Wolffian body; or it is possible, as Marchand's theory would make plausible, that they may arise from the separate development of one segmentation cell. According to the experimental studies of Roux and others each one of the primitive segmentation products of the ovum-that is, each segmentation cell-is capable of producing an entire embryo; thus the possibility arises that an isolated segmentation cell in course of progressive development may form a group of embryonic tissues, which be- 
cause of irregular nutrition succeed in their later growth in forming only an amorphous body of all sorts of organic structures mixed together. This, it is likely, enclosed within the individual formed from the other segmentation cells, remains without further development, as a tumor. [Many of the so-called ovarian dermoid cysts met in human beings are properly of this last type, complex teratomata, frequently containing tissues evidently from two or all three of the embryoblastic layers. It has been suggested in addition to the abore explanations for such teratomata in the sexual organs that they may be the result of segmentation and derelopment of an unfertilized orum directly. within the ovary, a parthenogenetic development. This idea has no wide acceptance, but it should be recalled that parthenogenetic development is well known in many lower forms of life, and that Jacques Loeb has succeeded in causing the development of unfertilized ora of sea-urchins by chemical stimulation. It should be added moreover that L. Loeb (Archiz. f. mikrosk. Anat. u. Entricklungsgeschicht. Bd. 65, 1905) has demonstrated karyokinetic changes and segmentation of the orum in the ovary of the unfertilized guinea-pig. The theoretical possibility of this mode of origin of ovarian teratomata should therefore be accepted; although eridence of its actual prevalence is not clear.] 


\section{FUNCTIONAL ABNORMALITIES}

\section{Nervous Disturbances-Disturbances of Motion.}

The nervous system regulates the functions of the organs and is the intermediary between the corporeal and psychical functions of the polycellular animal organism. The nervous matter may be excited by both external and internal agencies (stimuli), and being thus stimulated gives to the tissues the impulse for the manifestation of the powers peculiar to them. Alterations in the excitability of the nervous system and abnormal stimuli which occasion such variations, may result in functional disturbances of the nervous apparatus and the organs dependent upon it. Where the whole central system is altered, these disturbances are general and may manifest themselves in the widest possible range (gencral ncrious symptoms) of abnormal nervous phenomena (sensory, motor, secretory, reflex). Where definite localities in the central nervous system are subjected to injury, we speak of contral local involiement and focal symptoms. In this connection, too, nuclear involvement where the nucleus is affected, is differentiated from supra-nuclcar involvement, where the course of the conduction within the central system-that is, above the level of the nucleus-is the part disturbed; and according as the focus affected lies within the brain, in the medulla oblongata or in the spinal cord the lesion is said to be crrbral, bulbar or spinal. Where a nerve is injured in its course beyond its point of exit from the brain or spinal cord to its area of distribution or within the latter, peripheral affections ensue.

General Nervous Disturbances.-The brain and medulla together constitute the central organ presiding over the whole category of functional activities, and therefore everything which causes depression or excitation of cerebral activity must of necessity bring about a great variety and general distribution of disturbances. Every lesion of the deeper centres of the medulla 
oblongata, in which the circulatory and respiratory regulative apparatus are located, must necessarily inhibit or depress these most vitally important functions and thus threaten life. Lesions of the cerebral cortex bring about varying grades of disturbance of consciousness, which, including all phases of mentality, may be collectively spoken of as psychical disturbances.

The most severe of the psychic paralyses is cercbro-spinal concussion (commotio cercbri et medulla). After more or less severe concussion of the head or the whole body by a fall, blow or kick, the functions of the brain and cord cease. Anatomically: there is often nothing to be found in such a case, beyond slight hemorthagic effusions upon the membranes and the skull, and gross lesions of the nervous structures are absent. The severe symptom complex can only be explained by supposing that through the influence of the concussion waves upon the exceedingly complicated mechanism of the nervous system (compression alternating with stretching force) minute structural changes must be induced, as destruction of the delicate dendrites of the nerve cells (Krehl).

Cessation of the cerebral function is manifested by complete loss of conscionsness, loss of all voluntary muscular motility, loss of pupillary reactions, weak pulse and coolness of the external surface; and tends usually, in a few minutes, hours or days to death. In other and milder instances only a transient loss of sensibility cccurs. When the cord is the main part involved by the concussion there are met paralysis of the extremities, loss of sensation in the latter and in the trunk, and disturbances of respiration, as the principal symptoms. Sudden and usually brief disturbance of equilibrium and inability to maintain an upright posture (dizzincss, acrtigo), associated with loss of conscionsness (faint, slncope), occur from disturbance of the blood circulation in the cerebral cortex and in the cerebellum: they may result too from trivial grades of general ancmia of the brain, as a decrease in the arterial supply, and also occur in passive congestion (cardiac disease, compression of jugular veins) or from anatomical changes of the cerebellum, aural disease and toxic influences. In uncomplicated loss of consciousness the cerebral cortex is alone the seat of involvement; the animal suddenly begins to go slowly, stops for a moment, staggets, leans against the shafts or other support, turns around in a circle and falls; then, after remaining motionless for a few minutes, attempts with labored movements of the limbs to get up, and is gradually able to rise and 
resume consciousness. Sometimes vomiting (in dogs) and involuntary discharge of urine and feces ensue.

(For details see Friedberger-Fröhner, Veterinary Pathology, Amer. Ed., 1904. W. T. Keener \& Co., Chicago.)

Persistent unconsciousness, coma ( кoúá to lull to sleep), or sopor (sopor. a sleeping draught), is a state of insensibility which is met in serious brain affections, intoxications (uræmia) and particularly in connection with hiemorrhages from the cerebral vessels; in the last when it occurs suddenly and paralysis results, the process is usually called a brain or nerious stroke (apoplevia cercbralis, nerzosa: $\dot{a} \pi \circ \pi \lambda \dot{\eta} \sigma \sigma \epsilon \nu$, to strike down). When unconsciousness occurs paroxysmally, accompanied by muscular spasms and loss of sensacion it is known as falling sickness or epilcpsy ( $\dot{\eta} \dot{\epsilon} \pi i \lambda \eta \psi(s)$; it depends upon some functional disturbance of the cortical centres, the real cause of which is, however, unknown. Compression of the intracranial structures, as from blood clots, tumors, increased pressure of the cerebro-spinal fluid, or collections of exudate, give rise to the symptoms of compression of the brain (brain depression). These consist of general dulling of the senses, a sommolent, lethargic attitude, staggering and awkward gait and diminution of sensibility. The animal stands as though deaf, leans against some adjacent body for support, hangs its head or supports it against the wall or manger, and the eyelids are closed as if in sleep and the pupils dilated. The animal may be hard to move irom one spot and may continue for hours at a time in some abnormal posture (legs crossed or kneeling), or may run about with staggering gait into the wall and all sorts of obstacles, or may continually move about in a ring; it is apathetic when spoken to, touched, whipped, bitten by flies, etc.; takes no foor, or holds its food in the mouth without chewing, pushes its nose deep into the water in drinking; and may show other special pressure symptoms according to the particular part of the brain affected (reversed movements, riding paces, focal movements, dancing or rolling movements, or pressing toward one side). In the man and the dog there are also observed peculiar alterations of the eye-ground, so-called [choke disc] papillary congestion (really a papillitis, inflammation of the papilla and optic nerve): in horses with blind staggers these are not noted (merely an anæmia recognized-Friedberger-Fröhner). These symptoms depend upon the fact that in the first place in the 
conditions indicated disturhances of the blood circulation arise,

* as compresison of the large veins before opening into the rigid walled sinuses, with resultant stasis, interference with the escape of venous blood and cerebro-spinal fluid, and the production of increased pressure upon the brain substance; that, further, in the conditions mentioned increased formation of this fluid and special tissue changes in the brain may obtain, and perhaps, too, toxic influences (bacterial toxines, autotoxines).

Psychical excitement has its causative factors in hyperæmia of the brain, cerebral inflammatory changes, heat or toxic influences, and occurs in a number of different degrees of severity. The main symptoms are manifested as attacks of insanity (mania, delirium furibundum). The animals in these attacks suddenly lose their docility, try to free themselves from their fastenings, bite their own bodies, make attacks in whatever way is natural to them (strike out, kick, try to bite), run into obstacles and violently knock them over, stand up on their hind legs and throw themselves about with shrill cries, grit their teeth and froth at the mouth; the eyes become staring and wild, the conjunctive injected; and finally the animals collapse and go into spasms and convulsions. Especially in dogs of usually affectionate and lively disposition, well-trained and possessed of special intelligence we meet with the widest range of mental disturbances, from melancholia to hallucinations (fancied perceptions), marked confusion and insane outbursts, as in rabies (depressed stupid condition, snapping at the air, purposeless wandering about, sudden attacks of desire to bite, tearing of the animal's own flesh), or in poisoning with deadly night-shade or in sunstroke. Similar symptoms of excitement are seen in dogs in the autointoxication produced by the portal blood, that is, the absorbed products of albuminous decomposition in the intestine, in Eck's fistula [fistula made between the portal vein and vena cava with diversion of the portal blood from the liver]. Special nervous excitement and reflex psychic conditions occur in some of the diseases of the genital organs (ovarian cysts, ovarian tuberculosis, uterine affections, undescended testicle), the affected animals manifesting (uterine, ovarian, orchitic psychoses) an exaggerated sexual desire (nymphomania, satyriasis, erotomania, rutting, sexual insanity).

There are, too, a number of vague nervous conditions which lẹad to perversions of taste (perversions of appetite) manifested 
in animals, especially by the group of symptoms known as "licking" or pica (wool-eating sheep), the animals showing an inexpressible desire to lick all sorts of objects, even nauseating substances, and to eat them: an ultimately fatal cachexia developing in the chronic forms of these morbid appetites. [Analogous to the pica of chlorosis and other ancmias in man, and the dirt-eating habit of the anæmic hosts of uncinaria; probably many cases in animals are really expressions of a similar parasitism.] In rabid dogs there is a strong tendency to swallow foreign bodies. In sows the habit of eating the pigs and in ruminants of devouring the placenta are expressions of psychic abnormalities.

(For fuller details see Friedberger-Fröhner, Veterinary Pathology. Amer. Ed., I904. IV. T. Keener \& Co., Chicago.)

Motor Disturbances. - Muscular movements are normally-

I, Voluntary, stimulated by the psycho-motor elements of the gray cortical substance of the brain; 2 , automatic, caused by chemical stimuli, independently of voluntary influence, occurring rhythmically and at all times present; 3, refler, performed unconsciously and caused by transmission of sensory excitation to the motor apparatus; 4. co-ordinated and combined, the rapid succession and repetition of voluntary and involuntary movements as group actions, represented by facility of movement acquired from practice (Samuel). Disturbances of novement (motility) depend either upon pathological changes in the muscles, tendons, bones and joints, or upon changes in the motor nerves. The motor tract begins in the large ganglionic cells of the central convolutions, passes by the long pyramidal fibres to the cells of the anterior horns of the spinal cord and thence pass peripherally by the nerves distributed to the muscles. In addition those fibres and cells of the cerebral cortex which are concerned in perception, ideation and volitional stimulation are more or less involved in the production of voluntary movements. The complicated and widely distributed tract may be exposed at any point in its entire length to influences which are capable of interfering with its functions. Such disturbances manifest themselves either as pathological exaggeration or pathological impairment of motility.

Pathological exaggeration of motility is manifested by spasms; spasm or hyperkinesis ( $\dot{\eta} \kappa i \nu \eta \sigma s)$ may be defined as a paroxysmal muscular contraction which is occasioned by pathological stimulation or which, if caused by ordinary stimuli, exceeds normal 
degrees of contraction. When the members of the body are thrown into motion by these contractions alternating with relaxation the spasm is said to be clonic ( $\dot{\delta} \kappa \lambda b v o s$, violent movement) or altcrnating; when the contraction persists for a longer time, causing maintenance of rigidity in one position, it is said to be tonic (ó róvos, tension). Tonus and clonus may succeed each other alternately. The mildest form of spasm is tremor, really a quick succession of contractions of single muscles or muscle groups, the coarser types evident as visible and palpable jerkings. These muscular contractions or spasms are spoken of as convulsions when they give rise to excessive movement of the body and limbs, or violent tremors of the whole body (Samuel). Tonic spasm of individual muscles or of only one group of muscles is also termed a cramp (as of the jaw-muscles, trismus, from rpisw, to grit); the powerful and long continued contractions of the greater part of the musculature, tetanus ( $\tau \epsilon i \nu \omega$, to strain); a general body rigidity, without any important contraction (merely the ordinary contraction to maintain position), catalepsy; longcontinued shortening of individual groups of muscles, with permament deformity as a consequence, contracture (distinguished as actize, spastic or reflexophile in distinction from the passiz'c contractures not of nervous origin met in disease of joints). Convulsions when accompanied by loss of conscionsness are described as cpilcptic or epilcptiform.

We know in connection with the causes of spasms that they may be induced by a group of poisons or chemical irritants (tetanotoxine, strychnine, ergotin, lead, carbonic acid gas), and may be produced by poisonous substances arising as metabolic products in the body (in uræmia), and that muscular contractions may result (directly or reflexly) from mechanical excitation (section, torsion, crushing). In certain instances the spasms are caused by local lesions of the peripheral nerves, of the cord or of the brain (focal lesions), as the convulsions of suffocation by lack of oxygen supplied to the medulla oblongata, those of tetanus by the chemical combination of the toxine with the nervous substance of the spinal cord. The more minute lesions which take place in such examples are as yet not known, and the extremely confused ideas we possess of the relations between processes of excitation and inhibition prevent, for the present, any satisfactory explanation of the conditions involved.

The results of spasms vary with the location of the causative influence in the system. When the cause is directly in the 
muscles the effects of the spasms are of little importance, except that in long standing contracture atrophy is likely to develop. Spasm of the respiratory muscles of necessity canses fatal suffocation because of interference with respiratory movements. Spasms of the muscles of mastication cause starvation. In other examples a fatal termination may be explained by the primary or scme collateral action exerted by the poisons cansing the convulsions, the tetanus poison for example acting both by the lesions induced in the ganglionic cells and by a hemolytic action. It should be kept in mind that the spasms themselves are nothing more than the symptoms of the actual nervons disturbances.

(For details v. Friedberger-Fröhner, Lchrbuch der klinischin Vutersuchungsmethoden f. Ticrarzte, III. Aufl. Stuttgart. F. Enke.)

Loss of muscular power is termed palsy (akinesis [a. prü.,] $\eta \kappa i \nu \eta \sigma s$, movement) and may be differentiated into a complete loss of molility (paralysis), or partial loss (paresis) in which the power of the muscles to perform movements is only reduced in degree. In case of deficiency in co-ordination, where the movenents are performed clumsily and hesitatingly, the term afaria (a priv., $\dot{\eta} \tau \dot{\alpha} \xi_{\iota s}$, arrangement) is employed. Loss of motion occurs in pathological changes which directly inhibit muscular contractility (atrophy, inflammation, rupture, degenerations), or interfere with the movements of the bones (ankylosis of joints), or in conditions of inefficient innervation which induce palsy both indirectly by reducing the stimulus for contraction and by causing local changes [in the muscle].

A faulty innervation itself is usually the result of anatomical changes involving the ganglionic cells or nerve fibres and thus interfering with the formation of impulses or interrupting their transmission, as for example stretching, disintegration of the nervous substance by pressure (tumors), or destruction and degeneration from poisons (toxines, lead), or disturbances of blood supply. The location of the changes may be in the central motor apparatus, cerebral cortex or cord (cerebral or spinal palsy), or in the nerve trunks and their terminations (peripheral palsy).

The central and some of the peripheral palsies are usually unilateral (hemiplegia) because they are isually due to focal lesions, to local changes, in one of the bilaterally placed nerve ganglia or nerves; while spinal palsies generally involve both sides of the body (paraplegia) because in the comparatively small transverse area of the spinal cord the lesion causing the anatomical 
changes is apt to occupy the whole extent of the transverse section (transi'erse spinal palsy).

Inhibition of the motor centres may also be caused by excessive stimulation of the sensory nerves in a reflex manner (socalled refle.r paralysis or neurolytic palsy'), as where complete paralysis of the posteripr part of the body occurs after forcible compression of the kidney, bladder, ureter or a portion of the bowel (Samuel).

Results and terminations of palsies. The results of motor loss vary with the duration, extent of disturbance, the importance

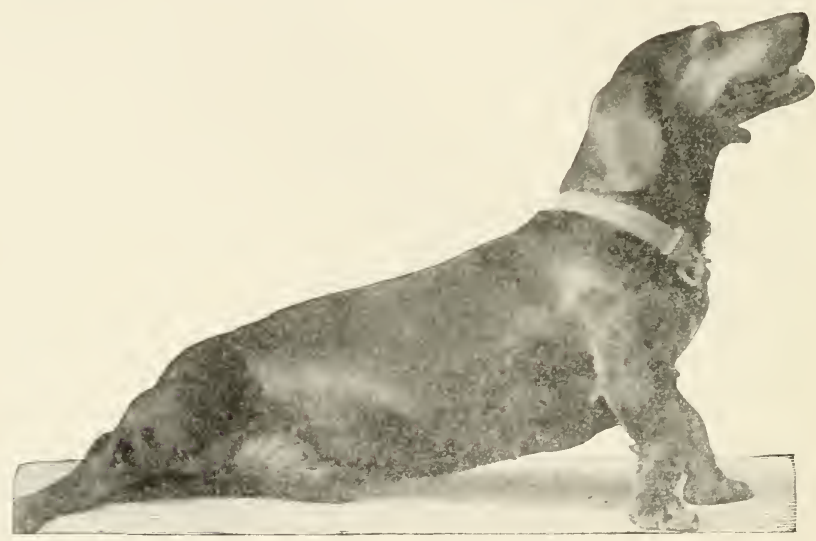

Fig. 131.

l'aralysis of the posterior part of the body: transverse spinal palsy. (Plotograph by $D:$. Jakob.)

of the affected muscles and of the causative lesion. In paralysis of the heart and respiratory muscles death at once ensues from cessation of circulation and gas interchange. Indirectly palsies of the pharyngeal muscles, the bladder, the intestine or the extremities may result in death because of the derelopment of various possible complications (aspiration of fluid in the air passages causing aspiration pneumonia; retention of urine and fecal material with production of dilatation, inflammation and erosion of the mucous membrane: hypostases and decubitus with bloor poisoning, etc.). In case of paresis of the sphincter of the bladder the urine constantly dribbles and the same symptom con- 
stantly occurs with palsy of the sphincter and detrusor muscles; when the detrusor alone is paralyzed and the sphincter is intact the urine collects in the bladder. Paralyses due to destruction of ganglion cells are irreparable; but peripheral palsies may sometimes recover by regeneration of nerve fibres. When regeneration of nerves does not occur in the spinal palsies due to destruction of the gray matter and anterior nerve roots, the paralyzed muscles undergo atrophy and degencration, with rapid loss of electrical irritability. In case of central palsies atrophy does not occur (Samuel).

Sensory Disturbances.-The sensory nerves transmit pressure and temperature sensations, special sensations (smell, taste, sight, hearing), and the so-called general sensations (hunger, thirst, special cutaneous sensations, sense of position). It is assumed that just as for the special senses there are particular nerves, there are also for every other sort of sensation special nerves; and it is known that nerves sensitive to pressure, pain, cold and heat liave entirely specialized and characteristic endings (v. Frey, Krehl). From these local receptive endorgans the sensation is conveyed centripetally to the perceptive psycho-sensory parts of the brain. where proper distribution is made to the tracts and innumerable connections occur with the reflex and autonatic nerves and ganglia. Disturbances of sensation may be considered as of two types, pathological crcitation and pathological depression of sensation. Pathological cxcitation of sensory nerves gives rise to symptoms of sensory irritation of the most varied forms and grades, according to the kind and position of the terminal organ and the lesions. Sensation of pain is caused by stimulation of the nerves of pain, which are distributed in profusion and have their terminations in the skin, at the mucous membrane orifices, serous and synovial membranes; these peripheral parts of the body therefore being capable of manifesting the most severe attacks of pain. Mechanical, chemical and thermic stimuli induce this symptom. If the nerve be in a condition of exaggerated irritability (hypercsthesia) ordinary physiological variations (muscular action, tension of tissue, congestion) are sufficient to bring on pain. Certain forms of pain apparently develop spontaneously (neuralgia), that is, without demonstrable anatomical lesions and from unknown changes. The tracts extending from the muscles, bones and glands and into the central nervous system are less sensitive to pain; although painful symptoms may be noticed arising from 
such locations also, as in affections of the spinal cord. In the brain, as indicated by the absence of pain during operative procedures, the sensibility to pain is very low, at least in the cortical areas. Only hairs, hoof, and cartilage, provided the matrix be not also involved, are entirely devoid of the sense of pain, because of the absence of nerves from these structures.

The existence of pain is shown in animals partly by general symptoms (restlessness, pawing, stamping, running about, rolling over, groaning, sighing, gritting the teeth, whining and crying shrilly), partly by local symptoms which suggest and more or less clearly indicate the location of the painful trouble (scratching, hobbling, protective movements when touched, striking against the abdomen, looking around toward the posterior part of the body, arching of the back, symptoms of colic).

Itching, indicated by the tendency to scratch, is met in cutaneous affections, in some intoxications and general disturbances: it must be due to an irritation of the nerve endings (pressure points, Kreh1), but the mode of origin is unknown.

The irritative simptoms, "formication," "going to sleep" of some part, which are caused by pressure upon nerve trunks (parasthesia), light and color phenomena from irritation in the optic tract (photopsia, chromopsia), abnormal temperature sensations, cannot be determined in animals, as they are subjective perceptions.

Pathological diminution of sinsation. In lesions which interrupt the course of the nerves at any position or render functionless the parts subjected to irritation, sensation is entirely lost or diminished or its transmission slowed. Scnsory palsy (anasthesia) may be induced by traumatic destruction of continuity (nerve section, crushing), by freezing, cauterizing the endings, by local anæmia, nedema, degeneration or atrophy of the peripheral nerves or brain and cord segments, as well as by the local and general action of certain medicaments (anodynes and anæsthetics, as morphine, chloroform, ether, chloral hydrate). Paralysis of sensation may be total, so that practically every kind of sensation is lost: or it may be partial, as when the sense of pain is abolished and the pressure sense or sensitiveness to electrical irritation is retained, or where the special senses are destroyed but the sense of pain is intact.

The results of disturbed sensation depend upon the importance of the physiological functions abolished. Anæsthesias of 
the optic system cause visual weakness (amblyopia) or complete blindness (amaurosis). Anestlesias of the autlitory nerves produce nervous difficulty of hearing (hypacusis) or complete deafness (anacusis). Loss of the sensory power of peripheral nerves is sometimes a source of danger for the tissues (for "the senses are the guardians of health"), which become conscious of every injury from the stimulus of pain and are thus able to call into action every protective means and measure the body possesses. In anesthesia of the conjunctiva the entrance of foreign bodies to the eye or conjunctival sac is not realized, and such substances are not carried off by the How of tears. Ifter section of the sensory nerves of the foot, penetration of a nail into the frog is not evinced by lameness, and the ensuing inflammation and gangrene of the hoof proceed unnoticed-conditions of serious consequence to the life of the subject. Interruption of the sensory portion of the reflex are puts an end to the tendon reflexes, which cease in anæsthesia of the peripheral nerves because the path of conduction to the reflex centre (pyramicls) is broken. If. however, the latter be intact and the communication between the brain and ganglion be destroyed the tendon reflexes are $\mathrm{cx}-$ aggerated (central inhibition may also exist). (Krehl.)

Loss of the so-called motor senses destroys the coordination of movements and causes the appearance of simptoms of vertigo ( $\mathrm{r}$. p. 42t). The sense of station of the limbs, which renders possible the acts of walking, standing and especially of orientation in space. depends upon coordination of the optic and auditory organs, and especially those portions of the latter enclosed in the petrons portion of the temporal bone, as well as of the centripetal sensations from the muscles, tendons, bones, joints, fascice and skin.

Where there is loss of a single sense, as that of sight, oricntation may be possible from increased acuteness of other senses (tactile sense, hearing) up to a certain degree Loss of sensory muscle impulses or deficient transmission of these impulses of anxsthesiat of the cerebral or spinal centres cause more or less loss of power of bodily motion. Disturbances of equtilibration of the boty occur especialiy in diseases of the organs in the semicircular canals, in the saccule and utricle, as well as of the central communications of these structures with the cerebellum. How far in these instances we have to deal with a pathological stimulatiou or paralysis, whether there exist a destructive phenomenon or one caused by deficicnt transmission of impulses, are questions difficult to decide lecause of the 
complexity of the nervous mechanism and even more because of the great variety of processes and causes possible in case of such disturbances.

Trophic, vasomotor and secretory disturbances depending upon nervous influences.-Of the vast number of cells which make up the animal body, there are, it is true, some cells, which are independent of the nervous system, acquiring their nutrition and performing their function by means of their own parenchyma alone. These are the isolated blood cells and lymph cells. All the other cells and tissues, however, in the matter of their metabolism, growth and maintenance of existence, depend upon the nervous system; and die if they be separated from it. The part played by the nerves in this connection is known as the trophic influence. This is most clearly apparent in nerve fibres themselves, which invariably degenerate if divided from their ganglia or if the latter be the seat of disease. In warm-blooded animals disintegration in the form of fatty degeneration sets in as early as from four to six days. A nerve fibre may be looked upon as the elongated process of a ganglion cell, and in this conception disintegration is easily understood when it is recalled that, as taught by Verworn and Krehl, any part of a cell which has been separated from its nucleus is sure to die. There is, it is true, a certain degree of individuality of the different segments of the nerve fibres so far as concerns their nutrition, metabolism and diseases, but permanent maintenance of their integrity is only assured by their continuity with the ganglion cells. And reversely the central portions of a neuron (that is to say, the ganglion cell) suffer if their connection with the peripheral nerves is broken; for example, after section of a nerve atrophy of the central portion also occurs, perhaps extending to the ganglion. It is recognized that the integrity of the ganglia and nerves is dependent upon the exercise of their function, that functional stimulation is essential for maintenance of their nutrition and life. Atrophy usually appears in the voluntary muscles as soon as they lose the influence of their proper ganglion cells in the anterior horns of the cord or of the equally: important cerebral parts; that is, when these become diseased or the communicating paths are interrupted or injured. The muscles, thereafter incapable of voluntary movements, are thus rendered immobile (inactive, or paralyzed), become decreased in volume from diminution in size of their individual fibres (simple atrophy, atrophy from disease), or may perhaps undergo granular and fragmentary disintegration of their contractile material (degeneration atrophy); a rapid shrinkage resulting, and transformation of the muscle into flat, 
thin bands of fibrous connective tissue. To what degree this atrophy may be due simply to the motor inactivity or is dependent upon a loss of proper nutritive influences as the principal factor is not certain. In simple disuse of muscles, as that obtaining in case of disease of the joints and bones or artificial fixation, atrophy may not appear for a long time, and the structure and electrical irritability remain unchanged, because the motionless muscle is still in connection with its unaltered ganglion cell, performs other functions as heat production and metabolisn, and continues to receive impulses of various kinds. In a neuropathic paralysis, however, the muscle no longer receives stimuli from the nerves and its metabolism no longer is as active: and atrophy may set in as early as within the first week after the nerve lesion, and may be so marked that in the course of a month the muscle may be reduced to half its former thickness. From these considerations it is evident that not mere inactivity alone, but the loss of some special trophic relation between the nerves and muscle, determine the issue.

The most common example of such neuropathic atrophy is seen in the shrinkage of the posterior crico-arytenoid muscle in laryngeal hemiplegia (laryngeal wheezing in the horse).

(For details v. Friedberger-Fröhner, Tetcrinary Pathology', Amer. Ed., IV. T. Keener \& Co., Chicago: Dexler, Die Nerienkrankhciten des Pfordes, ${ }_{1}$ sog (Denticke's Verl.); Thomassen, Monatsheft f. prakt. Ticrhcilkundc.)

In the same way in case of disease or loss of continuity of the nerves nutritional disturbances develop in the bones (rarefaction. atrophy), the salivary glands and testicles (diminution in size, degeneration of the parenchymatous cells). The effect of castration upon the development of the body and the change of physical habit is presumably also to be attributed to some trophic influence of the nervous system.

Secretory disturbances resulting from nervous lesions conform closely to the known physiology of glandular activity. It is well known that by direct or indirect stimulation of the secretory nerves of the salivary glands (facial nerve, gustatory nerve, trigeminus and branch of glosso-pharyngeal: stimulation by digitalin, pilocarpin. or inflammation) an increased, sometimes permanently increased. secretion of saliva results. Similarly a flow of tears is induced by stimulation of the lachrymal nerve, cervical sympathetic, trigeminus, and, too, reflexly by irritation of the nasal mucous memlorane.

Vasomotor disturbances (angioncuroses) manifest themselves by abnormal dilatation or constriction of the vessels, the former deter- 
mining a hyperæmia, the latter an anæmia of the organ affected. Centres for the vascular nerves are located in the floor of the fourth ventricle, in the dorsal and lumbar cord and in the ganglionic and nervous plexuses which regulate the tone or ordinary state of contraction of the arteries and the dilatation or constriction of the vessels to any calibre, and which are located about the walls of the vessels themselves. Stimulation of the fibres regarded as vasoconstrictor causes diminution of calibre, anæmia and lowered temperature of the organ involved; afterwards passing into paralysis, from which dilatation results. Paralysis of the vascular nervous centres also induces dilatation of the vessels and rapid lowering of blood pressure (section of cord, paralyzing poisons). Stimulation of the vasodilators canses marked dilatation of arteries, capillaries and veins and is induced especially by local toxic influences.

\section{Disturbances of Digestion.}

Diseases of the mouth, tongue, jaws and teeth all interfere with the taking of food and with mastication as well as the insalivation of the food within the mouth. The supply of nutritive material is thus diminished according to the grade and duration of such faults, and conditions of failure of nutrition and starvation are brought about. As the secretion of the gastric glands is in reflex associated relation with the movements of chewing so as to insure the immediate bathing of the swallowed bolus in proper juices (Pawlow), it is to be expected that in case of insufficient mastication the food taken into the stomach will be provided with a deficient amount of gastric juice and will therefore of necessity remain a longer time in the stomach.

The movements of mastication mechanically cleanse the mouth of its mucus and bacteria (immediately after prolonged chewing of food the mouth is almost aseptic): anything which causes diminution of these movements favors marked accumulation of microorganisms, as a result of which acid fermentations take place in the mouth, facilitating decay of the teeth, accumulation of tartar on the teeth, pharyngeal catarrh, etc.

In various diseases of the mouth (as foot and mouth disense), in a number of toxic disturbances (mercurialism) and nervous affections (trigeminus.), there ensues from direct or indirect nervous irritation an increased flow of saliva, the secretion dribbling from the mouth (salization, ptyalism). Dribbling of saliva may also be a symptom of paralysis of the lips. 
Diseases of the pharynx and the adjacent structures may, on the one hand, give rise to faults of obtaining food (painful inflammations), and on the other hand if swallowing is interfered with by such conditions important complications may arise as consequences. As the respiratory and alimentary canals cross in the pharynx and the epiglottal orifice must be closed in the act of swallowing and the nasal cavity must also be shut off from the mouth by the superior pharyngeal wall, there arises a danger in all affections which dininish the contractile power of the musculature of the pharyngeal walls (peripharyngeal œelema, inflammations, etc.) that particles of food and saliva may find their way into the nose or into the larynx and lungs. Sometimes the food and drink runs back (regurgitates) into the nose, or in case of pharyngeal palsy remains in the pharynx itselif; and under such circumstances drops and particles of the pharyngeal mucus and food may be drawn into the respiratory passages (aspirated). The fluids of the pharynx and mouth are apt to contain great numbers of bacteria, and these foreign agents ma: cause in the lungs the most serious inflammatory disturbances, terminating in gangrene (gangrenous inhalation pucumonia).

Diseases of the 0esophagus may cause important interference with nutrition. Both by constrictions and, too, by pathological dilatations of the gullet the food is prevented from passing regularly into the stomach. The larger fragments lodge at the place of stenosis and in any dilated parts, or in case of paralysis lie stagnant in the lumen of the tube and block the passage. This induces retching movements (antiperistaltic contractions) on the part of the cesophageal musculature, or if the cesophageal muscles are paralyzed voniting movements on the part of the stomach (pressure by the stagnant mass and by the resophagus, stuffed full like a sausage, upon the vagus nerves). Furthermore these stagnating masses of food undergo fermentation, cause injury to the mucous membrane and may in this way lead to rupture of the cesophagus; and in the latter event. from escape of septic materials, the surrounding tissue (cellular tisstie of neck, mediastinum and thoracic (avity) is apt to undergo suppuration and gangrene. In ruminants from partial or complete obstruction of the nesophagus (new growths, pressure from enlarged mediastinal lymph norles) regurgitation of the gastric contents and ertictation of gases from the stomach are interfered with, with the resultant development of chronic flatulence.

Diseases of the Stomach.-The efficiency of the gastric func- 
tion is disturbed in two ways in disease, the gastric secretion being affected in the first place and in the sccond the gastric motility being more or less affected. Usually both factors are involved at the same time, being directly or indirectly dependent upon each other. Disturbances of secretion are for the most part characterized by such features as diminution of the gastric juice, decrease in the proportion of acid (subacidity) and of pepsin resulting from degeneration of the glandular epithelium in acute and chronic catarrhs, anæmia, etc., or by continuous secretion of gastric juice without intervals oi rest (caused by local inflammatory stimulation of the nerves of the nutcous membrane). In the latter condition the secrction is apt to be poor in its hydrochloric acid and peptic enzyme, and its increased volume accounted for by hypersecretion of mucus. The secretion under such circumstances is likely to be of diminished functional value, or to be entirely incapable of digestive action, such a condition being termed dyspepsia. Although the passage of the gastric contents into the intestine is the principal factor in preventing serious prevalence of bacteria in the stomach, the acid of the gastric juice has a part in restricting the growth of bacteria and the occurrence of putrefactive changes in the viscus. A gastric juice poor in acid must be decidedly diminished in its antiseptic action, and for this reason a number of types of bacteria grow luxuriantly in the stomach in dyspeptic conditions. The gastric contents are often, in these instances, found to be neutral or alkaline in reaction, and putrefactive fermentation is present; where in spite of deficiency of hydrochloric acid an acid reaction prevails (dyspepsia acida), this is due to the formation of acetic acid (from alcohol, yeast fermentation) and lactic and butyric acids (from glucose, through the activity of bacteria).

In other cases there nay exist a hypersecretion with excessive hydrochloric acid production, or the latter (hyperacidity) may obtain without any increase in the digestive fluid (as a result of special conditions of irritation), with the effect of inhibiting starch digestion and causing gastric pain and vomiting (Krehl).

The products of fermentation and putrefaction of the gastric contents act as toxic irritants upon the mucous membrane, and may occasion further degenerative and inflammatory changes in that tissue. Under these and other circumstances, which induce (whether from circulatory disturbances or from the local lesions and toxic influences) paralysis or weakness of gastric peristalsis 
(gastric atony), the churning and mixing of the gastric contents with the secretion are not thoroughly accomplished. When the ingested matter is thus incficiently dealt with the undigested material is apt as a result to reniain stagnant in the viscus, and this in turn favors increased fermentation and putrefaction. As gases are very likely to be present among the products of these processes, flatulence of the stomach (meteorism, from $\mu \epsilon \tau \epsilon \omega \rho$ i $\xi \epsilon \nu$, to inflate) may be expected, varying in its intensity according as to whether the gas finds its way through the pylorus, is absorbed or finds an outlet through the gullet in belching, or whether it fails to escape.

Delay in emptying of the stomach or retention of the contents favors gastric dilatation. A distended stomach because of its increased volume causes pressure inflitnces upon the adjacent parts, compresses the liver, vena cava, etc., interferes with the diaphragmatic movements and thus renders respiration difficult. Even in moderate degrees of distension the absorptive powers of the gastric mucous membrane is diminished because of the tension of the lymphatics and blood ressels. The distended wall of the stomach may rupture and fatal hæmorrhage from the torn blood ressels quickly terminate life. Digestive faults in the first stomach of ruminants occur in the same general way as above, but with the difference that there is here no secretion from the gastric mucous membrane and we have to consider merely the macerating properties of the fluid swallowed, mixed with saliva. In these animals accunuliation of gas and tremendous distension invariably occur, either suddenly or gradually, when the contractile power of the wall of the paunch is lost. This may be caused by a number of influences, as chilling of the mucous surface of the paunch by frozen or rain-drenched food, overfeeding or overwork withont allowance of time for rumination, or toxic substances capable of causing paresis of the gastric wall and met in various food stuffs, as lucerne or clover. Meteorisn inay also be noticed when belching of gas is prevented by constrictions of the cesophagus.

Exaggerated gastric movennents occur especially in connection with pyloric obstruction, or from the influences of chemical materials which directly irritate the nucous membrane of the viscus or the smooth muscle of its walls, or may be caused indirectly by infuences acting on the medulla oblongata (reflexly and by irritation of the vagus). The violent contractions of the muscle 
of the organ may cause it to be rapidly emplied into the intes. tine, or, in association with the muscles of respiration (spasmorlic contraction of the muscles of the abdominal wall and diaphragm), may cause vomiting (comitus). It may be safely assumed that, as in man, more or less gastric pain is caused also in animals by varions affections. Distension of the stomach, the marked stretching of the wall, give rise to a sense of pressure; and the local effects of poisons, of increased proportions of acid, and of toxic hacterial products upon the sensory nerve fibres of the mucous membrane, and especially the occurrence of spasms of the gastric muscles, must surely often cause intense pain. It is known that in man migraine and vertigo as well as epileptiform convulsions may be of gastric origin, "apparently from vagus irritation; and some of the nervous simptom groups, as distemper of dogs, may perhaps be expletined by gastric changes.

Diseases of the Intestines. - In all intestinal diseases the micro-organisms, which teem in the contents and cause all sorts of disintegrative changes in the matcrial, play an important part. Bacteria of many types, yeasts and hypomycetes, as well as infusoria. are to be found in the intestine of the healthy human being and animal, being introduced witil the focd. Pasteur and Schottelius have pointed out that the presence of these conmensualists is an absolute necessity for the herbiverous animal, the bacterial processes being adijuvants in the digestion of the food (it has been inpossible to keep alive chicks hatched in sterile surromelings upon artificially sterilized food); although young guinea pigs have shown their alility to live and thrive on pure milk ciiet without hacteria in thcir intestine (Thierfelder and Nuttal).

The harmlessness of the ordinary intestinal bacteria is due to the fact that the liealthy epithelinm of the gastric and intestinal mucous membrane acts as an impenetrable barrier against them: the protective means possessed by these cells being apparently their strongly acid, albumen precipitating nucleinic acids (Klemperer). Again it is possible that the intestinal epithelium, as well as the rest of the tissue in every individual, may become accustomed to the toxic products of the ordinary intestinal bacteria, just as the epithelium of the stomach and upper part of the intestine is acid proof; or it may be that an immunity is obtained by the formation of antibodies. Then too the aciel gastric and small intestinal juice, as well as the bile, 
surely possess some degree of influence inhibiting the multiplication of the microphytes taken in with the foos. Marked excessive prevalenor of bacteria is, however, manly prevented by the comparatively rapid passage of the chyme through the small intestine and the discharge of large quantities of microorganisms periodically with the dejecta from the large bowel in regular defecation.

Ir conditions which interfere with the normal passage of the elyme through the intestinal tract, as in stagnation of the intestinal contents from obstruction, strictures, etc., or where the epithelium of the mucous membrane has undergone changes (degeneration, necrosis, desquamation), even the ordinary intestinal bacteria are capable of causing harm; and this is obviously possible where other types of microphytes having special toxic properties are introduced, these perhaps causing epithelial necroses or their toxic products being absorbed. We should regard, at any rate, as harmful products of ordinary intestinal bacteria the products of acid fermentation of the carbohydrates (lactic acid, butyric acid, acetic acid), these when present in large antount causing erosion of the mucous membrane (especially the delicate mucous membranes of young individuals), and gases, as hydrogen and methane and sulphuretted hydrogen, arising in albumen putrefaction.

The metabolic products of the bacteria themselves are even more numerous and their inflicuces varicd. But little is known of their relation with the pathology of the intestine, and there is so much confusion as to the nature of these sometimes poisonous substances, and their relation to the alkaloids, albuminates, enzymes, etc., tlat it is at present best to be satisfied with their tentative classification into toxines and endotoxines. We can only speak in a general way of the bacterial processes in the intestinal canal as the sonrce of pathogenic and fatal changes, and say that on the one hand they occasion local lesions iike inflammations, hamorrhages or necroses, and on the other hand give rise to general intoxications. Similar effects may be produced by the ingestion of various cliemical substances (poisons taken in with the food).

In many intestinal affections disturbances of the absorptive power of the mucous membrane ensue. This is especially true of the diffuse lesions; although in circumscribed changes the intervening portions of mucous membrane remaining unaltered 
continue to act and to transmit as usual the sugar and albumen to the blood and the fat to the chylous vessels. The causes of impairment of absorbent power lie primarily in the alterations of the intestinal epithelium, which normally acts in fact by attracting the nutrient substance to it (Krehl), and in the second place in the disturbances in the lymph follicles of the intestine; and, too, the passage of the intestinal contents may be too rapid to favor absorption, the period of contact of the nutrient material with the intestinal mucous surface being curtailed. The terms looscness of the bow'els or diarrhara are applied to the accelerated passage of the chyme through the intestine, apparent from the fluid or thin intestinal discharges. The usual cause of this condition is a heightened peristaltic action of the intestinal musculature; which may prevail throughout the intestinal tract or be restricted to the large intestine, and is due to irritation of the sensory nerve filaments in the nucous nembrane, with transmission of the impulse to the motor nerves of the intestinal muscles. According to Cohnheim extensive and general movements of the intestine can be aroused, starting from any level of the gastro-intestinal canal, by merely moderately strong irritation. Hand in hard with the increased movement hypersecretion from the mucous membrane may take place, a transudation from the blooci vessels, as another effect of local irritation. The irritant substances are of innumerable chemical types, including materials ingested from the outside of the body (drastic purgatives) or decomposition products formed in the bowel itself. According to Krehi the organic acids and gases (carbonic acid, hydrogen) are especially efficient in stimvlating intestinal movements. When the intestinal mucous membrane is already inflamed and hyperæmic even an ordinary amount of irritation is sufficient to cause increased peristalsis, as the nerves are even then subjected to pressure from the distended blood vessels and transudate (Cohnheim) or are deprived of their protective covering because of the defects in the mucous membrane. Diarrhneas may also be caused, however, by prevention of absorption of water from the intestine, which may be the case when salines are present in the gut, these substances having a strong tendency to combine with water and being themselves difficult of absorption (saline laxatives). Sudden changes of the blood currents and the blood gases, soaking or chilling the skin, or putrid blood poisoning are also factors 
which directly or indirectly are liable to cause spasmodic contractions of the intestinal muscles (dysperistalsis).

As already stated the absorption of nutritive material is disturbed in diarrhoeas; but in addition the digestive secretions and the digestible constituents of the food are carried out of the body by the frequent dejections. Besides there are losses of the epithelinm of the mucous membrane, losses of the inflammatory albuminous transudate or of blood; so that the body suffers also a loss of its circulatory and organic albumens. For these reasons diarrhoas weaken the strength of the subject, bring about a state of inanition and may perhaps be fatal. Although resorption is reduced in diarrhceas it is not entirely checked, being maintained in this or that segment of the bowel; because of this it is possible, in addition to the disturbances mentioned, that resorption of toxic substances may prevail, this feature adding complications to the morbid condition and aiding in bringing ahout a fatal termination.

There are a variety of influences which may rotard the passage of the chyme or completely stop its movement. Infrequent defecation, protracted retention of the chyme in the large gut, obstipation or constipation (constipare, to rencler compact), or simple stoppage of the bowel, is usually due to the character of the food, which may cause the chyme to be too thick because of insufficient presence of water or which does not stimulate the mucons membrane enough and causes an atony of the muscles of the intestine (from feeding bones to dogs, bran to horses). A constriction or complete closure of the intestinal lumen. stenosis, obturation or occlusion.* may be due to presence of foreign bodies (enteroliths, concretions, swallowed stones, cork stoppers, etc.), to tumors or to cicatrices. Or the intestine may be compressed or squeezed from without (strangulation of intestine, incarceration) when caught in some opening (rupture, hernia), or surrounded by some band: may be twisted (intestinal taist, voliulus) : or become impermeable because one segment becomes invaginated in another (intestinal intussusception, incagination). The obstruction of the lumen in all such cases causes a retention of the chyme with accumulation toward the stomach, and the possibility of passage of the intestina! contents, both food refuse and gases, to the anus is interrupted. The chyme stagnating above the obstruction, in which the processes of bacterial decom-

*obstruere, to barricade. stop up; occludere, to close up; obturare, to divert, according to Roth, probably frem $\theta v \rho \alpha$, door. 
position are continually going on, distcnds the intestine, particularly by gaseous inflation. If the lumen is merely narrowed and not completely obstructed and if the obstructing cause is slow in development, a compensatory hypertrophy of the musculature may ensue (v. p. 255), and for a time overcome the difficulty; the strengthened wall by its more forceful contractions succeeding in forcing the fluid chyme through the narrowed passage. Where occlusion is sudden and complcte, however, the retained contents accumulate in such amounts and the gaseous distension becomes so intense that serious symptoms and results are induced. The pressure, whether from without or from within the gut, upon the nerves of the intestinal wall and mucous membrane and their irritation by the chemical substances in the contents, cause violent contractions, spasms of the smooth muscles; which, either by anti-peristaltic movements or because of mere distension of the tube, and because from vagus irritation retching movements are set up, induce overflow and regurgitation of the

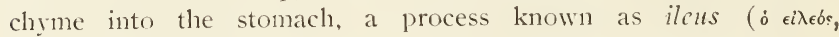
from $\epsilon i \lambda \dot{\epsilon} \omega$, I close, or $\epsilon i \lambda \epsilon^{\prime} \omega$. I turn, according to Roth) or miscrere, because of the frightful torture which it causes. The violent muscular spasms of the intestine are accompanied by severe pains, colicky pains or griping ( $\dot{\eta} \kappa \omega \lambda \iota \kappa \dot{\eta}$, sup-

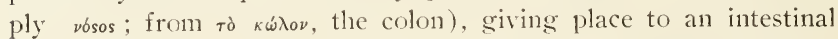
paralysis due to fatigue of the nuscular tissues and the stretching of the wall from gas accumulation. The distension as well as the spasmodic muscular contractions may lead to rupture of the intestine, along with pressure upon the neighboring organs and the large hlood ressels of the abdominal cavity. The stretching of the nerve fibres (splanchnic) with its reflex effect upon the heart, the continuous vomiting and the absorption of toxic substances, make up a group of processes which, with rapid loss of strength, cause the death of the animal. In conditions in which strangulation of the bowel causes the occlusion there are added to the above phenomena disturbances of the blood circulation in the intestine, venous congestion, congestive hremorrhages and transudates, and gangrene. Gangrene necessarily must result from the penetration of the intestinal bacteria into the tissues which are engorged with blood, without the least circulation and therefore necrosing.

Diseases of the Liver.-The manifold functions of the liver make it easily realized that any affection of this organ which is 
associated with destruction of its secretory parenchyma or with disturbances in the entrance or exit of blood, should be followed by numerous reactive effects upon the scneral body. The chemical function of the liver does not extend merely to the production of bile and furnishing of glyogen, but is directed to the production of a large number of cuzymes having for their purpose the dissolution, transformation. precipitation or complete destruction of toxic material circulating througin the blood, but brought to the liver especially from the intestine. The liver possesses, therefore, an antitoxic function, and is a toxolytic organ of the greatest inportance. According to Bangger and Zschokke the following enzymes have been isolated from the liver or bile; maltase, glykase, a proteolytic ferment (similar to trypsin), nucleinic ferment, aldehydase, lipase, fibrin ferment, a milk curdling ferment: and there have also been recognized a ferment capable of forming ammonium and urea from the amido acirls, one forming glycogen from sugar or the reverse change (of sugar from glycogen), another forming iron and bilirubin from hrmoglobin, and finally one causing the combination of cholic acid with glycocol and taurin, and of phenol with ethylsulphuric acid. A large number of alkaloids are rendered completely or partly inert by the liver. Schiff proved experimentally that a dose of nicotine, which is fatal if injected into a peripheral vein, is without effect if injected throngh the portal vein, and that an infusion of nicotine made into an emulsion with fresh liver tissue loses its toxic properties, while if rubbed up with muscle or nervous tissue quickly evinces its toxicity. In the same way strychine, cicutine, veratrine, caffeine, atropine, curare and animal toxines. extracts from putrefied tissue, have been found to be less toxic if mixed with liver tissue or injected by way of the portal vein so that they must first pass through the liver. Roger has shown that the portal blood before coming to the liver contains a considerable amount of toxic substances apparently absorhed from the intestine: the hlood of the other veins of the body, on the contrary, showing a far less toxic quality in spite of the fact that it carries with it varions metabolic prodnets from the tissues. The intestine is a chemical laboratory in which a great quantity of bodies of the lizost varied character are formerl from the combination of the digestive juices with the food: and then, too. the myriads of bacteria which inhabit the intestine give origin to products of more or less toxic nature. 
Bile, peptones, ferments, various vegetable acids, gases, the intestinal putrefaction substances, various alkaloids and proteid bodies are to be met; and are, it is true, largely carried out of the system with the feces, but to a certain degree are also absorbed by the portal venous radicles. The liver serves as a cleansing apparatus, and frees the portal blood from these harmful ingredients. As soon as the liver becomes affected by diseases which reduce its antitoxic functional ability, these substances must necessarily pass through the organ without being transformed and gain entrance to the general circulation and be changed possibly by other tissues or be eliminated by the kidneys, lungs and perspiration. The kidney is particularly capable of acting in place of the liver in purifying the blood. It has been shown that the urine in hepatic diseases and in conditions which cause an increased albumen disintegration (whether in the liver itself or elsewliere) acquires decidedly increased toxic properties and contains instead of or along with urea large amounts of ammoniacal compounds or acid bodies, as amido acids (leucin, tyrosin), aromatic oxyacirls, acetone and albumoses. Such materials are, however, injurious to the kidneys and for this reason disease of the liver is frequently complicated by pathological changes in the kidneys. In case of failure of the compensatory function of the kidneys, and especially if poisonous substances no longer neutralized by the liver parenchyma are passing into the blood, there may ensue symptoms of general intoxication acting particularly upon the central nervous system. These phenomena may be met in varying grades of severity, as convulsive or stuporous conditions of acute or protracted course, according to the extent of destruction of liver cells, the increase of albuminous disintegration in the liver and the access of infections factors. The most important poisonous material which occurs in case of destruction of the hepatic function concerned in the transformation of albuminates into urea and uric acid, is carbamic acid: the entrance of this material into the blood, as seen in animals deprived of the liver or having an Eck's fistula (Minkowski*), causes very intense cerebral disturbances.

Stagnation of bile, besides causing biliary staining of the liver and the rest of the body structures, the so-called ictcrus (v. p. 212), is also responsible for cerebral toxic symptoms (spasms and stupor), because of complex conditions of intoxication simi-

* For details see Kitt, Pathol. Anatomie der Hausticre, II Aufl. 1 Bd., p. 595. 
lar to the above. The eholic acid especially is toxic to the nervous structures, but the biliary pigments are not entirely inert; and hemolysis also plays a part (cholcmia). Obstruction of bile from the intestise and, too, diminished or completely checked production of bile (oligocholia, acholia), because of degeneration of the hepatic parenchyma, disturb the formation of the chyme; absorbtion of fat is made difficult in the absence of bile and the loss of the antiseptic influence of bile may cause intestinal catarrh from putrefaction of the ingesta.

Hepatic diseases also affect the nutrition of the tissues, from bringing about changes in the character of the blood. The liver is the storehouse for the sugar of the blood (E. Toit), which it elaborates from the material brought to it from the intestine by the portal vein; it stores up fat, and consumes the debris of broken down corpuscles; and in ease of loss of these functions the body must suffer from the abnormalities in some of its important sources of energy. Further there may be mentioned alterations which are brought about by mechanical interference with the blood circulation, as in diffuse induration of the liver; all diseases which tend to occlude the blood capillaries and cause compression and narrowing of the innumerable intrahepatic ranifications of the portal vein bring about passive congestion of the organs in which the portal vein originates, the stomach, intestine, spleen, and the visceral portion of the peritoneum. From this condition, dropsical transudation, ascites, is apt to develop.

Diseases of the Pancreas.-Diseases of this organ causing atrophic changes of the glandular parenchyma, render the formation and discharge (into the intestine) of its secretion impossible, have an important influence upon digestion of proteids and absorbtion of fats, may be followed by diabetes, and lead to serious and progressive emaciation. Especially in case of the horse, an animal possessing a relatively small stomach and only a short period of gastric digestion, the loss of the pancreatic function eannot be compensated for by other glands and gives origin to a fatal eachexia (Siedamgrotzky). Observations on dogs, cats, hogs, rabbits, pigeons, birds of prey, after extirpation of the gland or restriction of its secretion from the intestine by ligature of the duct, have shown that in case of failure of the pancreatic juice fats are no longer absorbed (only in natural emulsion is fat absorbed to the extent of thirty to forty per cent.: all the neutral fat reappears in the feces: and only part of the fats is 
split) ; only about forty-four per cent. of the proteids are absorbed (Abelmann, Krehl), and sixty to eighty per cent. of the carbohydrates. The latter and the sugar formed within the body from the albumens are excreted uncharged in the urine (Minkowski). The diabetes thus produced varies in its severity according as to whether the gland has been completely or only partially removed in operation, whether it is generally involved by disease, or whether portions of the gland remain in functionating condition. In dogs and cats after total ablation of the organ, the urine usually contains as high as eight to ten per cent. of sugar; and the animal may be expected to die from the progressive cachexia in the course of fifteen to twenty days. Diabetes fails, if, in experimental removal, a sufficient amount of the gland is left or transplanted under the skin; and occlusion of the duct (ligature, obstruction by calculi) does not invariably cause diabetes, as the secretory substance may then be absorbed through the lymphatics and be conveyed to the blood. The active agent involved is believed to be a ferment whose function is the destruction of the sugar in the blood; if this be wanting the sugar necessarily accumulates in the blood and passes unchanged into the urine. It is of further interest to recall that in diffuse carcinomatosis of the pancreas diabetes may similarly be absent, apparently because the tumor cells, as the offspring of the secreting gland cells, are capable of carrying on the function of the latter to an extent sufficient for the metabolic demands in this direction.

The effects of failure of the pancreatic juice to pass into the intestine may, in normal conditions of the intestine, be practically compensated (not in the horse--v. supra) by the fat-splitting power of the bile and the intestinal bacteria, the latter causing proteid putrefaction at the same time.

\section{Disturbances of Respiration.}

The constant intake of oxygen and output of carbonic acid gas is the first vital requirement of the cells of the animal body. In fœtal life this interchange of gases is performed by means of the placental circulation, in post-natal life by the entrance of atmospheric air to the respiratory surfaces of the lungs; and the red blood corpuscles act as essential agents in causing the interchange. The inspiration of the atmospheric air and the output of that which has been rendered unfit, constituting what is known as the crtcrnal respiration, may be impaired by closure 
and constriction of the respiratory passages, diminution of the respiratory surfaces of the lungs, and by any interference with the movements of the respiratory muscles. In conditions of obstruction of the nasal passages (swelling of the nasal mucous membrane, tumors), breathing may for the time be still accomplished through the mouth; obstruction of the larnyx and tra.chea with its branches, by foreign bodies or tumors in the lumen, spasm of the glottis, external pressure (thyroid tumors, œsophageal diverticula, strangulation), or collection of exudates in the tubes, causes difficulty of respiration (dyspnoa, air hunger) in proportion to the extent of the obstruction. Proportionately to the degree of diminution of entrance of air to the lungs the blood becomes impoverished in oxygen and its carbonic acid content increases. Lack of oxygen and excess of carbonic acid in the blood act to stimulate the respiratory centre in the medulla oblongata (point of exit of the vagus); this stimulation, and probably the mechanical influence of incomplete filling of the pulmonary alveoli, which directly affects the fibres of the vagus, bring about an increased contraction of the inspiratory muscles. Inspiration becomes stronger, more powerful, deeper and longer in duration, because the narrowed lumen of the tubes does not admit the external air to pass readily into the alveoli and the demand for oxygen in the medulla oblongata is not at once satisfied (Krehl). The air becomes rarified in the lung and the intra-alveolar pressure less than the external pressure upon the outside of the body; and for this reason inspiratory retractions of the intercostal tissues may be noted. Because the air cannot pass out through the narrowed portions of the tract with the usual ease, the lung does not collapse as rapidly as normally and expiration is also prolonged (Krehl).

Contraction of the diaphragm is the main factor of the inspiratory forces, the contraction of this broad muscular sheet, arching anteriorly because of the pressure of the abdominal viscera, causes it to assume a flatter plane and thus increases the thoracic space, the elevation of the chest wall, especially the lower ribs (by the leiator costarum muscles) aiding in producing this effect. Expiration is due to cessation of the diaphragmatic contraction (termination of its tension) and that of the levator muscles, as well as to the elastic contraction of the lung expanded in inspiration. Ruptures of the diaphragm (excluding here the question of hrmorrhage from the ruptured blood vessels and hernia of 
the abdominal viscera into the thoracic cavity) usually rapidly cause death because of the resulting impossibility of breathing. Difficulty of respiration is similarly occasioned by embarrassment of and pressure upon the diaphragm by meteorism of the abdominal organs, tumors, etc., where the alteration of contracting movements and resting stages is lost in consequence of tetanic spasm of the thoracic muscles and diaphragm. When air gains entrance to one of the pleural spaces because of a wound of the thoracic wall or rupture of the lung (perforating wounds, rupture of suppurating foci), the lung affected contracts from the action of its elastic tissue and, because being no longer impervious to air, it must adapt itself to the intrathoracic conditions (the mediastinum is pressed toward the opposite side by the entering air and may even be ruptured); expansion of the lung is, therefore, no longer possible, as the organ cannot follow the movements of the chest wall and lies loosely (that is, fixed only at its root) in the chest cavity. The thorax and lungs cannot properly expand and collapse if the chest cavities are occupied by fluid exudates (dropsy, pleurisy), or if the respiratory surfaces of the lungs are lessened, if a greater or less portion of the pulmonary alveoli are filled up or solidified by pressure of collections of exuded material, tumors or parasites and thus deprived of their air. However, even on the contrary if the lungs are excessively inflated, overdistended, a condition known as emplyysema, aeration of the organs and interclange of gases with the blood are impaired; the lung not collapsing properly in this condition, the air escaping with difficulty (the carbonic acid is unable to pass out by diffusion to a sufficient (legree), and the capillaries narrowed by being stretched and not permitting the blood to flow readily through their lumina. Under any such condition breathing becomes labored and air hunger becomes apparent. In addition circulatory disturbances are induced, sometimes due to pressure upon the thin walled veins by accumulations of fluid in the chest cavity and by the uninterrupted enlargement of volume of the lungs, this causing congestion in practically all of the veins (v. dropsy); the factor of aspiration of venous blood fails; and as terminal Ceatures difficulties of discharge of the blood from the right heart grow apace (resistance in pulmonary arterial area), if the vascular distribution in the lungs be embarrassed by pressure or obstructive infuences (secondary cardiac hypertrophy and dilatation). 
In some affections the respiratory movements are accelerated and more or less superficial. Increased frequence of respiration is met particularly in febrile affections and inflammatory affections involving the bronchioles. The causes underlying rapid and at the same time superficial breathing are not understood; it is supposed that the responsible infuences are related with the increased internal temperature or some irritation of the sensory vagus filaments. Respiratory disturbances may also be brought about by cerebral lesions. Affections of the nervous substance of the brain which do not induce paralysis of the medulla oblongata, accumulation of fluid, parasites, hemorrhages or toxic and infectious irritants, may cause an increase of intra-cerebral pressure, this apparently disturbing the stimulation of the respiratory centre, respiration becoming slowed even though the air is not in any way obstructed in its passage to the alveoli.

When the inspired air contains poisonous gases, it is to be expected that according to the cliaracter of these substances and their quantitative admixture in the atmosphere there will be manifested either violent coughing or suffocative symptoms (ammonia for example) : and inflammatory local disturbances will be induced (inhalation of formol vapor); or that some blood change (as in carbon monoxide poisoning) wiil be brought about by limitation of the atmospheric oxygen and alteration of the hrmoglobin.

The respiratory variations are the result of regulative processes set into activity automatically and reflexly by the respiratory centre. Deeper or accelerated respiratory movements may. to a greater or less degree, compensate for faults of gaseous interchange of the blood and the tissues. With cessation of the fault, as by removal of an obstruction to the entrance of air to the lungs. and coincidently with the moment when the medulla oblongata again receives a proper amount of oxygenated blood, the special stimulation of the centre ends and the respiratory movements again become normal. Where the causes, however, continue operative and become more marked, and even the excited respiratory movements finally become incapable of relieving the air hunger of the tissues, asphyria (suffocation) must ensue. Is long as the brain retains its normal irritability the labored and deep respiratory efforts indicate medullary irritation, and in the latter case the rate of cardiac action is also slowed from the marked irritation of the vagus, and the cutaneous vessels are dilated because of irritation of the vasomotor center (Krehl): after a time tonic- 
clonic contulsions occur and death follows after a short period of paralysis (Krehl). Where the irritability of the central nervous system is diminished (hæmorrhages into the brain, increased intracranial pressure, toxic or infectious influences) the symptoms caused by diminution in oxygen supply are less violent; the gradual and progressive increase of carbon dioxide produces a cerebral narcosis, the respiratory movements become gradually weaker and life ebbs away witi the advancing paralysis of the central nervous organs (Krehl).

The respiratory tubes are provided with a number of protective mechanisms, which serve to remove foreign particles which have gotten into the air passages. The passage to the trachea and lungs leads over an area, the naso-pharynx, richly inhabited by bacteria and often the point of lodgment of foreign bodies; the narrow laryngeal opening is the threshold separating this septic territory from the tracheal system. Of course, the larger foreign particles which get into the nose and pharynx and which irritate the nerves of the mucous surface and thus induce sneezing and snorting, are from time to time discharged, and the entrances for air are kept free for the passage of air; but motile virulent organisms may easily pass in, and aspiration of small drops of septic nucus and saliva or even food particles may readily occur. In this case (these substances having passed into the trachea and bronchi) the continued activity of the ciliated cells serves to remove these forcign particles, carrying them back to the trachea and larynx. The mucus secreted over the whole surface of the tubes serves to envelop the foreign elements, and, too, dilutes the poisonous substances and renders them inert. Noreover, the numerous depots of lymphoid cells may make an efficient phagocytosis possible and thus facilitate the removal of very minute foreign particles. In addition the reflex production of coughing, which forcibly discharges foreign objects, the collections of mucus to which they are adherent and any other injurious contents of the trachea, is manifested and acts to cleanse the upper portion of the tubes. Excitation of cough is principally induced by local irritation of the sensory vagus fibres in the larynx and trachea, and may also originate in the pleura, a special group of movements of the respiratory muscles being excited reflexly through the medulla oblongata. Cough begins with a deep inspiration, followed by a forcible expiration; the air under high pressure in the lungs forces its way out through the opening 
of the opposing rima glottidis and drives everything in the larynx and trachea (to a certain extent, too, that which is in the pharynx) out into the mouth (Krehl). Foreign bodies, exudates, etc., lodged below the bifurcation of the wind pipe may also at times be carried out; but there is a chance that objects in this position may be driven deeper into the bronchial tubes (Krehl). Expectoration, removal of such substances from the chest (or better, the lungs) is accomplished primarily by the ciliated epithelium and the upward convection, by this means, of the mucous vehicle; the stimulus to congh acting ipon the expectorate (sputum) only from the level of the bifurcation upwards.

These defensive mechanisms may be of no efficience if the harmfu! chemical, bacterial or mechanical agents are of such a character that they paralyze and destroy the epithelial cells and their ciliary action, and the phagocytes or the smooth muscles of the bronchial tubes, or if the foreign bodies are held firmly because of swelling of the bronchial mucous membrane, or if the obstruction be tightly fixed because of its size; and may be ineffective because of lesion or disease of the motor or sensory apparatus involved in coughing movements. The retention of foreign bodies and pathological secretions may be followed by asphyxia, because of the obstruction to the air passages; or may be the cause of such serious effects as gangrene of the lung, etc., as the obstructing substances usually carry bacteria with them. (Under normal conditions the pulmonary alveoli, the bronchial tubes and trachea are sterile, because in the first place the bacteria entering with the air lodge on the mucous membrane of the nose and pharynx. where the current of air first strikes at an angle and sweeps over the moist surface, and in the second place because they are carried up and out by the activity of the ciliated cells.)

The act of coughing may itself be dangerous. In conditions which increase the irritability of the respiratory mucous membrane or of the pleura (inflammations) frequent coughing is excited without there being anything requiring removal in the air passages. The violent expiratory force of coughing raises the intra-pulmonary air pressure and may thus cause pulmonary dis. tension (emphysema) : it interferes with the return flow of venous blood into the thorax and heart. to the extent that the latter may be practically empty, raises the arterial pressure all over the body, and may cause rupture of the vessels (Krehl). The 
violent respiratory paroxysms by increasing the abdominal pressure may force the abdominal viscera into abnormally or normally existing openings and thus cause displacements [hernia].

\section{Disturbances of the Urinary Excretion.}

The excretion from the blood of material no longer useful to the system through the kidneys, is subject to pathological faults if the quantity and quality of the blood passing through these organs are seriously changed, and if the renal parenchyma is the seat of pathological lesions. Increase in the quantity of the urine (polyuria), especially of the proportion of water in it, is met when the quantity of blood passing through the kidneys is greater than usual, that is, where arterial pressure is increased without any narrowing of the renal vessels, or where with the ordinary blood pressure the renal vessels are dilated. A more or less marked polyuria is met in a nun:ber of the acute and most of the chronic inflammations of the kidneys, and, too, after administration of diuretic drugs, in conrection with certain intoxications (mouldy oats, cantharides, colchicum, oil of turpentine), after resorption of transudates and exudates, in the stage of crisis in rebrile infections disease and in the conditions spoken of as diabetes insipicus and diabetes mellitus. It may be readily understood that to a certain degree this phenomenon is due to chemical stimulation of the renal parenchyma with secondarily increased permeability of the dilated blood vessels, or to increased secretory action of the vascular endothlium and renal epithelium; on the other hand the causes of the increase of blood pressure, apart from increased ingestion of fluids, are as yet by no means clear. Cardiac hypertrophy, vaso-motor disturbances originating in the medulla, and paralysis of the renal nerves are worthy of consideration in this relation. The causative factors underlying the large output of urine occurring in so-called urinary fux or diabetes isipidus (oraßaivw, to pass through: insipidus, tasteless-in distinction from diabetes mellitus) are equally obscure.

Better information obtains in regard to the canses of dimint1tion of the quantity of urine (oliguria) and of total suppression of urine (anuria). It must be obvious that all conditions in which the urinary passages are obstructed, as the urethra and ureters, by calculi, tumors, strictures, occlusion of the collecting tubules of the renal papilla by the shrinkage of the connective tissue about them or the retention of urinary casts, plugs of 
exuclate, etc., must offer a resistance to the outflow of urine; and any severe renal inflammations in which the epithelial cells become degenerated must reduce or stop entirely the secretory power of the organ. In the first group the urine is danmed back and pressure atrophy of the renal parenchyma takes place, the cortical portion continuing to secrete for a time and urine being unable to escape; and in the other type of cases the passages are open, but the original secretory disturbance determines a diminished production and a pathological composition of the urine. In case of unilateral disease such faults may be compensated by the opposite organ which remains functionally capable, which receives a larger proportion of the substance requiring excretion in the urine, functionates more freely and becomes hypertrophied. The secretion of urine must decrease also in conditions in which a diminished amount of blood flows into the kidneys as the result of narrowing of their blood vessels, as from shrinkage of the renal capillaries and atrophy of the glomeruli (in chronic interstitial nephritis), from thrombosis of the renal arteries, or notably from lowering of blood pressure as in cardiac failure and a number of the above mentioned conditions. In the same way a reduction in the output of urine takes place when large quantities of fluid escape from the body by other routes or where the fluid is kept back in the tissues as in fevers, diarrhœeas, or formation of fluid exudates and transudates in the body cavities.

One of the most important pathological phenomena is the excretion of albumen with the urine, albuminuria. In normal conditions, at least in states in which it is impossible to clearly distinguish between normal and pathological conditions, it is true there is a very small quantity of the blood albumen passed through the kidneys, so small that it can only be recognized in Durposefully concentrated urine and by special methods of determination, as after fatigue from physical exertion (so-called páysiological and accidental albuminurias). When the amount of albumen passed in the nriile beconies sufficient to be recognized by the ordinary albumen reagents, there is reason to apprehend the pathological disturbance of the renal function. This may be of a quickly transitory nature, of no particnlar importance and indistinguishable from the above mentioned non-pathogenic symptomatic conditions; but as a rule it is an indication of some lesion of the renal parenchyma.

All sorts of poisonous substances, both those of external 
source (metallic salts, ethereal oils, etc.), and especially the toxines of infectious diseases and the products of intracorporeal tissue disintegration and metabolism, may give rise to lesions of the renal epithelial cells and their basement membranes, making it possible for the albumen of the blood to pass through them. In most cases an inflammation of the organ is also present, this factor introducing additional features of increased permeability of the blood ressels, exudation, desquamation of the epithelium and the formation of albuminous casts. blood casts, etc. Some authors look upon every albuminuria as the result of an exudative nephritis of some degree oi severity, now unimportant, now serious.

(For fuller details v. Friedberger-Fröhner, Lelhrbuch d. klin. Untcrsuchungsmethoden. Verlag von F. Enke, Stuttgart, III. Auff.)

Another important symptom, often met in man, but uncommon in animals, is the excretion of sugar in the urine. Although the blood contains a considerable amount of sugar (as much as 0.2 per cent.). only minute traces pass into the urine normally: In conditions in which unusual amounts of sugar are brought into the blood (alimentary hyperglycamia), and the liver and muscles are no longer able to use it up, the kidneys excrete the excess (glycosuria): milk sugar passes most readily and in order thereafter levulose. cane sugar and grape sugar (Krehl). It was discovered by Mering that the administration of the glucoside phloridzin, which contains about forty per cent. of sugar, is followed by a glycosuria in which not only does a considerable amount of the unchanged glucoside pass off in the urine. but also sugar from the blood (dextrose) and from the liver (glycogen), probably because of some secondary toxic lesion of the renal epithelial cells.

A transitory glycosuria, ending in the course of few hours, occurs after puncture of Clande Bernard's glycosuric point, a procedure consisting in making a fine puncture in a certain spot in the floor of the fourth ventricle of the brain. A similar glycosuria may also be observed after lesions in other parts of the central nervous system and in a number of intoxications and infectious diseases (as in morphine poisoning, curare poisoning and rabies). If the splanchnic be cut at the same time, or the liver be extirpated, or if fasting animals are employed, the glycosuric puncture does not succeed or is uncertain, and therefore the 
quantity of glycogen in the liver has something to do with the glycosuria; the precise character of the nervous infuence is, however, unknown.

Where the excretion is a persistent and, too, a progressive symptom, provided no unusual quantities of sugar are ingested with the food, there may be said to exist a special disturbance of metabolism, diabetes mellitus ( $m e l$, honey; sıaßaivw, to pass through). In this affection even the ingestion of starch causes the appearance of grape sugar in the urine, so markedly, in fact, that the greatest portion of the starch instead of being assimilated passes off as sugar; and the urine only remains free from sugar if carbohydrates are entirely excluded from the food (Krehl and others). While in the milder cases of this disease the amount of proteid ingested has no infuence upon the glycosuria, although the sugar is formed normally from proteid also, and while in these cases an increased supply of albuminous diet seems to be quite advantageous to the nutrition of the body, there are cases of a severe type in which even a strict meat diet and even abstinence from all food do not permit the urinc to become free from sugar. Of what nature the metabolic disturbances may be which are responsible for the imperfect manner in which the carbohydrates and the glycogen formed from proteid are dealt with is still rather obscure and confused (cf. Krehl). In a measure the liver normally prevents the direct passage of carbohydrates into the circulation and has the power of removing sugar from the portal blood and of storing glycogen. Diseases of the liver affecting this function permit a hyperglycæmia to develop; (according to Bimes it ought to be possible to recognize the existence of hepatic diseace by feeding molasses, as in case the liver were normal the urine would remain free from sugar while otherwise glucose would become demonstrable in it). Especial information has been obtained from the excellent investigations of Mering and Minkowski in relation to the pancreas, which have shown that diabetes mellitus occurs when this gland is lost (extirpation degeneration) and that either the pancreatic juice or the glandular parenchyma, the blood from which passes to the liver by the portal vein, has in common with the liver a regulative influence upon the amount of sugar in the blood or some power of fixation of excesses of sigar (v. p. 448). TThe generally favored view at present among the profession would refer the pancreatic fault to some disease of the islands of Langerhans (Opie). It is be- 
lieved that these special structures secrete and give into the blood of the pancreatic vein a substance which is either itself a glycolytic ferment or which is not a ferment, but a more simple substance acting to energize a glycolytic ferment in the muscles and other tissues of the body. This glycolytic ferment is regarded as essential to the final transformations and chemical decomposition of the sugar in the system in order that it may be assimilater: in its absence assimilation 'is impossible and the body suffers by its inability to make use of the carbohydrates furnished to it, and at the same time the sugar in the blood accumulates and when in excess (above o.t per cent.) is excreted in the urine.]

In its chronic form diabetes, an expression of metabolic fault and at times accompanied by faults of the liver and pancreas, canses more or less harm to the whole body. When the incomplete assimilation of carbohydrates is not compensated by the supply of albuminates and fat, especially when the associated diseases do not pernit the assinilation of these last named substances, the general 111trition nutust of necessity fail. In serious cases there is apt to be present, as well, a marked proteid disintegration, and albuminuria appears as a symptom, together with degeneration of the lens and retina, disease of the blood ressel walls and a weakened resistive state of the tissues, which induces 2. special predisposition to tuberculosis and gangrene (in man).

Disturbances of micturition include difficult micturition (dysuria) caused by strictures and obstructions to the path of outflow (the dribbling forced passage, with tenesmus and frequent call to urinate, is called strangury) : complete repression of micturition (ischuria, retention of urine), in which case the bladder is full because of spasm of the sphincter or paralysis of the detrusor musculature; and inability to retain the urine (cnurcsis, incontincntia urinc), due to a paralysis of the sphincter of the bladder developing in disease of the spiral cord and of the bladder itself. dcn.)

(For details cf. Friedberger-Fröhner, Klinische Untersuchungsmetho-

Diseases of the urinary apparatus are more or less painful: affections of the passages being particularly apt to cause intense pains, to be attributed in part to muscle spasms, partly to mechanical irritation (foreign bodies) of the sensory filaments of the mucous membrane or to specially induced irritability (uicers, abrasions, inflammations) of these filaments. 
The effect of disturbances of the uropoictic system upon the general body depends upon the extent and the kind of the changes. All the bacterial processes in the kidneys and in the collecting receptacles (bladder, renal pclves) as well as the damming back of the urine in the ureters bilaterally, sooner or later bring about symptoms of intoxication, manifesting themselves especially by symptoms of cerebral depression, less frequently cerebral excitation, included collectively under the term uremia. This is partly referable to the impaired excretion from the blood of subsiances no longer of use in the economy and having toxic influences when retained, as the products of proteid decomposition particulanly; and the kidney is itself a place in which special metabolic processes are carried on, the products of which, as hippuric acid (Bunge and Schniedeberg), if retained may apparently take part in the production of urenia. The regulation of the proportion of water in the blood is apt to be disturbed in chronic renal affections: and, too, because of shrinkage of the comparatively large capillary area of the kidneys, a certain anount of resistance is likely to develop to the arterial blood flow; in these chronic affections, therefore, hydremia, cardiac hypertrophy and general dropsy may be cxpected as secondary features.

\section{Disturbances of the Thyroid Function.}

Destruction of the thyroid tissue by atrophy, degenerative processes or heterologous tumors, involves the loss of an organic function of vital importance; disturbances of nutrition, loss of physical strength and spasmodic attacks being induced, indicative of a general autointoxication of the body and leading to a fatal termination. In case of total extirpation of the thyroid gland [including the parathyroids] intense nervous symptoms (delirium, tetanic convulsions, and so-called tetany) quickly appear, as observed in man. Dogs usually die after such operations in from three to twenty-eight days and, too, under circumstances where injury to nerves, etc., cannot be held responsible. After removal of large portions of the thyroid there is also danger of autointoxication. Where the symptoms develop slowly, as is particularly true in pathological atrophy of the glandular tissue, the resultant condition is spoken of as cachetia strumipriza or athyrea; and besides the nervous symptoms there may be observed spasmodic 
dyspnœa, disturbances of growth, dilution of the blood and cutarieous changes (falling out of hair, thickening and mucoid degeneration of the subcutaneous tissue-my.rodcma atrophicum, strumosum, operatizum). Practically the same results are obtained from experiments on monkeys, sheep, goats, hogs and dogs as are observed in man after goitre operations (Hofmeister and Eiselberg).

[The acute results mentioned, tetany, dyspnœa, convulsions, palsies and death in a few days after ablation, are now generally attributed to loss of the parathyroid glandules; removal of the thyroid alone is not essentially fatal, and is followed by chronic metabolic disturbances in aninals comparable to myxœema in man. The precise interrelation which probably exists between the thyroid and parathyroids is not understood.]

It has been assumed (Rogowitsch) that the thyroid gland has as its function the removal or net:tralization of metaboiic products the retention of which in the system would produce toxic effects, that the organ probably, therefore, elaborates a secretory substance essential to the regulation of metabolism, which is apparently carried into the blood from the follicles by way of the extremely delicate lymph channels (Lubarsch). This assumption is founded upon the fact that a fatal result of operative removal may be avoided by removing only one lobe of the gland and by leaving some remnant of thyroid tissue or by favoring restoration of the tissue by artificial means (transplantation of thyroid, injection of thyroid extract), or if the parathyroids remain and take up a vicarious [?] activity. It seems probable that the hypophysis is able in some degree to compensate for loss of the thyroid by vicarious hypertrophy; at least in thyroidectomized rabbits enlargements of the pituitary body have been observed (Rogowitsch, Stieda, Hofmeister).

In connection with enlargements of the thyroid (struma hyperplastica and, too, carcinosa) a type of intoxication has been observed, apparently the very opposite to that following extirpation of the organ, the so-called Basedoz's disease (hyperthyrea, dysthyre( ) : this condition is very like the symptoms of intoxication which occur both in heaithy human beings and animals after large doses of thyroid extract, as accelerated and energetic cardiac action (tachycardia), cardiac dilatation and cardiac hypertrophy, epileptiform seizures, prominence of the eyeballs (goggle- 
eyes, exophthalnus), and various results of these disturbances. The condition apparently depends, as pointed out by Möbius, Greenfield, Rehn and others, upon a functional disturbance of the organ which occasions either a quantitative or qualitative change in the secretion (an excess of the sccretion or one in which there is a chemical change); but there are numerous uncertainties in relation to these points, as for example, the failure of a case of Basedow's disease to recover after extirpation or atrophy of the gland (Libarsch). Jewsejenko claims to have met this last disease also in animals (dog, horse); Reynard and Rongieux have observed stupidity in dogs and horses after development of goitre.

Cretinism and chondrodystrophy have been regarded as dependent upon the thyroid function, but according to Lubarsch's studies there is apparently no retiological relationship.

\section{Disturbances of the Sexual Function.}

Diseases and congenital anomalies of the sexual glands render the individual impotent to produce viable offspring, in males preventing formation of semen or procreation (impotence, impotentia generandi ct cocundi), in female animals interfering with ovulation, fertilization of the ovum and maturation of the embryo (barrenness, stcrility). Even general diseases (as fever, various painful affections of the muscles and joints, diseases of the spinal cord) may indirectly influence the sexual function (loss of excitability, mechanical interferences). According to Zschokke even if only one ovary is diseased there is, as a rule, a disturbance of the general sexual vitality. A number of affections of the sexual glands, as tuberculosis of the cvaries, chronic inflammation or cystic changes of these organs, or undescended testicle, are apt to be accompanied by special excitability of the sexual appetite (constant rutting, bullir.g, horsing, nymphomania, satyriasis), sometimes with violence of temper. In other cases, however, destruction of the sexual glands by disease or removal by castration is followed by suppression of sexual vitality, alterations in the state of nutrition, and changes in the bodily structure (change in the type of horns in oxen, loss of antlers in stag and roe-deer, shape of body approximating female type, fat accumulation, gentle disposition in castrated male animals). 1900.)

(For details cf. Zschokke, ,Dic Unfruchtbarkeit des Rindes, Zurich, 
Local abnormalities of the uterus may, in a mechanical way, determine the development of embryonal monstrosities or the death and premature expulsion of the fotus (miscarriage, abortion).

In cows and goats, less frequently in hogs, a form of paralysis not infrequently appears within the first few days after parturition, at the time of establishment of lactation, the so-called paralytic calf-fecer or paralysis of parturition. The cause of the condition, as Schmidt (Kolding) has pointed out, depends on an autointoxication related with the formation of colostrum. The colostrum milk is very rich in disintegrating epithelium of the mammary gland and apparently contains decomposition products from these elements, which, if absorbed in large amounts, are capable of giving rise to toxic effects. The condition is met especially in animals producing large amounts of milk, in case of unusually marked colostrum fornation. The excellent results of therapentic measures directed to reduce the milk secretion and causing oxidation of the poisonous products by introducing oxygen into the udder and thus rendering them inert, permit the acceptance of Schmidt's theory as well founded.

\section{Disturbances of the Cutaneous Function.}

The skin, with its epidermal covering, is an organ of defense to the body against the harmful influences of the exterior. Any traumatic denudation of the papillary layer or deeper wound destroys the protection it affords, and not only exposes the denuded surface to the drying influences of the air, but serves as a focus of entrance of infectious agents as well, since the injury opens easy access into the lymphatic paths. Long continued soaking of the epiderm causes its maceration (by nasal discharge, urine, etc.), continuous pressure leads to callosites or by contusion at places which overlie bony prominences to ruptures of vessels, hæmorrhages, and in this way to disturbances of nutrition which may cause necrosis of the skin (decubitus, decubital gangrene). The loss of albuminates occasioned by leakage of lymph in case of denudation of the epiderm (running sores of the skin and mouth) may cause material deterioration of the state of nutrition of the body (Landois).

To what extent disturbances may be caused by suppression of the cutaneous interchange of gases (output of water and car- 
bonic acid gas in the perspiration) we have but little knowledge. According to Ellenberger in horses, which were shaved and then covered with a coat of varnish, there appeared slowing of respiration, acceleration of pulse, a fall of the body temperature of one to one and a half degrees centigrade, symptoms of uneasiness, muscular twitchings, polyuria and increased excretions of urea, these symptoms soon disappearing; the animals took their food without being at all urged and at the end of the experiment showed a greater body weight than at the start. Hogs and dogs, after being varnished, showed 110 disturbances of health beyond a depression of the body temperature (cited from Schindelka). Sheep, however, after being coated with varnish, apparently suffer considerably (Ellenberger), and rabbits die if no more than oneeighth of their body surface be covered with varnish, apparently because of excessive heat dissipation (Landois, Fourcault, Becquerel and Brechet); in case the whole surface of the body is varnished the temperature at once drops (to $19^{\circ} \mathrm{C}$.).

For consideration of chilling of the skin, cf. p. 45 .

Because of the vast numbers of sensory nerves ending in the skin the least denudation of the papillary layer and inflammatory swelling of the cutis, as well as contact with foreign bodies, oceasion marked sensations of pain.

Pathological increase of perspiration, hyperidrosis (iopów, to sweat). may be met in a great variety of conditions which give rise to an excited state of the perspiratory centres in the medulla oblongata and cord (excess of carbon dioxide in the blood, deficiency of oxygen, sensory and psychic stimulation). Sweating may be general or local, sometimes unilateral, or limited by a transverse border strictly to the posterior portion of the body. In these latter examples disturbances in the nervous distribution are ustally at the bottom of the trouble; the abnormality is especially striking in dogs which do not sweat normally.

Diminished secretion from the glands allied to sweat glands, which are met in the muzzles of the ruminants, the snout in pigs and nose in dogs, as a result of which these parts, otherwise always moist, come to feel diry, is a marked symptom in all febrile diseases.

In case of the entrance of urine into the peritoneal cavity after rupture of the bladder, or in urinaty infiltration of the cellular tissue after rupture of the urethra, the perspiration may take 
on a urine-like odor; and in distemper in dogs and in the pock of sheep the skin gives off an unpleasant, offensive odor.

A rough, bristling appearance of the hair, brittle, dry wool, is a symptom of chronic nutritive faults; the same may be said of diminution of the subcutaneous fat, when it becames tight, inelastic and adherent to the underlying fascir (so-called hidebinding of the skin).

(For details v. Friedberger-Fröhner, Lehrbuch d. klinisch. Untersuchungsmethoden f. Tierarate, Verl. von Ferd. Enke, Stuttgart, III. Aull.) 
1.

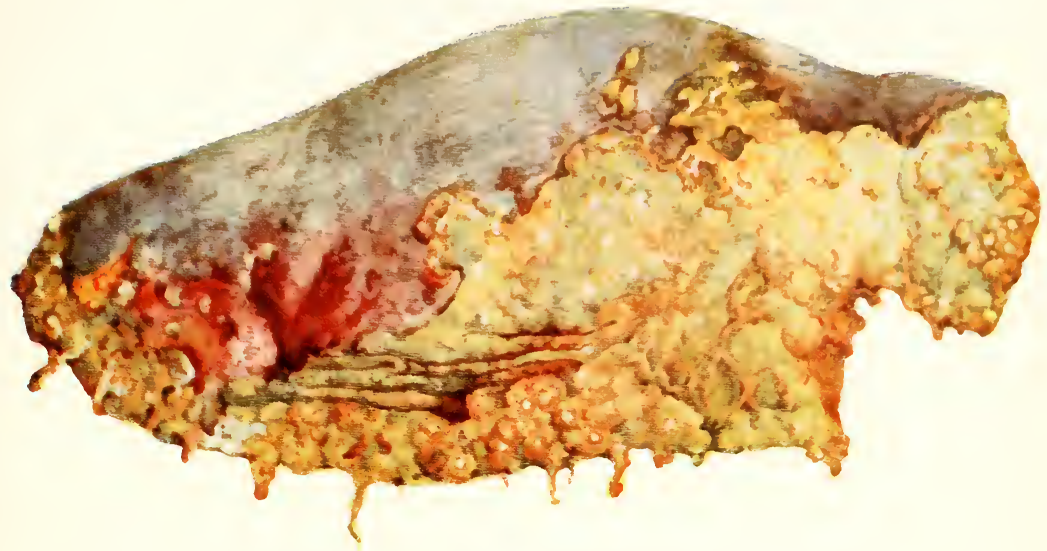

Fig. 1. Acute fibrinous pleurisy of horse. Yellow fibrinous exudate spread over tne reddened, somewhat clouded, pleura of the right lung.

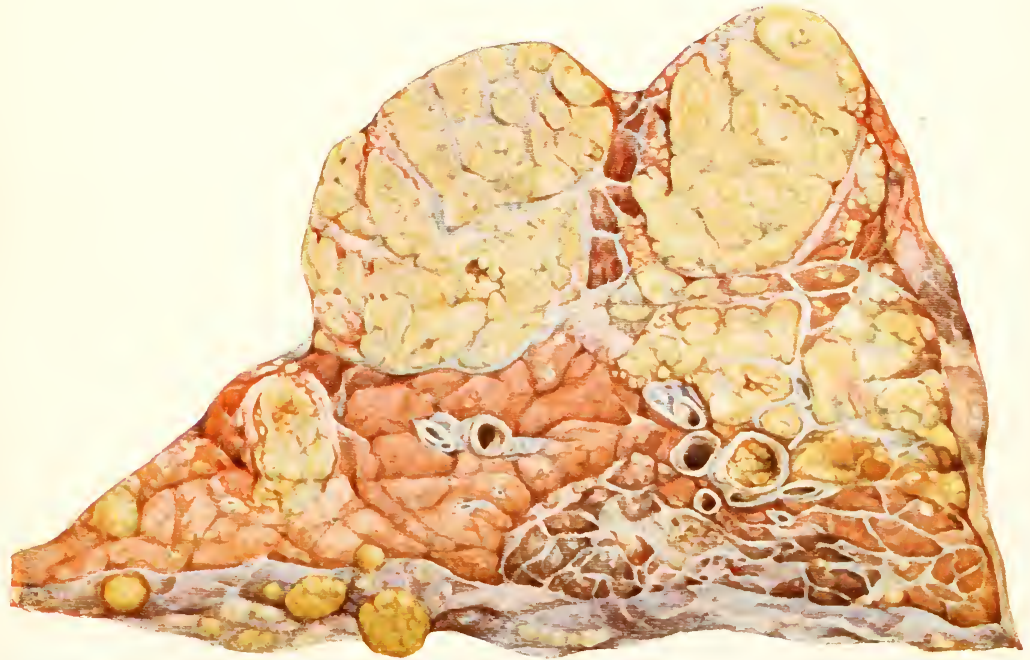

Fig. 2. Chronic chees tuberculous pneumonia. Section of lobe of cow's lung. 


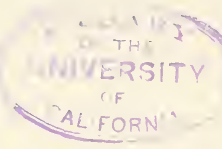


1.

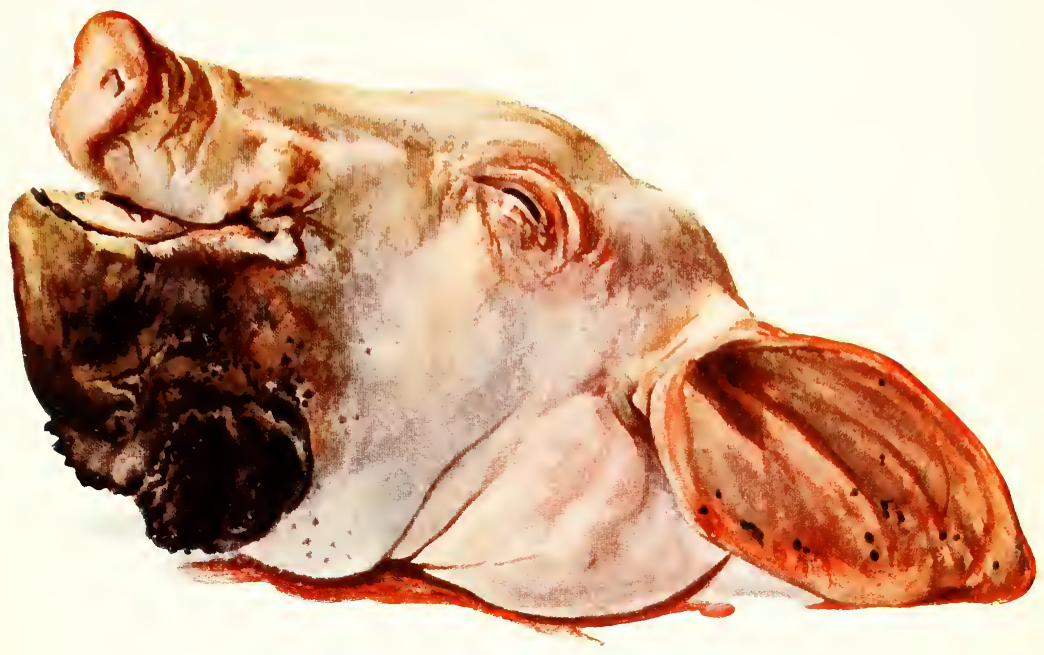

Fig. 1. Gangrene of skin of lower jaw of hog.

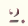

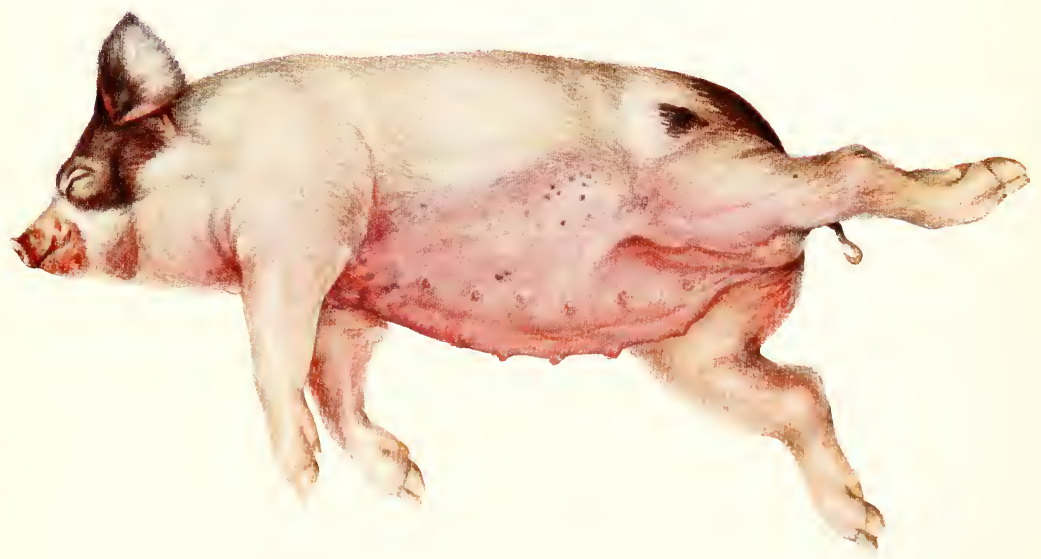

Fig. 2. Hyperæmia of skin in swine erysipelas. 



$$
\text { (1) }
$$





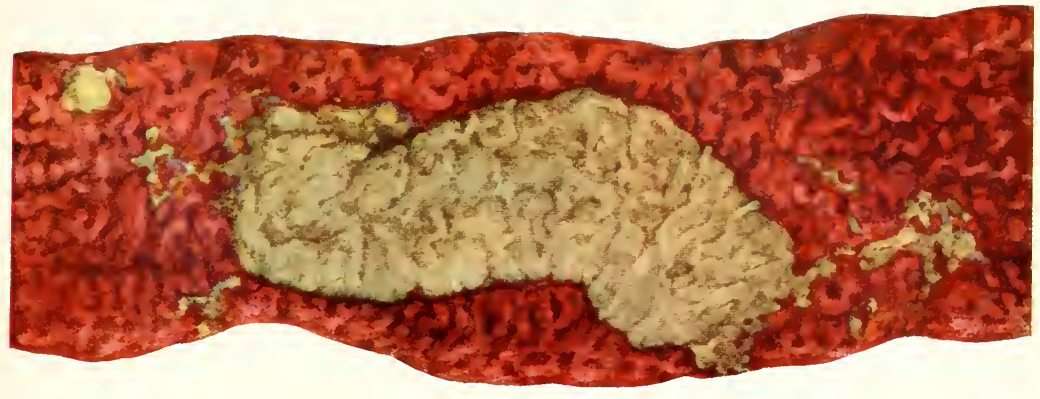

Fig. 1. Acute croupous enteritis: a portion of cow's intestine laid opey: the mucous membrane is the seat of hyperzmia and false membrane formation.

2.

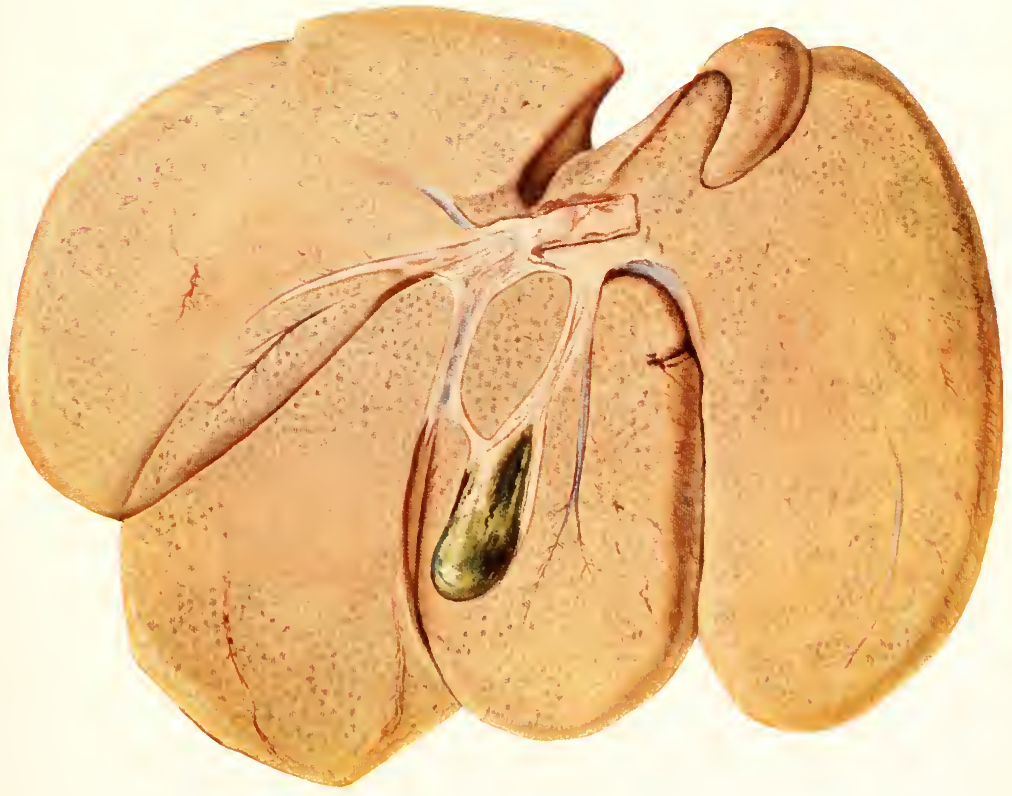

Fig. 2. Fatty liver of hog. 
$\Leftrightarrow$ 


\section{INDEX}

A

Actinomycosis ............... 3 IS

Fungous Growths of 320

Fungus of ....... 321

Act!nomycotic Abscesses...... 320

Nodules ....... 320

Active Hyperæmia ........... II 2

Immunization ......... 28

Acute Diseases ............. 95

Adenocarcinoma .........391, 407

Adenocystoma ............... fis

Adenofibroma Pericaniliculare . 390

Adenoma ................. 388 . Etiology of ........ 39 i

Varieties of ....... 389

Adenosarcoma .............. 39I

Agglutinins .............. 26

Air Embolism .............. It9

Hunger ................. 449

Akinesis ................ 429

Albuminous Degeneration ..... 190

Albuminuria ............... 455

Alexins ................. 19

Algor Mortis .............. 10.4

Alimentation, Disturbances of... 36

Amatrosis .............. 433

Amblyopia ................ 433

Amboceptor .............. 25

Amputation Neuroma .....250, 358

Amyloid Degeneration ........ 205 " " of Liver ...207 " " . ". Spleen .. 208

“ Reaction ........... 206

Anæmia ............... IIS, 157

Anæmic Infarct ............ I 45

Anæsthesia $\ldots \ldots \ldots \ldots \ldots \ldots \ldots 432$

Anamnesis .............. 93

Anasarca .............. I 30

Anatomical-pathological Processes .................... 3

Anatomical Types of. Inflammation and Fxudate......... 272

Angioneurosis ............ 435

Angioplasts .............. 2.40

Animal Parasites ............ 76

Poisons ........... si

Ante-mortem Clots ......... I32

Anthracosis ............... 215

Antibodies .............. 22
Antitoxine PAGE

Antria .................. 454

Apoplexy .............. 425

Apparent Death ............... I05

Argyria ........ ... .. 215

Aristotole ............. II

Ascites .............. . 30,447

Asphyxia ............... 38,451 as Cause of Death.... 39

Ataxia .................. 429

Athyrea ................ 459

Atony of Stomach............ 439

Atrophy ................ 185

Causes of .......... 186

Autoblastomata ............. 325

Autochthonous Melanosis ...... 209

Thrombi ...... 136

$\mathrm{B}$

Bacillıs Mallei ............ 3 I I

Bacteria ............... 54 Mode of Invasion of. . $6 \mathbf{r}$

Specific Action of.... 67

Bacteriæmia .............. 60

Basedow's Disease .......... +60

Bile, Abnormal Secretion of.... 46

Biliary Stasis ...........212, 446

Blood Corpuscles, Regeneration

of .....................25I

Blood, Imporerishment of ...... 157

" Pathological Changes of. I56

" Variations in ......... II I

" Vessels, Regeneration of. 240

Bone, Regeneration of........ 246

Botryomycosis .............. 324 Fungus of .... 324

Brain, Compression of ........ 425 ". Symptoms of 425 " Stroke .............. +25

Buffy Coat ............. I3I

Burns ................... 42

" Fatality of .......... 43

Cachectic Edema ........... I $2 \&$

Cachexia ................ I90

" of Cancer .......... 4If

Strumipriva ....... +59 
P.IGE

Cadaveric Clots ........... I32

Calcification ..................... 2 I6

Calculi .............216, 219

" Biliary ............ 223

Intestinal ........... 226

" Required Conditions of. 2 I9

" Salivary ........... 225

" Urinary ............. 220

Callus ................... 247

Cancer ................................

Cachexia of ........4 4 I

Cellular Morphology of. 402

Colloid ..............4 412

Cylindrical Cell ...... 406

Dissemination of ..... 400

Glandular Cell ....... 406

Gross Appearance of ... 410

Metastasis of ........ foI

Origin of ............. 396

Pearls ............. 405

Scirrhus .......... 413

Transplantation of .... 398

Varieties of ........ 403

Carcinoma ................. 394

Cardiac Hypertrophy ........ Io 6

Insufficiency .......... Ion

Cardinal Symptoms ......... 260

Cartilage, Regeneration of..... 245

Caseation ................. I79

Catalepsy ................. 428

Catarrhal Exudate ..............................

Catching Cold .............. 45

Causes of Disease......... 35

Cavernous Hamangioma ...362, 363

Cellular Pathology .......... I4

Cerebro-spinal Concussion .... 424

Cheesy Tuberculous Infiltration. 306

Chemical Agents ........... 50

Chemico-pathological Processes. 4

Chemotaxis ............ I8, I6I

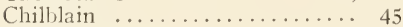

Cholemia ............... 447

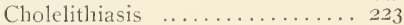

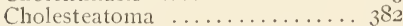

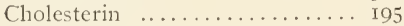

Chondroma ......................... 350

Chronic Diseases ........... 95

Chronic Inflammation ........ 284

Circulation, Disturbances of.... I06

Circulation, Schema of ......... I 40

Classification of Inflammatory

Processes ................ 288

Clinical Observation ..........

Clonic Spasms .............. 428

Clot, Absorption of ......... I25

Formation ........... I3I

Cloudy Swelling ............ I9o

Coagulation Necrosis......... I79

Coagulins ............... 26

Cohnheim's Theory ......... 336

Colic .................. 444

Collateral Circulation ......... I43

CEdema .......... 128

Colloid Degeneration ......... 204

Substances .......... 204

Coma .................. 425

Commotio Cerebri .......... 424

Comparative Pathology ....... I4

Compensation for Circulatory Abnormalities ........... 106

Compensatory Hypertrophy .... 255

Complements ......................... 25

Complications ......................... 99

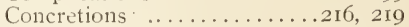

Concussion ..............50, 424

Congenital Diseases ......... 30

Comnective Tissue, Regeneration

of $\ldots \ldots \ldots \ldots \ldots \ldots \ldots \ldots \ldots \ldots \ldots \ldots \ldots$

Consciousness, Loss of. ...... 424

Consecutive Thrombi ......... I36

Constipation $\ldots \ldots \ldots \ldots \ldots \ldots \ldots 443$

Constitutional Predisposition ... 3 I

Contagious Diseases ........ 7 I

Continued Fever ............. 173

Convalescence .............. I73

Convulsions .............. 428

Corpora Amylacea .......... 208

Flava ...................... 208

Versicolorata ....... 208

Corrosive Poisons ........... 52

Cough ................. 452

Cramp .......................... 428

Croupous Exudate ......... 275 Inflammation ....... 275

Cruor Sanguinis .......... I3I

Cylindrical Cell Cancer........ 406

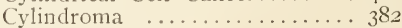

Cystic Tumors ............ 4 ${ }^{\mathrm{I}} 5$

Cystoma Penniferum .....................

Cytolysins .............. 25

D

Death $\ldots \ldots \ldots \ldots \ldots \ldots$ IOI

“ Agony ............ 103

" Causes of .......... 102

" Local .............. I

" Sigms of $\ldots \ldots \ldots \ldots \ldots \ldots$ IO 4

Decubitus ............... 462

Degenerative Processes-

Albuminous ............ 190

Amyloid ............... 205

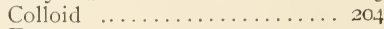

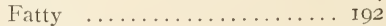

Hyaline ................ I99

Mucoid ................. 202

Delirium ................ 426

Demarcating Inflammation ... I84, 269

Depression of the Brain....... 425

Dermoid Cysts ...........4 416 
PAGE

Diabetes Insipidus . . . . . . . . . 454

$$
\text { Mellitus } \ldots \ldots \ldots+48,457
$$

Diagnosis ............... 9 I, 93

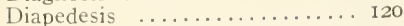

Diarrhøea ............... 442

Digestive Disturbances ...... 436

Dilatation of the Heart........ I09

Diphtheritic Inflammation ..... 276

Direct Symptoms ........... 92

Disease $\ldots \ldots \ldots \ldots \ldots \ldots \ldots$ I

Causes of $\ldots \ldots \ldots \ldots \ldots 35$

Conception of ........ 2

Course of ........9. 94

Periods of .......... 96

Recovery from ........ 100

Symptoms of ......91, 99

Termination of ....... 99

Diseases, Congenital and Inherited ................. 30

Diseases of First Stomach..... 439

$$
\text { Intestines ....... } 440
$$

"Jaw ...........446

" Liver .......... 444

“ Nouth ......... 436

“ Esophagus ...... 437

" Pancreas ........ 447

"Pharynx ........ 437

"Stomach ........ 437

"Teeth .......... 436

Dissemination of Germs in the Body ................... $6_{5}$

Disturbances of Alimentation and Nutrition............ 36

Disturbances of Circulation..... 106

Disturbances of Excretion of Urine ............... 454

Disturbances of Function of Skin 462

Disturbances of Metabolism .... I65

Disturbances of Respiration .... 448

Disturbances of Sexual Function ................ $46 \mathrm{I}$

Disturbances of Thyroid Function .................. 459

Dizziness ................ 424

Dropsical Transudate ........ I 29

Dropsy ................. I 26

Dwarfism ...................... 3I

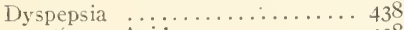

$$
\text { Acida } \ldots \ldots \ldots \ldots \ldots .438
$$

Dysperistalsis ............. 443

Dyspnœa ............... 419

Dysuria $\ldots \ldots \ldots \ldots \ldots \ldots \ldots \ldots \ldots 5^{8}$

\section{E}

Ecchondroma $\ldots \ldots \ldots \ldots \ldots \ldots 35$ I

Ecchymoses ............. 122

Ectogenous and Entogenous Infectious Agents ........70, 7 I

Ehrlich's Theory .......... 22

Electrical Intluences ......... 48
131, 138,149

Course of ......... I39

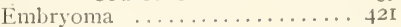

Emphysema ...........450, 453

Empyema .............. 28I

Enchondroma ...................... 35 I

Endarteries ................ 144

Endemic Disease .......... 72

Endothelioma .............. 380

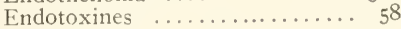

Enostoses .............. 354

Enteroliths ............. 227

Enuresis ................... 458

Eosinophilia .............. I63

Ephemeral Fever........... 173

Epidemic Disease ........... 73

Epiderm and Fpithelium, Regeneration of ............ 242 Epilepsy .............. 425,428

Epithelial Cysts ........... 4I5

Exhaustion .............. 40

Exitus Lethalis ............ IOI

Exogenous Pigmentation ...... 215

Exostoses ................... 354

Expectoration ............. 453

Experimental Pathology ....... 8

External Respiration......... 448

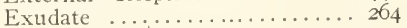

Eye in Death.............. I05

\section{F}

Fainting ......................... 424

Falling Sickness .......... 425

False Membrane ............ 274

Fat Embolism ............. I50

Fatty Changes ............ I92 .: Degeneration ........ I93, I98

.. Infiltration .......... I92

Faults of Heat Regulation...... I65

Fecal Concretions .......... 226

Fever .................. 165

" Production of ......... 168

" Stages of........... I I I

. Types of ............ 170

Fibrino-purulent Exudate ...... 278

Fibrinous Exudate .......... 273

Fibroblasts .............. 237

Fibroma .................. 34 I

Durum .............. 34

Growth of $\ldots \ldots \ldots \ldots .345$

Molle ............ 342

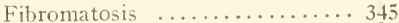

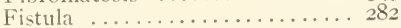

Flatulence ................ 439

Food, Deprivation of........ 36

Freezing ............. 44

Functional Abnormalities ..... 423

Overstimulation .... 40

Pathological Disturbances $\ldots \ldots \ldots \ldots$. 4 
G

PAGE

Galen I2

Gall Stones ............... 22,3

" " Composition of .... 223

Gangrene ................. I8I

Gangrenous Emphysema ....... 183

Gastric Atony .............. 439

Gelatinous Cancer .......... 4 42

General Nervous Disturbances.. 423 Pathology .......... 5

Germinal Variation .......... 32

Giant Cells ............... 239

" Cell Sarcoma .........................

Gigantism ................3I, 254

Glanders ................ 3I I

Abscesses ........... 315

Bacillus of ......... 3I I

Indurations ......... $3{ }^{15}$

Nodules ........... 3I

Ulcers ............3 314

Glands, Regeneration of ....... 244

Glandular Cell Cancers........ 406

Glioma ................... 360

Retinal ............ 36r

Glycogenic Infiltration ....... 205

Glycosuria $\ldots \ldots \ldots \ldots \ldots \ldots .456$

Gout ................... 229

Granulation Tissue ...24 $1,28_{3}, 285$

$\mathrm{H}$

Hremangioma $\ldots \ldots \ldots \ldots \ldots \ldots .362$

Hrmatemesis .............. 122

Hrmatocele ............. I22

Hæmatogenous Pigmentation ... 210

Hæmatoma ................ 122

Hæmaturia ............... 122

Hæmic Poisons ............. 53

Hxmochromatosis .......... 210

Hæmoglobinæmia .......... 160, 210

Hæmoglobinuria .......... I60, 2 I I

Hrmolysins .............. 25

Hæmopericardium ........... I22

Hremoptysis .............. 122

Hæmorrhage $\ldots \ldots \ldots \ldots \ldots \ldots$ II

Results of ...... I 24

Symptoms of..... I22

Varieties of ..... I2I

Hæmorrhagic Anæmia ......... 158

" Infarct ......... I45

Inflammation .... 283

Hæmosiderin .............. 2II

Hamothorax ............. 122

Haptophore $\ldots \ldots \ldots \ldots \ldots \ldots \ldots .23$

Health, Definition of ......... I

Heart, Condition of, in Disease. 106

Heat Regulation, Faults of..... I65

Stroke ............. 4I

Hemiplegia ............... 429

Hereditary Defects of Animals. 34

Man .... 33
PAGE

Heredity, Atavistic .......... 33

Collateral .......... 33

Direct ............. 33

Latent ........... 33

Heterotopic, Heterochronic and

Heterometric Processes ...... 3

Hippocrates .............. II

History of Pathology......... 10

Hodgkin's Disease ........... 373

Homogeneous Thrombi ....... I34

Hoof, Regeneration of........ 243

Host of a Parasite.......... 77

Humoral Pathology ........... II

Hyalin Degeneration ........ I99

Hydræmia ................. I6I

Hydrocele ................. I 30

Hydrocephalus .............. I30

Hydropericardium ........... I30

Hydrops ................. I26

Hydrothorax ................ I30

Hyperacidity $\ldots \ldots \ldots \ldots \ldots \ldots .438$

Hyperæmia $\ldots \ldots \ldots \ldots \ldots \ldots$ II2

Hyperæsthesia ............. 43I

Hypercholia ............. 213

Hyperchromatosis .......... 209

Hyperglycæmia $\ldots \ldots \ldots \ldots \ldots \ldots .45^{6}$

Hyperidrosis ............... 463

Hyperkinesis ............. 427

Hyperlencocytosis $\ldots \ldots \ldots \ldots \ldots$. I62

Hypernephroma ….....393, 397

Hyperthermia ............4I, 165

Hypertrophy Passive ......... 160

I

Icterus ............... 2 2, 446

Idiosyncrasy ............... 20

Ileus ..................... 444

Immunity $\ldots \ldots \ldots \ldots \ldots \ldots \ldots$ I6

Acquired $\ldots \ldots \ldots \ldots$ 2I

Active ........... 28

Natural .......... 24

Passive ......... 28

Relative ......... 20

Impotence ................ 46r

Incubation, Period of ......... 64

Indirect Symptoms .......... 92

Infarction $\ldots \ldots \ldots \ldots \ldots \ldots \ldots$ I 45

Infection $\ldots \ldots \ldots \ldots \ldots \ldots \ldots \ldots, \quad 54$

Embolic ........... 65

General ............. 66

Local ............. 65

Regional ......... 65

Infections Agencies .......... 54

Diseases and Their

Agents, Table of .. 73

Inflammation .............. 5 . 55

Anatomical Types of 272

Cardiac Valves .... 272 
PAGE

Inflammation, Cardinal Symptoms of ...........260

Causes of .......258

Chronic .........284

Corneal ......... 27I

Interstitial ....... 285

Nomenclature and Classification .... 288

Phenomena of ....260

Productive ........ 284

Specific .........286

Suppurative ...... 277

Termination of .... 289

Inflammatory Edena ....... 128, 273

Inherited Diseases .......... 30

Insanity ................ 426

Intermittent Disease ......... 95

Intestinal Calculi ............. 226

Intestines, Diseases of ........ $44^{\circ}$

Fermentation in .... H H

Intoxication $\ldots \ldots \ldots \ldots \ldots \ldots$ 50

Intranterine Disease, Origin of. . 31

Intussusception ............. 443

Irritability, Psychic............426

Ischænia ................ II 8

Itching $\ldots \ldots \ldots \ldots \ldots \ldots \ldots, 432$

\section{J}

Jaundice ................ 212

Sequels to.......... 2It

L

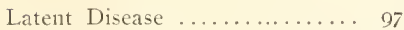

Leionyoma ............... 357

Letucocytosis ............... 162

Lencopenia ................ 162

Leukrmia ................. 163

"Licking" ................ 427

Lightning Stroke ........... 48

Lipoma ................... 346

Occurrence of ...... 348

Liquefaction Necrosis ........ I8 I

Liver, Diseases of .......... 444

". Functions of......... 445

Local Variations in Amount of Blood ................... III

Low Temperatures, Effects of. . 43

Lungs, Diseases of.......... 448

Lymphangioma .............. 364

Lymphatic Thrombosis ........ 154

Lymphatismus ............. 372

Lymphogenous Embolism ...... I54

Lymphoma ................ 372

\section{II}

Malignant Embolism .......... I5I

Mania ..................

PAGE

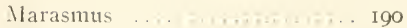

Mechanical Influences a........ 49

Melanin ............. ..... 209

Melanona .................... 377

Melanosarcoma $\ldots . \ldots \ldots \ldots \ldots \ldots . . .377$

Melanosis .................. 209

Metabolism in Fever......... I73

Metastasis ................ I5I

Meteorism ................ 439

Methanoglobin ............ 160

Metrorrhagia .............. I22

Miasmatic Disease ......... 7 I

Microbes ............... 54

Microphytes ............. I7

Microzoa $\ldots \ldots \ldots \ldots \ldots \ldots \ldots \ldots$ I7

Miliary Tubercle .........300, 303

Mineral Poisons.............................

Miscarriage $\ldots \ldots \ldots \ldots \ldots \ldots \ldots \ldots . \ldots \ldots 2$

Miserere ................. 444

Mixed Infection.............. 69

. Thrombi ............ 135

Motor Disturbances ......... 427

". " Automatic . 427

.

.. $\quad . . \quad$ Reflex ... 427

Mucoid Degeneration

II mmmification . . . . . . . . . . . 179

Muscle, Regeneration of....... 245

Myelogenous Sarcoma ....... 369

Myoma .................. 356

Iyositis Ossificans Progressiva. 355

Myxcedenta ............... 460

Myxona $\ldots \ldots \ldots \ldots \ldots \ldots \ldots \ldots . .349$

Myxosarcoma ............. 369

\section{N}

Necrosis ................. 176

" Causes of .......... 177

.. Symptoms of ....... 183

. Terminations of ..... 184

. with Coagulation...... 179

.. ". Desiccation .... 179

" " Putrefaction ... I8I

". Softening ...... I8 I

Neoplasms .............. 325

Nerves, Regeneration of....... 248

Nervous Disturbances .......... 423

$\begin{array}{ccc}\text { " Hypertrophy } \ldots \ldots \ldots & 257 \\ \text { Neuralgia } \ldots \ldots \ldots \ldots \ldots \ldots \ldots & 43 \mathrm{I}\end{array}$

Neurofibroma .............. 359

Neuroma .................. 358

“ Amputation ....250, 358

Neuromy xoma …........... 359

New Growths .............. 325

Nosology ................ 5

Nutrition, Disturbances of ..... 36

Nymphomania .............. +26 
$\mathrm{O}$

PAGE

Obesity ................ 197

Obstruction of Intestines....... 443

Blood Vessels... I3I

Occluding Thrombi .......... I36

Odontoblastoma ............. 4 I8

Odontoma .................. 418

“ Durum .......... 419

Mixtum ......... 419

Edema .................... 126

Results of .......... I30

Oligæmia ............... I57

Oligocythæmia ............. I57

Oliguria $\ldots \ldots \ldots \ldots \ldots \ldots \ldots, 454$

Oncology ................ 326

Organization of Thrombi...... I38

Osteoclasts ............... 248

Osteocystoma $\ldots \ldots \ldots \ldots \ldots \ldots, 420$

Osteoma .................. 353

Varieties of ........ 353

Osteo-sarcoma ............... 353

Oxygen Supply, Deprivation of.. 38

\section{$\mathrm{P}$}

Pain $43 \mathrm{I}$

Palsy ....................... 429

Results of ...........4 430

Pancreas, Diseases of .......... 447

Pandemic Disease ........... 73

Papillona ................. 383

Paradoxical Embolism .......... I42

Paræsthesia $\ldots \ldots \ldots \ldots \ldots \ldots \ldots . .432$

Paralysis .................... 429

Paralytic Calf Fever.......... 462

Paraplegia ................ 429

Parasites .................... 76

Table of $\ldots \ldots \ldots \ldots \ldots .82$

Parasitic Diseases ............... 76

Parenchymatous Inflammation.. 287 Poisons ..... 53

Passive Congestion .........115, 127 " Hyperæmia .......... II Hyperthermia .......... 165

Pathogenesis ...............

Pathogenic Microorganisms .... 55

Pathognomonic Symptoms..... 92

Pathognomy ............... 5

Pathological Changes of the

Blood ................... $1_{5} 6$

Pathological Processes, Classes

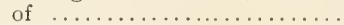

Pathology $\ldots \ldots \ldots \ldots \ldots \ldots \ldots \ldots \ldots \ldots \ldots \ldots$ I

" History of ........ I0

Periostoses ................. 354

Perithelioma ............... 382

Petechiæ .................. 122

Petrification ................ 216

Phagocytosis ….............. 18 , 161

Pharynx, Diseases of ......... 437

Phlegmon ................ 282

Pigmentation .............. 209

Pigmented Tumor ............ 377
Placental Origin of Disease.... PAGE 30

Plethora ................. 157

Poikilocytosis .............. I59

Poisons .................. 50

Classification of ...... 52

Polychromatophilia ........... I59

Polymorphocellular Sarcoma.... 369

Polyuria ................. 454

Post-mortem Clots .......... I32

Precipitins $\ldots \ldots \ldots \ldots \ldots \ldots \ldots .26$

Predisposition ............. I6

Pressure Atrophy ............ I87

Primary Lesion ............... g8

Thrombi ........... I 36

Processes of Repair and New

Formation ............... 232

Productive Inflammation ...... 284

Prognosis ................. 94

Progressive Inflanmatory Ostei-

tosis ................. 355

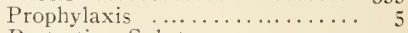

Protective Substances ......... 21

Proud Flesh .................286

Psammoma ................. 382

Psychical Disturbances ........ 424

Excitement ....... 426

Ptyalism ................ 436

Pus ..................... 277

. Concretions ............. 228

Putrefaction After Death...... I05

of Exudate ......283

Putrid Necrosis ............ I8I

\section{$\mathrm{R}$}

Ray Fungus .............. 32I

Receptor ............... 22

Recovery from Disease....... I00

Red Thrombi .............. 135

Regeneration .............. 232

Capacity for ...... 235

of Blood and Lympli $25 \mathrm{I}$

"Blood Vessels ... 240

"Bone and Cartilage ......... 246

" Connective 1 issue 237

"Corneal Epithelium $\ldots \ldots \ldots .243$

"Epiderm and Epithelium ...... 242

" Glands ....... 244

" Hair .......... 24.3

"Horny Tissue... 243

“ Muscle ....... 245

- Nerves ....... 218

"Tendon Tissue. 246

Remittent Disease ........... 95

Resistance to Disease........ I6

Respiration, Disturbances of.... 448

Respiratory Faults ........... 38

Retrograde Embolism ......... I42

Rhabdomyoma ............. 356 
PAGE

Riding Embolıs ............ I39

Rigor Mortis ............. I04

Round Cell Sitconna........ 367

\section{S}

Sago Spleen .............. 208

Salivary Calculi ............ 225

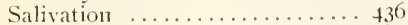

Sapremia ................. I85

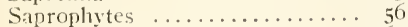

Sarcoma ........................ 365

Cells of .............. 365

Classes of .......... 366

Development and . Etiology of $\ldots \ldots \ldots \ldots 37 \mathrm{I}$

Gross Appearances of. 370

Scars Following Infarction.... I47

Scirrhus Cancers .......... 4 I 3

Secondary Lesions .......... 98 Thrombi .......... 136

Secretory Abnormalities........ 435

Sensation, Pathological Diminution of .............. 432

Sensory Disturbances ........ 43I

Sequestration ........... I78, I84

Sero-fibrinous Exudate ....... 275

Serous Inflammation ......... 273 Exudate ............ 27.3

Sexual Organs, Diseases of.... $46 \mathrm{I}$

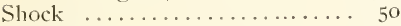

Side-chains ............... 22

Skin Diseases ............ 462

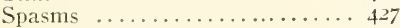

Results of ......... 429

Special Pathology .......... 5

Spindle Cell Sarcoma........ 368

Squamous Epithelioma ....... 404

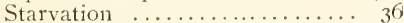

Stellate Cell Sarcoma......... 369

Sterility .............. 46 I

Stomach, Diseases of........ 4.37

Strangulation of Intestine...... 443

Stratificd Thrombi .......... I35

Struma Petrificans .......... 2 I0

Sudden Death ............ I0.3

Suftocation ............. 45i

Suffusion of Blood............ 122

Suppuration .............. 277

Symptomatology . . . . . . . . 9I

Symptoms of Death.......... I04

Syncope .................4 424

\section{$T$}

Tendon Tissuc, Regeneration of. 246

Teratoma ............415, 421

Terminations of Disease....... 99

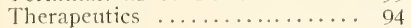

Therapy $\ldots \ldots \ldots \ldots \ldots \ldots \ldots \ldots . \ldots \ldots$

Thermic Influences ......... 4 I

Thermometry ............ I75

Thrombosis .............. I3I
Thrombosis, Catuses of . . ....... 132

Results of ....... I $4^{2}$

Thyroid Function, Disturbances

of .................... 459

Tissue Immunity .......... 63

Predisposition ......... 63

Tonic Spasms ............................ 428

Toxalbumens ............. 5I

Toxines ................ 5 I, 57

Toxophore .............. 23

Transplantation ............ 25I

Trammatic Influcuecs ........ 49

Trophic Disturbances ........ 4.34

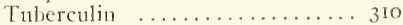

Tuberculosis ..............291

Anatomical Lesions of ........... 298 Bacillus of ...... 292 Heredity of ....... 295

Mode of Contagion. 294 Symptoms of ...... 310

Tuberculous Cavities ......... 307 Granıloma ................ 305

Ulcers ................ 305

Tumors ..................... 325

" Etiology of ......... 333

Cohnhein's

Theory . . 335

Classification of ...... 340

External Shape of..... 328

Growth of ......... 328

Pathological Significance

of $\ldots \ldots \ldots \ldots \ldots \ldots .329$

\section{U}

Ubiquitous Disease .......... 72

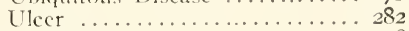

Jundernutrition $\ldots \ldots \ldots \ldots \ldots \ldots .38$

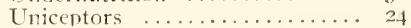

Uramia ................. 459

Urinary Calculi ............ 220

" Gravel ............ 220

" Organs, Diseases of... 454

Urolithiasis ............ 220

\section{V}

Valvular Thrombi .......... 136

Vasomotor Disturbances ...... 435

Vegetable Poisons ........... $5 \mathrm{I}$

Vertigo .................. 424

Vibices ................. 122

Virmlence of Toxines.......... 8 , 57

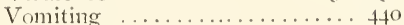

Vomitus .............. 440

IV

Wart ..................... $38_{5}$

Water, Withdrawal of ....... 37

White Thrombi ............. I,35

Work Iypertrophy .......... 254 



\section{W. T. KEENER \& COMPANY \\ Medical Publishers and Importers \\ 90 WABASH AVENUE. - - CHICAGO}

TEXT-BOCK F GTIFARATIVE GENERAI PATHOLOGI FOR THE USE OE PRACTITIONERS AND STIDENTS F TETERINARI IIEDICINE.

BI ERUE DR TH KITT. OF MUX - H

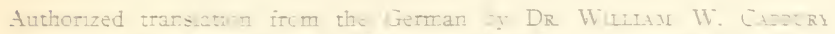

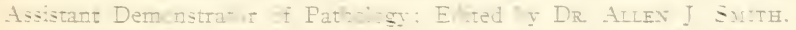

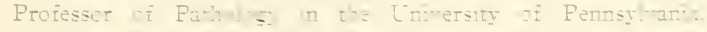

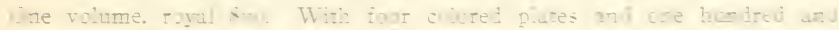

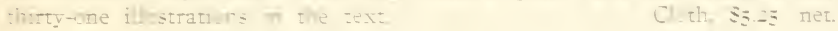

Kitt's work on ieneral Fathong is the wly ishal textbiek on Teterinary Fathoing published Heretifore students and veterinary surcems have had te consult muths yn human medicine in orter $\mathrm{t}$, ieam the foundations of nathol $\mathrm{s}$ : this volume, homever is nnten with special reference to the stident of animal medicine.

After an introduct ry review wi the zeneral conceptions of disease and of the listory if patholos. the ca rses of disease are discussed: then pathologic disturbances un ler four main headings. viz.: I Circulatory Disorders: 1 2, Metabolic Disorders. Desenerations and Necroses: 3 ' Reparatory Processes and Proliferation: 4 Functional Disonlers. All of the most common and characteristic processes are illustrated by a number it original drawings and colored plates.

The style of the book is terse and concise and all the definitions are clear cut: the work is thorcughly up $t$ ) date. as all the results of the recent investigations with the pathol-s and etiolses of disease are included in the text and are described in a very simple and popular manner. The practicins reterinarian will find in this book a modern work. convenient for ready reference, and containing everything that we know to-day regarding Veterinary Pathology.

W. T. Keever \& Co., Chicago. 


\section{SURGICAL DISEASES OF THE DOG AND CAT.}

With Chapters on Anæsthetics and Obstetrics.

(Second Edition of Canine and Feline Surgery.)

By FREDERICK T. G. HOBDAY, F. R. C. V. S., F. R. S. E.

Member of the Board of Examiners of the Royal College of Veterinary Surgeons; Late Professor in the Royal Veterinary College, London: Consulting Veterinary Surgeon to the British Bulldog and Griffon Bruxellois Clubs; Honorary Veterinary Surgeon to the Ladies' Kennel Association, the Fox-Terrier Clul, etc.

One volume, 366 pages, $5 \frac{3}{4} \times 8 \times 3 / 4$ inches. With $24 \mathrm{I}$ illetstrations.

Cloth, $\$ 3.25$ net.

"The reception accorded to the first edition has proved that a small text-book of the kind was distinctly wanted, and in presenting a second and enlarged edition the author regrets that illness and other causes have allowed the first to remain so long out of print.

By the request of numerous friends, the work has been enlarged to include symptoms and general details of treatment other than that which may be described as purely surgical, although the original idea of the author to keep the book as a handy manual for their final-year student has still been adhered to.

The introduction of antiseptics has revolutionized all of the old-fashioned ideas, and has benefited the animal world to almost as great an extent as that of man.

It is true that we have not quite the same advantages as our confreres in human surgical practice, but it is astonishing what can be done by strict attention to the principles introduced by the discoveries of Pasteur, and applied to surgery by the teachings of Lister.

Operations which only a few years ago were looked upon beforehand with dread are now done every day, and owners of pet animals have learned to appreciate the value of modern antiseptic methods almost as much as the members of the profession themselves."-Extract from Preface.

W. T. Keener \& Co., Chilcago. 
After-treatment consists merely in the application of a little antiseptic lotion (boric acid, chinosol, or permanganate of potash) several times a day.

Sarcoma or Carcinoma-Symptoms.-As a general rule the first signs will merely be a capricious appetite and difficulty in swallowing, the animal appearing to have a very sore throat. There is salivation, and, after the trouble has existed

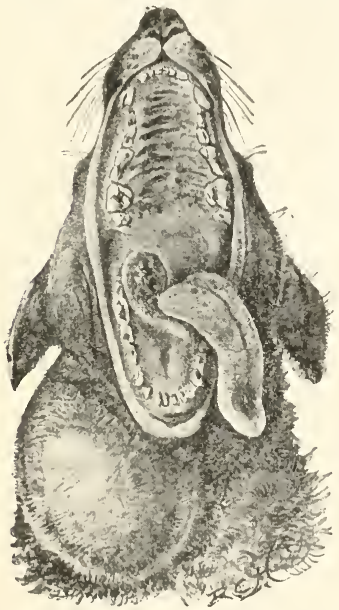

Fig. 102. - Carcinoma of the Pharynx and Cervical Glands of a Terrier.

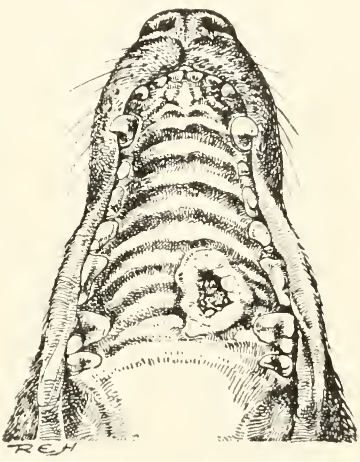

Fig. 103. - Endothelioma perforating the Palate of a Great Dane.

for a few weeks, a gradual emaciation of the body will be observed. It is not uncommon upon discovering a sore at the back of the mouth, such as is illustrated in Fig. I02, for a diagnosis to be made of an injury from a sharp bone or a piece of stick. This had been actually done in each of the cases from which Figs. I02 and 103 were sketched. All doubt on this question can be set aside if the wound has a ragged, ulcerated appearance, shows no tendency to heal after careful 


\title{
FRIEDBERGER \& FROHNER'S VETERINARY PATHOLOGY.
}

(Authorized Translation)

\author{
BY M. H. HAYES, F. R. C. V. S.
}

Author of "Points of the Horse," "Veterinary Notes for Horse Owners," etc. Edited by JOHN DUNSTAN, M. R. C. Y. S.

Professor of Surgery and Therapentics at the Royal (Dick) Veterinary College, Edinburgh.

There has long been wanting in Veterinary literature a comprehensive text-book dealing with the Diseases of Animals from their clinical, pathological and therapeutic standpoints and which might have some claim to the term "modern." This gap was efficiently filled in Germany by Frierberger and Frohner's splendid production, the value of which was soon correctly estimated by veterinary surgeons of all nations.

The late Captain Hayes, the most prolific English veterinary writer of modern times and editor of many valuable works on veterinary and equine matters, ever quick to appreciate merit, bought the right to translate the work into English. Two editions of the first volume were published during his life, and he was engaged on the translation of the second volume at the time of his death. Dr. Dunstan was requested by Mrs. Hayes to complete this volume. The work has also been translated into French and Russian and is the recognized text-book in American and European Veterinary Colleges.

Two volumes, 8 vo. I,285 pages. Cloth. Price per set, $\$ 8.00$ net.

\section{CONTENTS.}

VOLUME $I$.

Chapter I, Infective Diseases; II, Miscellaneous Infective Diseases; III, Chronic Constitutional Diseases; IV, Addenda to Infective Diseases; $\mathrm{V}$, Notes on Bacteriology; VI, The Terms Infection and Contagium. 
IOLUAE II.

Chapter I, Diseases of the Organs of I)igestion; Il, Diseases of the Esophagus; III, Discases of the Stomach; IV, Diseases of the Liver; 1, Diseases of the Peritonemin; VI, Diseases of the Spleen; VII, 1)iseases of the Urinary Organs: VIII, Diseases of the Sexual Organs; IX, Diseases of the Heart and Larger Blood Vessels: X, Skin Diseases; XI, Diseases of the Locomotory Organs: XII, Trichinosis in Swine; XIII, The Measles of Domestic Animals; XIV, Niescher's Tubes or Rainey's Corpuscles: XV. Diseases of the Nervous System: XVI, Diseases of the Spinal Cord and of Its Membranes: XVII, Diseases of the Peripheral Nerves; XVIII, Nenrosis without Known Anatomical Basis; XIX, Appendix to Diseases of the Nervous System: XX, Diseases of the Respiratory System; XXI, Diseases of the Accessory Cavities of the Nose; XXII, Diseases of the Larynx: XXIII. Diseases of the Trachea and Bronchi; XXIV. Diseases of the Lungs: XXV, Diseases of the Plenra: XXVI, Appendix to Diseases of the Respiratory Organs.

\section{OPINIONS OF FRIEDBERGER \& FROHNER.}

"It is this work that I recommend to my students, and believe that of all works on Veterinary Medicine, it is the best adapted to class room use." Leonard Pearson. Dean and Professor of Practice Veterinary Medicine, Department of Veterinary Medicine, Tniversity of Pennsylvania.

"Hope you will be amply repaid for its production-this, the most modern and best text-hook we have."-J. L. Robertson. Professor of Veterinary Medicine, New York Veterinary College

"I take pleasure in making a verr favorable report, after a careful review. on Veterinary. Pathology by Friedherger and Frohner, translated by M. H. Hayes, F. R. C. V. S. and eonsider it the hest work to-dar on theory and nractice of Veterinary Medicine for students use. and have recommended it to the students of the Chieago Teterinarr College."
(Signed)
A. H. B $+K E R$. V. S..

Dean of Faculty and Professor of Theory and Practice.

"I find that the print is excellent. thet the translator h.1s put the technical statements into free and easily understood English langmage, making the text easily comprehended by the average student. You will note by the catalogue malled you this day this work has been adopted as the text-book of this college."-S. Stewart. M. D., D. V. S. Professor of Veterinary Medicine. Kins'is City- Veterinary College.

"For many years Friedberger and Frohner's 'Lehrbueh der speciellen Pathologie und Therapie der Hausthiere' las been considered by competent judges to be the best treatise on diseases of animals and their treatment. It is not alone of inestimable value to the practitioner, but it must be regarded as an important addition to medical literature, to which the research scholar glady turns. The work has alreadv been translated into French and Russian, and is used as a text-book in those countries. An English translation should, therefore, be hailed with delight, as there is no really good English work covering the Practice of Veterinary Medicine."-Boston Medical and Surgical Journal.

In Ordering FRIEDBERGER and FROHNER to ensure latest edltion specify Hayes' Translation.

W. T. Keener \& Co., Cinicago. 
The only strictly uf-to-date Veterinary Physiology in the English language.

\title{
ESSENTIALS OF PHYSIOLOGY FOR VETERINARY STUDENTS.
}

\author{
By D. NOEL PATON, M. D., B. Sc, F. R. C. P., ED.
}

Superintendent of Research Laboratory of the Royal College of Physicians of Edinburgh; Lecturer on Physiology, School of Nedicine of the Royal College, Edinburgh: Examiner in Physiology in the University of Glasgow and for the Royal College of Plysicians, Edinburgh, and late Examiner in the University of Edinlurgh; Examiner in Physiology for the Royal College of Veterinary Surgeons; Late Professor of Physiology, Royal Dick V'eterinary College, Edinburgh.

One volume, 428 pages, $53 / 4 \times 83 / 4$ inches. Profusely illustrated.

Cloth, \$3.00 net.

Between the Physiology of man and that of domestic animals there is no fundamental difference and most of our knowledge of human physiology has been acquired from experiments upon the lower animals. But while the tissues of a man, a dog and a horse act much in the same manner, the mode of nutrition of these tissues is somewhat different and requires special attention in the case of each.

In this volume the attempt is made to give the Esscntials of General Physiology and of the special physiology of the domestic animals in a form suitable to the requirements of students and practitioners of veterinary medicine.

\section{OPINION.}

"The examination which we have been able to give this work has left a very favorable impression, as it appears that the essentials have been included, with nothing onitted except the padding. which in some text-books of physiology require a vast amount of reading to extract them, resulting in much confusion to students and those who do not have the time nor inclination to make the search for facts.

"We recommend the work heartily in the sense that it reduces the study of physiology to a 'reasonable' basis, and the author has done for the reader what is a difficult task upon his own account. Well printed, well bound, intelligently illustrated, Messrs. Keener have placed a 'reasonable' physiology before the profession at a reasonable price."-Am. T'ct. Rerieci. 


\section{THE FOOD AND DIGESTION}

up and prepared for digestion. In the horse the lips are long and prehensile, and are essential for the taking of food. Into the mouth three pairs of compound glands-the Salivary Glands-open. The parotid, lined entirely by enzyme-secreting epithelium, opens on the side of the cheek, while the submaxillary gland, composed partly of acini with enzyme-secreting, and partly of acini with mucinsecreting epithelium, and the sub-lingual, composed entirely of mucin-secreting acini, open under the tongue (S.C.).

The tongue in the horse is smooth, but in the ox, and especially in the cat, it is covered with a fine fur of processes, the filiform papillæ, which are of use in passing the food backwards along its surface in the act of swallowing. (For Organs of Taste see p. 132.)

Posteriorly, the mouth opens into the pharynx $(P h$.) or upper part of the gullet. In the horse the soft palate is very long, reaching to the base of the epiglottis, and, unless during swallowing, shutting off the mouth from the pharynx (Fig. 137). On each side,

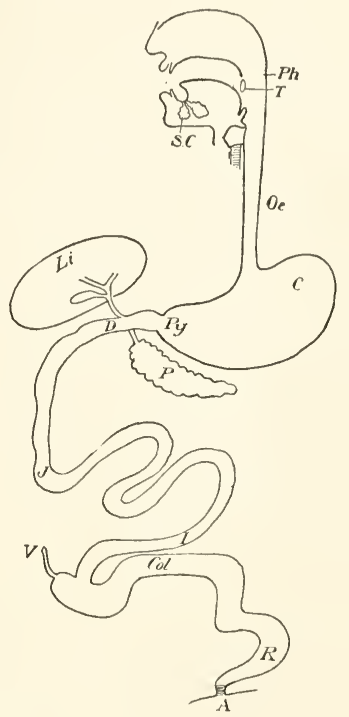

FIG. 138.-Diagram of the Parts of the Alimentary Canal, from Mouth to Anus. T., Tonsils; Ph., Pharynx; S.C., Salivary Glands; $O c .$, Esophagus ; C., Cardiac ; Py., Pyloric Portion of Stomach; D., Duodenum; $L i$., Liver; $P$., Pancreas; J., Jejunum; $I$., Ileum; $V$., Vermiform Appendix; Col., Colon; R., Rectum. between the mouth and the pharynx, is the tonsil (T.), an almond-like mass of lymphoid tissue. The pharynx is a cavity which can be shut off above from the posterior nares by raising the soft palate, and by pulling forward the posterior pharyngeal wall. It is surrounded by three constrictor muscles, which, by contracting 


\section{ANNOUNCEMENT}

\section{PATHOLOGY OF \\ METABOLISM}

Edited by

CARL VON NOORDEN

FIRST MEDICAL CLINIC, VIENNA. LATE OF FRANKFORT.

\section{Contributions by}

ADALBERT CZERNY (Breslau), CARL DAPPER (Kissingen', FR. KRAUS (Berlin), OTTO LOWEI (Vienna), ADOLF MAGNUS-LEVY (Berlin), M. MATTHES Cologne), LEO MORH (Berlin), CARL NEUBERG (Berlin), HUGO SALOMON (Frankfort', AD. SCHMIDT (Dresden), FRANZ STEINITZ (Breslau), H. STRAUSS (Berlin), W. WEINTRAUD (Wiesbaden).

\section{SECOND EDITION}

Authorized English Translation. Edited with Additions.

By

I. WALKER HALL, M. D.

Professor of Pathology, University College, Bristol.

THREE VOLUMES, 8vo. - $\quad$ - $\quad$ CLOTH, $\$ 16.00$

Payable, $\$ 4.00$ on receipt of Volume One; $\$ 6.00$ on receipt of Volume Two and $\$ 6.00$ on receipt of Volume Three

W. T. KEENER \& CO., CHICAGO

1906 


\section{PREFACE TO THE_ENGLISH EDITION.}

An English Edition of von Noorden's well-known "Lehrbuch Stoffirechsel" does not require any specific introduction.

Altrough the arrangement of the sections and their contents is the same as that of the first edition, the present text is mainly due to the co-operation of a number of workers in this branch of Medical Science. In his preface to the first German Edition, von Noorden acknowledged the encouragement and assistance of Gerhardt, von Jurgensen and Riegel; in his introduction to the second edition, he expresses his grateful thanks to the collaborators, "whose unflagging and zealous work alone has made it possible to bring out, instead of a second edition of my text-book, a hand-book of the pathology of metabolism" worthy of the extent and importance of the subject.

The length and style of the German original has restricted the addition of the more recent work in detail. The additions made to both the text and bibliography have conformed to the general principle of the work in presenting a compilation of facts and a critical discussion of hypothesis rather than the enunciation of theories; they have also been selected in harmony with the view of von Noorden and others, that experiments upon animals should be cited only in connection with acute conditions; as it is so difficult to compare them with the conditions which obtain in the chronic diseases of human beings, these addenda have been incorporated in the text.

It is a pleasure to express my thanks to my colleagues for their translations, to iny friend Professor T. H. Milroy, for the many valuable suggestions and to the University College Demonstrator in Pathology, Dr. Carey Coombs, for his careful preparation of the indices.

\section{CONTENTS OF THE VOLUMES.}

Volume I.

The Physiology of Metabolism.

By ADOLPH MAGNUS LEVY.

Voluan II.

Subject. Author. Translator.

Hunger and Chronic Inanition... v. Noorden ........ A. Jex Blake, M. D. Over Feeding .............. Noorden ........ R. W. Marsden, M. D. 
Fever and Infection.

F. Kraus J. O. Wakelin Barrett, D. Sc. M. D.

Stomach and Intestinal Diseases ................ Ad. Schmidt .....H. French, M. D., F. R. C. P.

Liver Diseases ............W. Weintrand ....R. W. Webster, M. D. Respiratory and Circulatory

Diseases .................. Matthes ........ Jex Blake, M. D. Blood Diseases .............. H. Strauss ....... J. A. Milroy, M. A., II. D.

Renal „Diseases ............ v. Noorden ....... C. Edgewortì, M. D.

Volume III.

Subject.

Author.

Translator.

Diabetes . Carl von Noorden...H. French, M. D., F. R. C. F.

Obesity ........................ Sarl von Noorden.. D. Spence, Ph. D.

Gout $\ldots \ldots \ldots \ldots \ldots \ldots \ldots \ldots$ Carl von Noorden...R. IV. IVebster, M. D.

Diabetes Insipidns and Addison's Disease ............ Leo Mohr ........ T. J. Horder, M. D. Thyroid Gland ............... Magnus Lery ... A. Jex Blake, M. D. Carbohydraturia ............. Carl Neuberg ..... J. Dixon Mann, M. D. F. R. C. P.

Cystinuria.

Alcaptonuria, Oxaluria, Phos-

phaturia .................Carl Neuberg .....W. H. Hurtley, D. Sc.

Nervous System

Muscles .................. Leo Vohr ......... Edwards

Bones

Cancerous Affections ......... Adolf Schmidt .... IV. Cramer, Ph. D.

Skin Diseases ............... Salomen and

Childhood von Noorden ....J. A. Nixon, M. B. Czerny and

Steinitz ........J. Fortescue Brickdale, Mi. D.

Influence of Drugs and Poisons. Otto Lowei ........J. Fortescue Brickdale, M. D.

Bath and Climate............ Natthes .......... A. Nixon, M. B. Phototherapentics ............. Salomen ........ Edwards.

Volume I will be published in the late autumn of 1906 . Volumes II and III in 1907. 




\section{DAY USE \\ RETURN TO DESK FROM WHICH BORROWED BIOLOGY LIBRARY}

TEL. NO. 642-2532

This book is due on the last date stamped below, or on the date to which renewed.

Renewed books are subject to immediate recall.

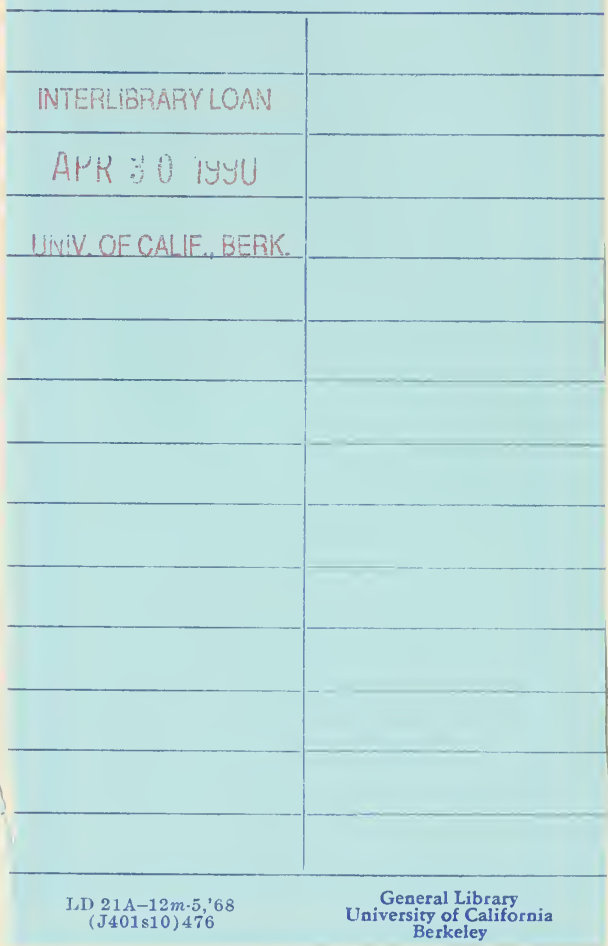


$\varepsilon \cdot \partial \varepsilon$ r.w 
